\title{
Hydrologic Data for Long Valley Caldera, Mono County, California, 1994-96
}

\author{
By James F. Howle and Christopher D. Farrar
}

U.S. GEOLOGICAL SURVEY

Open-File Report 00-230

N
ํ.
N 


\title{
U.S. DEPARTMENT OF THE INTERIOR \\ GALE A. NORTON, Secretary
}

\author{
U.S. GEOLOGICAL SURVEY \\ Charles G. Groat, Director
}

The use of firm, trade, and brand names in this report is for identification purposes only and does not constitute endorsement by the U.S. Geological Survey

For additional information write to:

District Chief

U.S. Geological Survey

Placer Hall

$6000 \mathrm{~J}$ Street

Sacramento, CA 95819-6129
Copies of this report can be purchased from:

U.S. Geological Survey

Information Services

Box 25286

Federal Center

Denver, CO 80225 


\section{CONTENTS}

Abstract
Introduction
Description of Study Area
Purpose and Scope
Report Format
Acknowledgments
Methods of Data Collection
$\quad$ Fround-Water Levels and Temperature Profiles
$\quad$ Water and Gas Temperatures
$\quad$ Water Equivalence of Snowpack
Water Samples for Chemical and Isotopic Analyses

\section{FIGURES}

1-3. Maps showing:

1. Location of Long Valley Caldera study area, Mono County, California ........................................................ 2

2. Key geographic features of Long Valley Caldera........................................................................................ 3

3. Locations of wells monitored by U.S. Geological Survey, 1994-96, Long Valley Caldera ............................ 13

4-12. Graphs showing:

4. Instantaneous water levels, well MWD M-14, 1988-96 ........................................................................ 14

5. Instantaneous water levels, well SC-2, 1983-96 ............................................................................... 14

6. Daily mean water levels, Lookout Mountain well (LKT), 1987-96 and 1994-96 _...................................... 15

7. Daily mean and instantaneous water levels, Lookout Mountain well (LKT), 1994, 1995, and 1996 ............. 16

8. Instantaneous water levels, well CW-3, 1988-91, and daily mean water levels, 1992-96 and 1994-96 ....... 22

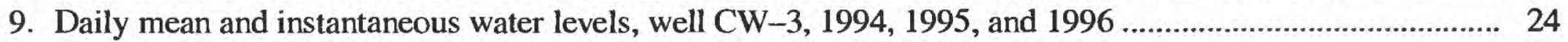

10. Daily mean and instantaneous water levels, well ESN, 1996 ..................................................................... 30

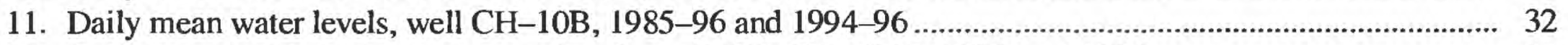

12. Daily mean and instantaneous water levels, well $\mathrm{CH}-10 \mathrm{~B}, 1994,1995$, and 1996..................................... 34

13. Map and histogram showing ground-water level changes in 36 selected wells, July 1994-96 ............................ 40

14-19. Graphs showing:

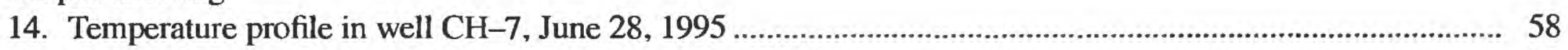

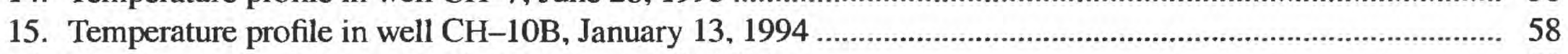

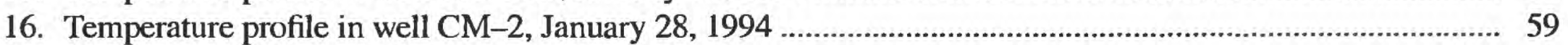

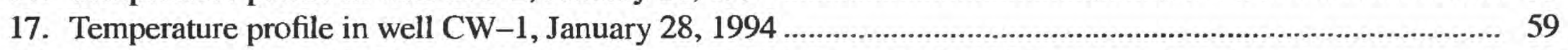

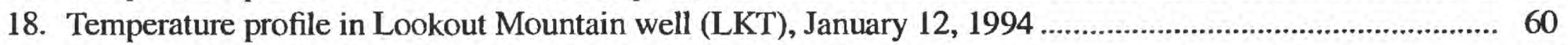

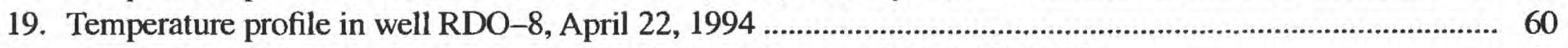

20. Map showing location of springs monitored by U.S. Geological Survey, 1994-96 ........................................... 61

21. Graph showing daily mean discharge, Little Hot Creek Spring (LHC-SP), 1994-95 ......................................... 62

22. Map showing location of data-collection sites at Hot Creek Fish Hatchery ......................................................... 65

23-38. Graphs showing:

23. Daily mean discharge, Fish Hatchery Spring AB (FHAB-WEIR), 1990-96 ............................................. 65

24. Daily mean discharge, Fish Hatchery Spring AB (FHAB-WEIR), 1994, 1995, and 1996 ......................... 66

25. Daily mean water temperature, Fish Hatchery Spring AB (FHAB-WEIR), 1992-96 …............................. 72

26. Daily mean water temperature, Fish Hatchery Spring AB (FHAB-WEIR), 1994, 1995, and $1996 \ldots \ldots \ldots \ldots \ldots . . .72$

27. Daily mean discharge, Fish Hatchery Spring CD (FHCD-Q), 1995-96 ……............................................. 78

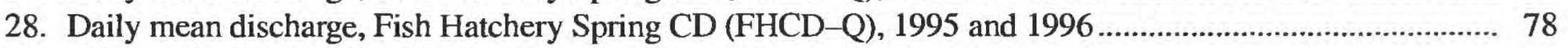


29. Daily mean water temperature, Fish Hatchery Spring CD (FHCD-T), 1988-96..

30. Daily mean water temperature, Fish Hatchery Spring CD (FHCD-T), 1994, 1995, and 1996.

31. Daily mean discharge, Fish Hatchery Spring 23 (FH23-Q), 1987-96.

32. Daily mean discharge, Fish Hatchery Spring 23 (FH23-Q), 1994, 1995, and 1996.

33. Daily mean water temperature, Fish Hatchery Spring 23 (FH23-T), 1988-96.

34. Daily mean water temperature, Fish Hatchery Spring 23 (FH23-T), 1994, 1995, and 1996

35. Daily mean discharge, Fish Hatchery Springs AB, CD, and 23 (FHAB-WEIR, FHCD-Q, and FH23-Q), 1995-96

36. Monthly mean water temperature, Fish Hatchery Springs AB, CD, and 23 (FHAB-WEIR, FHCD-T, and $\mathrm{FH} 23-\mathrm{T}$ ), 1988-96

37. Daily mean and instantaneous measured gage heights, Hot Bubbling Pool (HBP), 1989-96 ....................... 101

38. Daily mean and instantaneous measured gage heights, Hot Bubbling Pool (HBP), 1994, 1995, and $1996 \ldots 102$

39. Map showing location of streamflow sites monitored by U.S. Geological Survey, 1994-96................................ 108

40-43. Graphs showing:

40. Daily mean discharge, Hot Creek Flume (HCF), 1987-96.

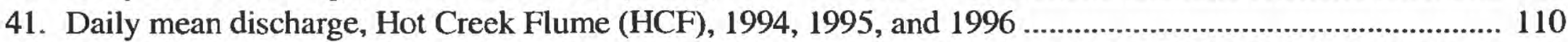

42. Daily mean discharge, Little Hot Creek downstream from Little Hot Creek Spring (LHC-SW), 1990-95 ... 116

43. Daily mean discharge, Little Hot Creek downstream from Little Hot Creek Spring (LHC-SW), 1994, 1995, and 1996

44. Map showing location of Mammoth Mountain Fumarole (MMF) ................................................................... 123

45-46. Graphs showing:

45. Daily mean vent gas temperature, Mammoth Mountain Fumarole (MMF), 1991-95 ................................... 124

46. Daily mean vent gas temperature, Mammoth Mountain Fumarole (MMF), 1994 and 1995-96 .................... 124

47. Map showing location of precipitation gages and snowpillow sites .................................................................... 129

48-51. Graphs showing:

48. Annual precipitation at Lookout Mountain (LKT-P), Mammoth Ranger Station (MRS), and Old Mammoth Meadow (OMM), July 1, 1994, to June 30, 1996.

49. Daily mean water equivalence of snowpack, Horseshoe Lake snowpillow site (HSL), 1995-96 and 1996-97

50. Daily mean water equivalence of snowpack, Lookout Mountain (LKT-P), 1994-95 and 1995-96

51. April 1 water equivalence of snowpack, Mammoth Pass Snow Course 205 (MP), 1981-96, near Mammoth Lakes

52. Map showing location of water-quality sampling sites

\section{TABLES}

1. Descriptions of data-collection sites, Long Valley Caldera, Mono County, California

2-4. Daily mean water levels, Lookout Mountain well (LKT):

2. 1994

3. 1995

4. 1996

5-7. Daily mean water levels, well CW-3:

5. 1994

6. 1995

7. 1996

8. Daily mean water levels, well ESN, 1996

9-11. Daily mean water levels, well $\mathrm{CH}-10 \mathrm{~B}$ :

9. 1994

10. 1995

11. 1996

12. Instantaneous ground-water levels in selected wells, 1994-96

3-14. Daily mean discharge, Little Hot Creek Spring (LHC-SP):

13. 1994

14. 1995 
15-17. Daily mean discharge, Fish Hatchery Spring AB (FHAB-WEIR):

15. 1994

16. 1995

17. 1996

18-20. Daily mean water temperature, Fish Hatchery Spring AB (FHAB-WEIR):

18. 1994

19. 1995

20. 1996

Daily mean discharge, Fish Hatchery Spring CD (FHCD-Q):

21. 1995

22. 1996

21-22. Daily mean water temperature, Fish Hatchery Spring CD (FHCD-T):

23. 1994

24. 1995

25. 1996

26-28. Daily mean discharge, Fish Hatchery Spring 23 (FH23-Q):

26. 1994

27. 1995

28. 1996

29-31. Daily mean water temperature, Fish Hatchery Spring 23 (FH23-T):

29. 1994

30. 1995

31. 1996

32-34. Daily mean gage height, Hot Bubbling Pool (HBP):

32. 1994

33. 1995

34. 1996

35-37. Daily mean discharge, Hot Creek Flume (HCF):

35. 1994

36. 1995

37. 1996

38-40. Daily mean discharge, Little Hot Creek downstream from Little Hot Creek Spring (LHC-SW):

38. 1994

39. 1995

40. 1996

41. Instantaneous discharge measurements, Mammoth Creek at Highway 395 (MC395), 1994-96.

42. Instantaneous discharge measurements, Mammoth Creek upstream of Hot Creek Fish Hatchery (MCAF), 1994-96

43. Instantaneous discharge measurements and gage heights, Hot Creek upstream of Gorge Geyser (HCA), 1994-96

44-46. Daily mean vent gas temperature, Mammoth Mountain Fumarole (MMF):

44. 1994

45. 1995

46. 1996

47-49. Daily precipitation, Lookout Mountain gage (LKT-P):

47. 1994

48. 1995

49. 1996

50. Monthly precipitation, Mammoth Ranger Station gage (MRS), 1994-96 ..................................................... 134

51-53. Daily precipitation, Old Mammoth Meadow gage (OMM):

51. 1994

52. 1995

53. 1996 
54-55. Daily mean water equivalence of snowpack, Horseshoe Lake snowpillow (HSL):

54. December 1995 to May 1996

55. November 1996 to May 1997

56-57. Daily mean water equivalence of snowpack, Lookout Mountain snowpillow (LKT-P):

56. November 1994 to May 1995

57. December 1995 to April 1996

58. Chemical and isotopic analyses of water samples collected at selected well, spring, and stream sites, 1994-96. 144

59. Partial chemical analyses of water samples collected at selected spring and stream sites, 1994-96 150

CONVERSION FACTORS, ABBREVIATIONS, AND VERTICAL DATUM

\section{Conversion Factors}

\begin{tabular}{rcl}
\hline Multiply & By & To obtain \\
\hline inch (in.) & 25.4 & millimeter \\
foot $(\mathrm{ft})$ & .3048 & meter \\
mile $(\mathrm{mi})$ & 1.609 & kilometer \\
square foot $\left(\mathrm{ft}^{2}\right)$ & .09290 & square meter \\
cubic foot per second $\left(\mathrm{ft}^{3} / \mathrm{s}\right)$ & .02832 & cubic meter per second \\
\hline
\end{tabular}

Temperature in degrees Celsius $\left({ }^{\circ} \mathrm{C}\right)$ may be converted to degrees Fahrenheit $\left({ }^{\circ} \mathrm{F}\right)$ as follows:

$$
{ }^{\circ} \mathrm{F}=\left(1.8 \times{ }^{\circ} \mathrm{C}\right)+32
$$

\section{Abbreviations}

Specific conductance is given in microsiemens per centimeter at 25 degrees Celsius $\left(\mu \mathrm{S} / \mathrm{cm}\right.$ at $\left.25^{\circ} \mathrm{C}\right)$.

Concentrations of chemical constituents in water are given either in milligrams per liter $(\mathrm{mg} / \mathrm{L})$ or micrograms per liter $(\mu \mathrm{g} / \mathrm{L})$.

$\mu \mathbf{m}=$ micrometer

$\mathbf{m} \mathbf{L}=$ milliliter

Vertical Datum

Sea level: In this report, "sea level" refers to the National Geodetic Vertical Datum of 1929 (NGVD of 1929)-a geodetic datum derived from a general adjustment of the first-order level nets of both the United States and Canada, formerly called Sea Level Datum of 1929.

Altitude, as used in this report, refers to distance above or below sea level.

Horizontal datum: In this report, latitudes and longitudes are referenced to North American Datum of 1927. 


\title{
Hydrologic Data for Long Valley Caldera, Mono County, California, 1994-96
}

\author{
By James F. Howle and Christopher D. Farrar
}

\section{ABSTRACT}

Hydrologic data were collected during 1994-96 as part of the U.S. Geological Survey's long-term Volcanic Hazards Monitoring Program of the Long Valley Caldera, Mono County, California, and the Long Valley Hydrologic Advisory Committee monitoring program. Hydrologic data collected include continuous record of ground-water levels in 4 wells; instantaneous measurements of ground-water levels in 53 wells; continuous record of discharge at 2 surface-water sites and 4 springs; continuous record of stage at 1 thermal pool; instantaneous discharge measurements at 3 surface-water sites; ground-water temperature profiles of 5 thermal wells and 1 nonthermal well; continuous record of water temperature in 3 springs; vent gas temperature at 1 fumarole; and chemical and isotopic analyses of water samples collected at 5 wells, 7 springs, and 5 surface-water sites. Precipitation amounts at 3 sites, and water equivalence of snowpack at 3 sites also are included. The data are presented in graphs or tables.

\section{INTRODUCTION}

Long Valley Caldera (fig. 1) is a site of geologically recent volcanism (Bailey, 1989). The high rates of seismicity and crustal deformation recorded in Long Valley Caldera since 1980 probably are related to magmatic intrusions (Rundle and Hill, 1988). The volcanic history and recent crustal unrest have been the motivation for the U.S. Geological Survey and other agencies to monitor a number of geologic and geophysical parameters to assess the potential of volcanic eruption in the near term (Hill and others, 1991).

The volcanic activity has provided heat for a hydrothermal system that underlies the western, southern, and eastern parts of Long Valley Caldera. Ground-water temperatures greater than $200^{\circ} \mathrm{C}$ have been measured in the western part of the caldera. Flow in the hydrothermal system is from west to east along the hydraulic gradient (Sorey and others, 1991). Thermal springs located in the southern and eastern parts of the caldera are natural discharge points for the hydrothermal system.

The thermal springs provide unique environments for wildlife and plants and are used for recreational bathing by thousands of tourists each year. The largest direct use of geothermal resources in the study area is at the Hot Creek Fish Hatchery (fig. 2), operated by the State of California since 1932. The hatchery uses between 15 and $25 \mathrm{ft}^{3} / \mathrm{s}$ of water from low-temperature $\left(15-20^{\circ} \mathrm{C}\right)$ thermal springs for raising trout. Use of geothermal resources for electric power generation began in 1985. By 1992, power production totaled about 40 megawatts from three plants located at Casa Diablo (fig. 2), and this production level has continued to date (2000).

A long-term hydrologic monitoring program was started in 1982 as part of the U.S. Geological Survey's Volcanic Hazards Monitoring Program. The purpose of the hydrologic monitoring is to document changes in the hydrologic system that are related to volcanic processes and seismicity. Hydrologic data collected for this purpose include ground-water levels; discharge rates of streams and springs; ground-water temperature profiles; water temperatures of springs; vent gas temperatures of fumaroles; precipitation amounts; and chemical and isotopic analyses of ground water and surface water. The number of data-collection sites and frequency of data collection have varied over the years in response to changes in hydrologic conditions, the level of geologic unrest, fulfilling the needs for baseline records, and funding levels. 


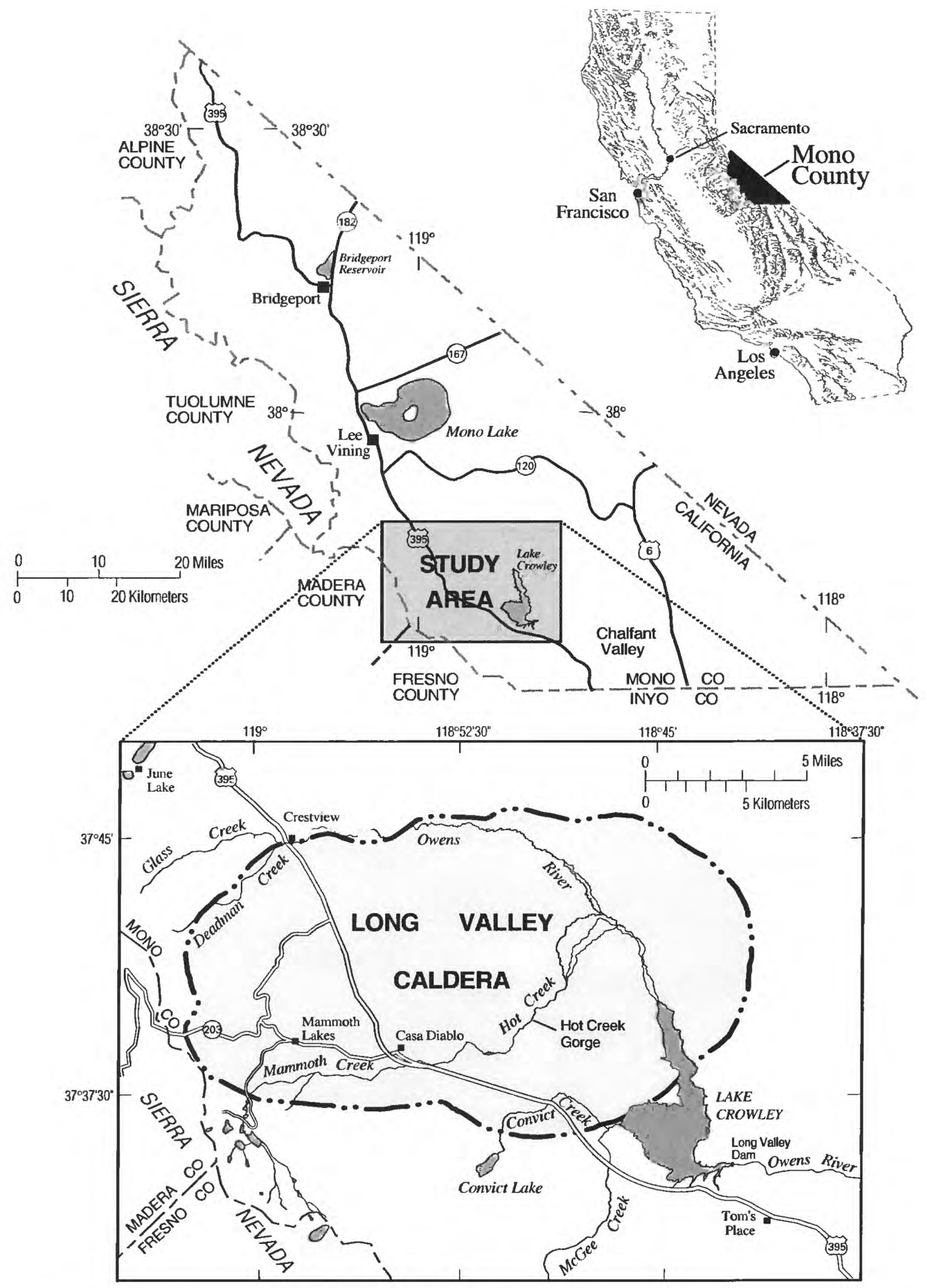

Figure 1. Location of Long Valley Caldera study area, Mono County, California. 


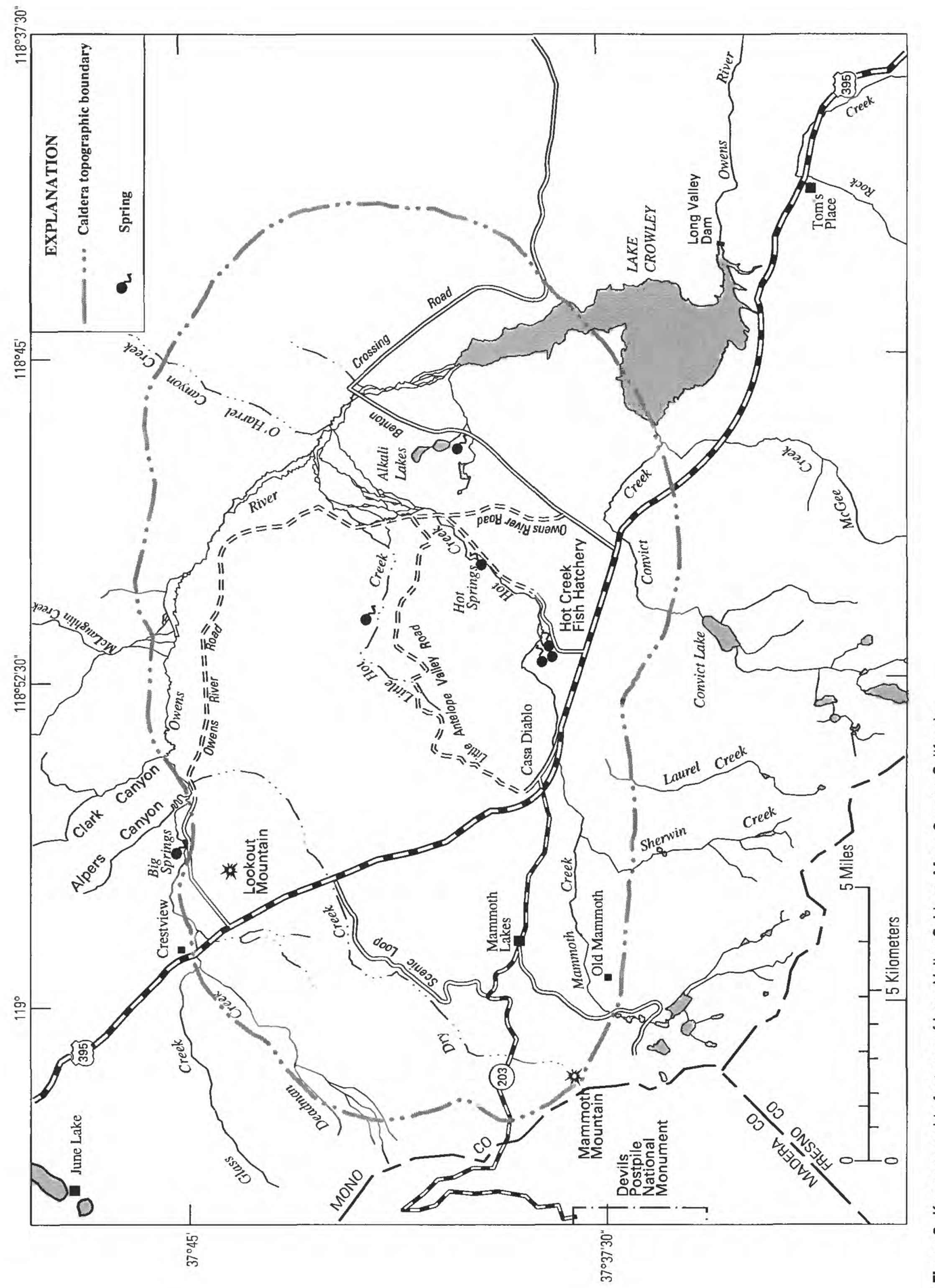

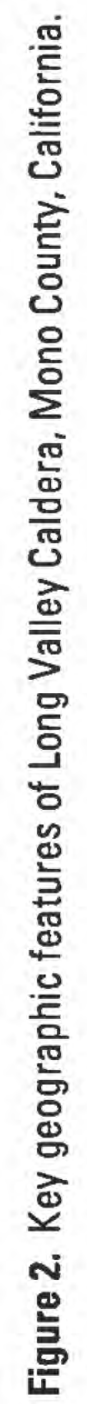


A second hydrologic monitoring program began in 1988 in cooperation with Mono County, California. The monitoring program was implemented to provide data to the Long Valley Hydrologic Advisory Committee (LVHAC). The LVHAC was formed in 1986 to provide Mono County with advice concerning hydrologic environmental issues related to resource developments within Long Valley Caldera (Farrar and Lyster, 1990). The monitoring program parallels the volcanic hazard monitoring, but is specifically directed at detecting hydrologic changes that are caused by geothermal resource utilization and ground-water pumping for potable water supply for the town of Mammoth Lakes.

The LVHAC has identified three key hydrologic features of concern for the county: Mammoth Creek, the thermal springs at Hot Creek Fish Hatchery, and the thermal springs in Hot Creek Gorge (fig. 1). Hydrologic monitoring carried out by the U.S. Geological Survey for the LVHAC and Mono County is primarily at sites along Mammoth Creek, Hot Creek, and at Hot Creek Fish Hatchery.

\section{Description of Study Area}

Long Valley Caldera is in Mono County in eastern California along the steep eastern front of the Sierra Nevada about 20 mi south-southeast of Mono Lake (fig. 1). The caldera is an oval-shaped topographic depression measuring approximately $18 \mathrm{mi}$ from east to west and about $12 \mathrm{mi}$ north to south. The topographic depression formed as the result of a massive volcanic eruption (Bailey, 1989) about 760,000 years ago (Sarna-Wojcicki and Pringle, 1992) and subsequent erosion. All monitoring sites for which data are presented in this report lie within or are proximal to the boundary of the Long Valley Caldera.

The caldera floor has a gentle topographic relief in the eastern part where altitudes range from about 6,800 to $7,200 \mathrm{ft}$ above sea level. The central and western parts are more mountainous with altitudes that range up to about $9,000 \mathrm{ft}$. Prominent mountains, reaching altitudes of over $13,000 \mathrm{ft}$, enclose the caldera on the south, west, and north. The southeastern boundary is of lower relief.

The caldera forms a well-defined surface-water drainage basin with a single outlet by way of Owens River in the southeastern part of the area (fig. 2). A number of perennial and intermittent streams drain from the high mountains that border the southern and western sides of the caldera. Fewer streams drain the northern mountains because precipitation amounts are less. These peripheral streams drain toward one of two main east-flowing streams on the caldera floor, Mammoth Creek on the south and Owens River on the north. Lake Crowley is an artificial reservoir formed by the Long Valley Dam across the Owens River. The reservoir receives water from Owens River, Mammoth Creek, and a few streams that drain smaller areas. In addition, the reservoir receives water imported from the Mono Basin located north of Long Valley Caldera. The imported water is transported $11 \mathrm{mi}$ through a tunnel and discharged into the Owens River about 15 mi upstream of Lake Crowley. The reservoir is part of the water-supply system for the City of Los Angeles.

\section{Purpose and Scope}

This report provides a compilation of hydrologic data collected in Long Valley Caldera by the U.S. Geological Survey during 1994-96. Data collected for both the volcanic hazards monitoring program and the LVHAC are presented in this report. The data include ground-water levels; discharge measurements; water temperatures in wells, streams, and springs; vent gas temperature; precipitation amounts; and chemical and isotopic analyses of water samples collected at selected sites. Hydrologic records for most of the monitoring sites span many more years than the reporting period; in some cases, 9-14 years of record are presented. In many cases, these multiyear records indicate annual cycles and long-term trends.

Although no interpretations of the data are provided in this report, the data will be useful to researchers who may want to relate hydrologic changes to geodetic and geophysical measurements collected during the study of volcanic and seismic processes active during recent years. The report also provides a summary of hydrologic data relevant to some of the environmental concerns of local, State, and Federal agencies, as well as individuals.

Hydrologic data from the volcanic hazards monitoring program prior to 1994 have been published in reports by Farrar and others $(1985,1987,1989)$ and Howle and Farrar (1996). Other significant data compilations include California Department of Water Resources (1967, 1973); Lewis (1974); Willey and others (1974); Mariner and 
Willey (1976); and Setmire (1984). In addition, the U.S. Forest Service has published annual reports containing hydrologic data for 1980 through 1985.

\section{Report Format}

Each site has been assigned a short alpha or alphanumeric identifier that is used in figures and tables. Table 1 lists all sites, grouped by site type, and provides the U.S. Geological Survey station identifier, station number, latitude, longitude, altitude, and information about the data presented in this report. Data tables and figures are grouped by site type (wells, springs, streams, fumarole, and precipitation gages) and are further subdivided into nonthermal or thermal within the groups for wells and springs. Individual maps show the locations of sites by site type.

The presentation of data differs according to site type and the level of variability of the data over the collection period. In general, each graph is followed by the table of data used to construct the graph. For sites with many years of record and where the variability of data is great, graphs for individual years are provided in addition to the multi-year graphs.

This report is accessible on the Internet at website http://ca.water.usgs.gov.

\section{Acknowledgments}

The cooperation of many well owners and landowners in allowing access to collect data is greatly appreciated. Thom Heller, U.S. Forest Service, provided the precipitation data for the Mammoth Ranger Station gage. Gary Sisson was especially helpful in providing information and access to some of the Mammoth Community Water District wells. Chris Boone and Dennis Redfern, California Department of Fish and Game, cooperated in many ways that assisted data collection at Hot Creek Fish Hatchery.

\section{METHODS OF DATA COLLECTION}

\section{Ground-Water Levels and Temperature Profiles}

Ground-water levels were measured three times per year in a network of approximately 53 wells (tables 1 and 12 and fig. 3, at back of report). The actual number of wells varied from year to year because of access conditions and the addition of new wells. The measurements generally were made in April, July, and November. These three months were chosen to provide values reasonably close to actual maximum and minimum water levels and to avoid difficult access conditions in the winter months. Water levels were measured monthly in 13 wells selected from the larger network (table 12).

The water levels were measured using chalked steel tapes or electric sounding devices. Electric sounding devices were used only when conditions in the well bore prevented accurate steel tape measurements. Conditions that required the use of electric sounders were high water temperatures $\left(>30^{\circ} \mathrm{C}\right)$ at the water surface, which would cause a high evaporation rate as a tape was withdrawn from the well, and wet casings above the water level, which would give false indications of the true water level. The general procedures for making accurate water-level measurements, as discussed in a report by Garber and Koopman (1968), were followed.

The water levels were measured with reference to a convenient measuring point on the well head and then were adjusted to land-surface datum using a constant difference between the measuring point and the land surface at the well. Land-surface altitudes were determined for each well site by spirit leveling from a point of known altitude or approximated from topographic maps (table 1). Altitudes that have a precision of less than $1 \mathrm{ft}$ are from leveling; other altitudes were estimated from topographic maps.

Daily mean values of water levels were computed from data recorded at hourly or shorter intervals. Water levels were recorded by a variety of means (table 1). A mechanical system consisting of a float, tape, and counter weight suspended over a pulley was used for shallow water levels (less than $20 \mathrm{ft}$ ). The data were recorded electronically using a shaft-encoder and data logger. For deeper water levels (more than $20 \mathrm{ft}$ ), pressure transducers 
were used to sense the depth of water above an arbitrary datum. In wells that had water temperatures at the water surface of less than $50^{\circ} \mathrm{C}$, submersible transducers were used. The transducers were suspended by weighted cables that held the sensing port at a constant depth. In wells that had water temperatures greater than $50^{\circ} \mathrm{C}$, a bubbler system was used with the transducer at the surface. The system consisted of a nitrogen tank, pressure regulator, and tubing. One end of the tubing was set at a fixed depth below land surface, and nitrogen was allowed to bubble out of the tube below the water surface. The back-pressure on this tube was sensed by a transducer and was related to the height of the water column above the tube orifice. The data for all systems that sensed pressure were recorded by a data logger and were converted to water levels in feet below land surface.

Temperature profiles in wells were made using portable logging equipment consisting of a thermistor attached to a cable and a digital meter to display the resistance. Resistance was converted to the equivalent temperature using a polynomial equation derived from calibration measurements performed in a laboratory. The profiles consist of a number of readings made at discrete points, variably spaced depending on the temperature gradient and depth of the well. Temperatures are accurate to $0.1^{\circ} \mathrm{C}$ or better.

\section{Flow Measurements in Springs and Streams}

Measurements of flow from springs and at cross sections in streams were made using current-velocity meters, weirs, and flumes. Procedures used are described in reports by Buchanan and Somers (1969), and Kilpatrick and Schneider (1983).

Daily mean values of flow were computed from stage records and a stage-discharge relation equation (Rantz and others, 1982; Kennedy, 1983, 1984). Stage was measured using a float, tape, and counter weight suspended over a pulley and was recorded on digital punch tape or graphic chart, or was recorded electronically using a shaftencoder and data logger.

\section{Water and Gas Temperatures}

Daily mean values of water temperature in springs and streams, and vent gas temperatures for fumaroles were computed from values recorded at hourly or more frequent intervals. The temperatures were sensed by using either thermistors or platinum resistance devices, and the data were recorded using data loggers. The accuracy of the temperature values is about $0.2^{\circ} \mathrm{C}$ for the sites at Hot Creek Fish Hatchery. The accuracy of the values for Mammoth Mountain Fumarole (location shown in fig. 44) is about $0.5^{\circ} \mathrm{C}$. Water temperatures were measured using the guidelines given in a report by Stevens and others (1975).

At Fish Hatchery Spring AB (FHAB-WEIR) (location shown in figs. 20 and 22), several spring vents discharge subaqueously to a pool about $400 \mathrm{ft}$ long and 10 to $25 \mathrm{ft}$ wide. At this site, the temperature recorded is a flow-weighted average temperature from all the individual vents, and is measured near the weir at the outflow point. This flow-weighted temperature is affected by variations in ambient air and soil temperatures, as well as variations in water temperatures at the spring vents.

Fish Hatchery Spring CD (FHCD) (location shown in figs. 20 and 22) is similar to Fish Hatchery Spring AB, in that several spring vents discharge subaqueously to a collection pool about $700 \mathrm{ft}$ in length. Temperature measurements at FHCD-T were made at the west end of the pool. A thermistor was placed in the subaqueous discharge stream of an individual vent that issues from fractured basalt lining the pool side.

At Fish Hatchery Spring 23 (FH23-T) (location shown in figs. 20 and 22), several springs, both subaqueous and subaerial, discharge to a collection pool. A thermistor was placed in a subaerial vent along the southeast side of the pool.

Temperature of the gas discharged from Mammoth Mountain Fumarole (MMF) (location shown in fig. 44) was measured by placing a sensor in a 1/2-in.-diameter pipe inserted into the side of the vent at a depth of approximately $\mathbf{l} \mathrm{ft}$ below land surface. The discharge point for the main flow of gas shifted a few inches from time to time because of minor slumping of the soil or disruption of the vent by curious visitors. At times, these disruptions of the vents left the temperature sensor a few inches from the main discharge stream. When the sensor was not positioned in the main discharge stream, recorded temperatures deviated from temperatures measured directly in the vent by as much as $2^{\circ}$ or $3^{\circ} \mathrm{C}$. 


\section{Water Equivalence of Snowpack}

Daily mean values of water equivalence of snowpack were computed from 15-minute values of pressure transducer output at three sites (locations shown in fig. 47). For each site a standard rating was developed that relates pressure transducer output (millivolts) to snow water content in inches. Field calibration of the rating was performed monthly when access and conditions permitted. Calibration checks were made by coring the snowpack in six predetermined locations adjacent to the snowpillow and comparing an average value (in inches of water) to the standard rating. For a thorough discussion of snow course field methods, equipment, and data reduction, refer to "Snow Survey Sampling Guide, U.S. Department of Agriculture, Soil Conservation Service, Agriculture Handbook No. 169, December 1984."

\section{Water Samples for Chemical and Isotopic Analyses}

Water samples from subaerial springs were collected by dipping a polyethylene container into the discharge stream as near the vent as possible. Samples from subaqueous springs and streams were collected using a depthintegrated sampler and equal-width stationing. Well water samples were collected from the discharge pipe while the wells were being pumped.

Aliquots of the samples were used to determine alkalinity, $\mathrm{pH}$, and specific conductance in the field. Aliquots for laboratory analyses were prepared in the field by filtering through $0.45-\mu \mathrm{m}$ capsulated filters. Filtered, unacidified water was placed in polyethylene bottles for anion analyses, and filtered water acidified with nitric acid was placed in polyethylene bottles for cation analyses. Water for mercury analysis was placed in flint glass bottles to which $10 \mathrm{~mL}$ of nitric acid and potassium dichromate was added. Samples for nitrogen and phosphorous analyses were placed in opaque, brown polyethylene bottles and chilled to $4^{\circ} \mathrm{C}$. This procedure was done to inhibit biologic activity. Laboratory methods used for analyses are described by Fishman and Friedman (1989).

\section{REFERENCES CITED}

Bailey, R.A., 1989, Geologic map of Long Valley caldera, Mono-Inyo Craters Volcanic Chain, and vicinity, eastern California: U.S. Geological Survey Map Series I-1933, 1 sheet plus 11 p. of text, scale 1:62,500.

Buchanan, T.J., and Somers, W.P., 1969, Discharge measurements at gaging stations: U.S. Geological Survey Techniques of Water-Resources Investigations, book 3, chap. A8, 65 p.

California Department of Water Resources, 1967, Investigation of geothermal waters in the Long Valley area, Mono County: California Department of Water Resources, 141 p.

California Department of Water Resources, 1973, Mammoth Basin water resources environmental study (final report): California Department of Water Resources, 70 p.

Farrar, C.D., and Lyster, D.L., 1990, Monitoring the hydrologic system for potential effects of geothermal and ground-water development in the Long Valley caldera, Mono County, California, U.S.A.: Geothermal Resources Council Transactions, v. 14 , pt. 1, p. 669-674.

Farrar, C.D., Sorey, M.L., Rojstaczer, S.A., Janik, C.J., Mariner, R.H., Winnett, T.L., and Clark, M.D., 1985, Hydrologic and geochemical monitoring in Long Valley Caldera, Mono County, California: U.S. Geological Survey Water-Resources Investigations Report 85-4183, 137 p.

Farrar, C.D., Sorey, M.L., Rojstaczer, S.A., Janik, C.J., Winnett, T.L., and Clark, M.D., 1987, Hydrologic and geochemical monitoring in Long Valley caldera, Mono County, California, 1985: U.S. Geological Survey Water-Resources Investigations Report 87-4090, 71 p.

Farrar, C.D., Sorey, M.L., Rojstaczer, S.A., Steinemann, A.C., and Clark, M.D., 1989, Hydrologic and geochemical monitoring in Long Valley caldera, Mono County, California, 1986: U.S. Geological Survey Water-Resources Investigations Report 89-4033, 69 p.

Fishman, M.J., and Friedman, L.C., 1989, Methods for determination of inorganic substances in water and fluvial sediments (3d ed): U.S. Geological Survey Techniques of Water-Resources Investigations, book 5, chap. A1, 545 p.

Garber, M.S., and Koopman, F.C., 1968, Methods of measuring water levels in deep wells: U.S. Geological Survey Techniques of Water-Resources Investigations, book 8, chap. A1, $23 \mathrm{p}$.

Hill, D.P., Johnston, M.J.S., Langbein, J.O., McNutt, S.R., Miller, C.D., Mortensen, C.E., Pitt, A.M., and Rojstaczer, S.A., 1991, Response plans for volcanic hazards in the Long Valley caldera and Mono Craters area, California: U.S. Geological Survey Open-File Report 91-270, 64 p. 
Howle, J.F., and Farrar, C.D., 1996, Hydrologic data for Long Valley Caldera, Mono County, California: U.S. Geological Survey Open-File Report 96-382, 286 p.

Kennedy, E.J., 1983, Computation of continuous records of streamflow: U.S. Geological Survey Techniques of WaterResources Investigations, book 3, chap. A13, 53 p.

Kennedy, E.J., 1984, Discharge ratings at gaging stations: U.S. Geological Survey Techniques of Water-Resources Investigations, book 3 , chap. A10, 59 p.

Kilpatrick, F.A., and Schneider, V.R., 1983, Use of flumes in measuring discharge: U.S. Geological Survey Techniques of Water-Resources Investigations, book 3, chap. A14, 46 p.

Lewis, R.E., 1974, Data on wells, springs, and thermal springs in Long Valley, Mono County, California: U.S. Geological Survey Open-File Report, 52 p.

Mariner, R.H., and Willey, L.M., 1976, Geochemistry of thermal waters in Long Valley, Mono County, California: Journal of Geophysical Research, v. 81, no. 5, p. 792-800.

Rantz, S.E., and others, 1982, Measurement and computation of streamflow: Volume 2. Computation of discharge: U.S. Geological Survey Water-Supply Paper 2175,631 p.

Rundle, J.B., and Hill, D.P., 1988, The geophysics of a restless caldera-Long Valley, California: Palo Alto, Calif., Annual Reviews Inc., Annual Review of Earth and Planetary Sciences, v. 16, p. 251-271.

Sarna-Wojcicki, A.M., and Pringle, M.S., Jr., 1992, Laser-fusion ${ }^{41} \mathrm{Ar} /{ }^{39} \mathrm{Ar}$ ages of the Tuff of Taylor Canyon and Bishop Tuff, E. California-W. Nevada: Eos, American Geophysical Union, Transactions, v. 73, no. 43, p. 633.

Setmire, J.G., 1984, Water-quality appraisal of Mammoth Creek and Hot Creek, Mono County, California: U.S. Geological Survey Water-Resources Investigations Report 84-4060, 50 p.

Stevens, H.H., Jr., Ficke, J.F., and Smoot, G.F., 1975, Water temperature-influential factors, field measurement, and data presentation: U.S. Geological Survey Techniques of Water-Resources Investigations, book 1, chap. D1, 65 p.

Sorey, M.L., Suemnicht, G.A., Sturchio, N.C., and Nordquist, G.A., 1991, New evidence on the hydrothermal system in Long Valley caldera, California, from wells, fluid sampling, electrical geophysics, and age determinations of hot-spring deposits: Journal of Volcanology and Geothermal Research, v. 48, p. 229-263.

U.S. Department of Agriculture, Soil Conservation Service, 1984, Snow Survey Sampling Guide, Agriculture Handbook No. 169,32 p.

U.S. Forest Service, 1980-1985, Water resources data for Mammoth Ranger District, Inyo National Forest. California: Published annually.

Willey, L.M., O'Neil, J.R., and Rapp, J.B., 1974, Chemistry of thermal waters in Long Valley, Mono County, California: U.S. Geological Survey Open-File Report, 19 p. 
FIGURES 3-52

AND

TABLES 1-59 
Tabla 1. Descriptions of data-collection sites, Long Valley Caldera, Mono County, California

[Station identifier: Short alphanumeric code used as reference in figures and tables. USGS station number: Unique numeric code used in U.S. Geological Survey databases. Data types: The types of data shown in this report are denoted by codes (AW-April 1 water equivalence of snowpack, F-miscellaneous discharge measurements, FD-daily discharge, $\mathrm{L}$-miscellaneous ground-water levels, $\mathrm{LD}$-daily ground-water levels computed from values recorded at least hourly, PD—daily precipitation, PM-monthly precipitation (daily values available from U.S. Forest Service), Q-chemical or isotopic analyses, SD — daily gage height, $\mathrm{T}$ - miscellaneous temperatures, TD—daily water temperatures, $\mathrm{TP}$-temperature profiles in a well, WD—daily water equivalence of snowpack). Equipment types: The types of equipment used at recording sites to collect data are given by codes (DR-digital recorder, GR - graphic chart recorder, PT-pressure transducer with data logger, SE—-shaft encoder with data logger, SP-snowpillow with pressure transducer and data logger, T-thermister with data logger. TB-tipping bucket gage with data logger. W-weir or flume. WB-weighing bucket gage and data logger)]

\begin{tabular}{cccccc}
\hline $\begin{array}{c}\text { Station } \\
\text { identifier }\end{array}$ & $\begin{array}{c}\text { USGS } \\
\text { station } \\
\text { No. }\end{array}$ & Latitude & Longitude & $\begin{array}{c}\text { Altitude of } \\
\text { land } \\
\text { surface } \\
\text { (feet above } \\
\text { sea level) }\end{array}$ & $\begin{array}{c}\text { Data type } \\
\text { Data on } \\
\text { type }\end{array}$ \\
\hline
\end{tabular}

\begin{tabular}{|c|c|c|c|c|c|c|c|}
\hline \multirow[b]{2}{*}{$2 \mathrm{~A}$} & \multicolumn{6}{|c|}{$\begin{array}{c}\text { Well sites } \\
\text { (See fig. } 3 \text { for site locations) }\end{array}$} & \multirow[b]{2}{*}{42} \\
\hline & 374315118441201 & $37^{\circ} 43^{\prime} 15^{\prime \prime}$ & $118^{\circ} 44^{\prime} 12^{\prime \prime}$ & 6,922 & $\mathbf{L}$ & & \\
\hline $27 \mathrm{R} 1$ & 374420118515501 & $37^{\circ} 44^{\prime} 20^{\prime \prime}$ & $118^{\circ} 51^{\prime} 55^{\prime \prime}$ & $7,039.18$ & $\mathbf{L}$ & & 42 \\
\hline $28 \mathrm{Pl}$ & 374420118532501 & $37^{\circ} 44^{\prime} 20^{\prime \prime}$ & $118^{\circ} 53^{\prime} 25^{\prime \prime}$ & $7,109.02$ & $\mathrm{~L}$ & & 42 \\
\hline $30 \mathrm{~N} 2$ & 374420118493301 & $37^{\circ} 44^{\prime} 20^{\prime \prime}$ & $118^{\circ} 49^{\prime} 33^{\prime \prime}$ & $6,913.21$ & $\mathbf{L}$ & & 42 \\
\hline $31 \mathrm{P} 1$ & 374332118491001 & $37^{\circ} 43^{\prime} 32^{\prime \prime}$ & $118^{\circ} 49^{\prime} 10^{\prime \prime}$ & $6,914.01$ & $\mathbf{L}$ & & 43 \\
\hline $31 \mathrm{P} 2$ & 374332118491002 & $37^{\circ} 43^{\prime} 32^{\prime \prime}$ & $118^{\circ} 49^{\prime} 10^{\prime \prime}$ & $6,915.7$ & $\mathbf{L}$ & & 43 \\
\hline $35 \mathrm{~N} 1$ & 374327118445901 & $37^{\circ} 43^{\prime} 27^{\prime \prime}$ & $118^{\circ} 44^{\prime} 59^{\prime \prime}$ & 6,914 & $\mathbf{L}$ & & 43 \\
\hline $36 \mathrm{P} 1$ & 374335118434101 & $37^{\circ} 43^{\prime} 35^{\prime \prime}$ & $118^{\circ} 43^{\prime} 41^{\prime \prime}$ & 6,995 & L & & 43 \\
\hline AP & 373737118501701 & $37^{\circ} 37^{\prime} 37^{\prime \prime}$ & $118^{\circ} 50^{\prime} 17^{\prime \prime}$ & 7,093 & $\mathrm{~L}$ & & 44 \\
\hline $\mathrm{CD}-2$ & 373847118545802 & $37^{\circ} 38^{\prime} 47^{\prime \prime}$ & $118^{\circ} 54^{\prime} 58^{\prime \prime}$ & 7,316 & $\mathbf{L}$ & & 44 \\
\hline $\mathrm{CH}-1$ & 374045118491001 & $37^{\circ} 40^{\prime} 45^{\prime \prime}$ & $118^{\circ} 49^{\prime} 10^{\prime \prime}$ & $6,969.93$ & $\mathrm{~L}$ & & 44 \\
\hline $\mathrm{CH}-10$ & 373932118491501 & $37^{\circ} 39^{\prime} 32^{\prime \prime}$ & $118^{\circ} 49^{\prime} 15^{\prime \prime}$ & $7,074.88$ & $\mathrm{~L}$ & & 44 \\
\hline $\mathrm{CH}-10 \mathrm{~A}$ & 373930118491601 & $37^{\circ} 39^{\prime} 30^{\prime \prime}$ & $118^{\circ} 49^{\prime} 16^{\prime \prime}$ & $7,078.80$ & $\mathrm{~L}$ & & 45 \\
\hline $\mathrm{CH}-10 \mathrm{~B}$ & 373930118491602 & $37^{\circ} 39^{\prime} 30^{\prime \prime}$ & $118^{\circ} 49^{\prime} 16^{\prime \prime}$ & $7,078.99$ & $\mathrm{~L}, \mathrm{LD}, \mathrm{TP}$ & PT & $32-39,45,58$ \\
\hline $\mathrm{CH}-11 \mathrm{~A}$ & 373846119023101 & $37^{\circ} 38^{\prime} 46^{\prime \prime}$ & $119^{\circ} 02^{\prime} 31^{\prime \prime}$ & 9,090 & $\mathrm{Q}$ & & $144-146$ \\
\hline $\mathrm{CH}-3$ & 373929118455405 & $37^{\circ} 39^{\prime} 29^{\prime \prime}$ & $118^{\circ} 45^{\prime} 54^{\prime \prime}$ & 6,870 & L & & 45 \\
\hline $\mathrm{CH}-5$ & 373759118474101 & $37^{\circ} 37^{\prime} 59^{\prime \prime}$ & $118^{\circ} 47^{\prime} 41^{\prime \prime}$ & 6,918 & $\mathrm{~L}$ & & 46 \\
\hline CH-6 & 374040118522501 & $37^{\circ} 40^{\prime} 40^{\prime \prime}$ & $118^{\circ} 52^{\prime} 25^{\prime \prime}$ & $7,248.23$ & $\mathrm{~L}$ & & 46 \\
\hline $\mathrm{CH}-7$ & 374000118483102 & $37^{\circ} 40^{\prime} 00^{\prime \prime}$ & $118^{\circ} 48^{\prime} 31^{\prime \prime}$ & $6,956.89$ & $\mathrm{~L}, \mathrm{TP}$ & & 46.58 \\
\hline CHURCH & 373707118491701 & $37^{\circ} 37^{\prime} 07^{\prime \prime}$ & $118^{\circ} 49^{\prime} 17^{\prime \prime}$ & 7,035 & L & & 46 \\
\hline CM-2 & 373906118522301 & $37^{\circ} 39^{\prime} 06^{\prime \prime}$ & $118^{\circ} 52^{\prime} 23^{\prime \prime}$ & 7,077 & L.TP & & 47.59 \\
\hline CR & 373816118532001 & $37^{\circ} 38^{\prime} 16^{\prime \prime}$ & $118^{\circ} 53^{\prime} 20^{\prime \prime}$ & $7,161.76$ & $\mathbf{L}$ & & 47 \\
\hline CT & 374511118585801 & $37^{\circ} 45^{\prime} 11^{\prime \prime}$ & $118^{\circ} 58^{\prime} 58^{\prime \prime}$ & 7,520 & L & & 47 \\
\hline CW-1 & 373850118513601 & $37^{\circ} 38^{\prime} 50^{\prime \prime}$ & $118^{\circ} 51^{\prime} 36^{\prime \prime}$ & $7,084.99$ & L.TP & & 47.59 \\
\hline CW-3 & 373849118513001 & $37^{\circ} 38^{\prime \prime} 49^{\prime \prime}$ & $118^{\circ} 51^{\prime} 30^{\prime \prime}$ & $7,062.20$ & $\mathrm{~L}, \mathrm{LD}$ & SE & $22-29,48$ \\
\hline ESN & 373818118515001 & $37^{\circ} 38^{\prime} 18^{\prime \prime}$ & $118^{\circ} 51^{\prime} 50^{\prime \prime}$ & 7,085 & L.LD & SE & $30-31.48$ \\
\hline ESO & 373818118514502 & $37^{\circ} 38^{\prime} 18^{\prime \prime}$ & $118^{\circ} 51^{\prime} 45^{\prime \prime}$ & 7,090 & $\mathbf{L}$ & & 48 \\
\hline HSL-1 & 373650119011301 & $37^{\circ} 36^{\prime} 50^{\prime \prime}$ & $119^{\circ} 01^{\prime} 13^{\prime \prime}$ & 9,040 & Q & & $144-146$ \\
\hline
\end{tabular}


Table 1. Descriptions of data-collection sites, Long Valley Caldera, Mono County, California - Continued

\begin{tabular}{|c|c|c|c|c|c|c|c|}
\hline $\begin{array}{l}\text { Station } \\
\text { identifier }\end{array}$ & $\begin{array}{c}\text { USGS } \\
\text { station } \\
\text { No. }\end{array}$ & Latitude & Longitude & $\begin{array}{l}\text { Altitude of } \\
\text { land } \\
\text { surface } \\
\text { (feet above } \\
\text { sea level) }\end{array}$ & Data type & $\begin{array}{l}\text { Equipment } \\
\text { type }\end{array}$ & $\begin{array}{l}\text { Data on } \\
\text { page(s) in this } \\
\text { report }\end{array}$ \\
\hline LAV-1 & 374051118523301 & $37^{\circ} 40^{\prime} 51^{\prime \prime}$ & $118^{\circ} 52^{\prime} 33^{\prime \prime}$ & 7,200 & L & & 49 \\
\hline LB-1 & 373634119004801 & $37^{\circ} 36^{\prime} 34^{\prime \prime}$ & $11^{\circ} 00^{\prime} 48^{\prime \prime}$ & 8,970 & Q & & $144-146$ \\
\hline LKT & 374300118554401 & $37^{\circ} 43^{\circ} 00^{\prime \prime}$ & $118^{\circ} 55^{\circ} 44^{\prime \prime}$ & 7,350 & L,LD,PD,TP & PT,SE & $15-21,49,60$ \\
\hline LV-2 & 373729118532802 & $37^{\circ} 37^{\prime} 29^{\prime \prime}$ & $118^{\circ} 53^{\prime} 28^{\prime \prime}$ & $7,167.39$ & $\mathbf{L}$ & & 49 \\
\hline LV-15 & 373913118551401 & $37^{\circ} 39^{\prime} 13^{\prime \prime}$ & $118^{\circ} 55^{\prime} 14^{\prime \prime}$ & 7,340 & L & & 50 \\
\hline LV-18 & 373918118481901 & $37^{\circ} 39^{\prime} 18^{\prime \prime}$ & $118^{\circ} 48^{\prime} 19^{\prime \prime}$ & $6,966.00$ & $\mathbf{L}$ & & 50 \\
\hline LV-19 & 373754118501701 & $37^{\circ} 37^{\prime} 54^{\prime \prime}$ & $118^{\circ} 50^{\prime} 17^{\prime \prime}$ & $7,090.40$ & $\mathbf{L}$ & & 50 \\
\hline LV-25 & 374233118482701 & $37^{\circ} 42^{\prime} 33^{\prime \prime}$ & $118^{\circ} 48^{\prime} 27^{\prime \prime}$ & $6,873.81$ & L & & 51 \\
\hline LV-30 & 374109118484101 & $37^{\circ} 41^{\prime} 09^{\prime \prime}$ & $118^{\circ} 48^{\prime} 41^{\prime \prime}$ & $6,895.62$ & $\mathbf{L}$ & & 51 \\
\hline LV-44 & 373917118551402 & $37^{\circ} 39^{\prime} 17^{\prime \prime}$ & $118^{\circ} 55^{\prime} 14^{\prime \prime}$ & 7,340 & $\mathbf{L}$ & & 51 \\
\hline LV-45 & 373914118472201 & $37^{\circ} 39^{\prime} 14^{\prime \prime}$ & $118^{\circ} 47^{\prime} 22^{\prime \prime}$ & $6,985.38$ & $\mathbf{L}$ & & 51 \\
\hline MMSA-1 & 373904119021501 & $37^{\circ} 39^{\prime} 04^{\prime \prime}$ & $119^{\circ} 02^{\prime} 15^{\prime \prime}$ & 8,890 & Q & & $144-146$ \\
\hline MMSA-2A & 373901119012901 & $37^{\circ} 39^{\prime} 01^{\prime \prime}$ & $119^{\circ} 01^{\prime} 29^{\prime \prime}$ & 8,790 & Q & & $144-146$ \\
\hline MW-1 & 373816118523901 & $37^{\circ} 38^{\prime} 16^{\prime \prime}$ & $118^{\circ} 52^{\prime} 39^{\prime \prime}$ & $7,046.43$ & $\mathbf{L}$ & & 52 \\
\hline MW-2 & 373841118512902 & $37^{\circ} 38^{\prime} 41^{\prime \prime}$ & $118^{\circ} 51^{\prime} 29^{\prime \prime}$ & $7,057.08$ & $\mathbf{L}$ & & 52 \\
\hline MW-4 & 373907118503801 & $37^{\circ} 39^{\prime} 07^{\prime \prime}$ & $118^{\circ} 50^{\prime} 38^{\prime \prime}$ & $7,081.26$ & $\mathbf{L}$ & & 52 \\
\hline MW-5 & 373841118512901 & $37^{\circ} 38^{\circ} 41^{\prime \prime}$ & $118^{\circ} 51^{\prime} 29^{\prime \prime}$ & $7,054.12$ & $\mathbf{L}$ & & 53 \\
\hline MWD 5 & 373757118591801 & $37^{\circ} 37^{\prime} 57^{\prime \prime \prime}$ & $118^{\circ} 59^{\prime} 18^{\prime \prime}$ & $7,985.28$ & $\mathbf{L}$ & & 53 \\
\hline MWD M-10 & 373719118585301 & $37^{\circ} 37^{\prime} 19^{\prime \prime}$ & $118^{\circ} 58^{\prime} 53^{\prime \prime}$ & $7,939.63$ & $\mathbf{L}$ & & 53 \\
\hline MWD M-11 & 373705118585601 & $37^{\circ} 37^{\prime} 05^{\prime \prime}$ & $118^{\circ} 58^{\prime} 56^{\prime \prime}$ & $7,968.56$ & $\mathbf{L}$ & & 53 \\
\hline MWD M-12 & 373714118585501 & $37^{\circ} 37^{\prime} 14^{\prime \prime}$ & $118^{\circ} 58^{\prime} 55^{\prime \prime}$ & $7,952.92$ & $\mathbf{L}$ & & 54 \\
\hline MWD M-14 & 373739118582101 & $37^{\circ} 37^{\prime} 39^{\prime \prime}$ & $118^{\circ} 58^{\prime} 21^{\prime \prime}$ & $7,877.79$ & $\mathbf{L}$ & & 14,54 \\
\hline PD & 373732118490001 & $37^{\circ} 37^{\prime} 32^{\prime \prime}$ & $118^{\circ} 49^{\prime} 00^{\prime \prime}$ & 7,014 & $\mathbf{L}$ & & 54 \\
\hline PLV-2 & 374249118584501 & $37^{\circ} 42^{\prime} 49^{\prime \prime}$ & $118^{\circ} 58^{\prime} 45^{\prime \prime}$ & 7,734 & $\mathbf{L}$ & & 55 \\
\hline RDO-8 & 373924118571201 & $37^{\circ} 39^{\prime} 24^{\prime \prime}$ & $118^{\circ} 57^{\prime} 14^{\prime \prime}$ & $7,788.4$ & L,TP & & 55,60 \\
\hline SC-1 & 373745118554001 & $37^{\circ} 37^{\prime} 45^{\prime \prime}$ & $118^{\circ} 55^{\prime} 40^{\prime \prime}$ & $7,469.84$ & $\mathbf{L}$ & & 55 \\
\hline SC-2 & 373745118554002 & $37^{\circ} 37^{\prime} 45^{\prime \prime}$ & $118^{\circ} 55^{\prime} 40^{\prime \prime}$ & $7,470.65$ & $\mathbf{L}$ & & 14,56 \\
\hline SNARL & 373654118495401 & $37^{\circ} 36^{\prime} 54^{\prime \prime}$ & $118^{\circ} 49^{\prime} 54^{\prime \prime}$ & 7,095 & $\mathbf{L}$ & & 56 \\
\hline SQ & 373742118515703 & $37^{\circ} 37^{\prime} 42^{\prime \prime}$ & $118^{\circ} 51^{\prime} 57^{\prime \prime}$ & $7,102.22$ & $\mathbf{L}$ & & 56 \\
\hline \multirow[t]{2}{*}{ SS-2 } & 373818118533202 & $37^{\circ} 38^{\prime} 18^{\prime \prime}$ & $118^{\circ} 53^{\prime} 32^{\prime \prime}$ & $7,179.13$ & $\mathbf{L}$ & & 57 \\
\hline & & \multicolumn{3}{|c|}{$\begin{array}{c}\text { Spring sites } \\
\text { (See fig. } \mathbf{2 0} \text { for site locations) }\end{array}$} & & & \\
\hline FHAB-WEIR & 373822118514401 & $37^{\circ} 38^{\prime} 22^{\prime \prime}$ & $118^{\circ} 51^{\prime} 44^{\prime \prime}$ & 7,050 & FD,TD,Q & SE,T & $\begin{array}{l}65-77,100,144 \\
146,150-151\end{array}$ \\
\hline FHCD-Q & 373818118513301 & $37^{\circ} 38^{\prime} 18^{\prime \prime}$ & $118^{\circ} 51^{\prime} 33^{\prime \prime}$ & 7,050 & FD,Q & SE,W & $\begin{array}{l}78-81,100,144 \\
146,151-153\end{array}$ \\
\hline FHCD-T & 373817118514101 & $37^{\circ} 38^{\prime} 17^{\prime \prime}$ & $118^{\circ} 51^{\prime} 41^{\prime \prime}$ & 7,053 & TD & $\mathbf{T}$ & $82-87,100$ \\
\hline
\end{tabular}


Table 1. Descriptions of data-collection sites, Long Valley Caldera, Mono County, California - Continued

\begin{tabular}{|c|c|c|c|c|c|c|c|}
\hline $\begin{array}{l}\text { Station } \\
\text { identifier }\end{array}$ & $\begin{array}{c}\text { USGS } \\
\text { station } \\
\text { No. }\end{array}$ & Latitude & Longitude & $\begin{array}{l}\text { Altitude of } \\
\text { land } \\
\text { surface } \\
\text { (feet above } \\
\text { sea levell }\end{array}$ & Data type & $\begin{array}{c}\text { Equipment } \\
\text { type }\end{array}$ & $\begin{array}{l}\text { Data on } \\
\text { page(s) in this } \\
\text { report }\end{array}$ \\
\hline FH23-Q & 373829118505801 & $37^{\circ} 38^{\prime} 29^{\prime \prime}$ & $118^{\circ} 50^{\prime} 56^{\prime \prime}$ & 7,050 & FD & GR,SE,W & $88-93,100$ \\
\hline FH23-T & 373829118505801 & $37^{\circ} 38^{\prime} 29^{\prime \prime}$ & $118^{\circ} 50^{\prime} 58^{\prime \prime}$ & 7,065 & $\mathrm{TD}, \mathrm{Q}$ & $\mathrm{T}$ & $\begin{array}{l}94-99,100,147- \\
149,153\end{array}$ \\
\hline HBP & 373850118513501 & $37^{\circ} 38^{\prime} 50^{\prime \prime}$ & $118^{\circ} 51^{\prime} 35^{\prime \prime}$ & 7,070 & SD & PT & $101-107$ \\
\hline $\mathrm{HC}-4$ & 373941118494301 & $37^{\circ} 39^{\prime} 41^{\prime \prime}$ & $118^{\circ} 49^{\prime} 37^{\prime \prime}$ & 6,960 & $\mathrm{Q}$ & & 153 \\
\hline HC-6L & 373915118493701 & $37^{\circ} 39^{\prime} 15^{\prime \prime}$ & $118^{\circ} 49^{\prime} 37^{\prime \prime}$ & 6,960 & Q & & $153-154$ \\
\hline LHC-SP & 374125118502501 & $37^{\circ} 41^{\prime} 25^{\prime \prime}$ & $118^{\circ} 50^{\prime} 25^{\prime \prime}$ & 7,035 & FD & GR,W & $62-64$ \\
\hline LS & 373701118533701 & $37^{\circ} 37^{\prime} 01^{\prime \prime}$ & $118^{\circ} 53^{\prime} 37^{\prime \prime}$ & 7,200 & Q & & $147-149$ \\
\hline MCS & 373708119040601 & $37^{\circ} 37^{\prime} 08^{\prime \prime}$ & $119^{\circ} 04^{\prime} 06^{\prime \prime}$ & 7,940 & Q & & $147-149$ \\
\hline \multicolumn{8}{|c|}{$\begin{array}{l}\text { Stream sites } \\
\text { (See fig. } 39 \text { for site locations) }\end{array}$} \\
\hline HCA & 10265147 & $37^{\circ} 39^{\prime} 27^{\prime \prime}$ & $118^{\circ} 49^{\prime} 54^{\prime \prime}$ & 7,035 & $\mathrm{~F}, \mathrm{Q}$ & & $122,154-155$ \\
\hline $\mathrm{HCF}$ & 10265150 & $37^{\circ} 40^{\prime} 08^{\prime \prime}$ & $118^{\circ} 49^{\prime} 00^{\prime \prime}$ & 6,950 & $\mathrm{FD}, \mathrm{Q}$ & GR,SE,W & $109-115,155$ \\
\hline LHC-SW & 10265160 & $37^{\circ} 41^{\prime} 25^{\prime \prime}$ & $118^{\circ} 50^{\prime} 31^{\prime \prime}$ & 6,990 & FD & DR,W & $116-121$ \\
\hline MC395 & 10265130 & $37^{\circ} 38^{\prime} 16^{\prime \prime}$ & $118^{\circ} 54^{\prime} 10^{\prime \prime}$ & 7,200 & $\mathrm{~F}, \mathrm{Q}$ & & $122,147-149$ \\
\hline MCAF & 10265143 & $37^{\circ} 38^{\prime} 37^{\prime \prime}$ & $118^{\circ} 51^{\prime} 11^{\prime \prime}$ & 7,052 & F,Q & & $122,147-149$ \\
\hline $\mathrm{MT}$ & 374525118532701 & $37^{\circ} 45^{\prime} 25^{\prime \prime}$ & $118^{\circ} 53^{\prime} 27^{\prime \prime}$ & 7,040 & $Q^{1}$ & & $147-149$ \\
\hline \multicolumn{8}{|c|}{$\begin{array}{c}\text { Fumarole site } \\
\text { (See for fig. } 44 \text { for site location) }\end{array}$} \\
\hline MMF & 373819119014201 & $37^{\circ} 38^{\prime} 19^{\prime \prime}$ & $119^{\circ} 01^{\prime} 42^{\prime \prime}$ & 9,900 & $\mathrm{TD}$ & $\mathrm{T}$ & $124-128$ \\
\hline \multicolumn{8}{|c|}{$\begin{array}{c}\text { Precipitation sites } \\
\text { (See fig. } 47 \text { for site locations) }\end{array}$} \\
\hline HSL & 373646119011401 & $37^{\circ} 36^{\prime} 46^{\prime \prime}$ & $119^{\circ} 01^{\prime} 14^{\prime \prime}$ & 9,040 & WD & SP & $138-139$ \\
\hline LKT-P & 374300118554401 & $37^{\circ} 43^{\prime} 00^{\prime \prime}$ & $118^{\circ} 55^{\prime} 44^{\prime \prime}$ & 7,350 & PD, WD & WB,SP & $\begin{array}{l}130,131-133 \\
140-141\end{array}$ \\
\hline MRS & none & $37^{\circ} 38^{\prime} 52^{\prime \prime}$ & $118^{\circ} 57^{\prime} 35^{\prime \prime}$ & 7,800 & PM & WB & 130,134 \\
\hline OMM & 373714118585502 & $37^{\circ} 37^{\prime} 14^{\prime \prime}$ & $118^{\circ} 58^{\prime} 55^{\prime \prime}$ & $7,953.0$ & PD & $\mathrm{TB}$ & $130,135-137$ \\
\hline MP & none & $37^{\circ} 36^{\circ} 42$ & $119^{\circ} 01^{\circ} 52$ & 9,500 & AW & SP & 142 \\
\hline
\end{tabular}

'Flow data available from Los Angeles Department of Water and Power; location shown in figure 52. 


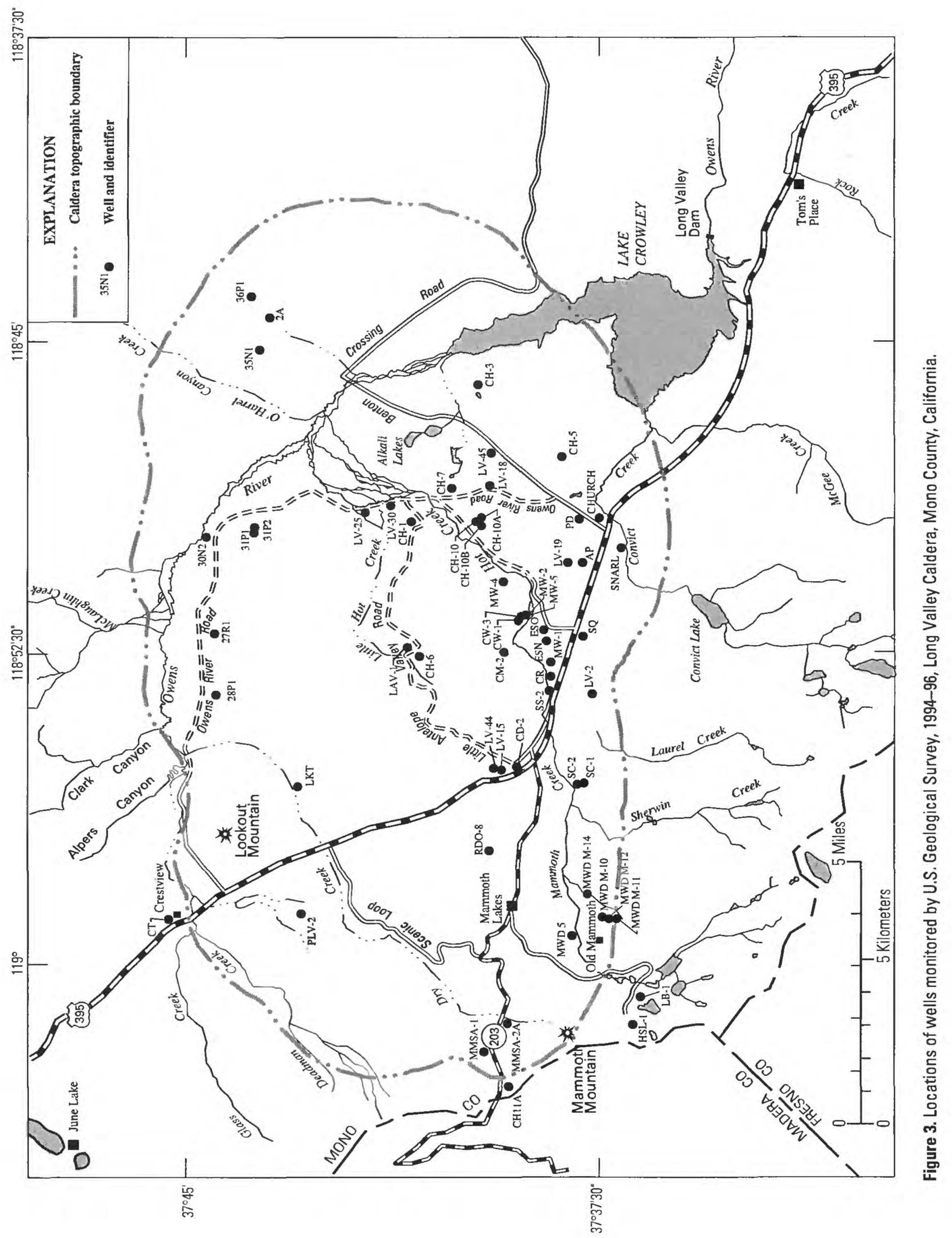




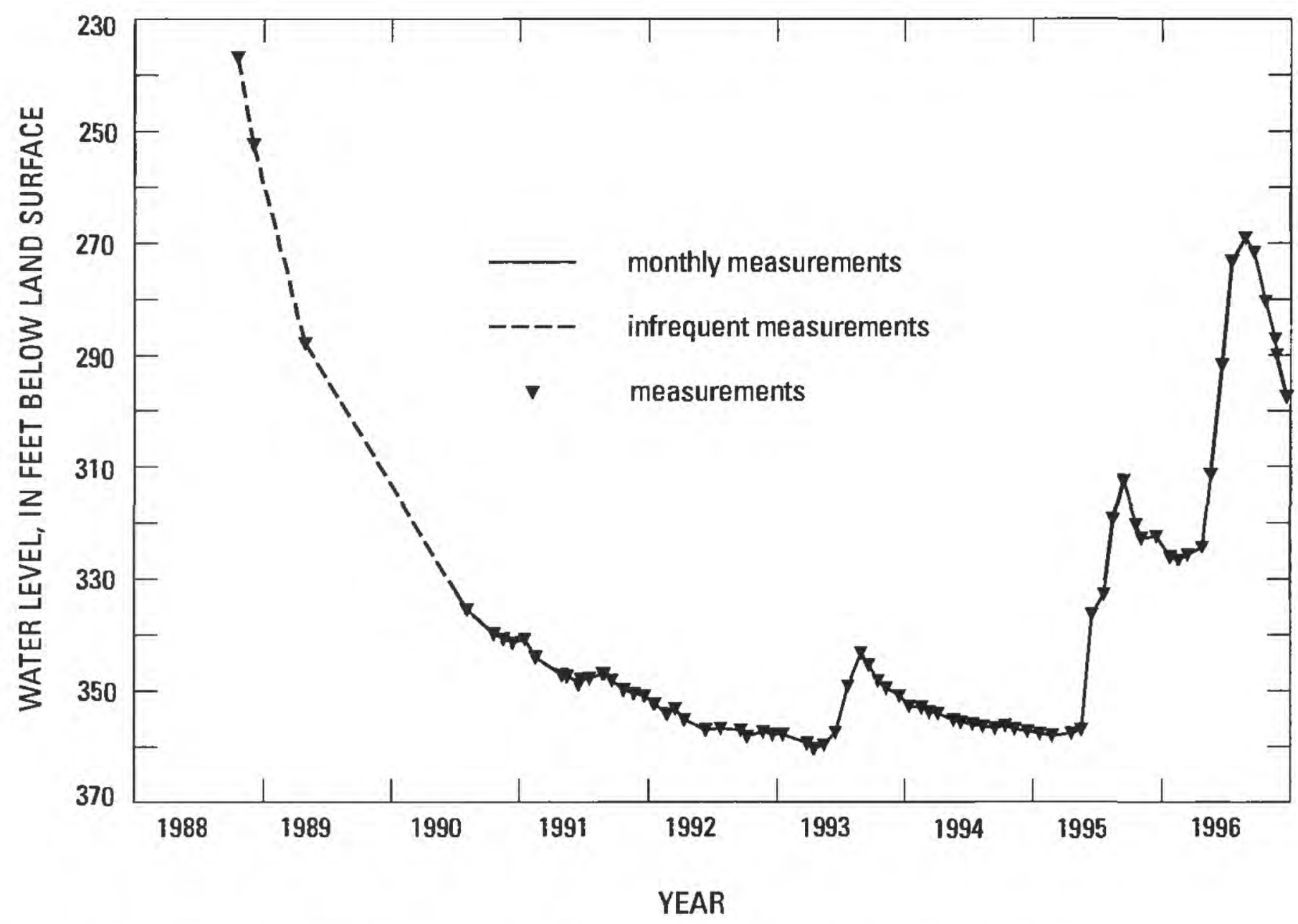

Figure 4. Instantaneous water levels, well MWD M-14, 1988-96, Long Valley Caldera, Mono County, California.

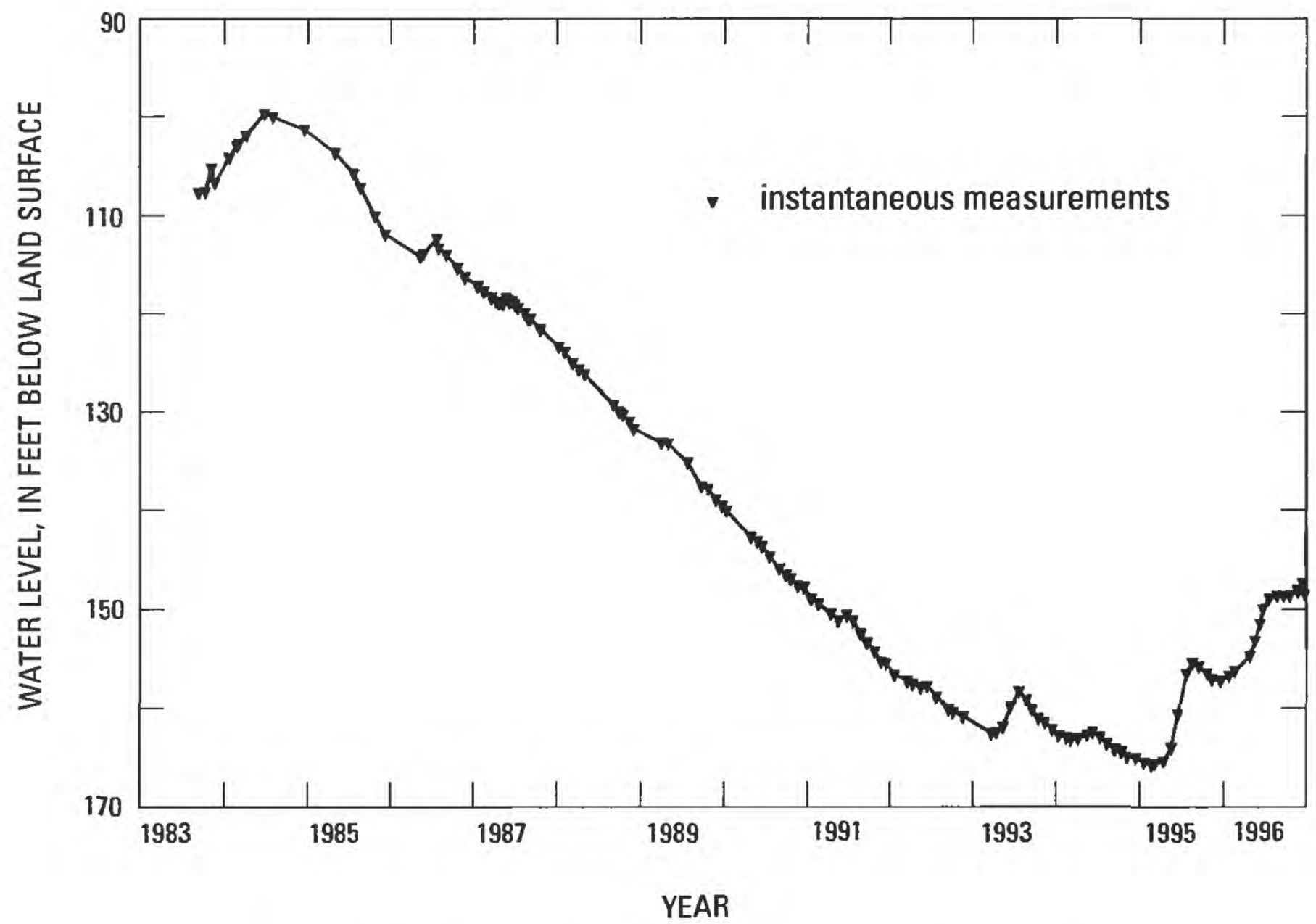

Figure 5. Instantaneous water levels, well SC-2, 1983-96, Long Valley Caldera, Mono County, California. 


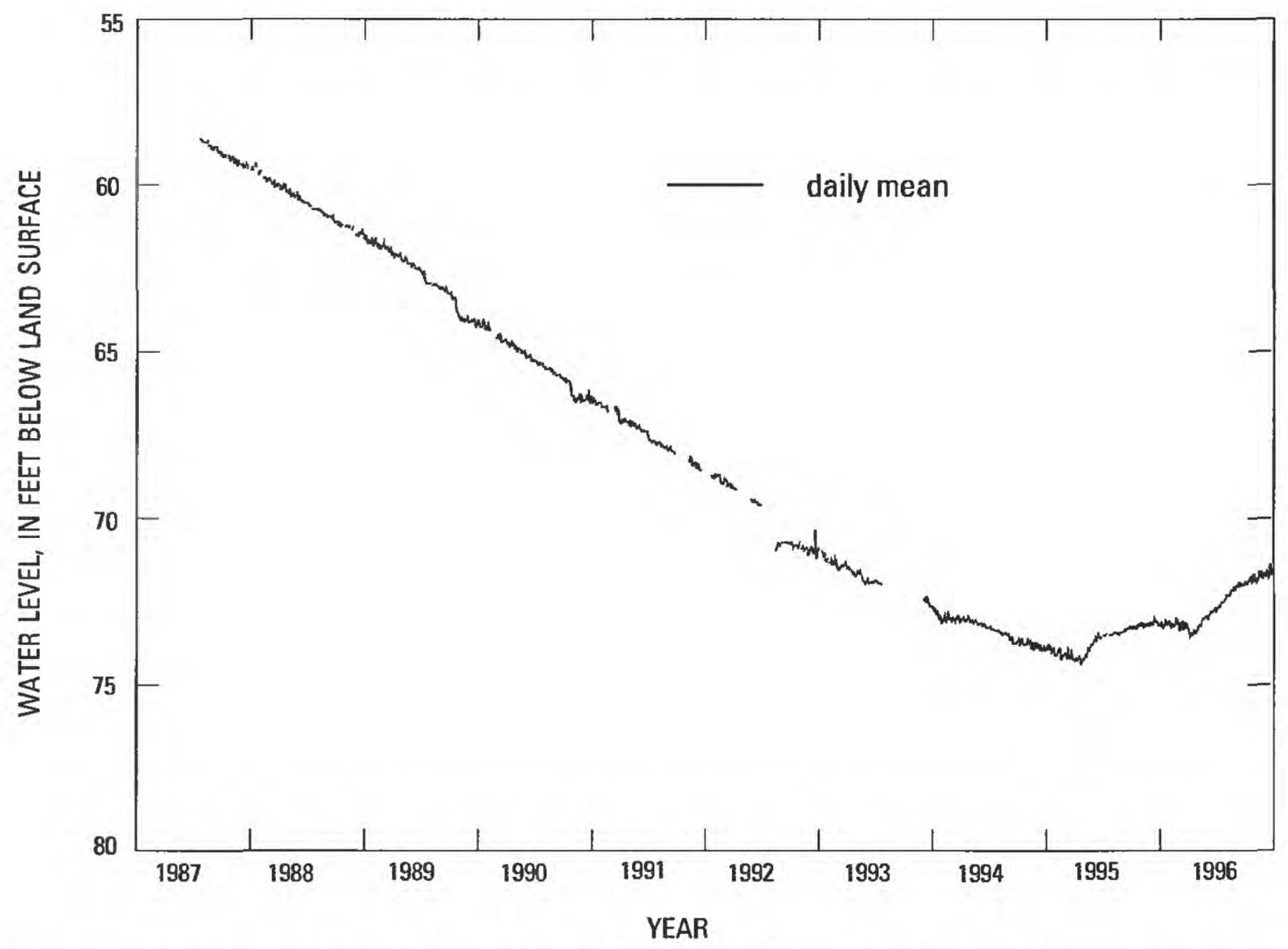

Figure 6. (A) Daily mean water levels, Lookout Mountain well (LKT), 1987-96, Long Valley Caldera, Mono County, California.

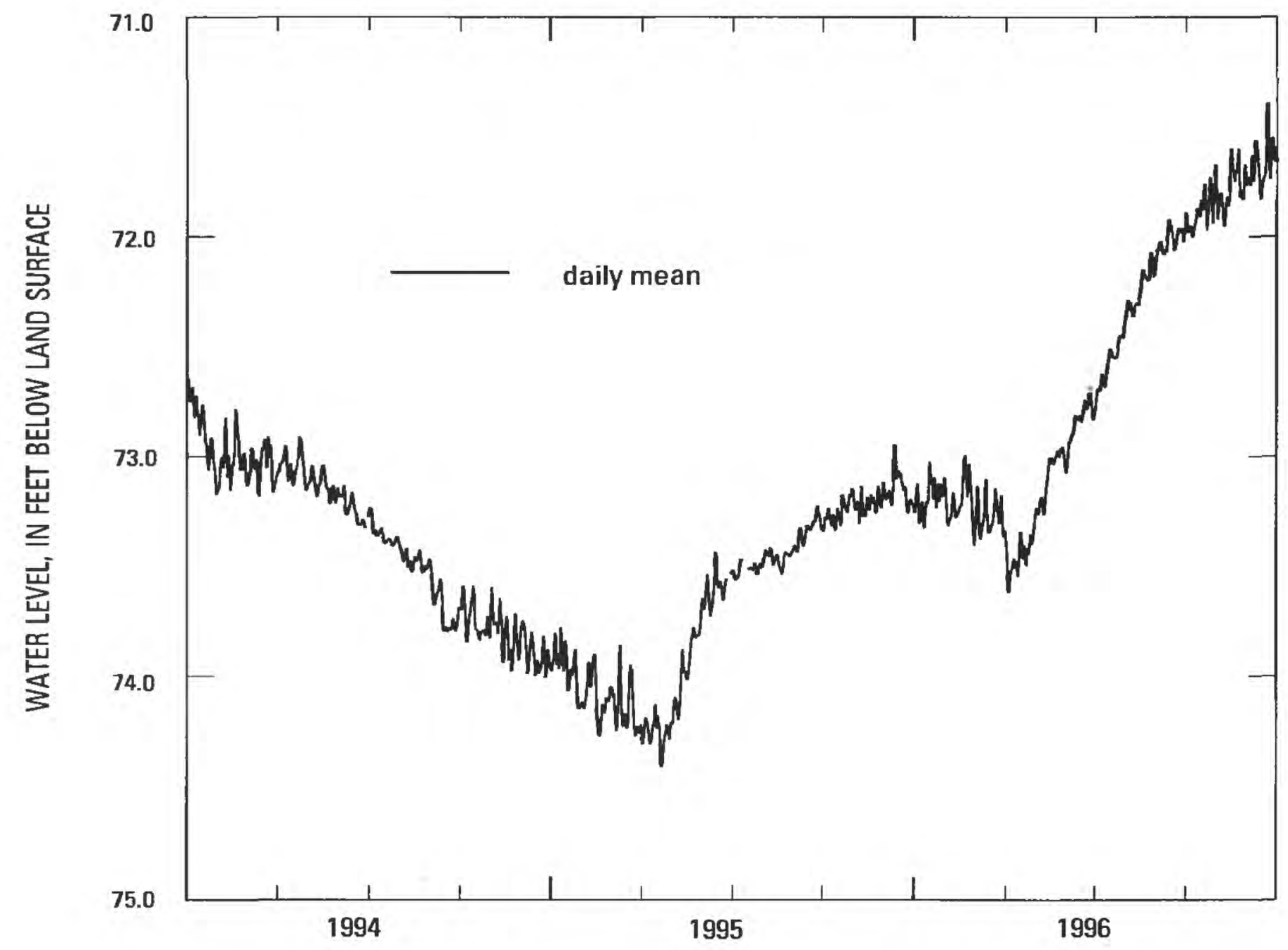

Figure 6. (B) Daily mean water levels, Lookout Mountain well (LKT), 1994-96, Long Valley Caldera, Mono County, California. 


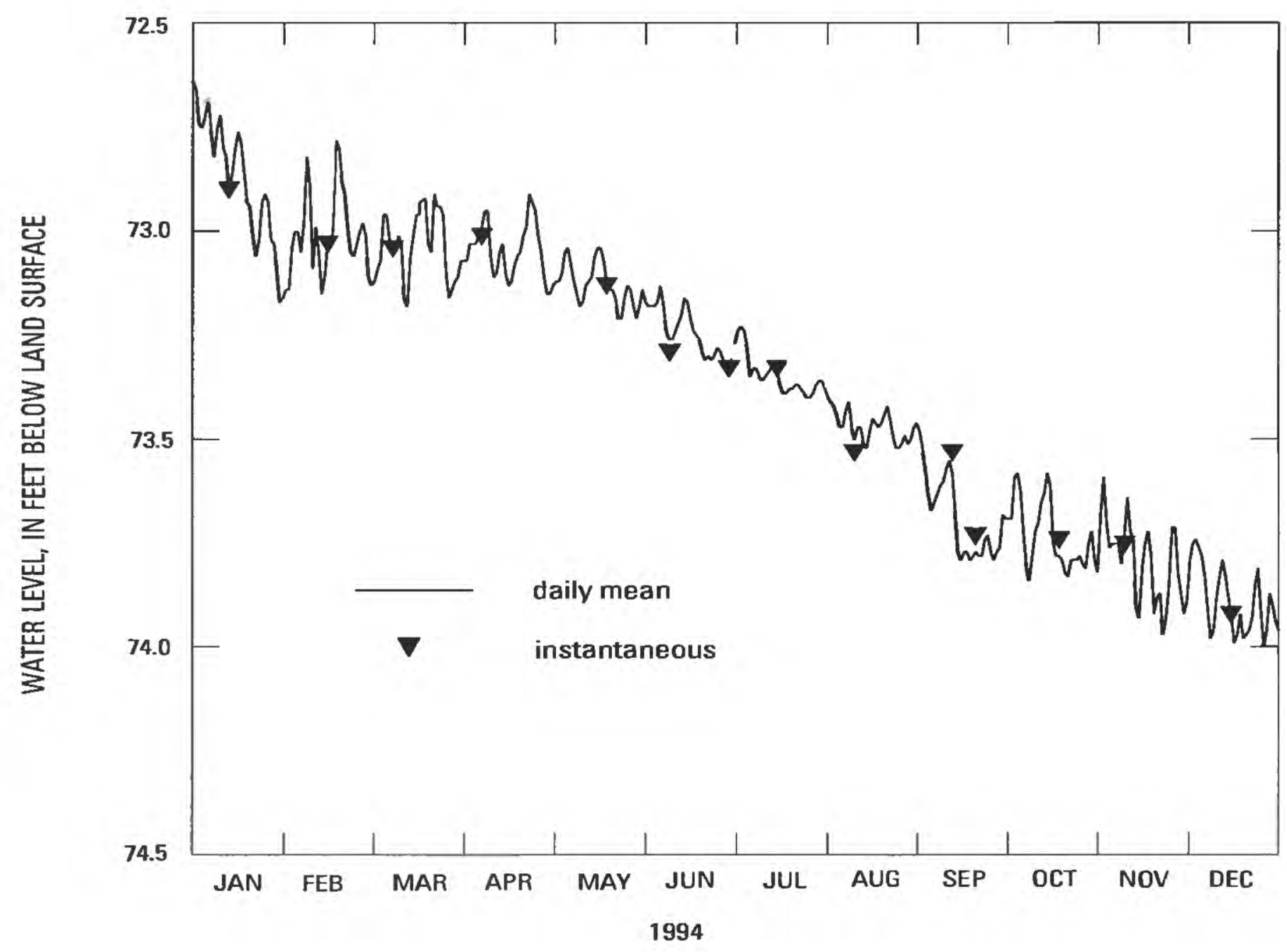

Figure 7. (A) Daily mean and instantaneous water levels, Lookout Mountain well (LKT), 1994, Long Valley Caldera, Mono County, California. 
Table 2. Daily mean water levels, Lookout Mountain well (LKT), 1994, Long Valley Caldera, California

[Values are in feet below land surface. —, no data; Max, maximum; Min, minimum]

\begin{tabular}{|c|c|c|c|c|c|c|c|c|c|c|c|c|}
\hline Day & Jan & Feb & Mar & Apr & May & Jun & Jul & Aug & Sep & Oct & Nov & Dec \\
\hline 1 & 72.66 & 73.14 & 73.13 & 73.07 & 73.13 & 73.17 & 73.27 & 73.40 & 73.48 & 73.69 & 73.68 & 73.79 \\
\hline 2 & 72.74 & 73.04 & 73.12 & 73.07 & 73.12 & 73.18 & 73.24 & 73.41 & 73.52 & 73.69 & 73.59 & 73.75 \\
\hline 3 & 72.75 & 73.00 & 73.09 & 73.03 & 73.12 & 73.18 & 73.23 & 73.42 & 73.57 & 73.59 & 73.70 & 73.74 \\
\hline 4 & 72.72 & 73.00 & 73.07 & 73.03 & 73.10 & 73.18 & 73.24 & 73.44 & 73.63 & 73.58 & 73.76 & 73.76 \\
\hline 5 & 72.68 & 73.05 & 72.96 & 73.03 & 73.05 & 73.17 & 73.28 & 73.47 & 73.67 & 73.62 & 73.75 & 73.78 \\
\hline 6 & 72.76 & 72.98 & 72.96 & 73.01 & 73.04 & 73.13 & 73.35 & 73.47 & 73.65 & 73.72 & 73.75 & 73.83 \\
\hline 7 & 72.82 & 72.82 & 73.02 & 72.99 & 73.08 & 73.18 & 73.33 & 73.43 & 73.63 & 73.80 & 73.75 & 73.91 \\
\hline 8 & 72.75 & 72.89 & 73.05 & 72.95 & 73.12 & 73.24 & 73.33 & 73.41 & 73.61 & 73.84 & 73.80 & 73.98 \\
\hline 9 & 72.72 & 73.09 & 73.05 & 72.95 & 73.15 & 73.26 & 73.35 & 73.47 & 73.60 & 73.78 & 73.73 & 73.96 \\
\hline 10 & 72.80 & 72.99 & 73.01 & 73.06 & 73.18 & 73.26 & 73.36 & 73.50 & 73.57 & 73.72 & 73.64 & 73.88 \\
\hline 11 & 72.82 & 73.05 & 73.03 & 73.11 & 73.17 & 73.24 & 73.35 & 73.47 & 73.55 & 73.70 & 73.72 & 73.83 \\
\hline 12 & 72.90 & 73.15 & 73.16 & 73.10 & 73.13 & 73.22 & 73.34 & 73.47 & 73.58 & 73.65 & 73.74 & 73.79 \\
\hline 13 & 72.86 & 73.11 & 73.18 & 73.05 & 73.12 & 73.20 & 73.33 & 73.51 & 73.67 & 73.63 & 73.90 & 73.83 \\
\hline 14 & 72.80 & 73.03 & 73.06 & 73.03 & 73.11 & 73.16 & 73.32 & 73.52 & 73.77 & 73.58 & 73.93 & 73.88 \\
\hline 15 & 72.76 & 73.04 & 73.01 & 73.10 & 73.06 & 73.17 & 73.34 & 73.48 & 73.79 & 73.61 & 73.81 & 73.93 \\
\hline 16 & 72.79 & 72.98 & 72.96 & 73.13 & 73.04 & 73.21 & 73.37 & 73.45 & 73.77 & 73.75 & 73.75 & 73.99 \\
\hline 17 & 72.86 & 72.78 & 72.96 & 73.12 & 73.04 & 73.24 & 73.39 & 73.46 & 73.77 & 73.78 & 73.72 & 73.97 \\
\hline 18 & 72.93 & 72.80 & 72.93 & 73.08 & 73.07 & 73.25 & 73.39 & 73.47 & 73.79 & 73.78 & 73.78 & 73.92 \\
\hline 19 & 72.94 & 72.88 & 72.92 & 73.06 & 73.12 & 73.26 & 73.38 & 73.46 & 73.78 & 73.79 & 73.92 & 73.98 \\
\hline 20 & 73.01 & 72.91 & 73.03 & 73.05 & 73.14 & 73.29 & 73.38 & 73.44 & 73.77 & 73.82 & 73.88 & 73.97 \\
\hline 21 & 73.06 & 72.99 & 73.05 & 73.01 & 73.14 & 73.31 & 73.37 & 73.42 & 73.78 & 73.83 & 73.87 & 73.96 \\
\hline 22 & 73.02 & 73.05 & 72.91 & 72.99 & 73.16 & 73.30 & 73.37 & 73.45 & 73.78 & 73.79 & 73.97 & 73.93 \\
\hline 23 & 72.93 & 73.06 & 72.94 & 72.91 & 73.21 & 73.31 & 73.38 & 73.49 & 73.74 & 73.79 & 73.93 & 73.85 \\
\hline 24 & 72.91 & 73.03 & 72.94 & 72.93 & 73.21 & 73.30 & 73.39 & 73.52 & 73.73 & 73.79 & 73.85 & 73.81 \\
\hline 25 & 72.93 & 73.00 & 72.96 & 72.95 & 73.16 & 73.28 & 73.40 & 73.52 & 73.77 & 73.78 & 73.71 & 73.91 \\
\hline 26 & 73.02 & 72.98 & 73.10 & 73.01 & 73.13 & 73.29 & 73.40 & 73.51 & 73.79 & 73.80 & 73.71 & 74.00 \\
\hline 27 & 73.03 & 73.01 & 73.16 & 73.05 & 73.14 & 73.31 & 73.39 & 73.49 & 73.77 & 73.81 & 73.82 & 73.96 \\
\hline 28 & 73.09 & 73.11 & 73.14 & 73.11 & 73.18 & 73.32 & 73.37 & 73.51 & 73.76 & 73.75 & 73.87 & 73.87 \\
\hline 29 & 73.17 & - & 73.12 & 73.15 & 73.21 & - & 73.36 & 73.50 & 73.68 & 73.72 & 73.92 & 73.90 \\
\hline 30 & 73.16 & - & 73.11 & 73.15 & 73.18 & - & 73.36 & 73.47 & 73.69 & 73.79 & 73.89 & 73.94 \\
\hline 31 & 73.14 & - & 73.07 & - & 73.14 & - & 73.38 & 73.46 & - & 73.82 & - & 73.96 \\
\hline Mean & 72.89 & 73.00 & 73.04 & 73.04 & 73.13 & 73.24 & 73.35 & 73.47 & 73.69 & 73.74 & 73.79 & 73.89 \\
\hline Max & 73.17 & 73.15 & 73.18 & 73.15 & 73.21 & 73.32 & 73.40 & 73.52 & 73.79 & 73.84 & 73.97 & 74.00 \\
\hline Min & 72.66 & 72.78 & 72.91 & 72.91 & 73.04 & 73.13 & 73.23 & 73.40 & 73.48 & 73.58 & 73.59 & 73.74 \\
\hline
\end{tabular}




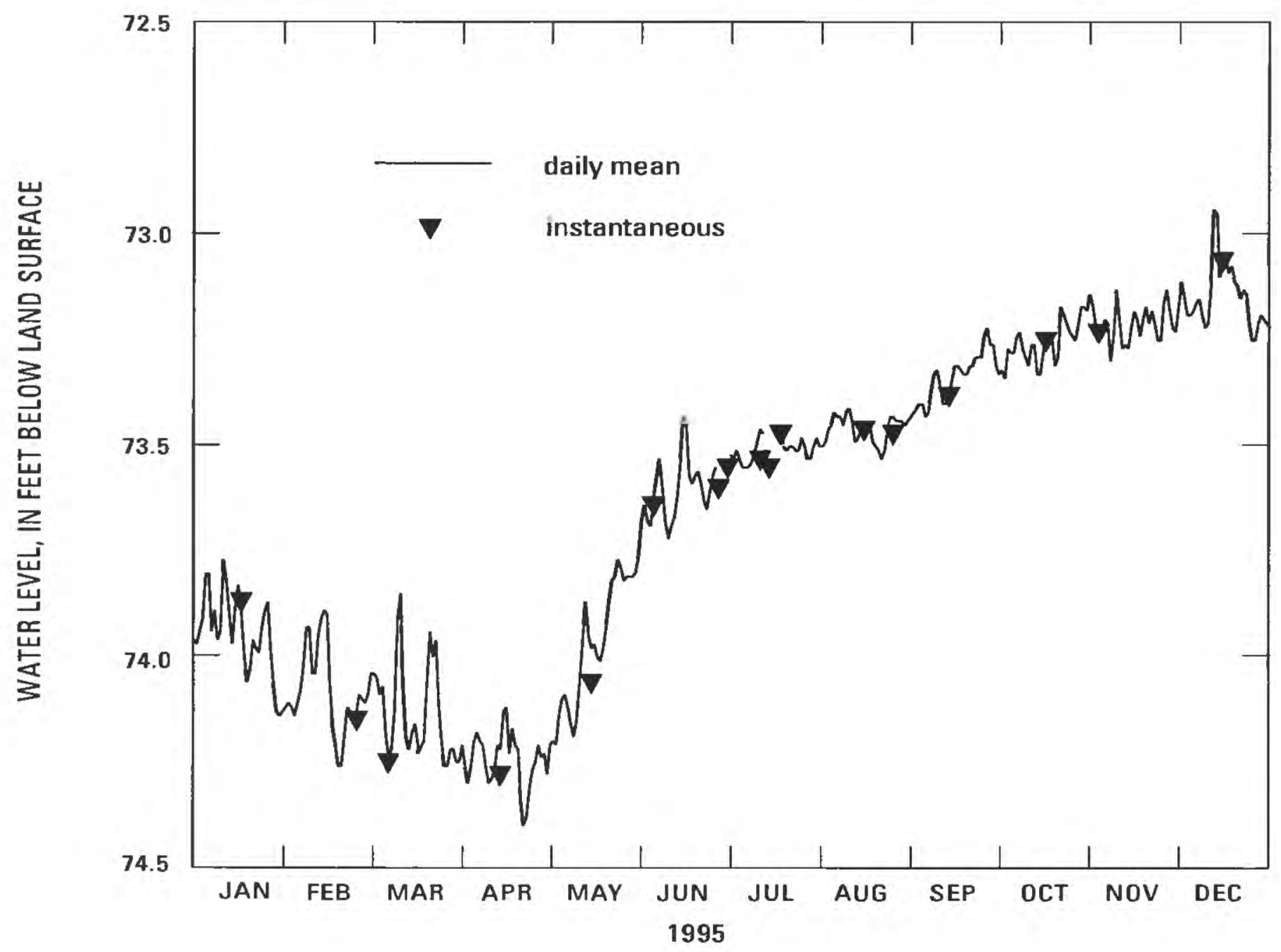

Figure 7. (B) Daily mean and instantaneous water levels, Lookout Mountain well (LKT), 1995, Long Valley Caldera, Mono County, California. 
Table 3. Daily mean water levels, Lookout Mountain well (LKT), 1995, Long Valley Caldera, Mono County, California

[Values are in feet below land surface. —, no data; Max, maximum; Min, minimum]

\begin{tabular}{|c|c|c|c|c|c|c|c|c|c|c|c|c|}
\hline Day & Jan & Feb & Mar & Apr & May & Jun & Jul & Aug & Sep & Oct & Nov & Dec \\
\hline 1 & 73.97 & 74.11 & 74.04 & 74.21 & 74.21 & 73.68 & 73.52 & 73.50 & 73.42 & 73.32 & 73.17 & 73.11 \\
\hline 2 & 73.94 & 74.12 & 74.04 & 74.26 & 74.20 & 73.64 & 73.53 & 73.49 & 73.41 & 73.34 & 73.22 & 73.15 \\
\hline 3 & 73.91 & 74.14 & 74.05 & 74.30 & 74.21 & 73.68 & 73.51 & 73.46 & 73.40 & 73.27 & 73.24 & 73.19 \\
\hline 4 & 73.80 & 74.11 & 74.09 & 74.26 & 74.14 & 73.69 & 73.53 & 73.45 & 73.40 & 73.28 & 73.23 & 73.19 \\
\hline 5 & 73.80 & 74.08 & 74.07 & 74.20 & 74.10 & 73.63 & 73.55 & 73.42 & 73.43 & 73.28 & 73.20 & 73.18 \\
\hline 6 & 73.94 & 74.02 & 74.18 & 74.18 & 74.09 & 73.58 & 73.55 & 73.43 & 73.42 & 73.24 & 73.21 & 73.16 \\
\hline 7 & 73.89 & 73.93 & 74.24 & 74.20 & 74.12 & 73.53 & 73.55 & 73.43 & 73.37 & 73.23 & 73.30 & 73.15 \\
\hline 8 & 73.96 & 73.93 & 74.22 & 74.21 & 74.16 & 73.60 & 73.54 & 73.45 & 73.33 & 73.27 & 73.24 & 73.19 \\
\hline 9 & 73.94 & 74.04 & 74.13 & 74.26 & 74.19 & 73.68 & 73.52 & 73.42 & 73.32 & 73.29 & 73.13 & 73.22 \\
\hline 10 & 73.77 & 74.04 & 73.91 & 74.30 & 74.15 & 73.72 & 73.49 & 73.41 & 73.36 & 73.31 & 73.20 & 73.21 \\
\hline 11 & 73.82 & 73.95 & 73.85 & 74.29 & 74.06 & 73.69 & 73.46 & 73.44 & 73.40 & 73.26 & 73.27 & 73.12 \\
\hline 12 & 73.90 & 73.91 & 74.07 & 74.27 & 73.96 & 73.67 & 73.47 & 73.49 & 73.40 & 73.26 & 73.26 & 72.94 \\
\hline 13 & 73.97 & 73.89 & 74.19 & 74.21 & 73.87 & 73.62 & - & 73.48 & 73.38 & 73.33 & 73.27 & 72.95 \\
\hline 14 & 73.88 & 73.90 & 74.22 & 74.22 & 73.95 & 73.54 & - & 73.46 & 73.34 & 73.33 & 73.22 & 73.10 \\
\hline 15 & 73.83 & 74.07 & 74.18 & 74.13 & 73.98 & 73.43 & - & 73.45 & 73.31 & 73.28 & 73.18 & 73.06 \\
\hline 16 & 73.89 & 74.17 & 74.16 & 74.12 & 73.97 & 73.45 & - & 73.44 & 73.31 & 73.23 & 73.20 & 73.06 \\
\hline 17 & 73.99 & 74.21 & 74.21 & 74.23 & 74.00 & 73.57 & - & 73.45 & 73.32 & 73.26 & 73.24 & 73.09 \\
\hline 18 & 74.06 & 74.26 & 74.23 & 74.17 & 74.01 & 73.59 & - & 73.49 & 73.33 & 73.25 & 73.20 & 73.07 \\
\hline 19 & 74.03 & 74.26 & 74.20 & 74.21 & 73.98 & 73.57 & 73.50 & 73.50 & 73.33 & 73.31 & 73.17 & 73.11 \\
\hline 20 & 73.96 & 74.19 & 74.06 & 74.22 & 73.93 & 73.56 & 73.51 & 73.51 & 73.31 & 73.29 & 73.21 & 73.12 \\
\hline 21 & 73.98 & 74.12 & 73.94 & 74.34 & 73.87 & 73.59 & 73.50 & 73.53 & 73.31 & 73.17 & 73.18 & 73.15 \\
\hline 22 & 73.99 & 74.13 & 74.00 & 74.40 & 73.82 & 73.63 & 73.50 & 73.51 & 73.29 & 73.19 & 73.21 & 73.13 \\
\hline 23 & 73.93 & 74.16 & 73.96 & 74.38 & 73.81 & 73.65 & 73.51 & 73.46 & 73.29 & 73.21 & 73.25 & 73.14 \\
\hline 24 & 73.89 & 74.14 & 74.11 & 74.31 & 73.77 & 73.61 & 73.51 & 73.43 & 73.29 & 73.23 & 73.25 & 73.21 \\
\hline 25 & 73.87 & 74.09 & 74.20 & 74.27 & 73.79 & 73.57 & 73.48 & 73.43 & 73.24 & 73.24 & 73.16 & 73.25 \\
\hline 26 & 73.99 & 74.10 & 74.26 & 74.25 & 73.82 & 73.55 & 73.50 & 73.44 & 73.22 & 73.25 & 73.13 & 73.25 \\
\hline 27 & 74.07 & 74.11 & 74.26 & 74.21 & 73.81 & - & 73.53 & 73.44 & 73.26 & 73.21 & 73.18 & 73.21 \\
\hline 28 & 74.13 & 74.09 & 74.22 & 74.24 & 73.81 & - & 73.53 & 73.44 & 73.26 & 73.17 & 73.22 & 73.19 \\
\hline 29 & 74.14 & - & 74.22 & 74.23 & 73.81 & - & 73.50 & 73.45 & 73.31 & 73.17 & 73.23 & 73.20 \\
\hline 30 & 74.13 & - & 74.25 & 74.28 & 73.80 & - & 73.48 & 73.44 & 73.33 & 73.18 & 73.17 & 73.21 \\
\hline 31 & 74.12 & - & 74.25 & - & 73.76 & - & 73.50 & 73.43 & - & 73.14 & - & 73.22 \\
\hline Mean & 73.95 & 74.08 & 74.13 & 74.25 & 73.97 & 73.60 & 73.51 & 73.46 & 73.34 & 73.25 & 73.21 & 73.15 \\
\hline Max & 74.14 & 74.26 & 74.26 & 74.40 & 74.21 & 73.72 & 73.55 & 73.53 & 73.43 & 73.34 & 73.30 & 73.25 \\
\hline Min & 73.77 & 73.89 & 73.85 & 74.12 & 73.76 & 73.43 & 73.46 & 73.41 & 73.22 & 73.14 & 73.13 & 72.94 \\
\hline
\end{tabular}




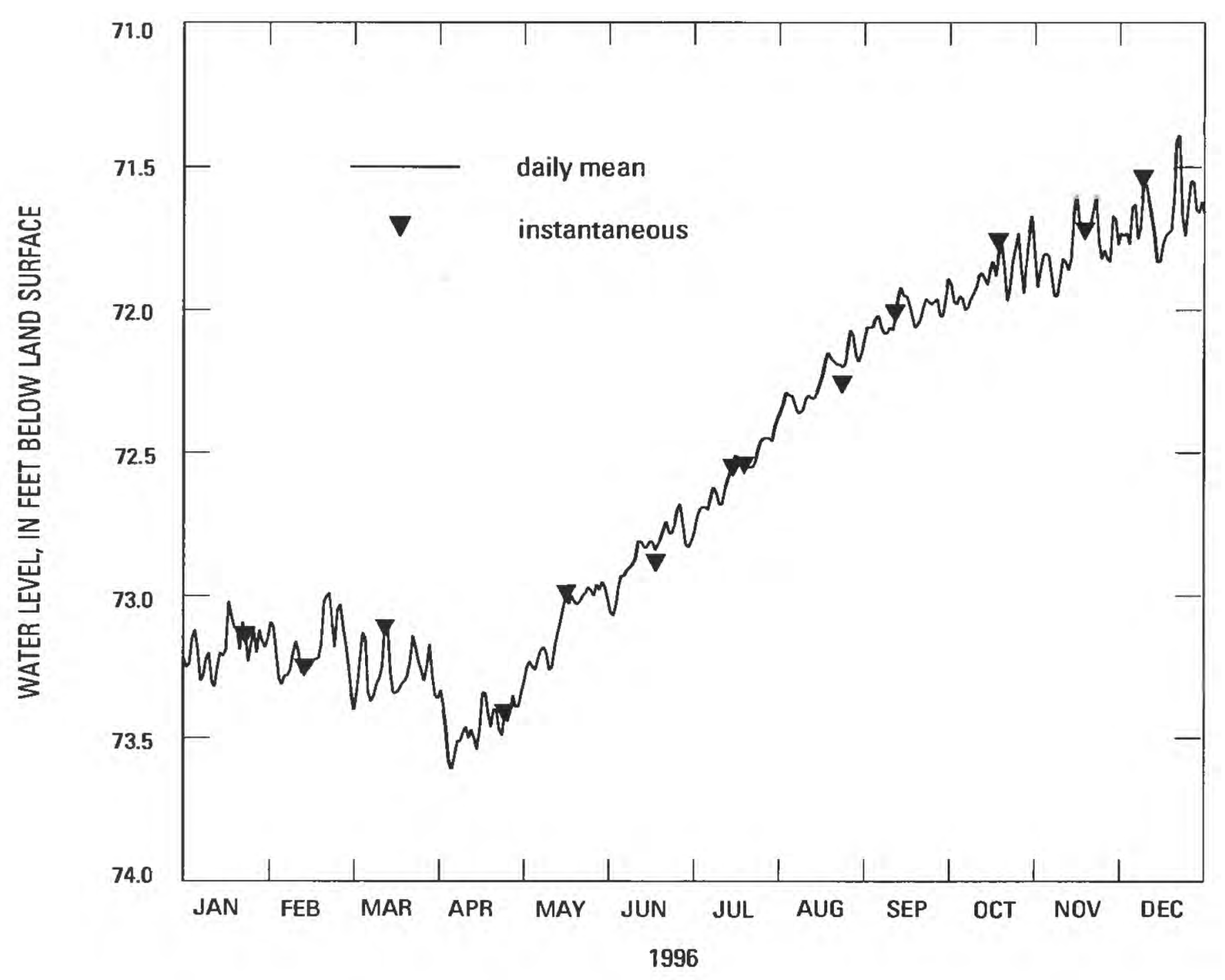

Figure 7. (C) Daily mean and instantaneous water levels, Lookout Mountain well (LKT), 1996, Long Valley Caldera, Mono County, California. 
Table 4. Daily mean water levels, Lookout Mountain well (LKT), 1996, Long Valley Caldera, Mono County, California

[Values are in feet below land surface. —, no data; Max, maximum; Min, minimum]

\begin{tabular}{|c|c|c|c|c|c|c|c|c|c|c|c|c|}
\hline Day & Jan & Feb & Mar & Apr & May & Jun & Jul & Aug & Sep & Oct & Nov & Dec \\
\hline 1 & 73.25 & 73.11 & 73.40 & 73.33 & 73.30 & 73.06 & 72.78 & 72.36 & 72.06 & 71.91 & 71.92 & 71.73 \\
\hline 2 & 73.24 & 73.20 & 73.33 & 73.39 & 73.25 & 73.07 & 72.73 & 72.33 & 72.06 & 71.97 & 71.86 & 71.74 \\
\hline 3 & 73.15 & 73.29 & 73.23 & 73.47 & 73.23 & 73.03 & 72.70 & 72.29 & 72.06 & 71.98 & 71.81 & 71.73 \\
\hline 4 & 73.12 & 73.31 & 73.13 & 73.58 & 73.25 & 72.96 & 72.69 & 72.30 & 72.03 & 71.95 & 71.80 & 71.77 \\
\hline 5 & 73.19 & 73.28 & 73.15 & 73.61 & 73.26 & 72.93 & 72.69 & 72.30 & 72.02 & 71.96 & 71.81 & 71.64 \\
\hline 6 & 73.30 & 73.28 & 73.34 & 73.56 & 73.22 & 72.93 & 72.70 & 72.33 & 72.06 & 72.00 & 71.88 & 71.63 \\
\hline 7 & 73.28 & 73.26 & 73.37 & 73.51 & 73.19 & 72.91 & 72.66 & 72.36 & 72.08 & 71.99 & 71.95 & 71.75 \\
\hline 8 & 73.22 & 73.20 & 73.35 & 73.51 & 73.18 & 72.90 & 72.62 & 72.36 & 72.08 & 71.96 & 71.95 & 71.71 \\
\hline 9 & 73.20 & 73.16 & 73.31 & 73.48 & 73.20 & 72.89 & 72.64 & 72.35 & 72.06 & 71.94 & 71.89 & 71.57 \\
\hline 10 & 73.30 & 73.20 & 73.29 & 73.46 & 73.26 & 72.87 & 72.68 & 72.31 & 72.07 & 71.92 & 71.82 & 71.56 \\
\hline 11 & 73.32 & 73.27 & 73.25 & 73.50 & 73.25 & 72.81 & 72.68 & 72.30 & 72.03 & 71.88 & 71.83 & 71.61 \\
\hline 12 & 73.25 & 73.27 & 73.10 & 73.47 & 73.18 & 72.81 & 72.63 & 72.31 & 71.96 & 71.87 & 71.86 & 71.67 \\
\hline 13 & 73.20 & 73.26 & 73.10 & 73.50 & 73.13 & 72.83 & 72.60 & 72.31 & 71.92 & 71.89 & 71.82 & 71.73 \\
\hline 14 & 73.21 & 73.25 & 73.27 & 73.54 & 73.09 & 72.83 & 72.57 & 72.29 & 71.95 & 71.91 & 71.67 & 71.83 \\
\hline 15 & 73.19 & 73.23 & 73.34 & 73.47 & 73.04 & 72.81 & 72.54 & 72.26 & 71.95 & 71.86 & 71.60 & 71.83 \\
\hline 16 & 73.02 & 73.22 & 73.34 & 73.34 & 73.00 & 72.81 & 72.51 & 72.23 & 71.98 & 71.83 & 71.69 & 71.77 \\
\hline 17 & 73.07 & 73.22 & 73.33 & 73.34 & 73.03 & 72.84 & 72.52 & 72.18 & 72.02 & 71.88 & 71.70 & 71.74 \\
\hline 18 & 73.11 & 73.17 & 73.31 & 73.41 & 73.00 & 72.82 & 72.54 & 72.15 & 72.06 & 71.81 & 71.75 & 71.73 \\
\hline 19 & 73.12 & 73.02 & 73.30 & 73.46 & 73.02 & 72.80 & 72.55 & 72.17 & 72.05 & 71.76 & 71.71 & 71.72 \\
\hline 20 & 73.19 & 73.00 & 73.28 & 73.40 & 73.03 & 72.77 & 72.55 & 72.18 & 72.03 & 71.85 & 71.70 & 71.61 \\
\hline 21 & 73.09 & 72.99 & 73.23 & 73.40 & 73.02 & 72.74 & 72.55 & 72.19 & 71.99 & 71.97 & 71.65 & 71.41 \\
\hline 22 & 73.14 & 73.09 & 73.14 & 73.47 & 73.00 & 72.78 & 72.55 & 72.19 & 71.96 & 71.92 & 71.60 & 71.39 \\
\hline 23 & 73.23 & 73.18 & 73.18 & 73.49 & 72.99 & 72.78 & 72.53 & 72.20 & 71.97 & 71.83 & 71.76 & 71.68 \\
\hline 24 & 73.17 & 73.05 & 73.23 & 73.41 & 72.97 & 72.75 & 72.49 & 72.19 & 71.98 & 71.78 & 71.82 & 71.74 \\
\hline 25 & 73.12 & 73.03 & 73.26 & 73.44 & 72.98 & 72.70 & 72.46 & 72.13 & 71.97 & 71.73 & 71.79 & 71.64 \\
\hline 26 & 73.20 & 73.11 & 73.30 & 73.41 & 73.00 & 72.68 & 72.45 & 72.07 & 71.96 & 71.85 & 71.82 & 71.55 \\
\hline 27 & 73.12 & 73.17 & 73.25 & 73.35 & 72.96 & 72.75 & 72.45 & 72.09 & 72.02 & 71.94 & 71.83 & 71.55 \\
\hline 28 & 73.16 & 73.25 & 73.17 & 73.39 & 72.98 & 72.82 & 72.45 & 72.16 & 72.02 & 71.83 & 71.67 & 71.65 \\
\hline 29 & 73.18 & 73.34 & 73.28 & 73.39 & 72.95 & 72.83 & 72.46 & 72.18 & 71.96 & 71.74 & 71.68 & 71.66 \\
\hline 30 & 73.15 & - & 73.35 & 73.34 & 72.97 & 72.81 & 72.41 & 72.15 & 71.89 & 71.67 & 71.77 & 71.62 \\
\hline 31 & 73.09 & - & 73.36 & - & 73.01 & - & 72.38 & 72.10 & - & 71.80 & - & 71.66 \\
\hline Mean & 73.18 & 73.19 & 73.27 & 73.45 & 73.09 & 72.84 & 72.57 & 72.24 & 72.01 & 71.88 & 71.78 & 71.67 \\
\hline Max & 73.32 & 73.34 & 73.40 & 73.61 & 73.30 & 73.07 & 72.78 & 72.36 & 72.08 & 72.00 & 71.95 & 71.83 \\
\hline Min & 73.02 & 72.99 & 73.10 & 73.33 & 72.95 & 72.68 & 72.38 & 72.07 & 71.89 & 71.67 & 71.60 & 71.39 \\
\hline
\end{tabular}




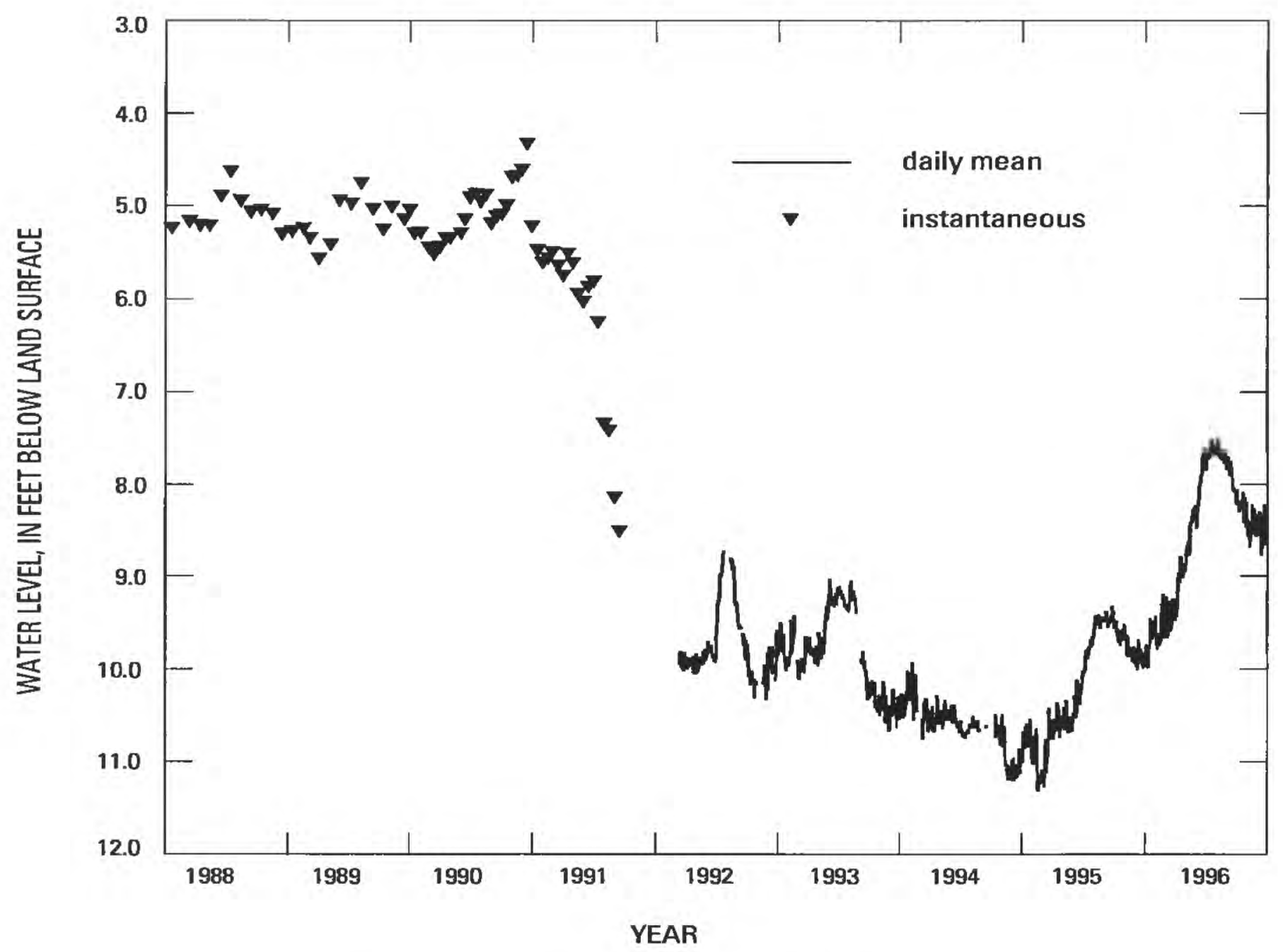

Figure 8. (A) Instantaneous water levels, well CW-3, 1988-91, and daily mean water levels, 1992-96, Long Valley Caldera, Mono County, California. 


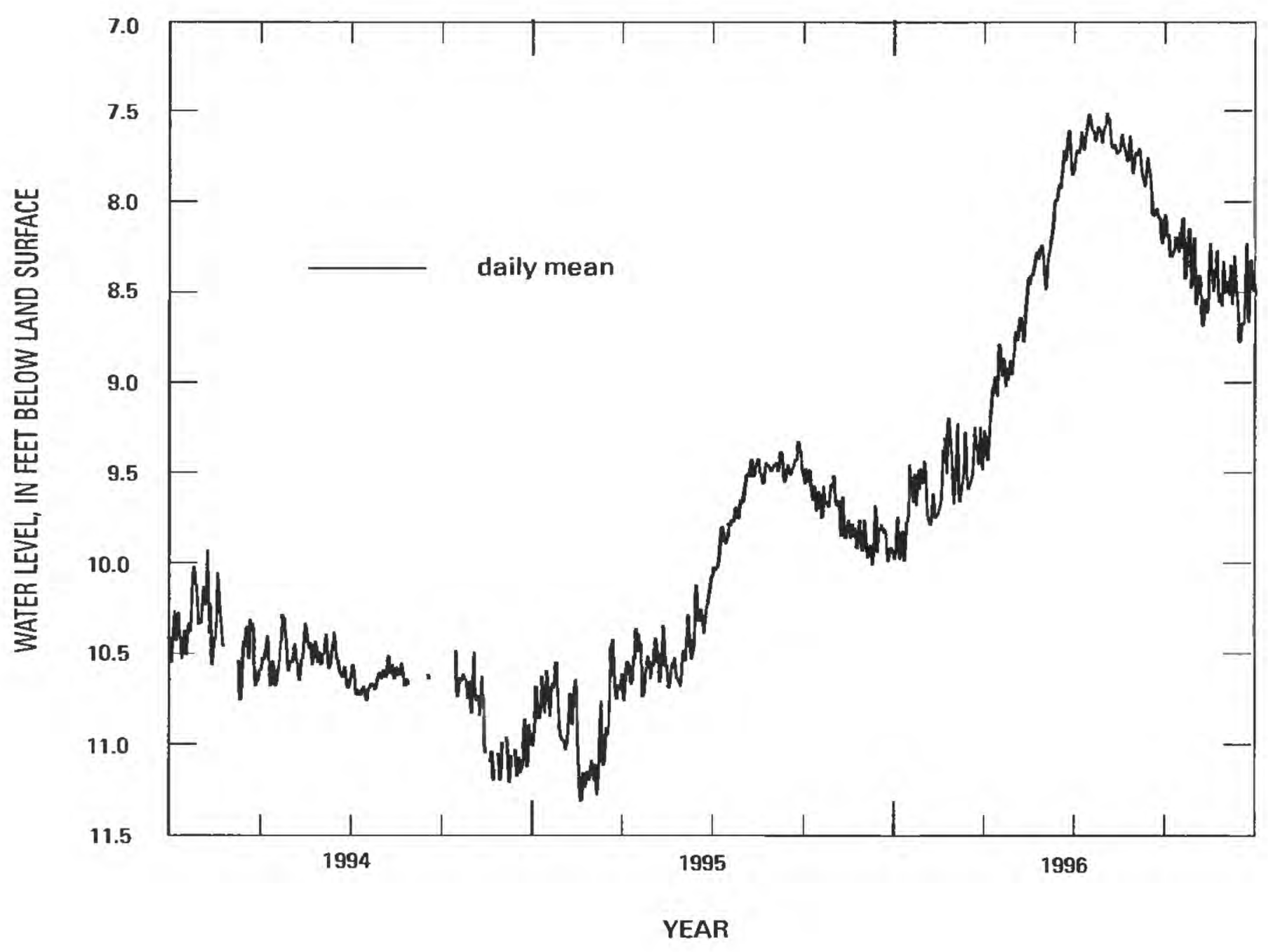

Figure 8. (B) Daily mean water levels, well CW-3, 1994-96, Long Valley Caldera, Mono County, California. 


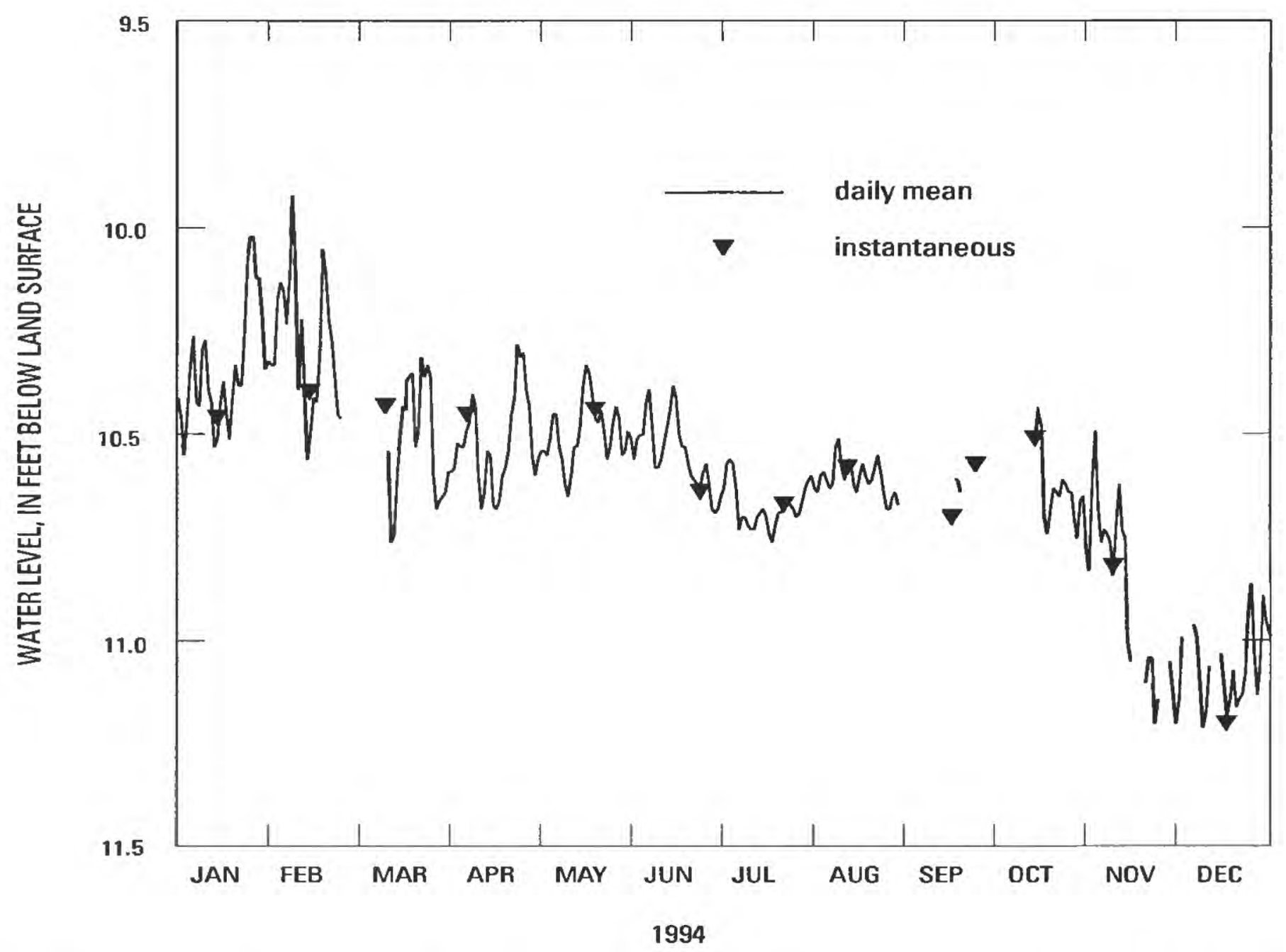

Figure 9. (A) Daily mean and instantaneous water levels, well CW-3, 1994, Long Valley Caldera, Mono County, California. 
Table 5. Daily mean water levels, well CW-3, 1994, Long Valley Caldera, California

[Values are in feet below land surface. —, no data: Max, maximum: Min. minimum]

\begin{tabular}{|c|c|c|c|c|c|c|c|c|c|c|c|c|}
\hline Day & Jan & Feb & Mar & Apr & May & Jun & Jul & Aug & Sep & Oct & Nov & Dec \\
\hline 1 & 10.46 & 10.33 & - & 10.59 & 10.54 & 10.56 & 10.63 & 10.64 & - & - & 10.61 & 10.99 \\
\hline 2 & 10.55 & 10.17 & - & 10.58 & 10.54 & 10.51 & 10.57 & 10.60 & - & - & 10.49 & - \\
\hline 3 & 10.45 & 10.13 & - & 10.52 & 10.55 & 10.50 & 10.56 & 10.59 & - & - & 10.68 & - \\
\hline 4 & 10.33 & 10.15 & - & 10.53 & 10.51 & 10.50 & 10.57 & 10.61 & - & - & 10.76 & - \\
\hline 5 & 10.26 & 10.23 & - & 10.53 & 10.45 & 10.42 & 10.63 & 10.63 & - & - & 10.73 & 10.96 \\
\hline 6 & 10.42 & 10.12 & - & 10.49 & 10.45 & 10.39 & 10.73 & 10.62 & - & - & 10.74 & 10.99 \\
\hline 7 & 10.43 & 9.92 & - & 10.47 & 10.53 & 10.48 & 10.70 & 10.53 & - & - & 10.76 & 11.10 \\
\hline 8 & 10.29 & 10.10 & - & 10.40 & 10.56 & 10.58 & 10.70 & 10.51 & - & - & 10.84 & 11.21 \\
\hline 9 & 10.27 & 10.39 & - & 10.43 & 10.62 & 10.58 & 10.72 & 10.58 & - & - & 10.72 & 11.17 \\
\hline 10 & 10.39 & 10.22 & - & 10.60 & 10.65 & 10.56 & 10.73 & 10.61 & - & - & 10.62 & 11.06 \\
\hline 11 & 10.42 & 10.39 & 10.54 & 10.68 & 10.60 & 10.52 & 10.73 & 10.57 & - & - & 10.73 & - \\
\hline 12 & 10.53 & 10.56 & 10.76 & 10.64 & 10.53 & 10.48 & 10.70 & 10.56 & - & - & 10.75 & - \\
\hline 13 & 10.51 & 10.49 & 10.74 & 10.54 & 10.53 & 10.44 & 10.69 & 10.62 & - & - & 11.00 & - \\
\hline 14 & 10.42 & 10.41 & 10.59 & 10.55 & 10.48 & 10.38 & 10.68 & 10.64 & - & - & 11.05 & 11.03 \\
\hline 15 & 10.37 & 10.42 & 10.50 & 10.67 & 10.38 & 10.41 & 10.70 & 10.60 & - & 10.48 & - & 11.10 \\
\hline 16 & 10.45 & 10.31 & 10.43 & 10.68 & 10.33 & 10.50 & 10.74 & 10.57 & 10.61 & 10.70 & - & 11.18 \\
\hline 17 & 10.51 & 10.05 & 10.44 & 10.66 & 10.35 & 10.53 & 10.76 & 10.60 & 10.61 & 10.74 & - & 11.14 \\
\hline 18 & 10.41 & 10.11 & 10.37 & 10.60 & 10.40 & 10.53 & 10.72 & 10.62 & 10.64 & 10.68 & - & 11.07 \\
\hline 19 & 10.33 & 10.22 & 10.35 & 10.58 & 10.46 & 10.57 & 10.69 & 10.61 & - & 10.63 & 11.10 & 11.16 \\
\hline 20 & 10.38 & 10.27 & 10.53 & 10.54 & 10.47 & 10.60 & 10.69 & 10.58 & - & 10.64 & 11.04 & 11.14 \\
\hline 21 & 10.38 & 10.36 & 10.49 & 10.45 & 10.44 & 10.61 & 10.67 & 10.55 & - & 10.65 & 11.04 & 11.13 \\
\hline 22 & 10.25 & 10.45 & 10.31 & 10.41 & 10.49 & 10.62 & 10.66 & 10.59 & - & 10.61 & 11.20 & 11.08 \\
\hline 23 & 10.08 & 10.46 & 10.36 & 10.28 & 10.56 & 10.63 & 10.67 & 10.64 & - & 10.62 & 11.14 & 10.94 \\
\hline 24 & 10.02 & - & 10.33 & 10.31 & 10.53 & 10.59 & 10.68 & 10.68 & - & 10.64 & - & 10.86 \\
\hline 25 & 10.02 & - & 10.36 & 10.30 & 10.47 & 10.57 & 10.70 & 10.68 & - & 10.64 & - & 11.03 \\
\hline 26 & 10.12 & - & 10.60 & 10.39 & 10.43 & 10.62 & 10.69 & 10.65 & - & 10.71 & - & 11.13 \\
\hline 27 & 10.12 & - & 10.68 & 10.43 & 10.46 & 10.68 & 10.66 & 10.64 & - & 10.75 & 11.05 & 11.05 \\
\hline 28 & 10.21 & - & 10.66 & 10.55 & 10.55 & 10.69 & 10.63 & 10.67 & - & 10.66 & 11.12 & 10.89 \\
\hline 29 & 10.34 & - & 10.65 & 10.60 & 10.54 & 10.68 & 10.61 & - & - & 10.65 & 11.20 & 10.95 \\
\hline 30 & 10.32 & - & 10.64 & 10.56 & 10.49 & 10.65 & 10.60 & - & - & 10.77 & 11.14 & 10.98 \\
\hline 31 & 10.33 & - & 10.59 & - & 10.51 & - & 10.63 & - & - & 10.83 & - & 11.00 \\
\hline Mean & 10.33 & 10.27 & 10.52 & 10.52 & 10.50 & 10.55 & 10.67 & 10.61 & 10.62 & 10.67 & 10.89 & 11.05 \\
\hline Max & 10.55 & 10.56 & 10.76 & 10.68 & 10.65 & 10.69 & 10.76 & 10.68 & 10.64 & 10.83 & 11.20 & 11.21 \\
\hline Min & 10.02 & 9.92 & 10.31 & 10.28 & 10.33 & 10.38 & 10.56 & 10.51 & 10.61 & 10.48 & 10.49 & 10.86 \\
\hline
\end{tabular}




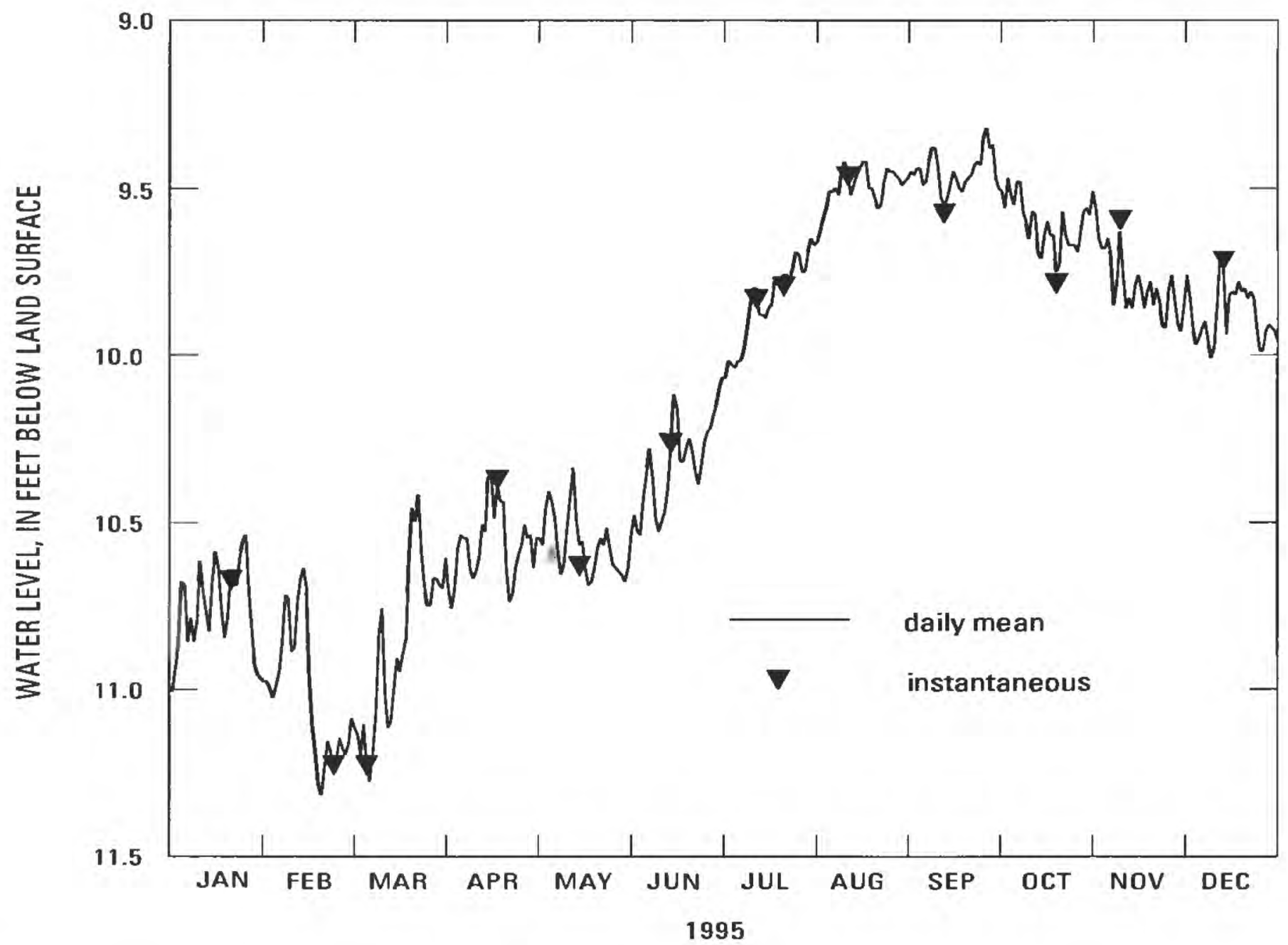

Figure 9. (B) Daily mean and instantaneous water levels, well CW-3, 1995, Long Valley Caldera, Mono County, California. 
Table 6. Daily mean water levels, well CW-3, 1995, Long Valley Caldera, California

[Values are in feet below land surface. —, no data; Max, maximum; Min, minimum]

\begin{tabular}{|c|c|c|c|c|c|c|c|c|c|c|c|c|}
\hline Day & Jan & Feb & Mar & Apr & May & Jun & Jul & Aug & Sep & Oct & Nov & Dec \\
\hline 1 & 11.01 & 10.98 & 11.09 & 10.61 & 10.55 & 10.54 & 10.07 & 9.66 & 9.45 & 9.51 & 9.56 & 9.76 \\
\hline 2 & 10.94 & 11.00 & 11.12 & 10.71 & 10.55 & 10.48 & 10.07 & 9.63 & 9.46 & 9.56 & 9.65 & 9.83 \\
\hline 3 & 10.87 & 11.03 & 11.14 & 10.76 & 10.57 & 10.53 & 10.02 & 9.59 & 9.44 & 9.47 & 9.68 & 9.92 \\
\hline 4 & 10.68 & 10.99 & 11.20 & 10.70 & 10.46 & 10.54 & 10.03 & 9.56 & 9.44 & 9.52 & 9.68 & 9.97 \\
\hline 5 & 10.69 & 10.95 & 11.11 & 10.58 & 10.41 & 10.43 & 10.04 & 9.51 & 9.49 & 9.55 & 9.65 & 9.95 \\
\hline 6 & 10.86 & 10.86 & 11.23 & 10.54 & 10.44 & 10.36 & 10.02 & 9.51 & 9.48 & 9.48 & 9.70 & 9.92 \\
\hline 7 & 10.79 & 10.72 & 11.28 & 10.55 & 10.49 & 10.28 & 10.02 & 9.50 & 9.42 & 9.48 & 9.85 & 9.90 \\
\hline 8 & 10.86 & 10.73 & 11.20 & 10.55 & 10.59 & 10.37 & 9.99 & 9.52 & 9.38 & 9.57 & 9.78 & 9.96 \\
\hline 9 & 10.80 & 10.89 & 11.08 & 10.64 & 10.66 & 10.49 & 9.93 & 9.45 & 9.38 & 9.60 & 9.63 & 10.01 \\
\hline 10 & 10.62 & 10.88 & 10.84 & 10.67 & 10.63 & 10.53 & 9.86 & 9.42 & 9.44 & 9.65 & 9.76 & 9.98 \\
\hline 11 & 10.71 & 10.74 & 10.76 & 10.65 & 10.52 & 10.50 & 9.80 & 9.46 & 9.53 & 9.57 & 9.86 & 9.85 \\
\hline 12 & 10.77 & 10.67 & 11.01 & 10.61 & 10.43 & 10.47 & 9.80 & 9.52 & 9.55 & 9.58 & 9.83 & 9.68 \\
\hline 13 & 10.83 & 10.64 & 11.12 & 10.51 & 10.34 & 10.40 & 9.88 & 9.49 & 9.53 & 9.69 & 9.86 & 9.74 \\
\hline 14 & 10.68 & 10.70 & 11.10 & 10.53 & 10.49 & 10.27 & 9.88 & 9.46 & 9.49 & 9.71 & 9.79 & 9.94 \\
\hline 15 & 10.59 & 10.96 & 11.00 & 10.37 & 10.57 & 10.12 & 9.89 & 9.44 & 9.45 & 9.64 & 9.76 & 9.82 \\
\hline 16 & 10.64 & 11.11 & 10.91 & 10.36 & 10.56 & 10.16 & 9.86 & 9.42 & 9.47 & 9.60 & 9.80 & 9.81 \\
\hline 17 & 10.76 & 11.19 & 10.95 & 10.49 & 10.64 & 10.32 & 9.85 & 9.42 & 9.50 & 9.64 & 9.86 & 9.82 \\
\hline 18 & 10.85 & 11.29 & 10.94 & 10.39 & 10.69 & 10.32 & 9.78 & 9.50 & 9.51 & 9.64 & 9.81 & 9.78 \\
\hline 19 & 10.79 & 11.32 & 10.85 & 10.44 & 10.68 & 10.28 & 9.79 & 9.50 & 9.48 & 9.75 & 9.78 & 9.81 \\
\hline 20 & 10.66 & 11.24 & 10.61 & 10.44 & 10.64 & 10.25 & 9.79 & 9.53 & 9.47 & 9.73 & 9.85 & 9.80 \\
\hline 21 & 10.69 & 11.16 & 10.46 & 10.64 & 10.58 & 10.29 & 9.76 & 9.56 & 9.46 & 9.57 & 9.80 & 9.83 \\
\hline 22 & 10.69 & 11.19 & 10.50 & 10.74 & 10.55 & 10.35 & 9.76 & 9.55 & 9.43 & 9.64 & 9.84 & 9.81 \\
\hline 23 & 10.61 & 11.24 & 10.42 & 10.72 & 10.57 & 10.39 & 9.77 & 9.48 & 9.42 & 9.67 & 9.91 & 9.83 \\
\hline 24 & 10.56 & 11.21 & 10.59 & 10.64 & 10.52 & 10.33 & 9.74 & 9.44 & 9.43 & 9.67 & 9.92 & 9.92 \\
\hline 25 & 10.54 & 11.15 & 10.69 & 10.60 & 10.58 & 10.26 & 9.69 & 9.45 & 9.35 & 9.67 & 9.80 & 9.99 \\
\hline 26 & 10.71 & 11.18 & 10.75 & 10.57 & 10.63 & 10.23 & 9.70 & 9.45 & 9.32 & 9.69 & 9.76 & 9.99 \\
\hline 27 & 10.83 & 11.20 & 10.75 & 10.51 & 10.64 & 10.22 & 9.75 & 9.46 & 9.38 & 9.63 & 9.84 & 9.93 \\
\hline 28 & 10.93 & 11.17 & 10.67 & 10.55 & 10.65 & 10.18 & 9.75 & 9.47 & 9.37 & 9.57 & 9.91 & 9.91 \\
\hline 29 & 10.96 & - & 10.67 & 10.54 & 10.66 & 10.15 & 9.69 & 9.49 & 9.47 & 9.56 & 9.93 & 9.92 \\
\hline 30 & 10.97 & - & 10.69 & 10.64 & 10.68 & 10.10 & 9.65 & 9.48 & 9.50 & 9.58 & 9.85 & 9.93 \\
\hline 31 & 10.98 & - & 10.70 & - & 10.64 & - & 9.67 & 9.47 & - & 9.51 & - & 9.96 \\
\hline Mean & 10.77 & 11.01 & 10.88 & 10.57 & 10.57 & 10.34 & 9.85 & 9.50 & 9.45 & 9.60 & 9.79 & 9.88 \\
\hline Max & 11.01 & 11.32 & 11.28 & 10.76 & 10.69 & 10.54 & 10.07 & 9.66 & 9.55 & 9.75 & 9.93 & 10.01 \\
\hline Min & 10.54 & 10.64 & 10.42 & 10.36 & 10.34 & 10.10 & 9.65 & 9.42 & 9.32 & 9.47 & 9.56 & 9.68 \\
\hline
\end{tabular}




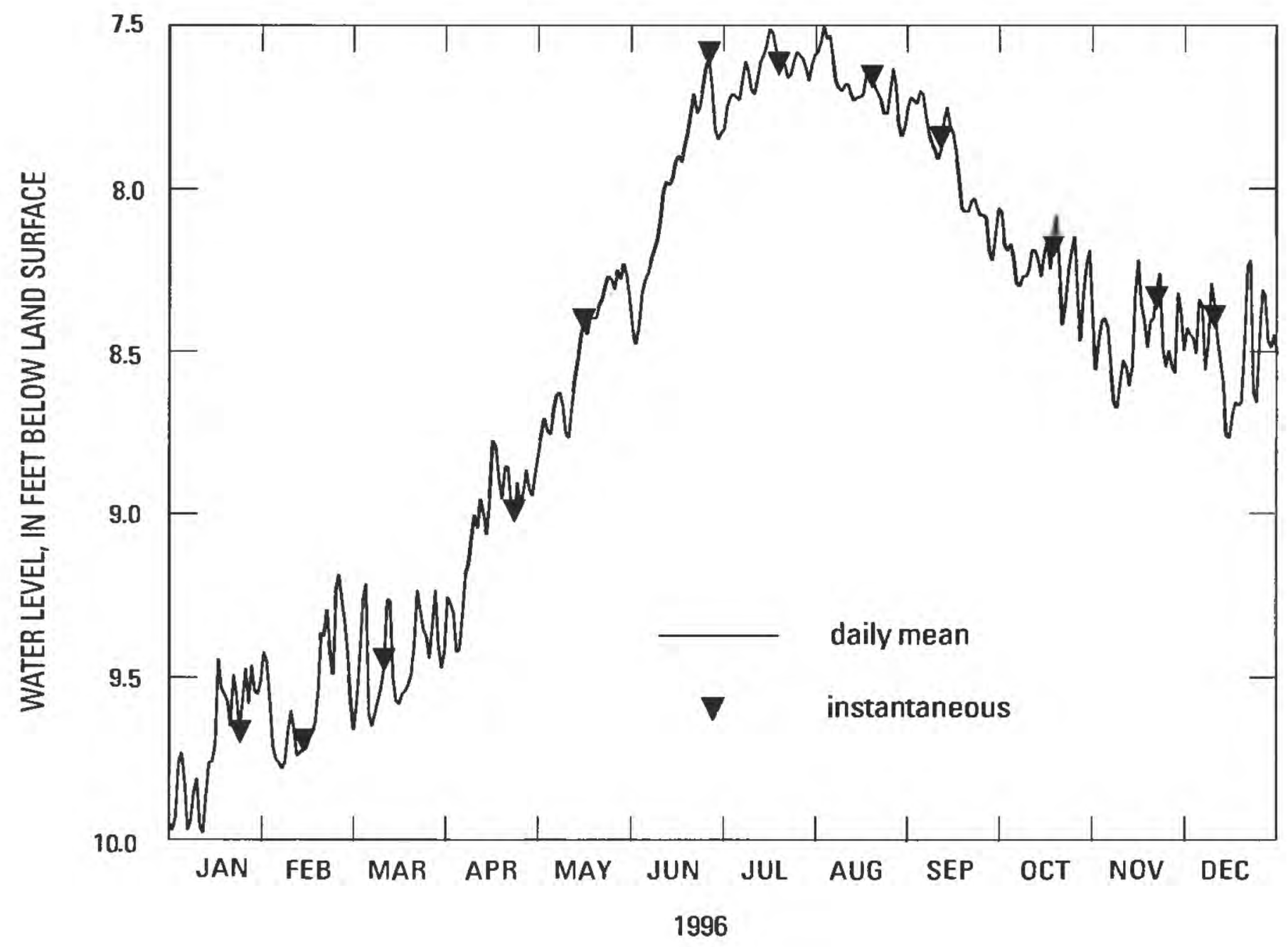

Figure 9. (C) Daily mean and instantaneous water levels, well CW-3, 1996, Long Valley Caldera, Mono County, California. 
Table 7. Daily mean water levels, well CW-3, 1996, Long Valley Caldera, California

[Values are in feet below land surface. —, no data; Max, maximum; Min, minimum]

\begin{tabular}{|c|c|c|c|c|c|c|c|c|c|c|c|c|}
\hline Day & Jan & Feb & Mar & Apr & May & Jun & Jul & Aug & Sep & Oct & Nov & Dec \\
\hline 1 & 9.98 & 9.46 & 9.67 & 9.26 & 8.82 & 8.44 & 7.82 & 7.58 & 7.72 & 8.07 & 8.56 & 8.43 \\
\hline 2 & 9.94 & 9.58 & 9.58 & 9.28 & 8.76 & 8.48 & 7.75 & 7.55 & 7.73 & 8.17 & 8.47 & 8.45 \\
\hline 3 & 9.77 & 9.72 & 9.44 & 9.31 & 8.71 & 8.42 & 7.72 & 7.50 & 7.74 & 8.19 & 8.41 & 8.46 \\
\hline 4 & 9.74 & 9.76 & 9.27 & 9.43 & 8.75 & 8.32 & 7.71 & 7.54 & 7.70 & 8.17 & 8.40 & 8.51 \\
\hline 5 & 9.83 & 9.77 & 9.22 & 9.42 & 8.76 & 8.28 & 7.72 & 7.53 & 7.71 & 8.21 & 8.43 & 8.34 \\
\hline 6 & 9.98 & 9.79 & 9.62 & 9.31 & 8.69 & 8.26 & 7.73 & 7.60 & 7.78 & 8.29 & 8.55 & 8.36 \\
\hline 7 & 9.95 & 9.77 & 9.66 & 9.19 & 8.64 & 8.22 & 7.67 & 7.67 & 7.83 & 8.30 & 8.66 & 8.56 \\
\hline 8 & 9.86 & 9.68 & 9.62 & 9.16 & 8.63 & 8.19 & 7.61 & 7.69 & 7.86 & 8.27 & 8.68 & 8.48 \\
\hline 9 & 9.82 & 9.61 & 9.58 & 9.08 & 8.67 & 8.16 & 7.64 & 7.70 & 7.88 & 8.27 & 8.60 & 8.29 \\
\hline 10 & 9.97 & 9.67 & 9.54 & 9.01 & 8.76 & 8.10 & 7.70 & 7.68 & 7.91 & 8.25 & 8.53 & 8.35 \\
\hline 11 & 9.99 & 9.75 & 9.48 & 9.05 & 8.77 & 8.01 & 7.71 & 7.68 & 7.88 & 8.19 & 8.55 & 8.46 \\
\hline 12 & 9.87 & 9.74 & 9.27 & 8.96 & 8.67 & 7.98 & 7.66 & 7.71 & 7.79 & 8.19 & 8.61 & 8.53 \\
\hline 13 & 9.77 & 9.73 & 9.27 & 9.01 & 8.59 & 7.99 & 7.61 & 7.73 & 7.75 & 8.22 & 8.55 & 8.60 \\
\hline 14 & 9.77 & 9.73 & 9.51 & 9.07 & 8.53 & 7.97 & 7.59 & 7.72 & 7.81 & 8.27 & 8.32 & 8.76 \\
\hline 15 & 9.71 & 9.70 & 9.58 & 8.95 & 8.46 & 7.92 & 7.56 & 7.72 & 7.83 & 8.20 & 8.22 & 8.77 \\
\hline 16 & 9.45 & 9.68 & 9.59 & 8.78 & 8.41 & 7.90 & 7.51 & 7.71 & 7.89 & 8.16 & 8.36 & 8.70 \\
\hline 17 & 9.54 & 9.65 & 9.56 & 8.80 & 8.45 & 7.92 & 7.52 & 7.65 & 7.97 & 8.25 & 8.41 & 8.66 \\
\hline 18 & 9.56 & 9.55 & 9.55 & 8.90 & 8.40 & 7.87 & 7.57 & 7.62 & 8.06 & 8.14 & 8.49 & 8.67 \\
\hline 19 & 9.58 & 9.37 & 9.53 & 8.96 & 8.40 & 7.83 & 7.60 & 7.66 & 8.07 & 8.08 & 8.41 & 8.66 \\
\hline 20 & 9.66 & 9.38 & 9.50 & 8.86 & 8.40 & 7.77 & 7.60 & 7.69 & 8.07 & 8.24 & 8.40 & 8.49 \\
\hline 21 & 9.50 & 9.30 & 9.41 & 8.86 & 8.36 & 7.71 & 7.62 & 7.71 & 8.04 & 8.42 & 8.31 & 8.24 \\
\hline 22 & 9.56 & 9.43 & 9.24 & 8.99 & 8.34 & 7.77 & 7.66 & 7.73 & 8.03 & 8.35 & 8.26 & 8.22 \\
\hline 23 & 9.68 & 9.50 & 9.30 & 9.02 & 8.30 & 7.75 & 7.65 & 7.77 & 8.06 & 8.25 & 8.49 & 8.63 \\
\hline 24 & 9.58 & 9.24 & 9.36 & 8.91 & 8.27 & 7.69 & 7.61 & 7.77 & 8.08 & 8.19 & 8.55 & 8.66 \\
\hline 25 & 9.48 & 9.19 & 9.38 & 8.98 & 8.28 & 7.63 & 7.58 & 7.70 & 8.08 & 8.14 & 8.50 & 8.47 \\
\hline 26 & 9.59 & 9.27 & 9.45 & 8.94 & 8.31 & 7.60 & 7.59 & 7.63 & 8.09 & 8.34 & 8.55 & 8.31 \\
\hline 27 & 9.47 & 9.34 & 9.35 & 8.87 & 8.25 & 7.69 & 7.60 & 7.68 & 8.19 & 8.47 & 8.57 & 8.33 \\
\hline 28 & 9.55 & 9.44 & 9.24 & 8.93 & 8.28 & 7.82 & 7.63 & 7.80 & 8.22 & 8.32 & 8.32 & 8.47 \\
\hline 29 & 9.56 & 9.58 & 9.41 & 8.95 & 8.23 & 7.85 & 7.67 & 7.84 & 8.14 & 8.23 & 8.38 & 8.49 \\
\hline 30 & 9.52 & - & 9.48 & 8.88 & 8.27 & 7.83 & 7.62 & 7.81 & 8.06 & 8.19 & 8.50 & 8.45 \\
\hline 31 & 9.43 & - & 9.43 & - & 8.35 & - & 7.59 & 7.75 & - & 8.40 & - & 8.51 \\
\hline Mean & 9.70 & 9.56 & 9.45 & 9.04 & 8.50 & 7.98 & 7.64 & 7.68 & 7.93 & 8.24 & 8.47 & 8.49 \\
\hline Max & 9.99 & 9.79 & 9.67 & 9.43 & 8.82 & 8.48 & 7.82 & 7.84 & 8.22 & 8.47 & 8.68 & 8.77 \\
\hline Min & 9.43 & 9.19 & 9.22 & 8.78 & 8.23 & 7.60 & 7.51 & 7.50 & 7.70 & 8.07 & 8.22 & 8.22 \\
\hline
\end{tabular}




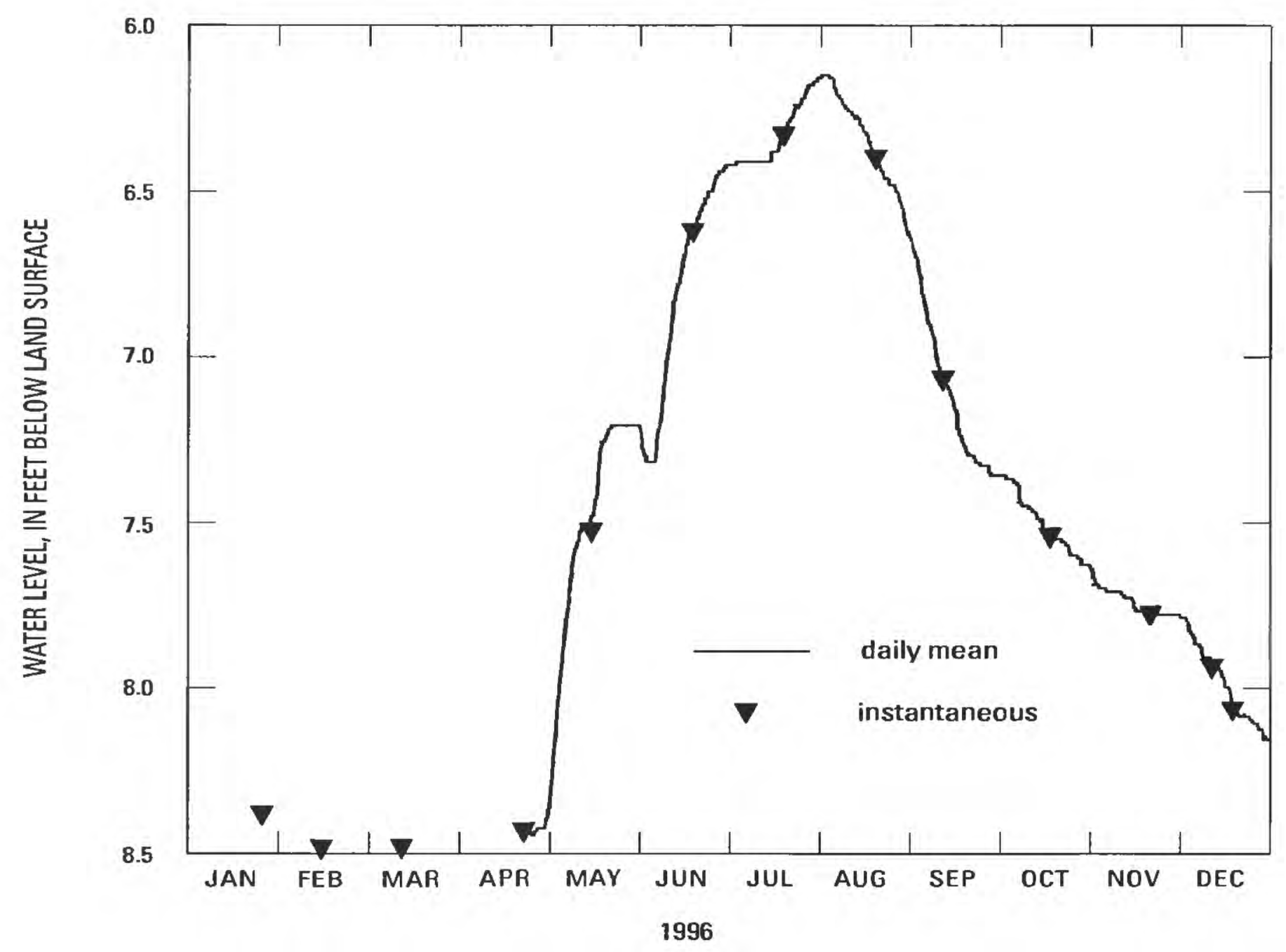

Figure 10. Daily mean and instantaneous water levels, well ESN, 1996, Long Valley Caldera, Mono County, California. 
Table 8. Daily mean water levels, well ESN, 1996, Long Valley Caldera, California

[Values are in feet below land surface. - , no data; Max, maximum; Min, minimum]

\begin{tabular}{|c|c|c|c|c|c|c|c|c|c|c|c|c|}
\hline Day & Jan & Feb & Mar & Apr & May & Jun & Jul & Aug & Sep & Oct & Nov & Dec \\
\hline 1 & - & - & - & - & 8.38 & 7.23 & 6.42 & 6.16 & 6.66 & 7.36 & 7.65 & 7.79 \\
\hline 2 & - & - & - & - & 8.30 & 7.29 & 6.42 & 6.15 & 6.69 & 7.36 & 7.68 & 7.79 \\
\hline 3 & - & - & - & - & 8.19 & 7.31 & 6.42 & 6.15 & 6.72 & 7.37 & 7.69 & 7.80 \\
\hline 4 & - & - & - & - & 8.08 & 7.32 & 6.41 & 6.16 & 6.78 & 7.37 & 7.70 & 7.82 \\
\hline 5 & - & - & - & - & 7.97 & 7.32 & 6.41 & 6.17 & 6.83 & 7.37 & 7.70 & 7.84 \\
\hline 6 & - & - & - & - & 7.88 & 7.31 & 6.41 & 6.20 & 6.87 & 7.38 & 7.71 & 7.86 \\
\hline 7 & - & - & - & - & 7.80 & 7.23 & 6.41 & 6.21 & 6.90 & 7.40 & 7.71 & 7.87 \\
\hline 8 & - & - & - & - & 7.72 & 7.19 & 6.41 & 6.23 & 6.93 & 7.44 & 7.71 & 7.89 \\
\hline 9 & - & - & - & - & 7.63 & 7.10 & $6.4 I$ & 6.24 & 6.98 & 7.45 & 7.71 & 7.91 \\
\hline 10 & - & - & - & - & 7.58 & 7.01 & 6.41 & 6.25 & 7.03 & 7.45 & 7.71 & 7.91 \\
\hline 11 & - & - & - & - & 7.55 & 6.95 & 6.41 & 6.26 & 7.07 & 7.46 & 7.72 & 7.93 \\
\hline 12 & - & - & - & - & 7.52 & 6.87 & 6.41 & 6.27 & 7.09 & 7.47 & 7.73 & 7.94 \\
\hline 13 & - & - & - & - & 7.51 & 6.81 & 6.41 & 6.28 & 7.09 & 7.48 & 7.73 & 7.94 \\
\hline 14 & - & - & - & - & 7.51 & 6.78 & 6.41 & 6.29 & 7.11 & 7.49 & 7.73 & 7.95 \\
\hline 15 & - & - & - & - & 7.50 & 6.73 & 6.40 & 6.31 & 7.14 & 7.51 & 7.75 & 7.97 \\
\hline 16 & - & - & - & - & 7.47 & 6.69 & 6.38 & 6.32 & 7.18 & 7.53 & 7.77 & 7.99 \\
\hline 17 & - & - & - & - & 7.43 & 6.65 & 6.38 & 6.34 & 7.23 & 7.53 & 7.77 & 8.00 \\
\hline 18 & - & - & - & - & 7.33 & 6.61 & 6.36 & 6.37 & 7.25 & 7.54 & 7.77 & 8.04 \\
\hline 19 & - & - & - & - & 7.27 & 6.61 & 6.33 & 6.39 & 7.28 & 7.55 & 7.78 & 8.08 \\
\hline 20 & - & - & - & - & 7.25 & 6.59 & 6.31 & 6.41 & 7.30 & 7.55 & 7.78 & 8.08 \\
\hline 21 & - & - & - & - & 7.23 & 6.57 & 6.30 & 6.43 & 7.30 & 7.55 & 7.79 & 8.09 \\
\hline 22 & - & - & - & - & 7.22 & 6.54 & 6.28 & 6.45 & 7.30 & 7.56 & 7.78 & 8.09 \\
\hline 23 & - & - & - & 8.43 & 7.21 & 6.52 & 6.26 & 6.46 & 7.32 & 7.57 & 7.78 & 8.09 \\
\hline 24 & - & - & - & 8.44 & 7.21 & 6.51 & 6.24 & 6.47 & 7.33 & 7.58 & 7.78 & 8.09 \\
\hline 25 & - & - & - & 8.45 & 7.21 & 6.50 & 6.24 & 6.48 & 7.33 & 7.60 & 7.78 & 8.10 \\
\hline 26 & - & - & - & 8.45 & 7.21 & 6.47 & 6.22 & 6.49 & 7.33 & 7.60 & 7.78 & 8.11 \\
\hline 27 & - & - & - & 8.43 & 7.21 & 6.45 & 6.20 & 6.51 & 7.34 & 7.61 & 7.78 & 8.12 \\
\hline 28 & - & - & - & 8.43 & 7.21 & 6.44 & 6.18 & 6.53 & 7.36 & 7.62 & 7.78 & 8.13 \\
\hline 29 & - & - & - & 8.43 & 7.21 & 6.44 & 6.18 & 6.57 & 7.36 & 7.63 & 7.78 & 8.15 \\
\hline 30 & - & - & - & 8.42 & 7.21 & 6.42 & 6.17 & 6.61 & 7.36 & 7.63 & 7.78 & 8.16 \\
\hline 31 & - & - & - & - & 7.21 & - & 6.16 & 6.63 & - & 7.63 & - & 8.16 \\
\hline Mean & - & - & - & 8.43 & 7.52 & 6.82 & 6.33 & 6.35 & 7.12 & 7.50 & 7.74 & 7.99 \\
\hline Max & - & - & - & 8.45 & 8.38 & 7.32 & 6.42 & 6.63 & 7.36 & 7.63 & 7.79 & 8.16 \\
\hline Min & - & - & - & 8.42 & 7.21 & 6.42 & 6.16 & 6.15 & 6.66 & 7.36 & 7.65 & 7.79 \\
\hline
\end{tabular}




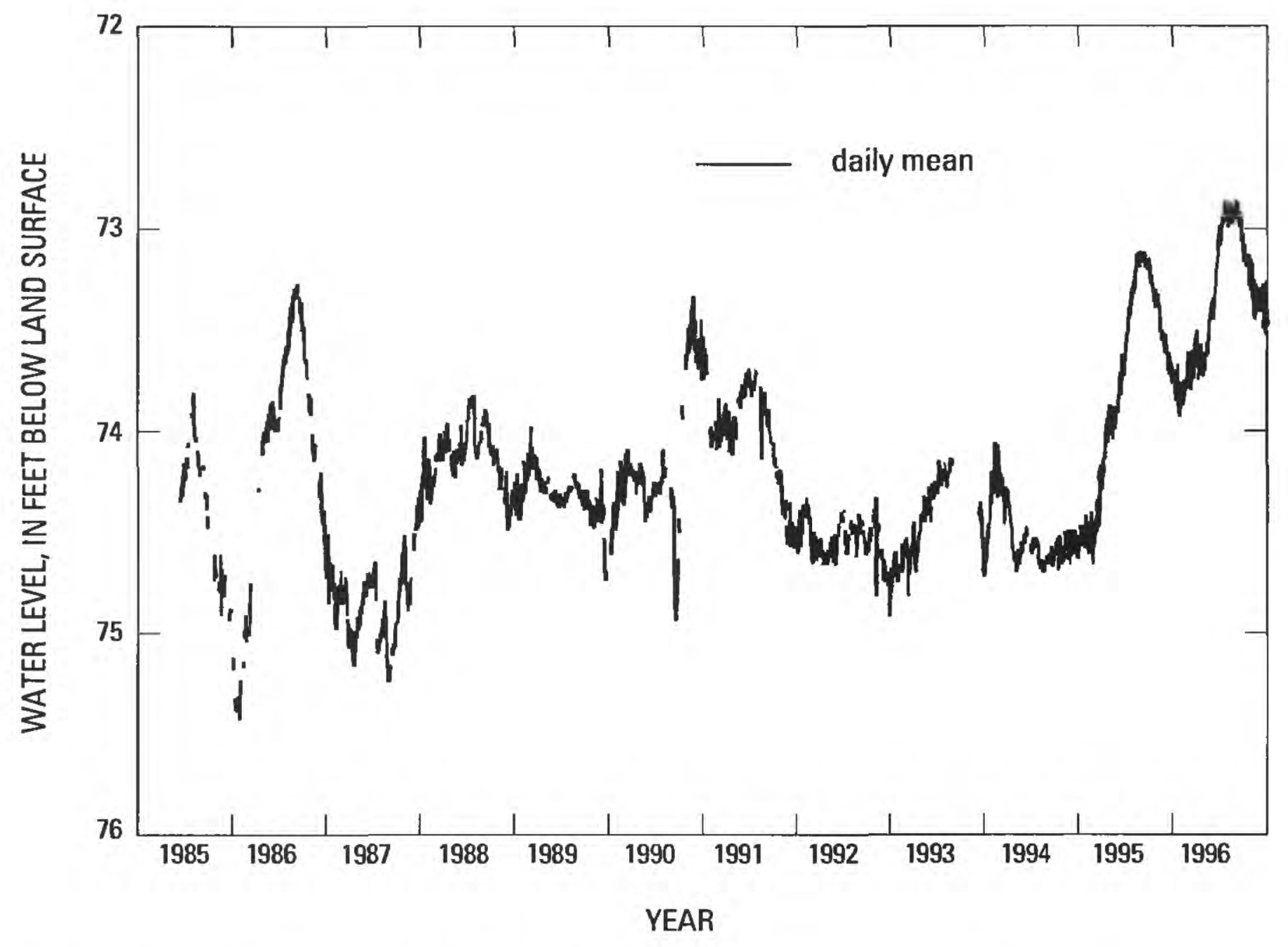

Figure 11. (A) Daily mean water levels, well $\mathrm{CH}-10 \mathrm{~B}$, 1985-96, Long Valley Caldera, Mono County, California. 


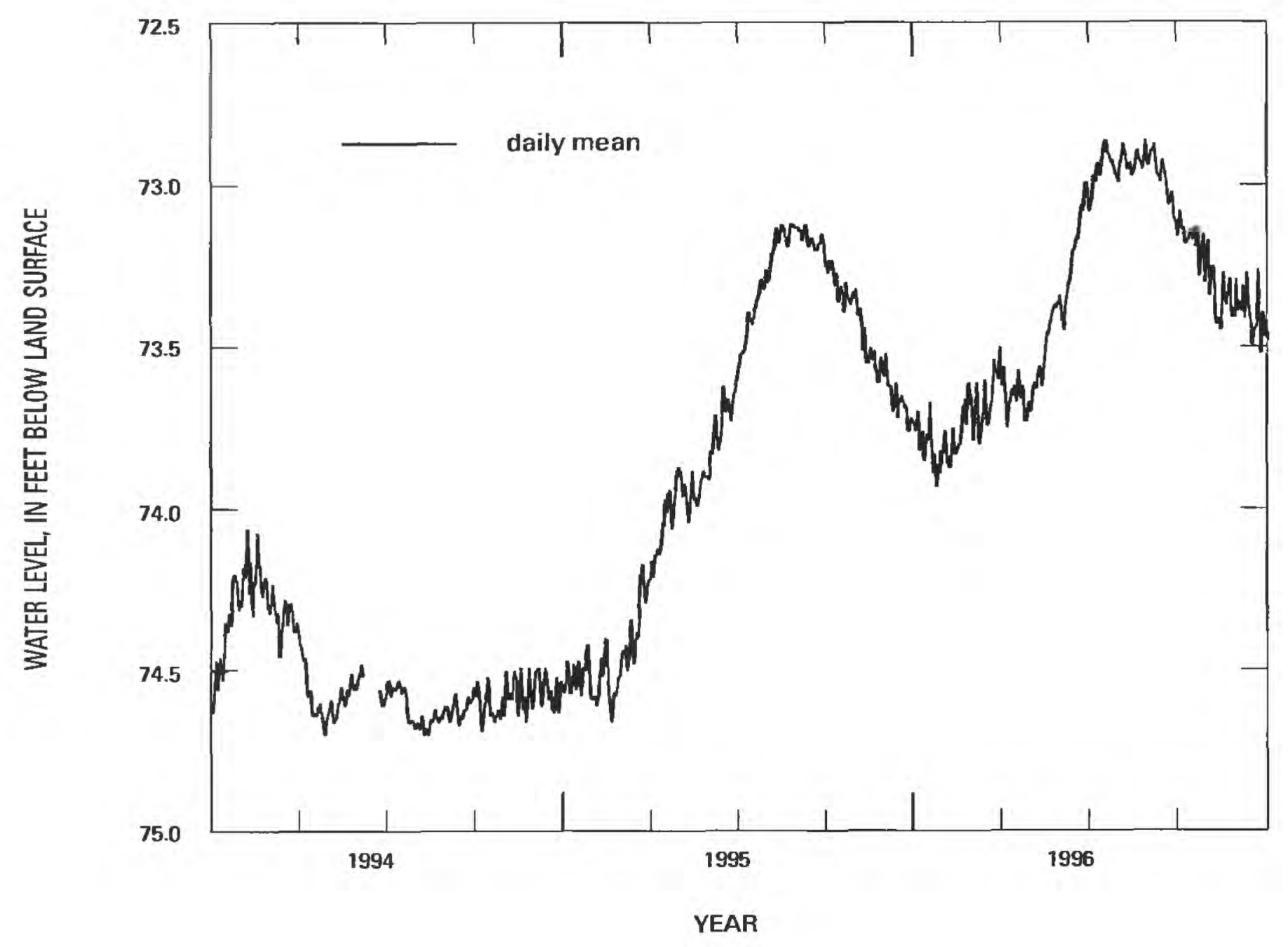

Figure 11. (B) Daily mean water levels, well CH-10B, 1994-96, Long Valley Caldera, Mono County, California. 


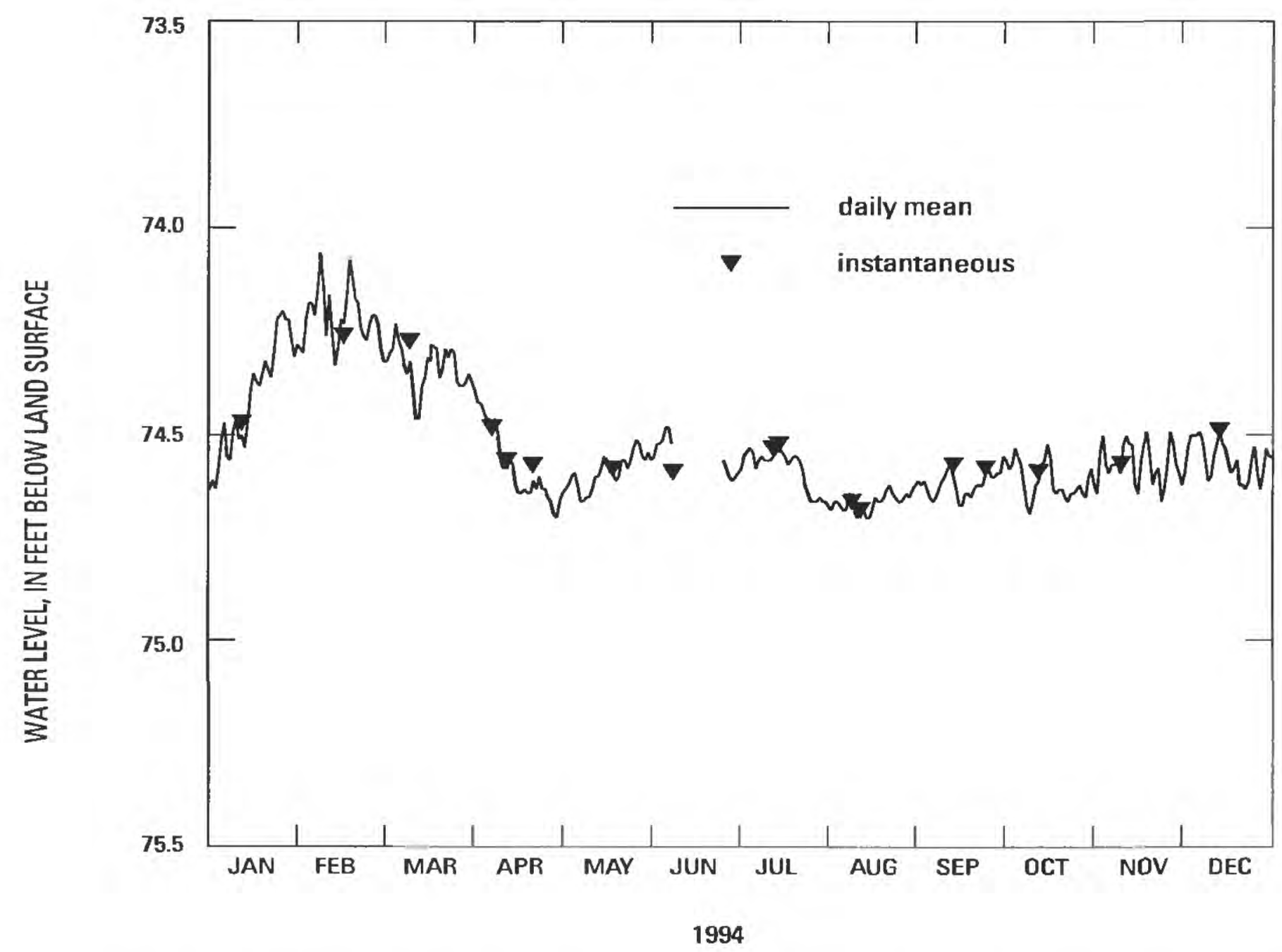

Figure 12. (A) Daily mean and instantaneous water levels, well CH-10B, 1994, Long Valley Caldera, Mono County, California. 
Table 9. Daily mean water levels, well CH-10B, 1994, Long Valley Caldera, California

[Values are in feet below land surface. —, no data; Max, maximum; Min, minimum]

\begin{tabular}{|c|c|c|c|c|c|c|c|c|c|c|c|c|}
\hline Day & Jan & Feb & Mar & Apr & May & Jun & Jul & Aug & Sep & Oct & Nov & Dec \\
\hline 1 & 74.61 & 74.30 & 74.32 & 74.39 & 74.64 & 74.55 & 74.58 & 74.68 & 74.62 & 74.58 & 74.54 & 74.53 \\
\hline 2 & 74.63 & 74.22 & 74.32 & 74.42 & 74.63 & 74.52 & 74.55 & 74.66 & 74.61 & 74.57 & 74.50 & 74.50 \\
\hline 3 & 74.58 & 74.18 & 74.30 & 74.42 & 74.62 & 74.52 & 74.54 & 74.66 & 74.63 & 74.53 & 74.57 & 74.50 \\
\hline 4 & 74.51 & 74.18 & 74.29 & 74.44 & 74.60 & 74.51 & 74.53 & 74.67 & 74.65 & 74.55 & 74.59 & 74.50 \\
\hline 5 & 74.47 & 74.21 & 74.23 & 74.46 & 74.59 & 74.48 & 74.54 & 74.68 & 74.66 & 74.57 & 74.57 & 74.49 \\
\hline 6 & 74.55 & 74.15 & 74.27 & 74.46 & 74.62 & 74.48 & 74.58 & 74.68 & 74.65 & 74.62 & 74.57 & 74.51 \\
\hline 7 & 74.56 & 74.06 & 74.29 & 74.47 & 74.66 & 74.52 & 74.56 & 74.64 & 74.63 & 74.67 & 74.57 & 74.56 \\
\hline 8 & 74.49 & 74.14 & 74.33 & 74.46 & 74.66 & - & 74.55 & 74.65 & 74.61 & 74.69 & 74.59 & 74.61 \\
\hline 9 & 74.46 & 74.26 & 74.35 & 74.50 & 74.65 & - & 74.56 & 74.68 & 74.60 & 74.66 & 74.52 & 74.60 \\
\hline 10 & 74.51 & 74.16 & 74.32 & 74.56 & 74.65 & - & 74.56 & 74.70 & 74.58 & 74.62 & 74.50 & 74.55 \\
\hline 11 & 74.50 & 74.25 & 74.38 & 74.58 & 74.63 & - & 74.56 & 74.68 & 74.57 & 74.61 & 74.52 & 74.52 \\
\hline 12 & 74.53 & 74.33 & 74.46 & 74.58 & 74.60 & - & 74.55 & 74.68 & 74.60 & 74.57 & 74.52 & 74.49 \\
\hline 13 & 74.47 & 74.29 & 74.46 & 74.56 & 74.60 & - & 74.54 & 74.70 & 74.63 & 74.56 & 74.62 & 74.52 \\
\hline 14 & 74.39 & 74.22 & 74.38 & 74.57 & 74.58 & - & 74.53 & 74.70 & 74.67 & 74.52 & 74.64 & 74.53 \\
\hline 15 & 74.35 & 74.23 & 74.36 & 74.62 & 74.55 & - & 74.54 & 74.68 & 74.67 & 74.55 & 74.56 & 74.56 \\
\hline 16 & 74.37 & 74.17 & 74.31 & 74.64 & 74.56 & - & 74.55 & 74.65 & 74.64 & 74.63 & 74.52 & 74.59 \\
\hline 17 & 74.38 & 74.07 & 74.32 & 74.64 & 74.59 & 一 & 74.57 & 74.66 & 74.64 & 74.64 & 74.49 & 74.58 \\
\hline 18 & 74.35 & 74.12 & 74.28 & 74.63 & 74.59 & - & 74.56 & 74.66 & 74.65 & 74.63 & 74.55 & 74.56 \\
\hline 19 & 74.32 & 74.17 & 74.29 & 74.64 & 74.61 & - & 74.55 & 74.65 & 74.63 & 74.63 & 74.62 & 74.62 \\
\hline 20 & 74.34 & 74.18 & 74.36 & 74.64 & 74.60 & - & 74.55 & 74.63 & 74.62 & 74.65 & 74.59 & 74.62 \\
\hline 21 & 74.36 & 74.24 & 74.34 & 74.61 & 74.56 & - & 74.56 & 74.62 & 74.62 & 74.66 & 74.58 & 74.63 \\
\hline 22 & 74.30 & 74.26 & 74.29 & 74.63 & 74.56 & - & 74.58 & 74.64 & 74.62 & 74.64 & 74.66 & 74.61 \\
\hline 23 & 74.22 & 74.27 & 74.31 & 74.60 & 74.58 & - & 74.62 & 74.65 & 74.59 & 74.64 & 74.63 & 74.56 \\
\hline 24 & 74.21 & 74.23 & 74.29 & 74.63 & 74.56 & - & 74.64 & 74.66 & 74.58 & 74.63 & 74.57 & 74.52 \\
\hline 25 & 74.20 & 74.21 & 74.30 & 74.63 & 74.53 & 74.56 & 74.66 & 74.66 & 74.60 & 74.62 & 74.49 & 74.59 \\
\hline 26 & 74.22 & 74.21 & 74.37 & 74.65 & 74.51 & 74.58 & 74.66 & 74.65 & 74.60 & 74.64 & 74.52 & 74.63 \\
\hline 27 & 74.22 & 74.23 & 74.38 & 74.66 & 74.52 & 74.60 & 74.66 & 74.64 & 74.59 & 74.65 & 74.57 & 74.59 \\
\hline 28 & 74.27 & 74.29 & 74.38 & 74.69 & 74.55 & 74.61 & 74.65 & 74.65 & 74.59 & 74.60 & 74.59 & 74.53 \\
\hline 29 & 74.31 & - & 74.37 & 74.70 & 74.56 & 74.60 & 74.66 & 74.63 & 74.55 & 74.58 & 74.62 & 74.55 \\
\hline 30 & 74.28 & - & 74.35 & 74.66 & 74.54 & 74.59 & 74.66 & 74.62 & 74.56 & 74.62 & 74.59 & 74.55 \\
\hline 31 & 74.29 & - & 74.37 & - & 74.56 & - & 74.67 & 74.61 & - & 74.64 & - & 74.56 \\
\hline Mean & 74.40 & 74.21 & 74.33 & 74.57 & 74.59 & 74.55 & 74.58 & 74.66 & 74.62 & 74.61 & 74.57 & 74.56 \\
\hline Max & 74.63 & 74.33 & 74.46 & 74.70 & 74.66 & 74.61 & 74.67 & 74.70 & 74.67 & 74.69 & 74.66 & 74.63 \\
\hline Min & 74.20 & 74.06 & 74.23 & 74.39 & 74.51 & 74.48 & 74.53 & 74.61 & 74.55 & 74.52 & 74.49 & 74.49 \\
\hline
\end{tabular}




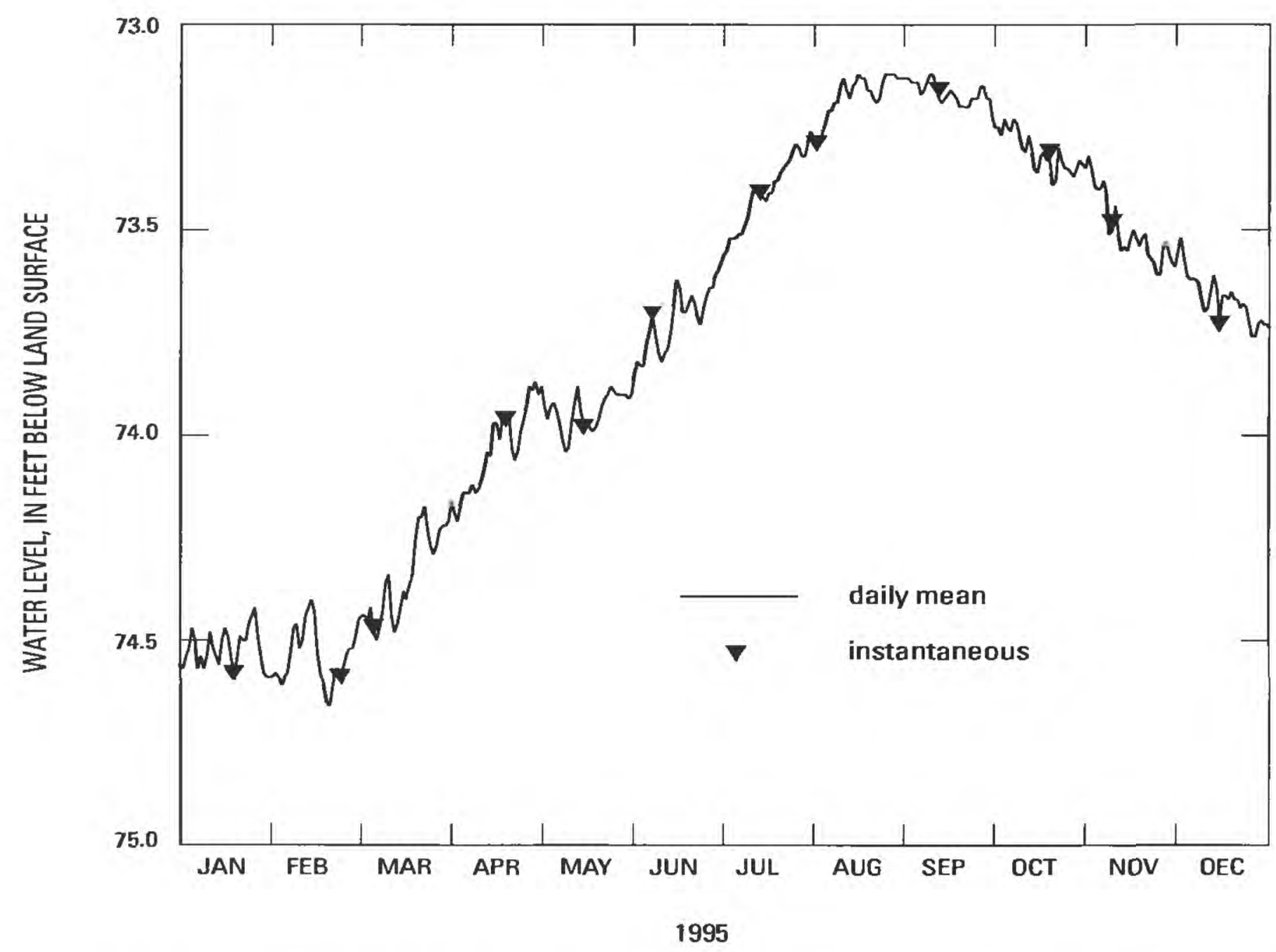

Figure 12. (B) Daily mean and instantaneous water levels, well CH-10B, 1995, Long Valley Caldera, Mono County, California. 
Table 10. Daily mean water levels, well CH-10B, 1995, Long Valley Caldera, California

[Values are in feet below land surface. - , no data; Max, maximum; Min, minimum]

\begin{tabular}{|c|c|c|c|c|c|c|c|c|c|c|c|c|}
\hline Day & Jan & Feb & Mar & Apr & May & Jun & Jul & Aug & Sep & Oct & Nov & Dec \\
\hline 1 & 74.57 & 74.58 & 74.45 & 74.16 & 73.88 & 73.85 & 73.56 & 73.30 & 73.13 & 73.25 & 73.35 & 73.52 \\
\hline 2 & 74.54 & 74.59 & 74.44 & 74.19 & 73.93 & 73.82 & 73.55 & 73.29 & 73.14 & 73.27 & 73.39 & 73.57 \\
\hline 3 & 74.52 & 74.61 & 74.44 & 74.21 & 73.96 & 73.83 & 73.52 & 73.26 & 73.14 & 73.23 & 73.40 & 73.61 \\
\hline 4 & 74.47 & 74.59 & 74.45 & 74.17 & 73.93 & 73.83 & 73.52 & 73.24 & 73.14 & 73.25 & 73.40 & 73.62 \\
\hline 5 & 74.50 & 74.58 & 74.42 & 74.14 & 73.92 & 73.78 & 73.52 & 73.21 & 73.17 & 73.26 & 73.38 & 73.62 \\
\hline 6 & 74.57 & 74.53 & 74.48 & 74.14 & 73.94 & 73.75 & 73.51 & 73.21 & 73.16 & 73.23 & 73.41 & 73.62 \\
\hline 7 & 74.54 & 74.47 & 74.50 & 74.14 & 73.97 & 73.71 & 73.51 & 73.19 & 73.14 & 73.24 & 73.51 & 73.63 \\
\hline 8 & 74.57 & 74.46 & 74.47 & 74.12 & 74.01 & 73.76 & 73.49 & 73.19 & 73.12 & 73.27 & 73.50 & 73.67 \\
\hline 9 & 74.54 & 74.52 & 74.43 & 74.14 & 74.04 & 73.80 & 73.47 & 73.15 & 73.12 & 73.30 & 73.44 & 73.70 \\
\hline 10 & 74.48 & 74.50 & 74.36 & 74.13 & 74.03 & 73.82 & 73.43 & 73.13 & 73.15 & 73.31 & 73.51 & 73.69 \\
\hline 11 & 74.52 & 74.44 & 74.34 & 74.11 & 73.97 & 73.80 & 73.40 & 73.16 & 73.18 & 73.27 & 73.55 & 73.65 \\
\hline 12 & 74.54 & 74.42 & 74.44 & 74.08 & 73.92 & 73.79 & 73.39 & 73.18 & 73.19 & 73.30 & 73.54 & 73.61 \\
\hline 13 & 74.56 & 74.40 & 74.48 & 74.04 & 73.88 & 73.75 & 73.42 & 73.15 & 73.18 & 73.35 & 73.55 & 73.64 \\
\hline 14 & 74.50 & 74.43 & 74.46 & 74.05 & 73.94 & 73.69 & 73.42 & 73.14 & 73.17 & 73.36 & 73.52 & 73.72 \\
\hline 15 & 74.47 & 74.53 & 74.42 & 73.97 & 73.97 & 73.62 & 73.43 & 73.12 & 73.16 & 73.32 & 73.50 & 73.66 \\
\hline 16 & 74.49 & 74.58 & 74.38 & 73.97 & 73.96 & 73.64 & 73.41 & 73.13 & 73.17 & 73.31 & 73.52 & 73.66 \\
\hline 17 & 74.54 & 74.61 & 74.40 & 74.01 & 73.98 & 73.70 & 73.41 & 73.13 & 73.18 & 73.33 & 73.54 & 73.67 \\
\hline 18 & 74.58 & 74.65 & 74.39 & 73.95 & 73.99 & 73.70 & 73.38 & 73.16 & 73.20 & 73.33 & 73.52 & 73.65 \\
\hline 19 & 74.55 & 74.66 & 74.34 & 73.96 & 73.98 & 73.68 & 73.38 & 73.16 & 73.20 & 73.39 & 73.51 & 73.67 \\
\hline 20 & 74.49 & 74.61 & 74.25 & 73.94 & 73.96 & 73.66 & 73.36 & 73.18 & 73.20 & 73.38 & 73.56 & 73.67 \\
\hline 21 & 74.50 & 74.57 & 74.20 & 74.03 & 73.93 & 73.68 & 73.35 & 73.19 & 73.20 & 73.30 & 73.57 & 73.69 \\
\hline 22 & 74.50 & 74.57 & 74.20 & 74.06 & 73.91 & 73.71 & 73.34 & 73.18 & 73.18 & 73.33 & 73.58 & 73.68 \\
\hline 23 & 74.46 & 74.58 & 74.17 & 74.04 & 73.90 & 73.73 & 73.33 & 73.14 & 73.18 & 73.35 & 73.61 & 73.69 \\
\hline 24 & 74.44 & 74.56 & 74.23 & 73.99 & 73.88 & 73.69 & 73.31 & 73.12 & 73.18 & 73.35 & 73.61 & 73.73 \\
\hline 25 & 74.42 & 74.53 & 74.27 & 73.96 & 73.89 & 73.66 & 73.29 & 73.12 & 73.15 & 73.36 & 73.54 & 73.76 \\
\hline 26 & 74.49 & 74.52 & 74.29 & 73.93 & 73.90 & 73.64 & 73.30 & 73.12 & 73.15 & 73.37 & 73.53 & 73.76 \\
\hline 27 & 74.54 & 74.52 & 74.27 & 73.88 & 73.90 & 73.64 & 73.32 & 73.12 & 73.18 & 73.35 & 73.56 & 73.73 \\
\hline 28 & 74.58 & 74.49 & 74.23 & 73.89 & 73.90 & 73.61 & 73.32 & 73.13 & 73.18 & 73.33 & 73.58 & 73.72 \\
\hline 29 & 74.59 & - & 74.22 & 73.87 & 73.90 & 73.60 & 73.29 & 73.13 & 73.23 & 73.34 & 73.59 & 73.73 \\
\hline 30 & 74.59 & - & 74.22 & 73.90 & 73.91 & 73.58 & 73.26 & 73.13 & 73.25 & 73.35 & 73.55 & 73.73 \\
\hline 31 & 74.59 & - & 74.21 & - & 73.90 & - & 73.28 & 73.13 & - & 73.32 & - & 73.74 \\
\hline Mean & 74.52 & 74.54 & 74.35 & 74.04 & 73.94 & 73.72 & 73.40 & 73.17 & 73.17 & 73.31 & 73.51 & 73.67 \\
\hline Max & 74.59 & 74.66 & 74.50 & 74.21 & 74.04 & 73.85 & 73.56 & 73.30 & 73.25 & 73.39 & 73.61 & 73.76 \\
\hline Min & 74.42 & 74.40 & 74.17 & 73.87 & 73.88 & 73.58 & 73.26 & 73.12 & 73.12 & 73.23 & 73.35 & 73.52 \\
\hline
\end{tabular}




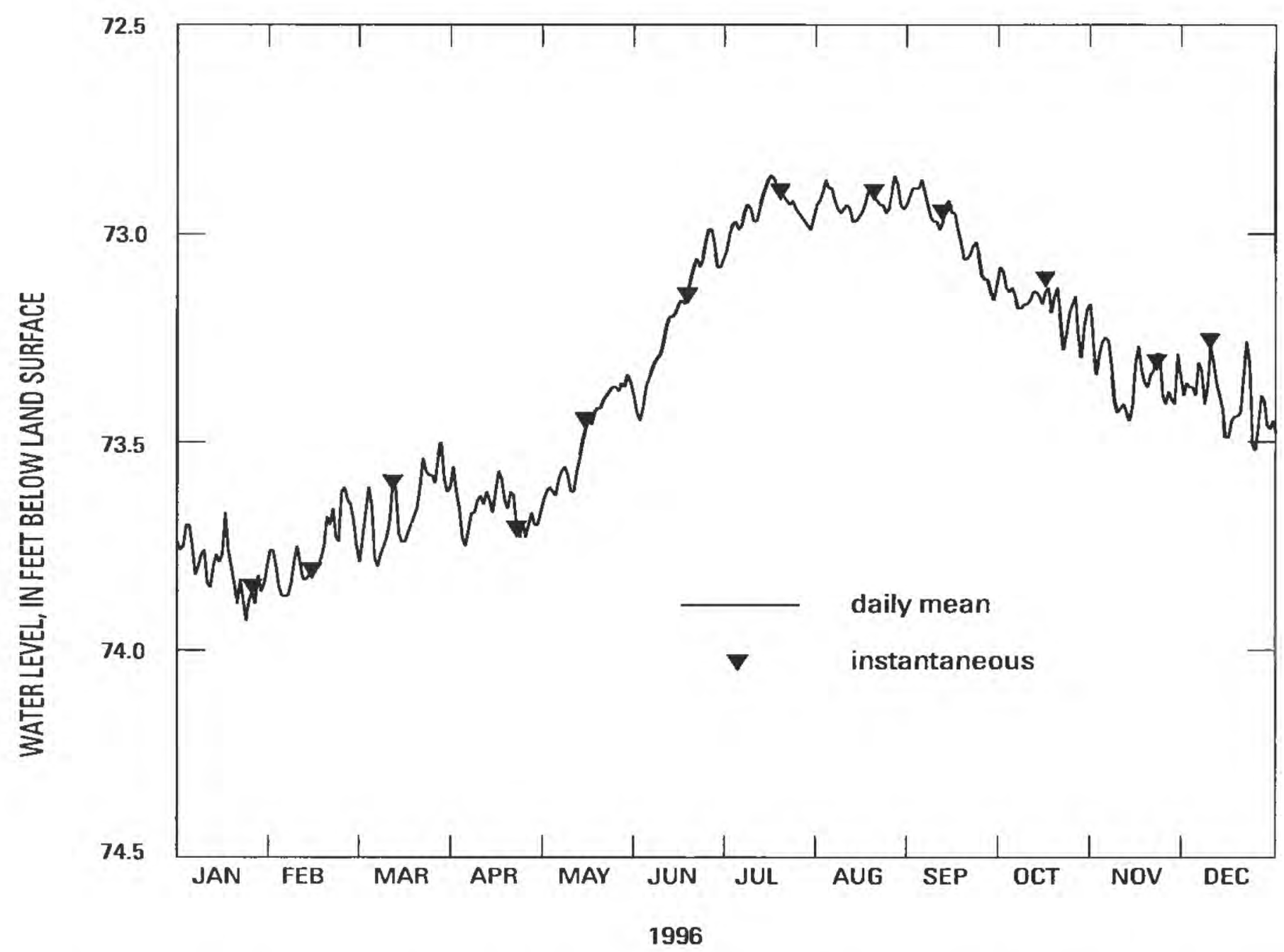

Figure 12. (C) Daily mean and instantaneous water levels, well $\mathrm{CH}-10 \mathrm{~B}$, 1996, Long Valley Caldera, Mono County, California. 
Table 11. Daily mean water levels, well $\mathrm{CH}-10 \mathrm{~B}, 1996$, Long Valley Caldera, California

[Values are in feet below land surface. —, no data; Max, maximum; Min, minimum]

\begin{tabular}{|c|c|c|c|c|c|c|c|c|c|c|c|c|}
\hline Day & Jan & Feb & Mar & Apr & May & Jun & Jul & Aug & Sep & Oct & Nov & Dec \\
\hline 1 & 73.76 & 73.76 & 73.79 & 73.56 & 73.64 & 73.43 & 73.04 & 72.92 & 72.89 & 73.09 & 73.34 & 73.36 \\
\hline 2 & 73.75 & 73.80 & 73.73 & 73.62 & 73.62 & 73.45 & 73.00 & 72.90 & 72.89 & 73.13 & 73.29 & 73.37 \\
\hline 3 & 73.70 & 73.85 & 73.67 & 73.66 & 73.61 & 73.42 & 72.98 & 72.87 & 72.89 & 73.14 & 73.26 & 73.37 \\
\hline 4 & 73.70 & 73.87 & 73.61 & 73.73 & 73.62 & 73.37 & 72.97 & 72.89 & 72.87 & 73.13 & 73.25 & 73.39 \\
\hline 5 & 73.75 & 73.87 & 73.65 & 73.75 & 73.63 & 73.35 & 72.99 & 72.89 & 72.90 & 73.15 & 73.26 & 73.31 \\
\hline 6 & 73.82 & 73.87 & 73.78 & 73.71 & 73.59 & 73.33 & 72.98 & 72.92 & 72.93 & 73.18 & 73.32 & 73.33 \\
\hline 7 & 73.80 & 73.84 & 73.80 & 73.67 & 73.57 & 73.31 & 72.95 & 72.94 & 72.96 & 73.18 & 73.40 & 73.41 \\
\hline 8 & 73.77 & 73.79 & 73.77 & 73.67 & 73.56 & 73.30 & 72.93 & 72.95 & 72.97 & 73.17 & 73.43 & 73.37 \\
\hline 9 & 73.76 & 73.75 & 73.75 & 73.64 & 73.58 & 73.29 & 72.94 & 72.94 & 72.97 & 73.17 & 73.42 & 73.27 \\
\hline 10 & 73.84 & 73.79 & 73.73 & 73.63 & 73.62 & 73.26 & 72.97 & 72.93 & 72.99 & 73.16 & 73.41 & 73.31 \\
\hline 11 & 73.85 & 73.83 & 73.69 & 73.65 & 73.62 & 73.22 & 72.97 & 72.94 & 72.97 & 73.14 & 73.43 & 73.36 \\
\hline 12 & 73.80 & 73.83 & 73.60 & 73.62 & 73.57 & 73.20 & 72.94 & 72.97 & 72.93 & 73.14 & 73.45 & 73.39 \\
\hline 13 & 73.77 & 73.82 & 73.61 & 73.64 & 73.54 & 73.20 & 72.91 & 72.97 & 72.92 & 73.15 & 73.41 & 73.42 \\
\hline 14 & 73.79 & 73.81 & 73.72 & 73.67 & 73.50 & 73.19 & 72.89 & 72.96 & 72.95 & 73.17 & 73.31 & 73.49 \\
\hline 15 & 73.77 & 73.80 & 73.74 & 73.62 & 73.47 & 73.17 & 72.87 & 72.95 & 72.95 & 73.14 & 73.27 & 73.49 \\
\hline 16 & 73.67 & 73.80 & 73.74 & 73.57 & 73.45 & 73.16 & 72.86 & 72.93 & 72.99 & 73.13 & 73.33 & 73.45 \\
\hline 17 & 73.76 & 73.78 & 73.72 & 73.59 & 73.46 & 73.17 & 72.87 & 72.90 & 73.02 & 73.19 & 73.36 & 73.44 \\
\hline 18 & 73.80 & 73.75 & 73.70 & 73.64 & 73.43 & 73.14 & 72.90 & 72.89 & 73.06 & 73.15 & 73.37 & 73.44 \\
\hline 19 & 73.84 & 73.68 & 73.68 & 73.66 & 73.42 & 73.11 & 72.90 & 72.91 & 73.06 & 73.13 & 73.34 & 73.43 \\
\hline 20 & 73.89 & 73.70 & 73.66 & 73.62 & 73.42 & 73.08 & 72.91 & 72.92 & 73.05 & 73.20 & 73.33 & 73.35 \\
\hline 21 & 73.83 & 73.66 & 73.61 & 73.63 & 73.40 & 73.06 & 72.92 & 72.93 & 73.03 & 73.28 & 73.29 & 73.26 \\
\hline 22 & 73.88 & 73.73 & 73.54 & 73.71 & 73.39 & 73.08 & 72.93 & 72.93 & 73.02 & 73.24 & 73.29 & 73.31 \\
\hline 23 & 73.93 & 73.74 & 73.57 & 73.73 & 73.38 & 73.06 & 72.92 & 72.95 & 73.05 & 73.19 & 73.39 & 73.50 \\
\hline 24 & 73.88 & 73.62 & 73.58 & 73.69 & 73.37 & 73.02 & 72.94 & 72.94 & 73.10 & 73.17 & 73.41 & 73.52 \\
\hline 25 & 73.86 & 73.61 & 73.58 & 73.73 & 73.37 & 72.99 & 72.95 & 72.90 & 73.11 & 73.15 & 73.38 & 73.46 \\
\hline 26 & 73.89 & 73.64 & 73.60 & 73.70 & 73.38 & 72.99 & 72.96 & 72.86 & 73.11 & 73.25 & 73.40 & 73.39 \\
\hline 27 & 73.82 & 73.65 & 73.54 & 73.67 & 73.36 & 73.03 & 72.97 & 72.88 & 73.14 & 73.30 & 73.41 & 73.40 \\
\hline 28 & 73.86 & 73.69 & 73.50 & 73.70 & 73.37 & 73.08 & 72.98 & 72.93 & 73.16 & 73.22 & 73.29 & 73.46 \\
\hline 29 & 73.84 & 73.75 & 73.59 & 73.70 & 73.34 & 73.08 & 72.99 & 72.94 & 73.12 & 73.18 & 73.34 & 73.47 \\
\hline 30 & 73.80 & - & 73.62 & 73.67 & 73.36 & 73.06 & 72.96 & 72.93 & 73.08 & 73.17 & 73.39 & 73.45 \\
\hline 31 & 73.76 & - & 73.61 & - & 73.39 & - & 72.93 & 72.91 & - & 73.27 & - & 73.48 \\
\hline Mean & 73.80 & 73.76 & 73.66 & 73.66 & 73.48 & 73.19 & 72.94 & 72.92 & 73.00 & 73.18 & 73.35 & 73.40 \\
\hline Max & 73.93 & 73.87 & 73.80 & 73.75 & 73.64 & 73.45 & 73.04 & 72.97 & 73.16 & 73.30 & 73.45 & 73.52 \\
\hline Min & 73.67 & 73.61 & 73.50 & 73.56 & 73.34 & 72.99 & 72.86 & 72.86 & 72.87 & 73.09 & 73.25 & 73.26 \\
\hline
\end{tabular}




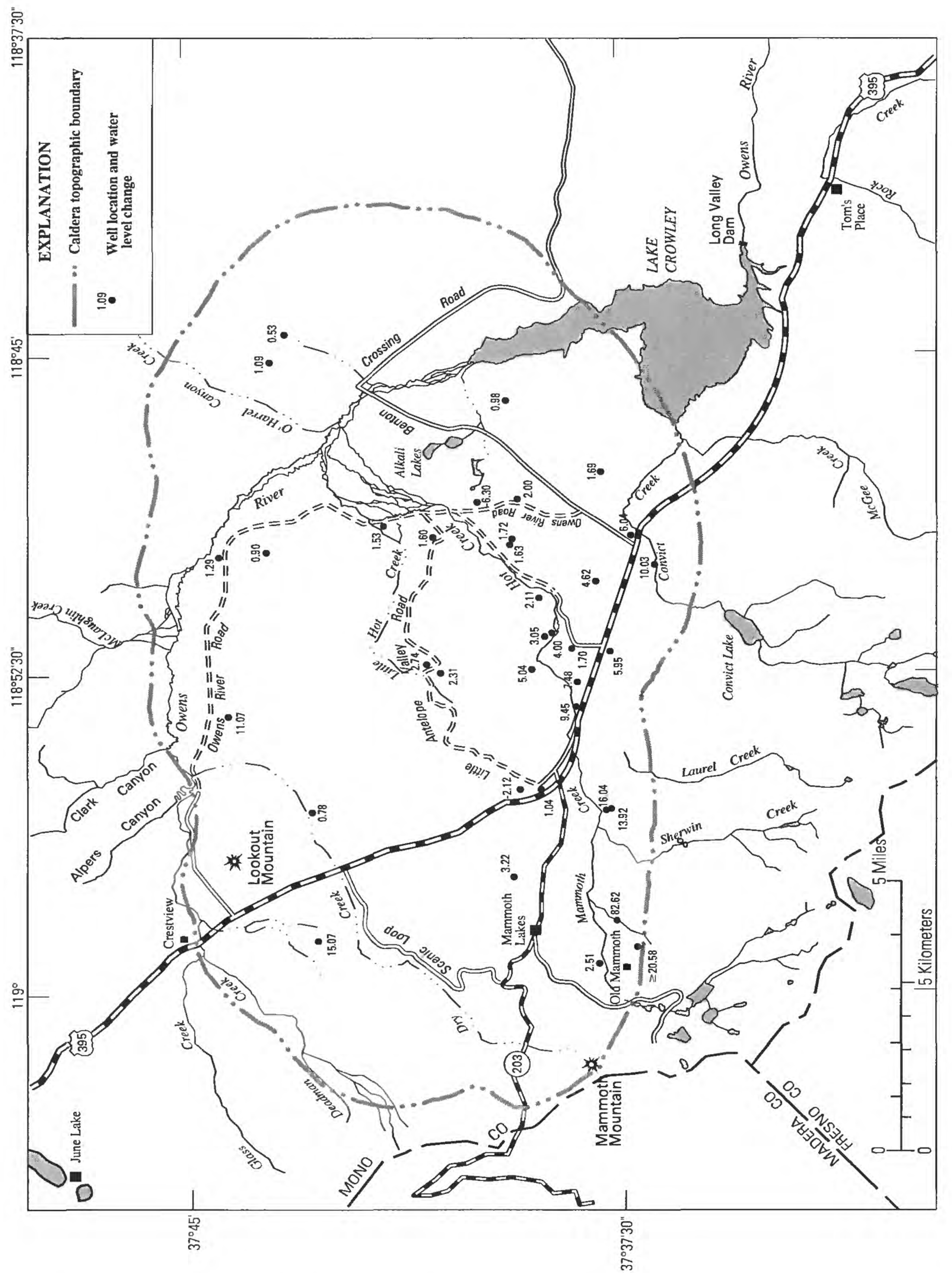

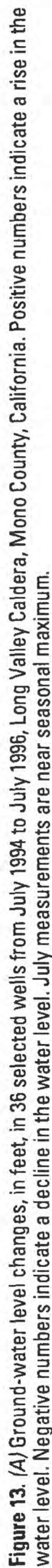




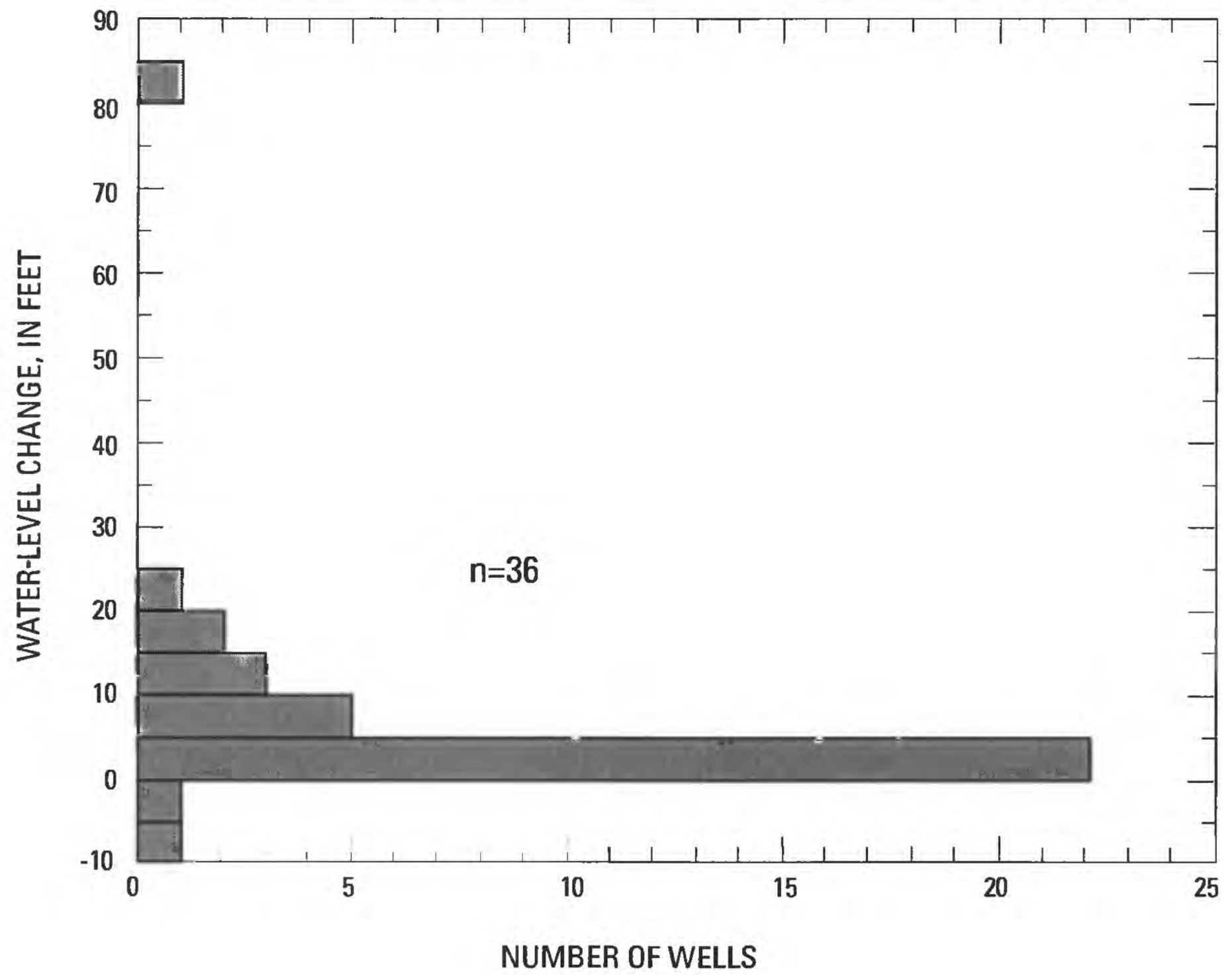

Figure 13. (B) Changes in water levels between July 1994 to July 1996 in 36 selected wells, Long Valley Caldera, Mono County, California. Data are from wells shown in figure 13a. Data are arranged in classes that span 5 feet of water-level change. Twenty-two wells are in the class that includes water-level increases of between 0.01 and 5.00 feet. 
Table 12. Instantaneous ground-water levels in selected wells, 1994-96, Long Valley Caldera, California

[Water levels are in feet below land-surface datum. Readings above land surface indicated by "+".

$\mathrm{M}$, method of measurement: $\mathrm{S}$, steel tape; $\mathrm{V}$, calibrated electric sounder;

W, status of well: D, dry; F, flowing; N, nearby pumping; O, obstruction; $\mathrm{P}$, pumping; R, recently pumped; $\mathrm{T}$, nearby recently pumped.

Highest and Lowest refer to static water levels; pumping levels (status P) or recently pumped (status R) are not considered]

\begin{tabular}{cccccc}
\hline Date & $\begin{array}{c}\text { Water } \\
\text { level MW }\end{array}$ & Date & $\begin{array}{c}\text { Water } \\
\text { level MW }\end{array}$ & Date & $\begin{array}{c}\text { Water } \\
\text { level MW }\end{array}$ \\
\hline
\end{tabular}

Site identifier: $2 \mathrm{~A}$

Site number: 374315118441201

About 13.7 miles northeast of Mammoth Lakes. Drilled livestock well. Diameter 8 inches, depth 32 feet. Altitude of land-surface datum 6,922 feet. Water-level records available 1982-96.

\begin{tabular}{|c|c|c|c|c|c|c|c|}
\hline 04-07-94 & & $70 \mathrm{~S}$ & 04-13-95 & $3.19 \mathrm{~S}$ & $04-25-96$ & $3.18 \mathrm{~S}$ & \\
\hline $07-21-94$ & & $15 \mathrm{~S}$ & $07-20-95$ & $4.03 \mathrm{VP}$ & $07-18-96$ & $3.92 \mathrm{~S}$ & \\
\hline $11-10-94$ & & $27 \mathrm{~S}$ & $11-07-95$ & $4.10 \mathrm{~S}$ & $11-21-96$ & $3.88 \mathrm{~S}$ & \\
\hline Highest & $04-2$ & & 3.18 & & & & \\
\hline Lowest & $07-2$ & & 4.45 & & & & \\
\hline Period of re & cord & Highest & $05-20-86$ & 3.08 & Lowest & $05-30-87$ & 5.59 \\
\hline Record beg & & $05-26-82$ & 38 Entries & & & & \\
\hline
\end{tabular}

Site identifier: 27R1

Site number: 374420118515501

About 8.0 miles northeast of Mammoth Lakes and 2.6 miles southeast of Arcularius Ranch. Drilled livestock well.

Diameter 6 inches, depth 44.3 feet. Altitude of land-surface datum 7,039.18 feet. Water-level records available $1982-96$.

\begin{tabular}{|c|c|c|c|c|c|c|}
\hline 04-07-94 & $38.18 \mathrm{~S}$ & $04-14-95$ & $38.46 \mathrm{~V}$ & $04-24-96$ & $37.92 \mathrm{~V}$ & \\
\hline $07-13-94$ & $40.85 \mathrm{VP}$ & $07-20-95$ & $38.18 \mathrm{~S}$ & $07-17-96$ & 43.04 VR & \\
\hline $11-10-94$ & $38.23 \mathrm{~V}$ & $11-07-95$ & $37.86 \mathrm{~V}$ & $11-20-96$ & $37.44 \mathrm{~V}$ & \\
\hline Highest & $11-20-96$ & 37.44 & & & & \\
\hline Lowest & $04-14-95$ & 38.46 & & & & \\
\hline Period of $\mathrm{r}$ & cord Highest & $07-16-85$ & 33.2 & Lowest & 04-14-95 & 38.46 \\
\hline Record beg & ins $\quad 11-18-82$ & 42 Entries & & & & \\
\hline
\end{tabular}

Site identifier: 28P1

Site number: 374420118532501

About 7.0 miles northeast of Mammoth Lakes and 1.4 miles southeast of Arcularius Ranch. Drilled livestock well.

Diameter 6 inches, depth 125 feet. Altitude of land-surface datum 7,109.02 feet. Water-level records available $1983-96$.

\begin{tabular}{|c|c|c|c|c|c|c|c|}
\hline $04-07-94$ & \multicolumn{2}{|c|}{$88.42 \mathrm{~V}$} & $04-14-95$ & $88.73 \mathrm{~V}$ & $04-24-96$ & $88.10 \mathrm{~V}$ & \\
\hline $07-13-94$ & \multicolumn{2}{|c|}{$97.57 \mathrm{VP}$} & $07-20-95$ & $88.64 \mathrm{~V}$ & $07-17-96$ & \multicolumn{2}{|l|}{$86.50 \mathrm{VR}$} \\
\hline $11-10-94$ & \multicolumn{2}{|c|}{$88.82 \mathrm{~V}$} & $11-07-95$ & $87.97 \mathrm{~V}$ & $11-20-96$ & \multicolumn{2}{|l|}{$87.88 \mathrm{~V}$} \\
\hline $\begin{array}{l}\text { Highest } \\
\text { Lowest }\end{array}$ & \multicolumn{2}{|c|}{$11-20-96$} & $\begin{array}{l}87.88 \\
88.82\end{array}$ & & & & \\
\hline $\begin{array}{l}\text { Period of re } \\
\text { Record begi }\end{array}$ & $\begin{array}{l}\text { ecord } \\
\text { ins }\end{array}$ & $\begin{array}{l}\text { Highest } \\
06-03-83\end{array}$ & $\begin{array}{l}\text { 07-25-84 } \\
40 \text { Entries }\end{array}$ & 75.28 & Lowest & $06-03-83$ & 116.55 \\
\hline
\end{tabular}

Site identifier: 30N2

Site number: 374420118493301

About 7.8 miles northeast of Mammoth Lakes and 4.8 miles southeast of Arcularius Ranch. Unused well. Diameter 8 inches, depth measured 31.8 feet in 1992. Altitude of land-surface datum 6,913.21 feet. Water-level records available 1972, 1982-96. Measurements from August 1989 through 1992 water year published as 003S029E19R001M.

\begin{tabular}{|c|c|c|c|c|c|c|}
\hline $04-07-94$ & $8.90 \mathrm{~S}$ & $04-13-95$ & $6.25 \mathrm{~V}$ & 04-24-96 & $6.08 \mathrm{~V}$ & \\
\hline $07-13-94$ & $9.40 \mathrm{~V}$ & $07-20-95$ & $7.48 \mathrm{~S}$ & $07-17-96$ & $8.11 \mathrm{~V}$ & \\
\hline $11-10-94$ & $9.79 \mathrm{~V}$ & $11-07-95$ & $9.30 \mathrm{~S}$ & $11-20-96$ & $9.41 \mathrm{~V}$ & \\
\hline Highest & $04-24-96$ & 6.08 & & & & \\
\hline Lowest & $11-10-94$ & 9.79 & & & & \\
\hline Period of record & Highest & $05-10-86$ & 2.95 & Lowest & $11-10-94$ & 9.79 \\
\hline Record begins & $05-11-72$ & 45 Entries & & & & \\
\hline
\end{tabular}


Table 12. Instantaneous ground-water levels in selected wells, 1994-96, Long Valley Caldera-Continued

\begin{tabular}{cccccccc}
\hline Date & $\begin{array}{c}\text { Water } \\
\text { level MW }\end{array}$ & Date & $\begin{array}{c}\text { Water } \\
\text { level MW }\end{array}$ & Date & $\begin{array}{c}\text { Water } \\
\text { level MW }\end{array}$ & Date & $\begin{array}{c}\text { Water } \\
\text { level MW }\end{array}$ \\
\hline
\end{tabular}

\section{Site identifier: 31P1}

\section{Site number: 374332118491001}

About 9.4 miles northeast of Mammoth Lakes and 5.4 miles southeast of Arcularius Ranch. Unused water-table well in alluvium of Quaternary age. Diameter 30 inches, depth 7.6 feet. Altitude of land-surface datum 6,914.01 feet. Water-level records available 1966. 1972-73, 1978-96.

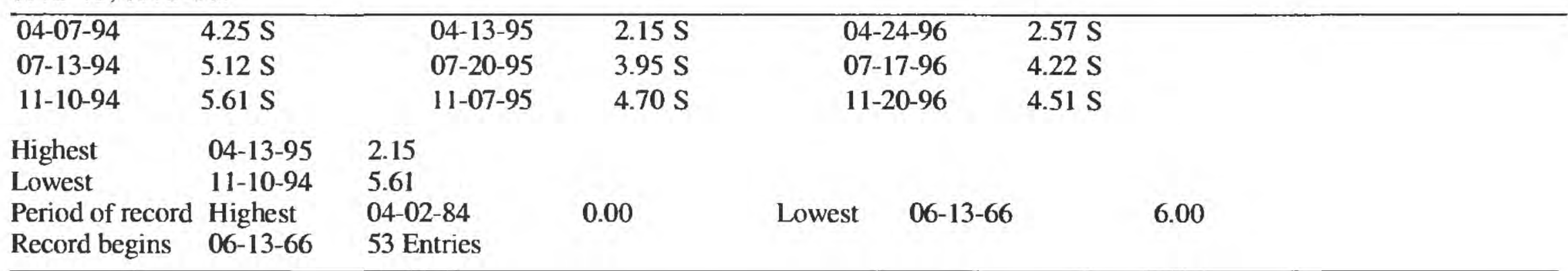

\section{Site identifier: 31P2}

\section{Site number: 374332118491002}

About 9.4 miles northeast of Mammoth Lakes and 5.4 miles southeast of Arcularius Ranch. Livestock well. Diameter 4 inches, depth unknown. Altitude of land-surface datum 6,915.7 feet. Water-level records available 1988-96.

\begin{tabular}{|c|c|c|c|c|c|c|c|}
\hline $04-07-94$ & $4.12 \mathrm{~S}$ & 04-13-95 & $2.07 \mathrm{~S}$ & & $4-96$ & $2.37 \mathrm{~S}$ & \\
\hline $07-13-94$ & $5.00 \mathrm{~S}$ & $07-20-95$ & $3.78 \mathrm{~S}$ & & $0-96$ & $4.36 \mathrm{~S}$ & \\
\hline $11-10-94$ & $5.50 \mathrm{~S}$ & $11-07-95$ & $4.55 \mathrm{~S}$ & & & & \\
\hline Highest & 04-13-95 & 2.07 & & & & & \\
\hline Lowest & $11-10-94$ & 5.50 & & & & & \\
\hline Period of record & Highest & 04-13-95 & 2.07 & Lowest & $07-20-93$ & & 9.70 \\
\hline Record begins & $04-28-88$ & 23 Entries & & & & & \\
\hline
\end{tabular}

Site identifier: $35 \mathrm{N1}$

Site number: 374327118445901

About 13 miles northeast of Mammoth Lakes. Drilled livestock well. Diameter unknown, depth 75 feet. Altitude of land-surface datum 6,914 feet. Water-level records available 1972, 1984-96.

\begin{tabular}{|c|c|c|c|c|c|c|}
\hline $04-07-94$ & $1.72 \mathrm{~S}$ & $04-13-95$ & $0.52 \mathrm{~S}$ & & $04-25-96$ & $0.62 \mathrm{~S}$ \\
\hline $07-21-94$ & $4.52 \mathrm{~S}$ & $07-20-95$ & $2.95 \mathrm{~S}$ & & $07-18-96$ & $3.43 \mathrm{~S}$ \\
\hline $11-10-94$ & $3.55 \mathrm{~S}$ & $11-07-95$ & $3.33 \mathrm{~S}$ & & $11-21-96$ & $2.83 \mathrm{~S}$ \\
\hline Highest & 04-13-95 & 0.52 & & & & \\
\hline Lowest & $07-21-94$ & 4.52 & & & & \\
\hline Period of record & Highest & 04-13-95 & 0.52 & Lowest & $07-21-94$ & 4.52 \\
\hline Record begins & $05-10-72$ & 39 Entries & & & & \\
\hline
\end{tabular}

\section{Site identifier: 36P1}

Site number: 374335118434101

About 14.2 miles northeast of Mammoth Lakes. Unused livestock well. Diameter unknown, depth measured 72 feet in 1992 . Altitude of land-surface datum 6,995 feet. Water-level records available 1986-96.

\begin{tabular}{llllll}
\hline $04-07-94$ & $69.25 \mathrm{~S}$ & $04-13-95$ & $69.30 \mathrm{~S}$ & $11-07-95$ & $69.24 \mathrm{~S}$ \\
$11-10-94$ & $69.30 \mathrm{~S}$ & $07-20-95$ & $69.19 \mathrm{~S}$ & $04-25-96$ & $69.22 \mathrm{~S}$
\end{tabular}

Highest $\quad 07-20-95 \quad 69.19$

Lowest 11-10-94, 04-13-95

69.30

05-30-87 $\quad 68.70$

Record begins $\quad 05-20-86 \quad 28$ Entries

$\begin{array}{llll}68.70 & \text { Lowest } & 11-29-86 & 71.51\end{array}$


Table 12. Instantaneous ground-water levels in selected wells, 1994-96, Long Valley Caldera-Continued

\begin{tabular}{lllllll}
\hline Date & $\begin{array}{c}\text { Water } \\
\text { level MW }\end{array}$ & Date & $\begin{array}{c}\text { Water } \\
\text { level MW }\end{array}$ & Date & $\begin{array}{c}\text { Water } \\
\text { level MW }\end{array}$ & Date \\
\hline
\end{tabular}

Site identifier: AP

Site number: 373737118501701

About 7 miles southeast of Mammoth Lakes, at the airport. Drilled public-supply well. Diameter 10 inches, depth 70 feet, perforated $52-66$ feet. Altitude of land-surface datum 7,093 feet. Water-level records available 1984-96.

\begin{tabular}{|c|c|c|c|c|c|c|c|c|}
\hline $04-07-94$ & $29.96 \mathrm{~S}$ & $04-13-95$ & $28.20 \mathrm{~S}$ & $11-10-95$ & 25.78 SR & & $07-18-96$ & $25.58 \mathrm{SR}$ \\
\hline $11-08-94$ & $30.05 \mathrm{~S}$ & $07-19-95$ & 25.96 SR & $04-25-96$ & $29.22 \mathrm{~S}$ & & $11-19-96$ & $26.43 \mathrm{~S}$ \\
\hline Highest & $11-19-96$ & 26.43 & & & & & & \\
\hline Lowest & $11-08-94$ & 30.05 & & & & & & \\
\hline Period of record & Highest & $08-30-86$ & 19.24 & Lowest & $11-08-94$ & 30.05 & & \\
\hline Record begins & $10-09-84$ & 37 Entries & & & & & & \\
\hline
\end{tabular}

Site identifier: CD-2

Site number: $\mathbf{3 7 3 8 4 7 1 1 8 5 4 5 8 0 2}$

About 2.5 miles east of Mammoth Lakes and 0.1 mile northwest of Casa Diablo Hot Springs. Drilled irrigation well. Diameter 6 inches, depth 75 feet. Altitude of land-surface datum 7,316 feet. Water-level records available 1990-96.

\begin{tabular}{|c|c|c|c|c|c|c|c|c|}
\hline $01-12-94$ & $47.06 \mathrm{~S}$ & $11-11-94$ & $50.65 \mathrm{~S}$ & $11-09-95$ & $48.45 \mathrm{~S}$ & & $09-17-96$ & $49.92 \mathrm{~S}$ \\
\hline $02-16-94$ & $47.36 \mathrm{~S}$ & $02-24-95$ & $50.82 \mathrm{~S}$ & $02-14-96$ & $49.60 \mathrm{~S}$ & & $11-15-96$ & $51.02 \mathrm{~S}$ \\
\hline 04-08-94 & $48.09 \mathrm{~S}$ & $03-24-95$ & $49.93 \mathrm{~S}$ & $03-13-96$ & $48.79 \mathrm{~S}$ & & $11-20-96$ & $50.90 \mathrm{~S}$ \\
\hline $05-19-94$ & $48.39 \mathrm{~S}$ & 04-13-95 & $40.22 \mathrm{~S}$ & $04-22-96$ & $44.90 \mathrm{~S}$ & & $12-18-96$ & $51.11 \mathrm{~S}$ \\
\hline $06-10-94$ & $47.96 \mathrm{~S}$ & $05-17-95$ & $37.94 \mathrm{~S}$ & $05-14-96$ & $44.25 \mathrm{~S}$ & & & \\
\hline $07-15-94$ & $47.80 \mathrm{~S}$ & $06-15-95$ & $41.21 \mathrm{~S}$ & $06-18-96$ & $45.27 \mathrm{~S}$ & & & \\
\hline $08-11-94$ & $49.88 \mathrm{~S}$ & $07-19-95$ & $43.90 \mathrm{~S}$ & $07-19-96$ & $46.76 \mathrm{~S}$ & & & \\
\hline $09-15-94$ & $50.28 \mathrm{~S}$ & $10-19-95$ & $48.60 \mathrm{~S}$ & $08-20-96$ & $50.05 \mathrm{~S}$ & & & \\
\hline Highest & $05-17-95$ & 37.94 & & & & \multirow{4}{*}{51.11} & & \\
\hline Lowest & $12-18-96$ & 51.11 & & \multirow{3}{*}{\multicolumn{2}{|c|}{$12-18-96$}} & & & \\
\hline Period of record & Highest & $08-29-90$ & 13.94 & & & & & \\
\hline Record begins & $08-29-90$ & 70 Entries & & & & & & \\
\hline
\end{tabular}

\section{Site identifier: $\mathrm{CH}-1$}

Site number: 374045118491001

About 8.1 miles northeast of Mammoth Lakes and 0.7 mile southwest of Cashbaugh Ranch. Drilled observation well. Diameter 1.25 inches, depth 973 feet, perforated 470-475 feet. Altitude of land-surface datum 6,969.93 feet. Water-level records available $1982-96$.

\begin{tabular}{|c|c|c|c|c|c|c|}
\hline $04-06-94$ & $40.67 \mathrm{~S}$ & 04-13-95 & $40.77 \mathrm{~V}$ & $04-24-96$ & $39.68 \mathrm{~V}$ & \\
\hline $07-21-94$ & $40.93 \mathrm{~S}$ & $07-12-95$ & $39.93 \mathrm{~V}$ & $07-17-96$ & $39.33 \mathrm{~V}$ & \\
\hline $11-10-94$ & $41.11 \mathrm{~S}$ & $11-07-95$ & $39.90 \mathrm{~V}$ & $11-20-96$ & $39.45 \mathrm{~S}$ & \\
\hline Highest & $07-17-96$ & 39.33 & & & & \\
\hline Lowest & 11-10-94 & 41.11 & & & & \\
\hline Period of record & Highest & $08-03-82$ & 33.67 & Lowest & $10-06-92$ & 41.36 \\
\hline Record begins & $08-03-82$ & 115 Entries & & & & \\
\hline
\end{tabular}

\section{Site identifier: $\mathbf{C H}-10$}

Site number: 373932118491501

About 7.8 miles east of Mammoth Lakes and 2 miles north of Whitmore Hot Springs. Drilled observation well. Diameter 1.25 inches, depth 175 feet, perforated 115-120 feet. Altitude of land-surface datum 7,074.88 feet. Water-level records available $1982-96$.

\begin{tabular}{|c|c|c|c|c|c|c|}
\hline $04-07-94$ & $70.22 \mathrm{~V}$ & 04-19-95 & $69.68 \mathrm{~V}$ & $04-25-96$ & $69.48 \mathrm{~V}$ & \\
\hline 08-08-94 & $70.39 \mathrm{~V}$ & $07-13-95$ & $69.14 \mathrm{~V}$ & $11-21-96$ & $69.04 \mathrm{~V}$ & \\
\hline $11-08-94$ & $70.32 \mathrm{~V}$ & $11-08-95$ & $69.20 \mathrm{~V}$ & & & \\
\hline Highest & $11-21-96$ & 69.04 & & & & \\
\hline Lowest & 08-08-94 & 70.39 & & & & \\
\hline Period of record & Highest & $11-03-83$ & 67.70 & Lowest & $08-30-87$ & 70.98 \\
\hline Record begins & $08-04-82$ & 41 Entries & & & & \\
\hline
\end{tabular}


Table 12. Instantaneous ground-water levels in selected wells, 1994-96, Long Valley Caldera-Continued

\begin{tabular}{lccccccc}
\hline Date & $\begin{array}{c}\text { Water } \\
\text { level MW }\end{array}$ & Date & $\begin{array}{c}\text { Water } \\
\text { level MW }\end{array}$ & Date & $\begin{array}{c}\text { Water } \\
\text { level MW }\end{array}$ & $\begin{array}{c}\text { Water } \\
\text { Date }\end{array}$ & level MW \\
\hline
\end{tabular}

\section{Site identifier: $\mathbf{C H}-10 \mathrm{~A}$}

Site number: 373930118491601

About 7.8 miles east of Mammoth Lakes and 2 miles north of Whitmore Hot Springs. Drilled observation well. Diameter 6 inches, depth 110 feet, perforated 90-1 10 feet. Altitude of land-surface datum 7,078.80 feet. Water-level records available 1983-96.

\begin{tabular}{lclllll}
\hline $04-07-94$ & $74.25 \mathrm{~V}$ & $04-19-95$ & $73.72 \mathrm{~V}$ & $02-14-96$ & $73.58 \mathrm{~V}$ & $11-21-96$ \\
$08-08-94$ & $74.42 \mathrm{~V}$ & $07-13-95$ & $73.18 \mathrm{~V}$ & $04-25-96$ & $73.54 \mathrm{~V}$ & \\
$11-08-94$ & $74.35 \mathrm{~V}$ & $11-08-95$ & $73.25 \mathrm{~V}$ & $07-19-96$ & $72.70 \mathrm{~V}$ & \\
& & & & & & \\
Highest & $07-19-96$ & 72.70 & & & & \\
Lowest & $08-08-94$ & 74.42 & & & & \\
Period of record & Highest & $09-28-83$ & 69.8 & Lowest & $08-30-87$ & \\
Record begins & $08-10-83$ & 56 Entries & & & & \\
\hline
\end{tabular}

Site Identifier: $\mathrm{CH}-10 \mathrm{~B}$

Site Number: $\mathbf{3 7 3 9 3 0 1 1 8 4 9 1 6 0 2}$

About 7.8 miles east of Mammoth Lakes and 2 miles north of Whitmore Hot Springs. Drilled observation well. Diameter 4 inches, depth 315 feet, open hole 300-315 feet. Altitude of land-surface datum 7,078.99 feet. Water-level records available 1983-96.

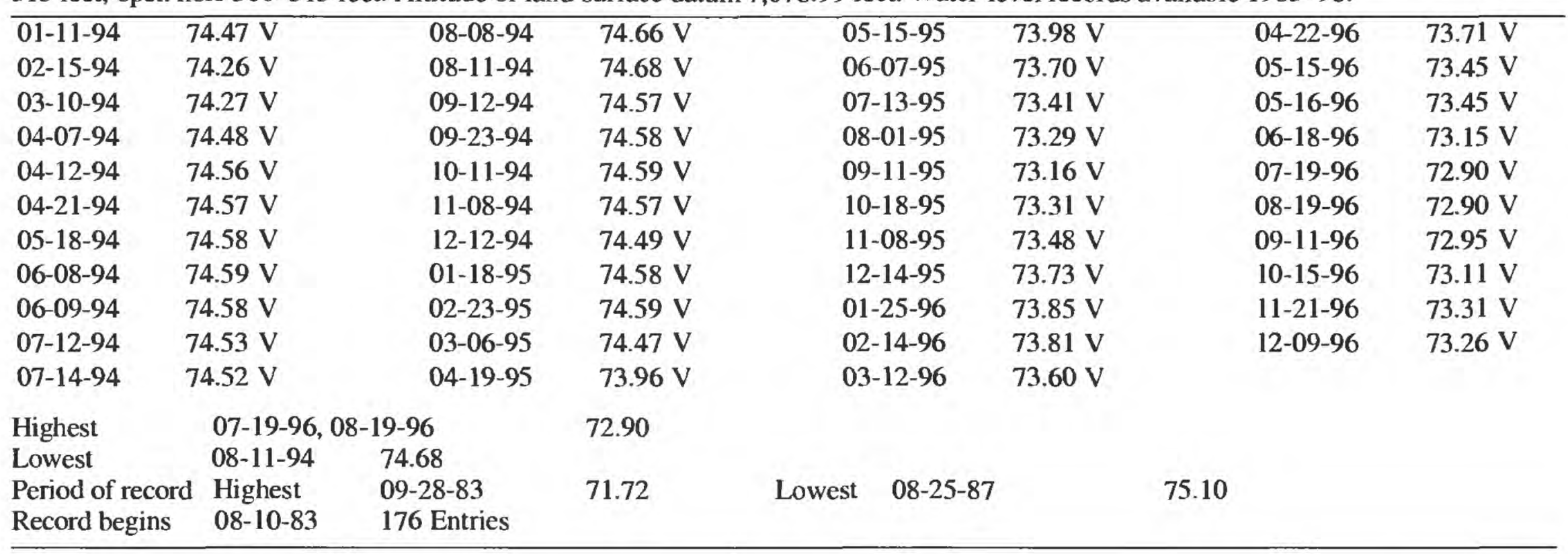

Site identifier: $\mathbf{C H}-\mathbf{3}$

Site number: 373929118455405

About 11.2 miles east of Mammoth Lakes and 3.2 miles northeast of Whitmore Hot Springs. Drilled observation well. Diameter 1.25 inches, depth 160 feet. Altitude of land-surface datum 6,870 feet. Water-level records available 1982-96.

\begin{tabular}{|c|c|c|c|c|c|c|c|}
\hline $04-07-94$ & $5.67 \mathrm{~S}$ & 04-13-95 & $4.86 \mathrm{~V}$ & 04 & $4-96$ & $4.80 \mathrm{~V}$ & \\
\hline $07-22-94$ & $5.88 \mathrm{~S}$ & 07-19-95 & $5.30 \mathrm{~S}$ & & $8-96$ & $4.90 \mathrm{~S}$ & \\
\hline $11-10-94$ & $5.65 \mathrm{~S}$ & $11-07-95$ & $4.95 \mathrm{~V}$ & & $1-96$ & $4.85 \mathrm{~V}$ & \\
\hline Highest & $04-24-96$ & 4.80 & & & & & \\
\hline Lowest & $07-22-94$ & 5.88 & & & & & \\
\hline Period of record & Highest & 04-24-96 & 4.80 & Lowest & $06-02-83$ & & 7.73 \\
\hline Record begins & $08-24-82$ & 43 Entries & & & & & \\
\hline
\end{tabular}


Table 12. Instantaneous ground-water levels in selected wells, 1994-96, Long Valley Caldera-Continued

\begin{tabular}{lllclll}
\hline Date & $\begin{array}{c}\text { Water } \\
\text { level MW }\end{array}$ & Date & $\begin{array}{c}\text { Water } \\
\text { level MW }\end{array}$ & Date & $\begin{array}{c}\text { Water } \\
\text { level MW }\end{array}$ & Date \\
\hline
\end{tabular}

\section{Site identifier: $\mathbf{C H}-5$ \\ Site number: 373759118474105}

About 9.9 miles east of Mammoth Lakes and 1.6 miles northeast of Whitmore Hot Springs. Drilled observation well. Diameter 1.25 inches, depth 875 feet. Altitude of land-surface datum 6,918 feet. Water-level records available 1982-96.

\begin{tabular}{|c|c|c|c|c|c|c|c|}
\hline $04-06-94$ & $44.92 \mathrm{~S}$ & $04-13-95$ & $44.97 \mathrm{~V}$ & & $4-96$ & $44.02 \mathrm{~V}$ & \\
\hline $07-22-94$ & $44.98 \mathrm{~S}$ & $07-14-95$ & $44.35 \mathrm{~S}$ & & $8-96$ & $43.29 \mathrm{~S}$ & \\
\hline $11-10-94$ & $45.35 \mathrm{~S}$ & $11-07-95$ & $43.76 \mathrm{~V}$ & & $1-96$ & $43.49 \mathrm{~V}$ & \\
\hline Highest & $07-18-96$ & 43.29 & & & & & \\
\hline Lowest & $11-10-94$ & 45.35 & & & & & \\
\hline Period of record & Highest & $11-12-84$ & 42.40 & Lowest & $11-20-92$ & & 45.96 \\
\hline Record begins & $08-24-82$ & 44 Entries & & & & & \\
\hline
\end{tabular}

Site identifier: $\mathrm{CH}-6$

Site number: 374040118522501

About 5.3 miles northeast of Mammoth Lakes in Little Antelope Valley. Drilled observation well. Diameter 1.25 inches, depth 677 feet, perforated 535-539 feet. Altitude of land-surface datum 7,248.23 feet. Water-level records available 1982-96.

\begin{tabular}{llllllll}
\hline $04-06-94$ & $208.66 \mathrm{~V}$ & $11-09-94$ & $208.73 \mathrm{~V}$ & $11-07-95$ & $208.20 \mathrm{~V}$ & $07-19-96$ & $206.70 \mathrm{~V}$ \\
$07-13-94$ & $209.01 \mathrm{~V}$ & $07-14-95$ & $208.41 \mathrm{~V}$ & $04-24-96$ & $207.40 \mathrm{~V}$ & $11-21-96$ & $207.04 \mathrm{~V}$ \\
& & & & & & & \\
Highest & $07-19-96$ & 206.70 & & & & & \\
Lowest & $07-13-94$ & 209.01 & & & & & \\
Period of record & Highest & $08-27-86$ & 202.55 & Lowest & $11-19-91$ & 209.25 & \\
Record begins & $08-24-82$ & 50 Entries & & & & & \\
\hline
\end{tabular}

\section{Site identifier: $\mathrm{CH}-7$}

Site number: 374000118483102

About 8.6 miles northeast of Mammoth Lakes and 1.2 miles south of Cashbaugh Ranch. Drilled observation well. Diameter 1.25 inches, depth 210 feet, perforated 198-203 feet. Obstruction at 72 feet below land surface. Altitude of land-surface datum 6,960 feet. Water-level records available 1982-96. Positive sign indicates water level is above land surface. Levels given when well status is $F$ (flowing) are height of casing in feet above land surface.

\begin{tabular}{|c|c|c|c|c|c|c|c|}
\hline $04-06-94$ & $+0.45 \mathrm{SF}$ & $02-23-95$ & $+1.75 \mathrm{~V}$ & $06-28-95$ & $5.68 \mathrm{~S}$ & $11-07-95$ & $5.93 \mathrm{~S}$ \\
\hline $07-12-94$ & $+0.45 \mathrm{SF}$ & $03-06-95$ & $+3.65 \mathrm{~S}$ & $07-13-95$ & $5.68 \mathrm{~S}$ & $02-14-96$ & $5.25 \mathrm{~S}$ \\
\hline $11-10-94$ & $+0.45 \mathrm{SF}$ & 04-13-95 & $+4.35 \mathrm{~S}$ & $07-18-95$ & $5.72 \mathrm{~S}$ & 04-24-96 & $5.09 \mathrm{~V}$ \\
\hline $12-12-94$ & $+0.45 \mathrm{SF}$ & 04-19-95 & $+4.35 \mathrm{~S}$ & $07-20-95$ & $5.75 \mathrm{~S}$ & $07-18-96$ & $5.85 \mathrm{~S}$ \\
\hline $12-15-94$ & $+3.33 \mathrm{~V}$ & 05-15-95 & $+7.77 \mathrm{~S}$ & 08-01-95 & $5.84 \mathrm{~S}$ & $11-20-96$ & $5.76 \mathrm{~S}$ \\
\hline $01-20-95$ & $1.75 \mathrm{~S}$ & $05-17-95$ & $+4.32 \mathrm{~V}$ & $09-12-95$ & $5.95 \mathrm{~S}$ & & \\
\hline Highest & $05-15-95$ & $+7.77 \mathrm{~S}$ & & & & & \\
\hline Lowest & $09-12-95$ & $5.95 \mathrm{~S}$ & & & & & \\
\hline Period of record & Highest & $05-15-95$ & $+7.77 \mathrm{~S}$ & Lowest & $11-17-82$ & 6.29 & \\
\hline Record begins & $08-03-82$ & 64 Entries & & & & & \\
\hline
\end{tabular}

\section{Site identifier: CHURCH}

\section{Site number: 373707118491701}

About 8 miles southeast of Mammoth Lakes and 1 mile southwest of Whitmore Hot Springs. Drilled public supply well. Diameter 6 inches, depth 45.5 feet. Altitude of land-surface datum 7,035 feet. Water-level records available 1989-96.

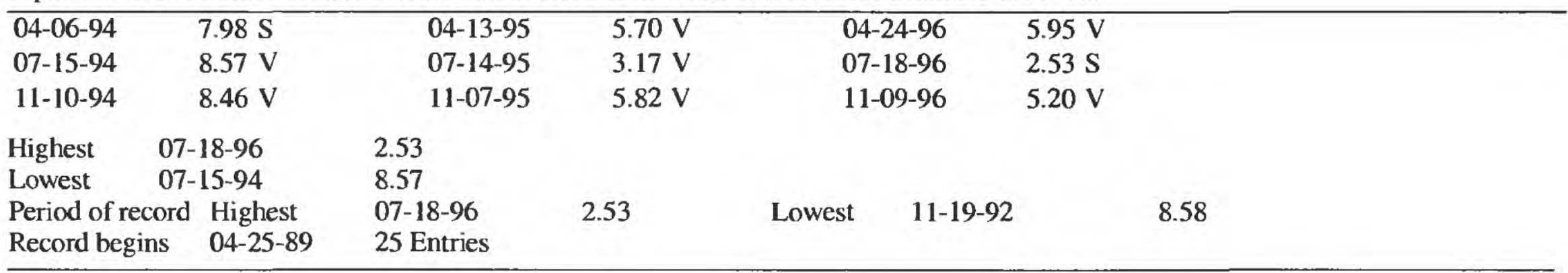


Table 12. Instantaneous ground-water levels in selected wells, 1994-96, Long Valley Caldera-Continued

\begin{tabular}{lllclccc}
\hline Date & $\begin{array}{c}\text { Water } \\
\text { level MW }\end{array}$ & Date & $\begin{array}{c}\text { Water } \\
\text { level MW }\end{array}$ & Date & $\begin{array}{c}\text { Water } \\
\text { level MW }\end{array}$ & Date & Water \\
level MW
\end{tabular}

\section{Site identifier: CM-2}

Site number: 373906118522301

About 4.9 miles east of Mammoth Lakes near Hot Creek Fish Hatchery. Drilled observation well. Diameter 4 inches, depth 152 feet. Altitude of land-surface datum 7,077.0 feet. Water-level records available 1985-96.

\begin{tabular}{|c|c|c|c|c|c|c|c|c|}
\hline 04-06-94 & $7.42 \mathrm{~S}$ & $11-08-94$ & $7.61 \mathrm{~S}$ & $11-09-95$ & $5.06 \mathrm{~S}$ & & $07-19-96$ & $1.86 \mathrm{~S}$ \\
\hline $07-21-94$ & $6.90 \mathrm{~S}$ & $07-12-95$ & $5.21 \mathrm{~S}$ & $04-23-96$ & 4.64 S & & $11-21-96$ & $3.88 \mathrm{~V}$ \\
\hline Highest & $07-19-96$ & 1.86 & & & & & & \\
\hline Lowest & $11-08-94$ & 7.61 & & & & & & \\
\hline Period of record & Highest & $08-27-86$ & 0.25 & Lowest & $11-08-94$ & 7.61 & & \\
\hline Record begins & 04-23-85 & 49 Entries & & & & & & \\
\hline
\end{tabular}

Site identifier: $\mathbf{C R}$

Site number: $\mathbf{3 7 3 8 1 6 1 1 8 5 3 2 0 0 1}$

About 4 miles east of Mammoth Lakes. Drilled domestic well. Diameter 6 inches, depth 80 feet. Altitude of land-surface datum 7,161.76 feet. Water-level records available 1986-95. Site discontinued November 10, 1995.

\begin{tabular}{lclllll}
\hline $07-22-94$ & $25.05 \mathrm{~S}$ & \multicolumn{1}{c}{$11-10-95$} & $17.23 \mathrm{~S}$ & & & \\
Highest & $11-10-95$ & 17.23 & & & \\
Lowest & $07-22-94$ & 25.05 & & Lowest & $07-29-88$ & 26.60 \\
Period of record & Highest & 05-21-86 & 10.40 & & \\
Record begins & $05-21-86$ & 17 Entries & & & \\
\hline
\end{tabular}

Site identifier: $\mathbf{C T}$

Site number: 374511118585801

About 7.5 miles north of Mammoth Lakes at the Caltrans Crestview Maintenance Station. Drilled public supply well. Diameter 10 inches, depth 490 feet. Altitude of land-surface datum 7,520 feet. Water-level records available 1990-96.

\begin{tabular}{llllll}
\hline $04-07-94$ & $128.90 \mathrm{~V}$ & $05-17-95$ & $125.75 \mathrm{~V}$ & $04-24-96$ & $110.83 \mathrm{~V}$ \\
$07-13-94$ & $124.76 \mathrm{~V}$ & $07-18-95$ & $102.36 \mathrm{~V}$ & $07-17-96$ & $128.89 \mathrm{~V}$ \\
$11-10-94$ & $130.99 \mathrm{~V}$ & $11-06-95$ & $106.76 \mathrm{~V}$ & $11-20-96$ & $109.20 \mathrm{~V}$
\end{tabular}

Highest $\quad 07-18-95 \quad 102.36$

Lowest $\quad 11-10-94 \quad 130.99$

Period of record Highest 07-18-95

Record begins $\quad 08-02-90 \quad 22$ Entries

$102.36 \quad$ Lowest $\quad 11-19-92 \quad 134.31$

\section{Site identifier: CW-1}

Site number: 373850118513601

About 5.6 miles east of Mammoth Lakes near Hot Creek Fish Hatchery. Drilled geothermal exploration well. Diameter 18 inches, drilled depth 805 feet, open hole 223-805 feet. Well plugged, abandoned, and buried, October 1997. Altitude of land-surface datum 7,084.99 feet. Water-level records available 1973, 1982-96.

\begin{tabular}{|c|c|c|c|c|c|c|c|}
\hline 04-06-94 & $15.70 \mathrm{~V}$ & $07-12-95$ & $15.00 \mathrm{~V}$ & $04-23-96$ & $14.15 \mathrm{~V}$ & $11-21-96$ & $13.47 \mathrm{~V}$ \\
\hline $11-08-94$ & $16.17 \mathrm{~V}$ & $11-09-95$ & $14.67 \mathrm{~V}$ & $07-19-96$ & $12.75 \mathrm{~V}$ & & \\
\hline Highest & $07-19-96$ & 12.75 & & & & & \\
\hline Lowest & $11-08-94$ & 16.17 & & & & & \\
\hline Period of record & Highest & $08-13-83$ & 6.77 & Lowest & $11-08-94$ & 16.17 & \\
\hline Record begins & $08-01-73$ & 51 Entries & & & & & \\
\hline
\end{tabular}


Table 12. Instantaneous ground-water levels in selected wells, 1994-96, Long Valley Caldera-Continued

\begin{tabular}{lcccccc}
\hline Date & $\begin{array}{c}\text { Water } \\
\text { level MW }\end{array}$ & Date & $\begin{array}{c}\text { Water } \\
\text { level MW }\end{array}$ & Date & $\begin{array}{c}\text { Water } \\
\text { level MW }\end{array}$ & Water \\
\hline
\end{tabular}

\section{Site identifier: $\mathrm{CW}-\mathbf{3}$ \\ Site number: 373849118513001}

About 5.7 miles east of Mammoth Lakes near Hot Creek Fish Hatchery. Drilled geothermal exploration well. Diameter 13 inches, accessible depth 420 feet, depth of hole 917 feet. Altitude of land-surface datum 7,062.20 feet. Water-level records available $1986-96$.

\begin{tabular}{|c|c|c|c|c|c|c|c|c|}
\hline $01-13-94$ & $10.48 \mathrm{~S}$ & $10-13-94$ & $10.51 \mathrm{~S}$ & $07-12-95$ & $9.83 \mathrm{~S}$ & & $04-23-96$ & $8.99 \mathrm{~S}$ \\
\hline $02-15-94$ & $10.42 \mathrm{~S}$ & $10-14-94$ & $10.44 \mathrm{~S}$ & $07-21-95$ & $9.79 \mathrm{~S}$ & & $05-16-96$ & $8.40 \mathrm{~S}$ \\
\hline $03-10-94$ & $10.43 \mathrm{~V}$ & $11-08-94$ & $10.82 \mathrm{~S}$ & $08-11-95$ & $9.46 \mathrm{~S}$ & & $06-25-96$ & $7.58 \mathrm{~V}$ \\
\hline 04-06-94 & $10.45 \mathrm{~V}$ & $12-16-94$ & $11.20 \mathrm{~S}$ & $09-12-95$ & $9.57 \mathrm{~V}$ & & $07-19-96$ & $7.62 \mathrm{~S}$ \\
\hline $05-19-94$ & $10.44 \mathrm{~V}$ & $01-20-95$ & $10.67 \mathrm{~S}$ & $10-19-95$ & $9.78 \mathrm{~V}$ & & $08-19-96$ & $7.65 \mathrm{~S}$ \\
\hline $06-23-94$ & $10.64 \mathrm{~V}$ & $02-23-95$ & $11.23 \mathrm{~V}$ & $11-09-95$ & $9.59 \mathrm{~V}$ & & $09-11-96$ & $7.84 \mathrm{~S}$ \\
\hline $07-21-94$ & $10.67 \mathrm{~V}$ & 03-06-95 & $11.23 \mathrm{~S}$ & $12-13-95$ & $9.71 \mathrm{~V}$ & & $10-18-96$ & $8.17 \mathrm{~S}$ \\
\hline $08-11-94$ & $10.58 \mathrm{~V}$ & $04-18-95$ & $10.37 \mathrm{~S}$ & $01-23-96$ & $9.67 \mathrm{~S}$ & & $11-21-96$ & $8.33 \mathrm{~S}$ \\
\hline $09-15-94$ & $10.70 \mathrm{~V}$ & $05-15-95$ & $10.63 \mathrm{~S}$ & $02-13-96$ & $9.70 \mathrm{~V}$ & & $12-10-96$ & $8.39 \mathrm{~S}$ \\
\hline $09-23-94$ & $10.57 \mathrm{~S}$ & $06-14-95$ & $10.26 \mathrm{~S}$ & $03-11-96$ & $9.45 \mathrm{~S}$ & & & \\
\hline Highest & $06-25-96$ & 7.58 & & \multirow{4}{*}{\multicolumn{2}{|c|}{$02-23-95,03-06-95$}} & & & \\
\hline Lowest & $02-23-95,03-$ & $06-95 \quad 11.23$ & & & & \multirow{3}{*}{11.23} & & \\
\hline Period of record & Highest & $09-01-86$ & 1.82 & & & & & \\
\hline Record begins & $05-10-86$ & 96 Entries & & & & & & \\
\hline
\end{tabular}

Site identifier: ESN

Site number: 373818118515001

About 5.5 miles east of Mammoth Lakes near Hot Creek Fish Hatchery. Drilled institutional well unused after 1980. Diameter 6 inches, depth 74 feet. Altitude of land-surface datum 7,080 feet. Water-level records available 1986-89, 1996.

\begin{tabular}{|c|c|c|c|c|c|c|c|c|}
\hline $04-22-96$ & $8.43 \mathrm{~S}$ & $06-17-96$ & $6.66 \mathrm{~V}$ & $08-19-96$ & $6.40 \mathrm{~S}$ & & $11-20-96$ & $7.78 \mathrm{~S}$ \\
\hline $04-23-96$ & $8.44 \mathrm{~S}$ & $06-18-96$ & $6.62 \mathrm{~S}$ & $09-11-96$ & $7.07 \mathrm{~S}$ & & $12-11-96$ & $7.94 \mathrm{~S}$ \\
\hline $05-15-96$ & $7.53 \mathrm{~V}$ & $07-15-96$ & $6.43 \mathrm{~V}$ & $09-16-96$ & $7.22 \mathrm{~S}$ & & $12-18-96$ & $8.07 \mathrm{~S}$ \\
\hline $05-16-96$ & $7.48 \mathrm{~S}$ & $07-19-96$ & $6.33 \mathrm{~S}$ & $10-15-96$ & $7.53 \mathrm{~S}$ & & & \\
\hline Highest & $07-19-96$ & 6.33 & & & & & & \\
\hline Lowest & 04-23-96 & 8.44 & & & & & & \\
\hline Period of record & Highest & $8-30-86$ & 2.48 & $11-28-88$ & & 8.67 & & \\
\hline Record begins & $08-30-86$ & 28 Entries & & & & & & \\
\hline
\end{tabular}

\section{Site identifier: ESO}

Site number: $\mathbf{3 7 3 8 1 8 1 1 8 5 1 4 5 0 2}$

About 5.5 miles east of Mammoth Lakes near Hot Creek Fish Hatchery. Drilled institutional well, unused after 1980. Diameter 6 inches, depth 44.3 feet. Altitude of land-surface datum 7,090 feet. Water-level records available 1985-96.

\begin{tabular}{|c|c|c|c|c|c|c|c|}
\hline $01-11-94$ & $20.27 \mathrm{~S}$ & $09-13-94$ & $20.58 \mathrm{~S}$ & $08-01-95$ & $17.43 \mathrm{~V}$ & $04-22-96$ & $19.56 \mathrm{~S}$ \\
\hline $02-15-94$ & $20.49 \mathrm{~V}$ & $10-13-94$ & $20.58 \mathrm{~S}$ & $08-15-95$ & $17.30 \mathrm{~V}$ & $05-14-96$ & $18.97 \mathrm{~S}$ \\
\hline $03-09-94$ & $20.45 \mathrm{~V}$ & $11-08-94$ & $20.63 \mathrm{~S}$ & $08-24-95$ & $17.38 \mathrm{~S}$ & $06-17-96$ & $18.29 \mathrm{~V}$ \\
\hline $04-07-94$ & $20.54 \mathrm{~S}$ & $12-15-94$ & $20.68 \mathrm{~V}$ & $09-12-95$ & $17.69 \mathrm{~S}$ & $07-15-96$ & $18.10 \mathrm{~V}$ \\
\hline $05-16-94$ & $20.30 \mathrm{~S}$ & 04-17-95 & $19.78 \mathrm{~S}$ & $10-17-95$ & $18.53 \mathrm{~S}$ & $08-19-96$ & $18.15 \mathrm{~S}$ \\
\hline 06-09-94 & $19.94 \mathrm{~V}$ & $05-17-95$ & $19.58 \mathrm{~S}$ & $11-09-95$ & $18.80 \mathrm{~S}$ & $09-16-96$ & $18.71 \mathrm{~S}$ \\
\hline $07-12-94$ & $19.80 \mathrm{~V}$ & $06-15-95$ & $18.68 \mathrm{~S}$ & $01-25-96$ & $19.55 \mathrm{~V}$ & $11-20-96$ & $19.11 \mathrm{~V}$ \\
\hline 08-09-94 & $20.30 \mathrm{~V}$ & $07-13-95$ & $17.79 \mathrm{~S}$ & $02-14-96$ & $19.62 \mathrm{~V}$ & $12-11-96$ & $19.19 \mathrm{~S}$ \\
\hline Highest & $08-15-95$ & 17.30 & & & & & \\
\hline Lowest & $12-15-94$ & 20.68 & & & & & \\
\hline Period of record & d Highest & $05-12-86$ & 16.95 & $12-17-92$ & 20.75 & & \\
\hline Record begins & 07-10-85 & 92 Entries & & & & & \\
\hline
\end{tabular}


Table 12. Instantaneous ground-water levels in selected wells, 1994-96, Long Valley Caldera-Continued

\begin{tabular}{|c|c|c|c|c|c|c|c|}
\hline Date & $\begin{array}{c}\text { Water } \\
\text { level MW }\end{array}$ & Date & $\begin{array}{c}\text { Water } \\
\text { level MW }\end{array}$ & Date & $\begin{array}{c}\text { Water } \\
\text { level MW }\end{array}$ & Date & $\begin{array}{c}\text { Water } \\
\text { level MW }\end{array}$ \\
\hline
\end{tabular}

\section{Site identifier: LAV-1}

Site number: 374051118523301

About 5.3 miles northeast of Mammoth Lakes in Little Antelope Valley. Observation well. Diameter 30 inches, depth 14 feet. Altitude of land-surface datum 7,200 feet. Water-level records available 1972-73, 1982-96.

\begin{tabular}{|c|c|c|c|c|c|c|}
\hline $04-06-94$ & $3.34 \mathrm{~S}$ & $04-20-95$ & $0.30 \mathrm{~S}$ & $04-24-96$ & $0.43 \mathrm{~S}$ & \\
\hline $07-13-94$ & $7.20 \mathrm{~V}$ & $07-14-95$ & $3.98 \mathrm{~S}$ & $07-19-96$ & $4.46 \mathrm{~S}$ & \\
\hline $11-09-94$ & $9.75 \mathrm{~S}$ & $11-07-95$ & $5.48 \mathrm{~S}$ & $11-21-96$ & $0.38 \mathrm{~S}$ & \\
\hline Highest & $04-20-95$ & 0.30 & & & & \\
\hline Lowest & $11-09-94$ & 9.75 & & & & \\
\hline Period of record & Highest & $05-10-86,08-27-86$ & +1.35 & Lowest & $11-09-94$ & 9.75 \\
\hline Record begins & $05-08-72$ & 39 Entries & & & & \\
\hline
\end{tabular}

Site identifier: LKT

Site number: 374300118554401

About 5.2 miles northeast of Mammoth Lakes and 1.3 miles east of Highway 395. Drilled geothermal exploration well. Diameter 6 inches, depth 3,500 feet, open hole 500-3,500 feet. Obstruction in well at 980 feet. Altitude of land-surface datum 7,350 feet. Water-level records available 1984-96.

\begin{tabular}{|c|c|c|c|c|c|c|c|c|}
\hline $01-12-94$ & $72.90 \mathrm{~S}$ & 09-20-94 & $73.73 \mathrm{~S}$ & $06-30-95$ & $73.55 \mathrm{~S}$ & & $04-24-96$ & $73.41 \mathrm{~S}$ \\
\hline 01-13-94 & $72.87 \mathrm{~S}$ & $10-18-94$ & $73.74 \mathrm{~S}$ & $07-11-95$ & $73.53 \mathrm{~S}$ & & $05-16-96$ & $72.99 \mathrm{~S}$ \\
\hline $02-14-94$ & $73.03 \mathrm{~S}$ & $11-09-94$ & $73.75 \mathrm{~S}$ & $07-18-95$ & $73.47 \mathrm{~S}$ & & $06-17-96$ & $72.88 \mathrm{~S}$ \\
\hline 03-08-94 & $73.04 \mathrm{~V}$ & $12-15-94$ & $73.92 \mathrm{~S}$ & $08-15-95$ & $73.46 \mathrm{~S}$ & & $07-15-96$ & $72.55 \mathrm{~S}$ \\
\hline 04-07-94 & $73.01 \mathrm{~S}$ & $01-16-95$ & $73.87 \mathrm{~S}$ & $08-25-95$ & $73.47 \mathrm{~S}$ & & $07-19-96$ & $72.54 \mathrm{~S}$ \\
\hline $05-19-94$ & $73.13 \mathrm{~S}$ & $02-24-95$ & $74.15 \mathrm{~S}$ & $09-13-95$ & $73.38 \mathrm{~S}$ & & $08-23-96$ & $72.26 \mathrm{~S}$ \\
\hline $06-09-94$ & $73.29 \mathrm{~S}$ & $03-07-95$ & $74.25 \mathrm{~S}$ & $10-17-95$ & $73.25 \mathrm{~S}$ & & $09-11-96$ & $72.01 \mathrm{~S}$ \\
\hline $06-29-94$ & $73.34 \mathrm{~S}$ & 04-14-95 & $74.28 \mathrm{~S}$ & $11-03-95$ & $73.23 \mathrm{~S}$ & & $10-18-96$ & $71.76 \mathrm{~S}$ \\
\hline $06-30-94$ & $73.30 \mathrm{~S}$ & $05-15-95$ & $74.06 \mathrm{~S}$ & $12-15-95$ & $73.06 \mathrm{~S}$ & & $11-18-96$ & $71.72 \mathrm{~S}$ \\
\hline $07-15-94$ & $73.33 \mathrm{~S}$ & $06-05-95$ & $73.64 \mathrm{~S}$ & $01-22-96$ & $73.14 \mathrm{~S}$ & & $12-09-96$ & $71.54 \mathrm{~S}$ \\
\hline $08-10-94$ & $73.53 \mathrm{~V}$ & $06-27-95$ & $73.60 \mathrm{~S}$ & $02-12-96$ & $73.25 \mathrm{~S}$ & & & \\
\hline $09-12-94$ & $73.53 \mathrm{~S}$ & $06-27-95$ & $73.56 \mathrm{~S}$ & $03-12-96$ & $73.11 \mathrm{~V}$ & & & \\
\hline Highest & $12-09-96$ & 71.54 & & & & & & \\
\hline Lowest & 04-14-95 & 74.28 & & & & & & \\
\hline Period of record & d Highest & $01-05-87$ & 57.61 & $04-14-95$ & & 74.28 & & \\
\hline Record begins & $11-12-84$ & 158 Entries & & & & & & \\
\hline
\end{tabular}

Site identifier: $\mathbf{L V}-2$

Site number: 373729118532802

About 4.5 miles southeast of Mammoth Lakes. Drilled observation well. Diameter 2 inches, drilled depth 44 feet, depth measured 41.5 feet in 1992, perforated 40-42 feet. Altitude of land-surface datum 7,167.39 feet. Water-level records available 1972-73, 1982-96.

\begin{tabular}{|c|c|c|c|c|c|c|c|}
\hline $04-07-94$ & SD & $06-15-95$ & $34.22 \mathrm{~V}$ & $04-25-96$ & $40.77 \mathrm{~V}$ & $09-17-96$ & $35.62 \mathrm{~S}$ \\
\hline $07-15-94$ & SD & $07-19-95$ & $26.05 \mathrm{~V}$ & $05-16-96$ & $37.10 \mathrm{~V}$ & $11-19-96$ & $39.51 \mathrm{~V}$ \\
\hline $11-08-94$ & SD & $08-15-95$ & $26.60 \mathrm{~V}$ & $06-04-96$ & $34.24 \mathrm{~V}$ & & \\
\hline 04-14-95 & SD & $09-12-95$ & $29.81 \mathrm{~V}$ & $07-18-96$ & $29.71 \mathrm{~V}$ & & \\
\hline $05-17-95$ & $41.35 \mathrm{~S}$ & $11-07-95$ & $36.44 \mathrm{~V}$ & $08-20-96$ & $32.43 \mathrm{~V}$ & & \\
\hline Highest & \multirow{2}{*}{\multicolumn{3}{|c|}{$\begin{array}{lr}07-19-95 & 26.05 \\
04-07-94,07-15-94,11-08-94,04-14-95\end{array}$}} & \multirow{2}{*}{\multicolumn{2}{|c|}{$>41.5$ (dry) }} & & \\
\hline Lowest & & & & & & & \\
\hline Period of record & d Highest & $05-26-82$ & \multirow[t]{2}{*}{24.07} & Lowest & \multirow[t]{2}{*}{$05-17-72$} & 42.65 & \\
\hline Record begins & $05-17-72$ & 56 Entries & & & & & \\
\hline
\end{tabular}


Table 12. Instantaneous ground-water levels in selected wells, 1994-96, Long Valley Caldera — Continued

\begin{tabular}{lllllll}
\hline Date & $\begin{array}{c}\text { Water } \\
\text { level MW }\end{array}$ & Date & $\begin{array}{c}\text { Water } \\
\text { level MW }\end{array}$ & Date & $\begin{array}{c}\text { Water } \\
\text { level MW }\end{array}$ & Date \\
\hline
\end{tabular}

\section{Site identifier: $\mathrm{LV}-15$}

Site number: 373913118551401

About 2.4 miles northeast of Mammoth Lakes and 0.9 mile north of junction of Highways 395 and 203. Drilled observation well. Diameter 2 inches, depth 57 feet, depth measured 14 feet in 1992, perforated 55-57 feet. Altitude of land-surface datum 7,340 feet. Water-level records available 1982-96.

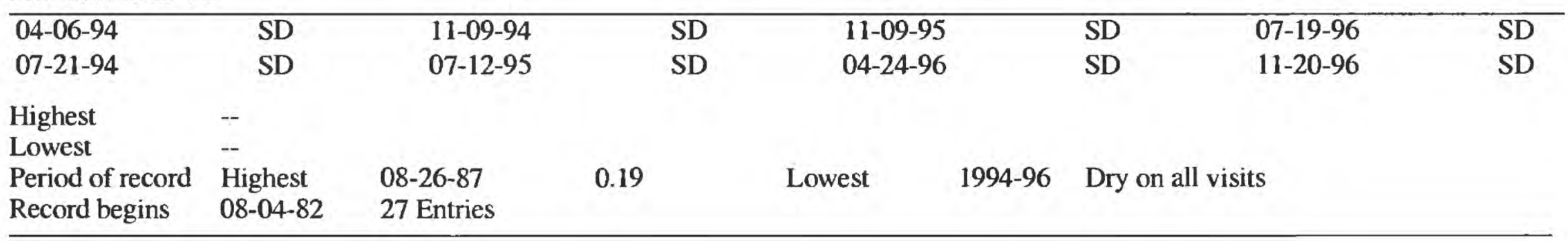

\section{Site identifier: $\mathrm{LV}-18$}

Site number: 373918118481901

About 8.8 miles east of Mammoth Lakes and 1.9 miles northeast of Whitmore Hot Springs. Drilled observation well. Diameter 2 inches, depth 71 feet, perforated 69-71 feet. Altitude of land-surface datum 6,966.0 feet. Water-level records available 1972-73, $1982-96$.

\begin{tabular}{lllllll}
\hline $04-06-94$ & $19.12 \mathrm{~S}$ & $04-13-95$ & $17.33 \mathrm{~S}$ & $04-25-96$ & $17.33 \mathrm{~S}$ & \\
$07-22-94$ & $19.38 \mathrm{~S}$ & $07-14-95$ & $17.34 \mathrm{~S}$ & $07-18-96$ & $17.38 \mathrm{~S}$ & \\
$11-10-94$ & $19.63 \mathrm{~S}$ & $11-07-95$ & $18.09 \mathrm{~S}$ & $11-20-96$ & $17.90 \mathrm{~S}$ & \\
Highest & $04-13-95,04-25-96$ & 17.33 & & & & \\
Lowest & $11-10-94$ & 19.63 & & & & \\
Period of record & Highest & $06-02-83$ & 12.44 & Lowest & $06-02-72$ & 21.0 \\
Record begins & $06-02-72$ & 48 Entries & & & & \\
\hline
\end{tabular}

Site identifier: $\mathbf{L V}-19$

Site number: 373754118501701

About 7 miles southeast of Mammoth Lakes and 1.4 miles west of Whitmore Hot Springs. Drilled observation well. Diameter 2 inches, depth 98.6 feet, perforated 96.6-98.6 feet. Altitude of land-surface datum 7,090.40 feet. Water-level records available 1972-73, 1986-96.

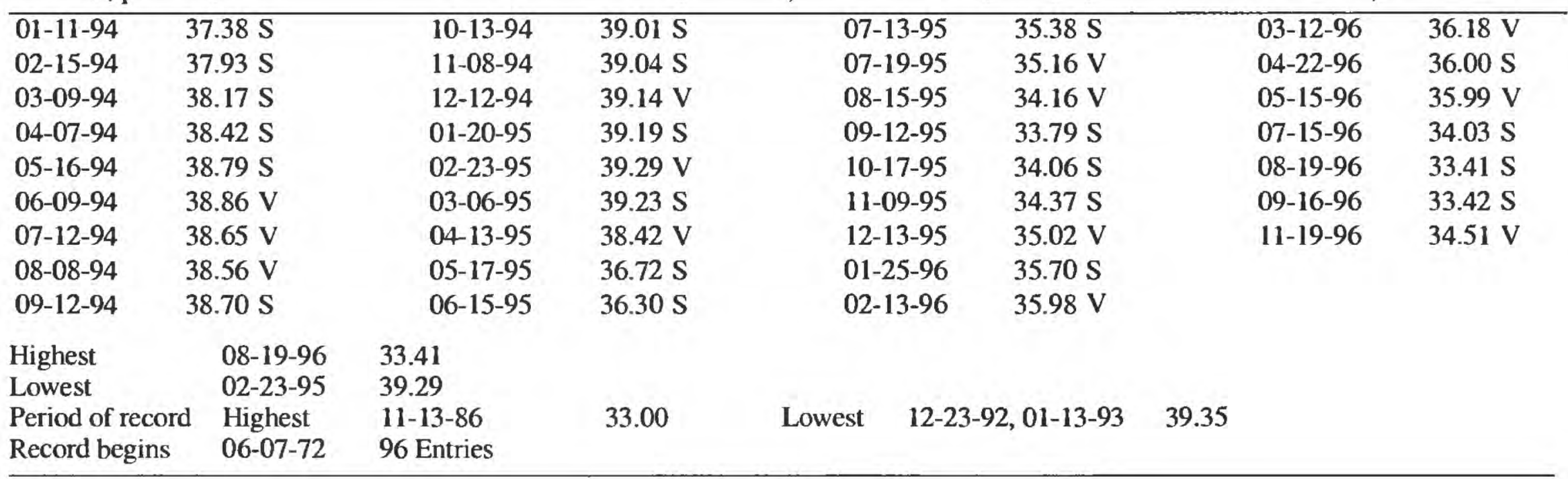


Table 12. Instantaneous ground-water levels in selected wells, 1994-96, Long Valley Caldera-Continued

\begin{tabular}{|c|c|c|c|c|c|c|c|}
\hline Data & $\begin{array}{l}\text { Water } \\
\text { level MW }\end{array}$ & Date & $\begin{array}{c}\text { Water } \\
\text { level MW }\end{array}$ & Date & $\begin{array}{c}\text { Water } \\
\text { level MW }\end{array}$ & Date & $\begin{array}{c}\text { Water } \\
\text { level MW }\end{array}$ \\
\hline
\end{tabular}

\section{Site identifier: $\mathbf{L V}-25$ \\ Site number: 374233118482701}

About 8.9 miles northeast of Mammoth Lakes and 0.8 mile north of Cashbaugh Ranch. Drilled observation well. Diameter 2 inches, depth 18 feet, depth measured 5.45 feet in 1992, perforated 16-18 feet. Altitude of land-surface datum $6,873.81$ feet. Water-level records available $1972-73,1982-96$.

\begin{tabular}{lllllll}
\hline $04-07-94$ & $2.58 \mathrm{~S}$ & $04-13-95$ & $1.17 \mathrm{~S}$ & $04-24-96$ & $1.59 \mathrm{~S}$ & \\
$07-13-94$ & $3.64 \mathrm{~S}$ & $07-20-95$ & $2.21 \mathrm{~S}$ & $07-17-96$ & $2.11 \mathrm{~S}$ & \\
$11-10-94$ & \multicolumn{1}{l}{$\mathrm{SD}$} & $11-07-95$ & $4.45 \mathrm{~S}$ & $11-20-96$ & $\mathrm{SD}$ & \\
Highest & $04-13-95$ & 1.17 & & & & \\
Lowest & $11-10-94,11-20-96$ & & & & & \\
Period of record & Highest & $06-03-83$ & 0.62 & Lowest & $07-25-72$ & \\
Record begins & $07-25-72$ & 47 Entries & & & & \\
\hline
\end{tabular}

Site identifier: $\mathbf{L V}-\mathbf{3 0}$

Site number: 374109118484101

About 8.9 miles northeast of Mammoth Lakes and 0.1 mile north of Cashbaugh Ranch. Drilled observation well. Diameter 2 inches, depth 9.1 feet, depth measured 5.9 feet in 1991, perforated 7.1-9.1 feet. Altitude of land-surface datum 6,895.62 feet. Water-level records available 1982-95.

\begin{tabular}{lclllll}
\hline $04-07-94$ & $1.16 \mathrm{~S}$ & \multicolumn{1}{c}{$04-13-95$} & $0.58 \mathrm{~V}$ & $07-20-95$ & $0.48 \mathrm{~S}$ & $11-07-95$ \\
$07-13-94$ & $4.19 \mathrm{~S}$ & & & & & \\
& & & & & & \\
Highest & $07-20-95$ & 0.48 & & & & \\
Lowest & $11-07-95$ & 5.50 & Lowest & $07-20-93$ & 5.56 & \\
$\begin{array}{l}\text { Period of record } \\
\text { Record begins }\end{array}$ & Highest & $06-03-83$ & 0.05 & & & \\
\hline
\end{tabular}

Site identifier: $\mathbf{L V}-\mathbf{4 4}$

Site number: 373917118551402

About 2.4 miles northeast of Mammoth Lakes and 1 mile north of junction of Highways 395 and 203. Drilled observation well. Diameter 2 inches, depth 79.7 feet, depth measured 55.2 feet in 1992, perforated 77.7-79.7 feet. Altitude of land-surface datum 7,340 feet. Waterlevel records available $1973-96$.

\begin{tabular}{lccrrrl}
\hline $04-06-94$ & $35.80 \mathrm{~S}$ & $04-20-95$ & $42.72 \mathrm{~V}$ & $04-24-96$ & $41.94 \mathrm{~V}$ \\
$07-21-94$ & $37.87 \mathrm{~S}$ & $07-12-95$ & $38.62 \mathrm{~V}$ & $07-19-96$ & $39.99 \mathrm{~V}$ & \\
$11-09-94$ & $40.28 \mathrm{~S}$ & $11-09-95$ & $39.51 \mathrm{~V}$ & $11-19-96$ & $41.09 \mathrm{~V}$ & \\
Highest & $04-06-94$ & 35.80 & & & & \\
Lowest & $04-20-95$ & 42.72 & & & & \\
Period of record & Highest & $05-21-86$ & +1.00 & Lowest & $04-20-95$ & 42.72 \\
Record begins & $08-07-73$ & 36 Entries & & & & \\
\hline
\end{tabular}

\section{Site identifier: $\mathrm{LV}-45$ \\ Site number: 373914118472201}

About 9.6 miles east of Mammoth Lakes and 2 miles northeast of Whitmore Hot Springs. Drilled observation well. Diameter 2 inches, depth 96 feet. Altitude of land-surface datum 6.985 .38 feet. Water-level records available 1982-96.

\begin{tabular}{|c|c|c|c|c|c|c|c|}
\hline $04-06-94$ & $40.15 \mathrm{~S}$ & $04-13-95$ & $40.23 \mathrm{~V}$ & & $4-96$ & $40.22 \mathrm{~V}$ & \\
\hline $07-12-94$ & $40.20 \mathrm{~V}$ & $07-14-95$ & $40.23 \mathrm{~V}$ & & $8-96$ & $40.17 \mathrm{~S}$ & \\
\hline $11-10-94$ & $40.23 \mathrm{~V}$ & $11-07-95$ & $40.25 \mathrm{~V}$ & & $0-96$ & $40.23 \mathrm{~V}$ & \\
\hline Highest & $04-06-94$ & 40.15 & & & & & \\
\hline Lowest & $11-07-95$ & 40.25 & & & & & \\
\hline Period of record & Highest & $10-19-85$ & 26.63 & Lowest & $11-0$ & & 4025 \\
\hline Record begins & $05-25-82$ & 44 Entries & & & & & \\
\hline
\end{tabular}


Table 12. Instantaneous ground-water levels in selected wells, 1994-96, Long Valley Caldera — Continued

\begin{tabular}{lllllll}
\hline Date & $\begin{array}{c}\text { Water } \\
\text { level MW }\end{array}$ & Date & $\begin{array}{c}\text { Water } \\
\text { level MW }\end{array}$ & Date & $\begin{array}{c}\text { Water } \\
\text { level MW }\end{array}$ & Date \\
\hline
\end{tabular}

Site identifier: MW-1

Site number: 373816118523901

About 4.6 miles east of Mammoth Lakes near Hot Creek Fish Hatchery. Drilled observation well. Diameter 6 inches $0-40$ feet, 2 inches 40-504 feet, depth 504 feet, perforated 450-504 feet. Well plugged, abandoned, and buried, October 1997. Altitude of land-surface datum 7046.43 feet. Water-level records available 1992-96.

\begin{tabular}{|c|c|c|c|c|c|c|c|c|}
\hline 01-12-94 & $34.55 \mathrm{~S}$ & 09-14-94 & $34.31 \mathrm{~V}$ & $07-19-95$ & $13.76 \mathrm{~V}$ & & $05-15-96$ & $23.44 \mathrm{~V}$ \\
\hline $02-16-94$ & $35.32 \mathrm{~S}$ & $10-13-94$ & $34.52 \mathrm{~V}$ & $09-12-95$ & $19.80 \mathrm{~S}$ & & $06-17-96$ & $17.79 \mathrm{~V}$ \\
\hline 03-09-94 & $35.61 \mathrm{~S}$ & $11-08-94$ & $35.15 \mathrm{~V}$ & $10-17-95$ & $25.80 \mathrm{~S}$ & & $07-15-96$ & $22.12 \mathrm{~V}$ \\
\hline 04-07-94 & $35.76 \mathrm{~S}$ & $12-15-94$ & $35.94 \mathrm{~V}$ & $11-09-95$ & $24.34 \mathrm{~S}$ & & $08-19-96$ & $19.79 \mathrm{~S}$ \\
\hline $05-16-94$ & $33.55 \mathrm{~S}$ & $02-23-95$ & $36.25 \mathrm{~V}$ & $01-25-96$ & $31.52 \mathrm{~V}$ & & $09-17-96$ & $31.23 \mathrm{~S}$ \\
\hline $06-09-94$ & $26.99 \mathrm{~V}$ & $03-23-95$ & $34.79 \mathrm{~S}$ & $02-14-96$ & $31.89 \mathrm{~V}$ & & & \\
\hline $07-12-94$ & $29.60 \mathrm{~V}$ & $05-17-95$ & $31.35 \mathrm{~V}$ & $03-12-96$ & $31.91 \mathrm{~S}$ & & & \\
\hline $08-10-94$ & $33.96 \mathrm{~V}$ & $06-15-95$ & $18.93 \mathrm{~V}$ & $04-22-96$ & $31.51 \mathrm{~S}$ & & & \\
\hline Highest & $07-19-95$ & 13.76 & & & & & & \\
\hline Lowest & $02-23-95$ & 36.25 & & & & & & \\
\hline Period of record & Highest & $07-19-95$ & 13.76 & $01-13-93$ & & 39.67 & & \\
\hline Record begins & $09-16-92$ & 40 Entries & & & & & & \\
\hline
\end{tabular}

Site identifier: MW-2

Site number: 373841118512902

About 5.6 miles east of Mammoth Lakes near Hot Creek Fish Hatchery. Drilled observation well. Diameter 5 inches 0-40 feet, 2 inches 40-255 feet, depth 255 feet, perforated 230-255 feet. Well plugged, abandoned, and buried, October 1997. Altitude of land-surface datum 7,057.08 feet. Water-level records available 1987-96.

\begin{tabular}{|c|c|c|c|c|c|c|c|c|}
\hline $04-06-94$ & $1.73 \mathrm{~S}$ & $11-08-94$ & $1.90 \mathrm{~S}$ & $11-09-95$ & $+0.10 \mathrm{SF}$ & & $07-19-96$ & $+0.34 \mathrm{SF}$ \\
\hline $07-21-94$ & $1.75 \mathrm{~S}$ & $07-12-95$ & $0.17 \mathrm{~S}$ & $04-23-96$ & $0.77 \mathrm{~S}$ & & $11-21-96$ & $+0.36 \mathrm{SF}$ \\
\hline Highest & $11-21-96$ & +0.36 & & & & & & \\
\hline Lowest & $11-08-94$ & 1.90 & & & & & & \\
\hline Period of record & Highest & $11-21-96$ & +0.36 & Lowest & & 2.11 & & \\
\hline Record begins & $04-19-87$ & 27 Entries & & & & & & \\
\hline
\end{tabular}

Site identifier: $\mathrm{MW}-4$

Site number: 373907118503801

About 6.5 miles east of Mammoth Lakes near Hot Creek Gorge. Drilled observation well. Diameter 6 inches 0-80 feet, 2 inches 80-340 feet, depth 340 feet, perforated 320-340 feet. Altitude of land-surface datum 7,081.26 feet. Water-level records available $1986-96$.

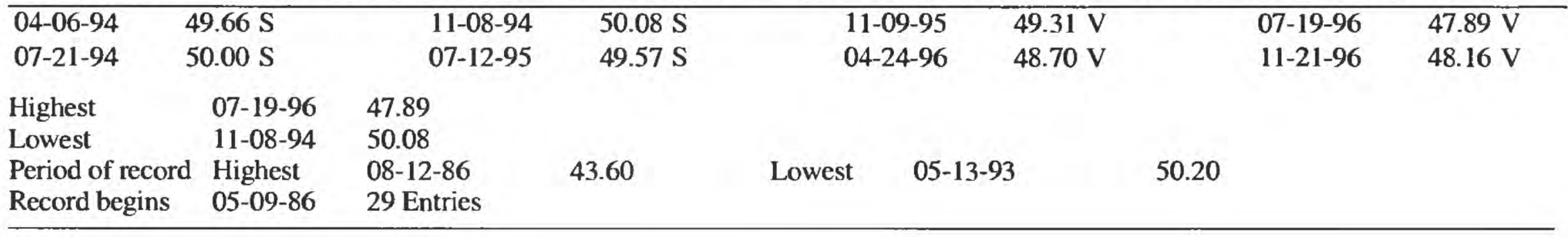


Table 12. Instantaneous ground-water levels in selected wells, 1994-96, Long Valley Caldera-Continued

\begin{tabular}{lcccccc}
\hline Date & $\begin{array}{c}\text { Water } \\
\text { level MW }\end{array}$ & Date & $\begin{array}{c}\text { Water } \\
\text { level MW }\end{array}$ & Date & $\begin{array}{c}\text { Water } \\
\text { level MW }\end{array}$ & $\begin{array}{c}\text { Water } \\
\text { level MW }\end{array}$ \\
\hline
\end{tabular}

\section{Site identifier: MW-5}

Site number: $\mathbf{3 7 3 8 4 1 1 1 8 5 1 2 9 0 1}$

About 5.6 miles east of Mammoth Lakes near Hot Creek Fish Hatchery. Drilled observation well. Diameter 6 inches 0-50 feet, 2 inches 50-496 feet, depth 496 feet, perforated 460-496 feet. Well plugged. abandoned. and buried. October 1997. Altitude of land-surface datum 7,054.12 feet. Water-level records available 1986-96.

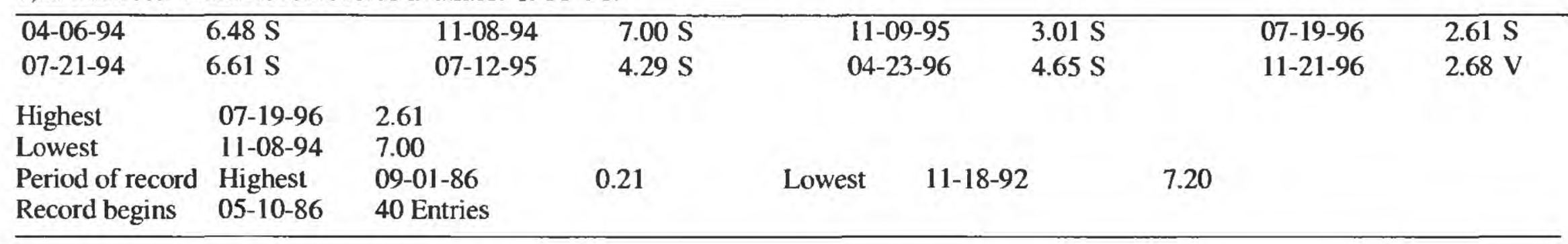

\section{Site identifier: MWD 5}

Site number: $\mathbf{3 7 3 7 5 7 1 1 8 5 9 1 8 0 1}$

In Old Mammoth near Mammoth Creek. Drilled unused well. Diameter 8 inches, depth 357.5 feet, perforated 59.8-112.7 and 132.7-357.5 feet. Altitude of land-surface datum 7,985.28 feet. Water-level records available 1988-96.

\begin{tabular}{|c|c|c|c|c|c|c|}
\hline 04-07-94 & $2.71 \mathrm{~S}$ & 04-19-95 & $0.87 \mathrm{~V}$ & & -96 & \\
\hline $07-22-94$ & $2.51 \mathrm{~S}$ & $07-19-95$ & $+0.30 \mathrm{~S}$ & & -96 & \\
\hline $11-08-94$ & $3.03 \mathrm{~S}$ & $11-02-95$ & $0.84 \mathrm{~S}$ & & -96 & \\
\hline Highest & $04-25-96$ & +1.82 & & & & \\
\hline Lowest & $11-08-94$ & 3.03 & & & & \\
\hline Period of record & Highest & $04-25-96$ & +1.82 & Lowest & $11-19-92$ & 7.10 \\
\hline Record begins & $03-03-88$ & 29 Entries & & & & \\
\hline
\end{tabular}

\section{Site identifier: MWD M-10}

Site number: 373719118585301

About 0.8 mile south of Mammoth Lakes near the Snow Creek development. Drilled observation well. Diameter 4 inches, depth measured 27.2 feet, perforated 7-27 feet. Altitude of land-surface datum 7,939.63 feet. Water-level records available 1988-96.

\begin{tabular}{llcrrr}
\hline $04-07-94$ & VD & $07-19-95$ & $26.90 \mathrm{~V}$ & $05-16-96$ & $23.91 \mathrm{~V}$ \\
$07-22-94$ & SD & $11-02-95$ & $26.71 \mathrm{~V}$ & $07-19-96$ & $27.00 \mathrm{~S}$ \\
$11-08-94$ & SD & $04-25-96$ & $26.88 \mathrm{~V}$ & $11-19-96$ & $26.90 \mathrm{~V}$ \\
Highest & $05-16-96$ & 23.91 & & & \\
Lowest & $04-07-94.07-22-94,11-08-94$ & $>27.2$ (dry) & & \\
Period of record & Highest & $07-28-88$ & 13.27 & Lowest & Dry on several visits \\
Record begins & $07-28-88$ & 30 Entries & & & \\
\hline
\end{tabular}

Site identifier: MWD M-11

Site number: 373705118585601

About 0.9 mile south of Mammoth Lakes near the Snow Creek development. Drilled observation well. Diameter 4 inches, depth 43 feet, perforated 5-43 feet. Altitude of land-surface datum 7,968.56 feet. Water-level records available 1988-96.

\begin{tabular}{|c|c|c|c|c|c|c|c|}
\hline $04-07-94$ & $2.31 \mathrm{~V}$ & $11-08-94$ & $32.48 \mathrm{~V}$ & $11-02-95$ & $17.68 \mathrm{~V}$ & $07-19-96$ & $11.22 \mathrm{~S}$ \\
\hline $07-22-94$ & $1.50 \mathrm{~S}$ & $07-19-95$ & $5.19 \mathrm{~V}$ & $04-25-96$ & $18.93 \mathrm{~V}$ & $11-19-96$ & $16.52 \mathrm{~V}$ \\
\hline Highest & $07-19-95$ & 5.19 & & & & & \\
\hline Lowest & $11-08-94$ & 32.48 & & & & & \\
\hline Period of record & Highest & $07-19-93$ & 4.26 & Lowest & $11-19-92$ & 34.40 & \\
\hline Record begins & $07-28-88$ & 31 Entries & & & & & \\
\hline
\end{tabular}


Table 12. Instantaneous ground-water levels in selected wells, 1994-96, Long Valley Caldera-Continued

\begin{tabular}{|c|c|c|c|c|c|c|c|}
\hline Date & $\begin{array}{l}\text { Watar } \\
\text { level MW }\end{array}$ & Date & $\begin{array}{c}\text { Water } \\
\text { level MW }\end{array}$ & Date & $\begin{array}{c}\text { Water } \\
\text { level MW }\end{array}$ & Date & $\begin{array}{c}\text { Water } \\
\text { level MW }\end{array}$ \\
\hline
\end{tabular}

Site identifier: MWD M-12

Site number: 373714118585501

About 0.9 mile south of Mammoth Lakes near the Snow Creek development. Drilled observation well. Diameter 4 inches, depth measured 26.45 feet in 1991, original depth 27 feet, perforated 7-27 feet. Altitude of land-surface datum 7,952.92 feet. Water-level records available 1988-96.

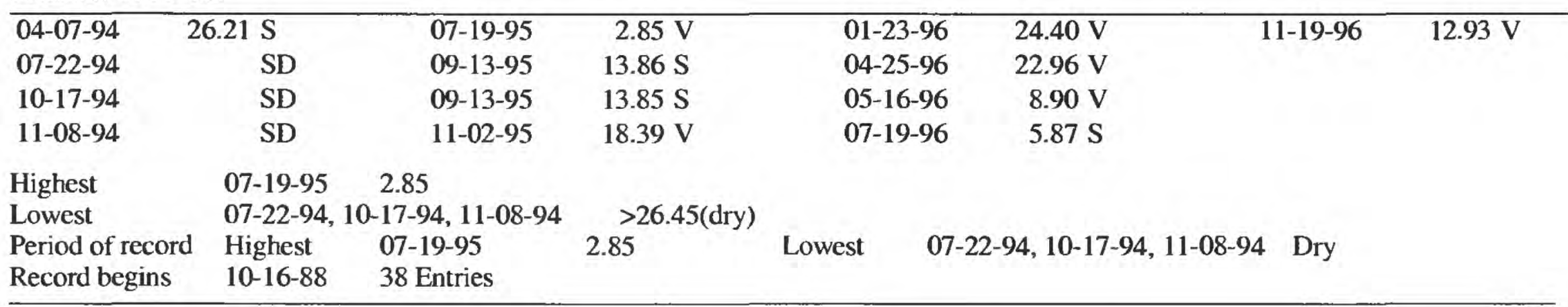

Site identifier: MWD M-14

Site number: 373739118582101

About 0.3 mile south of Mammoth Lakes in the Snow Creek development. Drilled observation well. Diameter 4 inches, depth 501 feet, perforated 100-501 feet. Altitude of land-surface datum 7.877.79 feet. Water-level records available 1988-96.

\begin{tabular}{|c|c|c|c|c|c|c|c|}
\hline 01-12-94 & $352.55 \mathrm{~V}$ & $10-13-94$ & $356.00 \mathrm{~V}$ & 08-15-95 & $319.12 \mathrm{~V}$ & 05-15-96 & $311.32 \mathrm{~V}$ \\
\hline $02-16-94$ & $352.78 \mathrm{~V}$ & $11-08-94$ & $356.61 \mathrm{~V}$ & $09-12-95$ & $312.30 \mathrm{~V}$ & $06-17-96$ & $291.60 \mathrm{~V}$ \\
\hline 03-10-94 & $353.60 \mathrm{~V}$ & $12-15-94$ & $357.01 \mathrm{~V}$ & $10-18-95$ & $320.15 \mathrm{~V}$ & $07-15-96$ & $273.10 \mathrm{~V}$ \\
\hline 04-04-94 & $353.79 \mathrm{~V}$ & 01-19-95 & $357.40 \mathrm{~V}$ & $11-03-95$ & $322.60 \mathrm{~V}$ & $08-22-96$ & $268.95 \mathrm{~V}$ \\
\hline 05-19-94 & $355.03 \mathrm{~V}$ & $02-23-95$ & $357.83 \mathrm{~V}$ & $12-14-95$ & $322.20 \mathrm{~V}$ & $09-16-96$ & $271.56 \mathrm{~V}$ \\
\hline $06-09-94$ & $355.45 \mathrm{~V}$ & $04-19-95$ & $357.40 \mathrm{~V}$ & $01-23-96$ & $325.90 \mathrm{~V}$ & $10-18-96$ & $280.21 \mathrm{~V}$ \\
\hline $07-12-94$ & $355.72 \mathrm{~V}$ & $05-17-95$ & $356.75 \mathrm{~V}$ & $02-15-96$ & $326.51 \mathrm{~V}$ & $11-15-96$ & $286.91 \mathrm{~V}$ \\
\hline 08-10-94 & $356.10 \mathrm{~V}$ & 06-15-95 & $336.22 \mathrm{~V}$ & $03-12-96$ & $325.49 \mathrm{~V}$ & $11-19-96$ & $289.85 \mathrm{~V}$ \\
\hline 09-14-94 & $356.43 \mathrm{~V}$ & 07-19-95 & $332.58 \mathrm{~V}$ & $04-22-96$ & $324.21 \mathrm{~V}$ & $12-18-96$ & $297.29 \mathrm{~V}$ \\
\hline Highest & $08-22-96$ & 268.95 & & \multirow{4}{*}{\multicolumn{2}{|c|}{$04-14-93$}} & & \\
\hline Lowest & 02-23-95 & 357.83 & & & & \multirow{3}{*}{360.12} & \\
\hline Period of recor & rd Highest & $10-16-88$ & 237.0 & & & & \\
\hline Record begins & $10-16-88$ & 83 Entries & & & & & \\
\hline
\end{tabular}

\section{Site identifier: PD}

Site number: 373732118490001

About 8.2 miles southeast of Mammoth Lakes at Mono County Probation Department Camp. Drilled institutional well. Diameter 6 inches, depth 150 feet. Altitude of land-surface datum 7,014 feet. Water-level records available 1986-95.

\begin{tabular}{lclllll}
\hline $04-06-94$ & $24.20 \mathrm{~S}$ & \multicolumn{1}{c}{$07-22-94$} & $24.92 \mathrm{SR}$ & & $04-13-95$ & $22.07 \mathrm{~S}$ \\
Highest & $04-13-95$ & 22.07 & & & & \\
Lowest & $04-06-94$ & 24.20 & & & & \\
Period of record & Highest & $08-30-86$ & 21.69 & Lowest & $04-25-89$ & 29.45 \\
Record begins & $05-21-86$ & 26 Entries & & & & \\
\hline
\end{tabular}


Table 12. Instantaneous ground-water levels in selected wells, 1994-96, Long Valley Caldera-Continued

\begin{tabular}{llllllll}
\hline Date & $\begin{array}{c}\text { Water } \\
\text { level MW }\end{array}$ & Date & $\begin{array}{c}\text { Water } \\
\text { level MW }\end{array}$ & Date & $\begin{array}{c}\text { Water } \\
\text { level MW }\end{array}$ & $\begin{array}{c}\text { Water } \\
\text { level MW }\end{array}$ \\
\hline
\end{tabular}

Site identifier: PLV-2

Site number: 374249118584501

About 4.7 miles north of Mammoth Lakes and 1.3 miles west of Highway 395. Drilled geothermal exploration well. Diameter 1.25 inches, depth 2,330 feet, perforated 1,845-1,855 feet. Altitude of land-surface datum 7,734 feet. Water-level records available $1984-96$.

\begin{tabular}{lclllll}
\hline $05-16-94$ & $442.51 \mathrm{~V}$ & $11-07-94$ & $444.74 \mathrm{~V}$ & $07-18-95$ & $445.30 \mathrm{~V}$ & $07-17-96$ \\
$07-13-94$ & $442.82 \mathrm{~V}$ & $04-20-95$ & $446.55 \mathrm{~V}$ & $11-06-95$ & $439.30 \mathrm{~V}$ & \\
Highest & $07-17-96$ & 427.75 & & & & \\
Lowest & $04-20-95$ & 446.55 & & & & \\
Period of record & Highest & $08-30-84$ & 398.00 & Lowest & $04-20-95$ & 446.55 \\
Record begins & $08-30-84$ & 45 Entries & & & & \\
\hline
\end{tabular}

\section{Site identifier: RDO-8}

Site number: 373924118571201

About 0.8 mile northeast of Mammoth Lakes and 0.7 mile north of U.S. Forest Service ranger station. Drilled observation well. Diameter 2.38 inches, depth 1,304 feet, original depth of hole 2,346 feet, perforated 1,100-1,110 feet. Altitude of land-surface datum 7,788.4 feet. Water-level records available 1986-96.

\begin{tabular}{|c|c|c|c|c|c|c|c|c|}
\hline $01-13-94$ & $435.82 \mathrm{~V}$ & $10-13-94$ & $435.97 \mathrm{~V}$ & $08-15-95$ & $435.72 \mathrm{~V}$ & & $06-17-96$ & $432.82 \mathrm{~V}$ \\
\hline $02-14-94$ & $435.00 \mathrm{~V}$ & 11-09-94 & $436.23 \mathrm{~V}$ & $09-12-95$ & $435.80 \mathrm{~V}$ & & $07-15-96$ & $433.48 \mathrm{~V}$ \\
\hline 03-08-94 & $435.20 \mathrm{~V}$ & $01-20-95$ & $438.77 \mathrm{~V}$ & $10-18-95$ & $435.10 \mathrm{~V}$ & & $08-19-96$ & $432.11 \mathrm{~V}$ \\
\hline 04-13-94 & $435.65 \mathrm{~V}$ & $02-23-95$ & $437.35 \mathrm{~V}$ & 11-03-95 & $435.02 \mathrm{~V}$ & & $08-22-96$ & $432.00 \mathrm{~V}$ \\
\hline $04-21-94$ & $435.72 \mathrm{~V}$ & $04-20-95$ & $436.45 \mathrm{~V}$ & $01-25-96$ & $434.30 \mathrm{~V}$ & & $11-19-96$ & $430.12 \mathrm{~V}$ \\
\hline $05-19-94$ & $436.20 \mathrm{~V}$ & $05-17-95$ & $436.05 \mathrm{~V}$ & $02-14-96$ & $434.35 \mathrm{~V}$ & & & \\
\hline $08-10-94$ & $436.70 \mathrm{~V}$ & $06-15-95$ & $435.63 \mathrm{~V}$ & $04-23-96$ & $434.80 \mathrm{~V}$ & & & \\
\hline $09-15-94$ & $436.55 \mathrm{~V}$ & $07-20-95$ & $435.80 \mathrm{~V}$ & $05-14-96$ & $435.14 \mathrm{~V}$ & & & \\
\hline Highest & $11-19-96$ & 430.12 & & \multirow{4}{*}{\multicolumn{2}{|c|}{ 06-07-89 }} & \multirow{4}{*}{448.40} & & \\
\hline Lowest & 01-20-95 & 438.77 & & & & & & \\
\hline Period of recorc & rd Highest & $11-19-96$ & 430.12 & & & & & \\
\hline Record begins & $10-21-86$ & 124 Entries & & & & & & \\
\hline
\end{tabular}

Site identifier: SC-1

Site number: 373745118554001

About 2.3 miles southeast of Mammoth Lakes. Drilled observation well. Diameter 2.5 inches, depth of hole 132 feet, open hole 40 to 132 feet. Altitude of land-surface datum 7,469.84 feet. Water-level records available 1982-96.

\begin{tabular}{|c|c|c|c|c|c|c|c|}
\hline $01-12-94$ & $118.20 \mathrm{~V}$ & 11-08-94 & $120.34 \mathrm{~V}$ & $09-12-95$ & $99.95 \mathrm{~V}$ & $07-15-96$ & $99.58 \mathrm{~V}$ \\
\hline $02-14-94$ & $119.61 \mathrm{~V}$ & $12-15-94$ & $118.97 \mathrm{~S}$ & $10-18-95$ & $108.67 \mathrm{~V}$ & $08-20-96$ & $106.61 \mathrm{~V}$ \\
\hline 03-08-94 & $120.03 \mathrm{~V}$ & $01-20-95$ & $120.64 \mathrm{~V}$ & $11-07-95$ & $110.98 \mathrm{~V}$ & 09-16-96 & $109.23 \mathrm{~V}$ \\
\hline 04-07-94 & $119.92 \mathrm{~V}$ & $02-23-95$ & $121.19 \mathrm{~V}$ & $12-14-95$ & $113.15 \mathrm{~V}$ & $10-15-96$ & $111.08 \mathrm{~V}$ \\
\hline $05-16-94$ & $117.68 \mathrm{~V}$ & 03-07-95 & $121.27 \mathrm{~V}$ & $01-23-96$ & $114.00 \mathrm{~V}$ & $11-15-96$ & $111.30 \mathrm{~V}$ \\
\hline $06-09-94$ & $114.30 \mathrm{~V}$ & 04-14-95 & $113.95 \mathrm{~V}$ & $02-16-96$ & $114.14 \mathrm{~V}$ & $11-19-96$ & $111.47 \mathrm{~V}$ \\
\hline $07-12-94$ & $115.62 \mathrm{~V}$ & $05-17-95$ & $111.18 \mathrm{~V}$ & $04-22-96$ & $111.23 \mathrm{~V}$ & $12-09-96$ & $110.87 \mathrm{~V}$ \\
\hline $08-10-94$ & $117.93 \mathrm{~V}$ & $06-15-95$ & $99.41 \mathrm{~V}$ & $05-15-96$ & $107.41 \mathrm{~V}$ & $12-18-96$ & $110.99 \mathrm{~V}$ \\
\hline $09-14-94$ & $119.34 \mathrm{~V}$ & $07-19-95$ & $81.06 \mathrm{~V}$ & 06-04-96 & $96.45 \mathrm{~V}$ & & \\
\hline $10-13-94$ & $119.85 \mathrm{~V}$ & 08-15-95 & $90.29 \mathrm{~V}$ & $06-17-96$ & $92.38 \mathrm{~V}$ & & \\
\hline Highest & $07-19-95$ & 81.06 & & \multirow{4}{*}{\multicolumn{2}{|c|}{$03-07-95$}} & \multirow{4}{*}{12127} & \\
\hline Lowest & 03-07-95 & 121.27 & & & & & \\
\hline Period of record & Highest & $07-06-83$ & 69.95 & & & & \\
\hline Record begins & $08-05-82$ & 122 Entries & & & & & \\
\hline
\end{tabular}


Table 12. Instantaneous ground-water levels in selected wells, 1994-96, Long Valley Caldera-Continued

\begin{tabular}{lllllll}
\hline Date & $\begin{array}{c}\text { Water } \\
\text { level MW }\end{array}$ & Date & $\begin{array}{c}\text { Water } \\
\text { level MW }\end{array}$ & Date & $\begin{array}{c}\text { Water } \\
\text { level MW }\end{array}$ & Date \\
\hline
\end{tabular}

Site identifier: $\mathrm{SC}-2$

Site number: 373745118554002

About 2.3 miles southeast of Mammoth Lakes. Drilled observation well. Diameter 4 inches, depth of hole 230 feet, open hole $215-230$ feet. Altitude of land-surface datum 7,470.65 feet. Water-level records available 1983-96.

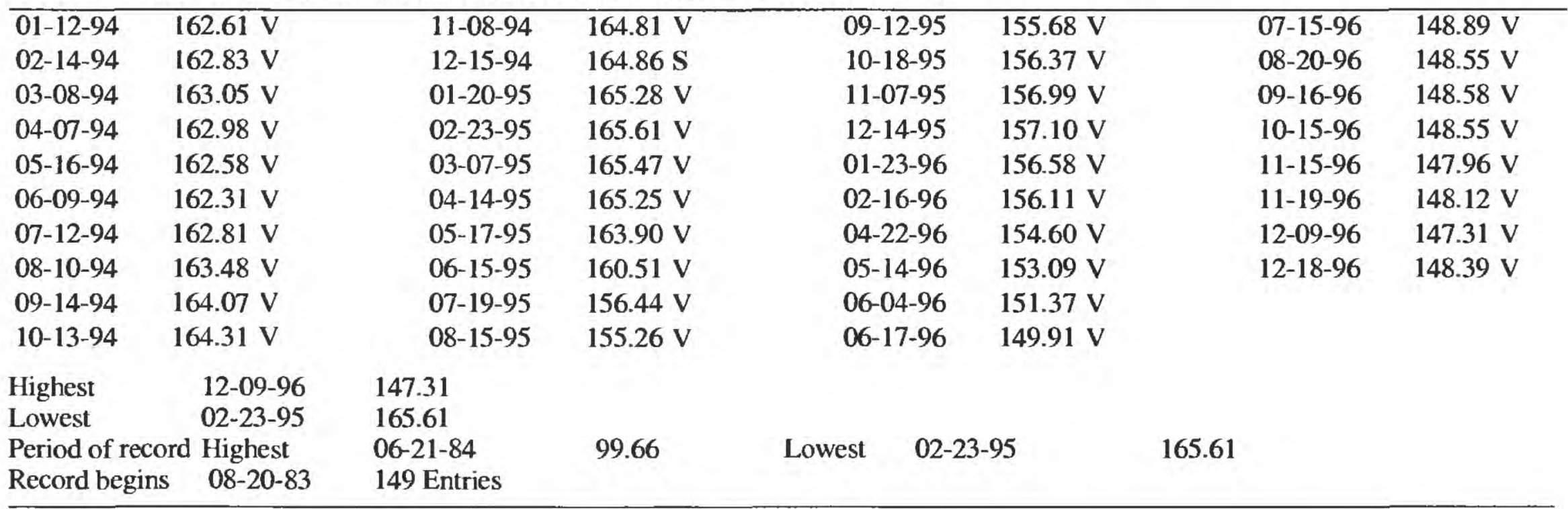

Site identifier: SNARI

Site number: 373654118495401

About 7.6 miles southeast of Mammoth Lakes at the Sierra Nevada Aquatic Research Laboratory. Drilled institutional well. Diameter 6 inches, depth 70 feet, perforated 34-70 feet. Altitude of land-surface datum 7,095 feet. Water-level records available $1959-96$.

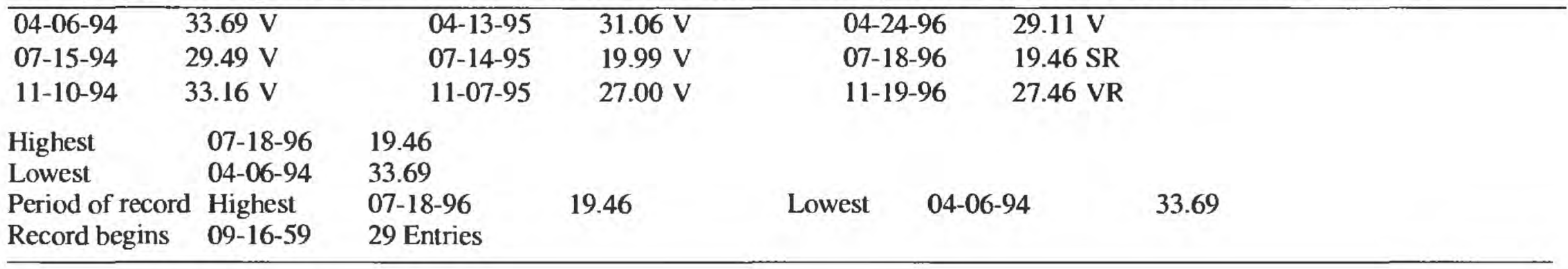

Site identifier: $\mathrm{SQ}$

Site number: 373742118515703

About 5.6 miles southeast of Mammoth Lakes. Drilled industrial well. Diameter 8 inches, depth 125 feet, perforated $27-125$ feet. Altitude of land-surface datum 7,102.22 feet. Water-level records available 1984-96.

\begin{tabular}{|c|c|c|c|c|c|c|c|c|}
\hline $01-11-94$ & $20.75 \mathrm{~S}$ & $12-13-94$ & $22.17 \mathrm{~V}$ & $10-17-95$ & $14.78 \mathrm{~S}$ & & $06-17-96$ & $14.31 \mathrm{~V}$ \\
\hline $02-15-94$ & $21.46 \mathrm{~V}$ & $02-23-95$ & $22.13 \mathrm{~V}$ & $11-07-95$ & $15.68 \mathrm{~V}$ & & $07-15-96$ & $13.65 \mathrm{~V}$ \\
\hline 03-09-94 & $21.32 \mathrm{~V}$ & $03-23-95$ & $20.96 \mathrm{~S}$ & 11-09-95 & $15.68 \mathrm{~S}$ & & $07-18-96$ & $13.52 \mathrm{~S}$ \\
\hline 04-07-94 & $21.65 \mathrm{~S}$ & $04-13-95$ & $19.92 \mathrm{~V}$ & $12-14-95$ & $17.39 \mathrm{~V}$ & & $08-19-96$ & $13.40 \mathrm{~S}$ \\
\hline $05-16-94$ & $21.23 \mathrm{~S}$ & $05-17-95$ & $18.65 \mathrm{~V}$ & $01-25-96$ & $18.31 \mathrm{~S}$ & & $09-16-96$ & $15.52 \mathrm{~S}$ \\
\hline 06-09-94 & $19.94 \mathrm{~V}$ & $06-15-95$ & $15.72 \mathrm{~V}$ & $02-14-96$ & $18.56 \mathrm{~S}$ & & $10-18-96$ & $16.43 \mathrm{~V}$ \\
\hline $07-12-94$ & $19.60 \mathrm{~V}$ & $07-13-95$ & $12.29 \mathrm{~S}$ & $03-12-96$ & $18.59 \mathrm{~S}$ & & $11-15-96$ & $17.07 \mathrm{~S}$ \\
\hline 08-08-94 & $20.87 \mathrm{~V}$ & $07-19-95$ & $11.65 \mathrm{~V}$ & $04-22-96$ & $18.40 \mathrm{~S}$ & & $11-19-96$ & $17.15 \mathrm{~V}$ \\
\hline $09-12-94$ & $21.68 \mathrm{~S}$ & 08-01-95 & $10.94 \mathrm{~V}$ & $05-14-96$ & $16.65 \mathrm{~S}$ & & $12-18-96$ & $17.76 \mathrm{~V}$ \\
\hline $10-13-94$ & $21.81 \mathrm{~S}$ & $08-15-95$ & $10.43 \mathrm{~V}$ & $05-16-96$ & $16.61 \mathrm{~V}$ & & & \\
\hline $11-08-94$ & $21.95 \mathrm{~S}$ & $09-12-95$ & $11.80 \mathrm{~S}$ & $06-04-96$ & $15.95 \mathrm{~V}$ & & & \\
\hline Highest & $08-15-95$ & 10.43 & & & & & & \\
\hline Lowest & $12-13-94$ & 22.17 & & & & & & \\
\hline Period of record & d Highest & $08-15-95$ & 10.43 & $12-16-92$ & & 22.34 & & \\
\hline Record begins & $07-17-84$ & 114 Entries & & & & & & \\
\hline
\end{tabular}


Table 12. Instantaneous ground-water levels in selected wells, 1994-96, Long Valley Caldera-Continued

\begin{tabular}{lllcllll}
\hline Date & $\begin{array}{c}\text { Water } \\
\text { level MW }\end{array}$ & Date & $\begin{array}{c}\text { Water } \\
\text { level MW }\end{array}$ & Date & $\begin{array}{c}\text { Water } \\
\text { level MW }\end{array}$ & Date & Water \\
level MW
\end{tabular}

Site identifier: SS-2

Site number: $\mathbf{3 7 3 8 1 8 1 1 8 5 3 3 2 0 2}$

About 3.9 miles east of Mammoth Lakes at former Mono County Sheriff substation. Drilled public-supply well. Diameter 6 inches, depth 100 feet, perforated 34-60 and 80-100 feet. Altitude of land-surface datum 7,179.13 feet. Water-level records available 1978, $1984-96$.

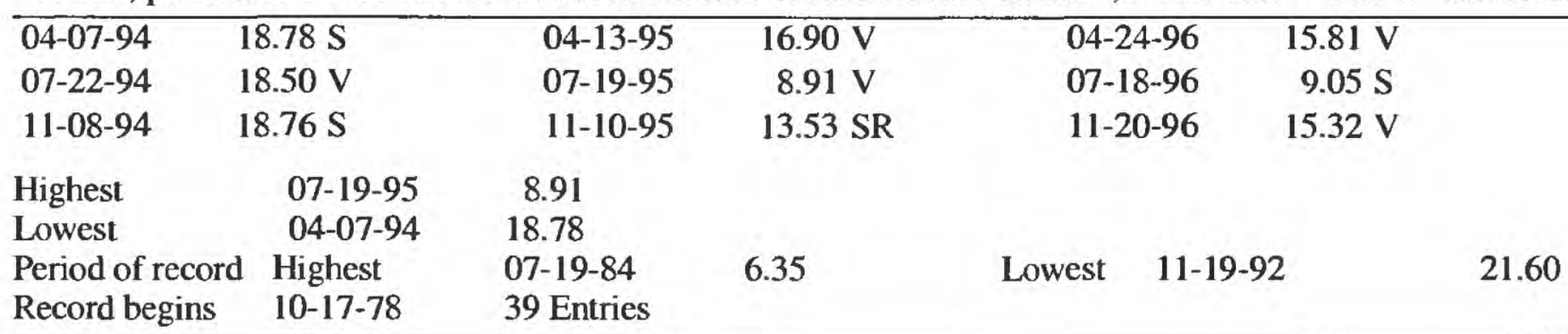




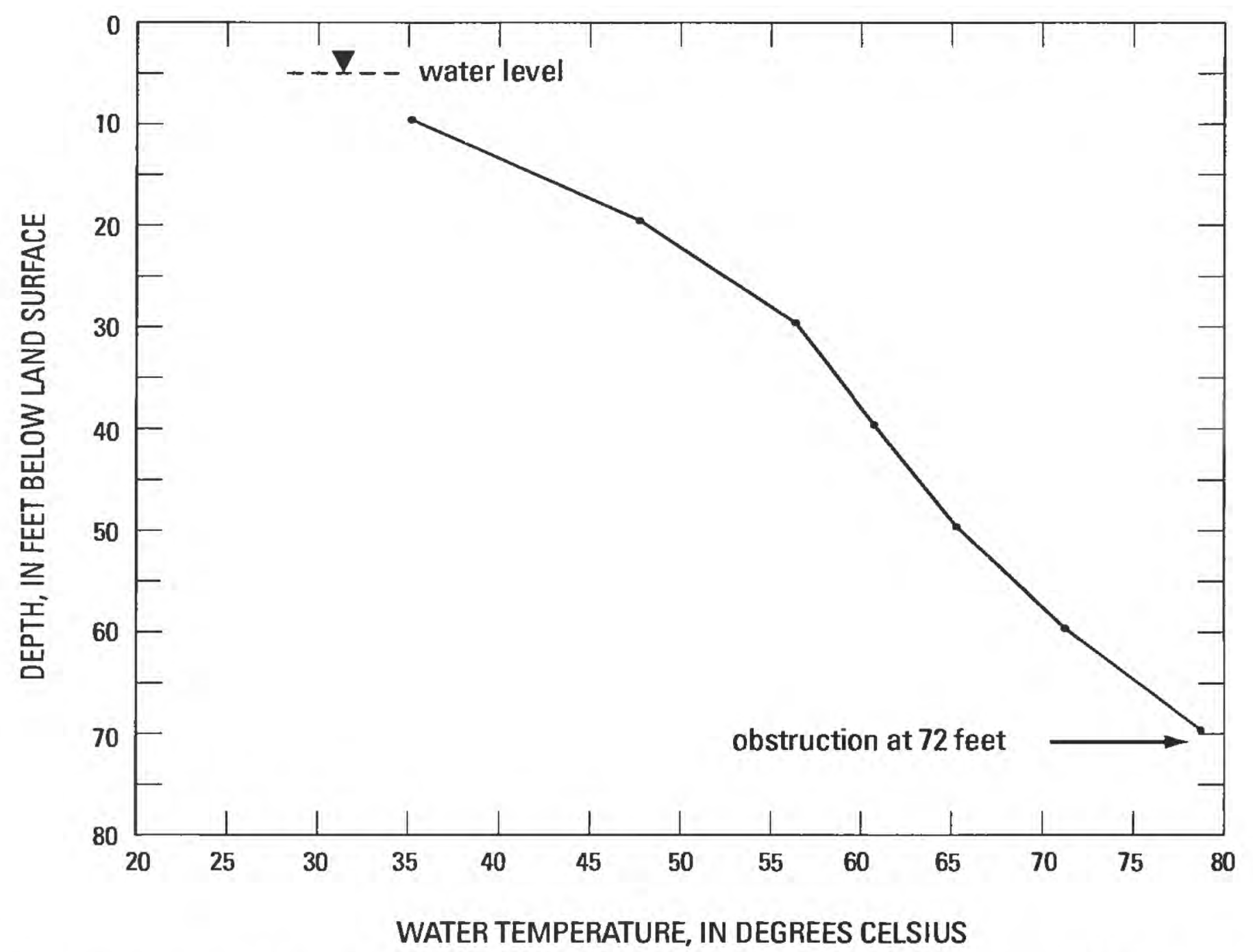

Figure 14. Temperature profile in well CH-7, June 28, 1995, Long Valley Caldera, Mono County, California. Total depth of well is 210 feet. Log ends at 70 feet because of obstruction at 72 feet.

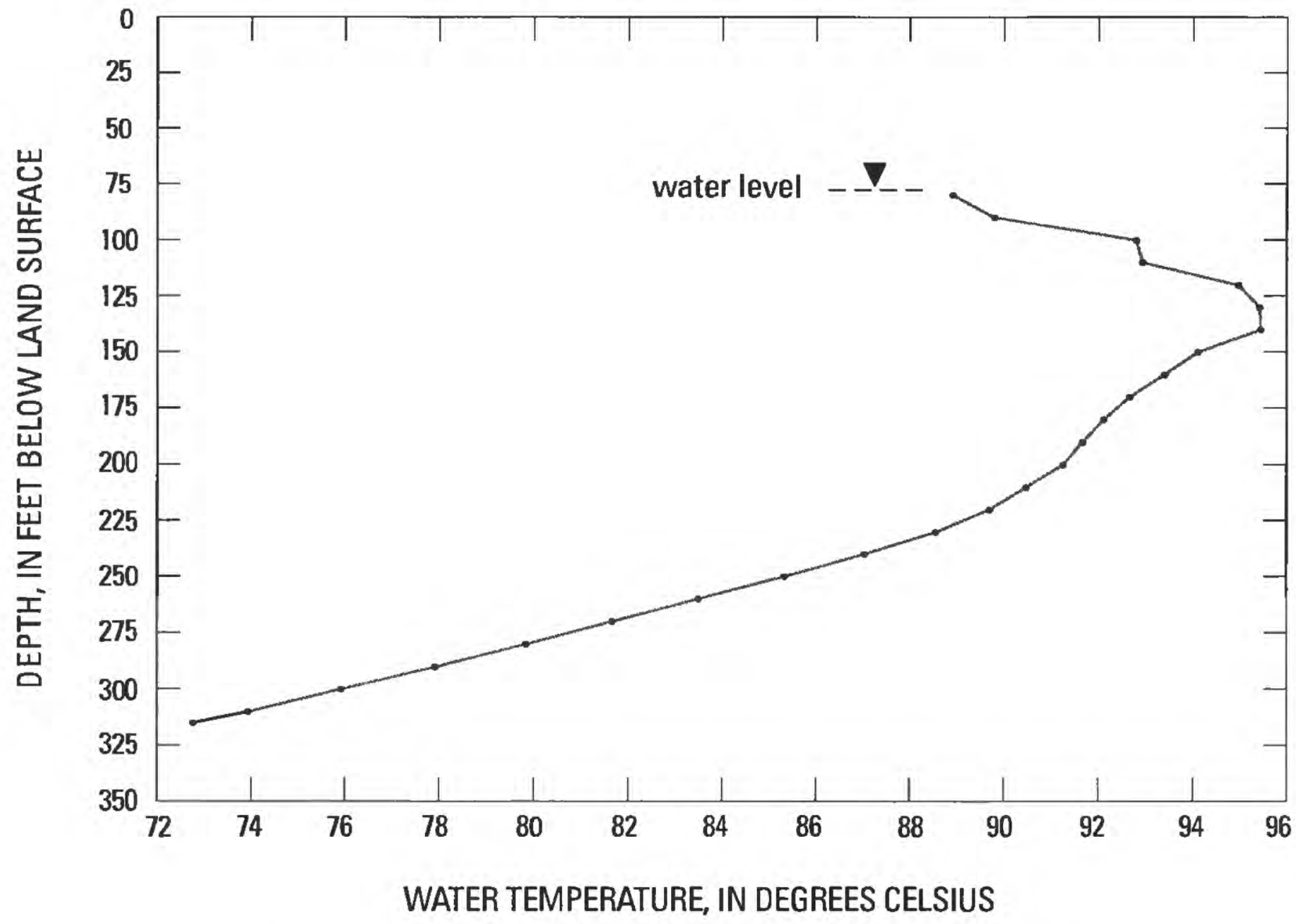

Figure 15. Temperature profile in well CH-10B, January 13, 1994, Long Valley Caldera, Mono County, California. 


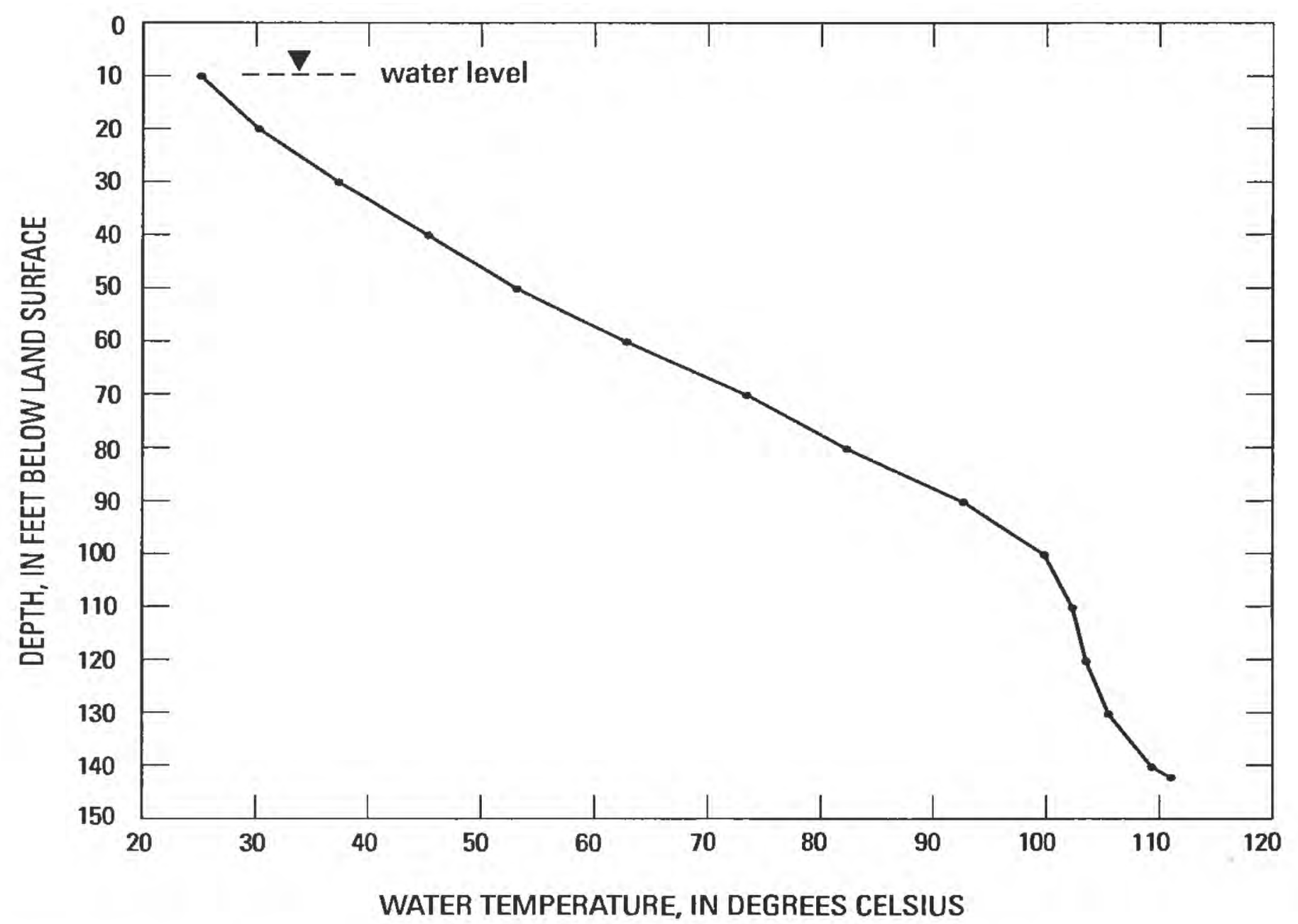

Figure 16. Temperature profile in well CM-2, January 28, 1994, Long Valley Caldera, Mono County, California.

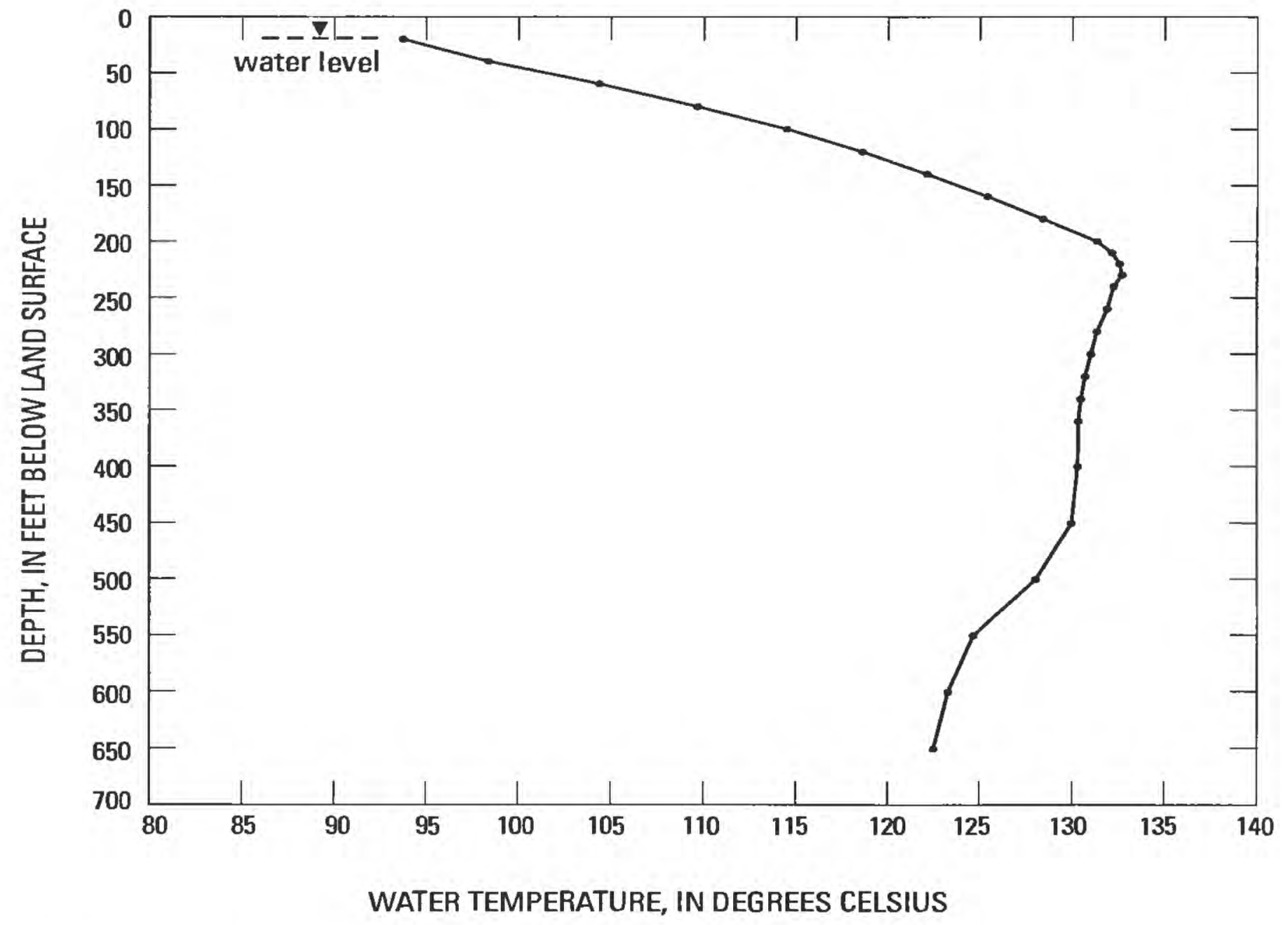

Figure 17. Temperature profile in well CW-1, January 28, 1994, Long Valley Caldera, Mono County, California. 


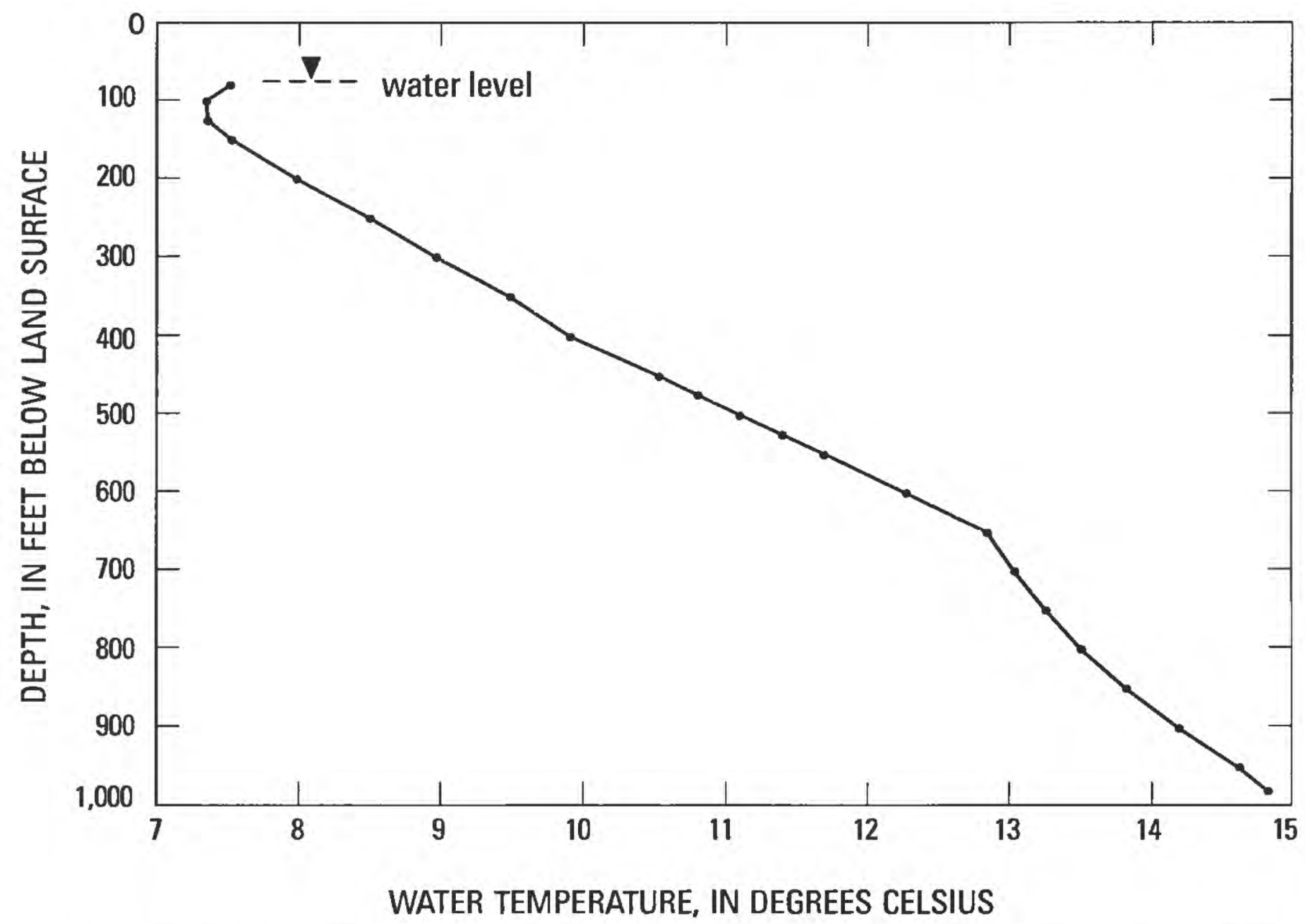

Figure 18. Temperature profile in Lookout Mountain well (LKT), January 12, 1994, Long Valley Caldera, Mono County, California.

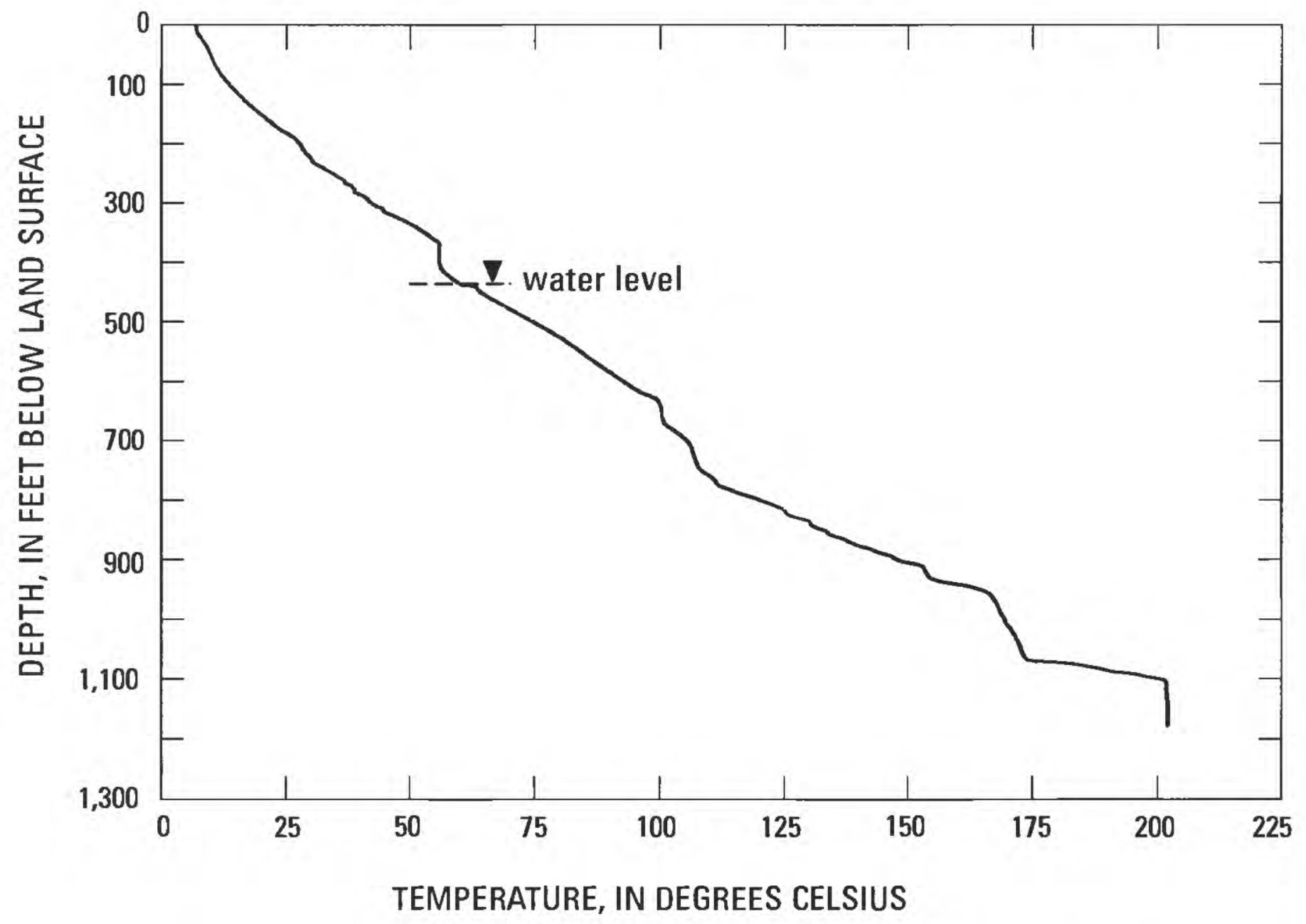

Figure 19. Temperature profile in well RD0-8, April 22, 1994, Long Valley Caldera, Mono County, California (Data provided by R. Jacobson, Sandia National Laboratories, New Mexico). 


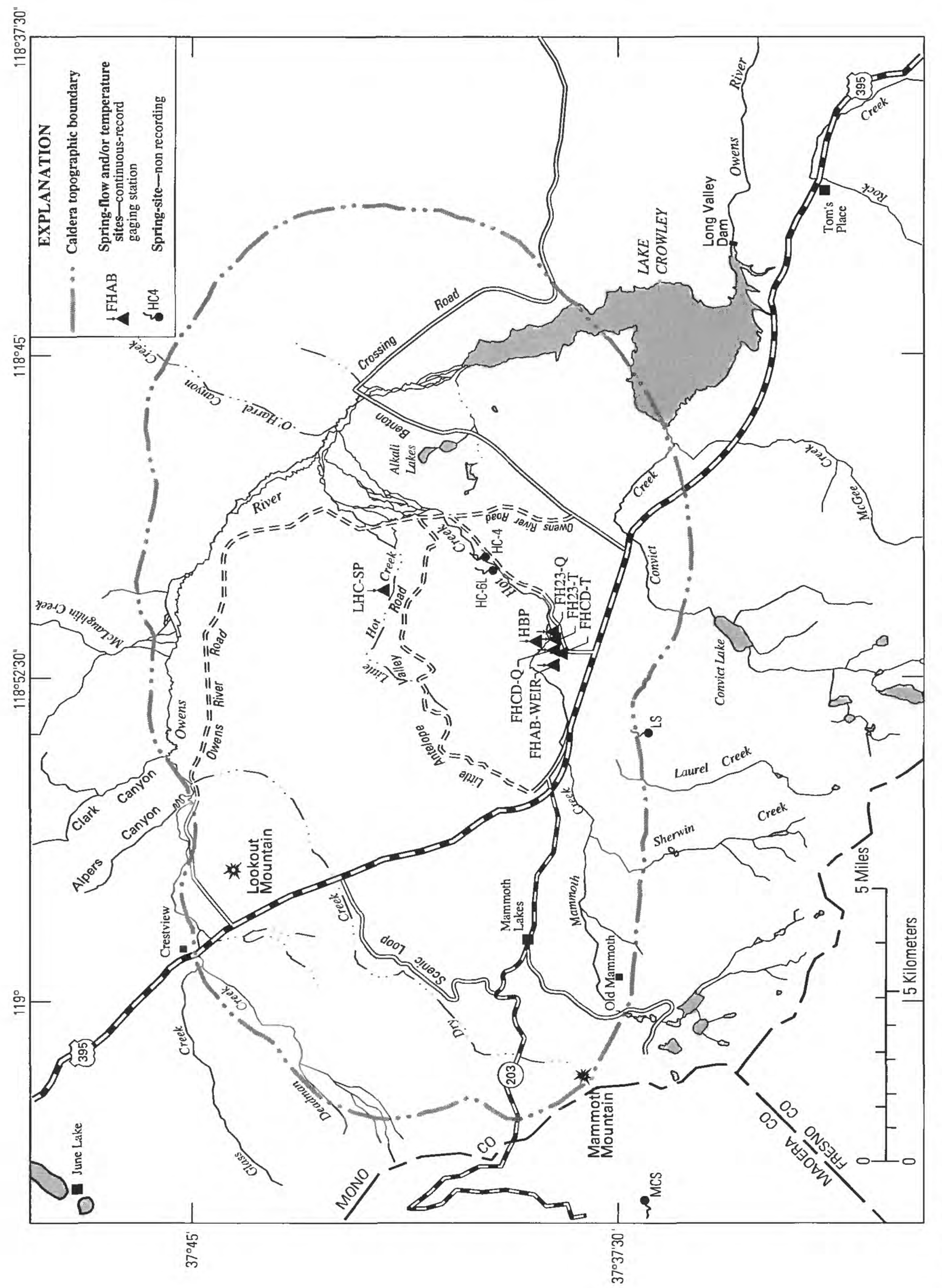

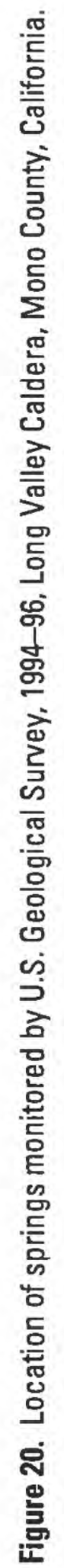




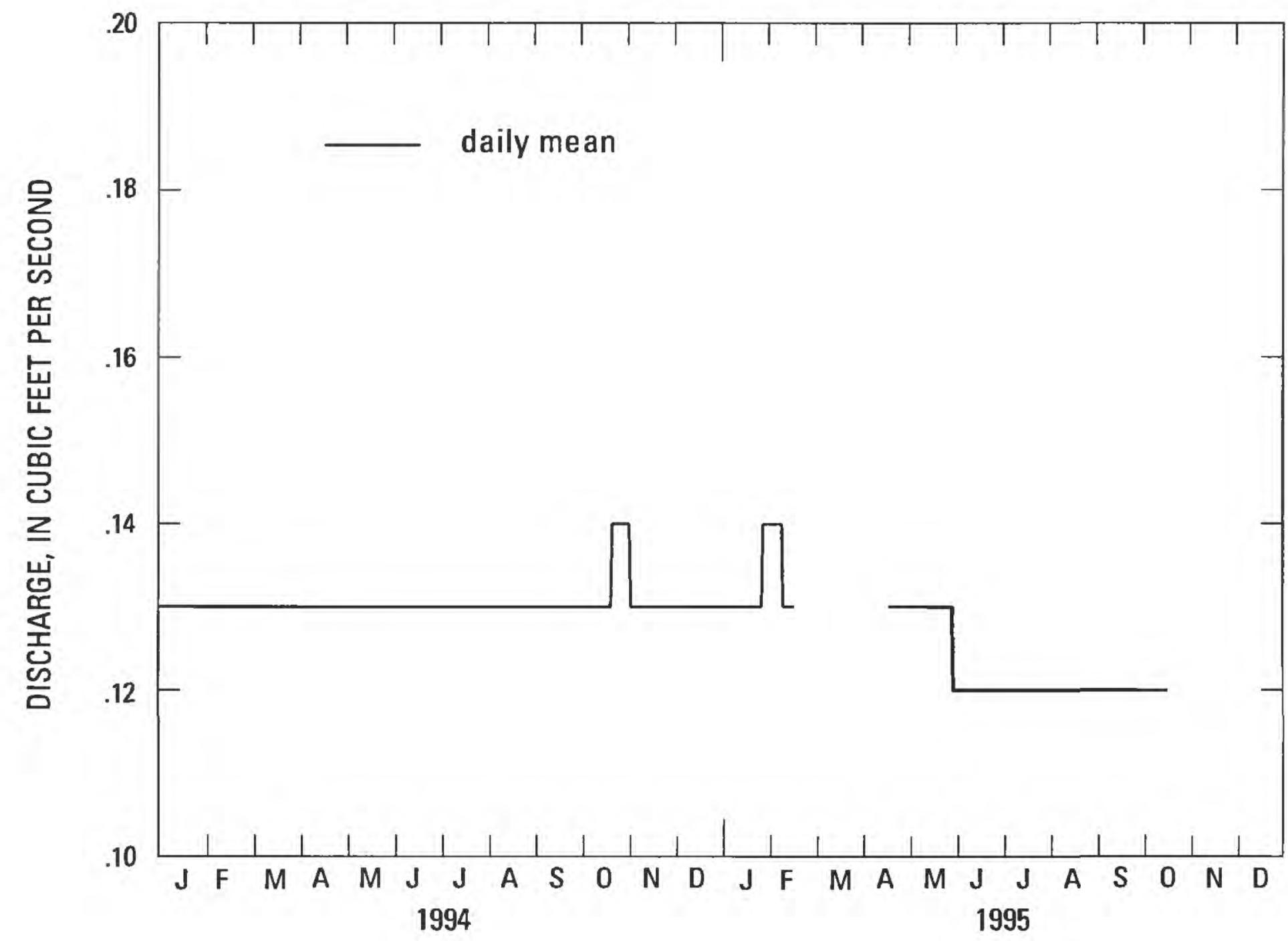

Figure 21. Daily mean discharge, Little Hot Creek Spring (LHC-SP), 1994-95, Long Valley Caldera, Mono County, California. Measurements discontinued October 17, 1995. 
Table 13. Daily mean discharge, Little Hot Creek Spring (LHC-SP), 1994, Long Valley Caldera, California

[Values are in cubic feet per second. -, no data; Max, maximum; Min, minimum]

\begin{tabular}{|c|c|c|c|c|c|c|c|c|c|c|c|c|}
\hline Day & Jan & Feb & Mar & Apr & May & Jun & Jul & Aug & Sep & Oct & Nov & Dec \\
\hline 1 & 0.13 & 0.13 & 0.13 & 0.13 & 0.13 & 0.13 & 0.13 & 0.13 & 0.13 & 0.13 & 0.14 & 0.13 \\
\hline 2 & .13 & .13 & .13 & .13 & .13 & .13 & .13 & .13 & .13 & .13 & .13 & .13 \\
\hline 3 & .13 & .13 & .13 & .13 & .13 & .13 & .13 & .13 & .13 & .13 & .13 & .13 \\
\hline 4 & .13 & .13 & .13 & .13 & .13 & .13 & .13 & .13 & .13 & .13 & .13 & .13 \\
\hline 5 & .13 & .13 & .13 & .13 & .13 & .13 & .13 & .13 & .13 & .13 & .13 & .13 \\
\hline 6 & .13 & .13 & .13 & .13 & .13 & .13 & .13 & .13 & .13 & .13 & .13 & .13 \\
\hline 7 & .13 & .13 & .13 & .13 & .13 & .13 & .13 & .13 & .13 & .13 & .13 & .13 \\
\hline 8 & .13 & .13 & .13 & .13 & .13 & .13 & .13 & .13 & .13 & .13 & .13 & .13 \\
\hline 9 & .13 & .13 & .13 & .13 & .13 & .13 & .13 & .13 & .13 & .13 & .13 & .13 \\
\hline 10 & .13 & .13 & .13 & .13 & .13 & .13 & .13 & .13 & .13 & .13 & .13 & .13 \\
\hline 11 & .13 & .13 & .13 & .13 & .13 & .13 & .13 & .13 & .13 & .13 & .13 & .13 \\
\hline 12 & .13 & .13 & .13 & .13 & .13 & .13 & .13 & .13 & .13 & .13 & .13 & .13 \\
\hline 13 & .13 & .13 & .13 & .13 & .13 & .13 & .13 & .13 & .13 & .13 & .13 & .13 \\
\hline 14 & .13 & .13 & .13 & .13 & .13 & .13 & .13 & .13 & .13 & .13 & .13 & .13 \\
\hline 15 & .13 & .13 & .13 & .13 & .13 & .13 & .13 & .13 & .13 & .13 & .13 & .13 \\
\hline 16 & .13 & .13 & .13 & .13 & .13 & .13 & .13 & .13 & .13 & .13 & .13 & .13 \\
\hline 17 & .13 & .13 & .13 & .13 & .13 & .13 & .13 & .13 & .13 & .13 & .13 & .13 \\
\hline 18 & .13 & .13 & .13 & .13 & .13 & .13 & .13 & .13 & .13 & .13 & .13 & .13 \\
\hline 19 & .13 & .13 & .13 & .13 & .13 & .13 & .13 & .13 & .13 & .13 & .13 & .13 \\
\hline 20 & .13 & .13 & .13 & .13 & .13 & .13 & .13 & .13 & .13 & .13 & .13 & .13 \\
\hline 21 & .13 & .13 & .13 & .13 & .13 & .13 & .13 & .13 & .13 & .14 & .13 & .13 \\
\hline 22 & .13 & .13 & .13 & .13 & .13 & .13 & .13 & .13 & .13 & .14 & .13 & .13 \\
\hline 23 & .13 & .13 & .13 & .13 & .13 & .13 & .13 & .13 & .13 & .14 & .13 & .13 \\
\hline 24 & .13 & .13 & .13 & .13 & .13 & .13 & .13 & .13 & .13 & .14 & .13 & .13 \\
\hline 25 & .13 & .13 & .13 & .13 & .13 & .13 & .13 & .13 & .13 & .14 & .13 & .13 \\
\hline 26 & .13 & .13 & .13 & .13 & .13 & .13 & .13 & .13 & .13 & .14 & .13 & .13 \\
\hline 27 & .13 & .13 & .13 & .13 & .13 & .13 & .13 & .13 & .13 & .14 & .13 & .13 \\
\hline 28 & .13 & .13 & .13 & .13 & .13 & .13 & .13 & .13 & .13 & .14 & .13 & .13 \\
\hline 29 & .13 & - & .13 & .13 & .13 & .13 & .13 & .13 & .13 & .14 & .13 & .13 \\
\hline 30 & .13 & - & .13 & .13 & .13 & .13 & .13 & .13 & .13 & .14 & .13 & .13 \\
\hline 31 & .13 & - & .13 & - & .13 & - & .13 & .13 & - & .14 & - & .13 \\
\hline Mean & .13 & .13 & .13 & .13 & .13 & .13 & .13 & .13 & .13 & .13 & .13 & .13 \\
\hline Max & .13 & .13 & .13 & .13 & .13 & .13 & .13 & .13 & .13 & .14 & .14 & .13 \\
\hline Min & .13 & .13 & .13 & .13 & .13 & .13 & .13 & .13 & .13 & .13 & .13 & .13 \\
\hline
\end{tabular}


Table 14. Daily mean discharge, Little Hot Creek Spring (LHC-SP), 1995, Long Valley Caldera, California

[Values are in cubic feet per second. _, no data; Max, maximum; Min, minimum. Measurements discontinued after October 17, 1995]

\begin{tabular}{|c|c|c|c|c|c|c|c|c|c|c|c|c|}
\hline Day & Jan & Feb & Mar & Apr & May & Jun & Jul & Aug & Sep & Oct & Nov & Dec \\
\hline 1 & 0.13 & 0.14 & - & - & 0.13 & 0.12 & 0.12 & 0.12 & 0.12 & 0.12 & - & - \\
\hline 2 & .13 & .14 & - & - & .13 & .12 & .12 & .12 & .12 & .12 & - & - \\
\hline 3 & .13 & .14 & - & - & .13 & .12 & .12 & .12 & .12 & .12 & - & - \\
\hline 4 & .13 & .14 & - & - & .13 & .12 & .12 & .12 & .12 & .12 & - & - \\
\hline 5 & .13 & .14 & - & - & .13 & .12 & .12 & .12 & .12 & .12 & - & - \\
\hline 6 & .13 & .14 & - & - & .13 & .12 & .12 & .12 & .12 & .12 & - & - \\
\hline 7 & .13 & .14 & - & - & .13 & .12 & .12 & .12 & .12 & .12 & - & - \\
\hline 8 & .13 & .14 & - & - & .13 & .12 & .12 & .12 & .12 & .12 & - & - \\
\hline 9 & .13 & .13 & - & - & .13 & .12 & .12 & .12 & .12 & .12 & - & - \\
\hline 10 & .13 & .13 & - & - & .13 & .12 & .12 & .12 & .12 & .12 & - & - \\
\hline 11 & .13 & .13 & - & - & .13 & .12 & .12 & .12 & .12 & .12 & - & - \\
\hline 12 & .13 & .13 & - & - & .13 & .12 & .12 & .12 & .12 & .12 & - & - \\
\hline 13 & .13 & .13 & - & - & .13 & .12 & .12 & .12 & .12 & .12 & - & - \\
\hline 14 & .13 & .13 & - & - & .13 & .12 & .12 & .12 & .12 & .12 & - & - \\
\hline 15 & .13 & .13 & - & - & .13 & .12 & .12 & .12 & .12 & .12 & - & - \\
\hline 16 & .13 & .13 & - & - & .13 & .12 & .12 & .12 & .12 & .12 & - & - \\
\hline 17 & .13 & - & - & - & .13 & .12 & .12 & .12 & .12 & .12 & - & - \\
\hline 18 & .13 & - & - & - & .13 & .12 & .12 & .12 & .12 & - & - & - \\
\hline 19 & .13 & - & - & 0.13 & .13 & .12 & .12 & .12 & .12 & - & - & - \\
\hline 20 & .13 & - & - & .13 & .13 & .12 & .12 & .12 & .12 & - & - & - \\
\hline 21 & .13 & - & - & .13 & .13 & .12 & .12 & .12 & .12 & - & - & - \\
\hline 22 & .13 & - & - & .13 & .13 & .12 & .12 & .12 & .12 & - & - & - \\
\hline 23 & .13 & - & - & .13 & .13 & .12 & .12 & .12 & .12 & - & - & - \\
\hline 24 & .13 & - & - & .13 & .13 & .12 & .12 & .12 & .12 & - & - & - \\
\hline 25 & .13 & - & - & .13 & .13 & .12 & .12 & .12 & .12 & - & - & - \\
\hline 26 & .13 & - & - & .13 & .13 & .12 & .12 & .12 & .12 & - & - & - \\
\hline 27 & .14 & - & - & .13 & .13 & .12 & .12 & .12 & .12 & - & - & - \\
\hline 28 & .14 & - & - & .13 & .13 & .12 & .12 & .12 & .12 & - & - & - \\
\hline 29 & .14 & - & - & .13 & .13 & .12 & .12 & .12 & .12 & - & - & - \\
\hline 30 & .14 & - & - & .13 & .13 & .12 & .12 & .12 & .12 & - & - & - \\
\hline 31 & .14 & - & - & - & .12 & - & .12 & .12 & - & - & - & - \\
\hline Mean & .13 & .13 & - & .13 & .13 & .12 & .12 & .12 & .12 & .12 & - & - \\
\hline Max & .14 & .14 & - & .13 & .13 & .12 & .12 & .12 & .12 & .12 & - & - \\
\hline Min & .13 & .13 & - & .13 & .12 & .12 & .12 & .12 & .12 & .12 & - & - \\
\hline
\end{tabular}




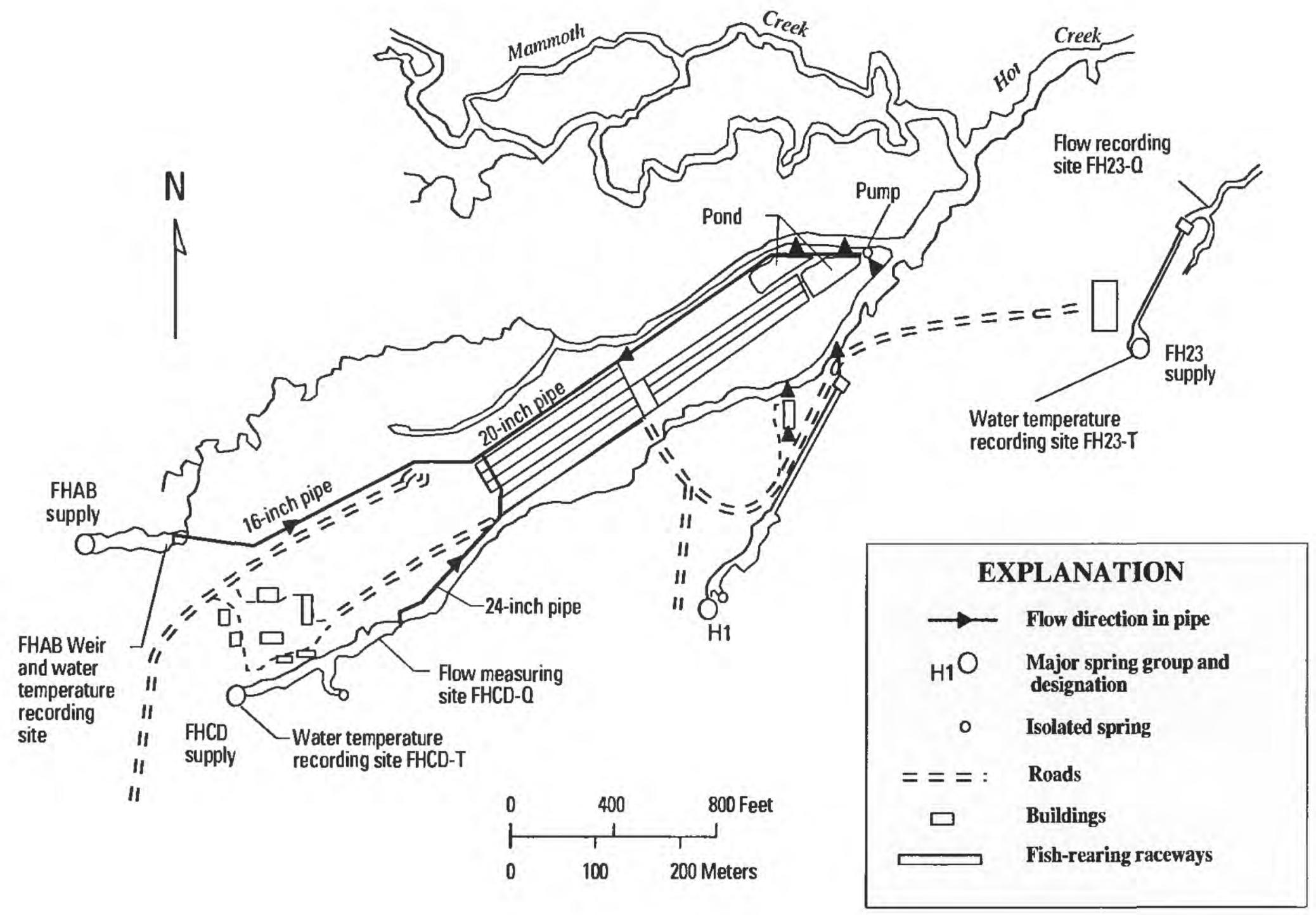

Figure 22. Location of data-collection sites at Hot Creek Fish Hatchery, Long Valley Caldera, Mono County, California.

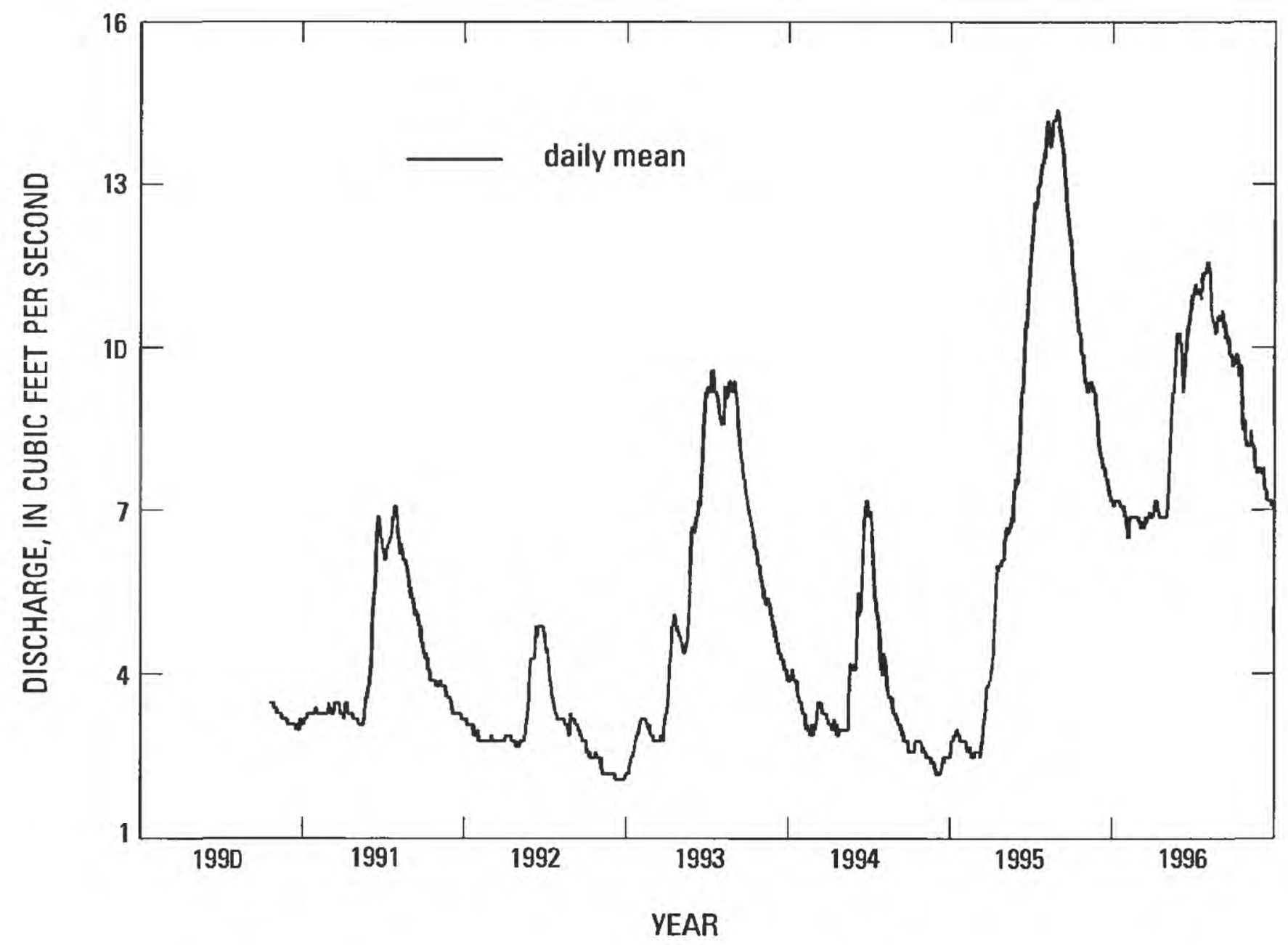

Figure 23. Daily mean discharge, Fish Hatchery Spring AB (FHAB-WEIR), 1990-96, Long Valley Caldera, Mono County, California. 


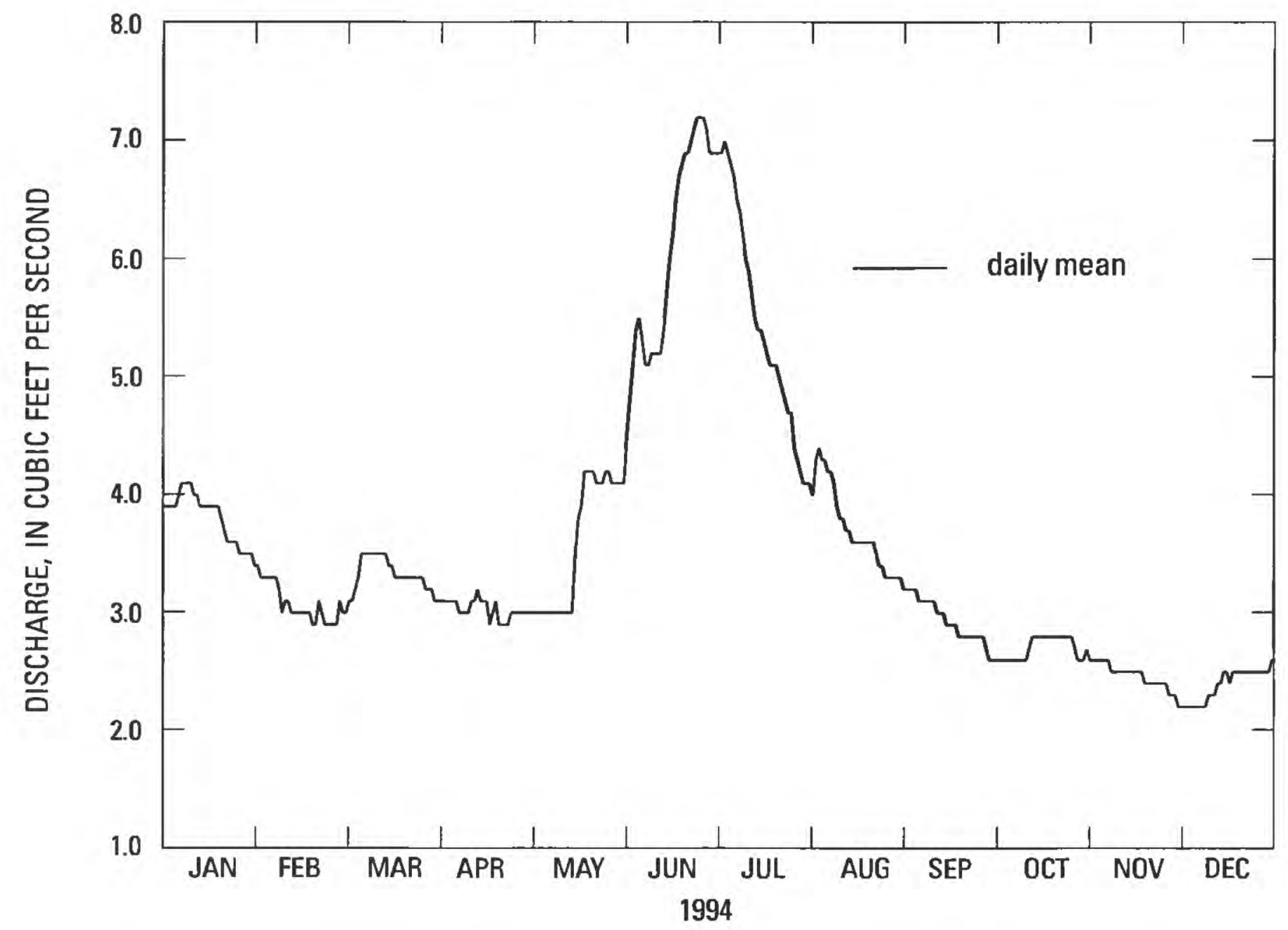

Figure 24. (A) Daily mean discharge, Fish Hatchery Spring AB (FHAB-WEIR), 1994, Long Valley Caldera, Mono County, California. 
Table 15. Daily mean discharge, Fish Hatchery Spring AB (FHAB-WEIR), 1994, Long Valley Caldera, California

[Values are in cubic feet per second. - . no data: Max. maximum; Min. minimum]

\begin{tabular}{|c|c|c|c|c|c|c|c|c|c|c|c|c|}
\hline Day & Jan & Feb & Mar & Apr & May & Jun & Jul & Aug & Sep & Oct & Nov & Dec \\
\hline 1 & 3.9 & 3.3 & 3.0 & 3.1 & 3.0 & 4.5 & 6.9 & 4.0 & 3.2 & 2.6 & 2.6 & 2.2 \\
\hline 2 & 3.9 & 3.3 & 3.1 & 3.1 & 3.0 & 4.8 & 6.9 & 4.3 & 3.2 & 2.6 & 2.6 & 2.2 \\
\hline 3 & 3.9 & 3.3 & 3.1 & 3.1 & 3.0 & 5.1 & 7.0 & 4.4 & 3.2 & 2.6 & 2.6 & 2.2 \\
\hline 4 & 3.9 & 3.3 & 3.2 & 3.1 & 3.0 & 5.4 & 6.9 & 4.3 & 3.2 & 2.6 & 2.6 & 2.2 \\
\hline 5 & 4.0 & 3.3 & 3.3 & 3.1 & 3.0 & 5.5 & 6.8 & 4.3 & 3.1 & 2.6 & 2.6 & 2.2 \\
\hline 6 & 4.1 & 3.3 & 3.5 & 3.1 & 3.0 & 5.3 & 6.7 & 4.2 & 3.1 & 2.6 & 2.6 & 2.2 \\
\hline 7 & 4.1 & 3.2 & 3.5 & 3.0 & 3.0 & 5.1 & 6.5 & 4.2 & 3.1 & 2.6 & 2.5 & 2.2 \\
\hline 8 & 4.1 & 3.0 & 3.5 & 3.0 & 3.0 & 5.1 & 6.4 & 4.1 & 3.1 & 2.6 & 2.5 & 2.2 \\
\hline 9 & 4.1 & 3.1 & 3.5 & 3.0 & 3.0 & 5.2 & 6.2 & 3.9 & 3.1 & 2.6 & 2.5 & 2.3 \\
\hline 10 & 4.0 & 3.1 & 3.5 & 3.0 & 3.0 & 5.2 & 6.0 & 3.8 & 3.1 & 2.6 & 2.5 & 2.3 \\
\hline 11 & 4.0 & 3.0 & 3.5 & 3.1 & 3.0 & 5.2 & 5.9 & 3.8 & 3.0 & 2.7 & 2.5 & 2.3 \\
\hline 12 & 3.9 & 3.0 & 3.5 & 3.1 & 3.0 & 5.2 & 5.7 & 3.7 & 3.0 & 2.8 & 2.5 & 2.4 \\
\hline 13 & 3.9 & 3.0 & 3.5 & 3.2 & 3.0 & 5.4 & 5.5 & 3.7 & 3.0 & 2.8 & 2.5 & 2.4 \\
\hline 14 & 3.9 & 3.0 & 3.5 & 3.1 & 3.0 & 5.7 & 5.4 & 3.6 & 2.9 & 2.8 & 2.5 & 2.5 \\
\hline 15 & 3.9 & 3.0 & 3.4 & 3.1 & 3.5 & 6.0 & 5.4 & 3.6 & 2.9 & 2.8 & 2.5 & 2.5 \\
\hline 16 & 3.9 & 3.0 & 3.4 & 3.1 & 3.8 & 6.2 & 5.3 & 3.6 & 2.9 & 2.8 & 2.5 & 2.4 \\
\hline 17 & 3.9 & 3.0 & 3.3 & 2.9 & 3.9 & 6.5 & 5.2 & 3.6 & 2.9 & 2.8 & 2.5 & 2.5 \\
\hline 18 & 3.9 & 2.9 & 3.3 & 3.0 & 4.2 & 6.7 & 5.1 & 3.6 & 2.8 & 2.8 & 2.4 & 2.5 \\
\hline 19 & 3.8 & 2.9 & 3.3 & 3.1 & 4.2 & 6.8 & 5.1 & 3.6 & 2.8 & 2.8 & 2.4 & 2.5 \\
\hline 20 & 3.7 & 3.1 & 3.3 & 2.9 & 4.2 & 6.9 & 5.1 & 3.6 & 2.8 & 2.8 & 2.4 & 2.5 \\
\hline 21 & 3.6 & 3.0 & 3.3 & 2.9 & 4.2 & 6.9 & 5.0 & 3.6 & 2.8 & 2.8 & 2.4 & 2.5 \\
\hline 22 & 3.6 & 2.9 & 3.3 & 2.9 & 4.1 & 7.0 & 4.9 & 3.5 & 2.8 & 2.8 & 2.4 & 2.5 \\
\hline 23 & 3.6 & 2.9 & 3.3 & 2.9 & 4.1 & 7.1 & 4.8 & 3.4 & 2.8 & 2.8 & 2.4 & 2.5 \\
\hline 24 & 3.6 & 2.9 & 3.3 & 3.0 & 4.1 & 7.2 & 4.7 & 3.4 & 2.8 & 2.8 & 2.4 & 2.5 \\
\hline 25 & 3.5 & 2.9 & 3.3 & 3.0 & 4.2 & 7.2 & 4.7 & 3.3 & 2.8 & 2.8 & 2.4 & 2.5 \\
\hline 26 & 3.5 & 2.9 & 3.3 & 3.0 & 4.2 & 7.2 & 4.4 & 3.3 & 2.8 & 2.7 & 2.3 & 2.5 \\
\hline 27 & 3.5 & 3.1 & 3.2 & 3.0 & 4.1 & 7.1 & 4.3 & 3.3 & 2.7 & 2.6 & 2.3 & 2.5 \\
\hline 28 & 3.5 & 3.0 & 3.2 & 3.0 & 4.1 & 6.9 & 4.2 & 3.3 & 2.6 & 2.6 & 2.3 & 2.5 \\
\hline 29 & 3.5 & - & 3.2 & 3.0 & 4.1 & 6.9 & 4.1 & 3.3 & 2.6 & 2.6 & 2.2 & 2.5 \\
\hline 30 & 3.4 & - & 3.1 & 3.0 & 4.1 & 6.9 & 4.1 & 3.3 & 2.6 & 2.7 & 2.2 & 2.6 \\
\hline 31 & 3.4 & - & 3.1 & - & 4.1 & - & 4.1 & 3.2 & - & 2.6 & - & 2.6 \\
\hline Mean & 3.79 & 3.06 & 3.32 & 3.03 & 3.59 & 6.07 & 5.46 & 3.70 & 2.92 & 2.70 & 2.45 & 2.40 \\
\hline Max & 4.1 & 3.3 & 3.5 & 3.2 & 4.2 & 7.2 & 7.0 & 4.4 & 3.2 & 2.8 & 2.6 & 2.6 \\
\hline Min & 3.4 & 2.9 & 3.0 & 2.9 & 3.0 & 4.5 & 4.1 & 3.2 & 2.6 & 2.6 & 2.2 & 2.2 \\
\hline
\end{tabular}




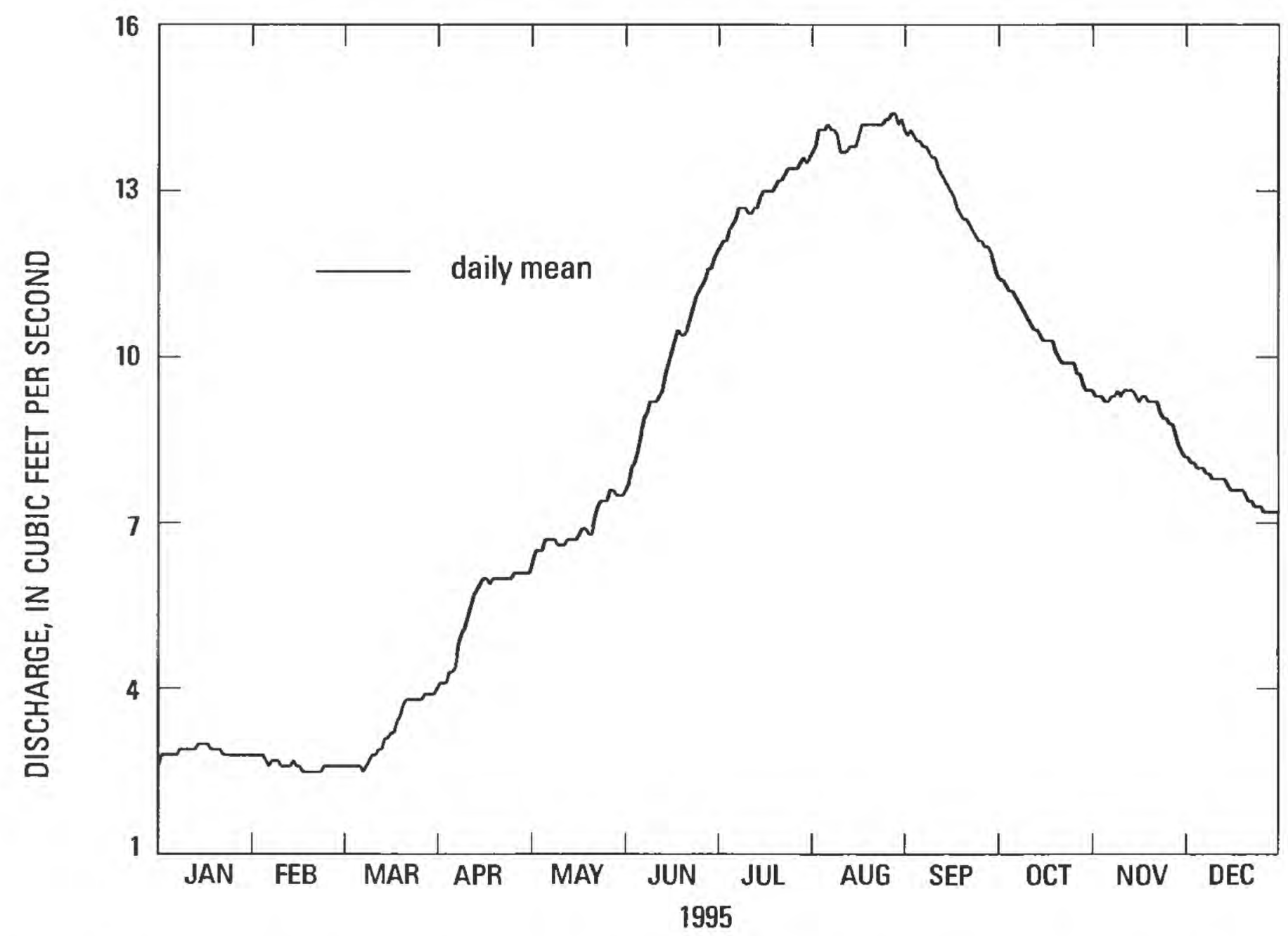

Figure 24. (B) Daily mean discharge, Fish Hatchery Spring AB (FHAB-WEIR), 1995, Long Valley Caldera, Mono County, California. 
Table 16. Daily mean discharge, Fish Hatchery Spring AB (FHAB-WEIR), 1995, Long Valley Caldera, California

[Values are in cubic feet per second. - . no data; Max, maximum; Min, minimum]

\begin{tabular}{|c|c|c|c|c|c|c|c|c|c|c|c|c|}
\hline Day & Jan & Feb & Mar & Apr & May & Jun & Jul & Aug & Sep & Oct & Nov & Dec \\
\hline 1 & 2.8 & 2.8 & 2.6 & 4.0 & 6.1 & 7.6 & 11.9 & 13.7 & 14.0 & 11.4 & 9.3 & 8.2 \\
\hline 2 & 2.8 & 2.8 & 2.6 & 4.1 & 6.3 & 7.7 & 12.0 & 13.8 & 14.1 & 11.4 & 9.3 & 8.1 \\
\hline 3 & 2.8 & 2.8 & 2.6 & 4.1 & 6.5 & 8.0 & 12.1 & 14.1 & 14.0 & 11.3 & 9.3 & 8.1 \\
\hline 4 & 2.8 & 2.7 & 2.6 & 4.1 & 6.5 & 8.1 & 12.1 & 14.1 & 13.9 & 11.2 & 9.2 & 8.0 \\
\hline 5 & 2.8 & 2.6 & 2.6 & 4.3 & 6.5 & 8.3 & 12.3 & 14.1 & 13.9 & 11.2 & 9.2 & 8.0 \\
\hline 6 & 2.8 & 2.7 & 2.6 & 4.3 & 6.7 & 8.6 & 12.4 & 14.2 & 13.8 & 11.1 & 9.3 & 8.0 \\
\hline 7 & 2.9 & 2.7 & 2.6 & 4.4 & 6.7 & 8.9 & 12.5 & 14.1 & 13.8 & 11.0 & 9.3 & 7.9 \\
\hline 8 & 2.9 & 2.7 & 2.5 & 4.8 & 6.7 & 9.0 & 12.7 & 14.1 & 13.7 & 10.9 & 9.4 & 7.9 \\
\hline 9 & 2.9 & 2.6 & 2.6 & 5.0 & 6.7 & 9.2 & 12.7 & 14.0 & 13.6 & 10.8 & 9.3 & 7.8 \\
\hline 10 & 2.9 & 2.6 & 2.7 & 5.1 & 6.6 & 9.2 & 12.7 & 13.7 & 13.6 & 10.7 & 9.4 & 7.8 \\
\hline 11 & 2.9 & 2.6 & 2.8 & 5.3 & 6.6 & 9.2 & 12.6 & 13.7 & 13.4 & 10.6 & 9.4 & 7.8 \\
\hline 12 & 2.9 & 2.6 & 2.8 & 5.5 & 6.6 & 9.3 & 12.6 & 13.7 & 13.3 & 10.5 & 9.4 & 7.8 \\
\hline 13 & 3.0 & 2.7 & 2.9 & 5.7 & 6.7 & 9.4 & 12.7 & 13.8 & 13.2 & 10.5 & 9.4 & 7.8 \\
\hline 14 & 3.0 & 2.6 & 2.9 & 5.8 & 6.7 & 9.7 & 12.7 & 13.8 & 13.1 & 10.4 & 9.3 & 7.7 \\
\hline 15 & 3.0 & 2.6 & 3.1 & 5.9 & 6.7 & 9.9 & 12.9 & 13.8 & 13.0 & 10.3 & 9.2 & 7.6 \\
\hline 16 & 3.0 & 2.5 & 3.1 & 6.0 & 6.7 & 10.1 & 13.0 & 14.0 & 12.9 & 10.3 & 9.3 & 7.6 \\
\hline 17 & 2.9 & 2.5 & 3.2 & 6.0 & 6.8 & 10.3 & 13.0 & 14.2 & 12.7 & 10.3 & 9.3 & 7.6 \\
\hline 18 & 2.9 & 2.5 & 3.2 & 5.9 & 6.9 & 10.5 & 13.0 & 14.2 & 12.6 & 10.3 & 9.2 & 7.6 \\
\hline 19 & 2.9 & 2.5 & 3.4 & 6.0 & 6.9 & 10.4 & 13.0 & 14.2 & 12.5 & 10.1 & 9.2 & 7.6 \\
\hline 20 & 2.9 & 2.5 & 3.5 & 6.0 & 6.8 & 10.4 & 13.1 & 14.2 & 12.5 & 10.0 & 9.2 & 7.5 \\
\hline 21 & 2.8 & 2.5 & 3.7 & 6.0 & 6.8 & 10.5 & 13.2 & 14.2 & 12.4 & 9.9 & 9.2 & 7.4 \\
\hline 22 & 2.8 & 2.5 & 3.8 & 6.0 & 7.1 & 10.7 & 13.2 & 14.2 & 12.3 & 9.9 & 9.0 & 7.4 \\
\hline 23 & 2.8 & 2.6 & 3.8 & 6.0 & 7.3 & 10.9 & 13.3 & 14.2 & 12.2 & 9.9 & 8.9 & 7.3 \\
\hline 24 & 2.8 & 2.6 & 3.8 & 6.0 & 7.4 & 11.1 & 13.4 & 14.2 & 12.1 & 9.9 & 8.9 & 7.3 \\
\hline 25 & 2.8 & 2.6 & 3.8 & 6.0 & 7.4 & 11.2 & 13.4 & 14.3 & 12.1 & 9.9 & 8.8 & 7.3 \\
\hline 26 & 2.8 & 2.6 & 3.8 & 6.1 & 7.4 & 11.3 & 13.4 & 14.3 & 12.0 & 9.7 & 8.8 & 7.2 \\
\hline 27 & 2.8 & 2.6 & 3.8 & 6.1 & 7.6 & 11.4 & 13.4 & 14.4 & 12.0 & 9.7 & 8.6 & 7.2 \\
\hline 28 & 2.8 & 2.6 & 3.9 & 6.1 & 7.6 & 11.6 & 13.5 & 14.4 & 11.9 & 9.5 & 8.4 & 7.2 \\
\hline 29 & 2.8 & - & 3.9 & 6.1 & 7.5 & 11.6 & 13.6 & 14.2 & 11.7 & 9.4 & 8.3 & 7.2 \\
\hline 30 & 2.8 & - & 3.9 & 6.1 & 7.5 & 11.8 & 13.5 & 14.3 & 11.5 & 9.4 & 8.2 & 7.2 \\
\hline 31 & 2.8 & - & 3.9 & - & 7.5 & - & 13.6 & 14.1 & - & 9.4 & - & 7.2 \\
\hline Mean & 2.86 & 2.61 & 3.19 & 5.43 & 6.90 & 9.86 & 12.9 & 14.1 & 12.9 & 10.4 & 9.10 & 7.62 \\
\hline Max & 3.0 & 2.8 & 3.9 & 6.1 & 7.6 & 11.8 & 13.6 & 14.4 & 14.1 & 11.4 & 9.4 & 8.2 \\
\hline Min & 2.8 & 2.5 & 2.5 & 4.0 & 6.1 & 7.6 & 11.9 & 13.7 & 11.5 & 9.4 & 8.2 & 7.2 \\
\hline
\end{tabular}




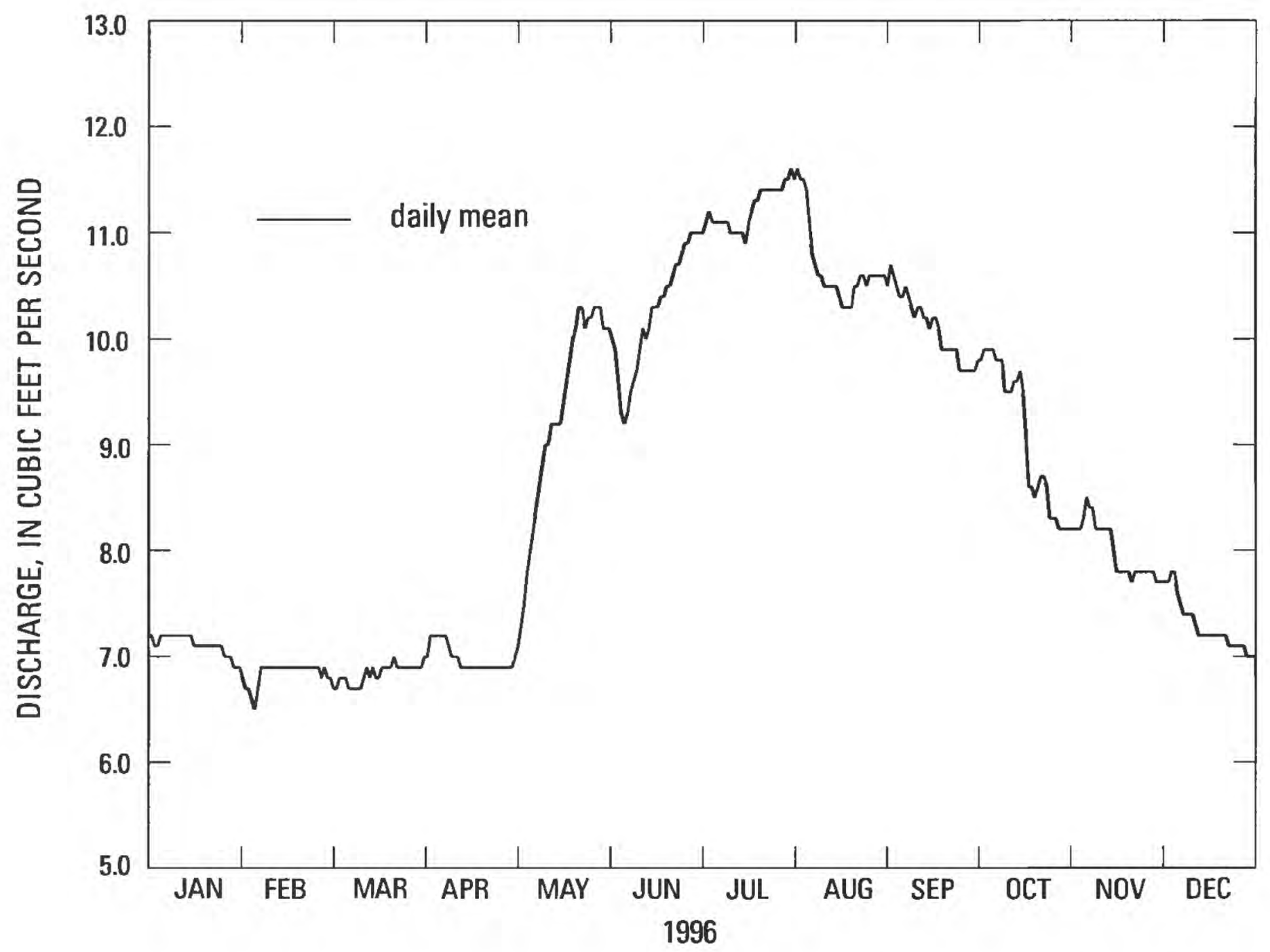

Figure 24. (C) Daily mean discharge, Fish Hatchery Spring AB (FHAB-WEIR), 1996, Long Valley Caldera, Mono County, California. 
Table 17. Daily mean discharge, Fish Hatchery Spring AB (FHAB-WEIR), 1996, Long Valley Caldera, California

[Values are in cubic feet per second. —, no data: Max, maximum: Min. minimum]

\begin{tabular}{|c|c|c|c|c|c|c|c|c|c|c|c|c|}
\hline Day & Jan & Feb & Mar & Apr & May & Jun & Jul & Aug & Sep & Oct & Nov & Dec \\
\hline 1 & 7.2 & 6.7 & 6.7 & 7.0 & 7.1 & 10.0 & 11.0 & 11.6 & 10.7 & 9.8 & 8.2 & 7.7 \\
\hline 2 & 7.1 & 6.7 & 6.7 & 7.2 & 7.3 & 9.9 & 11.1 & 11.5 & 10.6 & 9.9 & 8.2 & 7.7 \\
\hline 3 & 7.1 & 6.6 & 6.8 & 7.2 & 7.5 & 9.6 & 11.2 & 11.5 & 10.5 & 9.9 & 8.2 & 7.8 \\
\hline 4 & 7.2 & 6.5 & 6.8 & 7.2 & 7.8 & 9.3 & 11.1 & 11.4 & 10.4 & 9.9 & 8.3 & 7.8 \\
\hline 5 & 7.2 & 6.7 & 6.8 & 7.2 & 8.0 & 9.2 & 11.1 & 11.1 & 10.4 & 9.9 & 8.5 & 7.6 \\
\hline 6 & 7.2 & 6.9 & 6.7 & 7.2 & 8.2 & 9.3 & 11.1 & 10.8 & 10.5 & 9.8 & 8.4 & 7.5 \\
\hline 7 & 7.2 & 6.9 & 6.7 & 7.2 & 8.4 & 9.5 & 11.1 & 10.7 & 10.4 & 9.8 & 8.4 & 7.4 \\
\hline 8 & 7.2 & 6.9 & 6.7 & 7.1 & 8.6 & 9.6 & 11.1 & 10.6 & 10.3 & 9.8 & 8.2 & 7.4 \\
\hline 9 & 7.2 & 6.9 & 6.7 & 7.0 & 8.8 & 9.7 & 11.1 & 10.6 & 10.2 & 9.5 & 8.2 & 7.4 \\
\hline 10 & 7.2 & 6.9 & 6.7 & 7.0 & 9.0 & 9.9 & 11.0 & 10.5 & 10.3 & 9.5 & 8.2 & 7.4 \\
\hline 11 & 7.2 & 6.9 & 6.8 & 7.0 & 9.0 & 10.1 & 11.0 & 10.5 & 10.3 & 9.5 & 8.2 & 7.3 \\
\hline 12 & 7.2 & 6.9 & 6.9 & 6.9 & 9.2 & 10.0 & 11.0 & 10.5 & 10.2 & 9.6 & 8.2 & 7.2 \\
\hline 13 & 7.2 & 6.9 & 6.8 & 6.9 & 9.2 & 10.1 & 11.0 & 10.5 & 10.2 & 9.6 & 8.2 & 7.2 \\
\hline 14 & 7.2 & 6.9 & 6.9 & 6.9 & 9.2 & 10.3 & 11.0 & 10.5 & 10.1 & 9.7 & 8.0 & 7.2 \\
\hline 15 & 7.1 & 6.9 & 6.8 & 6.9 & 9.2 & 10.3 & 10.9 & 10.4 & 10.2 & 9.5 & 7.8 & 7.2 \\
\hline 16 & 7.1 & 6.9 & 6.8 & 6.9 & 9.4 & 10.3 & 11.1 & 10.3 & 10.2 & 9.1 & 7.8 & 7.2 \\
\hline 17 & 7.1 & 6.9 & 6.9 & 6.9 & 9.6 & 10.4 & 11.2 & 10.3 & 10.1 & 8.6 & 7.8 & 7.2 \\
\hline 18 & 7.1 & 6.9 & 6.9 & 6.9 & 9.8 & 10.4 & 11.3 & 10.3 & 9.9 & 8.6 & 7.8 & 7.2 \\
\hline 19 & 7.1 & 6.9 & 6.9 & 6.9 & 10.0 & 10.5 & 11.3 & 10.3 & 9.9 & 8.5 & 7.8 & 7.2 \\
\hline 20 & 7.1 & 6.9 & 6.9 & 6.9 & 10.1 & 10.5 & 11.4 & 10.5 & 9.9 & 8.6 & 7.7 & 7.2 \\
\hline 21 & 7.1 & 6.9 & 7.0 & 6.9 & 10.3 & 10.6 & 11.4 & 10.5 & 9.9 & 8.7 & 7.8 & 7.2 \\
\hline 22 & 7.1 & 6.9 & 6.9 & 6.9 & 10.3 & 10.7 & 11.4 & 10.6 & 9.9 & 8.7 & 7.8 & 7.1 \\
\hline 23 & 7.1 & 6.9 & 6.9 & 6.9 & 10.1 & 10.7 & 11.4 & 10.6 & 9.9 & 8.6 & 7.8 & 7.1 \\
\hline 24 & 7.1 & 6.9 & 6.9 & 6.9 & 10.2 & 10.8 & 11.4 & 10.5 & 9.7 & 8.3 & 7.8 & 7.1 \\
\hline 25 & 7.0 & 6.9 & 6.9 & 6.9 & 10.2 & 10.9 & 11.4 & 10.6 & 9.7 & 8.3 & 7.8 & 7.1 \\
\hline 26 & 7.0 & 6.8 & 6.9 & 6.9 & 10.3 & 10.9 & 11.4 & 10.6 & 9.7 & 8.3 & 7.8 & 7.1 \\
\hline 27 & 7.0 & 6.9 & 6.9 & 6.9 & 10.3 & 11.0 & 11.4 & 10.6 & 9.7 & 8.2 & 7.8 & 7.1 \\
\hline 28 & 6.9 & 6.8 & 6.9 & 6.9 & 10.3 & 11.0 & 11.5 & 10.6 & 9.7 & 8.2 & 7.7 & 7.0 \\
\hline 29 & 6.9 & 6.8 & 6.9 & 6.9 & 10.1 & 11.0 & 11.5 & 10.6 & 9.7 & 8.2 & 7.7 & 7.0 \\
\hline 30 & 6.9 & - & 6.9 & 7.0 & 10.1 & 11.0 & 11.6 & 10.6 & 9.8 & 8.2 & 7.7 & 7.0 \\
\hline 31 & 6.8 & - & 7.0 & - & 10.1 & - & 11.5 & 10.5 & - & 8.2 & - & 7.0 \\
\hline Mean & 7.10 & 6.84 & 6.84 & 6.98 & 9.28 & 10.2 & 11.2 & 10.7 & 10.1 & 9.06 & 7.99 & 7.28 \\
\hline Max & 7.2 & 6.9 & 7.0 & 7.2 & 10.3 & 11.0 & 11.6 & 11.6 & 10.7 & 9.9 & 8.5 & 7.8 \\
\hline Min & 6.8 & 6.5 & 6.7 & 6.9 & 7.1 & 9.2 & 10.9 & 10.3 & 9.7 & 8.2 & 7.7 & 7.0 \\
\hline
\end{tabular}




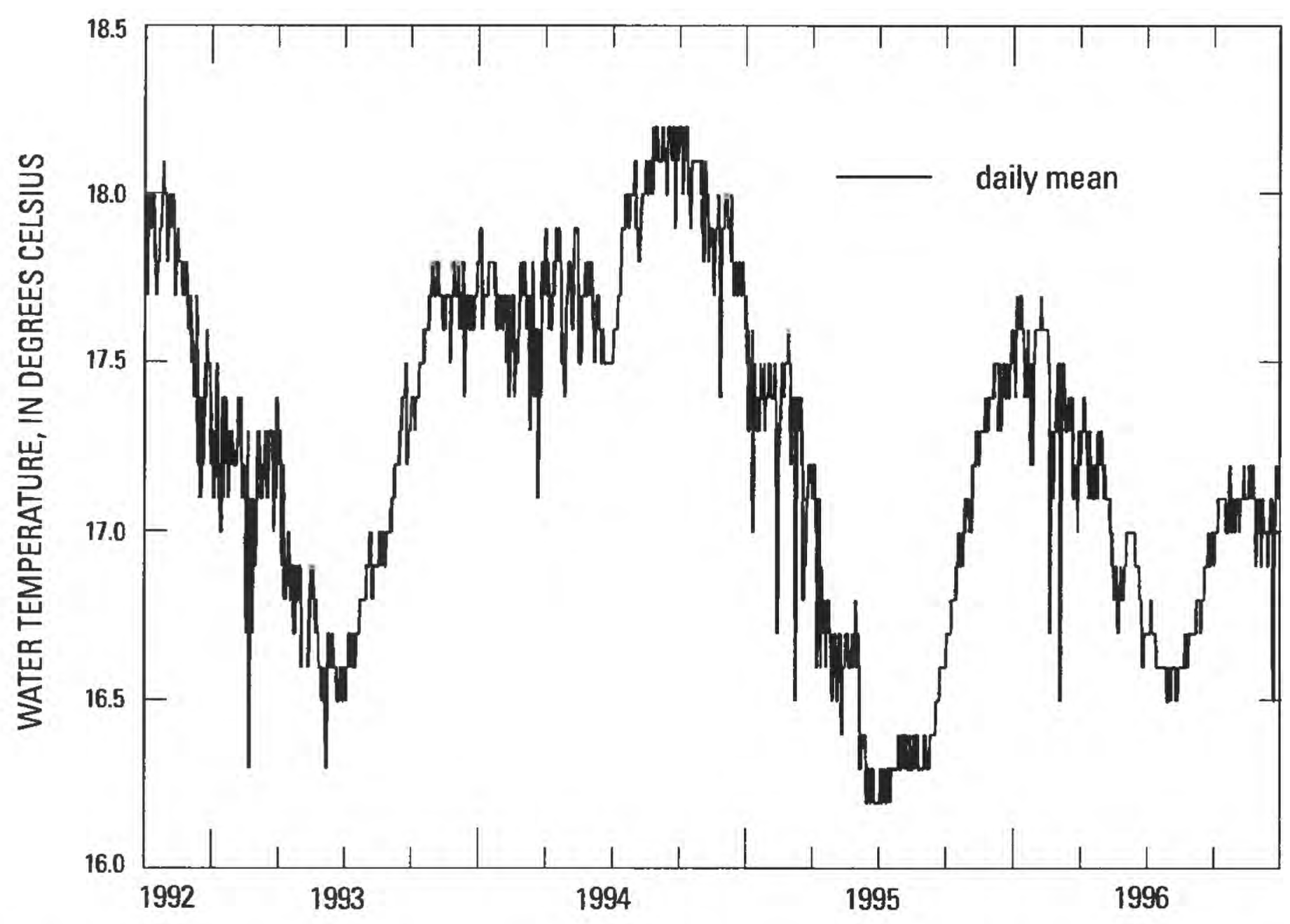

Figure 25. Daily mean water temperature, Fish Hatchery Spring AB (FHAB-WEIR), October 1992 to December 1996, Long Valley Caldera, Mono County, California.

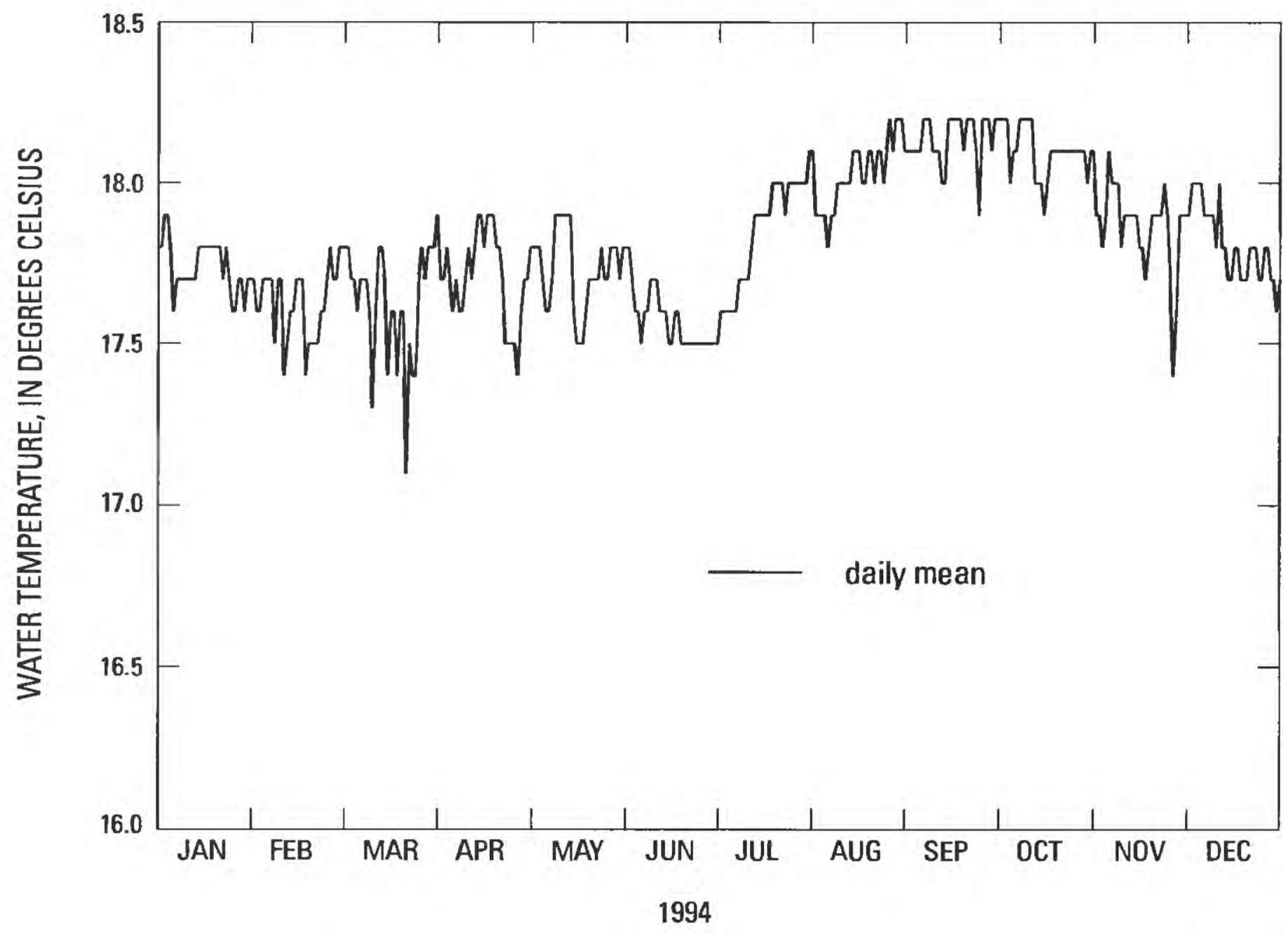

Figure 26. (A) Daily mean water temperature, Fish Hatchery Spring AB (FHAB-WEIR), 1994, Long Valley Caldera, Mono County, California. 
Table 18. Daily mean water temperature, Fish Hatchery Spring AB (FHAB-WEIR), 1994, Long Valley Caldera, California

[Values are in degrees Celsius. - , no data; Max, maximum; Min, minimum]

\begin{tabular}{|c|c|c|c|c|c|c|c|c|c|c|c|c|}
\hline Day & Jan & Feb & Mar & Apr & May & Jun & Jul & Aug & Sep & Oct & Nov & Dec \\
\hline 1 & 17.8 & 17.6 & 17.8 & 17.9 & 17.8 & 17.8 & 17.5 & 18.1 & 18.1 & 18.2 & 17.9 & 17.9 \\
\hline 2 & 17.9 & 17.6 & 17.8 & 17.7 & 17.8 & 17.8 & 17.6 & 17.9 & 18.1 & 18.2 & 17.9 & 18.0 \\
\hline 3 & 17.9 & 17.7 & 17.8 & 17.7 & 17.8 & 17.7 & 17.6 & 17.9 & 18.1 & 18.2 & 17.8 & 18.0 \\
\hline 4 & 17.8 & 17.7 & 17.7 & 17.8 & 17.8 & 17.6 & 17.6 & 17.9 & 18.1 & 18.0 & 17.9 & 18.0 \\
\hline 5 & 17.6 & 17.7 & 17.7 & 17.7 & 17.7 & 17.6 & 17.6 & 17.9 & 18.1 & 18.1 & 18.1 & 18.0 \\
\hline 6 & 17.7 & 17.7 & 17.6 & 17.6 & 17.6 & 17.5 & 17.6 & 17.8 & 18.2 & 18.1 & 18.0 & 17.9 \\
\hline 7 & 17.7 & 17.5 & 17.7 & 17.7 & 17.6 & 17.6 & 17.6 & 17.9 & 18.2 & 18.2 & 18.0 & 17.9 \\
\hline 8 & 17.7 & 17.7 & 17.7 & 17.6 & 17.7 & 17.6 & 17.7 & 17.9 & 18.2 & 18.2 & 18.0 & 17.9 \\
\hline 9 & 17.7 & 17.7 & 17.7 & 17.6 & 17.9 & 17.7 & 17.7 & 18.0 & 18.1 & 18.2 & 17.8 & 17.9 \\
\hline 10 & 17.7 & 17.4 & 17.6 & 17.7 & 17.9 & 17.7 & 17.7 & 18.0 & 18.1 & 18.2 & 17.9 & 17.8 \\
\hline 11 & 17.7 & 17.5 & 17.3 & 17.8 & 17.9 & 17.7 & 17.7 & 18.0 & 18.1 & 18.2 & 17.9 & 18.0 \\
\hline 12 & 17.7 & 17.6 & 17.6 & 17.7 & 17.9 & 17.6 & 17.8 & 18.0 & 18.0 & 18.0 & 17.9 & 17.8 \\
\hline 13 & 17.8 & 17.6 & 17.8 & 17.8 & 17.9 & 17.6 & 17.9 & 18.0 & 18.0 & 18.0 & 17.9 & 17.8 \\
\hline 14 & 17.8 & 17.7 & 17.8 & 17.9 & 17.9 & 17.6 & 17.9 & 18.1 & 18.2 & 18.0 & 17.9 & 17.7 \\
\hline 15 & 17.8 & 17.7 & 17.7 & 17.9 & 17.6 & 17.5 & 17.9 & 18.1 & 18.2 & 17.9 & 17.8 & 17.7 \\
\hline 16 & 17.8 & 17.7 & 17.4 & 17.8 & 17.5 & 17.5 & 17.9 & 18.1 & 18.2 & 18.0 & 17.8 & 17.8 \\
\hline 17 & 17.8 & 17.4 & 17.6 & 17.9 & 17.5 & 17.6 & 17.9 & 18.0 & 18.2 & 18.1 & 17.7 & 17.8 \\
\hline 18 & 17.8 & 17.5 & 17.6 & 17.9 & 17.5 & 17.6 & 17.9 & 18.0 & 18.2 & 18.1 & 17.8 & 17.7 \\
\hline 19 & 17.8 & 17.5 & 17.4 & 17.9 & 17.6 & 17.5 & 18.0 & 18.1 & 18.1 & 18.1 & 17.9 & 17.7 \\
\hline 20 & 17.8 & 17.5 & 17.6 & 17.8 & 17.7 & 17.5 & 18.0 & 18.1 & 18.2 & 18.1 & 17.9 & 17.7 \\
\hline 21 & 17.7 & 17.5 & 17.6 & 17.8 & 17.7 & 17.5 & 18.0 & 18.0 & 18.2 & 18.1 & 17.9 & 17.8 \\
\hline 22 & 17.8 & 17.6 & 17.1 & 17.7 & 17.7 & 17.5 & 18.0 & 18.1 & 18.2 & 18.1 & 17.9 & 17.8 \\
\hline 23 & 17.7 & 17.6 & 17.5 & 17.5 & 17.7 & 17.5 & 17.9 & 18.1 & 18.1 & 18.1 & 18.0 & 17.8 \\
\hline 24 & 17.6 & 17.7 & 17.4 & 17.5 & 17.8 & 17.5 & 18.0 & 18.0 & 17.9 & 18.1 & 17.9 & 17.7 \\
\hline 25 & 17.6 & 17.8 & 17.4 & 17.5 & 17.7 & 17.5 & 18.0 & 18.1 & 18.2 & 18.1 & 17.7 & 17.7 \\
\hline 26 & 17.7 & 17.7 & 17.7 & 17.5 & 17.7 & 17.5 & 18.0 & 18.2 & 18.2 & 18.1 & 17.4 & 17.8 \\
\hline 27 & 17.7 & 17.7 & 17.8 & 17.4 & 17.8 & 17.5 & 18.0 & 18.1 & 18.2 & 18.1 & 17.6 & 17.8 \\
\hline 28 & 17.6 & 17.8 & 17.7 & 17.6 & 17.8 & 17.5 & 18.0 & 18.2 & 18.1 & 18.1 & 17.9 & 17.7 \\
\hline 29 & 17.7 & - & 17.8 & 17.7 & 17.8 & 17.5 & 18.0 & 18.2 & 18.2 & 18.0 & 17.9 & 17.7 \\
\hline 30 & 17.7 & - & 17.8 & 17.7 & 17.7 & 17.5 & 18.0 & 18.2 & 18.2 & 18.1 & 17.9 & 17.6 \\
\hline 31 & 17.7 & - & 17.8 & - & 17.8 & - & 18.1 & 18.1 & - & 18.1 & - & 17.7 \\
\hline Mean & 17.7 & 17.6 & 17.6 & 17.7 & 17.7 & 17.6 & 17.8 & 18.0 & 18.1 & 18.1 & 17.9 & 17.8 \\
\hline Max & 17.9 & 17.8 & 17.8 & 17.9 & 17.9 & 17.8 & 18.1 & 18.2 & 18.2 & 18.2 & 18.1 & 18.0 \\
\hline Min & 17.6 & 17.4 & 17.1 & 17.4 & 17.5 & 17.5 & 17.5 & 17.8 & 17.9 & 17.9 & 17.4 & 17.6 \\
\hline
\end{tabular}




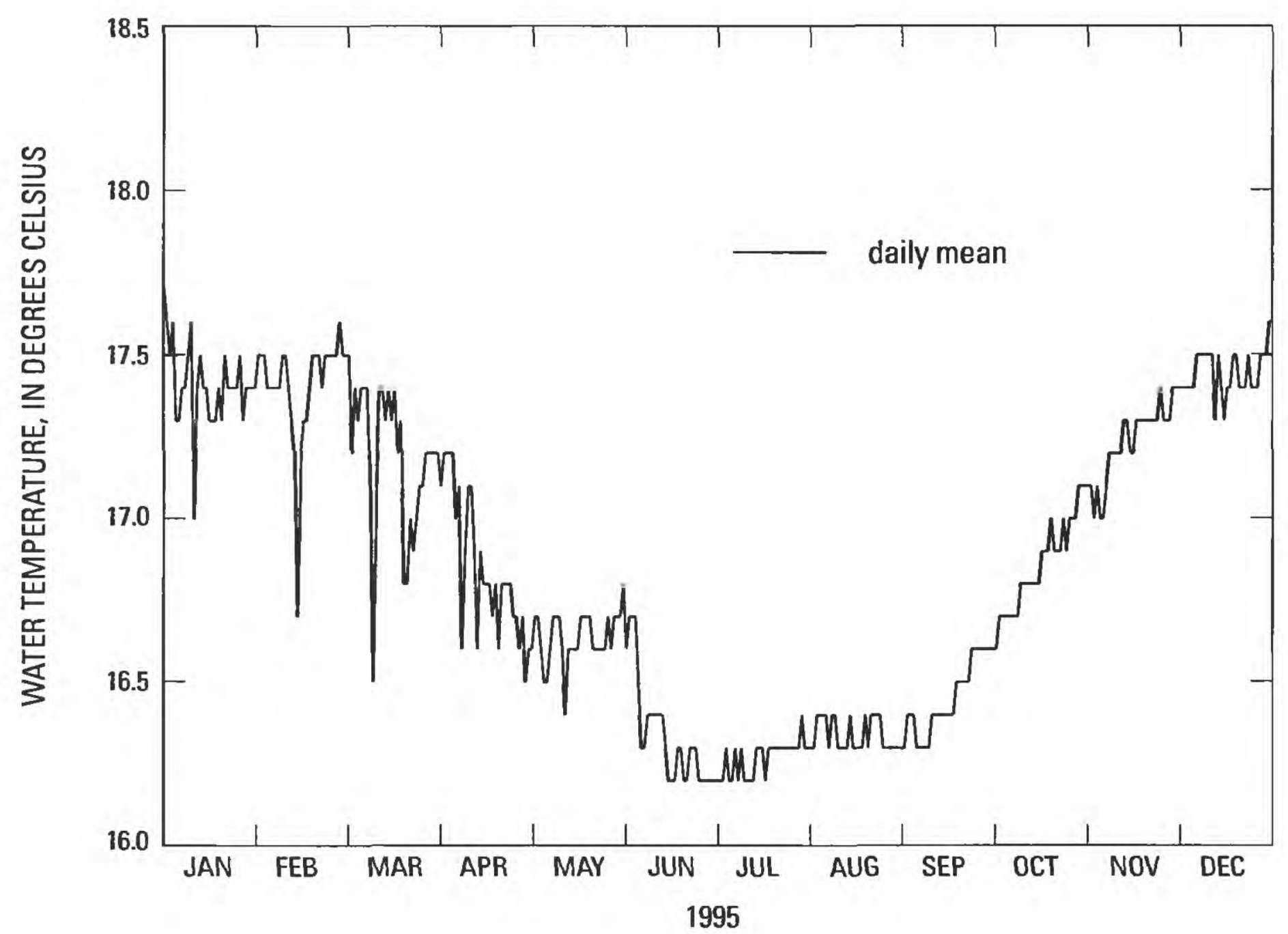

Figure 26. (B) Daily mean water temperature, Fish Hatchery Spring AB (FHAB-WEIR), 1995, Long Valley Caldera, Mono County, Califomia. 
Table 19. Daily mean water temperature, Fish Hatchery Spring AB (FHAB-WEIR), 1995, Long Valley Caldera, California

[Values are in degrees Celsius. _, no data; Max, maximum; Min, minimum]

\begin{tabular}{|c|c|c|c|c|c|c|c|c|c|c|c|c|}
\hline Day & Jan & Feb & Mar & Apr & May & Jun & Jul & Aug & Sep & Oct & Nov & Dec \\
\hline 1 & 17.6 & 17.5 & 17.5 & 17.1 & 16.6 & 16.6 & 16.2 & 16.3 & 16.3 & 16.6 & 17.1 & 17.4 \\
\hline 2 & 17.5 & 17.5 & 17.5 & 17.2 & 16.7 & 16.7 & 16.2 & 16.3 & 16.4 & 16.7 & 17.0 & 17.4 \\
\hline 3 & 17.6 & 17.4 & 17.2 & 17.2 & 16.7 & 16.7 & 16.2 & 16.4 & 16.4 & 16.7 & 17.1 & 17.4 \\
\hline 4 & 17.3 & 17.4 & 17.4 & 17.2 & 16.6 & 16.7 & 16.3 & 16.4 & 16.4 & 16.7 & 17.0 & 17.4 \\
\hline 5 & 17.3 & 17.4 & 17.3 & 17.2 & 16.5 & 16.5 & 16.2 & 16.4 & 16.3 & 16.7 & 17.0 & 17.4 \\
\hline 6 & 17.4 & 17.4 & 17.4 & 17.0 & 16.5 & 16.3 & 16.2 & 16.4 & 16.3 & 16.7 & 17.1 & 17.5 \\
\hline 7 & 17.4 & 17.4 & 17.4 & 17.1 & 16.6 & 16.3 & 16.3 & 16.3 & 16.3 & 16.7 & 17.2 & 17.5 \\
\hline 8 & 17.5 & 17.5 & 17.4 & 16.6 & 16.7 & 16.4 & 16.2 & 16.4 & 16.3 & 16.7 & 17.2 & 17.5 \\
\hline 9 & 17.6 & 17.5 & 17.1 & 16.9 & 16.7 & 16.4 & 16.3 & 16.4 & 16.3 & 16.8 & 17.2 & 17.5 \\
\hline 10 & 17.0 & 17.4 & 16.5 & 17.1 & 16.7 & 16.4 & 16.2 & 16.3 & 16.4 & 16.8 & 17.2 & 17.5 \\
\hline 11 & 17.4 & 17.3 & 17.1 & 17.1 & 16.6 & 16.4 & 16.2 & 16.3 & 16.4 & 16.8 & 17.2 & 17.5 \\
\hline 12 & 17.5 & 17.2 & 17.4 & 16.9 & 16.4 & 16.4 & 16.2 & 16.3 & 16.4 & 16.8 & 17.3 & 17.3 \\
\hline 13 & 17.4 & 16.7 & 17.4 & 16.6 & 16.6 & 16.4 & 16.2 & 16.3 & 16.4 & 16.8 & 17.3 & 17.5 \\
\hline 14 & 17.4 & 17.2 & 17.3 & 16.9 & 16.6 & 16.3 & 16.3 & 16.4 & 16.4 & 16.8 & 17.2 & 17.4 \\
\hline 15 & 17.3 & 17.3 & 17.4 & 16.8 & 16.6 & 16.2 & 16.3 & 16.3 & 16.4 & 16.8 & 17.2 & 17.3 \\
\hline 16 & 17.3 & 17.3 & 17.3 & 16.8 & 16.6 & 16.2 & 16.3 & 16.3 & 16.4 & 16.9 & 17.3 & 17.4 \\
\hline 17 & 17.3 & 17.4 & 17.4 & 16.8 & 16.7 & 16.2 & 16.2 & 16.3 & 16.4 & 16.9 & 17.3 & 17.4 \\
\hline 18 & 17.4 & 17.5 & 17.2 & 16.7 & 16.7 & 16.3 & 16.3 & 16.3 & 16.5 & 16.9 & 17.3 & 17.5 \\
\hline 19 & 17.3 & 17.5 & 17.3 & 16.8 & 16.7 & 16.3 & 16.3 & 16.4 & 16.5 & 17.0 & 17.3 & 17.5 \\
\hline 20 & 17.5 & 17.5 & 16.8 & 16.6 & 16.7 & 16.2 & 16.3 & 16.3 & 16.5 & 16.9 & 17.3 & 17.4 \\
\hline 21 & 17.4 & 17.4 & 16.8 & 16.8 & 16.6 & 16.2 & 16.3 & 16.4 & 16.5 & 16.9 & 17.3 & 17.4 \\
\hline 22 & 17.4 & 17.5 & 17.0 & 16.8 & 16.6 & 16.3 & 16.3 & 16.4 & 16.5 & 16.9 & 17.3 & 17.4 \\
\hline 23 & 17.4 & 17.5 & 16.9 & 16.8 & 16.6 & 16.3 & 16.3 & 16.4 & 16.6 & 17.0 & 17.3 & 17.5 \\
\hline 24 & 17.4 & 17.5 & 17.0 & 16.8 & 16.6 & 16.3 & 16.3 & 16.4 & 16.6 & 16.9 & 17.4 & 17.4 \\
\hline 25 & 17.5 & 17.5 & 17.1 & 16.7 & 16.6 & 16.2 & 16.3 & 16.3 & 16.6 & 17.0 & 17.3 & 17.4 \\
\hline 26 & 17.3 & 17.5 & 17.1 & 16.7 & 16.7 & 16.2 & 16.3 & 16.3 & 16.6 & 17.0 & 17.3 & 17.4 \\
\hline 27 & 17.4 & 17.6 & 17.2 & 16.6 & 16.6 & 16.2 & 16.3 & 16.3 & 16.6 & 17.0 & 17.3 & 17.5 \\
\hline 28 & 17.4 & 17.5 & 17.2 & 16.7 & 16.7 & 16.2 & 16.3 & 16.3 & 16.6 & 17.1 & 17.4 & 17.5 \\
\hline 29 & 17.4 & - & 17.2 & 16.5 & 16.7 & 16.2 & 16.4 & 16.3 & 16.6 & 17.1 & 17.4 & 17.5 \\
\hline 30 & 17.4 & - & 17.2 & 16.6 & 16.7 & 16.2 & 16.3 & 16.3 & 16.6 & 17.1 & 17.4 & 17.6 \\
\hline \multirow[t]{2}{*}{31} & 17.5 & - & 17.2 & - & 16.8 & - & 16.3 & 16.3 & - & 17.1 & - & 17.6 \\
\hline & & & & & & & " & & & & & \\
\hline Mean & 17.4 & 17.4 & 17.2 & 16.9 & 16.6 & 16.3 & 16.3 & 16.3 & 16.4 & 16.9 & 17.2 & 17.4 \\
\hline Max & 17.6 & 17.6 & 17.5 & 17.2 & 16.8 & 16.7 & 16.4 & 16.4 & 16.6 & 17.1 & 17.4 & 17.6 \\
\hline Min & 17.0 & 16.7 & 16.5 & 16.5 & 16.4 & 16.2 & 16.2 & 16.3 & 16.3 & 16.6 & 17.0 & 17.3 \\
\hline
\end{tabular}




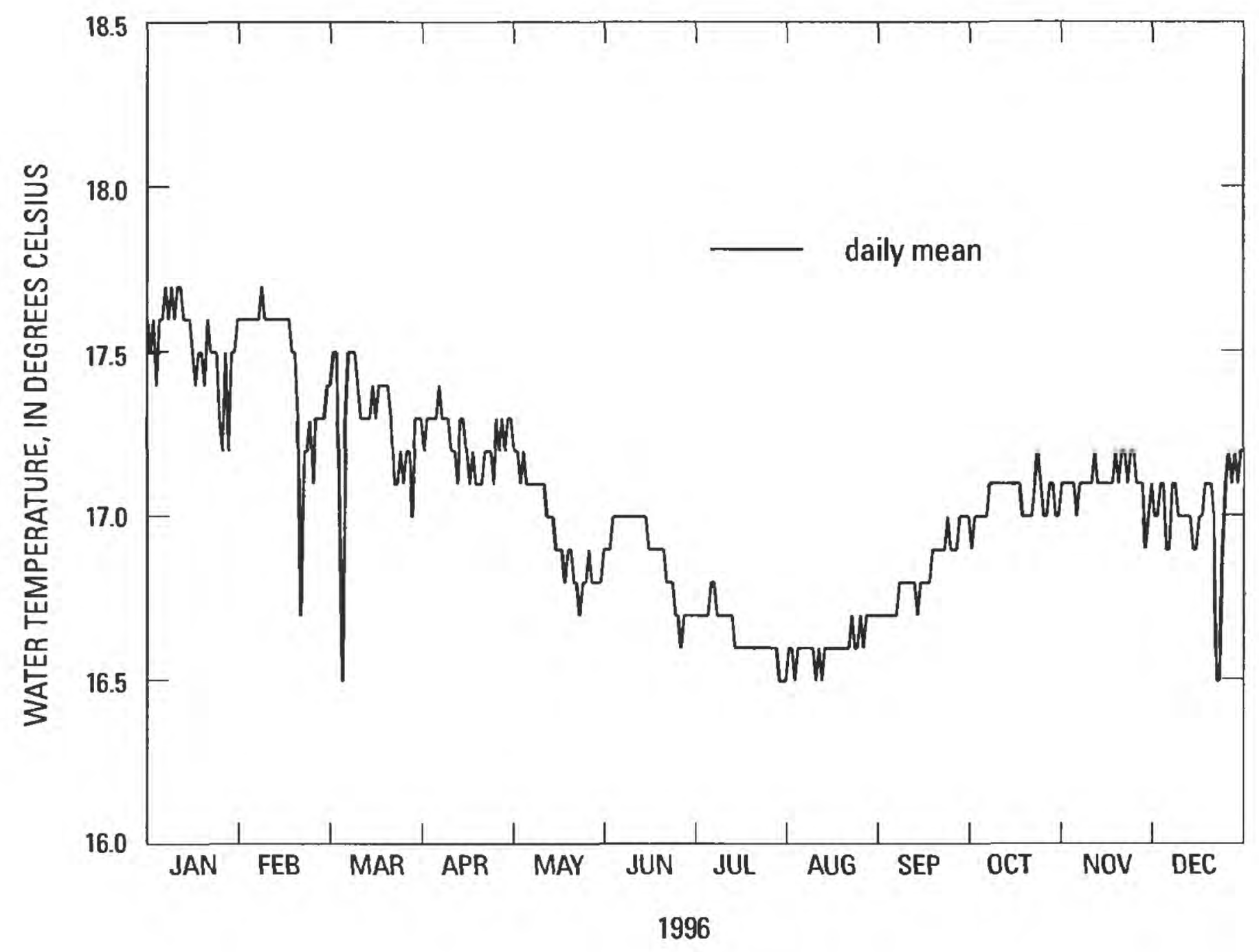

Figure 26. (C) Daily mean water temperature, Fish Hatchery Spring AB (FHAB-WEIR), 1996, Long Valtey Caldera, Mono County, California. 
Table 20. Daily mean water temperature, Fish Hatchery Spring AB (FHAB-WEIR), 1996, Long Valley Caldera, California

[Values are in degrees Celsius. —, no data: Max, maximum; Min, minimum]

\begin{tabular}{|c|c|c|c|c|c|c|c|c|c|c|c|c|}
\hline Day & Jan & Feb & Mar & Apr & May & Jun & Jul & Aug & Sep & Oct & Nov & Dec \\
\hline 1 & 17.5 & 17.6 & 17.4 & 17.2 & 17.2 & 16.9 & 16.7 & 16.6 & 16.7 & 16.9 & 17.1 & 17.0 \\
\hline 2 & 17.6 & 17.6 & 17.5 & 17.3 & 17.2 & 16.9 & 16.7 & 16.6 & 16.7 & 17.0 & 17.1 & 17.0 \\
\hline 3 & 17.4 & 17.6 & 17.5 & 17.3 & 17.1 & 17.0 & 16.7 & 16.5 & 16.7 & 17.0 & 17.1 & 17.1 \\
\hline 4 & 17.6 & 17.6 & 17.0 & 17.3 & 17.2 & 17.0 & 16.7 & 16.6 & 16.7 & 17.0 & 17.1 & 17.1 \\
\hline 5 & 17.6 & 17.6 & 16.5 & 17.3 & 17.1 & 17.0 & 16.7 & 16.6 & 16.7 & 17.0 & 17.0 & 16.9 \\
\hline 6 & 17.7 & 17.6 & 17.3 & 17.4 & 17.1 & 17.0 & 16.8 & 16.6 & 16.7 & 17.0 & 17.1 & 16.9 \\
\hline 7 & 17.6 & 17.7 & 17.5 & 17.3 & 17.1 & 17.0 & 16.8 & 16.6 & 16.8 & 17.1 & 17.1 & 17.1 \\
\hline 8 & 17.7 & 17.6 & 17.5 & 17.3 & 17.1 & 17.0 & 16.7 & 16.6 & 16.8 & 17.1 & 17.1 & 17.1 \\
\hline 9 & 17.6 & 17.6 & 17.5 & 17.3 & 17.1 & 17.0 & 16.7 & 16.6 & 16.8 & 17.1 & 17.1 & 17.0 \\
\hline 10 & 17.7 & 17.6 & 17.4 & 17.2 & 17.1 & 17.0 & 16.7 & 16.5 & 16.8 & 17.1 & 17.1 & 17.0 \\
\hline 11 & 17.7 & 17.6 & 17.3 & 17.2 & 17.1 & 17.0 & 16.7 & 16.6 & 16.8 & 17.1 & 17.2 & 17.0 \\
\hline 12 & 17.6 & 17.6 & 17.3 & 17.1 & 17.0 & 17.0 & 16.7 & 16.5 & 16.8 & 17.1 & 17.1 & 17.0 \\
\hline 13 & 17.6 & 17.6 & 17.3 & 17.3 & 17.0 & 17.0 & 16.7 & 16.6 & 16.7 & 17.1 & 17.1 & 17.0 \\
\hline 14 & 17.6 & 17.6 & 17.3 & 17.3 & 17.0 & 17.0 & 16.6 & 16.6 & 16.8 & 17.1 & 17.1 & 16.9 \\
\hline 15 & 17.5 & 17.6 & 17.4 & 17.2 & 16.9 & 16.9 & 16.6 & 16.6 & 16.8 & 17.1 & 17.1 & 16.9 \\
\hline 16 & 17.4 & 17.6 & 17.3 & 17.1 & 16.9 & 16.9 & 16.6 & 16.6 & 16.8 & 17.1 & 17.1 & 17.0 \\
\hline 17 & 17.5 & 17.5 & 17.4 & 17.2 & 16.9 & 16.9 & 16.6 & 16.6 & 16.8 & 17.1 & 17.1 & 17.0 \\
\hline 18 & 17.5 & 17.5 & 17.4 & 17.1 & 16.8 & 16.9 & 16.6 & 16.6 & 16.9 & 17.0 & 17.2 & 17.1 \\
\hline 19 & 17.4 & 17.3 & 17.4 & 17.1 & 16.9 & 16.9 & 16.6 & 16.6 & 16.9 & 17.0 & 17.1 & 17.1 \\
\hline 20 & 17.6 & 16.7 & 17.4 & 17.1 & 16.9 & 16.9 & 16.6 & 16.6 & 16.9 & 17.0 & 17.2 & 17.1 \\
\hline 21 & 17.5 & 17.2 & 17.3 & 17.2 & 16.8 & 16.8 & 16.6 & 16.6 & 16.9 & 17.0 & 17.2 & 17.0 \\
\hline 22 & 17.5 & 17.2 & 17.1 & 17.2 & 16.8 & 16.8 & 16.6 & 16.7 & 16.9 & 17.1 & 17.1 & 16.5 \\
\hline 23 & 17.5 & 17.3 & 17.1 & 17.2 & 16.7 & 16.8 & 16.6 & 16.6 & 17.0 & 17.2 & 17.2 & 16.5 \\
\hline 24 & 17.3 & 17.1 & 17.2 & 17.1 & 16.8 & 16.7 & 16.6 & 16.6 & 16.9 & 17.1 & 17.2 & 16.9 \\
\hline 25 & 17.2 & 17.3 & 17.1 & 17.3 & 16.8 & 16.7 & 16.6 & 16.7 & 16.9 & 17.0 & 17.1 & 17.1 \\
\hline 26 & 17.5 & 17.3 & 17.2 & 17.2 & 16.9 & 16.6 & 16.6 & 16.6 & 16.9 & 17.0 & 17.1 & 17.2 \\
\hline 27 & 17.2 & 17.3 & 17.2 & 17.3 & 16.8 & 16.7 & 16.6 & 16.7 & 17.0 & 17.1 & 17.1 & 17.1 \\
\hline 28 & 17.5 & 17.3 & 17.0 & 17.2 & 16.8 & 16.7 & 16.6 & 16.7 & 17.0 & 17.1 & 16.9 & 17.2 \\
\hline 29 & 17.5 & 17.4 & 17.3 & 17.3 & 16.8 & 16.7 & 16.5 & 16.7 & 17.0 & 17.0 & 17.0 & 17.1 \\
\hline 30 & 17.6 & - & 17.3 & 17.3 & 16.8 & 16.7 & 16.5 & 16.7 & 17.0 & 17.0 & 17.1 & 17.2 \\
\hline 31 & 17.6 & - & 17.3 & - & 16.9 & - & 16.5 & 16.7 & - & 17.1 & - & 17.2 \\
\hline Mean & 17.5 & 17.5 & 17.3 & 17.2 & 17.0 & 16.9 & 16.6 & 16.6 & 16.8 & 17.1 & 17.1 & 17.0 \\
\hline Max & 17.7 & 17.7 & 17.5 & 17.4 & 17.2 & 17.0 & 16.8 & 16.7 & 17.0 & 17.2 & 17.2 & 17.2 \\
\hline Min & 17.2 & 16.7 & 16.5 & 17.1 & 16.7 & 16.6 & 16.5 & 16.5 & 16.7 & 16.9 & 16.9 & 16.5 \\
\hline
\end{tabular}




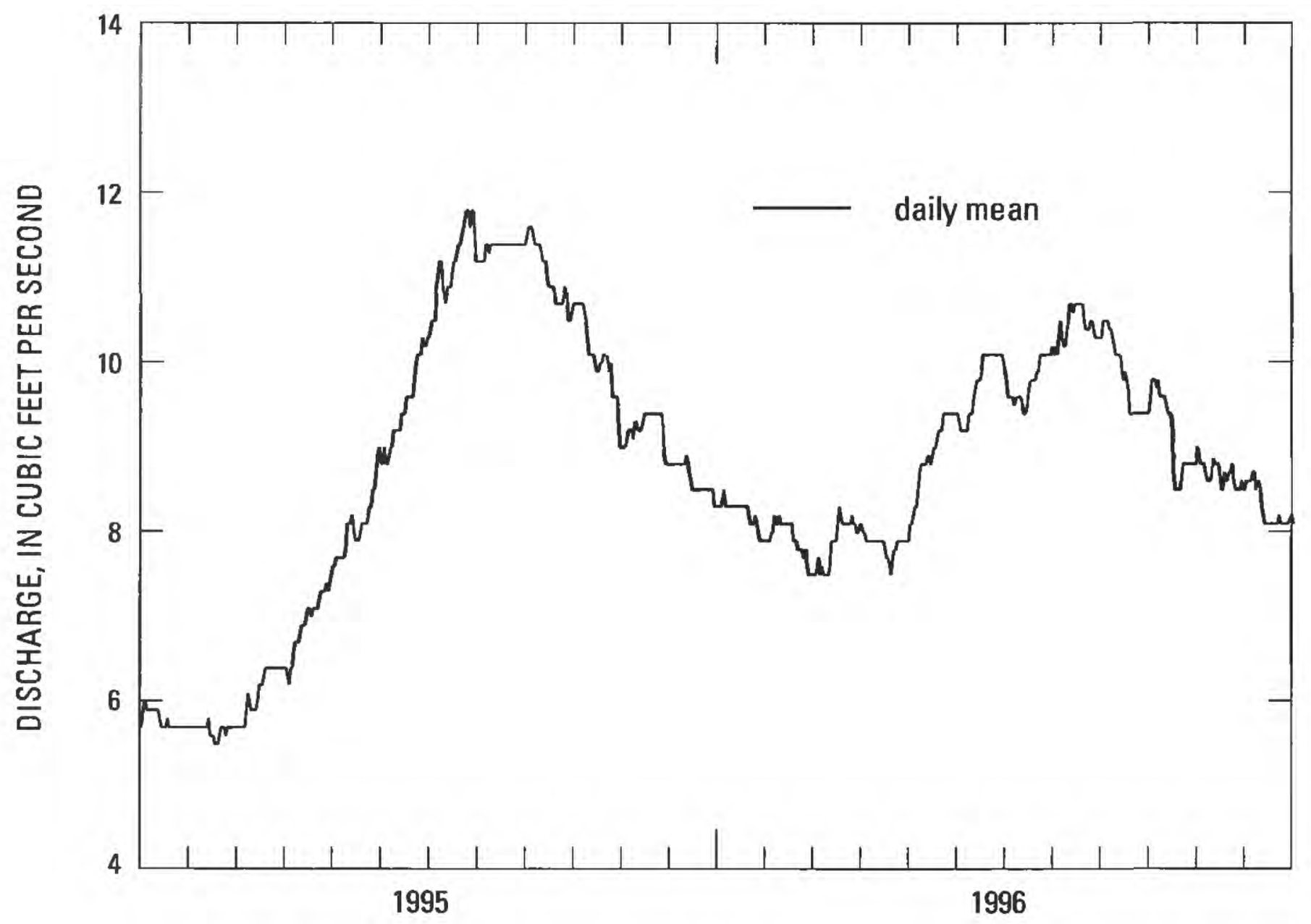

Figure 27. Daily mean discharge, Fish Hatchery Spring CD (FHCD-0), 1995-96, Long Valley Caldera, Mono County, California.

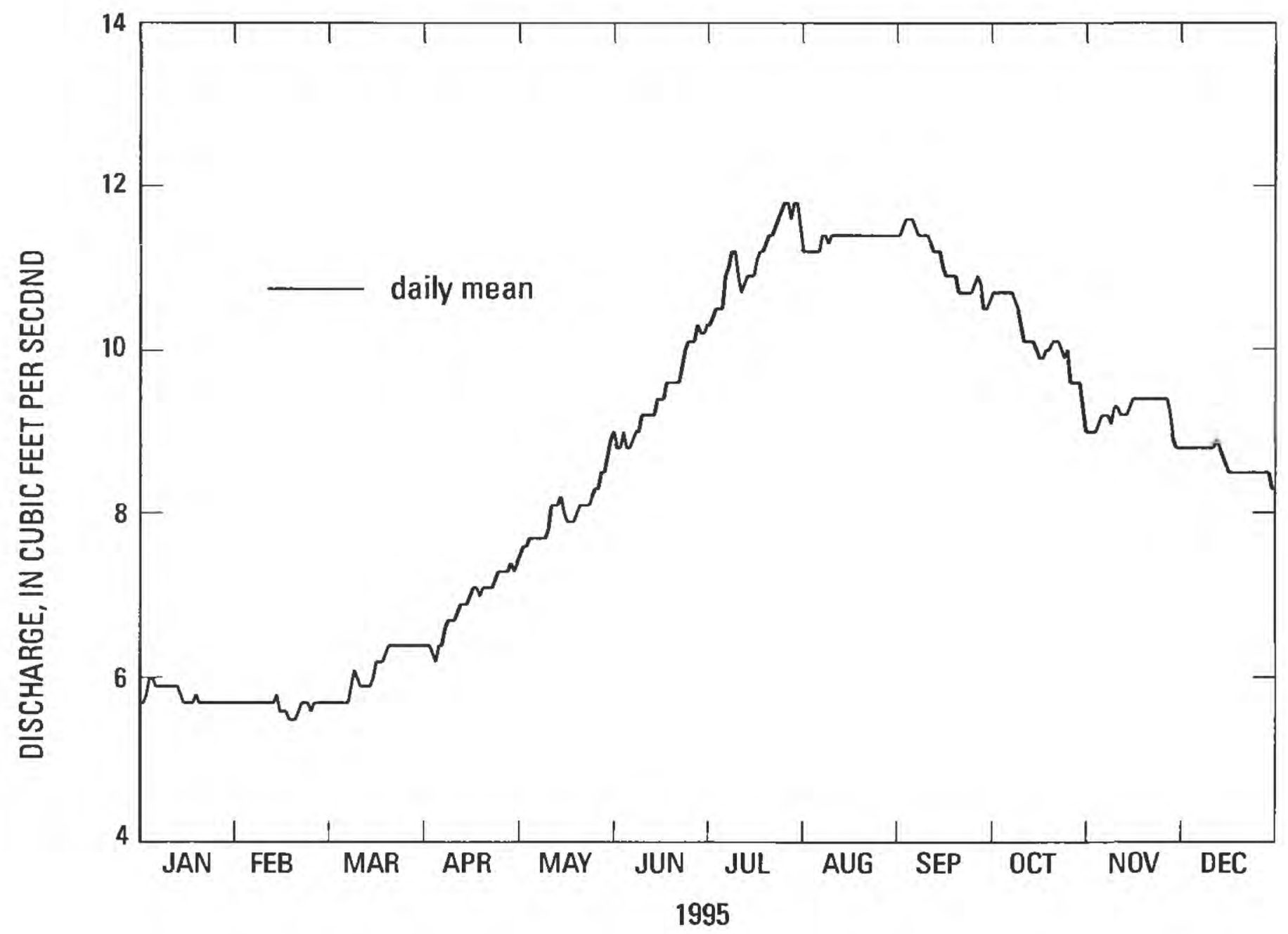

Figure 28. (A) Daily mean discharge, Fish Hatchery Spring CD (FHCD-0), 1995, Long Valley Caldera, Mono County, California. 
Table 21. Daily mean discharge, Fish Hatchery Spring CD (FHCD-0), 1995, Long Valley Caldera, California

[Values are in cubic feet per second. —, no data; Max, maximum: Min, minimum]

\begin{tabular}{|c|c|c|c|c|c|c|c|c|c|c|c|c|}
\hline Day & Jan & Feb & Mar & Apr & May & Jun & Jul & Aug & Sep & Oct & Nov & Dec \\
\hline 1 & 5.7 & 5.7 & 5.7 & 6.4 & 7.4 & 9.0 & 10.3 & 11.2 & 11.4 & 10.7 & 9.0 & 8.8 \\
\hline 2 & 5.8 & 5.7 & 5.7 & 6.4 & 7.5 & 8.8 & 10.3 & 11.2 & 11.5 & 10.7 & 9.0 & 8.8 \\
\hline 3 & 6.0 & 5.7 & 5.7 & 6.4 & 7.6 & 8.8 & 10.4 & 11.2 & 11.6 & 10.7 & 9.0 & 8.8 \\
\hline 4 & 6.0 & 5.7 & 5.7 & 6.3 & 7.6 & 9.0 & 10.5 & 11.2 & 11.6 & 10.7 & 9.1 & 8.8 \\
\hline 5 & 5.9 & 5.7 & 5.7 & 6.2 & 7.7 & 8.8 & 10.5 & 11.2 & 11.6 & 10.7 & 9.2 & 8.8 \\
\hline 6 & 5.9 & 5.7 & 5.7 & 6.4 & 7.7 & 8.8 & 10.5 & 11.2 & 11.5 & 10.7 & 9.2 & 8.8 \\
\hline 7 & 5.9 & 5.7 & 5.7 & 6.4 & 7.7 & 8.9 & 10.9 & 11.4 & 11.4 & 10.7 & 9.2 & 8.8 \\
\hline 8 & 5.9 & 5.7 & 5.7 & 6.6 & 7.7 & 9.0 & 11.0 & 11.4 & 11.4 & 10.6 & 9.1 & 8.8 \\
\hline 9 & 5.9 & 5.7 & 5.9 & 6.7 & 7.7 & 9.0 & 11.2 & 11.3 & 11.4 & 10.5 & 9.3 & 8.8 \\
\hline 10 & 5.9 & 5.7 & 6.1 & 6.7 & 7.7 & 9.2 & 11.2 & 11.4 & 11.4 & 10.3 & 9.3 & 8.8 \\
\hline 11 & 5.9 & 5.7 & 6.0 & 6.7 & 7.8 & 9.2 & 10.9 & 11.4 & 11.3 & 10.1 & 9.2 & 8.8 \\
\hline 12 & 5.9 & 5.7 & 5.9 & 6.8 & 8.1 & 9.2 & 10.7 & 11.4 & 11.2 & 10.1 & 9.2 & 8.9 \\
\hline 13 & 5.8 & 5.8 & 5.9 & 6.9 & 8.1 & 9.2 & 10.8 & 11.4 & 11.2 & 10.1 & 9.2 & 8.8 \\
\hline 14 & 5.7 & 5.6 & 5.9 & 6.9 & 8.1 & 9.2 & 10.9 & 11.4 & 11.2 & 10.1 & 9.3 & 8.7 \\
\hline 15 & 5.7 & 5.6 & 5.9 & 6.9 & 8.2 & 9.4 & 10.9 & 11.4 & 11.0 & 10.0 & 9.4 & 8.6 \\
\hline 16 & 5.7 & 5.6 & 6.0 & 7.0 & 8.0 & 9.4 & 10.9 & 11.4 & 10.9 & 9.9 & 9.4 & 8.5 \\
\hline 17 & 5.7 & 5.5 & 6.2 & 7.1 & 7.9 & 9.4 & 11.1 & 11.4 & 10.9 & 9.9 & 9.4 & 8.5 \\
\hline 18 & 5.8 & 5.5 & 6.2 & 7.1 & 7.9 & 9.6 & 11.2 & 11.4 & 10.9 & 10.0 & 9.4 & 8.5 \\
\hline 19 & 5.7 & 5.5 & 6.2 & 7.0 & 7.9 & 9.6 & 11.2 & 11.4 & 10.9 & 10.0 & 9.4 & 8.5 \\
\hline 20 & 5.7 & 5.6 & 6.3 & 7.1 & 8.0 & 9.6 & 11.3 & 11.4 & 10.7 & 10.1 & 9.4 & 8.5 \\
\hline 21 & 5.7 & 5.7 & 6.4 & 7.1 & 8.1 & 9.6 & 11.4 & 11.4 & 10.7 & 10.1 & 9.4 & 8.5 \\
\hline 22 & 5.7 & 5.7 & 6.4 & 7.1 & 8.1 & 9.6 & 11.4 & 11.4 & 10.7 & 10.1 & 9.4 & 8.5 \\
\hline 23 & 5.7 & 5.7 & 6.4 & 7.1 & 8.1 & 9.8 & 11.5 & 11.4 & 10.7 & 10.0 & 9.4 & 8.5 \\
\hline 24 & 5.7 & 5.6 & 6.4 & 7.2 & 8.1 & 10.0 & 11.6 & 11.4 & 10.7 & 9.9 & 9.4 & 8.5 \\
\hline 25 & 5.7 & 5.7 & 6.4 & 7.3 & 8.2 & 10.1 & 11.7 & 11.4 & 10.8 & 10.0 & 9.4 & 8.5 \\
\hline 26 & 5.7 & 5.7 & 6.4 & 7.3 & 8.3 & 10.1 & 11.8 & 11.4 & 10.9 & 9.6 & 9.4 & 8.5 \\
\hline 27 & 5.7 & 5.7 & 6.4 & 7.3 & 8.3 & 10.1 & 11.8 & 11.4 & 10.8 & 9.6 & 9.2 & 8.5 \\
\hline 28 & 5.7 & 5.7 & 6.4 & 7.3 & 8.5 & 10.3 & 11.6 & 11.4 & 10.5 & 9.6 & 8.9 & 8.5 \\
\hline 29 & 5.7 & - & 6.4 & 7.4 & 8.5 & 10.2 & 11.8 & 11.4 & 10.5 & 9.6 & 8.8 & 8.5 \\
\hline 30 & 5.7 & - & 6.4 & 7.3 & 8.7 & 10.2 & 11.8 & 11.4 & 10.6 & 9.3 & 8.8 & 8.3 \\
\hline 31 & 5.7 & - & 6.4 & - & 8.9 & - & 11.5 & 11.4 & - & 9.0 & - & 8.3 \\
\hline Mean & 5.78 & 5.66 & 6.08 & 6.88 & 8.00 & 9.44 & 11.1 & 11.4 & 11.1 & 10.1 & 9.23 & 8.63 \\
\hline Max & 6.0 & 5.8 & 6.4 & 7.4 & 8.9 & 10.3 & 11.8 & 11.4 & 11.6 & 10.7 & 9.4 & 8.9 \\
\hline Min & 5.7 & 5.5 & 5.7 & 6.2 & 7.4 & 8.8 & 10.3 & 11.2 & 10.5 & 9.0 & 8.8 & 8.3 \\
\hline
\end{tabular}




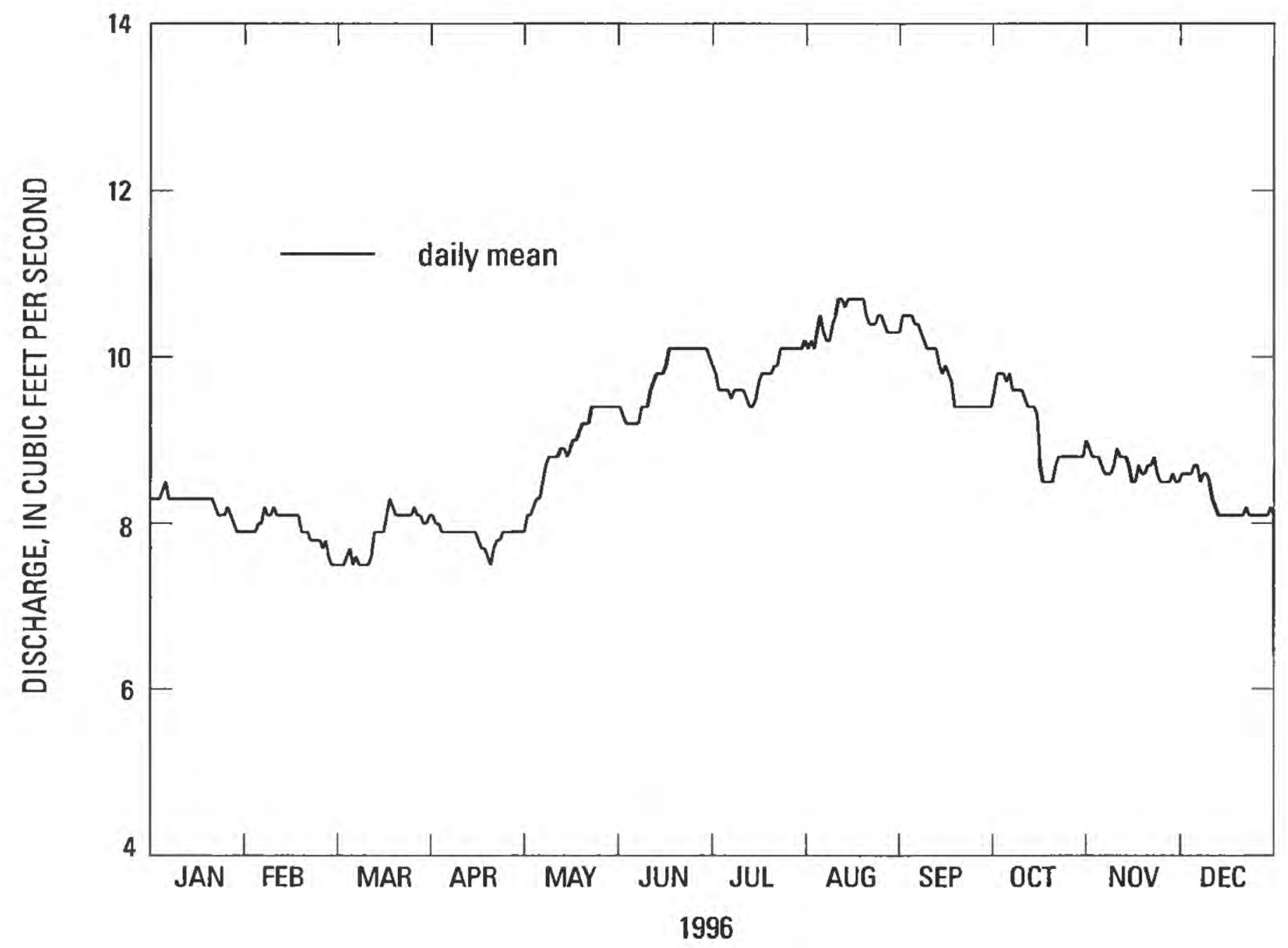

Figure 28. (B) Daily mean discharge, Fish Hatchery Spring CD (FHCD-0), 1996, Long Valley Caldera, Mono County, California. 
Table 22. Daily mean discharge, Fish Hatchery Spring CD (FHCD-0), 1996, Long Valley Caldera, California

[Values are in cubic feet per second. -, no data; Max, maximum; Min, minimum]

\begin{tabular}{|c|c|c|c|c|c|c|c|c|c|c|c|c|}
\hline Day & Jan & Feb & Mar & Apr & May & Jun & Jul & Aug & Sep & Oct & Nov & Dec \\
\hline 1 & 8.3 & 7.9 & 7.5 & 8.1 & 7.9 & 9.4 & 9.9 & 10.1 & 10.5 & 9.6 & 8.9 & 8.6 \\
\hline 2 & 8.3 & 7.9 & 7.5 & 8.0 & 8.1 & 9.3 & 9.8 & 10.2 & 10.5 & 9.8 & 8.8 & 8.6 \\
\hline 3 & 8.3 & 7.9 & 7.5 & 8.0 & 8.1 & 9.2 & 9.6 & 10.1 & 10.5 & 9.8 & 8.8 & 8.6 \\
\hline 4 & 8.4 & 8.0 & 7.6 & 7.9 & 8.2 & 9.2 & 9.6 & 10.3 & 10.5 & 9.8 & 8.8 & 8.6 \\
\hline 5 & 8.5 & 8.0 & 7.7 & 7.9 & 8.3 & 9.2 & 9.6 & 10.5 & 10.4 & 9.7 & 8.7 & 8.7 \\
\hline 6 & 8.3 & 8.2 & 7.5 & 7.9 & 8.3 & 9.2 & 9.6 & 10.3 & 10.4 & 9.8 & 8.6 & 8.7 \\
\hline 7 & 8.3 & 8.1 & 7.6 & 7.9 & 8.5 & 9.2 & 9.5 & 10.2 & 10.3 & 9.6 & 8.6 & 8.5 \\
\hline 8 & 8.3 & 8.1 & 7.5 & 7.9 & 8.7 & 9.4 & 9.6 & 10.2 & 10.2 & 9.6 & 8.6 & 8.6 \\
\hline 9 & 8.3 & 8.2 & 7.5 & 7.9 & 8.8 & 9.4 & 9.6 & 10.4 & 10.1 & 9.6 & 8.7 & 8.6 \\
\hline 10 & 8.3 & 8.1 & 7.5 & 7.9 & 8.8 & 9.4 & 9.6 & 10.5 & 10.1 & 9.6 & 8.9 & 8.5 \\
\hline 11 & 8.3 & 8.1 & 7.5 & 7.9 & 8.8 & 9.6 & 9.6 & 10.7 & 10.1 & 9.5 & 8.8 & 8.3 \\
\hline 12 & 8.3 & 8.1 & 7.6 & 7.9 & 8.8 & 9.7 & 9.5 & 10.7 & 10.1 & 9.4 & 8.8 & 8.2 \\
\hline 13 & 8.3 & 8.1 & 7.9 & 7.9 & 8.9 & 9.8 & 9.4 & 10.6 & 9.9 & 9.4 & 8.8 & 8.1 \\
\hline 14 & 8.3 & 8.1 & 7.9 & 7.9 & 8.9 & 9.8 & 9.4 & 10.7 & 9.8 & 9.4 & 8.7 & 8.1 \\
\hline 15 & 8.3 & 8.1 & 7.9 & 7.9 & 8.8 & 9.8 & 9.5 & 10.7 & 9.9 & 9.3 & 8.5 & 8.1 \\
\hline 16 & 8.3 & 8.1 & 7.9 & 7.8 & 8.9 & 9.9 & 9.7 & 10.7 & 9.8 & 8.7 & 8.5 & 8.1 \\
\hline 17 & 8.3 & 8.1 & 8.1 & 7.7 & 9.0 & 10.1 & 9.8 & 10.7 & 9.7 & 8.5 & 8.7 & 8.1 \\
\hline 18 & 8.3 & 7.9 & 8.3 & 7.7 & 9.0 & 10.1 & 9.8 & 10.7 & 9.4 & 8.5 & 8.6 & 8.1 \\
\hline 19 & 8.3 & 7.9 & 8.2 & 7.6 & 9.1 & 10.1 & 9.8 & 10.7 & 9.4 & 8.5 & 8.6 & 8.1 \\
\hline 20 & 8.3 & 7.9 & 8.1 & 7.5 & 9.2 & 10.1 & 9.8 & 10.5 & 9.4 & 8.5 & 8.7 & 8.1 \\
\hline 21 & 8.2 & 7.8 & 8.1 & 7.7 & 9.2 & 10.1 & 9.9 & 10.4 & 9.4 & 8.7 & 8.7 & 8.1 \\
\hline 22 & 8.1 & 7.8 & 8.1 & 7.8 & 9.2 & 10.1 & 9.9 & 10.4 & 9.4 & 8.8 & 8.8 & 8.2 \\
\hline 23 & 8.1 & 7.8 & 8.1 & 7.8 & 9.4 & 10.1 & 10.1 & 10.4 & 9.4 & 8.8 & 8.6 & 8.1 \\
\hline 24 & 8.1 & 7.8 & 8.1 & 7.9 & 9.4 & 10.1 & 10.1 & 10.5 & 9.4 & 8.8 & 8.5 & 8.1 \\
\hline 25 & 8.2 & 7.7 & 8.1 & 7.9 & 9.4 & 10.1 & 10.1 & 10.5 & 9.4 & 8.8 & 8.5 & 8.1 \\
\hline 26 & 8.1 & 7.8 & 8.2 & 7.9 & 9.4 & 10.1 & 10.1 & 10.4 & 9.4 & 8.8 & 8.5 & 8.1 \\
\hline 27 & 8.0 & 7.6 & 8.1 & 7.9 & 9.4 & 10.1 & 10.1 & 10.3 & 9.4 & 8.8 & 8.5 & 8.1 \\
\hline 28 & 7.9 & 7.5 & 8.1 & 7.9 & 9.4 & 10.1 & 10.1 & 10.3 & 9.4 & 8.8 & 8.6 & 8.1 \\
\hline 29 & 7.9 & 7.5 & 8.0 & 7.9 & 9.4 & 10.1 & 10.1 & 10.3 & 9.4 & 8.8 & 8.5 & 8.1 \\
\hline 30 & 7.9 & - & 8.0 & 7.9 & 9.4 & 10.0 & 10.1 & 10.3 & 9.4 & 8.8 & 8.5 & 8.2 \\
\hline 31 & 7.9 & - & 8.1 & - & 9.4 & - & 10.2 & 10.3 & - & 9.0 & - & 8.1 \\
\hline Mean & 8.22 & 7.93 & 7.86 & 7.86 & 8.91 & 9.76 & 9.79 & 10.4 & 9.83 & 9.15 & 8.66 & 8.28 \\
\hline Max & 8.5 & 8.2 & 8.3 & 8.1 & 9.4 & 10.1 & 10.2 & 10.7 & 10.5 & 9.8 & 8.9 & 8.7 \\
\hline Min & 7.9 & 7.5 & 7.5 & 7.5 & 7.9 & 9.2 & 9.4 & 10.1 & 9.4 & 8.5 & 8.5 & 8.1 \\
\hline
\end{tabular}




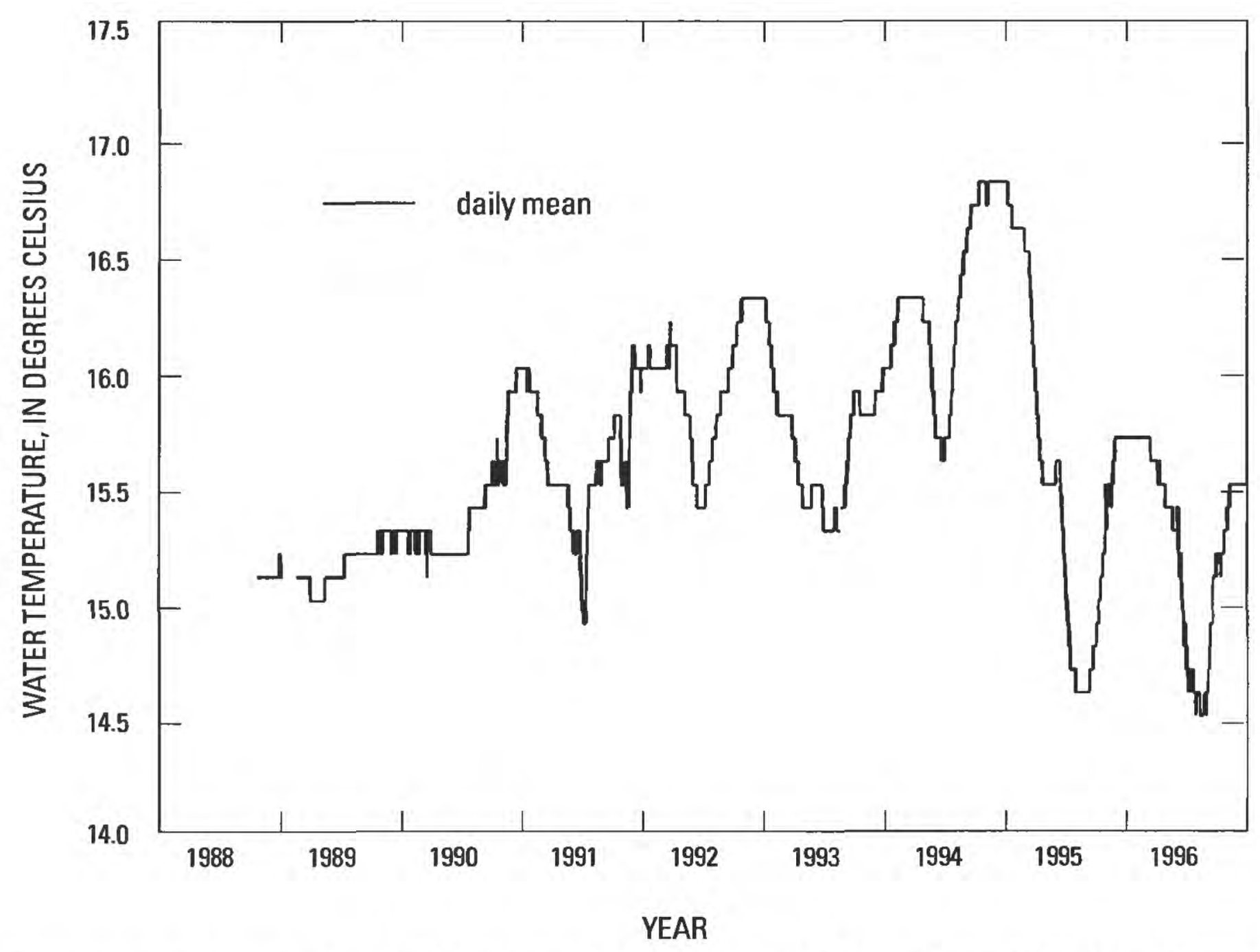

Figure 29. Daily mean water temperature, Fish Hatchery Spring CD (FHCD-T), 1988-96, Long Valley Caldera, Mono County, California.

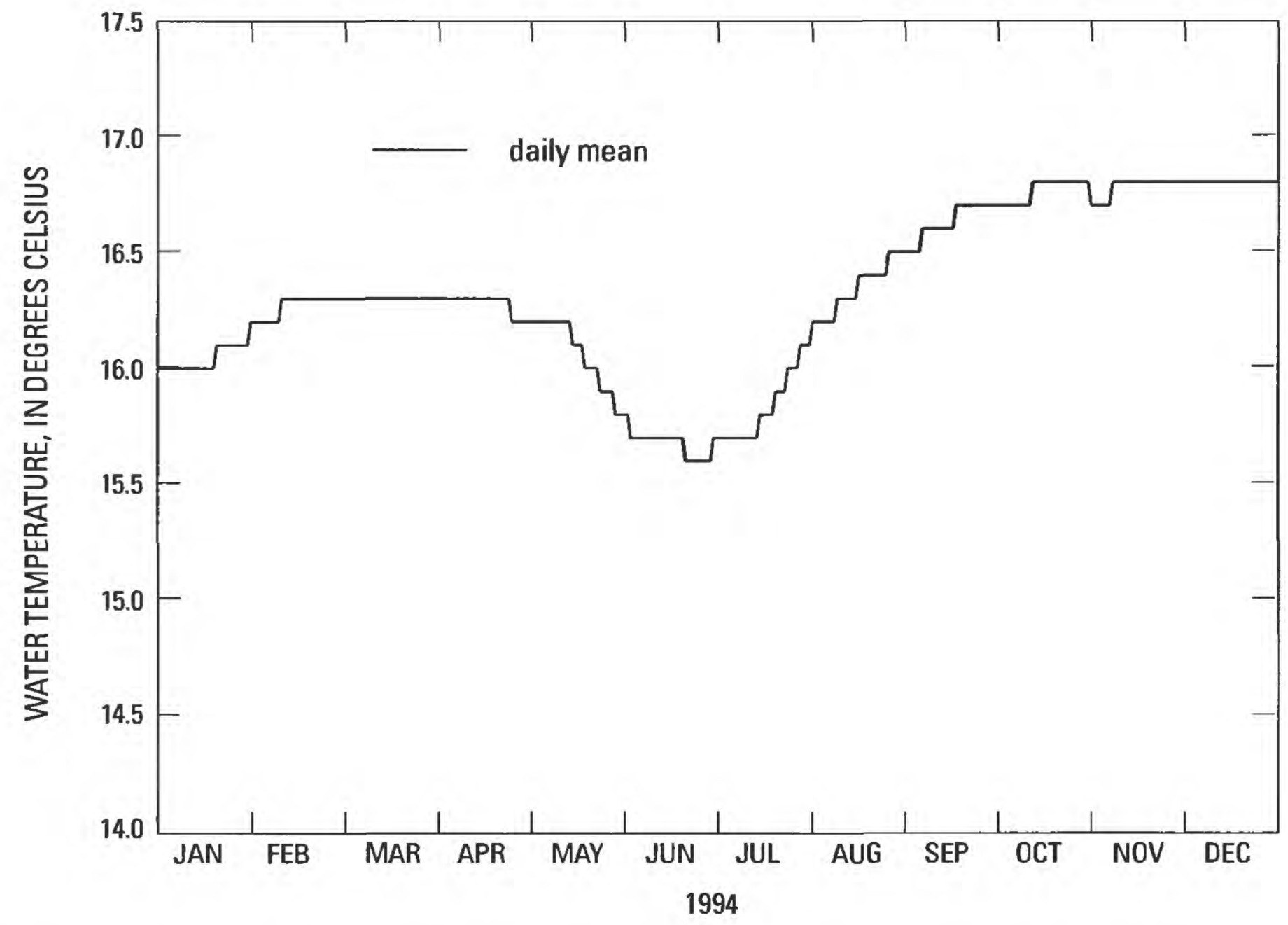

Figure 30. (A) Daily mean water temperature, Fish Hatchery Spring CD (FHCD-T), 1994, Long Valley Caldera, Mono County, California. 
Table 23. Daily mean water temperature, Fish Hatchery Spring CD (FHCD-T), 1994, Long Valley Caldera, California

[Values are in degrees Celsius. —, no data; Max, maximum; Min, minimum]

\begin{tabular}{|c|c|c|c|c|c|c|c|c|c|c|c|c|}
\hline Day & Jan & Feb & Mar & Apr & May & Jun & Jul & Aug & Sep & Oct & Nov & Dec \\
\hline 1 & 16.0 & 16.2 & 16.3 & 16.3 & 16.2 & 15.8 & 15.7 & 16.2 & 16.5 & 16.7 & 16.7 & 16.8 \\
\hline 2 & 16.0 & 16.2 & 16.3 & 16.3 & 16.2 & 15.8 & 15.7 & 16.2 & 16.5 & 16.7 & 16.7 & 16.8 \\
\hline 3 & 16.0 & 16.2 & 16.3 & 16.3 & 16.2 & 15.7 & 15.7 & 16.2 & 16.5 & 16.7 & 16.7 & 16.8 \\
\hline 4 & 16.0 & 16.2 & 16.3 & 16.3 & 16.2 & 15.7 & 15.7 & 16.2 & 16.5 & 16.7 & 16.7 & 16.8 \\
\hline 5 & 16.0 & 16.2 & 16.3 & 16.3 & 16.2 & 15.7 & 15.7 & 16.2 & 16.5 & 16.7 & 16.7 & 16.8 \\
\hline 6 & 16.0 & 16.2 & 16.3 & 16.3 & 16.2 & 15.7 & 15.7 & 16.2 & 16.6 & 16.7 & 16.7 & 16.8 \\
\hline 7 & 16.0 & 16.2 & 16.3 & 16.3 & 16.2 & 15.7 & 15.7 & 16.2 & 16.6 & 16.7 & 16.8 & 16.8 \\
\hline 8 & 16.0 & 16.2 & 16.3 & 16.3 & 16.2 & 15.7 & 15.7 & 16.2 & 16.6 & 16.7 & 16.8 & 16.8 \\
\hline 9 & 16.0 & 16.3 & 16.3 & 16.3 & 16.2 & 15.7 & 15.7 & 16.3 & 16.6 & 16.7 & 16.8 & 16.8 \\
\hline 10 & 16.0 & 16.3 & 16.3 & 16.3 & 16.2 & 15.7 & 15.7 & 16.3 & 16.6 & 16.7 & 16.8 & 16.8 \\
\hline 11 & 16.0 & 16.3 & 16.3 & 16.3 & 16.2 & 15.7 & 15.7 & 16.3 & 16.6 & 16.7 & 16.8 & 16.8 \\
\hline 12 & 16.0 & 16.3 & 16.3 & 16.3 & 16.2 & 15.7 & 15.7 & 16.3 & 16.6 & 16.8 & 16.8 & 16.8 \\
\hline 13 & 16.0 & 16.3 & 16.3 & 16.3 & 16.2 & 15.7 & 15.7 & 16.3 & 16.6 & 16.8 & 16.8 & 16.8 \\
\hline 14 & 16.0 & 16.3 & 16.3 & 16.3 & 16.2 & 15.7 & 15.7 & 16.3 & 16.6 & 16.8 & 16.8 & 16.8 \\
\hline 15 & 16.0 & 16.3 & 16.3 & 16.3 & 16.1 & 15.7 & 15.8 & 16.3 & 16.6 & 16.8 & 16.8 & 16.8 \\
\hline 16 & 16.0 & 16.3 & 16.3 & 16.3 & 16.1 & 15.7 & 15.8 & 16.4 & 16.6 & 16.8 & 16.8 & 16.8 \\
\hline 17 & 16.0 & 16.3 & 16.3 & 16.3 & 16.1 & 15.7 & 15.8 & 16.4 & 16.7 & 16.8 & 16.8 & 16.8 \\
\hline 18 & 16.0 & 16.3 & 16.3 & 16.3 & 16.1 & 15.7 & 15.8 & 16.4 & 16.7 & 16.8 & 16.8 & 16.8 \\
\hline 19 & 16.1 & 16.3 & 16.3 & 16.3 & 16.0 & 15.7 & 15.8 & 16.4 & 16.7 & 16.8 & 16.8 & 16.8 \\
\hline 20 & 16.1 & 16.3 & 16.3 & 16.3 & 16.0 & 15.7 & 15.9 & 16.4 & 16.7 & 16.8 & 16.8 & 16.8 \\
\hline 21 & 16.1 & 16.3 & 16.3 & 16.3 & 16.0 & 15.6 & 15.9 & 16.4 & 16.7 & 16.8 & 16.8 & 16.8 \\
\hline 22 & 16.1 & 16.3 & 16.3 & 16.3 & 16.0 & 15.6 & 15.9 & 16.4 & 16.7 & 16.8 & 16.8 & 16.8 \\
\hline 23 & 16.1 & 16.3 & 16.3 & 16.3 & 16.0 & 15.6 & 15.9 & 16.4 & 16.7 & 16.8 & 16.8 & 16.8 \\
\hline 24 & 16.1 & 16.3 & 16.3 & 16.3 & 15.9 & 15.6 & 16.0 & 16.4 & 16.7 & 16.8 & 16.8 & 16.8 \\
\hline 25 & 16.1 & 16.3 & 16.3 & 16.2 & 15.9 & 15.6 & 16.0 & 16.4 & 16.7 & 16.8 & 16.8 & 16.8 \\
\hline 26 & 16.1 & 16.3 & 16.3 & 16.2 & 15.9 & 15.6 & 16.0 & 16.5 & 16.7 & 16.8 & 16.8 & 16.8 \\
\hline 27 & 16.1 & 16.3 & 16.3 & 16.2 & 15.9 & 15.6 & 16.0 & 16.5 & 16.7 & 16.8 & 16.8 & 16.8 \\
\hline 28 & 16.1 & 16.3 & 16.3 & 16.2 & 15.9 & 15.6 & 16.1 & 16.5 & 16.7 & 16.8 & 16.8 & 16.8 \\
\hline 29 & 16.1 & - & 16.3 & 16.2 & 15.8 & 15.6 & 16.1 & 16.5 & 16.7 & 16.8 & 16.8 & 16.8 \\
\hline 30 & 16.2 & - & 16.3 & 16.2 & 15.8 & 15.7 & 16.1 & 16.5 & 16.7 & 16.8 & 16.8 & 16.8 \\
\hline 31 & 16.2 & - & 16.3 & - & 15.8 & - & 16.1 & 16.5 & - & 16.7 & - & 16.8 \\
\hline Mean & 16.0 & 16.3 & 16.3 & 16.3 & 16.1 & 15.7 & 15.8 & 16.3 & 16.6 & 16.8 & 16.8 & 16.8 \\
\hline $\operatorname{Max}$ & 16.2 & 16.3 & 16.3 & 16.3 & 16.2 & 15.8 & 16.1 & 16.5 & 16.7 & 16.8 & 16.8 & 16.8 \\
\hline Min & 16.0 & 16.2 & 16.3 & 16.2 & 15.8 & 15.6 & 15.7 & 16.2 & 16.5 & 16.7 & 16.7 & 16.8 \\
\hline
\end{tabular}




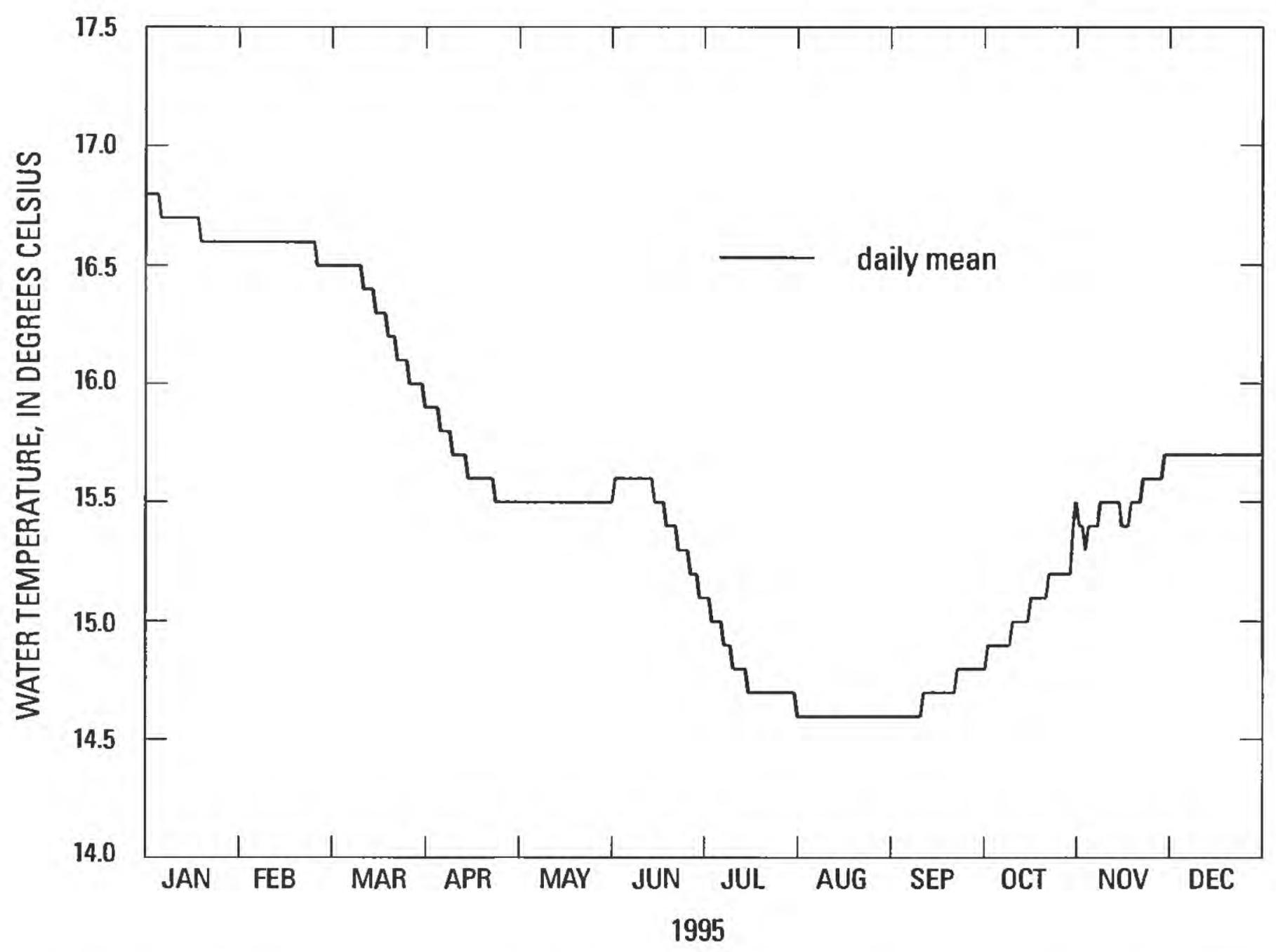

Figure 30. (B) Daily mean water temperature, Fish Hatchery Spring CD (FHCD-T), 1995, Long Valley Caldera, Mono County, California. 
Table 24. Daily mean water temperature, Fish Hatchery Spring CD (FHCD-T), 1995, Long Valley Caldera, California

[Values are in degrees Celsius. -, no data: Max, maximum: Min, minimum]

\begin{tabular}{|c|c|c|c|c|c|c|c|c|c|c|c|c|}
\hline Day & Jan & Feb & Mar & Apr & May & Jun & Jul & Aug & Sep & Oct & Nov & Dec \\
\hline 1 & 16.8 & 16.6 & 16.5 & 15.9 & 15.5 & 15.5 & 15.1 & 14.6 & 14.6 & 14.8 & 15.4 & 15.7 \\
\hline 2 & 16.8 & 16.6 & 16.5 & 15.9 & 15.5 & 15.6 & 15.1 & 14.6 & 14.6 & 14.9 & 15.4 & 15.7 \\
\hline 3 & 16.8 & 16.6 & 16.5 & 15.9 & 15.5 & 15.6 & 15.1 & 14.6 & 14.6 & 14.9 & 15.3 & 15.7 \\
\hline 4 & 16.8 & 16.6 & 16.5 & 15.9 & 15.5 & 15.6 & 15.0 & 14.6 & 14.6 & 14.9 & 15.4 & 15.7 \\
\hline 5 & 16.7 & 16.6 & 16.5 & 15.9 & 15.5 & 15.6 & 15.0 & 14.6 & 14.6 & 14.9 & 15.4 & 15.7 \\
\hline 6 & 16.7 & 16.6 & 16.5 & 15.8 & 15.5 & 15.6 & 15.0 & 14.6 & 14.6 & 14.9 & 15.4 & 15.7 \\
\hline 7 & 16.7 & 16.6 & 16.5 & 15.8 & 15.5 & 15.6 & 15.0 & 14.6 & 14.6 & 14.9 & 15.4 & 15.7 \\
\hline 8 & 16.7 & 16.6 & 16.5 & 15.8 & 15.5 & 15.6 & 14.9 & 14.6 & 14.6 & 14.9 & 15.5 & 15.7 \\
\hline 9 & 16.7 & 16.6 & 16.5 & 15.8 & 15.5 & 15.6 & 14.9 & 14.6 & 14.6 & 14.9 & 15.5 & 15.7 \\
\hline 10 & 16.7 & 16.6 & 16.5 & 15.7 & 15.5 & 15.6 & 14.9 & 14.6 & 14.6 & 15.0 & 15.5 & 15.7 \\
\hline 11 & 16.7 & 16.6 & 16.5 & 15.7 & 15.5 & 15.6 & 14.8 & 14.6 & 14.7 & 15.0 & 15.5 & 15.7 \\
\hline 12 & 16.7 & 16.6 & 16.4 & 15.7 & 15.5 & 15.6 & 14.8 & 14.6 & 14.7 & 15.0 & 15.5 & 15.7 \\
\hline 13 & 16.7 & 16.6 & 16.4 & 15.7 & 15.5 & 15.6 & 14.8 & 14.6 & 14.7 & 15.0 & 15.5 & 15.7 \\
\hline 14 & 16.7 & 16.6 & 16.4 & 15.7 & 15.5 & 15.6 & 14.8 & 14.6 & 14.7 & 15.0 & 15.5 & 15.7 \\
\hline 15 & 16.7 & 16.6 & 16.4 & 15.6 & 15.5 & 15.5 & 14.8 & 14.6 & 14.7 & 15.0 & 15.4 & 15.7 \\
\hline 16 & 16.7 & 16.6 & 16.3 & 15.6 & 15.5 & 15.5 & 14.7 & 14.6 & 14.7 & 15.1 & 15.4 & 15.7 \\
\hline 17 & 16.7 & 16.6 & 16.3 & 15.6 & 15.5 & 15.5 & 14.7 & 14.6 & 14.7 & 15.1 & 15.4 & 15.7 \\
\hline 18 & 16.6 & 16.6 & 16.3 & 15.6 & 15.5 & 15.5 & 14.7 & 14.6 & 14.7 & 15.1 & 15.5 & 15.7 \\
\hline 19 & 16.6 & 16.6 & 16.3 & 15.6 & 15.5 & 15.4 & 14.7 & 14.6 & 14.7 & 15.1 & 15.5 & 15.7 \\
\hline 20 & 16.6 & 16.6 & 16.2 & 15.6 & 15.5 & 15.4 & 14.7 & 14.6 & 14.7 & 15.1 & 15.5 & 15.7 \\
\hline 21 & 16.6 & 16.6 & 16.2 & 15.6 & 15.5 & 15.4 & 14.7 & 14.6 & 14.7 & 15.1 & 15.5 & 15.7 \\
\hline 22 & 16.6 & 16.6 & 16.2 & 15.6 & 15.5 & 15.4 & 14.7 & 14.6 & 14.8 & 15.2 & 15.6 & 15.7 \\
\hline 23 & 16.6 & 16.6 & 16.1 & 15.6 & 15.5 & 15.3 & 14.7 & 14.6 & 14.8 & 15.2 & 15.6 & 15.7 \\
\hline 24 & 16.6 & 16.6 & 16.1 & 15.5 & 15.5 & 15.3 & 14.7 & 14.6 & 14.8 & 15.2 & 15.6 & 15.7 \\
\hline 25 & 16.6 & 16.5 & 16.1 & 15.5 & 15.5 & 15.3 & 14.7 & 14.6 & 14.8 & 15.2 & 15.6 & 15.7 \\
\hline 26 & 16.6 & 16.5 & 16.1 & 15.5 & 15.5 & 15.3 & 14.7 & 14.6 & 14.8 & 15.2 & 15.6 & 15.7 \\
\hline 27 & 16.6 & 16.5 & 16.0 & 15.5 & 15.5 & 15.2 & 14.7 & 14.6 & 14.8 & 15.2 & 15.6 & 15.7 \\
\hline 28 & 16.6 & 16.5 & 16.0 & 15.5 & 15.5 & 15.2 & 14.7 & 14.6 & 14.8 & 15.2 & 15.6 & 15.7 \\
\hline 29 & 16.6 & - & 16.0 & 15.5 & 15.5 & 15.2 & 14.7 & 14.6 & 14.8 & 15.2 & 15.7 & 15.7 \\
\hline 30 & 16.6 & - & 16.0 & 15.5 & 15.5 & 15.1 & 14.7 & 14.6 & 14.8 & 15.4 & 15.7 & 15.7 \\
\hline 31 & 16.6 & - & 16.0 & - & 15.5 & - & 14.7 & 14.6 & - & 15.5 & - & 15.7 \\
\hline Mean & 16.7 & 16.6 & 16.3 & 15.7 & 15.5 & 15.5 & 14.8 & 14.6 & 14.7 & 15.1 & 15.5 & 15.7 \\
\hline Max & 16.8 & 16.6 & 16.5 & 15.9 & 15.5 & 15.6 & 15.1 & 14.6 & 14.8 & 15.5 & 15.7 & 15.7 \\
\hline Min & 16.6 & 16.5 & 16.0 & 15.5 & 15.5 & 15.1 & 14.7 & 14.6 & 14.6 & 14.8 & 15.3 & 15.7 \\
\hline
\end{tabular}




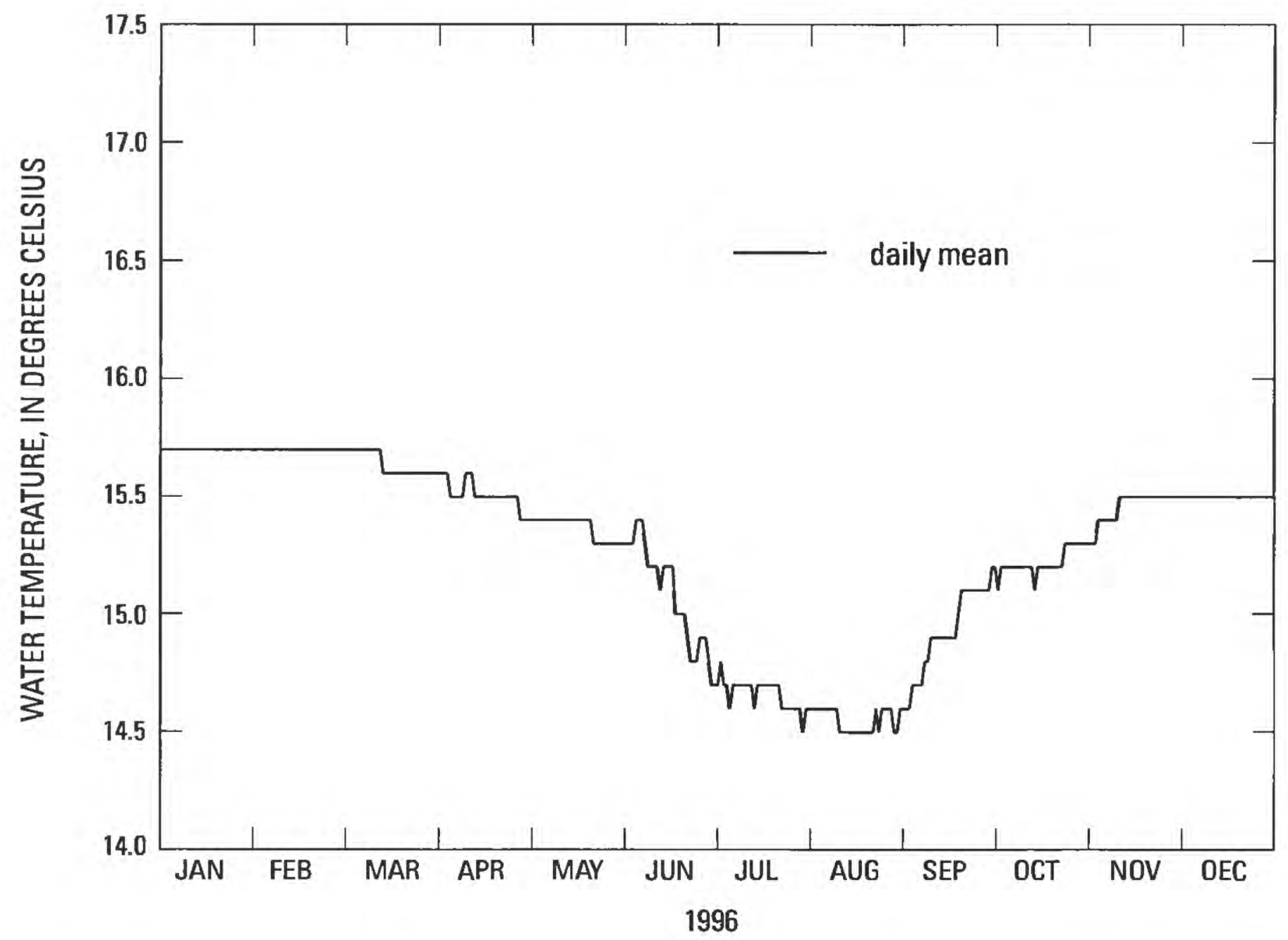

Figure 30. (C) Daily mean water temperature, Fish Hatchery Spring CD (FHCD-T), 1996, Long Valley Caldera, Mono County, California. 
Table 25. Daily mean water temperature, Fish Hatchery Spring CD (FHCD-T), 1996, Long Valley Caldera, California

[Values are in degrees Celsius. -, no data: Max, maximum: Min, minimum]

\begin{tabular}{|c|c|c|c|c|c|c|c|c|c|c|c|c|}
\hline Day & Jan & Feb & Mar & Apr & May & Jun & Jul & Aug & Sep & Oct & Nov & Dec \\
\hline 1 & 15.7 & 15.7 & 15.7 & 15.6 & 15.4 & 15.3 & 14.7 & 14.6 & 14.6 & 15.1 & 15.3 & 15.5 \\
\hline 2 & 15.7 & 15.7 & 15.7 & 15.6 & 15.4 & 15.3 & 14.8 & 14.6 & 14.6 & 15.2 & 15.3 & 15.5 \\
\hline 3 & 15.7 & 15.7 & 15.7 & 15.6 & 15.4 & 15.3 & 14.7 & 14.6 & 14.7 & 15.2 & 15.4 & 15.5 \\
\hline 4 & 15.7 & 15.7 & 15.7 & 15.5 & 15.4 & 15.4 & 14.7 & 14.6 & 14.7 & 15.2 & 15.4 & 15.5 \\
\hline 5 & 15.7 & 15.7 & 15.7 & 15.5 & 15.4 & 15.4 & 14.6 & 14.6 & 14.7 & 15.2 & 15.4 & 15.5 \\
\hline 6 & 15.7 & 15.7 & 15.7 & 15.5 & 15.4 & 15.4 & 14.7 & 14.6 & 14.7 & 15.2 & 15.4 & 15.5 \\
\hline 7 & 15.7 & 15.7 & 15.7 & 15.5 & 15.4 & 15.3 & 14.7 & 14.6 & 14.8 & 15.2 & 15.4 & 15.5 \\
\hline 8 & 15.7 & 15.7 & 15.7 & 15.5 & 15.4 & 15.2 & 14.7 & 14.6 & 14.8 & 15.2 & 15.4 & 15.5 \\
\hline 9 & 15.7 & 15.7 & 15.7 & 15.6 & 15.4 & 15.2 & 14.7 & 14.6 & 14.9 & 15.2 & 15.4 & 15.5 \\
\hline 10 & 15.7 & 15.7 & 15.7 & 15.6 & 15.4 & 15.2 & 14.7 & 14.5 & 14.9 & 15.2 & 15.5 & 15.5 \\
\hline 11 & 15.7 & 15.7 & 15.7 & 15.6 & 15.4 & 15.2 & 14.7 & 14.5 & 14.9 & 15.2 & 15.5 & 15.5 \\
\hline 12 & 15.7 & 15.7 & 15.7 & 15.5 & 15.4 & 15.1 & 14.7 & 14.5 & 14.9 & 15.2 & 15.5 & 15.5 \\
\hline 13 & 15.7 & 15.7 & 15.6 & 15.5 & 15.4 & 15.2 & 14.6 & 14.5 & 14.9 & 15.1 & 15.5 & 15.5 \\
\hline 14 & 15.7 & 15.7 & 15.6 & 15.5 & 15.4 & 15.2 & 14.7 & 14.5 & 14.9 & 15.2 & 15.5 & 15.5 \\
\hline 15 & 15.7 & 15.7 & 15.6 & 15.5 & 15.4 & 15.2 & 14.7 & 14.5 & 14.9 & 15.2 & 15.5 & 15.5 \\
\hline 16 & 15.7 & 15.7 & 15.6 & 15.5 & 15.4 & 15.2 & 14.7 & 14.5 & 14.9 & 15.2 & 15.5 & 15.5 \\
\hline 17 & 15.7 & 15.7 & 15.6 & 15.5 & 15.4 & 15.0 & 14.7 & 14.5 & 14.9 & 15.2 & 15.5 & 15.5 \\
\hline 18 & 15.7 & 15.7 & 15.6 & 15.5 & 15.4 & 15.0 & 14.7 & 14.5 & 15.0 & 15.2 & 15.5 & 15.5 \\
\hline 19 & 15.7 & 15.7 & 15.6 & 15.5 & 15.4 & 15.0 & 14.7 & 14.5 & 15.1 & 15.2 & 15.5 & 15.5 \\
\hline 20 & 15.7 & 15.7 & 15.6 & 15.5 & 15.4 & 15.0 & 14.7 & 14.5 & 15.1 & 15.2 & 15.5 & 15.5 \\
\hline 21 & 15.7 & 15.7 & 15.6 & 15.5 & 15.3 & 14.9 & 14.7 & 14.5 & 15.1 & 15.2 & 15.5 & 15.5 \\
\hline 22 & 15.7 & 15.7 & 15.6 & 15.5 & 15.3 & 14.8 & 14.6 & 14.6 & 15.1 & 15.2 & 15.5 & 15.5 \\
\hline 23 & 15.7 & 15.7 & 15.6 & 15.5 & 15.3 & 14.8 & 14.6 & 14.5 & 15.1 & 15.3 & 15.5 & 15.5 \\
\hline 24 & 15.7 & 15.7 & 15.6 & 15.5 & 15.3 & 14.8 & 14.6 & 14.6 & 15.1 & 15.3 & 15.5 & 15.5 \\
\hline 25 & 15.7 & 15.7 & 15.6 & 15.5 & 15.3 & 14.9 & 14.6 & 14.6 & 15.1 & 15.3 & 15.5 & 15.5 \\
\hline 26 & 15.7 & 15.7 & 15.6 & 15.5 & 15.3 & 14.9 & 14.6 & 14.6 & 15.1 & 15.3 & 15.5 & 15.5 \\
\hline 27 & 15.7 & 15.7 & 15.6 & 15.4 & 15.3 & 14.9 & 14.6 & 14.6 & 15.1 & 15.3 & 15.5 & 15.5 \\
\hline 28 & 15.7 & 15.7 & 15.6 & 15.4 & 15.3 & 14.8 & 14.6 & 14.5 & 15.1 & 15.3 & 15.5 & 15.5 \\
\hline 29 & 15.7 & 15.7 & 15.6 & 15.4 & 15.3 & 14.7 & 14.5 & 14.5 & 15.2 & 15.3 & 15.5 & 15.5 \\
\hline 30 & 15.7 & - & 15.6 & 15.4 & 15.3 & 14.7 & 14.6 & 14.6 & 15.2 & 15.3 & 15.5 & 15.5 \\
\hline 31 & 15.7 & - & 15.6 & - & 15.3 & - & 14.6 & 14.6 & - & 15.3 & - & 15.5 \\
\hline Mean & 15.7 & 15.7 & 15.6 & 15.5 & 15.4 & 15.1 & 14.7 & 14.6 & 14.9 & 15.2 & 15.5 & 15.5 \\
\hline Max & 15.7 & 15.7 & 15.7 & 15.6 & 15.4 & 15.4 & 14.8 & 14.6 & 15.2 & 15.3 & 15.5 & 15.5 \\
\hline Min & 15.7 & 15.7 & 15.6 & 15.4 & 15.3 & 14.7 & 14.5 & 14.5 & 14.6 & 15.1 & 15.3 & 15.5 \\
\hline
\end{tabular}




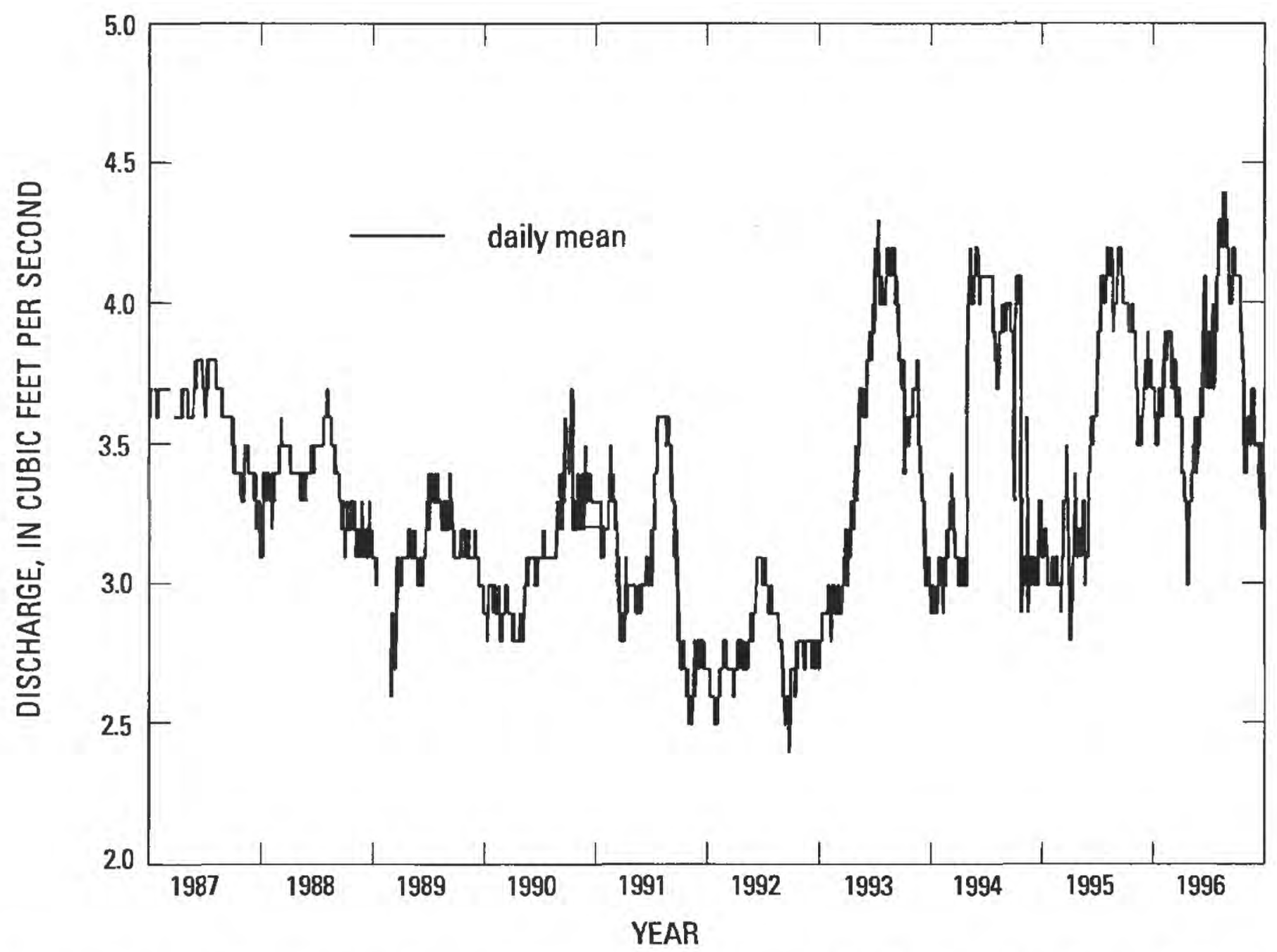

Figure 31. Daily mean discharge, Fish Hatchery Spring 23 (FH23-0), 1987-96, Long Valley Caldera, Mono County, California.

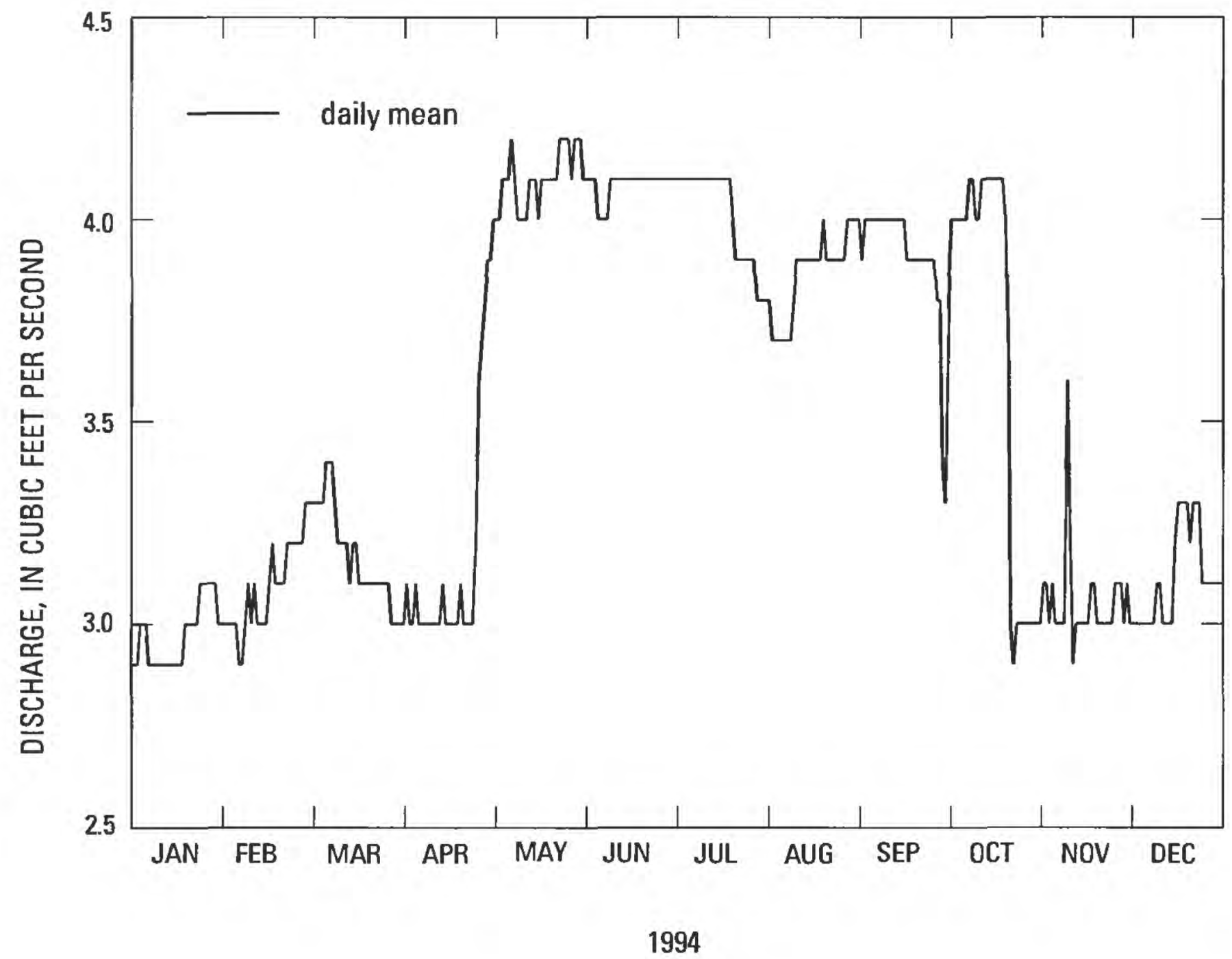

Figure 32. (A) Daily mean discharge, Fish Hatchery Spring 23 (FH23-0), 1994, Long Valley Caldera, Mono County, California. 
Table 26. Daily mean discharge, Fish Hatchery Spring 23 (FH23-0), 1994, Long Valley Caldera, California

[Values are in cubic feet per second. —, no data: Max, maximum: Min, minimum]

\begin{tabular}{|c|c|c|c|c|c|c|c|c|c|c|c|c|}
\hline Day & Jan & Feb & Mar & Apr & May & Jun & Jul & Aug & Sep & Oct & Nov & Dec \\
\hline 1 & 2.9 & 3.0 & 3.3 & 3.0 & 4.0 & 4.1 & 4.1 & 3.8 & 3.9 & 4.0 & 3.1 & 3.0 \\
\hline 2 & 2.9 & 3.0 & 3.3 & 3.1 & 4.0 & 4.1 & 4.1 & 3.7 & 4.0 & 4.0 & 3.1 & 3.0 \\
\hline 3 & 3.0 & 3.0 & 3.3 & 3.0 & 4.0 & 4.1 & 4.1 & 3.7 & 4.0 & 4.0 & 3.0 & 3.0 \\
\hline 4 & 3.0 & 3.0 & 3.3 & 3.0 & 4.1 & 4.1 & 4.1 & 3.7 & 4.0 & 4.0 & 3.1 & 3.0 \\
\hline 5 & 3.0 & 2.9 & 3.3 & 3.1 & 4.1 & 4.0 & 4.1 & 3.7 & 4.0 & 4.0 & 3.0 & 3.0 \\
\hline 6 & 2.9 & 2.9 & 3.4 & 3.0 & 4.1 & 4.0 & 4.1 & 3.7 & 4.0 & 4.0 & 3.0 & 3.0 \\
\hline 7 & 2.9 & 3.0 & 3.4 & 3.0 & 4.2 & 4.0 & 4.1 & 3.7 & 4.0 & 4.1 & 3.0 & 3.0 \\
\hline 8 & 2.9 & 3.1 & 3.4 & 3.0 & 4.1 & 4.0 & 4.1 & 3.7 & 4.0 & 4.1 & 3.0 & 3.0 \\
\hline 9 & 2.9 & 3.0 & 3.3 & 3.0 & 4.0 & 4.1 & 4.1 & 3.8 & 4.0 & 4.0 & 3.6 & 3.1 \\
\hline 10 & 2.9 & 3.1 & 3.2 & 3.0 & 4.0 & 4.1 & 4.1 & 3.9 & 4.0 & 4.0 & 3.2 & 3.1 \\
\hline 11 & 2.9 & 3.0 & 3.2 & 3.0 & 4.0 & 4.1 & 4.1 & 3.9 & 4.0 & 4.1 & 2.9 & 3.0 \\
\hline 12 & 2.9 & 3.0 & 3.2 & 3.0 & 4.0 & 4.1 & 4.1 & 3.9 & 4.0 & 4.1 & 3.0 & 3.0 \\
\hline 13 & 2.9 & 3.0 & 3.2 & 3.0 & 4.1 & 4.1 & 4.1 & 3.9 & 4.0 & 4.1 & 3.0 & 3.0 \\
\hline 14 & 2.9 & 3.0 & 3.1 & 3.1 & 4.1 & 4.1 & 4.1 & 3.9 & 4.0 & 4.1 & 3.0 & 3.0 \\
\hline 15 & 2.9 & 3.1 & 3.2 & 3.0 & 4.1 & 4.1 & 4.1 & 3.9 & 4.0 & 4.1 & 3.0 & 3.2 \\
\hline 16 & 2.9 & 3.2 & 3.2 & 3.0 & 4.0 & 4.1 & 4.1 & 3.9 & 3.9 & 4.1 & 3.0 & 3.3 \\
\hline 17 & 2.9 & 3.1 & 3.1 & 3.0 & 4.1 & 4.1 & 4.1 & 3.9 & 3.9 & 4.1 & 3.1 & 3.3 \\
\hline 18 & 3.0 & 3.1 & 3.1 & 3.0 & 4.1 & 4.1 & 4.1 & 3.9 & 3.9 & 4.1 & 3.1 & 3.3 \\
\hline 19 & 3.0 & 3.1 & 3.1 & 3.0 & 4.1 & 4.1 & 4.1 & 4.0 & 3.9 & 4.0 & 3.0 & 3.3 \\
\hline 20 & 3.0 & 3.1 & 3.1 & 3.1 & 4.1 & 4.1 & 4.0 & 3.9 & 3.9 & 3.7 & 3.0 & 3.2 \\
\hline 21 & 3.0 & 3.2 & 3.1 & 3.0 & 4.1 & 4.1 & 3.9 & 3.9 & 3.9 & 3.0 & 3.0 & 3.3 \\
\hline 22 & 3.0 & 3.2 & 3.1 & 3.0 & 4.1 & 4.1 & 3.9 & 3.9 & 3.9 & 2.9 & 3.0 & 3.3 \\
\hline 23 & 3.1 & 3.2 & 3.1 & 3.0 & 4.2 & 4.1 & 3.9 & 3.9 & 3.9 & 3.0 & 3.0 & 3.3 \\
\hline 24 & 3.1 & 3.2 & 3.1 & 3.0 & 4.2 & 4.1 & 3.9 & 3.9 & 3.9 & 3.0 & 3.0 & 3.1 \\
\hline 25 & 3.1 & 3.2 & 3.1 & 3.2 & 4.2 & 4.1 & 3.9 & 3.9 & 3.9 & 3.0 & 3.1 & 3.1 \\
\hline 26 & 3.1 & 3.2 & 3.1 & 3.6 & 4.2 & 4.1 & 3.9 & 3.9 & 3.8 & 3.0 & 3.1 & 3.1 \\
\hline 27 & 3.1 & 3.3 & 3.1 & 3.7 & 4.1 & 4.1 & 3.9 & 4.0 & 3.8 & 3.0 & 3.1 & 3.1 \\
\hline 28 & 3.1 & 3.3 & 3.0 & 3.8 & 4.2 & 4.1 & 3.8 & 4.0 & 3.4 & 3.0 & 3.0 & 3.1 \\
\hline 29 & 3.0 & - & 3.0 & 3.9 & 4.2 & 4.1 & 3.8 & 4.0 & 3.3 & 3.0 & 3.1 & 3.1 \\
\hline 30 & 3.0 & - & 3.0 & 3.9 & 4.2 & 4.1 & 3.8 & 4.0 & 3.8 & 3.0 & 3.0 & 3.1 \\
\hline 31 & 3.0 & - & 3.0 & - & 4.1 & - & 3.8 & 4.0 & - & 3.0 & - & 3.1 \\
\hline Mean & 2.97 & 3.09 & 3.17 & 3.15 & 4.10 & 4.09 & 4.01 & 3.87 & 3.90 & 3.66 & 3.05 & 3.11 \\
\hline $\operatorname{Max}$ & 3.1 & 3.3 & 3.4 & 3.9 & 4.2 & 4.1 & 4.1 & 4.0 & 4.0 & 4.1 & 3.6 & 3.3 \\
\hline Min & 2.9 & 2.9 & 3.0 & 3.0 & 4.0 & 4.0 & 3.8 & 3.7 & 3.3 & 2.9 & 2.9 & 3.0 \\
\hline
\end{tabular}




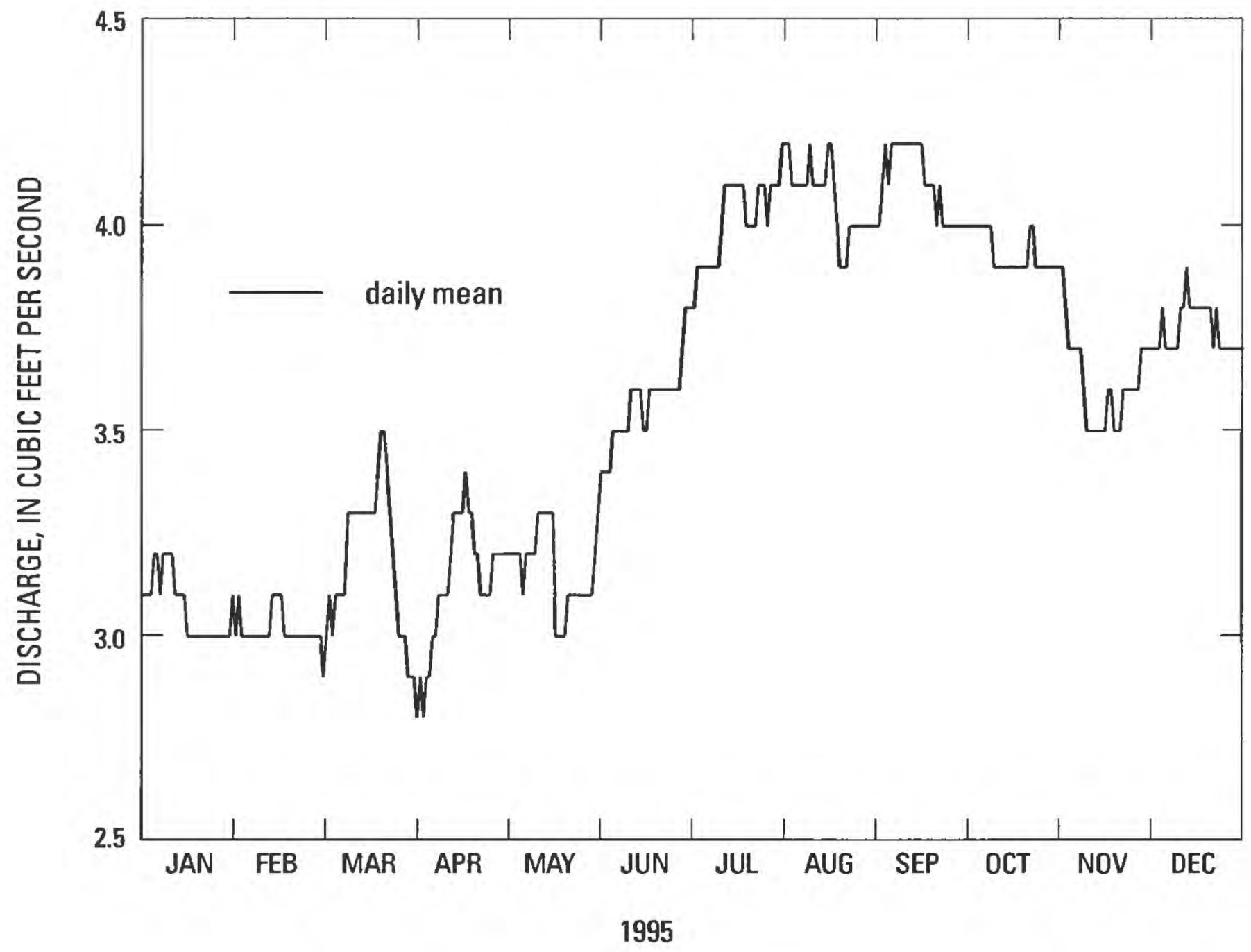

Figure 32. (B) Daily mean discharge, Fish Hatchery Spring 23 (FH23-Q), 1995, Long Valley Caldera, Mono County, California. 
Table 27. Daily mean discharge, Fish Hatchery Spring 23 (FH23-0), 1995, Long Valley Caldera, California

[Values are in cubic feet per second. —, no data: Max, maximum; Min, minimum]

\begin{tabular}{|c|c|c|c|c|c|c|c|c|c|c|c|c|}
\hline Day & Jan & Feb & Mar & Apr & May & Jun & Jul & Aug & Sep & Oct & Nov & Dec \\
\hline 1 & 3.1 & 3.1 & 2.9 & 2.8 & 3.2 & 3.4 & 3.8 & 4.2 & 4.0 & 4.0 & 3.9 & 3.7 \\
\hline 2 & 3.1 & 3.0 & 3.0 & 2.9 & 3.2 & 3.4 & 3.8 & 4.2 & 4.1 & 4.0 & 3.8 & 3.7 \\
\hline 3 & 3.1 & 3.0 & 3.1 & 2.8 & 3.2 & 3.4 & 3.9 & 4.1 & 4.2 & 4.0 & 3.7 & 3.7 \\
\hline 4 & 3.2 & 3.0 & 3.0 & 2.9 & 3.2 & 3.4 & 3.9 & 4.1 & 4.1 & 4.0 & 3.7 & 3.8 \\
\hline 5 & 3.2 & 3.0 & 3.1 & 2.9 & 3.2 & 3.5 & 3.9 & 4.1 & 4.2 & 4.0 & 3.7 & 3.7 \\
\hline 6 & 3.1 & 3.0 & 3.1 & 3.0 & 3.1 & 3.5 & 3.9 & 4.1 & 4.2 & 4.0 & 3.7 & 3.7 \\
\hline 7 & 3.2 & 3.0 & 3.1 & 3.0 & 3.2 & 3.5 & 3.9 & 4.1 & 4.2 & 4.0 & 3.7 & 3.7 \\
\hline 8 & 3.2 & 3.0 & 3.1 & 3.1 & 3.2 & 3.5 & 3.9 & 4.1 & 4.2 & 4.0 & 3.6 & 3.7 \\
\hline 9 & 3.2 & 3.0 & 3.3 & 3.1 & 3.2 & 3.5 & 3.9 & 4.2 & 4.2 & 3.9 & 3.5 & 3.7 \\
\hline 10 & 3.2 & 3.0 & 3.3 & 3.1 & 3.2 & 3.5 & 3.9 & 4.1 & 4.2 & 3.9 & 3.5 & 3.8 \\
\hline 11 & 3.1 & 3.0 & 3.3 & 3.1 & 3.3 & 3.6 & 4.0 & 4.1 & 4.2 & 3.9 & 3.5 & 3.8 \\
\hline 12 & 3.1 & 3.1 & 3.3 & 3.2 & 3.3 & 3.6 & 4.1 & 4.1 & 4.2 & 3.9 & 3.5 & 3.9 \\
\hline 13 & 3.1 & 3.1 & 3.3 & 3.3 & 3.3 & 3.6 & 4.1 & 4.1 & 4.2 & 3.9 & 3.5 & 3.8 \\
\hline 14 & 3.1 & 3.1 & 3.3 & 3.3 & 3.3 & 3.6 & 4.1 & 4.1 & 4.2 & 3.9 & 3.5 & 3.8 \\
\hline 15 & 3.0 & 3.1 & 3.3 & 3.3 & 3.3 & 3.5 & 4.1 & 4.2 & 4.2 & 3.9 & 3.5 & 3.8 \\
\hline 16 & 3.0 & 3.0 & 3.3 & 3.3 & 3.3 & 3.5 & 4.1 & 4.2 & 4.1 & 3.9 & 3.6 & 3.8 \\
\hline 17 & 3.0 & 3.0 & 3.3 & 3.4 & 3.0 & 3.6 & 4.1 & 4.1 & 4.1 & 3.9 & 3.6 & 3.8 \\
\hline 18 & 3.0 & 3.0 & 3.3 & 3.3 & 3.0 & 3.6 & 4.1 & 4.0 & 4.1 & 3.9 & 3.5 & 3.8 \\
\hline 19 & 3.0 & 3.0 & 3.4 & 3.3 & 3.0 & 3.6 & 4.0 & 3.9 & 4.1 & 3.9 & 3.5 & 3.8 \\
\hline 20 & 3.0 & 3.0 & 3.5 & 3.2 & 3.0 & 3.6 & 4.0 & 3.9 & 4.0 & 3.9 & 3.5 & 3.8 \\
\hline 21 & 3.0 & 3.0 & 3.5 & 3.2 & 3.1 & 3.6 & 4.0 & 3.9 & 4.1 & 4.0 & 3.6 & 3.7 \\
\hline 22 & 3.0 & 3.0 & 3.4 & 3.1 & 3.1 & 3.6 & 4.0 & 4.0 & 4.0 & 4.0 & 3.6 & 3.8 \\
\hline 23 & 3.0 & 3.0 & 3.3 & 3.1 & 3.1 & 3.6 & 4.1 & 4.0 & 4.0 & 3.9 & 3.6 & 3.7 \\
\hline 24 & 3.0 & 3.0 & 3.2 & 3.1 & 3.1 & 3.6 & 4.1 & 4.0 & 4.0 & 3.9 & 3.6 & 3.7 \\
\hline 25 & 3.0 & 3.0 & 3.1 & 3.1 & 3.1 & 3.6 & 4.1 & 4.0 & 4.0 & 3.9 & 3.6 & 3.7 \\
\hline 26 & 3.0 & 3.0 & 3.0 & 3.2 & 3.1 & 3.6 & 4.0 & 4.0 & 4.0 & 3.9 & 3.6 & 3.7 \\
\hline 27 & 3.0 & 3.0 & 3.0 & 3.2 & 3.1 & 3.6 & 4.1 & 4.0 & 4.0 & 3.9 & 3.7 & 3.7 \\
\hline 28 & 3.0 & 3.0 & 3.0 & 3.2 & 3.1 & 3.7 & 4.1 & 4.0 & 4.0 & 3.9 & 3.7 & 3.7 \\
\hline 29 & 3.0 & - & 2.9 & 3.2 & 3.1 & 3.8 & 4.1 & 4.0 & 4.0 & 3.9 & 3.7 & 3.7 \\
\hline 30 & 3.1 & - & 2.9 & 3.2 & 3.2 & 3.8 & 4.1 & 4.0 & 4.0 & 3.9 & 3.7 & 3.7 \\
\hline 31 & 3.0 & - & 2.9 & - & 3.3 & - & 4.2 & 4.0 & - & 3.9 & - & 3.7 \\
\hline Mean & 3.07 & 3.02 & 3.18 & 3.13 & 3.16 & 3.56 & 4.01 & 4.06 & 4.10 & 3.93 & 3.61 & 3.75 \\
\hline Max & 3.2 & 3.1 & 3.5 & 3.4 & 3.3 & 3.8 & 4.2 & 4.2 & 4.2 & 4.0 & 3.9 & 3.9 \\
\hline Min & 3.0 & 3.0 & 2.9 & 2.8 & 3.0 & 3.4 & 3.8 & 3.9 & 4.0 & 3.9 & 3.5 & 3.7 \\
\hline
\end{tabular}




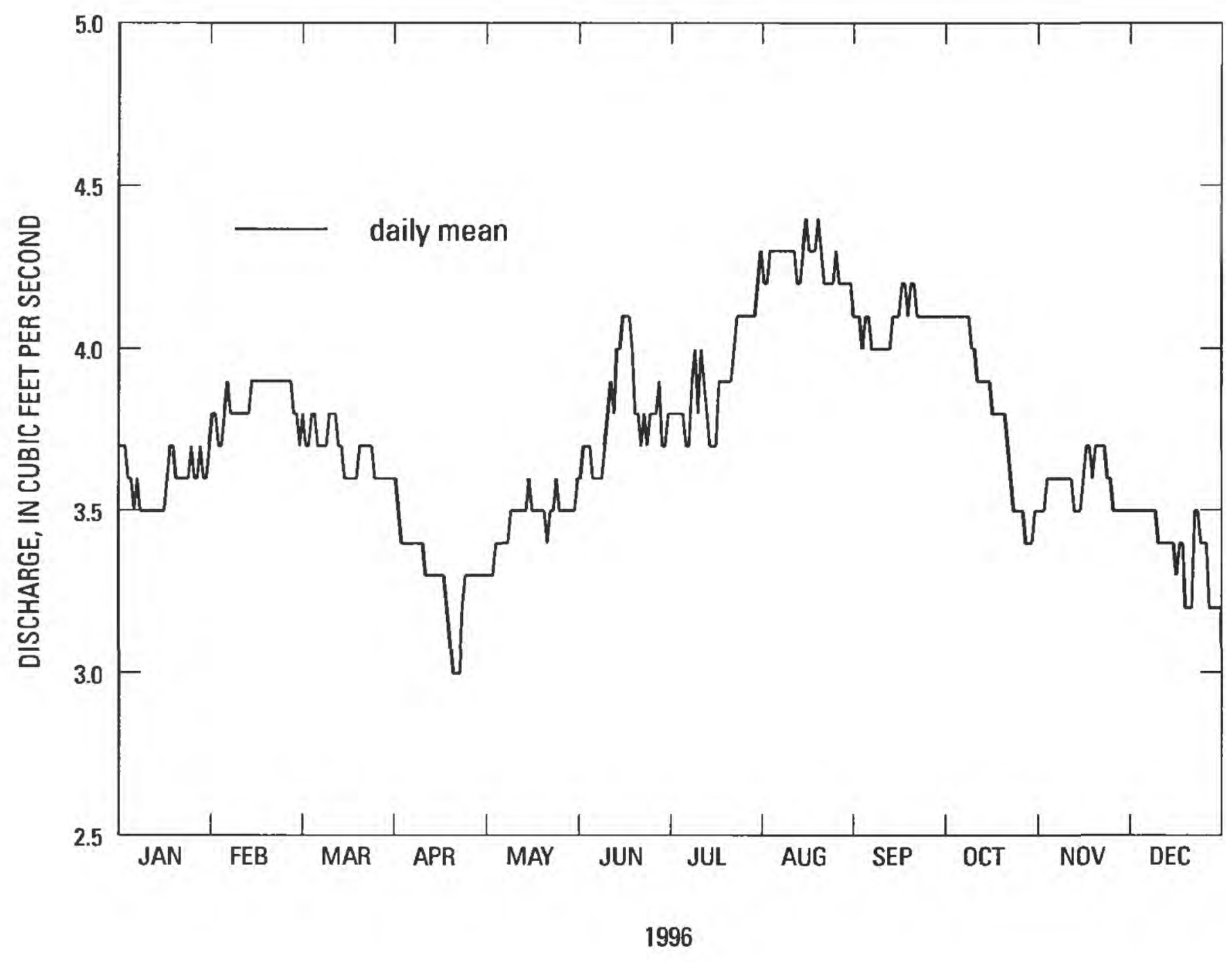

Figure 32. (C) Daily mean discharge, Fish Hatchery Spring 23 (FH23-0), 1996, Long Valley Caldera, Mono County, California. 
Table 28. Daily mean discharge, Fish Hatchery Spring 23 (FH23-0), 1996, Long Valley Caldera, California

[Values are in cubic feet per second. -, no data; Max, maximum; Min, minimum]

\begin{tabular}{|c|c|c|c|c|c|c|c|c|c|c|c|c|}
\hline Day & Jan & Feb & Mar & Apr & May & Jun & Jul & Aug & Sep & Oct & Nov & Dec \\
\hline 1 & 3.7 & 3.8 & 3.8 & 3.6 & 3.3 & 3.6 & 3.8 & 4.2 & 4.1 & 4.1 & 3.5 & 3.5 \\
\hline 2 & 3.7 & 3.7 & 3.7 & 3.5 & 3.3 & 3.7 & 3.8 & 4.2 & 4.1 & 4.1 & 3.5 & 3.5 \\
\hline 3 & 3.6 & 3.7 & 3.7 & 3.4 & 3.3 & 3.7 & 3.8 & 4.3 & 4.0 & 4.1 & 3.6 & 3.5 \\
\hline 4 & 3.6 & 3.8 & 3.8 & 3.4 & 3.4 & 3.7 & 3.8 & 4.3 & 4.1 & 4.1 & 3.6 & 3.5 \\
\hline 5 & 3.5 & 3.9 & 3.8 & 3.4 & 3.4 & 3.6 & 3.8 & 4.3 & 4.1 & 4.1 & 3.6 & 3.5 \\
\hline 6 & 3.6 & 3.8 & 3.7 & 3.4 & 3.4 & 3.6 & 3.7 & 4.3 & 4.0 & 4.1 & 3.6 & 3.5 \\
\hline 7 & 3.5 & 3.8 & 3.7 & 3.4 & 3.4 & 3.6 & 3.7 & 4.3 & 4.0 & 4.1 & 3.6 & 3.5 \\
\hline 8 & 3.5 & 3.8 & 3.7 & 3.4 & 3.4 & 3.6 & 3.9 & 4.3 & 4.0 & 4.1 & 3.6 & 3.5 \\
\hline 9 & 3.5 & 3.8 & 3.7 & 3.4 & 3.5 & 3.7 & 4.0 & 4.3 & 4.0 & 4.0 & 3.6 & 3.5 \\
\hline 10 & 3.5 & 3.8 & 3.8 & 3.4 & 3.5 & 3.8 & 3.8 & 4.3 & 4.0 & 4.0 & 3.6 & 3.4 \\
\hline 11 & 3.5 & 3.8 & 3.8 & 3.3 & 3.5 & 3.9 & 4.0 & 4.3 & 4.0 & 3.9 & 3.6 & 3.4 \\
\hline 12 & 3.5 & 3.8 & 3.8 & 3.3 & 3.5 & 3.8 & 3.9 & 4.2 & 4.0 & 3.9 & 3.5 & 3.4 \\
\hline 13 & 3.5 & 3.9 & 3.7 & 3.3 & 3.5 & 4.0 & 3.8 & 4.2 & 4.1 & 3.9 & 3.5 & 3.4 \\
\hline 14 & 3.5 & 3.9 & 3.7 & 3.3 & 3.5 & 4.0 & 3.7 & 4.3 & 4.1 & 3.9 & 3.5 & 3.4 \\
\hline 15 & 3.5 & 3.9 & 3.6 & 3.3 & 3.6 & 4.1 & 3.7 & 4.4 & 4.1 & 3.9 & 3.6 & 3.4 \\
\hline 16 & 3.6 & 3.9 & 3.6 & 3.3 & 3.5 & 4.1 & 3.7 & 4.3 & 4.2 & 3.8 & 3.7 & 3.3 \\
\hline 17 & 3.7 & 3.9 & 3.6 & 3.3 & 3.5 & 4.1 & 3.9 & 4.3 & 4.2 & 3.8 & 3.7 & 3.4 \\
\hline 18 & 3.7 & 3.9 & 3.6 & 3.2 & 3.5 & 4.0 & 3.9 & 4.3 & 4.1 & 3.8 & 3.6 & 3.4 \\
\hline 19 & 3.6 & 3.9 & 3.6 & 3.1 & 3.5 & 3.8 & 3.9 & 4.4 & 4.2 & 3.8 & 3.7 & 3.2 \\
\hline 20 & 3.6 & 3.9 & 3.7 & 3.0 & 3.5 & 3.8 & 3.9 & 4.3 & 4.2 & 3.8 & 3.7 & 3.2 \\
\hline 21 & 3.6 & 3.9 & 3.7 & 3.0 & 3.4 & 3.7 & 3.9 & 4.2 & 4.1 & 3.7 & 3.7 & 3.2 \\
\hline 22 & 3.6 & 3.9 & 3.7 & 3.0 & 3.5 & 3.8 & 4.0 & 4.2 & 4.1 & 3.6 & 3.7 & 3.5 \\
\hline 23 & 3.6 & 3.9 & 3.7 & 3.2 & 3.5 & 3.7 & 4.1 & 4.2 & 4.1 & 3.5 & 3.6 & 3.5 \\
\hline 24 & 3.7 & 3.9 & 3.7 & 3.3 & 3.6 & 3.8 & 4.1 & 4.2 & 4.1 & 3.5 & 3.6 & 3.4 \\
\hline 25 & 3.6 & 3.9 & 3.6 & 3.3 & 3.5 & 3.8 & 4.1 & 4.3 & 4.1 & 3.5 & 3.5 & 3.4 \\
\hline 26 & 3.6 & 3.9 & 3.6 & 3.3 & 3.5 & 3.8 & 4.1 & 4.2 & 4.1 & 3.5 & 3.5 & 3.4 \\
\hline 27 & 3.7 & 3.8 & 3.6 & 3.3 & 3.5 & 3.9 & 4.1 & 4.2 & 4.1 & 3.4 & 3.5 & 3.2 \\
\hline 28 & 3.6 & 3.8 & 3.6 & 3.3 & 3.5 & 3.7 & 4.1 & 4.2 & 4.1 & 3.4 & 3.5 & 3.2 \\
\hline 29 & 3.6 & 3.7 & 3.6 & 3.3 & 3.5 & 3.7 & 4.1 & 4.2 & 4.1 & 3.4 & 3.5 & 3.2 \\
\hline 30 & 3.7 & - & 3.6 & 3.3 & 3.5 & 3.8 & 4.2 & 4.2 & 4.1 & 3.5 & 3.5 & 3.2 \\
\hline 31 & 3.8 & - & 3.6 & - & 3.6 & - & 4.3 & 4.1 & - & 3.5 & - & 3.2 \\
\hline Mean & 3.60 & 3.84 & 3.68 & 3.30 & 3.47 & 3.80 & 3.92 & 4.26 & 4.09 & 3.80 & 3.58 & 3.38 \\
\hline Max & 3.8 & 3.9 & 3.8 & 3.6 & 3.6 & 4.1 & 4.3 & 4.4 & 4.2 & 4.1 & 3.7 & 3.5 \\
\hline Min & 3.5 & 3.7 & 3.6 & 3.0 & 3.3 & 3.6 & 3.7 & 4.1 & 4.0 & 3.4 & 3.5 & 3.2 \\
\hline
\end{tabular}




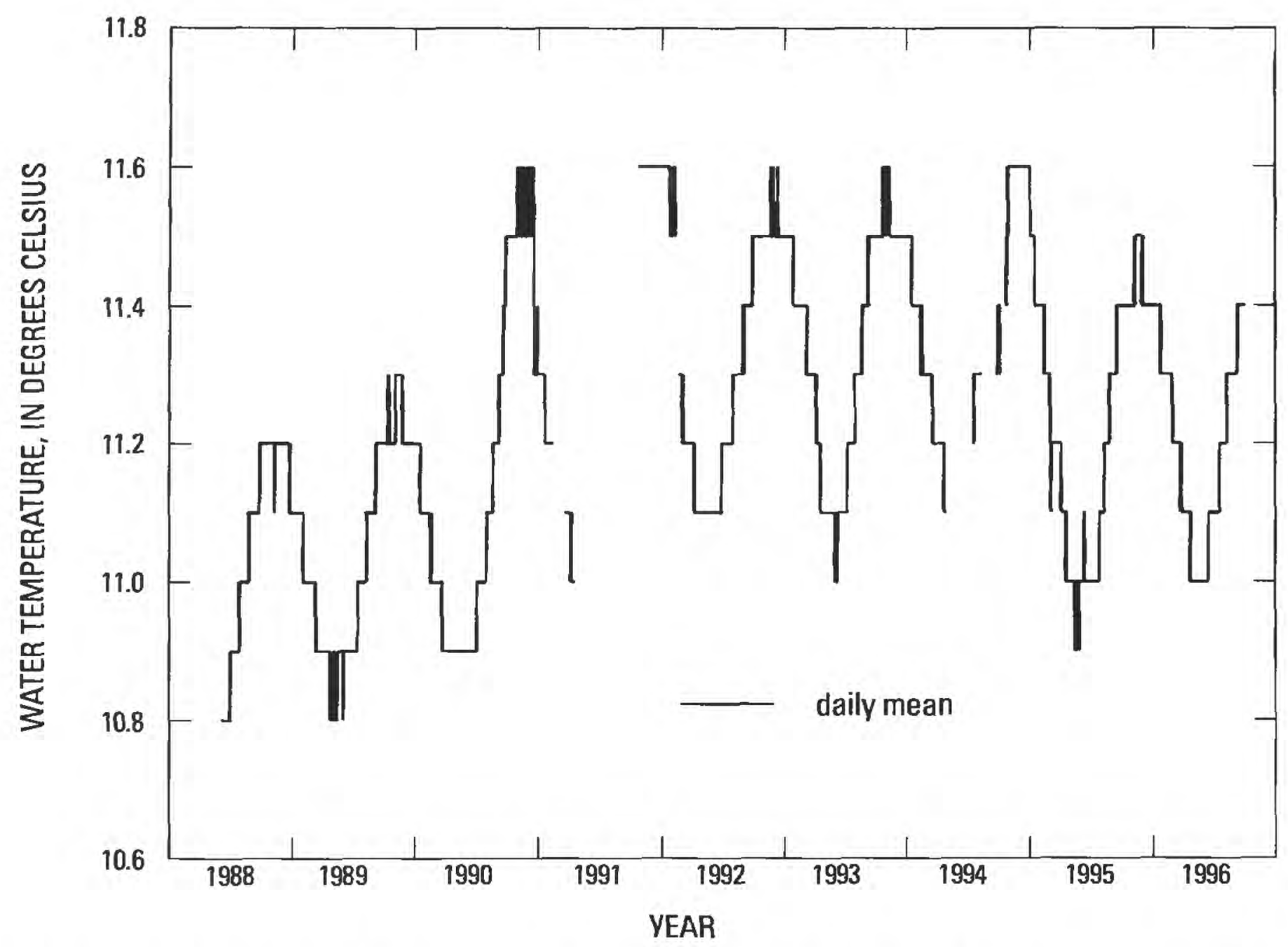

Figure 33. Daily mean water temperature, Fish Hatchery Spring 23 (FH23-T), 1988-96, Long Valley Caldera, Mono County, California.

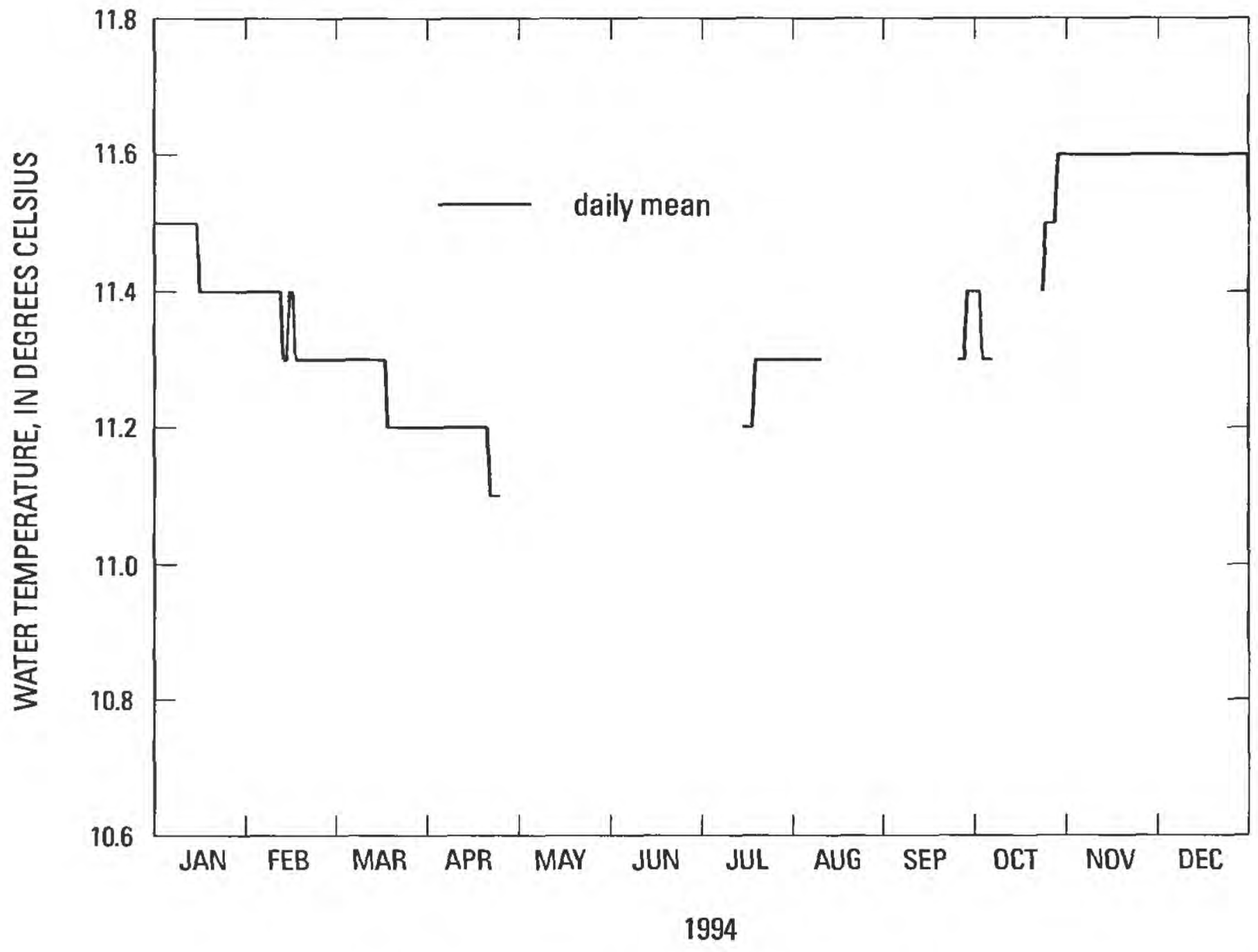

Figure 34. (A) Daily mean water temperature, Fish Hatchery Spring 23 (FH23-T), 1994, Long Valley Caldera, Mono County, California. 
Table 29. Daily mean water temperature, Fish Hatchery Spring 23 (FH23-T), 1994, Long Valley Caldera, California

[Values are in degrees Celsius. -, no data; Max, maximum; Min, minimum]

\begin{tabular}{|c|c|c|c|c|c|c|c|c|c|c|c|c|}
\hline Day & Jan & Feb & Mar & Apr & May & Jun & Jul & Aug & Sep & Oct & Nov & Dec \\
\hline 1 & 11.5 & 11.4 & 11.3 & 11.2 & - & - & - & 11.3 & - & 11.4 & 11.6 & 11.6 \\
\hline 2 & 11.5 & 11.4 & 11.3 & 11.2 & - & - & - & 11.3 & - & 11.4 & 11.6 & 11.6 \\
\hline 3 & 11.5 & 11.4 & 11.3 & 11.2 & - & - & - & 11.3 & - & 11.3 & 11.6 & 11.6 \\
\hline 4 & 11.5 & 11.4 & 11.3 & 11.2 & - & - & - & 11.3 & - & 11.3 & 11.6 & 11.6 \\
\hline 5 & 11.5 & 11.4 & 11.3 & 11.2 & - & - & - & 11.3 & - & 11.3 & 11.6 & 11.6 \\
\hline 6 & 11.5 & 11.4 & 11.3 & 11.2 & - & - & - & 11.3 & - & 11.3 & 11.6 & 11.6 \\
\hline 7 & 11.5 & 11.4 & 11.3 & 11.2 & - & - & - & 11.3 & - & - & 11.6 & 11.6 \\
\hline 8 & 11.5 & 11.4 & 11.3 & 11.2 & - & - & - & 11.3 & - & - & 11.6 & 11.6 \\
\hline 9 & 11.5 & 11.4 & 11.3 & 11.2 & - & - & - & 11.3 & - & - & 11.6 & 11.6 \\
\hline 10 & 11.5 & 11.4 & 11.3 & 11.2 & - & - & - & 11.3 & - & - & 11.6 & 11.6 \\
\hline 11 & 11.5 & 11.4 & 11.3 & 11.2 & - & - & - & - & - & - & 11.6 & 11.6 \\
\hline 12 & 11.5 & 11.3 & 11.3 & 11.2 & - & - & - & - & - & - & 11.6 & 11.6 \\
\hline 13 & 11.5 & 11.3 & 11.3 & 11.2 & - & - & - & - & - & - & 11.6 & 11.6 \\
\hline 14 & 11.5 & 11.4 & 11.3 & 11.2 & - & - & - & - & - & - & 11.6 & 11.6 \\
\hline 15 & 11.4 & 11.4 & 11.3 & 11.2 & - & - & 11.2 & - & - & - & 11.6 & 11.6 \\
\hline 16 & 11.4 & 11.3 & 11.3 & 11.2 & - & - & 11.2 & - & - & - & 11.6 & 11.6 \\
\hline 17 & 11.4 & 11.3 & 11.3 & 11.2 & - & - & 11.2 & - & - & - & 11.6 & 11.6 \\
\hline 18 & 11.4 & 11.3 & 11.3 & 11.2 & - & - & 11.2 & - & - & - & 11.6 & 11.6 \\
\hline 19 & 11.4 & 11.3 & 11.2 & 11.2 & - & - & 11.3 & - & - & - & 11.6 & 11.6 \\
\hline 20 & 11.4 & 11.3 & 11.2 & 11.2 & - & - & 11.3 & - & - & - & 11.6 & 11.6 \\
\hline 21 & 11.4 & 11.3 & 11.2 & 11.2 & - & - & 11.3 & - & - & - & 11.6 & 11.6 \\
\hline 22 & 11.4 & 11.3 & 11.2 & 11.1 & - & - & 11.3 & - & - & - & 11.6 & 11.6 \\
\hline 23 & 11.4 & 11.3 & 11.2 & 11.1 & - & - & 11.3 & - & - & 11.4 & 11.6 & 11.6 \\
\hline 24 & 11.4 & 11.3 & 11.2 & 11.1 & - & - & 11.3 & - & - & 11.5 & 11.6 & 11.6 \\
\hline 25 & 11.4 & 11.3 & 11.2 & 11.1 & - & - & 11.3 & - & 11.3 & 11.5 & 11.6 & 11.6 \\
\hline 26 & 11.4 & 11.3 & 11.2 & - & - & - & 11.3 & - & 11.3 & 11.5 & 11.6 & 11.6 \\
\hline 27 & 11.4 & 11.3 & 11.2 & - & - & - & 11.3 & - & 11.3 & 11.5 & 11.6 & 11.6 \\
\hline 28 & 11.4 & 11.3 & 11.2 & - & - & - & 11.3 & - & 11.4 & 11.6 & 11.6 & 11.6 \\
\hline 29 & 11.4 & - & 11.2 & - & - & - & 11.3 & - & 11.4 & 11.6 & 11.6 & 11.6 \\
\hline 30 & 11.4 & - & 11.2 & - & - & - & 11.3 & - & 11.4 & 11.6 & 11.6 & 11.6 \\
\hline 31 & 11.4 & - & 11.2 & - & - & - & 11.3 & - & - & 11.6 & - & 11.5 \\
\hline Mean & 11.4 & 11.3 & 11.3 & 11.2 & - & - & 11.3 & 11.3 & 11.3 & 11.5 & 11.6 & 11.6 \\
\hline Max & 11.5 & 11.4 & 11.3 & 11.2 & - & - & 11.3 & 11.3 & 11.4 & 11.6 & 11.6 & 11.6 \\
\hline Min & 11.4 & 11.3 & 11.2 & 11.1 & - & - & 11.2 & 11.3 & 11.3 & 11.3 & 11.6 & 11.5 \\
\hline
\end{tabular}




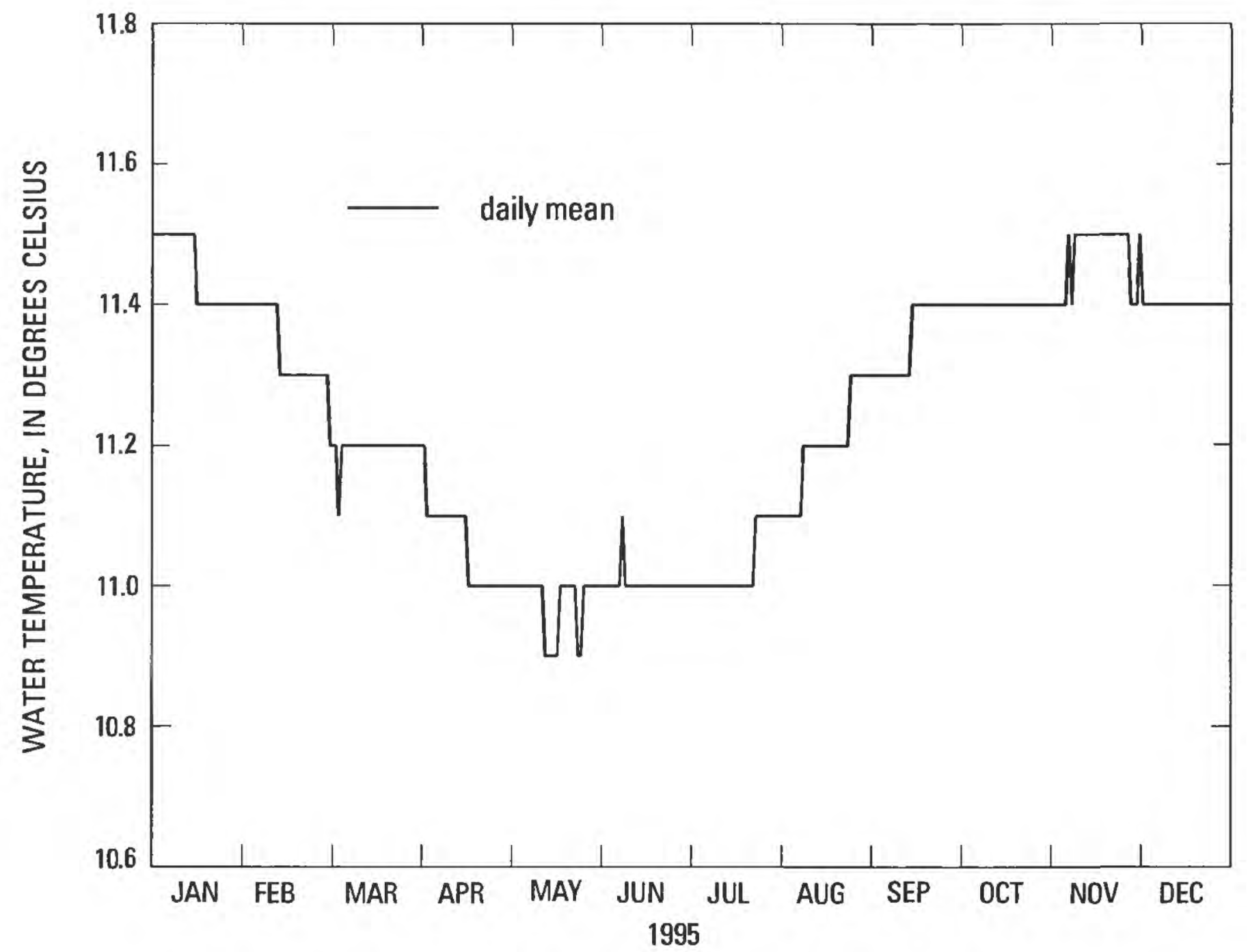

Figure 34. (B) Daily mean water temperature, Fish Hatchery Spring 23 (FH23-T), 1995, Long Valley Caldera, Mono County, California. 
Table 30. Daily mean water temperature, Fish Hatchery Spring 23 (FH23-T), 1995, Long Valley Caldera, California

[Values are in degrees Celsius. - , no data; Max, maximum; Min, minimum]

\begin{tabular}{|c|c|c|c|c|c|c|c|c|c|c|c|c|}
\hline Day & Jan & Fab & Mar & Apr & May & Jun & Jul & Aug & Sep & Oct & Nov & Dec \\
\hline 1 & 11.5 & 11.4 & 11.2 & 11.2 & 11.0 & 11.0 & 11.0 & 11.1 & 11.3 & 11.4 & 11.4 & 11.4 \\
\hline 2 & 11.5 & 11.4 & 11.2 & 11.2 & 11.0 & 11.0 & 11.0 & 11.1 & 11.3 & 11.4 & 11.4 & 11.4 \\
\hline 3 & 11.5 & 11.4 & 11.2 & 11.1 & 11.0 & 11.0 & 11.0 & 11.1 & 11.3 & 11.4 & 11.4 & 11.4 \\
\hline 4 & 11.5 & 11.4 & 11.1 & 11.1 & 11.0 & 11.0 & 11.0 & 11.1 & 11.3 & 11.4 & 11.4 & 11.4 \\
\hline 5 & 11.5 & 11.4 & 11.2 & 11.1 & 11.0 & 11.0 & 11.0 & 11.1 & 11.3 & 11.4 & 11.4 & 11.4 \\
\hline 6 & 11.5 & 11.4 & 11.2 & 11.1 & 11.0 & 11.0 & 11.0 & 11.1 & 11.3 & 11.4 & 11.5 & 11.4 \\
\hline 7 & 11.5 & 11.4 & 11.2 & 11.1 & 11.0 & 11.0 & 11.0 & 11.1 & 11.3 & 11.4 & 11.4 & 11.4 \\
\hline 8 & 11.5 & 11.4 & 11.2 & 11.1 & 11.0 & 11.1 & 11.0 & 11.2 & 11.3 & 11.4 & 11.5 & 11.4 \\
\hline 9 & 11.5 & 11.4 & 11.2 & 11.1 & 11.0 & 11.0 & 11.0 & 11.2 & 11.3 & 11.4 & 11.5 & 11.4 \\
\hline 10 & 11.5 & 11.4 & 11.2 & 11.1 & 11.0 & 11.0 & 11.0 & 11.2 & 11.3 & 11.4 & 11.5 & 11.4 \\
\hline 11 & 11.5 & 11.4 & 11.2 & 11.1 & 11.0 & 11.0 & 11.0 & 11.2 & 11.3 & 11.4 & 11.5 & 11.4 \\
\hline 12 & 11.5 & 11.3 & 11.2 & 11.1 & 11.0 & 11.0 & 11.0 & 11.2 & 11.3 & 11.4 & 11.5 & 11.4 \\
\hline 13 & 11.5 & 11.3 & 11.2 & 11.1 & 10.9 & 11.0 & 11.0 & 11.2 & 11.3 & 11.4 & 11.5 & 11.4 \\
\hline 14 & 11.5 & 11.3 & 11.2 & 11.1 & 10.9 & 11.0 & 11.0 & 11.2 & 11.4 & 11.4 & 11.5 & 11.4 \\
\hline 15 & 11.4 & 11.3 & 11.2 & 11.1 & 10.9 & 11.0 & 11.0 & 11.2 & 11.4 & 11.4 & 11.5 & 11.4 \\
\hline 16 & 11.4 & 11.3 & 11.2 & 11.1 & 10.9 & 11.0 & 11.0 & 11.2 & 11.4 & 11.4 & 11.5 & 11.4 \\
\hline 17 & 11.4 & 11.3 & 11.2 & 11.0 & 10.9 & 11.0 & 11.0 & 11.2 & 11.4 & 11.4 & 11.5 & 11.4 \\
\hline 18 & 11.4 & 11.3 & 11.2 & 11.0 & 11.0 & 11.0 & 11.0 & 11.2 & 11.4 & 11.4 & 11.5 & 11.4 \\
\hline 19 & 11.4 & 11.3 & 11.2 & 11.0 & 11.0 & 11.0 & 11.0 & 11.2 & 11.4 & 11.4 & 11.5 & 11.4 \\
\hline 20 & 11.4 & 11.3 & 11.2 & 11.0 & 11.0 & 11.0 & 11.0 & 11.2 & 11.4 & 11.4 & 11.5 & 11.4 \\
\hline 21 & 11.4 & 11.3 & 11.2 & 11.0 & 11.0 & 11.0 & 11.0 & 11.2 & 11.4 & 11.4 & 11.5 & 11.4 \\
\hline 22 & 11.4 & 11.3 & 11.2 & 11.0 & 11.0 & 11.0 & 11.0 & 11.2 & 11.4 & 11.4 & 11.5 & 11.4 \\
\hline 23 & 11.4 & 11.3 & 11.2 & 11.0 & 11.0 & 11.0 & 11.1 & 11.2 & 11.4 & 11.4 & 11.5 & 11.4 \\
\hline 24 & 11.4 & 11.3 & 11.2 & 11.0 & 10.9 & 11.0 & 11.1 & 11.3 & 11.4 & 11.4 & 11.5 & 11.4 \\
\hline 25 & 11.4 & 11.3 & 11.2 & 11.0 & 10.9 & 11.0 & 11.1 & 11.3 & 11.4 & 11.4 & 11.5 & 11.4 \\
\hline 26 & 11.4 & 11.3 & 11.2 & 11.0 & 11.0 & 11.0 & 11.1 & 11.3 & 11.4 & 11.4 & 11.5 & 11.4 \\
\hline 27 & 11.4 & 11.3 & 11.2 & 11.0 & 11.0 & 11.0 & 11.1 & 11.3 & 11.4 & 11.4 & 11.4 & 11.4 \\
\hline 28 & 11.4 & 11.3 & 11.2 & 11.0 & 11.0 & 11.0 & 11.1 & 11.3 & 11.4 & 11.4 & 11.4 & 11.4 \\
\hline 29 & 11.4 & - & 11.2 & 11.0 & 11.0 & 11.0 & 11.1 & 11.3 & 11.4 & 11.4 & 11.4 & 11.4 \\
\hline 30 & 11.4 & - & 11.2 & 11.0 & 11.0 & 11.0 & 11.1 & 11.3 & 11.4 & 11.4 & 11.5 & 11.4 \\
\hline 31 & 11.4 & - & 11.2 & - & 11.0 & - & 11.1 & 11.3 & - & 11.4 & - & 11.4 \\
\hline Mean & 11.4 & 11.3 & 11.2 & 11.1 & 11.0 & 11.0 & 11.0 & 11.2 & 11.4 & 11.4 & 11.5 & 11.4 \\
\hline $\operatorname{Max}$ & 11.5 & 11.4 & 11.2 & 11.2 & 11.0 & 11.1 & 11.1 & 11.3 & 11.4 & 11.4 & 11.5 & 11.4 \\
\hline Min & 11.4 & 11.3 & 11.1 & 11.0 & 10.9 & 11.0 & 11.0 & 11.1 & 11.3 & 11.4 & 11.4 & 11.4 \\
\hline
\end{tabular}




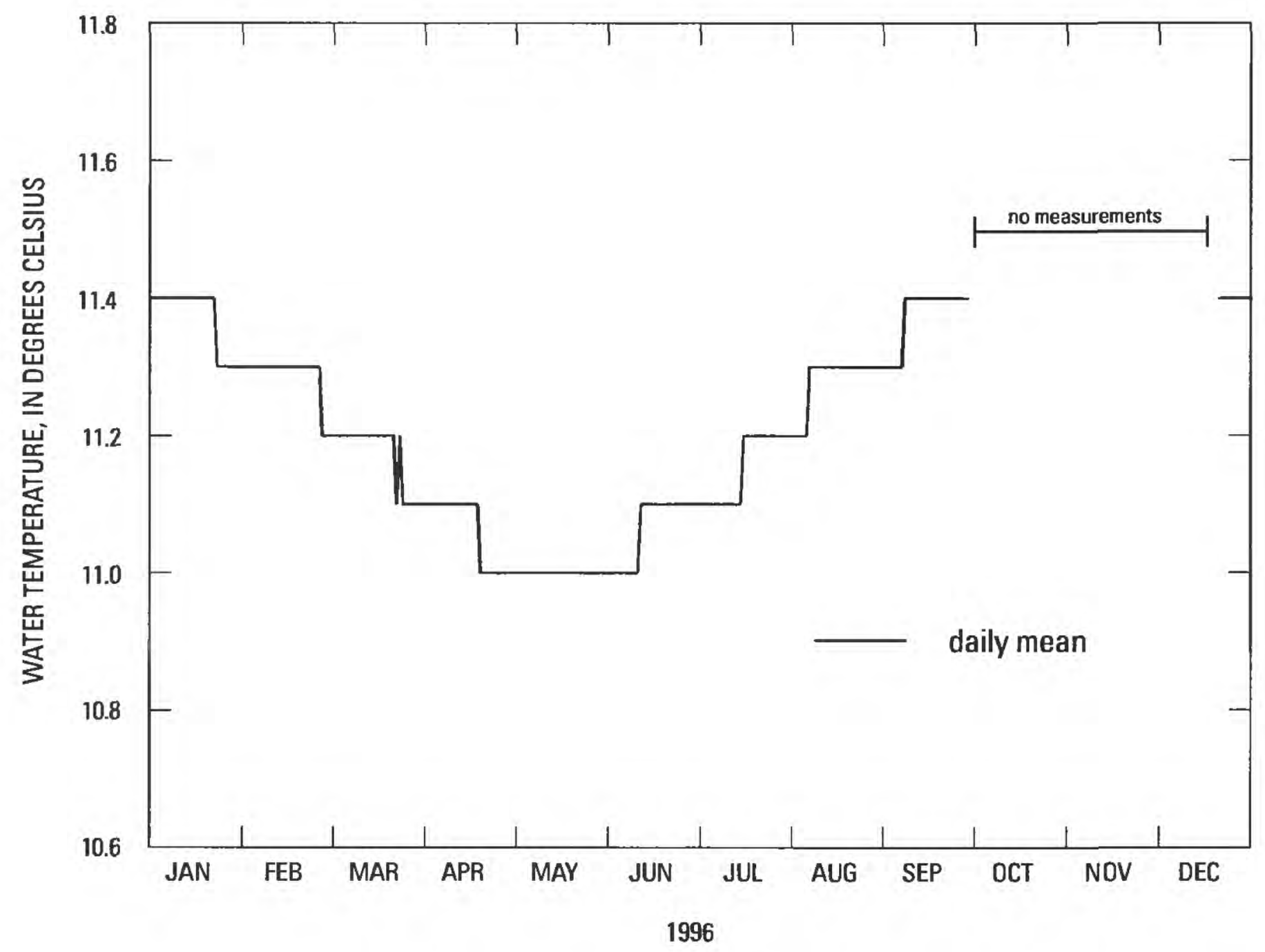

Figure 34. (C) Daily mean water temperature, Fish Hatchery Spring 23 (FH23-T), 1996, Long Valley Caldera, Mono County, California. 
Table 31. Daily mean water temperature, Fish Hatchery Spring 23 (FH23-T), 1996, Long Valley Caldera, California

[Values are in degrees Celsius. -, no data; Max, maximum; Min, minimum]

\begin{tabular}{|c|c|c|c|c|c|c|c|c|c|c|c|c|}
\hline Day & Jan & Feb & Mar & Apr & May & Jun & Jul & Aug & Sep & Oct & Nov & Dec \\
\hline 1 & 11.4 & 11.3 & 11.2 & 11.1 & 11.0 & 11.0 & 11.1 & 11.2 & 11.3 & - & - & - \\
\hline 2 & 11.4 & 11.3 & 11.2 & 11.1 & 11.0 & 11.0 & 11.1 & 11.2 & 11.3 & - & - & - \\
\hline 3 & 11.4 & 11.3 & 11.2 & 11.1 & 11.0 & 11.0 & 11.1 & 11.2 & 11.3 & - & - & - \\
\hline 4 & 11.4 & 11.3 & 11.2 & 11.1 & 11.0 & 11.0 & 11.1 & 11.2 & 11.3 & - & - & - \\
\hline 5 & 11.4 & 11.3 & 11.2 & 11.1 & 11.0 & 11.0 & 11.1 & 11.2 & 11.3 & - & - & - \\
\hline 6 & 11.4 & 11.3 & 11.2 & 11.1 & 11.0 & 11.0 & 11.1 & 11.3 & 11.3 & - & - & - \\
\hline 7 & 11.4 & 11.3 & 11.2 & 11.1 & 11.0 & 11.0 & 11.1 & 11.3 & 11.4 & - & - & - \\
\hline 8 & 11.4 & 11.3 & 11.2 & 11.1 & 11.0 & 11.0 & 11.1 & 11.3 & 11.4 & - & - & - \\
\hline 9 & 11.4 & 11.3 & 11.2 & 11.1 & 11.0 & 11.0 & 11.1 & 11.3 & 11.4 & - & - & - \\
\hline 10 & 11.4 & 11.3 & 11.2 & 11.1 & 11.0 & 11.0 & 11.1 & 11.3 & 11.4 & - & - & - \\
\hline 11 & 11.4 & 11.3 & 11.2 & 11.1 & 11.0 & 11.1 & 11.1 & 11.3 & 11.4 & - & - & - \\
\hline 12 & 11.4 & 11.3 & 11.2 & 11.1 & 11.0 & 11.1 & 11.1 & 11.3 & 11.4 & - & - & - \\
\hline 13 & 11.4 & 11.3 & 11.2 & 11.1 & 11.0 & 11.1 & 11.1 & 11.3 & 11.4 & - & - & - \\
\hline 14 & 11.4 & 11.3 & 11.2 & 11.1 & 11.0 & 11.1 & 11.1 & 11.3 & 11.4 & - & - & - \\
\hline 15 & 11.4 & 11.3 & 11.2 & 11.1 & 11.0 & 11.1 & 11.2 & 11.3 & 11.4 & - & - & - \\
\hline 16 & 11.4 & 11.3 & 11.2 & 11.1 & 11.0 & 11.1 & 11.2 & 11.3 & 11.4 & - & - & - \\
\hline 17 & 11.4 & 11.3 & 11.2 & 11.1 & 11.0 & 11.1 & 11.2 & 11.3 & 11.4 & - & - & - \\
\hline 18 & 11.4 & 11.3 & 11.2 & 11.1 & 11.0 & 11.1 & 11.2 & 11.3 & 11.4 & - & - & - \\
\hline 19 & 11.4 & 11.3 & 11.2 & 11.0 & 11.0 & 11.1 & 11.2 & 11.3 & 11.4 & - & - & - \\
\hline 20 & 11.4 & 11.3 & 11.2 & 11.0 & 11.0 & 11.1 & 11.2 & 11.3 & 11.4 & - & - & 11.4 \\
\hline 21 & 11.4 & 11.3 & 11.2 & 11.0 & 11.0 & 11.1 & 11.2 & 11.3 & 11.4 & - & - & - 11.4 \\
\hline 22 & 11.3 & 11.3 & 11.1 & 11.0 & 11.0 & 11.1 & 11.2 & 11.3 & 11.4 & - & - & 11.4 \\
\hline 23 & 11.3 & 11.3 & 11.2 & 11.0 & 11.0 & 11.1 & 11.2 & 11.3 & 11.4 & - & - & 11.4 \\
\hline 24 & 11.3 & 11.3 & 11.1 & 11.0 & 11.0 & 11.1 & 11.2 & 11.3 & 11.4 & - & - & 11.4 \\
\hline 25 & 11.3 & 11.3 & 11.1 & 11.0 & 11.0 & 11.1 & 11.2 & 11.3 & 11.4 & - & - & 11.4 \\
\hline 26 & 11.3 & 11.2 & 11.1 & 11.0 & 11.0 & 11.1 & 11.2 & 11.3 & 11.4 & - & - & 11.4 \\
\hline 27 & 11.3 & 11.2 & 11.1 & 11.0 & 11.0 & 11.1 & 11.2 & 11.3 & 11.4 & - & - & 11.4 \\
\hline 28 & 11.3 & 11.2 & 11.1 & 11.0 & 11.0 & 11.1 & 11.2 & 11.3 & 11.4 & - & - & 11.4 \\
\hline 29 & 11.3 & 11.2 & 11.1 & 11.0 & 11.0 & 11.1 & 11.2 & 11.3 & - & - & - & 11.4 \\
\hline 30 & 11.3 & - & 11.1 & 11.0 & 11.0 & 11.1 & 11.2 & 11.3 & $\ldots$ & - & - & 11.4 \\
\hline 31 & 11.3 & - & 11.1 & - & 11.0 & - & 11.2 & 11.3 & - & - & - & 11.4 \\
\hline Mean & 11.4 & 11.3 & 11.2 & 11.1 & 11.0 & 11.1 & 11.2 & 11.3 & 11.4 & - & - & 11.4 \\
\hline Max & 11.4 & 11.3 & 11.2 & 11.1 & 11.0 & I1.1 & 11.2 & 11.3 & 11.4 & - & - & 11.4 \\
\hline Min & 11.3 & 11.2 & 11.1 & 11.0 & 11.0 & 11.0 & 11.1 & 11.2 & 11.3 & - & - & 11.4 \\
\hline
\end{tabular}




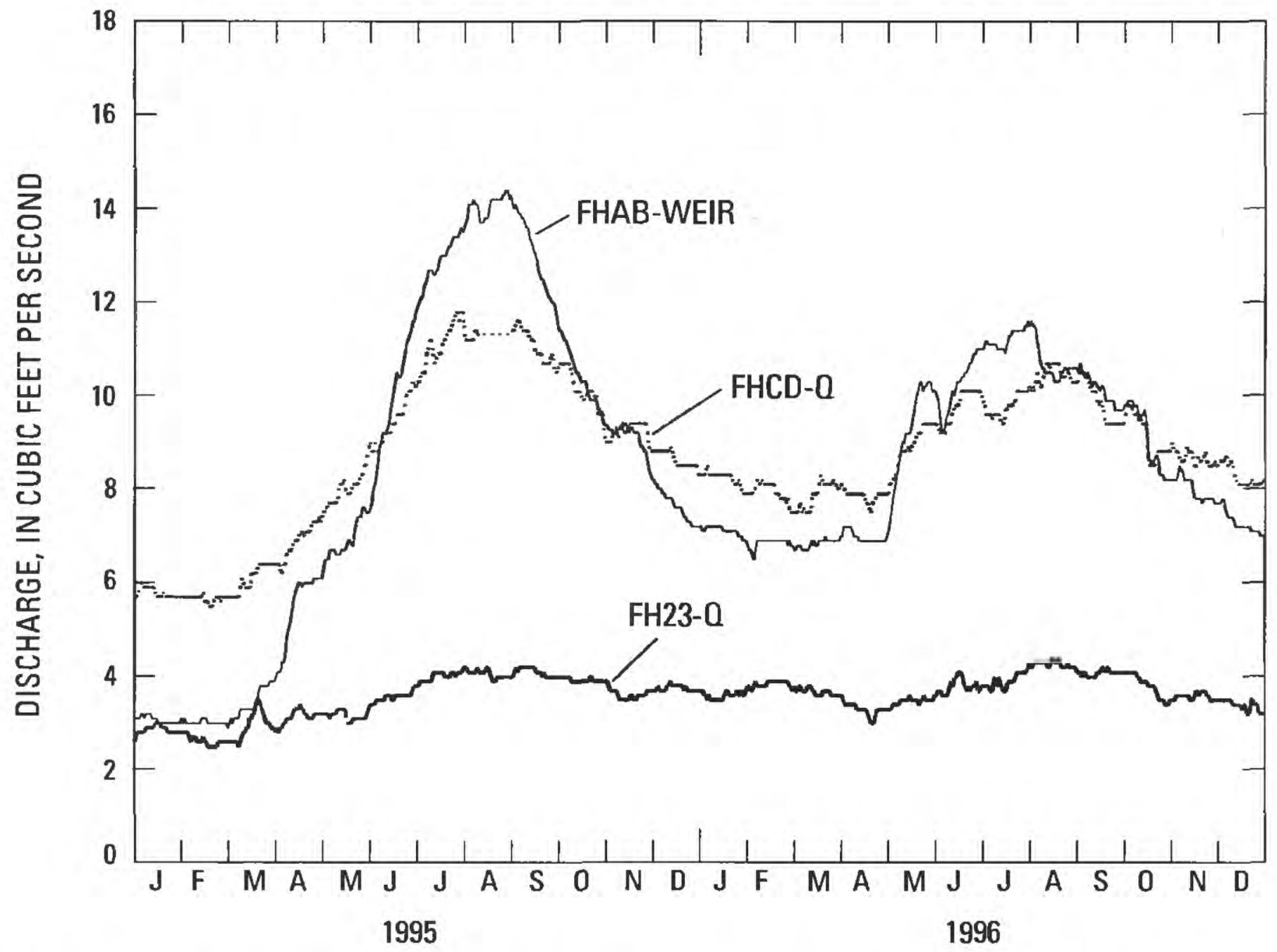

Figure 35. Daily mean discharge, Fish Hatchery Springs AB, CD, and 23 (FHAB-WEIR, FHCD-0, and FH23-0), 1995-96, Long Valley Caldera, Mono County, California.

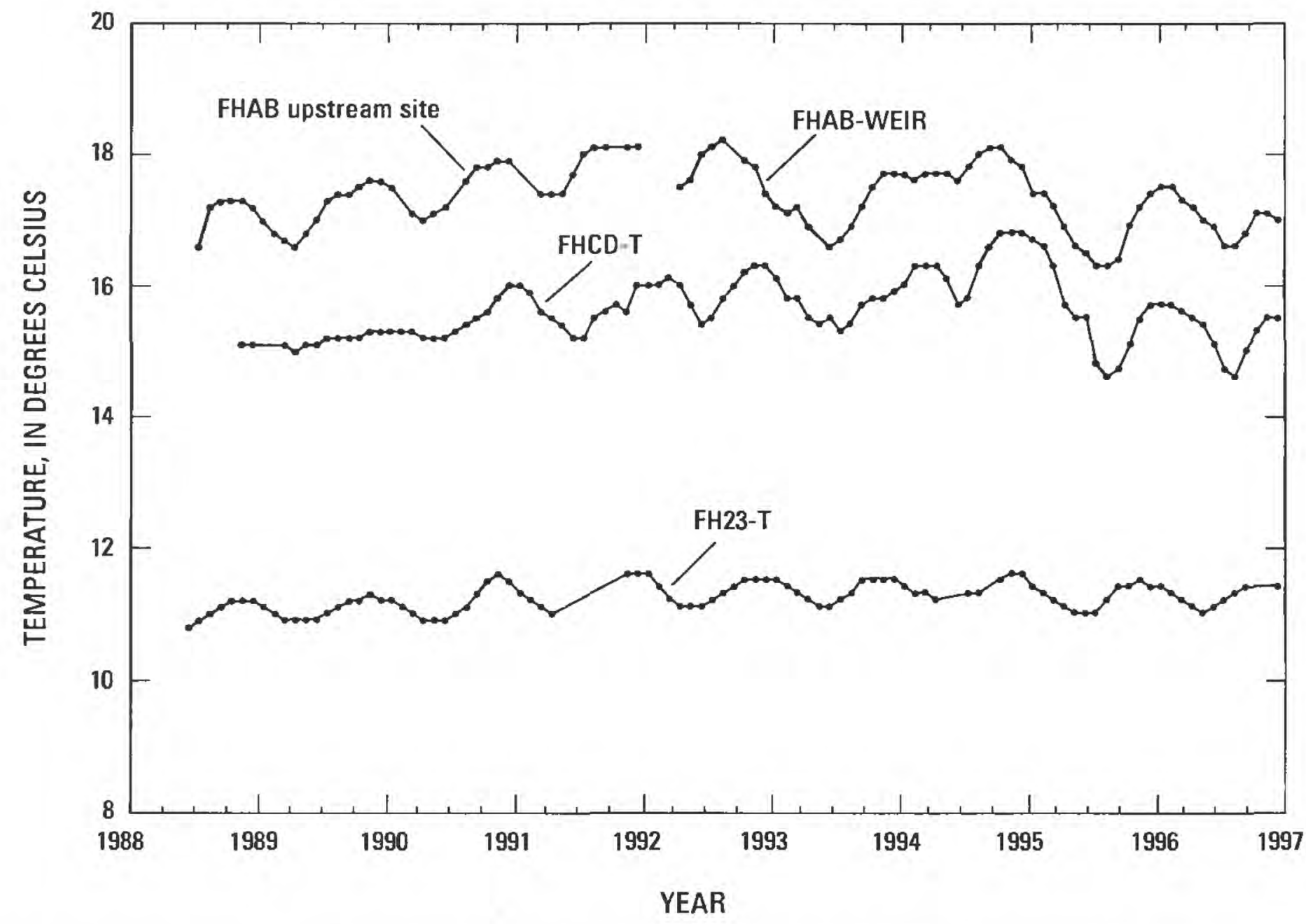

Figure 36. Monthly mean water temperature, Fish Hatchery Springs AB, CD, and 23 (FHAB, FHCD-T, and FH23-T), 1988-96, Long Valley Caldera, Mono County, California. 


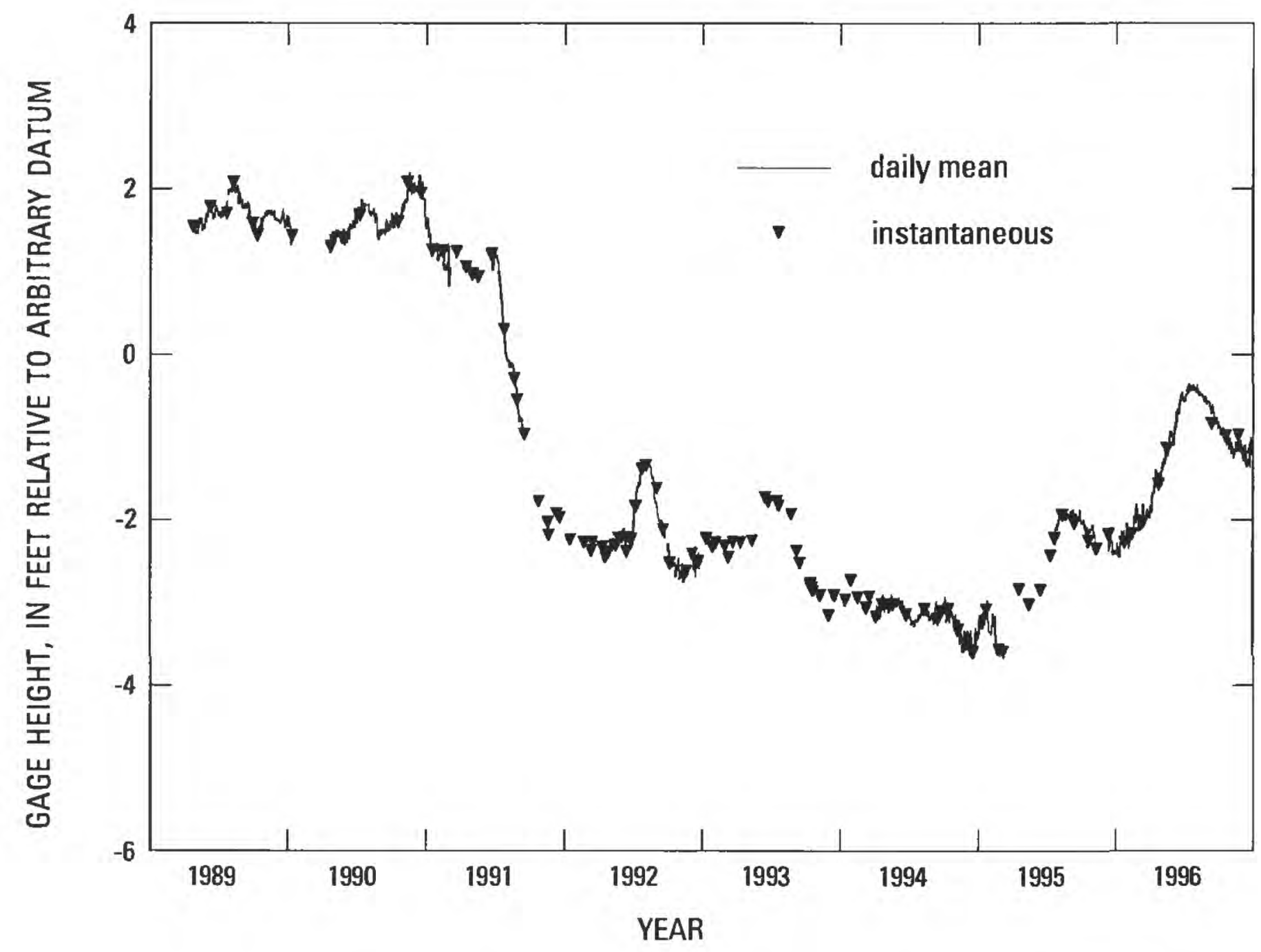

Figure 37. Daily mean and instantaneous measured gage heights, Hot Bubbling Pool (HBP), 1989-96, Long Valley Caldera, Mono County, California. 


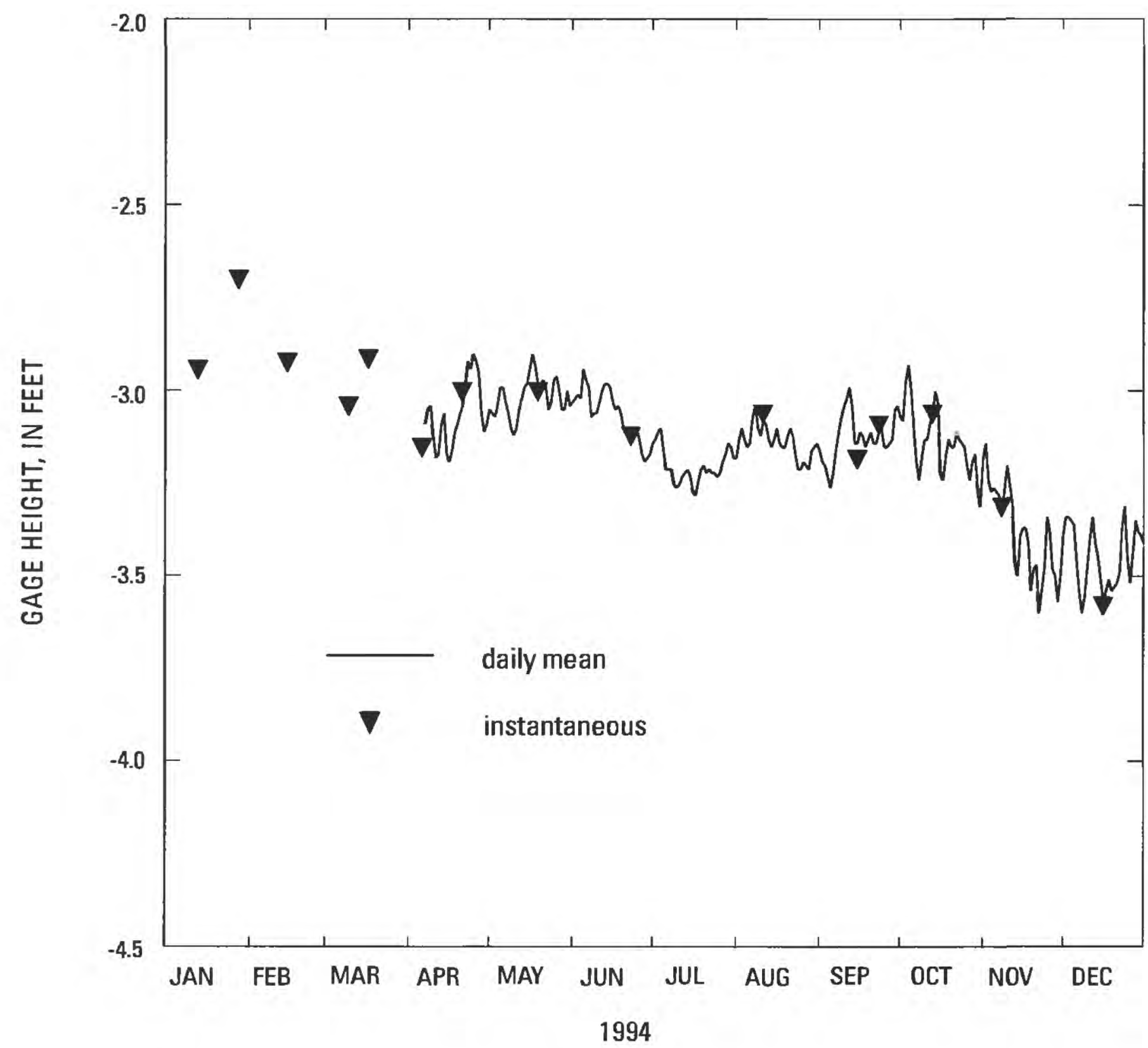

Figure 38. (A) Daily mean and instantaneous measured gage heights, Hot Bubbling Pool (HBP), 1994, Long Valley Caldera, Mono County, California. 
Table 32. Daily mean gage height, Hot Bubbling Pool (HBP), 1994, Long Valley Caldera, California

[Values are in feet relative to an arbitrary datum _ _, no data; Max, maximum; Min, minimum]

\begin{tabular}{|c|c|c|c|c|c|c|c|c|c|c|c|c|}
\hline Day & Jan & Feb & Mar & Apr & May & Jun & Jul & Aug & Sep & Oct & Nov & Dec \\
\hline 1 & - & - & - & - & -3.05 & -3.03 & -3.14 & -3.18 & -3.16 & -3.07 & -3.18 & -3.39 \\
\hline 2 & - & - & $\ldots$ & - & -3.06 & -3.02 & -3.13 & -3.13 & -3.19 & -3.08 & -3.14 & -3.34 \\
\hline 3 & - & - & - & - & -3.07 & -3.01 & -3.11 & -3.10 & -3.20 & -2.97 & -3.24 & -3.34 \\
\hline 4 & - & - & - & $\ldots$ & -3.04 & -3.02 & -3.10 & -3.13 & -3.23 & -2.93 & -3.27 & -3.35 \\
\hline 5 & - & - & - & - & -2.99 & -2.94 & -3.16 & -3.15 & -3.26 & -3.00 & -3.26 & -3.36 \\
\hline 6 & - & - & - & - & -2.99 & -2.97 & -3.21 & -3.14 & -3.22 & -3.10 & -3.27 & -3.46 \\
\hline 7 & - & - & - & -3.09 & -3.03 & -2.99 & -3.21 & -3.07 & -3.16 & -3.19 & -3.28 & -3.54 \\
\hline 8 & - & - & - & -3.05 & -3.06 & -3.07 & -3.21 & -3.04 & -3.11 & -3.24 & -3.33 & -3.60 \\
\hline 9 & - & - & - & -3.04 & -3.10 & -3.06 & -3.25 & -3.10 & -3.07 & -3.18 & -3.27 & -3.56 \\
\hline 10 & - & - & - & -3.12 & -3.12 & -3.06 & -3.26 & -3.12 & -3.04 & -3.13 & -3.20 & -3.48 \\
\hline 11 & - & - & - & -3.18 & -3.10 & -3.03 & -3.25 & -3.08 & -3.02 & -3.13 & -3.25 & -3.40 \\
\hline 12 & - & - & - & -3.17 & -3.05 & -3.00 & -3.23 & -3.09 & -2.99 & -3.09 & -3.30 & -3.34 \\
\hline 13 & - & - & - & -3.09 & -3.02 & -2.98 & -3.22 & -3.13 & -3.04 & -3.06 & -3.46 & -3.41 \\
\hline 14 & - & - & - & -3.06 & -2.99 & -2.98 & -3.21 & -3.15 & -3.14 & -3.00 & -3.50 & -3.45 \\
\hline 15 & - & - & - & -3.17 & -2.98 & -2.99 & -3.23 & -3.13 & -3.14 & -3.03 & -3.39 & -3.51 \\
\hline 16 & - & - & - & -3.19 & -2.94 & -3.03 & -3.27 & -3.10 & -3.11 & -3.22 & -3.37 & -3.57 \\
\hline 17 & - & - & - & -3.16 & -2.90 & -3.05 & -3.28 & -3.14 & -3.12 & -3.24 & -3.37 & -3.54 \\
\hline 18 & - & - & - & -3.11 & -2.93 & -3.04 & -3.24 & -3.15 & -3.15 & -3.17 & -3.41 & -3.51 \\
\hline 19 & - & - & - & -3.09 & -2.98 & -3.06 & -3.21 & -3.15 & -3.13 & -3.13 & -3.54 & -3.54 \\
\hline 20 & - & - & - & -3.06 & -2.98 & -3.10 & -3.20 & -3.12 & -3.11 & -3.15 & -3.48 & -3.53 \\
\hline 21 & - & - & - & -3.04 & -2.97 & -3.11 & -3.22 & -3.10 & -3.14 & -3.15 & -3.47 & -3.52 \\
\hline 22 & - & - & - & -2.99 & -2.99 & -3.12 & -3.21 & -3.12 & -3.14 & -3.11 & -3.60 & -3.49 \\
\hline 23 & - & - & - & -2.92 & -3.05 & -3.14 & -3.22 & -3.17 & -3.11 & -3.13 & -3.54 & -3.36 \\
\hline 24 & - & - & - & -2.94 & -3.03 & -3.11 & -3.22 & -3.21 & -3.10 & -3.14 & -3.48 & -3.31 \\
\hline 25 & - & - & - & -2.90 & -2.97 & -3.10 & -3.23 & -3.21 & -3.15 & -3.15 & -3.34 & -3.45 \\
\hline 26 & - & - & - & -2.92 & -2.96 & -3.13 & -3.22 & -3.19 & -3.15 & -3.20 & -3.38 & -3.52 \\
\hline 27 & - & - & - & -2.95 & -3.00 & -3.17 & -3.19 & -3.20 & -3.14 & -3.24 & -3.48 & -3.44 \\
\hline 28 & - & - & - & -3.06 & -3.05 & -3.19 & -3.17 & -3.21 & -3.13 & -3.19 & -3.50 & -3.35 \\
\hline 29 & - & - & - & -3.11 & -3.05 & -3.18 & -3.14 & -3.16 & -3.05 & -3.17 & -3.57 & -3.38 \\
\hline 30 & - & - & - & -3.09 & -3.00 & -3.17 & -3.15 & -3.15 & -3.04 & -3.26 & -3.50 & -3.39 \\
\hline 31 & - & - & - & - & -3.04 & - & -3.18 & -3.14 & - & -3.31 & - & -3.41 \\
\hline Mean & - & - & - & -3.06 & -3.02 & -3.06 & -3.20 & -3.14 & -3.12 & -3.13 & -3.38 & -3.45 \\
\hline Max & - & - & - & -2.90 & -2.90 & -2.94 & -3.10 & -3.04 & -2.99 & -2.93 & -3.14 & -3.31 \\
\hline Min & - & - & - & -3.19 & -3.12 & -3.19 & -3.28 & -3.21 & -3.26 & -3.31 & -3.60 & -3.60 \\
\hline
\end{tabular}




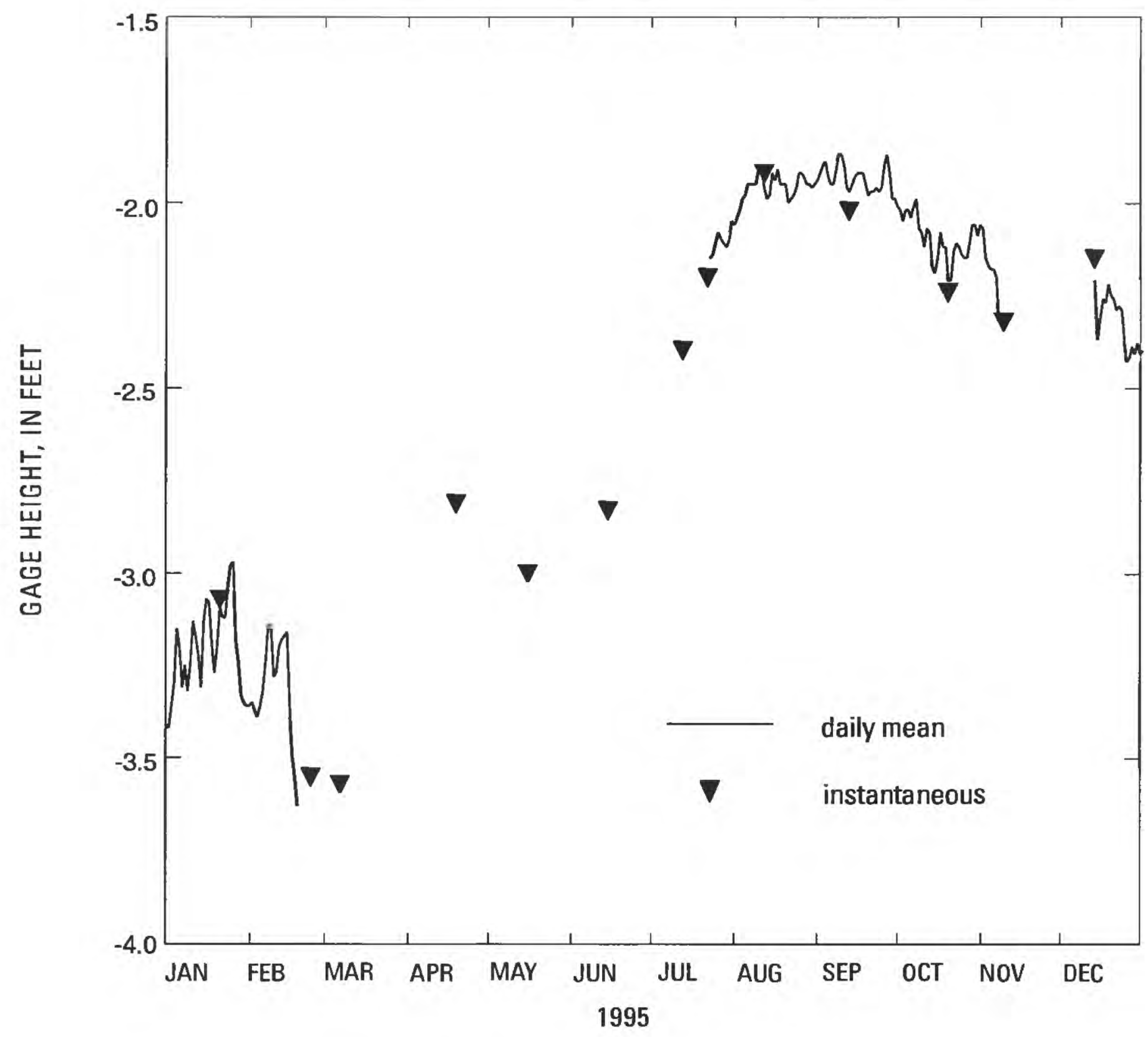

Figure 38. (B) Daily mean and instantaneous measured gage heights, Hot Bubbling Pool (HBP), 1995, Long Valley Caldera, Mono County, California. 
Table 33. Daily mean gage height, Hot Bubbling Pool (HBP), 1995, Long Valley Caldera, California

[Values are in feet relative to an arbitrary datum. - , no data; Max, maximum; Min, minimum]

\begin{tabular}{|c|c|c|c|c|c|c|c|c|c|c|c|c|}
\hline Day & Jan & Feb & Mar & Apr & May & Jun & Jul & Aug & Sep & Oct & Nov & Dec \\
\hline 1 & -3.42 & -3.35 & - & - & - & - & - & -2.04 & -1.92 & -2.02 & -2.07 & - \\
\hline 2 & -3.36 & -3.37 & - & - & - & - & - & -2.02 & -1.90 & -2.05 & -2.15 & - \\
\hline 3 & -3.30 & -3.39 & - & - & - & - & - & -1.99 & -1.89 & -2.02 & -2.17 & - \\
\hline 4 & -3.15 & -3.36 & - & - & - & - & - & -1.98 & -1.93 & -2.02 & -2.18 & - \\
\hline 5 & -3.20 & -3.32 & - & - & - & - & - & -1.95 & -1.95 & -2.04 & -2.18 & - \\
\hline 6 & -3.31 & -3.24 & - & - & - & - & - & -1.95 & -1.95 & -2.01 & -2.20 & - \\
\hline 7 & -3.25 & -3.14 & - & - & - & - & - & -1.95 & -1.91 & -1.99 & -2.32 & - \\
\hline 8 & -3.32 & -3.14 & - & - & - & - & - & -1.95 & -1.87 & -2.07 & -2.30 & - \\
\hline 9 & -3.25 & -3.28 & - & - & - & - & - & -1.91 & -1.87 & -2.08 & - & - \\
\hline 10 & -3.13 & -3.27 & - & - & - & - & - & -1.91 & -1.90 & -2.12 & - & - \\
\hline 11 & -3.17 & -3.20 & - & - & - & - & - & -1.96 & -1.96 & -2.07 & - & - \\
\hline 12 & -3.22 & -3.18 & - & - & - & - & - & -1.99 & -1.97 & -2.08 & - & - \\
\hline 13 & -3.31 & -3.17 & - & - & - & - & - & -1.98 & -1.95 & -2.17 & - & -2.21 \\
\hline 14 & -3.13 & -3.16 & - & - & - & - & - & -1.92 & -1.93 & -2.19 & - & -2.37 \\
\hline 15 & -3.07 & -3.36 & - & - & - & - & - & -1.94 & -1.92 & -2.15 & - & -2.31 \\
\hline 16 & -3.08 & -3.49 & - & - & - & - & - & -1.91 & -1.92 & -2.08 & - & -2.26 \\
\hline 17 & -3.19 & -3.55 & - & - & - & - & - & -1.95 & -1.92 & -2.12 & - & -2.27 \\
\hline 18 & -3.27 & -3.63 & - & - & - & - & - & -1.95 & -1.95 & -2.12 & - & -2.22 \\
\hline 19 & -3.20 & - & - & - & - & - & - & -1.95 & -1.98 & -2.21 & - & -2.25 \\
\hline 20 & -3.09 & - & - & - & - & - & - & -2.00 & -1.97 & -2.21 & - & -2.26 \\
\hline 21 & -3.12 & - & - & - & - & - & - & -1.99 & -1.97 & -2.13 & - & -2.29 \\
\hline 22 & -3.12 & - & - & - & - & - & -2.15 & -1.98 & -1.96 & -2.11 & - & -2.28 \\
\hline 23 & -3.04 & - & - & - & - & - & -2.14 & -1.96 & -1.97 & -2.12 & - & -2.29 \\
\hline 24 & -2.98 & - & - & - & - & - & -2.11 & -1.92 & -1.96 & -2.14 & - & -2.37 \\
\hline 25 & -2.97 & - & - & - & - & - & -2.08 & -1.92 & -1.90 & -2.15 & - & -2.43 \\
\hline 26 & -3.18 & - & - & - & - & - & -2.10 & -1.93 & -1.87 & -2.15 & - & -2.42 \\
\hline 27 & -3.24 & - & - & - & - & - & -2.11 & -1.95 & -1.92 & -2.11 & - & -2.39 \\
\hline 28 & -3.33 & - & - & - & - & - & -2.12 & -1.95 & -1.99 & -2.06 & - & -2.41 \\
\hline 29 & -3.35 & - & - & - & - & - & -2.10 & -1.96 & -1.99 & -2.06 & - & -2.38 \\
\hline 30 & -3.36 & - & - & - & - & - & -2.05 & -1.95 & -2.01 & -2.09 & - & -2.41 \\
\hline 31 & -3.36 & - & - & - & - & - & -2.06 & -1.94 & - & -2.06 & - & -2.40 \\
\hline Mean & -3.21 & -3.31 & - & - & - & - & -2.10 & -1.96 & -1.94 & -2.10 & -2.20 & -2.33 \\
\hline Max & -2.97 & -3.14 & - & - & - & - & -2.05 & -1.91 & -1.87 & -1.99 & -2.07 & -2.21 \\
\hline Min & -3.42 & -3.63 & - & - & - & - & -2.15 & -2.04 & -2.01 & -2.21 & -2.32 & -2.43 \\
\hline
\end{tabular}




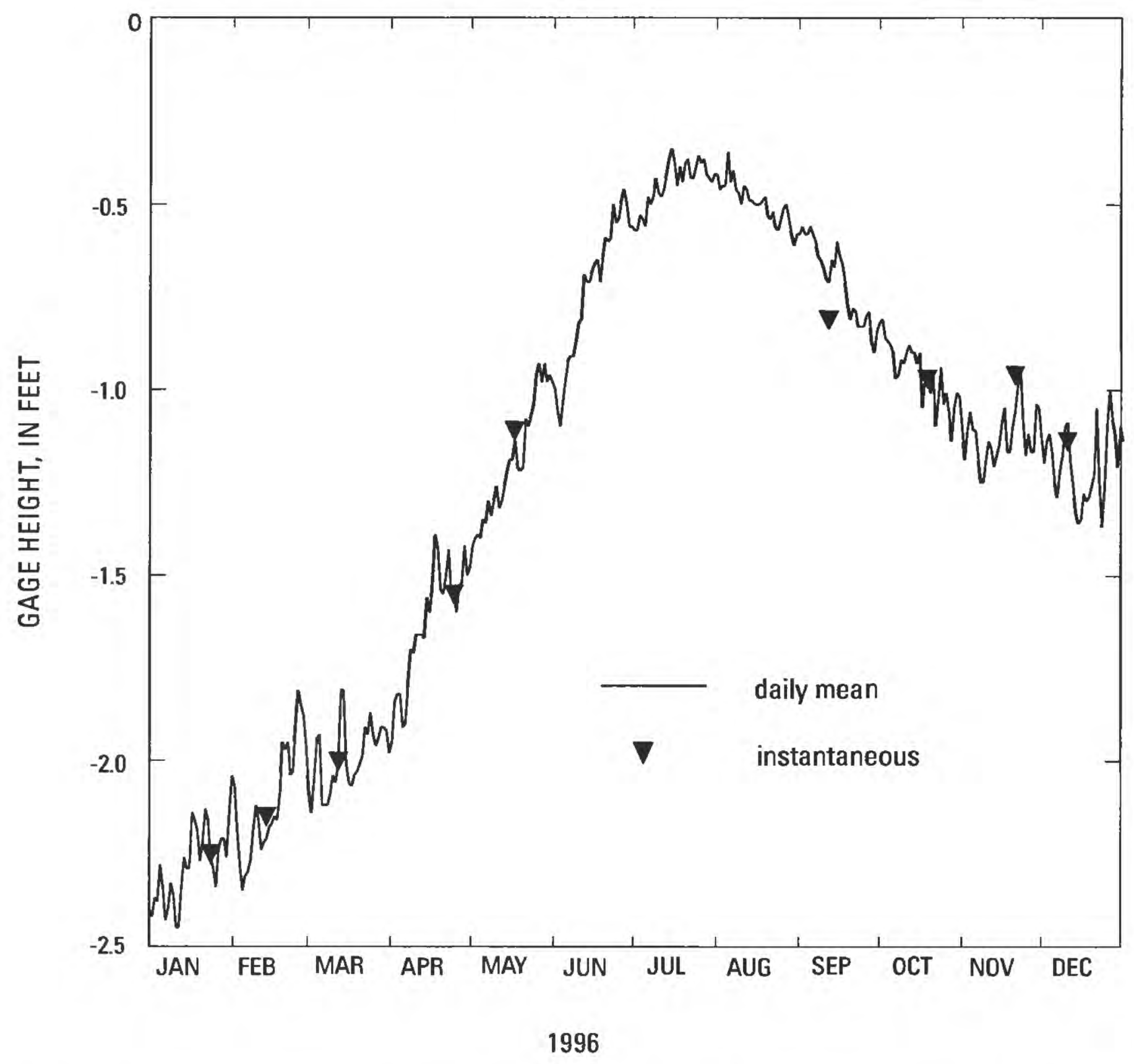

Figure 38. (C) Daily mean and instantaneous measured gage heights, Hot Bubbling Pool (HBP), 1996, Long Valley Caldera, Mono County, California. 
Table 34. Daily mean gage height, Hot Bubbling Pool (HBP), 1996, Long Valley Caldera, California

[Values are in feet relative to an arbitrary datum. -, no data; Max, maximum; Min. minimum]

\begin{tabular}{|c|c|c|c|c|c|c|c|c|c|c|c|c|}
\hline Day & Jan & Feb & Mar & Apr & May & Jun & Jul & Aug & Sep & Oct & Nov & Dec \\
\hline 1 & -2.42 & -2.07 & -2.14 & -1.84 & -1.40 & -1.06 & -0.57 & -0.46 & -0.56 & -0.81 & -1.19 & -1.20 \\
\hline 2 & -2.37 & -2.19 & -2.05 & -1.82 & -1.39 & -1.10 & -.53 & -.45 & -.58 & -.86 & -1.11 & -1.14 \\
\hline 3 & -2.38 & -2.28 & -1.94 & -1.82 & -1.40 & -1.03 & -.54 & -.45 & -.58 & -.87 & -1.06 & -1.12 \\
\hline 4 & -2.28 & -2.35 & -1.93 & -1.91 & -1.35 & -.97 & -.56 & -.36 & -.56 & -.88 & -1.11 & -1.17 \\
\hline 5 & -2.34 & -2.31 & -2.12 & -1.90 & -1.36 & -.92 & -.48 & -.44 & -.58 & -.90 & -1.11 & -1.25 \\
\hline 6 & -2.43 & -2.30 & -2.12 & -1.79 & -1.30 & -.91 & -.50 & -.41 & -.60 & -.97 & -1.18 & -1.29 \\
\hline 7 & -2.40 & -2.27 & -2.12 & -1.70 & -1.34 & -.91 & -.48 & -.46 & -.64 & -.96 & -1.25 & -1.22 \\
\hline 8 & -2.33 & -2.19 & -2.09 & -1.71 & -1.30 & -.87 & -.43 & -.47 & -.65 & -.92 & -1.25 & -1.18 \\
\hline 9 & -2.36 & -2.12 & -2.04 & -1.66 & -1.26 & -.82 & -.47 & -.50 & -.67 & -.93 & -1.19 & -1.10 \\
\hline 10 & -2.45 & -2.16 & -2.06 & -1.66 & -1.32 & -.81 & -.48 & -.45 & -.70 & -.90 & -1.14 & -1.09 \\
\hline 11 & -2.45 & -2.24 & -2.01 & -1.66 & -1.30 & -.69 & -.46 & -.46 & -.71 & -.88 & -1.16 & -1.20 \\
\hline 12 & -2.34 & -2.22 & -1.81 & -1.67 & -1.26 & -.71 & -.42 & -.49 & -.65 & -.90 & -1.21 & -1.26 \\
\hline 13 & -2.26 & -2.21 & -1.81 & -1.56 & -1.22 & -.71 & -.38 & -.49 & -.67 & -.90 & -1.18 & -1.33 \\
\hline 14 & -2.29 & -2.18 & -2.01 & -1.60 & -1.19 & -.68 & -.35 & -.50 & -.60 & -.93 & -1.15 & -1.36 \\
\hline 15 & -2.29 & -2.17 & -2.06 & -1.53 & -1.19 & -.66 & -.39 & -.50 & -.64 & -.90 & -1.09 & -1.35 \\
\hline 16 & -2.14 & -2.15 & -2.07 & -1.39 & -1.14 & -.65 & -.45 & -.50 & -.66 & -1.05 & -1.05 & -1.28 \\
\hline 17 & -2.16 & -2.16 & -2.04 & -1.43 & -1.21 & -.71 & -.40 & -.49 & -.71 & -.96 & -1.17 & -1.30 \\
\hline 18 & -2.19 & -2.09 & -2.03 & -1.54 & -1.22 & -.64 & -.44 & -.48 & -.77 & -.98 & -1.17 & -1.29 \\
\hline 19 & -2.27 & -1.95 & -2.01 & -1.55 & -1.21 & -.59 & -.39 & -.53 & -.81 & -1.01 & -1.10 & -1.26 \\
\hline 20 & -2.22 & -1.97 & -1.99 & -1.50 & -1.08 & -.60 & -.38 & -.54 & -.78 & -.96 & -1.06 & -1.23 \\
\hline 21 & -2.13 & -1.95 & -1.91 & -1.43 & -1.10 & -.59 & -.43 & -.52 & -.79 & -1.10 & -.99 & -1.05 \\
\hline 22 & -2.16 & -2.04 & -1.93 & -1.55 & -1.07 & -.50 & -.43 & -.56 & -.83 & -1.03 & -.94 & -1.25 \\
\hline 23 & -2.26 & -2.03 & -1.87 & -1.55 & -1.04 & -.55 & -.40 & -.57 & -.83 & -.94 & -1.09 & -1.37 \\
\hline 24 & -2.29 & -1.91 & -1.93 & -1.60 & -.96 & -.54 & -.37 & -.54 & -.83 & -1.04 & -1.18 & -1.26 \\
\hline 25 & -2.34 & -1.81 & -1.96 & -1.53 & -.93 & -.49 & -.39 & -.51 & -.80 & -1.01 & -1.12 & -1.10 \\
\hline 26 & -2.24 & -1.85 & -1.94 & -1.52 & -.98 & -.46 & -.38 & -.50 & -.79 & -1.06 & -1.17 & -1.00 \\
\hline 27 & -2.21 & -1.88 & -1.91 & -1.42 & -.93 & -.50 & -.42 & -.54 & -.87 & -1.14 & -1.17 & -1.08 \\
\hline 28 & -2.21 & -1.96 & -1.91 & -1.50 & -.98 & -.56 & -.43 & -.58 & -.90 & -1.05 & -1.04 & -1.12 \\
\hline 29 & -2.26 & -2.08 & -1.92 & -1.48 & -.96 & -.56 & -.44 & -.61 & -.84 & -1.01 & -1.05 & -1.21 \\
\hline 30 & -2.14 & - & -1.98 & -1.42 & -.98 & -.57 & -.42 & -.58 & -.82 & -1.02 & -1.13 & -1.10 \\
\hline 31 & -2.04 & - & -1.95 & - & -1.00 & - & -.42 & -.58 & - & -1.10 & - & -1.14 \\
\hline Mean & -2.28 & -2.11 & -1.99 & -1.61 & -1.17 & -.71 & -.44 & -.50 & -.71 & -.97 & -1.13 & -1.20 \\
\hline Max & -2.04 & -1.81 & -1.81 & -1.39 & -.93 & -.46 & -.35 & -.36 & -.56 & -.81 & -.94 & -1.00 \\
\hline Min & -2.45 & -2.35 & -2.14 & -1.91 & -1.40 & -1.10 & -.57 & -.61 & -.90 & -1.14 & -1.25 & -1.37 \\
\hline
\end{tabular}




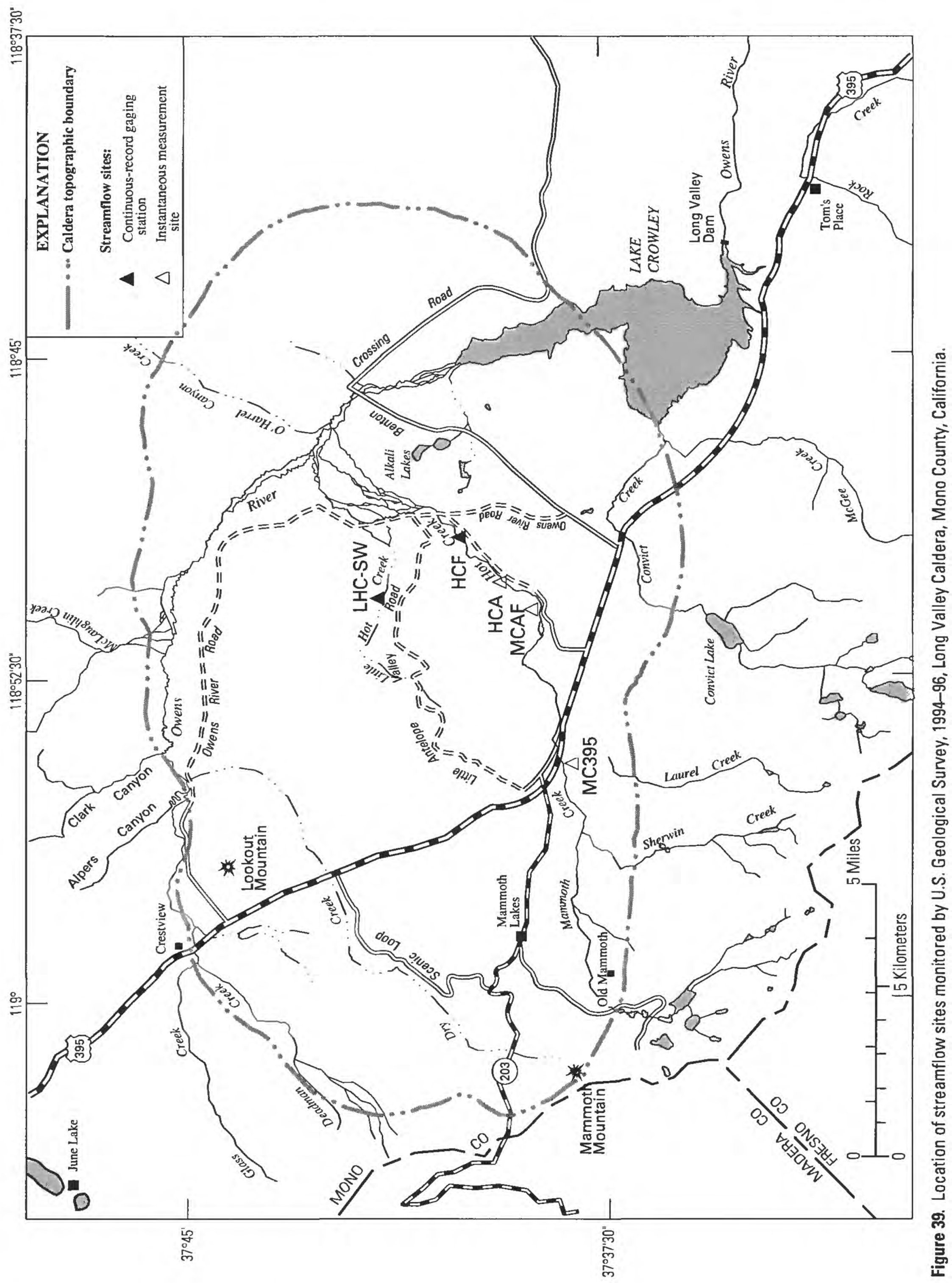




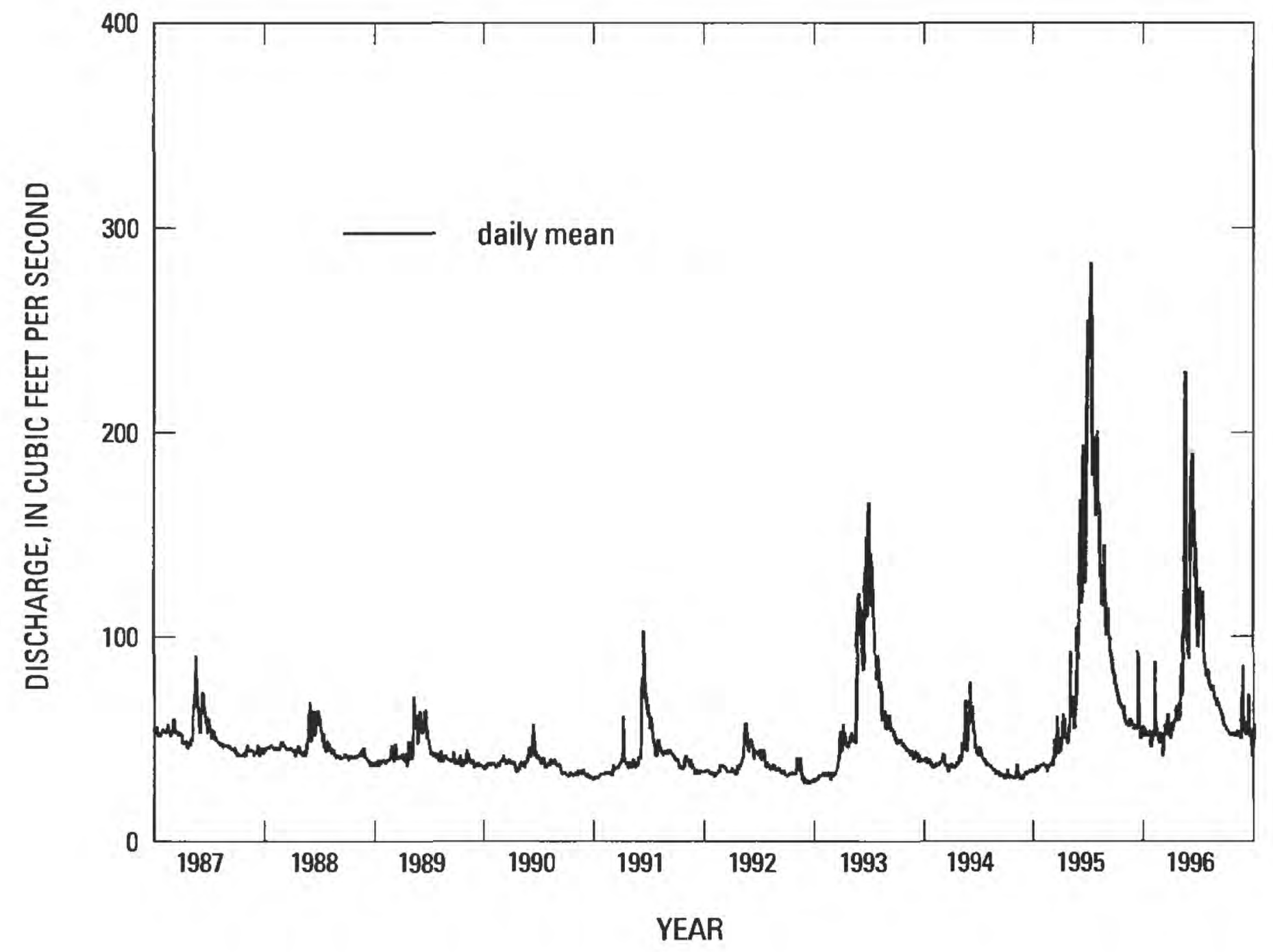

Figure 40. Daily mean discharge, Hot Creek Flume (HCF), 1987-96, Long Valley Caldera, Mono County, California. 


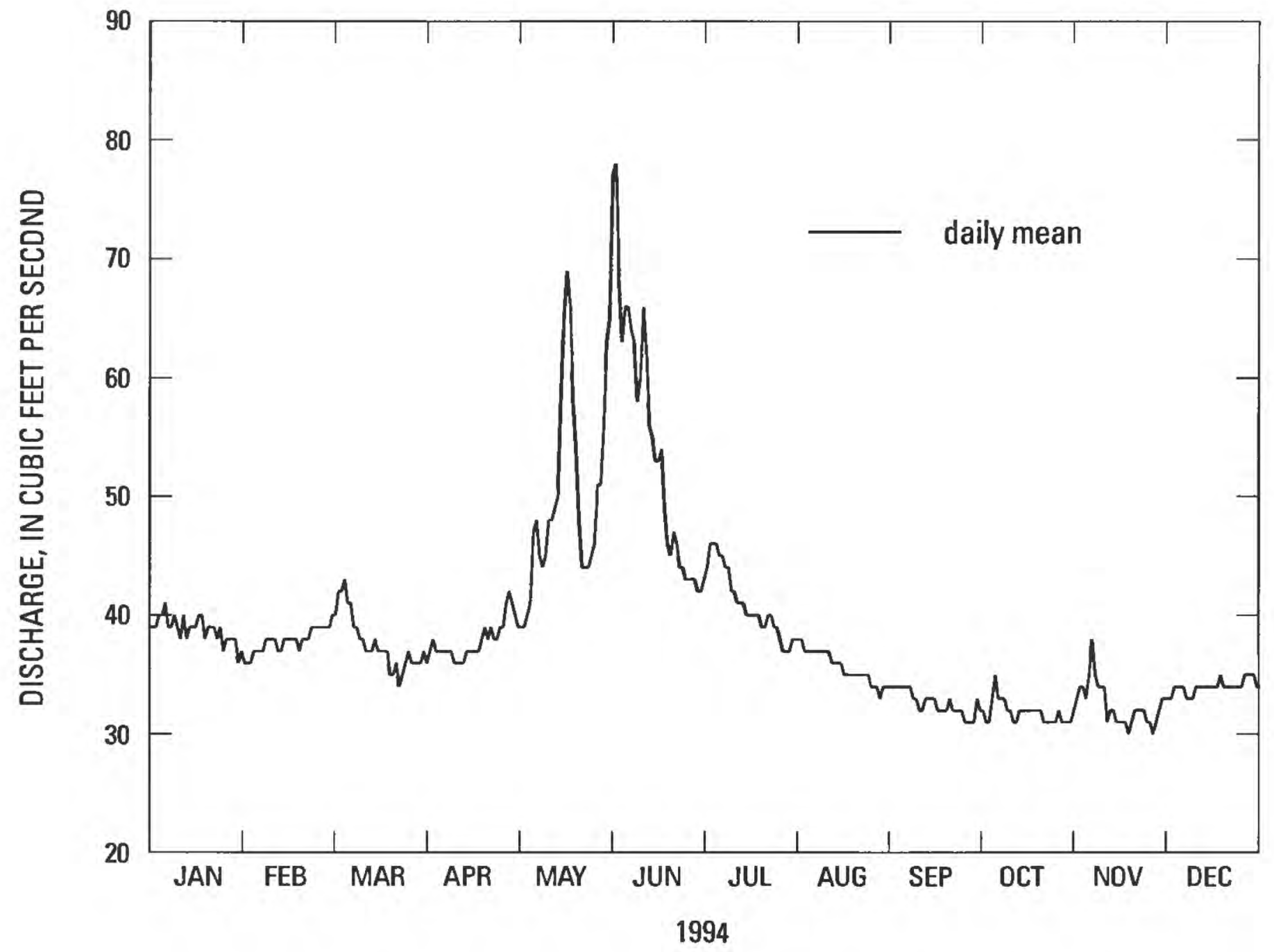

Figure 41. (A) Daily mean discharge, Hot Creek Flume (HCF), 1994, Long Valley Caldera, Mono County, California. 
Table 35. Daily mean discharge, Hot Creek Flume (HCF), 1994, Long Valley Caldera, California

[Values are in cubic feet per second. - , no data; Max, maximum; Min, minimum]

\begin{tabular}{|c|c|c|c|c|c|c|c|c|c|c|c|c|}
\hline Day & Jan & Feb & Mar & Apr & May & Jun & Jul & Aug & Sep & Oct & Nov & Dec \\
\hline 1 & 39 & 36 & 40 & 36 & 39 & 77 & 43 & 38 & 34 & 32 & 33 & 33 \\
\hline 2 & 39 & 36 & 40 & 37 & 39 & 78 & 44 & 38 & 34 & 31 & 34 & 33 \\
\hline 3 & 40 & 37 & 42 & 38 & 39 & 68 & 46 & 37 & 34 & 31 & 34 & 34 \\
\hline 4 & 40 & 37 & 42 & 37 & 40 & 63 & 46 & 37 & 34 & 33 & 33 & 34 \\
\hline 5 & 41 & 37 & 43 & 37 & 41 & 66 & 46 & 37 & 34 & 35 & 35 & 34 \\
\hline 6 & 39 & 37 & 41 & 37 & 47 & 66 & 45 & 37 & 34 & 33 & 38 & 34 \\
\hline 7 & 39 & 38 & 41 & 37 & 48 & 64 & 45 & 37 & 34 & 33 & 35 & 33 \\
\hline 8 & 40 & 38 & 39 & 37 & 45 & 63 & 44 & 37 & 33 & 33 & 34 & 33 \\
\hline 9 & 39 & 38 & 39 & 37 & 44 & 58 & 44 & 37 & 33 & 32 & 34 & 33 \\
\hline 10 & 38 & 38 & 38 & 36 & 45 & 60 & 42 & 37 & 32 & 32 & 34 & 34 \\
\hline 11 & 40 & 37 & 38 & 36 & 48 & 66 & 42 & 37 & 32 & 31 & 31 & 34 \\
\hline 12 & 38 & 37 & 37 & 36 & 48 & 62 & 41 & 36 & 33 & 31 & 32 & 34 \\
\hline 13 & 39 & 38 & 37 & 36 & 49 & 56 & 41 & 36 & 33 & 32 & 32 & 34 \\
\hline 14 & 39 & 38 & 37 & 37 & 50 & 55 & 41 & 36 & 33 & 32 & 31 & 34 \\
\hline 15 & 39 & 38 & 38 & 37 & 58 & 53 & 40 & 36 & 33 & 32 & 31 & 34 \\
\hline 16 & 40 & 38 & 37 & 37 & 65 & 53 & 40 & 35 & 32 & 32 & 31 & 34 \\
\hline 17 & 40 & 38 & 37 & 37 & 69 & 54 & 40 & 35 & 32 & 32 & 31 & 34 \\
\hline 18 & 38 & 37 & 37 & 37 & 66 & 49 & 40 & 35 & 32 & 32 & 30 & 35 \\
\hline 19 & 39 & 38 & 37 & 38 & 58 & 46 & 40 & 35 & 32 & 32 & 31 & 34 \\
\hline 20 & 39 & 38 & 35 & 39 & 54 & 45 & 39 & 35 & 33 & 32 & 32 & 34 \\
\hline 21 & 39 & 38 & 35 & 38 & 48 & 47 & 39 & 35 & 32 & 31 & 32 & 34 \\
\hline 22 & 38 & 39 & 36 & 39 & 44 & 46 & 40 & 35 & 32 & 31 & 32 & 34 \\
\hline 23 & 39 & 39 & 34 & 38 & 44 & 44 & 40 & 35 & 32 & 31 & 32 & 34 \\
\hline 24 & 37 & 39 & 35 & 38 & 44 & 44 & 39 & 35 & 32 & 31 & 31 & 34 \\
\hline 25 & 38 & 39 & 36 & 39 & 45 & 43 & 39 & 34 & 31 & 31 & 31 & 34 \\
\hline 26 & 38 & 39 & 37 & 39 & 46 & 43 & 38 & 34 & 31 & 32 & 30 & 35 \\
\hline 27 & 38 & 39 & 36 & 41 & 51 & 43 & 37 & 34 & 31 & 31 & 31 & 35 \\
\hline 28 & 38 & 39 & 36 & 42 & 51 & 43 & 37 & 33 & 31 & 31 & 32 & 35 \\
\hline 29 & 36 & - & 36 & 41 & 56 & 42 & 37 & 34 & 33 & 31 & 33 & 35 \\
\hline 30 & 37 & - & 36 & 40 & 63 & 42 & 38 & 34 & 32 & 31 & 33 & 34 \\
\hline 31 & 36 & - & 37 & - & 65 & - & 38 & 34 & - & 32 & - & 34 \\
\hline Mean & 38.7 & 37.9 & 37.7 & 37.8 & 50.0 & 54.6 & 41.0 & 35.6 & 32.6 & 31.8 & 32.4 & 34.0 \\
\hline $\operatorname{Max}$ & 41 & 39 & 43 & 42 & 69 & 78 & 46 & 38 & 34 & 35 & 38 & 35 \\
\hline Min & 36 & 36 & 34 & 36 & 39 & 42 & 37 & 33 & 31 & 31 & 30 & 33 \\
\hline
\end{tabular}




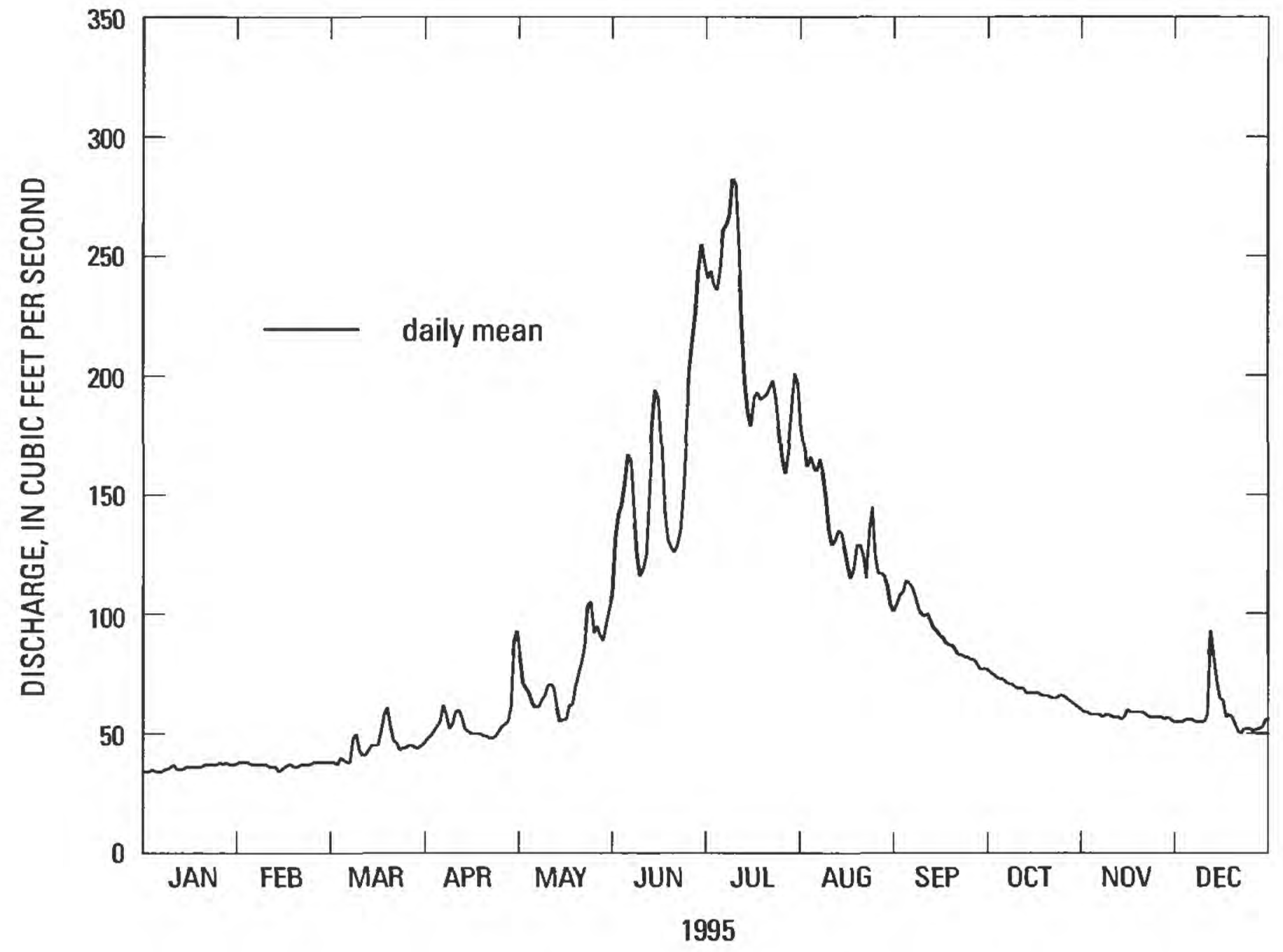

Figure 41. (B) Daily mean discharge, Hot Creek Flume (HCF), 1995, Long Valley Caldera, Mono County, California. 
Table 36. Daily mean discharge, Hot Creek Flume (HCF), 1995, Long Valley Caldera, California

[Values are in cubic feet per second. —, no data; Max, maximum; Min, minimum]

\begin{tabular}{|c|c|c|c|c|c|c|c|c|c|c|c|c|}
\hline Day & Jan & Feb & Mar & Apr & May & Jun & Jul & Aug & Sep & Oct & Nov & Dec \\
\hline 1 & 34 & 38 & 38 & 46 & 93 & 108 & 247 & 178 & 104 & 76 & 59 & 55 \\
\hline 2 & 34 & 38 & 38 & 48 & 82 & 132 & 241 & 170 & 108 & 75 & 59 & 55 \\
\hline 3 & 35 & 38 & 38 & 49 & 71 & 142 & 244 & 162 & 109 & 74 & 58 & 55 \\
\hline 4 & 34 & 37 & 37 & 51 & 69 & 146 & 238 & 166 & 114 & 73 & 58 & 56 \\
\hline 5 & 34 & 37 & 40 & 53 & 67 & 155 & 236 & 162 & 113 & 73 & 58 & 56 \\
\hline 6 & 34 & 37 & 39 & 55 & 63 & 167 & 245 & 160 & 111 & 72 & 58 & 56 \\
\hline 7 & 35 & 37 & 38 & 62 & 61 & 164 & 261 & 165 & 107 & 71 & 57 & 55 \\
\hline 8 & 35 & 37 & 38 & 58 & 61 & 143 & 263 & 159 & 102 & 71 & 58 & 55 \\
\hline 9 & 36 & 37 & 48 & 52 & 64 & 125 & 267 & 149 & 100 & 70 & 58 & 55 \\
\hline 10 & 37 & 36 & 50 & 54 & 66 & 116 & 283 & 135 & 99 & 69 & 57 & 55 \\
\hline 11 & 35 & 36 & 43 & 59 & 70 & 119 & 280 & 129 & 100 & 69 & 57 & 58 \\
\hline 12 & 35 & 36 & 41 & 60 & 71 & 125 & 254 & 131 & 97 & 69 & 57 & 93 \\
\hline 13 & 35 & 34 & 41 & 57 & 69 & 151 & 222 & 135 & 94 & 67 & 56 & 82 \\
\hline 14 & 36 & 35 & 43 & 52 & 61 & 179 & 198 & 134 & 93 & 67 & 57 & 72 \\
\hline 15 & 36 & 36 & 45 & 51 & 55 & 194 & 185 & 128 & 91 & 67 & 60 & 65 \\
\hline 16 & 36 & 37 & 45 & 50 & 56 & 190 & 179 & 121 & 90 & 67 & 59 & 64 \\
\hline 17 & 36 & 37 & 45 & 50 & 56 & 169 & 191 & 115 & 88 & 67 & 59 & 57 \\
\hline 18 & 36 & 36 & 51 & 50 & 62 & 142 & 193 & 119 & 87 & 66 & 59 & 58 \\
\hline 19 & 36 & 36 & 58 & 50 & 62 & 131 & 190 & 129 & 87 & 66 & 59 & 57 \\
\hline 20 & 37 & 37 & 61 & 49 & 70 & 128 & 191 & 129 & 85 & 66 & 59 & 54 \\
\hline 21 & 37 & 37 & 53 & 49 & 75 & 126 & 192 & 125 & 83 & 65 & 58 & 51 \\
\hline 22 & 37 & 37 & 47 & 48 & 80 & 129 & 195 & 115 & 83 & 65 & 57 & 50 \\
\hline 23 & 37 & 37 & 46 & 48 & 87 & 135 & 198 & 135 & 82 & 65 & 57 & 52 \\
\hline 24 & 37 & 38 & 43 & 49 & 103 & 152 & 190 & 145 & 82 & 66 & 57 & 52 \\
\hline 25 & 38 & 38 & 44 & 51 & 105 & 176 & 177 & 125 & 81 & 66 & 57 & 52 \\
\hline 26 & 37 & 38 & 44 & 53 & 92 & 202 & 165 & 117 & 81 & 65 & 57 & 51 \\
\hline 27 & 38 & 38 & 45 & 54 & 95 & 214 & 159 & 117 & 79 & 64 & 56 & 52 \\
\hline 28 & 37 & 38 & 45 & 55 & 91 & 225 & 170 & 116 & 77 & 63 & 57 & 52 \\
\hline 29 & 37 & - & 44 & 61 & 89 & 244 & 185 & 112 & 77 & 62 & 56 & 53 \\
\hline 30 & 37 & - & 44 & 88 & 96 & 255 & 201 & 104 & 77 & 61 & 55 & 56 \\
\hline 31 & 38 & - & 45 & - & 102 & - & 197 & 101 & - & 60 & - & 56 \\
\hline Mean & 36.0 & 36.9 & 44.4 & 53.7 & 75.6 & 159 & 214 & 135 & 92.7 & 67.6 & 57.6 & 57.7 \\
\hline Max & 38 & 38 & 61 & 88 & 105 & 255 & 283 & 178 & 114 & 76 & 60 & 93 \\
\hline Min & 34 & 34 & 37 & 46 & 55 & 108 & 159 & 101 & 77 & 60 & 55 & 50 \\
\hline
\end{tabular}




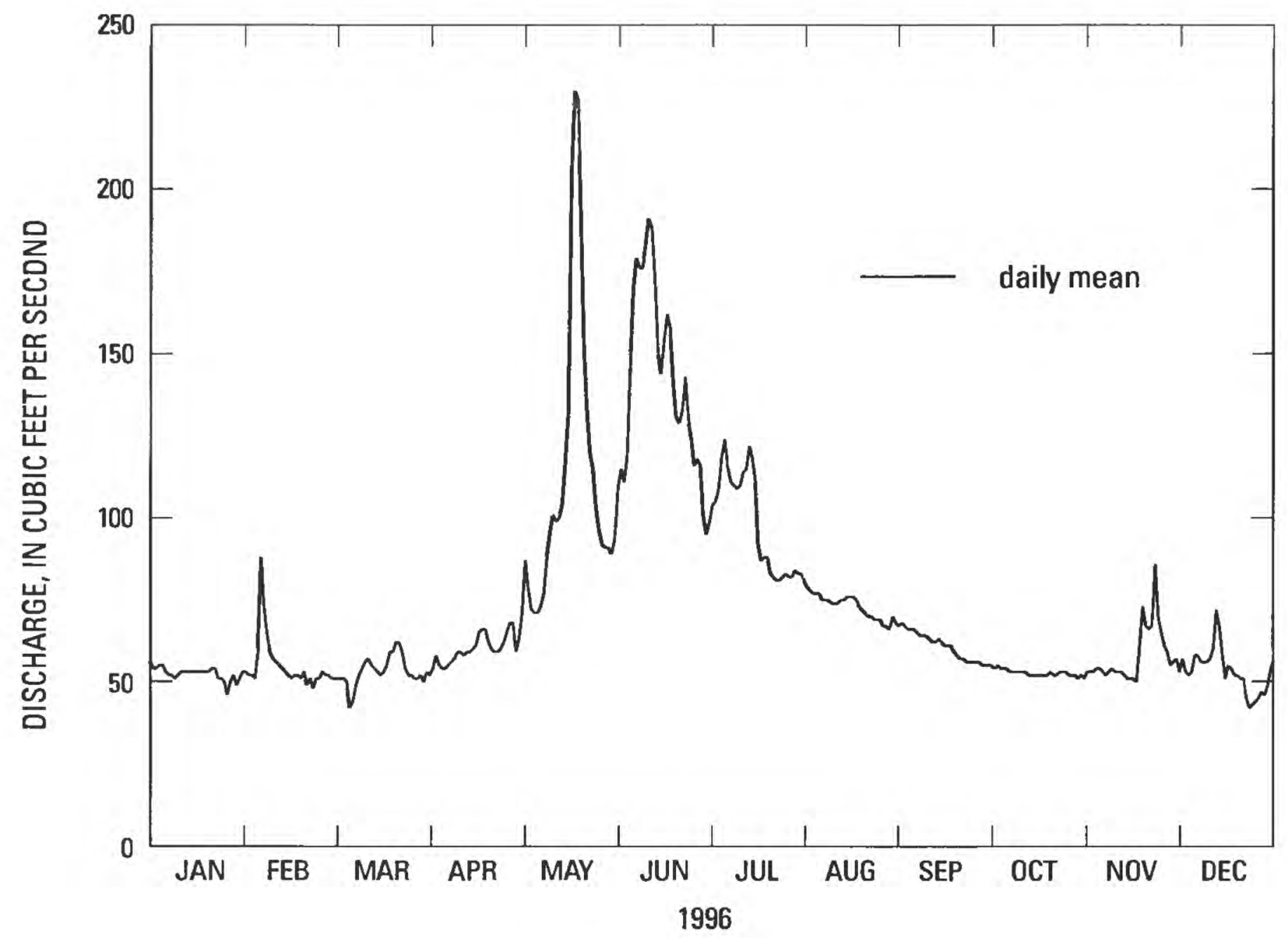

Figure 41. (C) Daily mean discharge, Hot Creek Flume (HCF), 1996, Long Valley Caldera, Mono County, California. 
Table 37. Daily mean discharge, Hot Creek Flume (HCF), 1996, Long Valley Caldera, California

[Values are in cubic feet per second. _, no data; Max, maximum; Min, minimum]

\begin{tabular}{|c|c|c|c|c|c|c|c|c|c|c|c|c|}
\hline Day & Jan & Feb & Mar & Apr & May & Jun & Jul & Aug & Sep & Oct & Nov & Dec \\
\hline 1 & 54 & 52 & 51 & 54 & 87 & 115 & 104 & 79 & 68 & 54 & 53 & 57 \\
\hline 2 & 54 & 52 & 51 & 58 & 78 & 111 & 105 & 78 & 67 & 55 & 53 & 53 \\
\hline 3 & 55 & 51 & 51 & 55 & 72 & 119 & 109 & 77 & 66 & 54 & 54 & 52 \\
\hline 4 & 55 & 59 & 50 & 54 & 71 & 146 & 119 & 77 & 66 & 54 & 54 & 53 \\
\hline 5 & 53 & 88 & 42 & 54 & 71 & 169 & 124 & 77 & 66 & 54 & 53 & 58 \\
\hline 6 & 52 & 72 & 44 & 55 & 73 & 179 & 115 & 75 & 65 & 53 & 52 & 58 \\
\hline 7 & 52 & 64 & 49 & 56 & 77 & 176 & 111 & 75 & 64 & 53 & 53 & 56 \\
\hline 8 & 51 & 59 & 52 & 57 & 88 & 176 & 110 & 75 & 64 & 53 & 54 & 56 \\
\hline 9 & 52 & 57 & 54 & 59 & 95 & 183 & 109 & 74 & 64 & 53 & 53 & 56 \\
\hline 10 & 53 & 56 & 56 & 59 & 101 & 191 & 110 & 74 & 63 & 53 & 53 & 57 \\
\hline 11 & 53 & 55 & 57 & 58 & 99 & 188 & 114 & 74 & 62 & 53 & 53 & 60 \\
\hline 12 & 53 & 54 & 55 & 59 & 100 & 172 & 115 & 75 & 62 & 52 & 52 & 72 \\
\hline 13 & 53 & 53 & 54 & 59 & 104 & 151 & 122 & 75 & 63 & 52 & 51 & 67 \\
\hline 14 & 53 & 52 & 53 & 60 & 117 & 144 & 118 & 76 & 62 & 52 & 51 & 58 \\
\hline 15 & 53 & 51 & 52 & 61 & 132 & 154 & 111 & 76 & 61 & 52 & 51 & 51 \\
\hline 16 & 53 & 52 & 53 & 65 & 205 & 162 & 92 & 76 & 61 & 52 & 50 & 55 \\
\hline 17 & 53 & 52 & 55 & 66 & 230 & 158 & 87 & 75 & 61 & 52 & 61 & 54 \\
\hline 18 & 53 & 51 & 59 & 66 & 227 & 142 & 88 & 73 & 59 & 52 & 73 & 52 \\
\hline 19 & 53 & 53 & 59 & 62 & 192 & 131 & 88 & 72 & 58 & 53 & 67 & 52 \\
\hline 20 & 54 & 49 & 62 & 60 & 152 & 129 & 83 & 71 & 57 & 52 & 66 & 51 \\
\hline 21 & 54 & 51 & 62 & 59 & 133 & 133 & 82 & 70 & 57 & 52 & 67 & 51 \\
\hline 22 & 51 & 48 & 59 & 59 & 120 & 143 & 81 & 70 & 56 & 53 & 86 & 45 \\
\hline 23 & 51 & 51 & 54 & 60 & 115 & 130 & 81 & 69 & 56 & 53 & 70 & 42 \\
\hline 24 & 50 & 51 & 52 & 62 & 103 & 124 & 82 & 69 & 56 & 53 & 65 & 43 \\
\hline 25 & 46 & 53 & 52 & 65 & 96 & 116 & 83 & 69 & 56 & 52 & 61 & 44 \\
\hline 26 & 50 & 52 & 51 & 68 & 92 & 118 & 82 & 67 & 56 & 52 & 59 & 45 \\
\hline 27 & 52 & 52 & 51 & 68 & 91 & 116 & 82 & 67 & 55 & 52 & 55 & 47 \\
\hline 28 & 49 & 51 & 52 & 59 & 91 & 101 & 84 & 66 & 55 & 51 & 56 & 46 \\
\hline 29 & 51 & 51 & 50 & 63 & 89 & 95 & 83 & 70 & 55 & 52 & 57 & 49 \\
\hline 30 & 53 & - & 53 & 71 & 94 & 99 & 83 & 68 & 55 & 51 & 53 & 54 \\
\hline 31 & 53 & - & 52 & - & 109 & - & 81 & 67 & - & 53 & - & 57 \\
\hline Mean & 52.3 & 54.9 & 53.1 & 60.4 & 113 & 142 & 98.0 & 72.8 & 60.5 & 52.6 & 57.9 & 53.3 \\
\hline Max & 55 & 88 & 62 & 71 & 230 & 191 & 124 & 79 & 68 & 55 & 86 & 72 \\
\hline Min & 46 & 48 & 42 & 54 & 71 & 95 & 81 & 66 & 55 & 51 & 50 & 42 \\
\hline
\end{tabular}




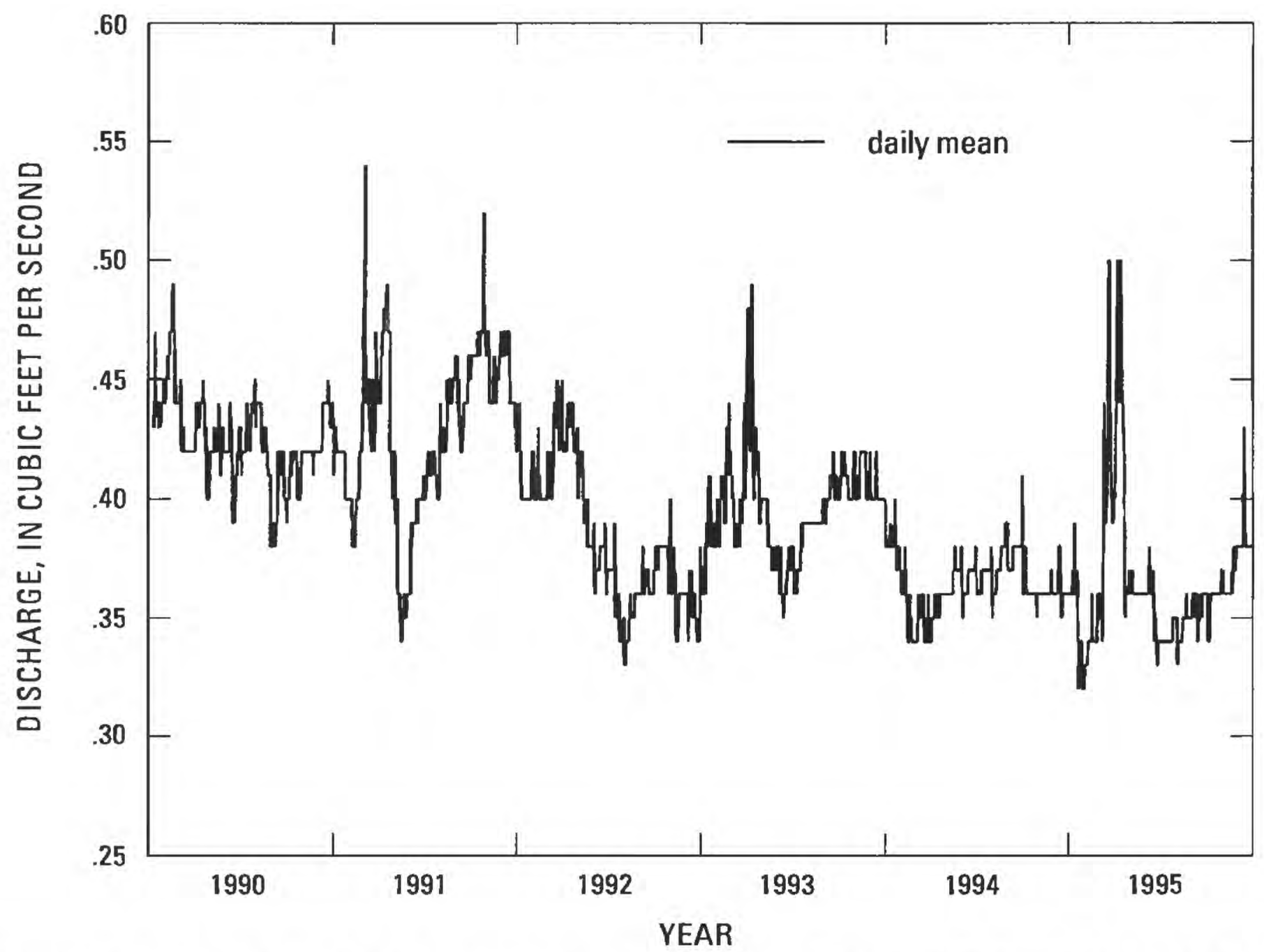

Figure 42. Daily mean discharge, Little Hot Creek downstream from Little Hot Creek Spring (LHC-SW), 1990-95, Long Valley Caldera, Mono County, California.

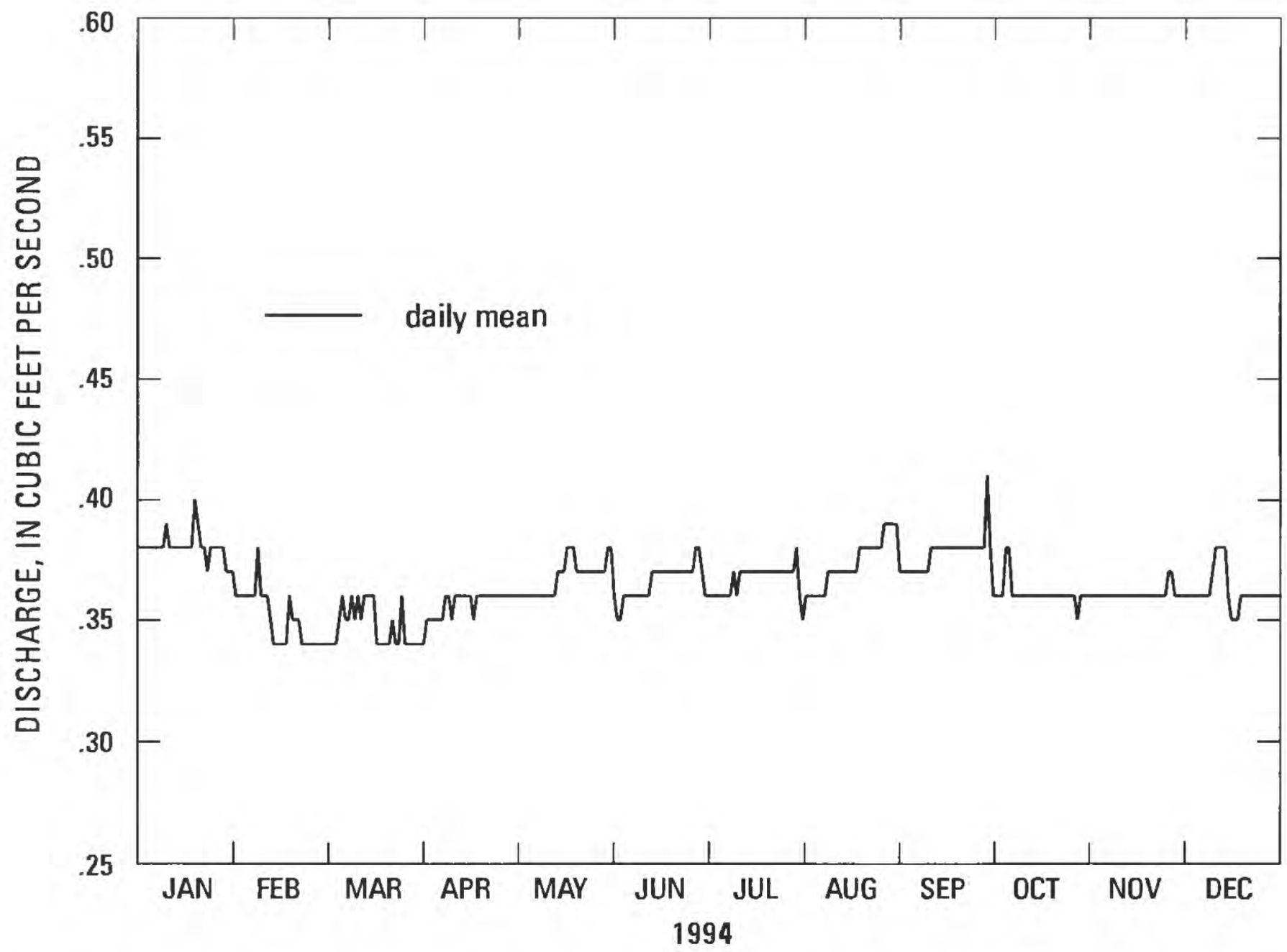

Figure 43. (A) Daily mean discharge, Little Hot Creek downstream from Little Hot Creek Spring (LHC-SW), 1994, Long Valley Caldera, Mono County, California. 
Table 38. Daily mean discharge, Little Hot Creek downstream from Little Hot Creek Spring (LHC-SW), 1994, Long Valley Caldera, California [Values are in cubic feet per second. —. no data; Max, maximum; Min. minimum]

\begin{tabular}{|c|c|c|c|c|c|c|c|c|c|c|c|c|}
\hline Day & Jan & Feb & Mar & Apr & May & Jun & Jul & Aug & Sep & Oct & Nov & Dec \\
\hline 1 & 0.38 & 0.36 & 0.34 & 0.34 & 0.36 & 0.36 & 0.36 & 0.36 & 0.37 & 0.36 & 0.36 & 0.36 \\
\hline 2 & .38 & .36 & .34 & .35 & .36 & .35 & .36 & .36 & .37 & .36 & .36 & .36 \\
\hline 3 & .38 & .36 & .34 & .35 & .36 & .35 & .36 & .36 & .37 & .36 & .36 & .36 \\
\hline 4 & .38 & .36 & .34 & .35 & .36 & .36 & .36 & .36 & .37 & .38 & .36 & .36 \\
\hline 5 & .38 & .36 & .35 & .35 & .36 & .36 & .36 & .36 & .37 & .38 & .36 & .36 \\
\hline 6 & .38 & .36 & .36 & .35 & .36 & .36 & .36 & .36 & .37 & .36 & .36 & .36 \\
\hline 7 & .38 & .38 & .35 & .35 & .36 & .36 & .36 & .36 & .37 & .36 & .36 & .36 \\
\hline 8 & .38 & .36 & .35 & .36 & .36 & .36 & .36 & .37 & .37 & .36 & .36 & .36 \\
\hline 9 & .39 & .36 & .36 & .36 & .36 & .36 & .37 & .37 & .37 & .36 & .36 & .37 \\
\hline 10 & .38 & .36 & .35 & .35 & .36 & .36 & .36 & .37 & .38 & .36 & .36 & .38 \\
\hline 11 & .38 & .35 & .36 & .36 & .36 & .36 & .37 & .37 & .38 & .36 & .36 & .38 \\
\hline 12 & .38 & .34 & .35 & .36 & .36 & .36 & .37 & .37 & .38 & .36 & .36 & .38 \\
\hline 13 & .38 & .34 & .36 & .36 & .36 & .37 & .37 & .37 & .38 & .36 & .36 & .38 \\
\hline 14 & .38 & .34 & .36 & .36 & .37 & .37 & .37 & .37 & .38 & .36 & .36 & .36 \\
\hline 15 & .38 & .34 & .36 & .36 & .37 & .37 & .37 & .37 & .38 & .36 & .36 & .35 \\
\hline 16 & .38 & .34 & .36 & .36 & .37 & .37 & .37 & .37 & .38 & .36 & .36 & .35 \\
\hline 17 & .38 & .36 & .34 & .35 & .38 & .37 & .37 & .37 & .38 & .36 & .36 & .35 \\
\hline 18 & .40 & .35 & .34 & .36 & .38 & .37 & .37 & .38 & .38 & .36 & .36 & .36 \\
\hline 19 & .39 & .35 & .34 & .36 & .38 & .37 & .37 & .38 & .38 & .36 & .36 & .36 \\
\hline 20 & .38 & .35 & .34 & .36 & .37 & .37 & .37 & .38 & .38 & .36 & .36 & .36 \\
\hline 21 & .38 & .34 & .34 & .36 & .37 & .37 & .37 & .38 & .38 & .36 & .36 & .36 \\
\hline 22 & .37 & .34 & .35 & .36 & .37 & .37 & .37 & .38 & .38 & .36 & .36 & .36 \\
\hline 23 & .38 & .34 & .34 & .36 & .37 & .37 & .37 & .38 & .38 & .36 & .36 & .36 \\
\hline 24 & .38 & .34 & .34 & .36 & .37 & .37 & .37 & .38 & .38 & .36 & .36 & .36 \\
\hline 25 & .38 & .34 & .36 & .36 & .37 & .37 & .37 & .38 & .38 & .36 & .37 & .36 \\
\hline 26 & .38 & .34 & .34 & .36 & .37 & .37 & .37 & .39 & .38 & .36 & .37 & .36 \\
\hline 27 & .38 & .34 & .34 & .36 & .37 & .38 & .37 & .39 & .38 & .35 & .36 & .36 \\
\hline 28 & .37 & .34 & .34 & .36 & .37 & .38 & .37 & .39 & .41 & .36 & .36 & .36 \\
\hline 29 & .37 & - & .34 & .36 & .37 & .37 & .38 & .39 & .38 & .36 & .36 & .36 \\
\hline 30 & .37 & - & .34 & .36 & .38 & .36 & .36 & .39 & .36 & .36 & .36 & .36 \\
\hline 31 & .36 & - & .34 & - & .38 & - & .35 & .37 & - & .36 & - & .36 \\
\hline Mean & .38 & .35 & .35 & .36 & .37 & .37 & .37 & .37 & .38 & .36 & .36 & .36 \\
\hline Max & .40 & .38 & .36 & .36 & .38 & .38 & .38 & .39 & .41 & .38 & .37 & .38 \\
\hline Min & .36 & .34 & .34 & .34 & .36 & .35 & .35 & .36 & .36 & .35 & .36 & .35 \\
\hline
\end{tabular}




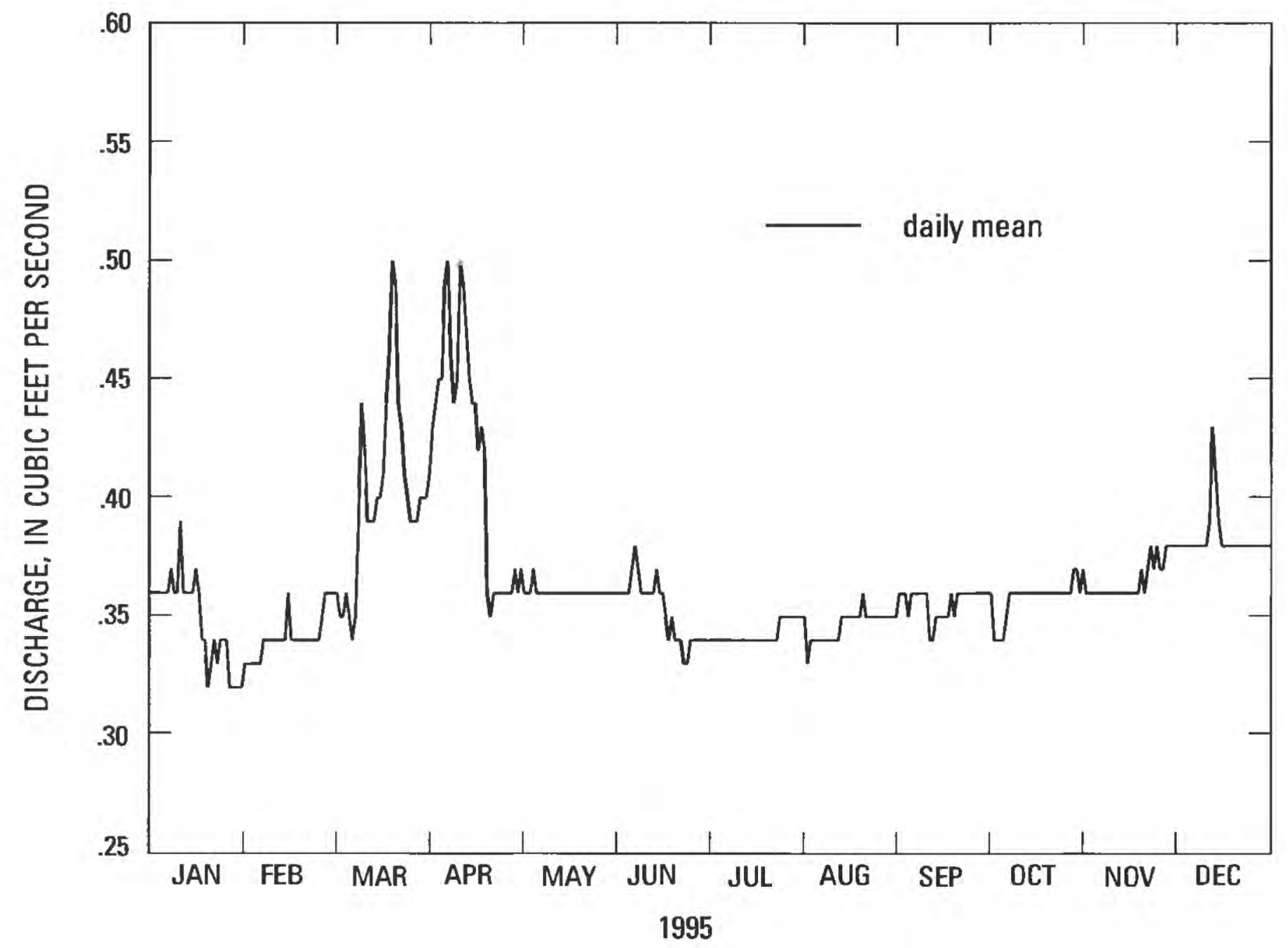

Figure 43. (B) Daily mean discharge, Little Hot Creek downstream from Little Hot Creek Spring (LHC-SW), 1995, Long Valley Caldera, Mono County, California. 
Table 39. Daily mean discharge, Little Hot Creek downstream from Little Hot Creek Spring (LHC-SW), 1995, Long Valley Caldera, California [Values are in cubic feet per second. - , no data; Max, maximum; Min, minimum]

\begin{tabular}{|c|c|c|c|c|c|c|c|c|c|c|c|c|}
\hline Day & Jan & Feb & Mar & Apr & May & Jun & Jul & Aug & Sep & Oct & Nov & Dec \\
\hline 1 & 0.36 & 0.33 & 0.36 & 0.41 & 0.37 & 0.36 & 0.34 & 0.35 & 0.36 & 0.36 & 0.36 & 0.38 \\
\hline 2 & .36 & .33 & .36 & .43 & .36 & .36 & .34 & .33 & .36 & .34 & .36 & .38 \\
\hline 3 & .36 & .33 & .35 & .44 & .36 & .36 & .34 & .34 & .36 & .34 & .36 & .38 \\
\hline 4 & .36 & .33 & .35 & .45 & .36 & .36 & .34 & .34 & .35 & .34 & .36 & .38 \\
\hline 5 & .36 & .33 & .36 & .45 & .37 & .36 & .34 & .34 & .36 & .34 & .36 & .38 \\
\hline 6 & .36 & .34 & .35 & .49 & .36 & .37 & .34 & .34 & .36 & .35 & .36 & .38 \\
\hline 7 & .37 & .34 & .34 & .50 & .36 & .38 & .34 & .34 & .36 & .36 & .36 & .38 \\
\hline 8 & .36 & .34 & .35 & .46 & .36 & .37 & .34 & .34 & .36 & .36 & .36 & .38 \\
\hline 9 & .36 & .34 & .39 & .44 & .36 & .36 & .34 & .34 & .36 & .36 & .36 & .38 \\
\hline 10 & .39 & .34 & .44 & .45 & .36 & .36 & .34 & .34 & .36 & .36 & .36 & .38 \\
\hline 11 & .36 & .34 & .42 & .50 & .36 & .36 & .34 & .34 & .34 & .36 & .36 & .39 \\
\hline 12 & .36 & .34 & .39 & .49 & .36 & .36 & .34 & .34 & .34 & .36 & .36 & .43 \\
\hline 13 & .36 & .34 & .39 & .47 & .36 & .36 & .34 & .35 & .35 & .36 & .36 & .41 \\
\hline 14 & .36 & .36 & .39 & .45 & .36 & .37 & .34 & .35 & .35 & .36 & .36 & .39 \\
\hline 15 & .37 & .34 & .40 & .44 & .36 & .36 & .34 & .35 & .35 & .36 & .36 & .38 \\
\hline 16 & .36 & .34 & .40 & .44 & .36 & .36 & .34 & .35 & .35 & .36 & .36 & .38 \\
\hline 17 & .34 & .34 & .41 & .42 & .36 & .35 & .34 & .35 & .35 & .36 & .36 & .38 \\
\hline 18 & .34 & .34 & .44 & .43 & .36 & .34 & .34 & .35 & .36 & .36 & .36 & .38 \\
\hline 19 & .32 & .34 & .46 & .42 & .36 & .35 & .34 & .35 & .35 & .36 & .37 & .38 \\
\hline 20 & .33 & .34 & .50 & .36 & .36 & .34 & .34 & .36 & .36 & .36 & .36 & .38 \\
\hline 21 & .34 & .34 & .49 & .35 & .36 & .34 & .34 & .35 & .36 & .36 & .37 & .38 \\
\hline 22 & .33 & .34 & .44 & .36 & .36 & .34 & .34 & .35 & .36 & .36 & .38 & .38 \\
\hline 23 & .34 & .34 & .43 & .36 & .36 & .33 & .34 & .35 & .36 & .36 & .37 & .38 \\
\hline 24 & .34 & .34 & .41 & .36 & .36 & .33 & .35 & .35 & .36 & .36 & .38 & .38 \\
\hline 25 & .34 & .35 & .40 & .36 & .36 & .34 & .35 & .35 & .36 & .36 & .37 & .38 \\
\hline 26 & .32 & .36 & .39 & .36 & .36 & .34 & .35 & .35 & .36 & .36 & .37 & .38 \\
\hline 27 & .32 & .36 & .39 & .36 & .36 & .34 & .35 & .35 & .36 & .36 & .38 & .38 \\
\hline 28 & .32 & .36 & .39 & .36 & .36 & .34 & .35 & .35 & .36 & .37 & .38 & .38 \\
\hline 29 & .32 & - & .40 & .37 & .36 & .34 & .35 & .35 & .36 & .37 & .38 & .38 \\
\hline 30 & .32 & - & .40 & .36 & .36 & .34 & .35 & .35 & .36 & .36 & .38 & .38 \\
\hline 31 & .33 & - & .40 & - & .36 & - & .35 & .35 & - & .37 & - & .38 \\
\hline Mean & .35 & .34 & .40 & .42 & .36 & .35 & .34 & .35 & .36 & .36 & .37 & .38 \\
\hline Max & .39 & .36 & .50 & .50 & .37 & .38 & .35 & .36 & .36 & .37 & .38 & .43 \\
\hline Min & .32 & .33 & .34 & .35 & .36 & .33 & .34 & .33 & .34 & .34 & .36 & .38 \\
\hline
\end{tabular}




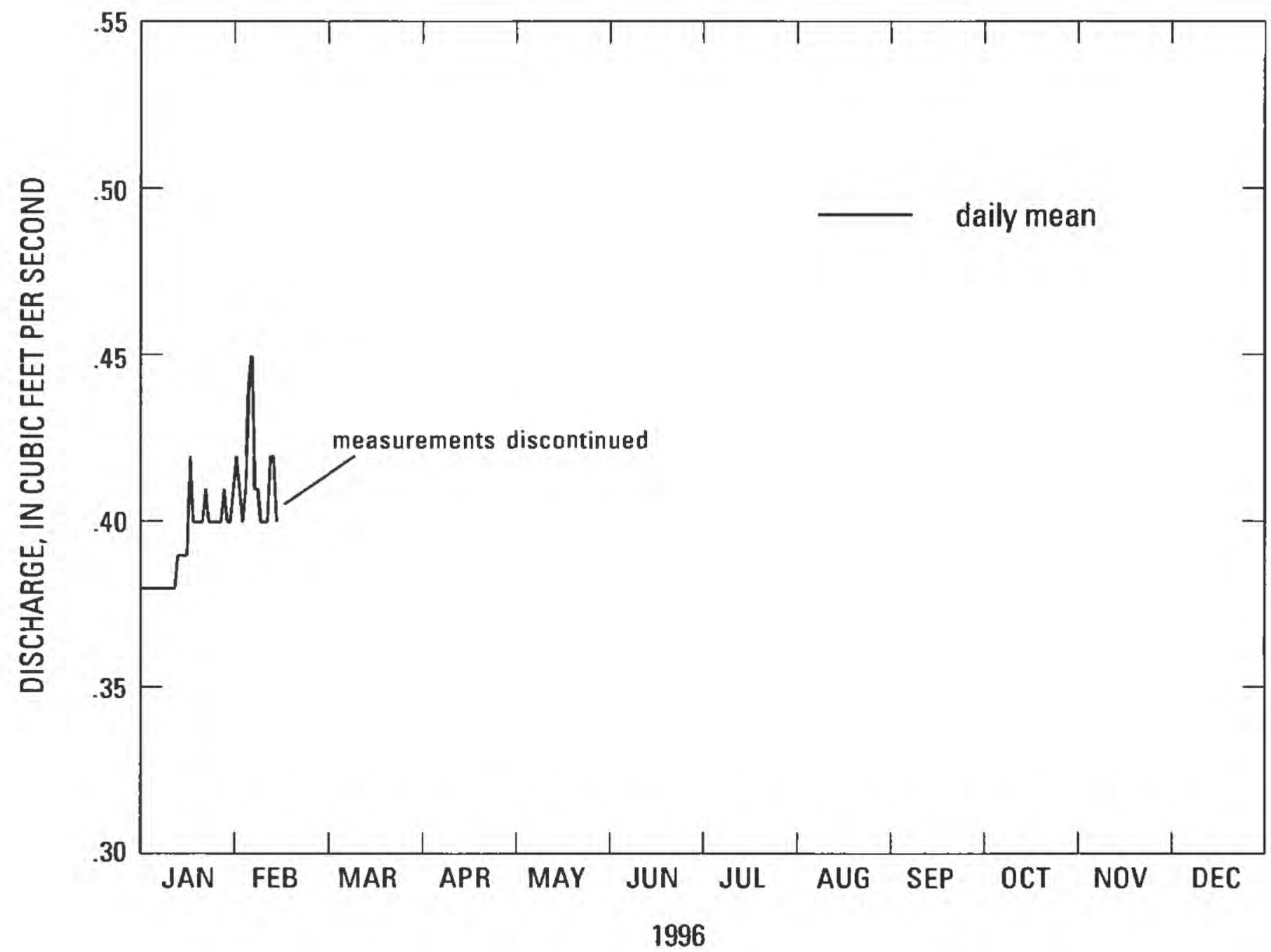

Figure 43. (C) Daily mean discharge, Little Hot Creek downstream from Little Hot Creek Spring (LHC-SW), 1996, Long Valley Caldera, Mono County, California. 
Table 40. Daily mean discharge, Little Hot Creek downstream from Little Hot Creek Spring (LHC-SW), 1996, Long Valley Caldera, California [Values are in cubic feet per second. -, no data; *, measurements discontinued. Max, maximum; Min, minimum]

\begin{tabular}{|c|c|c|c|c|c|c|c|c|c|c|c|c|}
\hline Day & Jan & Feb & Mar & Apr & May & Jun & Jul & Aug & Sep & Oct & Nov & Dec \\
\hline 1 & 0.38 & 0.41 & - & - & - & - & - & - & - & - & - & - \\
\hline 2 & .38 & .40 & - & - & - & - & - & - & - & - & - & - \\
\hline 3 & .38 & .41 & - & - & - & - & - & - & - & - & - & - \\
\hline 4 & .38 & .44 & - & - & - & - & - & - & - & - & - & - \\
\hline 5 & .38 & .45 & - & - & - & - & - & - & - & - & - & - \\
\hline 6 & .38 & .41 & - & - & - & - & - & - & - & - & - & - \\
\hline 7 & .38 & .41 & - & - & - & - & - & - & - & - & - & - \\
\hline 8 & .38 & .40 & - & - & - & - & - & - & - & - & - & - \\
\hline 9 & .38 & .40 & - & - & - & - & - & - & - & - & - & - \\
\hline 10 & .38 & .40 & - & - & - & - & - & - & - & - & - & - \\
\hline 11 & .38 & .42 & - & - & - & - & - & - & - & - & - & - \\
\hline 12 & .39 & .42 & - & - & - & - & - & - & - & - & - & - \\
\hline 13 & .39 & .40 & - & - & - & - & - & - & - & - & - & - \\
\hline 14 & .39 & $*$ & - & - & - & - & - & - & - & - & - & - \\
\hline 15 & .39 & - & - & - & - & - & - & - & - & - & - & - \\
\hline 16 & .42 & - & - & - & - & - & - & - & - & - & - & - \\
\hline 17 & .40 & - & - & - & - & - & - & - & - & - & - & - \\
\hline 18 & .40 & - & - & - & - & - & - & - & - & - & - & - \\
\hline 19 & .40 & - & - & - & - & - & - & - & - & - & - & - \\
\hline 20 & .40 & - & - & - & - & - & - & - & - & - & - & - \\
\hline 21 & .41 & - & - & - & - & - & - & - & - & - & - & - \\
\hline 22 & .40 & - & - & - & - & - & - & - & - & - & - & - \\
\hline 23 & .40 & - & - & - & - & - & - & - & - & - & - & - \\
\hline 24 & .40 & - & - & - & - & - & - & - & - & - & - & - \\
\hline 25 & .40 & - & - & - & - & - & - & - & - & - & - & - \\
\hline 26 & .40 & - & - & - & - & - & - & - & - & - & - & - \\
\hline 27 & .41 & - & - & - & - & - & - & - & - & - & - & - \\
\hline 28 & .40 & - & - & - & - & - & - & - & - & - & - & - \\
\hline 29 & .40 & - & - & - & - & - & - & - & - & - & - & - \\
\hline 30 & .41 & - & - & - & - & - & - & - & - & - & - & - \\
\hline 31 & .42 & - & - & - & - & - & - & - & - & - & - & - \\
\hline Mean & .39 & .41 & - & - & - & - & - & - & - & - & - & - \\
\hline Max & .42 & .45 & - & - & - & - & - & - & - & - & - & - \\
\hline Min & .38 & .40 & - & - & - & - & - & - & - & - & - & - \\
\hline
\end{tabular}


Table 41. Instantaneous discharge measurements, Mammoth Creek at Highway 395 (MC395), 1994-96, Long Valley Caldera, California

[Discharge is in cubic feet per second]

\begin{tabular}{lc}
\hline Date & Discharge \\
\hline $01-10-94$ & 5.19 \\
$04-12-94$ & 7.37 \\
$07-11-94$ & 10.1 \\
$10-19-94$ & 5.81 \\
$03-22-95$ & 8.10 \\
$04-17-95$ & 10.7 \\
$10-16-95$ & 18.7 \\
$01-24-96$ & 9.88 \\
$04-23-96$ & 17.8 \\
$07-16-96$ & 49.3 \\
$10-16-96$ & 9.82 \\
\hline
\end{tabular}

Table 42. Instantaneous discharge measurements, Mammoth Creek upstream of Hot Creek Fish Hatchery (MCAF), 1994-96, Long Valley Caldera, California

[Discharge is in cubic feet per second]

\begin{tabular}{lc}
\hline Date & Discharge \\
\hline $01-10-94$ & 3.30 \\
$04-12-94$ & 5.53 \\
$07-11-94$ & 6.74 \\
$10-19-94$ & 3.18 \\
$01-17-95$ & 8.94 \\
$04-18-95$ & 13.7 \\
$10-16-95$ & 19.4 \\
$01-24-96$ & 9.62 \\
$04-23-96$ & 17.4 \\
$07-16-96$ & 38.5 \\
$10-16-96$ & 7.11 \\
\hline
\end{tabular}

Table 43. Instantaneous discharge measurements and gage heights, Hot Creek upstream of Gorge Geyser (HCA), 1994-96, Long Valley Caldera, California

[Discharge is in cubic feet per second]

\begin{tabular}{lcc}
\hline Date & Gage height & Discherge \\
\hline $01-12-94$ & 0.75 & 29.7 \\
$02-15-94$ & .66 & 29.4 \\
$03-10-94$ & .65 & 29.9 \\
$04-13-94$ & .75 & 26.7 \\
$05-18-94$ & 1.08 & 59.9 \\
$06-21-94$ & .99 & 43.2 \\
$07-13-94$ & .98 & 35.8 \\
$08-09-94$ & .97 & 30.8 \\
$09-14-94$ & .87 & 29.3 \\
$10-20-94$ & .78 & 28.8 \\
$01-18-95$ & .62 & 28.4 \\
$04-19-95$ & .76 & 41.2 \\
$07-12-95$ & 1.55 & 231 \\
$10-17-95$ & .91 & 54.9 \\
$01-25-96$ & .72 & 29.5 \\
$04-24-96$ & 1.09 & 47.3 \\
$07-17-96$ & 1.12 & 73.8 \\
$10-17-96$ & .85 & 39.4 \\
\hline
\end{tabular}




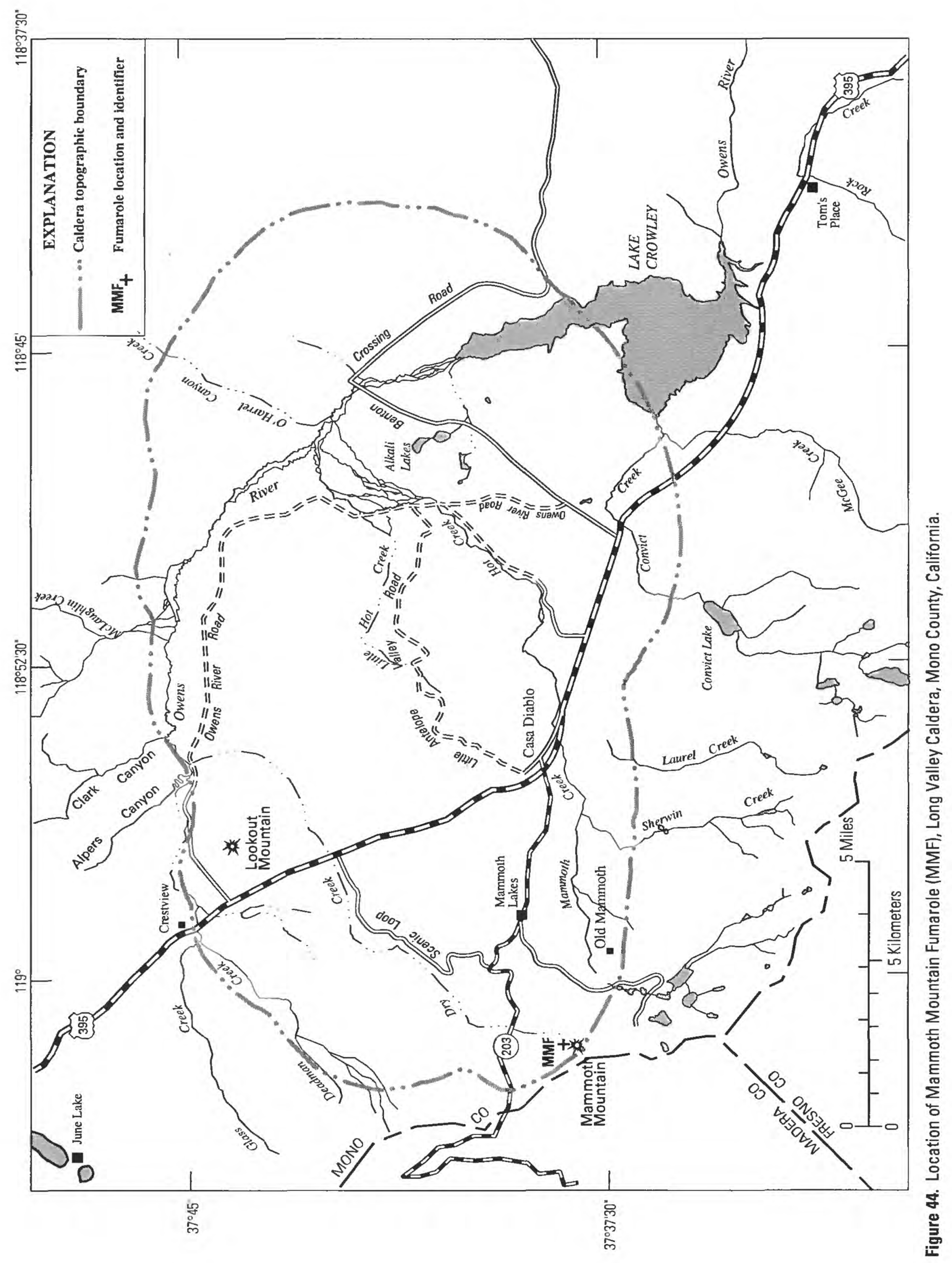




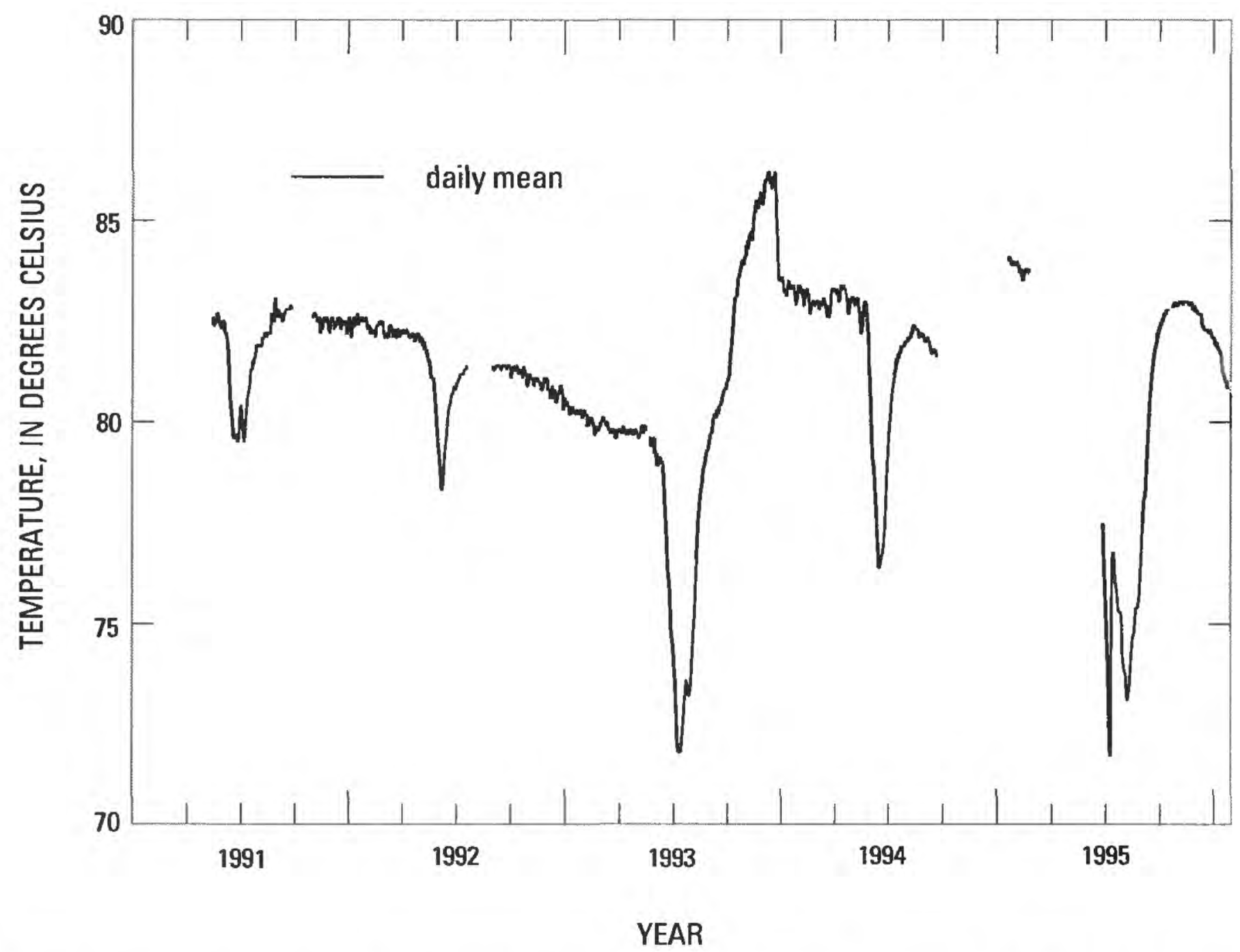

Figure 45. Daily mean vent gas temperature, Mammoth Mountain Fumarole (MMF), 1991-95, Long Valley Caldera, Mono County, California.

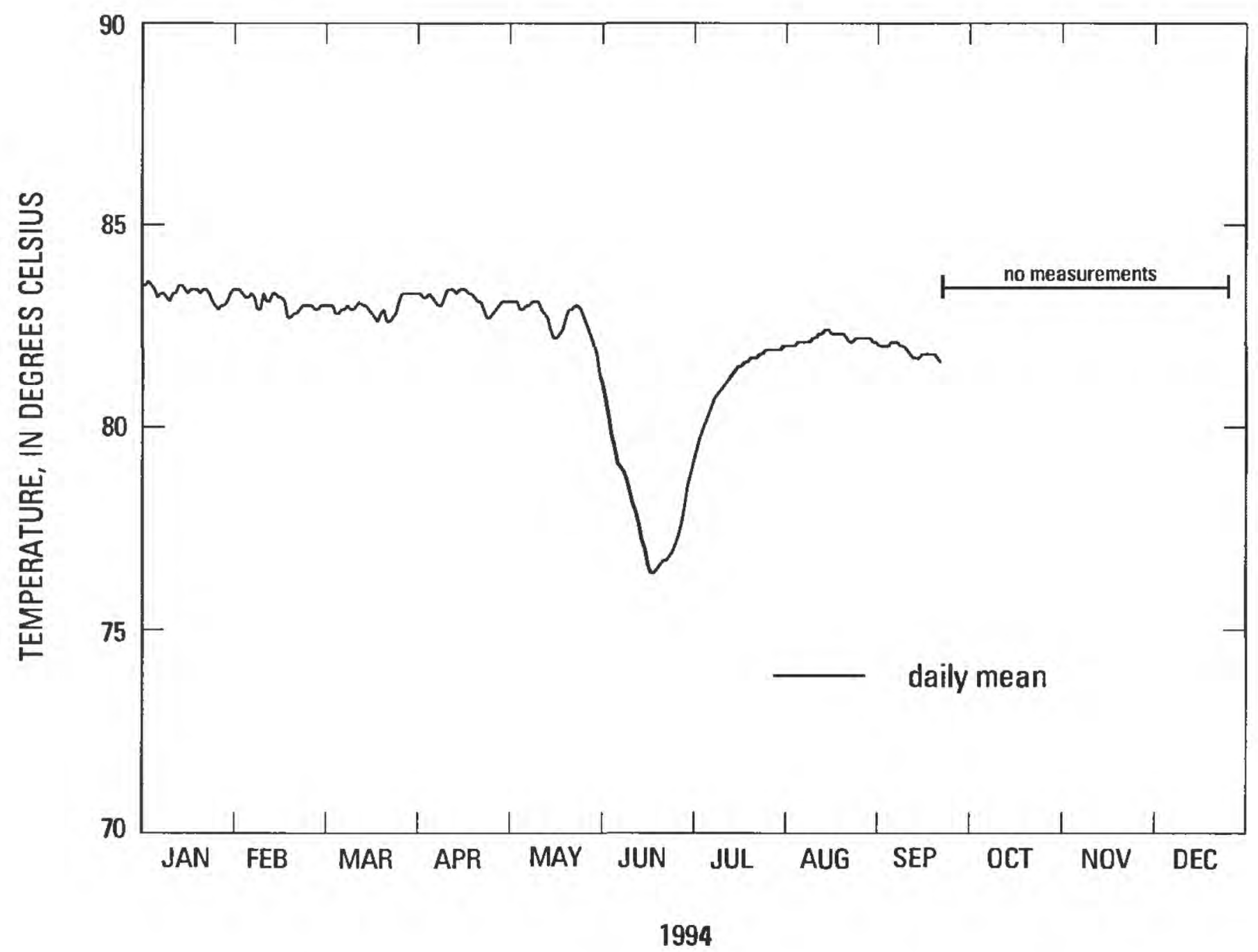

Figure 46. (A) Daily mean vent gas temperature, Mammoth Mountain Fumarole (MMF), 1994, Long Valley Caldera, Mono County, California. 
Table 44. Daily mean vent gas temperature, Mammoth Mountain Fumarole (MMF), 1994, Long Valley Caldera, California [Values are in degrees Celsius. _, no data; Max, maximum; Min, minimum. See fig. 44 for location of site MMF]

\begin{tabular}{|c|c|c|c|c|c|c|c|c|c|c|c|c|}
\hline Day & Jan & Feb & Mar & Apr & May & Jun & Jul & Aug & Sep & Oct & Nov & Dec \\
\hline 1 & 83.5 & 83.4 & 83.0 & 83.3 & 83.1 & 81.1 & 79.1 & 82.0 & 82.0 & - & - & - \\
\hline 2 & 83.6 & 83.3 & 83.0 & 83.3 & 83.1 & 80.7 & 79.4 & 82.0 & 82.0 & - & - & - \\
\hline 3 & 83.5 & 83.2 & 83.0 & 83.2 & 83.1 & 80.3 & 79.7 & 82.0 & 82.0 & - & - & - \\
\hline 4 & 83.4 & 83.2 & 83.0 & 83.2 & 83.1 & 79.8 & 79.9 & 82.0 & 82.0 & - & - & - \\
\hline 5 & 83.2 & 83.3 & 82.8 & 83.3 & 82.9 & 79.5 & 80.1 & 82.1 & 82.1 & - & - & - \\
\hline 6 & 83.3 & 83.2 & 82.8 & 83.2 & 82.9 & 79.1 & 80.3 & 82.1 & 82.1 & - & - & - \\
\hline 7 & 83.3 & 82.9 & 82.9 & 83.1 & 83.0 & 79.0 & 80.5 & 82.1 & 82.1 & - & - & - \\
\hline 8 & 83.2 & 82.9 & 82.9 & 83.0 & 83.0 & 78.9 & 80.7 & 82.1 & 82.0 & - & - & - \\
\hline 9 & 83.1 & 83.3 & 83.0 & 83.0 & 83.1 & 78.7 & 80.8 & 82.1 & 82.0 & - & - & - \\
\hline 10 & 83.3 & 83.1 & 82.9 & 83.2 & 83.1 & 78.4 & 80.9 & 82.2 & 81.9 & - & - & - \\
\hline 11 & 83.3 & 83.1 & 82.9 & 83.4 & 83.1 & 78.1 & 81.0 & 82.2 & 81.8 & - & - & - \\
\hline 12 & 83.5 & 83.3 & 83.0 & 83.4 & 82.9 & 77.9 & 81.1 & 82.3 & 81.7 & - & - & - \\
\hline 13 & 83.5 & 83.3 & 83.1 & 83.4 & 82.8 & 77.6 & 81.2 & 82.3 & 81.7 & - & - & - \\
\hline 14 & 83.4 & 83.2 & 83.0 & 83.3 & 82.7 & 77.2 & 81.3 & 82.4 & 81.7 & - & - & - \\
\hline 15 & 83.3 & 83.2 & 83.0 & 83.4 & 82.4 & 77.0 & 81.4 & 82.4 & 81.8 & - & - & - \\
\hline 16 & 83.4 & 83.1 & 82.9 & 83.4 & 82.2 & 76.6 & 81.5 & 82.3 & 81.8 & - & - & - \\
\hline 17 & 83.4 & 82.7 & 82.8 & 83.4 & 82.2 & 76.4 & 81.5 & 82.3 & 81.8 & - & - & - \\
\hline 18 & 83.4 & 82.7 & 82.7 & 83.3 & 82.3 & 76.4 & 81.6 & 82.3 & 81.8 & - & - & - \\
\hline 19 & 83.3 & 82.8 & 82.6 & 83.3 & 82.4 & 76.5 & 81.6 & 82.3 & 81.8 & - & - & - \\
\hline 20 & 83.4 & 82.8 & 82.8 & 83.2 & 82.7 & 76.6 & 81.7 & 82.3 & 81.7 & - & - & - \\
\hline 21 & 83.4 & 82.9 & 82.9 & 83.1 & 82.9 & 76.7 & 81.7 & 82.2 & 81.6 & - & - & - \\
\hline 22 & 83.3 & 83.0 & 82.6 & 83.1 & 82.9 & 76.7 & 81.7 & 82.1 & - & - & - & - \\
\hline 23 & 83.1 & 83.0 & 82.6 & 82.9 & 83.0 & 76.8 & 81.8 & 82.1 & - & - & - & - \\
\hline 24 & 83.0 & 83.0 & 82.7 & 82.7 & 83.0 & 76.9 & 81.8 & 82.2 & - & - & - & - \\
\hline 25 & 82.9 & 83.0 & 82.8 & 82.7 & 82.9 & 77.1 & 81.9 & 82.2 & - & - & - & - \\
\hline 26 & 83.0 & 82.9 & 83.1 & 82.8 & 82.7 & 77.3 & 81.9 & 82.2 & - & - & - & - \\
\hline 27 & 83.0 & 82.9 & 83.3 & 82.9 & 82.5 & 77.6 & 81.9 & 82.2 & - & - & - & - \\
\hline 28 & 83.1 & 83.0 & 83.3 & 83.0 & 82.3 & 78.0 & 81.9 & 82.2 & - & - & - & - \\
\hline 29 & 83.3 & - & 83.3 & 83.1 & 82.1 & 78.5 & 81.9 & 82.2 & - & - & - & - \\
\hline 30 & 83.4 & - & 83.3 & 83.1 & 81.8 & 78.8 & 81.9 & 82.1 & - & - & - & - \\
\hline 31 & 83.4 & - & 83.3 & - & 81.3 & - & 82.0 & 82.1 & - & - & - & - \\
\hline Mean & 83.3 & 83.1 & 82.9 & 83.2 & 82.7 & 78.0 & 81.2 & 82.2 & 81.9 & - & - & - \\
\hline Max & 83.6 & 83.4 & 83.3 & 83.4 & 83.1 & 81.1 & 82.0 & 82.4 & 82.1 & - & - & - \\
\hline Min & 82.9 & 82.7 & 82.6 & 82.7 & 81.3 & 76.4 & 79.1 & 82.0 & 81.6 & - & - & - \\
\hline
\end{tabular}




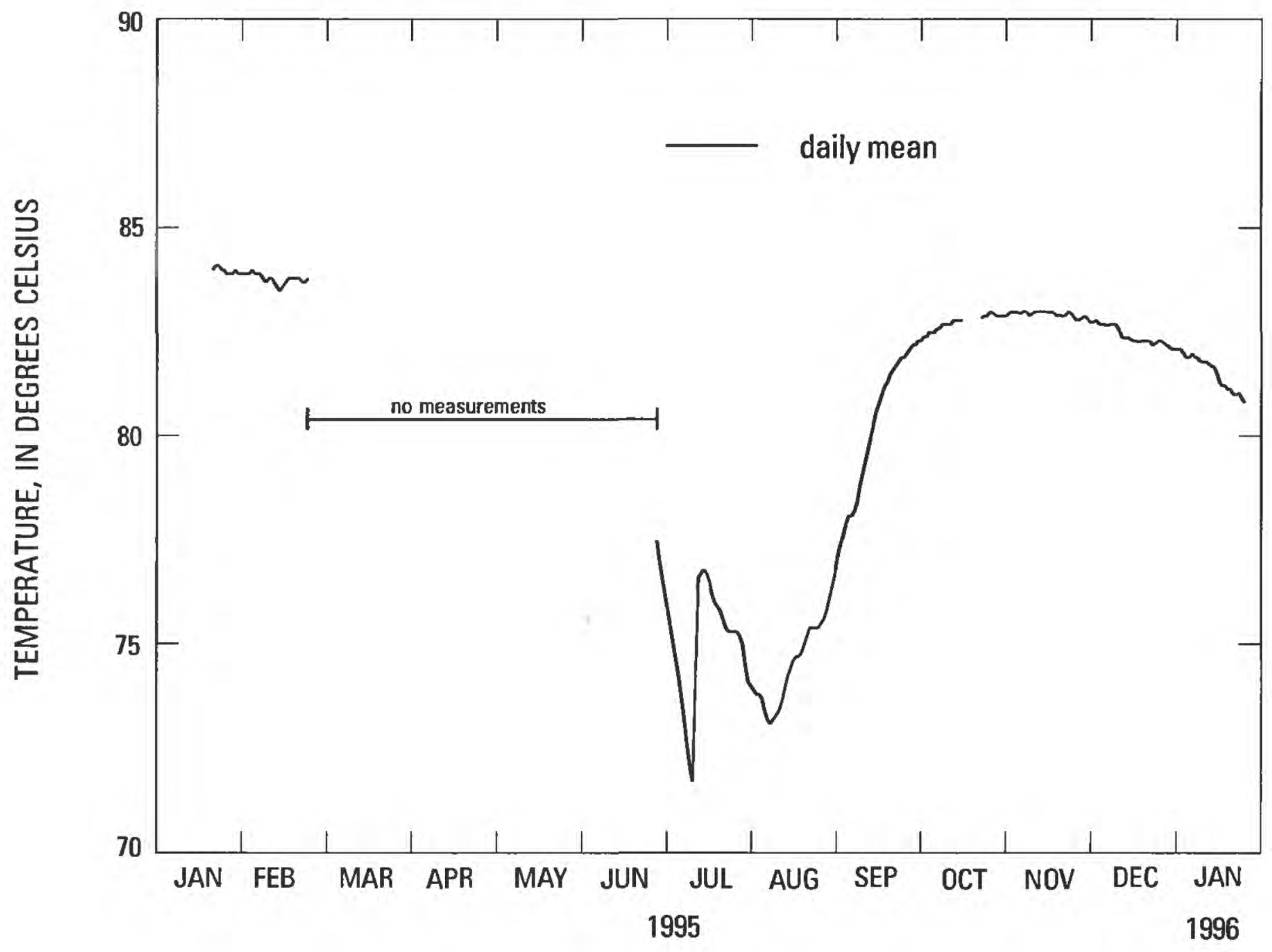

Figure 46. (B) Daily mean vent gas temperature, Mammoth Mountain Fumarole (MMF), 1995-96, Long Valley Caldera, Mono County, California. 
Table 45. Daily mean vent gas temperature, Mammoth Mountain Fumarole (MMF), 1995, Long Valley Caldera, California

[Values are in degrees Celsius. -, no data; Max, maximum; Min, minimum. See fig. 44 for location of site MMF]

\begin{tabular}{|c|c|c|c|c|c|c|c|c|c|c|c|c|}
\hline Day & Jan & Feb & Mar & Apr & May & Jun & Jul & Aug & Sep & Oct & Nov & Dec \\
\hline 1 & - & 83.9 & - & - & - & - & 76.2 & 74.0 & 77.1 & 82.3 & 82.9 & 82.7 \\
\hline 2 & - & 83.9 & - & - & - & - & 75.8 & 73.9 & 77.4 & 82.4 & 83.0 & 82.7 \\
\hline 3 & - & 84.0 & - & - & - & - & 75.4 & 73.8 & 77.6 & 82.4 & 83.0 & 82.8 \\
\hline 4 & - & 83.9 & - & - & - & - & 75.0 & 73.8 & 77.9 & 82.5 & 83.0 & 82.7 \\
\hline 5 & - & 83.9 & - & - & - & - & 74.6 & 73.7 & 78.1 & 82.5 & 83.0 & 82.7 \\
\hline 6 & - & 83.9 & - & - & - & - & 74.2 & 73.4 & 78.1 & 82.5 & 83.0 & 82.7 \\
\hline 7 & - & 83.8 & - & - & - & - & 73.7 & 73.2 & 78.2 & 82.6 & 83.0 & 82.7 \\
\hline 8 & - & 83.7 & - & - & - & - & 73.2 & 73.1 & 78.4 & 82.6 & 83.0 & 82.7 \\
\hline 9 & - & 83.8 & - & - & - & - & 72.6 & 73.2 & 78.8 & 82.7 & 82.9 & 82.7 \\
\hline 10 & - & 83.8 & - & - & - & - & 72.1 & 73.3 & 79.1 & 82.7 & 82.9 & 82.7 \\
\hline 11 & - & 83.7 & - & - & - & - & 71.7 & 73.4 & 79.4 & 82.7 & 83.0 & 82.6 \\
\hline 12 & - & 83.6 & - & - & - & - & 74.4 & 73.6 & 79.7 & 82.7 & 83.0 & 82.4 \\
\hline 13 & - & 83.5 & - & - & - & - & 76.6 & 73.9 & 80.0 & 82.8 & 83.0 & 82.4 \\
\hline 14 & - & 83.6 & - & - & - & - & 76.7 & 74.2 & 80.3 & 82.8 & 83.0 & 82.4 \\
\hline 15 & - & 83.7 & - & - & - & - & 76.8 & 74.4 & 80.6 & 82.8 & 83.0 & 82.3 \\
\hline 16 & - & 83.8 & - & - & - & - & 76.7 & 74.6 & 80.8 & 82.8 & 83.0 & 82.3 \\
\hline 17 & - & 83.8 & - & - & - & - & 76.5 & 74.7 & 81.0 & - & 83.0 & 82.3 \\
\hline 18 & - & 83.8 & - & - & - & - & 76.2 & 74.7 & 81.2 & - & 83.0 & 82.3 \\
\hline 19 & - & 83.8 & - & - & - & - & 76.0 & 74.8 & 81.3 & - & 82.9 & 82.3 \\
\hline 20 & 84.0 & 83.8 & - & - & - & - & 75.9 & 75.0 & 81.5 & - & 82.9 & 82.3 \\
\hline 21 & 84.1 & 83.7 & - & - & - & - & 75.8 & 75.2 & 81.6 & - & 82.9 & 82.3 \\
\hline 22 & 84.1 & 83.7 & - & - & - & - & 75.6 & 75.4 & 81.7 & - & 82.9 & 82.3 \\
\hline 23 & 84.0 & 83.8 & - & - & - & - & 75.4 & 75.4 & 81.8 & 82.9 & 83.0 & 82.2 \\
\hline 24 & 84.0 & - & - & - & - & - & 75.3 & 75.4 & 81.9 & 82.9 & 83.0 & 82.2 \\
\hline 25 & 83.9 & - & - & - & - & - & 75.3 & 75.4 & 81.9 & 82.9 & 82.9 & 82.3 \\
\hline 26 & 83.9 & - & - & - & - & - & 75.3 & 75.5 & 82.0 & 83.0 & 82.8 & 82.3 \\
\hline 27 & 83.9 & - & - & - & - & - & 75.3 & 75.6 & 82.1 & 83.0 & 82.8 & 82.2 \\
\hline 28 & 84.0 & - & - & - & - & 77.5 & 75.2 & 75.8 & 82.2 & 82.9 & 82.9 & 82.2 \\
\hline 29 & 83.9 & - & - & - & - & 77.0 & 75.0 & 76.1 & 82.2 & 82.9 & 82.9 & 82.1 \\
\hline 30 & 83.9 & - & - & - & - & 76.6 & 74.5 & 76.4 & 82.3 & 82.9 & 82.8 & 82.1 \\
\hline 31 & 83.9 & - & - & - & - & - & 74.1 & 76.7 & - & 82.9 & - & 82.1 \\
\hline Mean & 84.0 & 83.8 & - & - & - & 77.0 & 75.1 & 74.6 & 80.2 & 82.7 & 82.9 & 82.4 \\
\hline Max & 84.1 & 84.0 & - & - & - & 77.5 & 76.8 & 76.7 & 82.3 & 83.0 & 83.0 & 82.8 \\
\hline Min & 83.9 & 83.5 & - & - & - & 76.6 & 71.7 & 73.1 & 77.1 & 82.3 & 82.8 & 82.1 \\
\hline
\end{tabular}


Table 46. Daily mean vent gas temperature, Mammoth Mountain Fumarole (MMF), 1996, Long Valley Caldera, California [Values are in degrees Celsius. -, no data; Max, maximum; Min, minimum. See fig. 44 for location of site MMF]

\begin{tabular}{|c|c|c|c|c|c|c|c|c|c|c|c|c|}
\hline Day & Jan & Feb & Mar & Apr & May & Jun & Jul & Aug & Sep & Oct & Nov & Dec \\
\hline 1 & 82.1 & - & - & - & - & - & - & - & - & - & - & - \\
\hline 2 & 82.1 & - & - & - & - & - & - & - & - & - & - & - \\
\hline 3 & 82.0 & - & - & - & - & - & - & - & - & - & - & - \\
\hline 4 & 81.9 & - & - & - & - & - & - & - & - & - & - & - \\
\hline 5 & 81.9 & - & - & - & - & - & - & - & - & - & - & - \\
\hline 6 & 82.0 & - & - & - & - & - & - & - & - & - & - & - \\
\hline 7 & 81.9 & - & - & - & - & - & - & - & - & - & - & - \\
\hline 8 & 81.9 & - & - & - & - & - & - & - & - & - & 一 & - \\
\hline 9 & 81.8 & - & - & - & - & - & - & - & - & - & - & - \\
\hline 10 & 81.8 & - & - & - & - & - & - & - & - & - & - & - \\
\hline 11 & 81.8 & - & - & - & - & - & - & - & - & - & - & - \\
\hline 12 & 81.8 & - & - & - & - & - & - & - & - & - & - & - \\
\hline 13 & 81.7 & - & - & - & - & - & - & - & - & - & - & - \\
\hline 14 & 81.7 & - & - & - & - & - & - & - & - & - & - & - \\
\hline 15 & 81.5 & - & - & - & - & - & - & - & - & - & - & - \\
\hline 16 & 81.3 & - & - & - & - & - & - & - & - & - & - & - \\
\hline 17 & 81.2 & - & - & - & - & - & - & - & - & - & - & - \\
\hline 18 & 81.2 & - & - & - & - & - & - & - & - & - & - & - \\
\hline 19 & 81.1 & - & - & - & - & - & - & - & - & - & - & - \\
\hline 20 & 81.1 & - & - & - & - & - & - & - & - & - & - & - \\
\hline 21 & 81.0 & - & - & - & - & - & - & - & - & - & - & - \\
\hline 22 & 81.0 & - & - & - & - & - & - & - & - & - & - & - \\
\hline 23 & 81.1 & - & - & - & - & - & - & - & - & - & - & - \\
\hline 24 & 80.9 & - & - & - & - & - & - & - & - & - & - & - \\
\hline 25 & 80.8 & - & - & - & - & - & - & - & - & - & - & - \\
\hline 26 & - & - & - & - & - & - & - & - & - & - & - & - \\
\hline 27 & - & - & - & - & - & - & - & - & - & - & - & - \\
\hline 28 & - & - & - & - & - & - & - & - & - & - & - & - \\
\hline 29 & - & - & - & - & - & - & - & - & - & - & - & - \\
\hline 30 & - & - & - & - & - & - & - & - & - & - & - & - \\
\hline 31 & - & - & - & - & - & - & - & - & - & - & - & - \\
\hline Mean & 81.5 & - & - & - & - & - & - & - & - & - & - & - \\
\hline Max & 82.1 & - & - & - & - & - & - & - & - & - & - & - \\
\hline Min & 80.8 & - & - & - & - & - & - & - & - & - & - & - \\
\hline
\end{tabular}




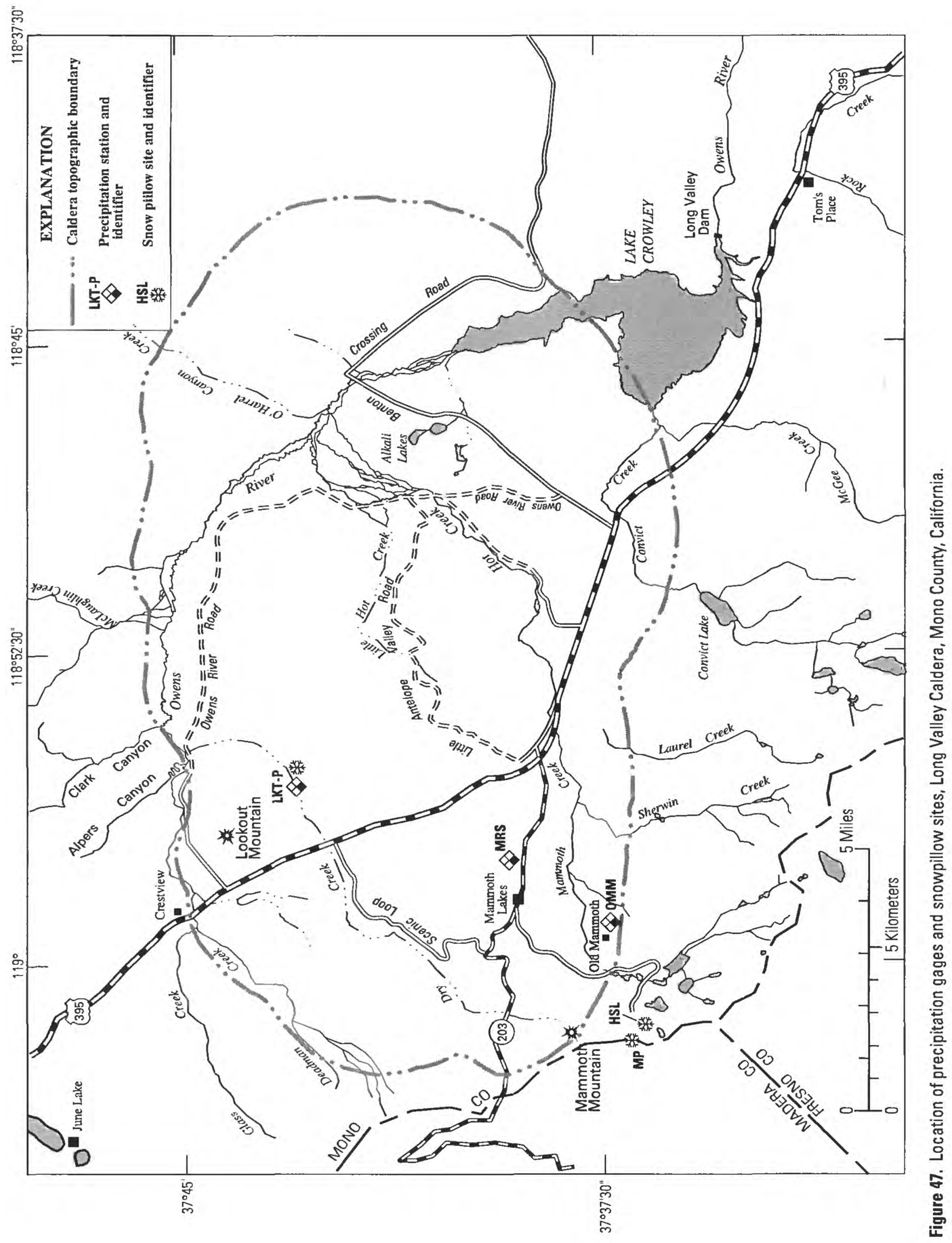




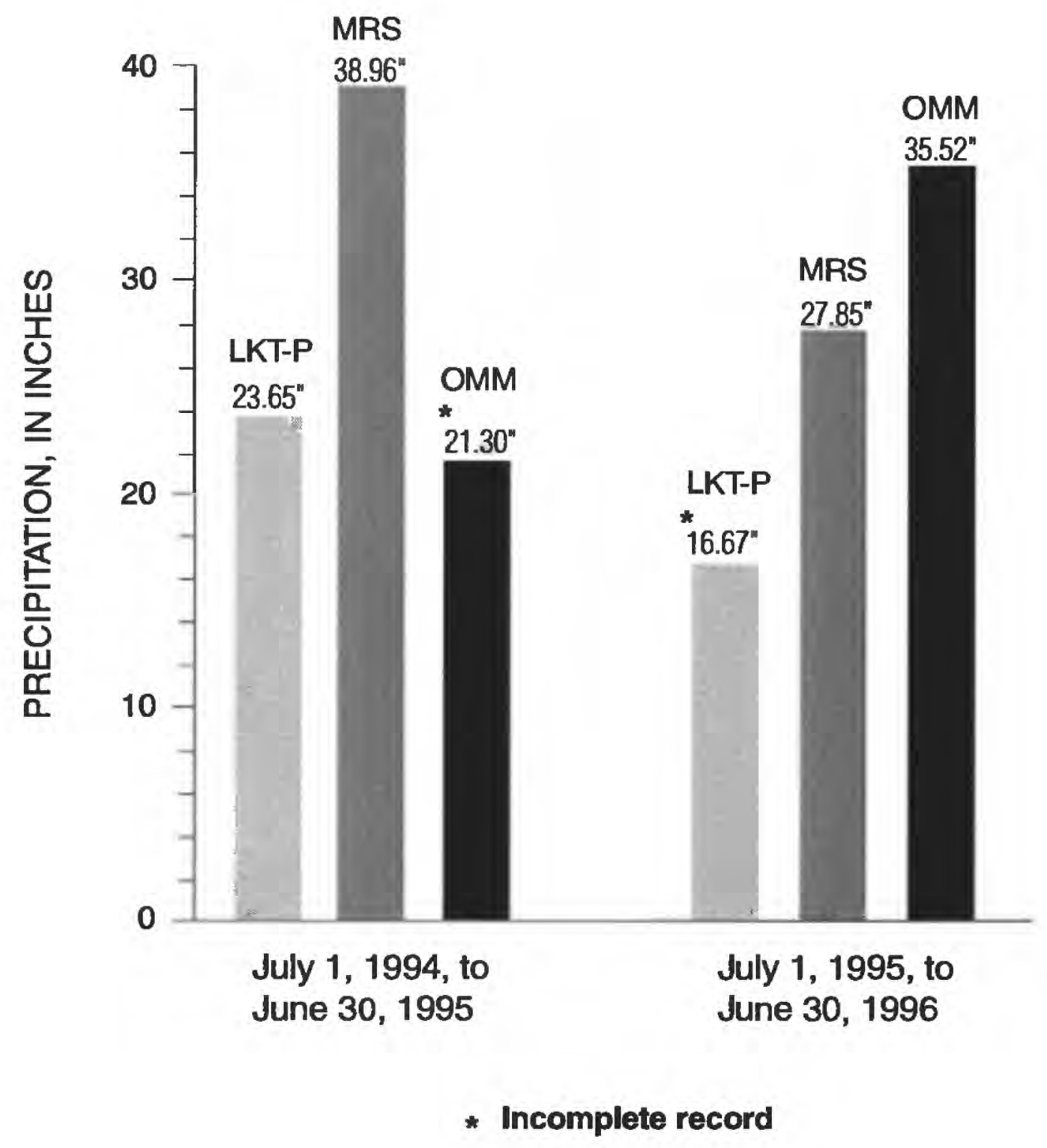

Figure 48. Annual precipitation at Lookout Mountain (LKT-P), Mammoth Ranger Station (MRS), and Old Mammoth Meadow (OMM), July 1 , 1994, to June 30, 1996, Long Valley Caldera, Mono County, California. 
Table 47. Daily precipitation, Lookout Mountain gage (LKT-P), 1994, Long Valley Caldera, California

[Values are in inches. - , no data]

\begin{tabular}{|c|c|c|c|c|c|c|c|c|c|c|c|c|}
\hline Day & Jan & Feb & Mar & Apr & May & Jun & Jul & Aug & Sep & Oct & Nov & Dec \\
\hline 1 & 0.00 & 0.00 & 0.00 & 0.00 & 0.08 & 0.04 & 0.00 & 0.00 & 0.00 & 0.04 & 0.00 & 0.04 \\
\hline 2 & .00 & .00 & .00 & .00 & .00 & .00 & .00 & .00 & .00 & .00 & .00 & .00 \\
\hline 3 & .04 & .00 & .00 & .00 & .04 & .00 & .00 & .00 & .00 & .00 & .00 & .00 \\
\hline 4 & .00 & .04 & .00 & .04 & .00 & .00 & .00 & .00 & .00 & .19 & .00 & .04 \\
\hline 5 & .00 & .00 & .00 & .00 & .00 & .00 & .00 & .00 & .00 & .62 & .00 & .04 \\
\hline 6 & .00 & .00 & .00 & .00 & .08 & .00 & .00 & .00 & .00 & .35 & .00 & .00 \\
\hline 7 & .00 & .66 & .04 & .04 & .19 & .00 & .00 & .00 & .00 & .08 & .00 & .00 \\
\hline 8 & .00 & .19 & - & .00 & .08 & .00 & .00 & .00 & .00 & .04 & .12 & .00 \\
\hline 9 & .00 & .04 & - & .04 & .16 & .00 & .00 & .00 & .00 & .00 & .00 & .00 \\
\hline 10 & .00 & .00 & - & .00 & .00 & .00 & .00 & .00 & .00 & .00 & .23 & .00 \\
\hline 11 & .00 & .08 & - & .12 & .00 & .00 & .00 & .00 & .00 & .00 & .00 & .00 \\
\hline 12 & .00 & .00 & - & .00 & .00 & .00 & .00 & .00 & .00 & .00 & .00 & .19 \\
\hline 13 & .04 & .00 & - & .00 & .00 & .00 & .00 & .00 & .00 & .00 & .00 & .12 \\
\hline 14 & .00 & - & - & .00 & .00 & .00 & .00 & .00 & .00 & .00 & .00 & .04 \\
\hline 15 & .00 & - & - & .04 & .00 & .00 & .00 & .00 & .00 & .00 & .00 & .00 \\
\hline 16 & .00 & - & - & .00 & .00 & .00 & .00 & .00 & .00 & .00 & .04 & .04 \\
\hline 17 & .00 & - & - & .00 & .00 & .00 & .00 & .00 & .00 & .04 & .04 & .00 \\
\hline 18 & .00 & - & - & .00 & .43 & .00 & .00 & .00 & .00 & .00 & .04 & .00 \\
\hline 19 & .00 & - & .00 & .00 & .00 & .00 & .00 & .00 & .00 & .00 & .08 & .00 \\
\hline 20 & .00 & - & .00 & .00 & .00 & .00 & .00 & .00 & - & .00 & .00 & .04 \\
\hline 21 & .00 & - & .00 & .00 & .04 & .00 & .00 & .00 & - & .04 & .00 & .00 \\
\hline 22 & .00 & - & .04 & .00 & .04 & .00 & .00 & .00 & .00 & .00 & .00 & .00 \\
\hline 23 & .04 & - & .00 & .00 & .00 & .00 & .00 & .00 & .00 & .00 & .00 & .00 \\
\hline 24 & .00 & - & .00 & .00 & .00 & .00 & .00 & .00 & .00 & .00 & .04 & .00 \\
\hline 25 & .08 & .00 & .19 & .00 & .00 & .00 & .00 & .00 & .00 & .00 & .55 & .12 \\
\hline 26 & .00 & .00 & .16 & .00 & .00 & .00 & .00 & .00 & .00 & .00 & .55 & .04 \\
\hline 27 & .00 & .00 & .00 & .00 & .00 & .00 & .00 & .00 & .00 & .00 & .04 & .00 \\
\hline 28 & .19 & .00 & .00 & .08 & .00 & .00 & .00 & .00 & .00 & .00 & .00 & .00 \\
\hline 29 & .08 & - & .00 & .00 & .00 & .00 & .00 & .00 & .00 & .00 & .00 & .08 \\
\hline 30 & .00 & - & .00 & .00 & .19 & .00 & .00 & .00 & .12 & .00 & .00 & .00 \\
\hline 31 & .00 & - & .00 & - & .23 & - & .00 & .00 & - & .00 & - & .00 \\
\hline Total & 0.47 & $1.01^{1}$ & $0.43^{1}$ & 0.36 & 1.56 & 0.04 & 0.00 & 0.00 & $0.12^{1}$ & 1.40 & 1.73 & 0.79 \\
\hline \multicolumn{13}{|c|}{ Calendar year total $7.91^{1}$} \\
\hline
\end{tabular}


Table 48. Daily precipitation, Lookout Mountain gage (LKT-P), 1995, Long Valley Caldera, California

[Values are in inches. - no data]

\begin{tabular}{|c|c|c|c|c|c|c|c|c|c|c|c|c|}
\hline Day & Jan & Feb & Mar & Apr & May & Jun & Jul & Aug & Sep & Oct & Nov & Dec \\
\hline 1 & 0.00 & 0.00 & 0.00 & 0.00 & 0.47 & 0.00 & - & - & - & - & - & 0.00 \\
\hline 2 & .00 & .00 & .00 & .00 & .04 & .04 & - & - & - & - & - & .00 \\
\hline 3 & .04 & .00 & .35 & .00 & .00 & .00 & - & - & - & - & - & .00 \\
\hline 4 & .78 & .00 & .00 & .00 & .00 & .00 & - & - & - & - & - & .00 \\
\hline 5 & .16 & .00 & .00 & .00 & .04 & .00 & - & - & - & - & - & .00 \\
\hline 6 & .27 & .00 & .00 & .00 & .04 & .16 & - & - & - & - & - & .00 \\
\hline 7 & .66 & .04 & .00 & .00 & .04 & .04 & - & - & - & - & 0.00 & .00 \\
\hline 8 & .19 & .00 & .00 & .00 & .00 & .04 & - & - & - & - & .00 & .00 \\
\hline 9 & .12 & .00 & 2.1 & .00 & .00 & .00 & - & - & - & - & .00 & .00 \\
\hline 10 & 2.1 & .00 & 1.9 & .00 & .00 & .00 & - & - & - & - & .00 & .00 \\
\hline 11 & .31 & .00 & 1.6 & .00 & .00 & .00 & - & - & - & - & .00 & 1.03 \\
\hline 12 & .12 & .04 & .00 & .00 & .00 & .00 & - & - & - & - & .00 & 2.17 \\
\hline 13 & .00 & .27 & .00 & .00 & .08 & .00 & - & - & - & - & .00 & .71 \\
\hline 14 & .55 & .27 & .00 & .08 & .08 & .00 & - & - & - & - & .00 & .58 \\
\hline 15 & .12 & .08 & .04 & .00 & .23 & .35 & - & - & - & - & .00 & - \\
\hline 16 & .04 & .00 & .00 & .00 & .04 & .04 & - & - & - & - & .00 & - \\
\hline 17 & .00 & .00 & .00 & .00 & .00 & .00 & - & - & - & - & .00 & - \\
\hline 18 & .00 & .00 & .04 & .00 & .00 & .00 & - & - & - & - & .00 & - \\
\hline 19 & .00 & .00 & .04 & .00 & .00 & .00 & - & - & - & - & .00 & - \\
\hline 20 & .00 & .00 & .94 & .00 & .00 & .00 & - & - & - & - & .00 & - \\
\hline 21 & .04 & .00 & .78 & .04 & .00 & .00 & - & - & - & - & .00 & - \\
\hline 22 & .12 & .00 & .58 & .00 & .16 & .00 & - & - & - & - & .00 & - \\
\hline 23 & .35 & .00 & .58 & .00 & .27 & .00 & - & - & - & - & .00 & - \\
\hline 24 & .31 & .00 & .12 & .00 & .19 & .00 & - & - & - & - & .00 & - \\
\hline 25 & .23 & .00 & .04 & .00 & .04 & .19 & - & - & - & - & .00 & - \\
\hline 26 & .04 & .00 & .08 & .00 & .04 & .00 & - & - & - & - & .00 & - \\
\hline 27 & .00 & .00 & .00 & .00 & .00 & 一 & - & - & - & - & .00 & - \\
\hline 28 & .00 & .00 & .00 & .00 & .00 & - & - & - & - & - & .00 & - \\
\hline 29 & .00 & - & .00 & .43 & .00 & - & 一 & - & - & - & .00 & - \\
\hline 30 & .00 & - & .00 & .00 & .00 & - & - & 一 & - & - & .00 & - \\
\hline 31 & .00 & - & .00 & - & .00 & - & - & - & - & - & - & - \\
\hline Total & 6.55 & 0.70 & 9.19 & 0.55 & 1.76 & $0.86^{1}$ & 一 & 一 & - & - & $0.00^{1}$ & $4.49^{1}$ \\
\hline \multicolumn{13}{|c|}{ Calendar year total $24.1^{1}$} \\
\hline
\end{tabular}


Table 49. Daily precipitation, Lookout Mountain gage (LKT-P), 1996, Long Valley Caldera, California

[Values are in inches. -, no data]

\begin{tabular}{|c|c|c|c|c|c|c|c|c|c|c|c|c|}
\hline Day & Jan & Feb & Mar & Apr & May & Jun & Jul & Aug & Sep & Oct & Nov & Dec \\
\hline 1 & - & 0.02 & - & 0.36 & 0.00 & 0.00 & 0.00 & 0.06 & 0.00 & 0.00 & 0.03 & 0.00 \\
\hline 2 & - & .00 & - & .10 & .00 & .00 & .04 & .00 & .00 & .00 & .28 & .01 \\
\hline 3 & - & .00 & - & .00 & .00 & .00 & .00 & .00 & .00 & .00 & .40 & .01 \\
\hline 4 & - & 1.14 & - & .00 & .00 & .00 & .00 & .00 & .00 & .00 & .01 & .00 \\
\hline 5 & - & .58 & - & .00 & .00 & .00 & .00 & .00 & .00 & .00 & .00 & .45 \\
\hline 6 & - & .00 & - & .00 & .00 & .00 & .00 & .00 & .00 & .00 & .01 & .15 \\
\hline 7 & - & .00 & - & .00 & .00 & .00 & .00 & .00 & .00 & .00 & .00 & .00 \\
\hline 8 & - & .00 & - & .00 & .00 & .00 & .00 & .00 & .00 & .00 & .00 & .00 \\
\hline 9 & - & .00 & 0.96 & .00 & .00 & .00 & .00 & .00 & .00 & .00 & .00 & .32 \\
\hline 10 & - & .00 & .00 & .06 & .00 & .00 & .00 & .00 & .00 & .00 & .00 & 1.00 \\
\hline 11 & - & .00 & .24 & .00 & .00 & .00 & .05 & .00 & .00 & .00 & .00 & .52 \\
\hline 12 & - & - & .42 & .00 & .00 & .00 & .26 & .00 & .00 & .00 & .00 & .02 \\
\hline 13 & - & - & .14 & .00 & .00 & .00 & .08 & .00 & .00 & .00 & .00 & .00 \\
\hline 14 & - & - & .00 & .00 & .00 & .00 & .31 & .00 & .00 & .00 & .00 & .00 \\
\hline 15 & - & - & .02 & .00 & .40 & .00 & .00 & .00 & .00 & .00 & .00 & .00 \\
\hline 16 & - & - & .00 & .81 & .58 & .00 & .00 & .00 & .00 & .00 & .00 & .00 \\
\hline 17 & - & - & .00 & .31 & - & - & .00 & .00 & .00 & .00 & 1.82 & .00 \\
\hline 18 & - & - & .00 & 1.74 & - & .00 & .00 & .00 & .00 & .14 & .00 & .00 \\
\hline 19 & - & - & .00 & .00 & - & .00 & .00 & .00 & .00 & .00 & .21 & .00 \\
\hline 20 & - & 3.04 & .00 & .00 & - & .00 & .00 & .00 & .00 & .00 & .00 & .00 \\
\hline 21 & - & - & .00 & .00 & - & .00 & .00 & .00 & .00 & .00 & 1.51 & 1.53 \\
\hline 22 & - & - & .00 & .00 & .00 & .00 & .00 & .00 & .00 & .00 & .24 & .97 \\
\hline 23 & - & - & .00 & .00 & .00 & .00 & .00 & .00 & .00 & .00 & .00 & .01 \\
\hline 24 & 0.14 & - & .00 & .00 & .03 & .00 & .00 & .00 & .00 & .04 & .02 & .00 \\
\hline 25 & .14 & - & .00 & .00 & .00 & .00 & .00 & .00 & .00 & .00 & .00 & .00 \\
\hline 26 & .00 & - & .00 & .00 & .01 & .00 & .00 & .00 & .00 & .00 & .00 & .12 \\
\hline 27 & .65 & - & .00 & .00 & .03 & .00 & .00 & .00 & .00 & .00 & .00 & .26 \\
\hline 28 & .00 & - & .00 & .00 & .00 & .00 & .00 & .00 & .00 & .03 & .05 & .00 \\
\hline 29 & .00 & - & .00 & .00 & .00 & .00 & .00 & .00 & .00 & .00 & .07 & .48 \\
\hline 30 & .00 & - & .00 & .00 & .00 & .00 & .00 & .00 & .00 & .03 & .02 & .10 \\
\hline 31 & .18 & - & .00 & - & .08 & - & .00 & .00 & - & .00 & - & .00 \\
\hline Total & $1.11^{1}$ & $4.78^{1}$ & $1.78^{1}$ & 3.38 & $1.13^{1}$ & $0.00^{1}$ & 0.74 & 0.06 & 0.00 & 0.24 & 4.67 & 5.95 \\
\hline \multicolumn{13}{|c|}{ Calendar year total $23.84^{1}$} \\
\hline
\end{tabular}


Table 50. Monthly precipitation, Mammoth Ranger Station gage (MRS), 1994-96, Long Valley Caldera, California [Data provided by Thom Heller, U.S. Forest Service. Values are in inches]

\begin{tabular}{|c|c|c|c|}
\hline Month & 1994 & 1995 & 1996 \\
\hline January & 0.48 & 10.40 & 4.33 \\
\hline February & 5.17 & 1.40 & 10.09 \\
\hline March & 1.03 & 11.79 & 2.67 \\
\hline April & .96 & .85 & 2.54 \\
\hline May & 1.84 & 3.27 & 2.85 \\
\hline June & .00 & 1.71 & .05 \\
\hline July & .03 & .00 & .78 \\
\hline August & .00 & .30 & .18 \\
\hline September & 1.20 & .16 & .00 \\
\hline October & 1.23 & .15 & 2.18 \\
\hline November & 6.09 & .00 & 4.77 \\
\hline December & .99 & 4.71 & 10.22 \\
\hline Total & 19.02 & 34.74 & 40.66 \\
\hline
\end{tabular}


Table 51. Daily precipitation, Old Mammoth Meadow gage (OMM), 1994, Long Valley Caldera, California

[Values are in inches. -, no data]

\begin{tabular}{|c|c|c|c|c|c|c|c|c|c|c|c|c|}
\hline Day & Jan & Fab & Mar & Apr & May & Jun & Jul & Aug & Sep & Oct & Nov & Dec \\
\hline 1 & - & - & - & - & - & - & - & - & - & - & 0.02 & 0.00 \\
\hline 2 & - & - & - & - & - & - & - & - & - & - & .12 & .01 \\
\hline 3 & - & - & - & - & - & - & - & - & - & - & .11 & .11 \\
\hline 4 & - & - & - & - & - & - & - & - & - & - & .03 & .02 \\
\hline 5 & - & - & - & - & - & - & - & - & - & - & 1.20 & .06 \\
\hline 6 & - & - & - & - & - & - & - & - & - & - & .14 & .00 \\
\hline 7 & - & - & - & - & - & - & - & - & - & - & .11 & .04 \\
\hline 8 & - & - & - & - & - & - & - & - & - & - & .06 & .00 \\
\hline 9 & - & - & - & - & - & - & - & - & - & - & .06 & .00 \\
\hline 10 & - & - & - & - & - & - & - & - & - & - & .42 & .01 \\
\hline 11 & - & - & - & - & - & - & - & - & - & - & .02 & .00 \\
\hline 12 & - & - & - & - & - & - & - & - & - & - & .01 & .54 \\
\hline 13 & - & - & - & - & - & - & - & - & - & - & .01 & .15 \\
\hline 14 & - & - & - & - & - & - & - & - & - & - & .00 & .03 \\
\hline 15 & - & - & - & - & - & - & - & - & - & - & .05 & .02 \\
\hline 16 & - & - & - & - & - & - & - & - & - & - & .04 & .00 \\
\hline 17 & - & - & - & - & - & - & - & - & - & - & .40 & .00 \\
\hline 18 & - & - & - & - & - & - & - & - & - & - & .13 & .00 \\
\hline 19 & - & - & - & - & - & - & - & - & - & - & .02 & .00 \\
\hline 20 & - & - & - & - & - & - & - & - & - & - & .00 & .00 \\
\hline 21 & - & - & - & - & - & - & - & - & - & (') & .01 & .00 \\
\hline 22 & - & - & - & - & - & - & - & - & - & 0.00 & .00 & .00 \\
\hline 23 & - & - & - & - & - & - & - & - & - & .00 & .00 & .00 \\
\hline 24 & - & - & - & - & - & - & - & - & - & .00 & .03 & .31 \\
\hline 25 & - & - & - & - & - & - & - & - & - & .00 & 1.16 & .11 \\
\hline 26 & - & - & - & - & - & - & - & - & - & .00 & .35 & .00 \\
\hline 27 & - & - & - & - & - & - & - & - & - & .00 & .01 & .01 \\
\hline 28 & - & - & - & - & - & - & - & - & - & .00 & .00 & .15 \\
\hline 29 & - & - & - & - & - & - & - & - & - & .00 & .00 & .01 \\
\hline 30 & - & - & - & - & - & - & - & - & - & .00 & .00 & .01 \\
\hline 31 & - & - & - & - & - & - & - & - & - & .00 & - & .00 \\
\hline Totals & - & - & - & - & - & - & - & - & - & $0.00^{2}$ & 4.51 & 1.59 \\
\hline \multicolumn{13}{|c|}{ Calendar year total $6.10^{2}$} \\
\hline
\end{tabular}

${ }^{1}$ Record begins October 21, 1994.

${ }^{2}$ Incomplete record. 
Table 52. Daily precipitation, Old Mammoth Meadow gage (OMM), 1995, Long Valley Caldera, California

[Values are in inches. -, no data]

\begin{tabular}{|c|c|c|c|c|c|c|c|c|c|c|c|c|}
\hline Day & Jan & Feb & Mar & Apr & May & Jun & Jul & Aug & Sep & Oct & Nov & Dec \\
\hline 1 & 0.00 & - & 0.00 & - & - & - & - & - & - & - & - & 0.04 \\
\hline 2 & .03 & - & .00 & - & - & - & - & - & - & - & 0.00 & .00 \\
\hline 3 & .31 & - & .58 & - & - & - & - & - & - & - & .00 & .00 \\
\hline 4 & 1.68 & - & .00 & - & - & - & - & - & - & - & .00 & .00 \\
\hline 5 & .33 & - & .00 & - & - & - & - & - & - & - & .00 & .08 \\
\hline 6 & .43 & - & .00 & - & - & - & - & - & - & - & .00 & .04 \\
\hline 7 & .64 & - & .00 & - & - & - & - & - & - & - & .00 & .00 \\
\hline 8 & .31 & - & - & - & - & - & - & - & - & - & .00 & .00 \\
\hline 9 & .37 & - & - & - & - & - & - & - & - & - & .00 & .00 \\
\hline 10 & 2.71 & - & - & - & - & - & - & - & - & - & .00 & .00 \\
\hline 11 & .21 & - & - & - & - & - & - & - & - & - & .00 & 2.22 \\
\hline 12 & .00 & - & - & - & - & - & - & - & - & - & .00 & 3.28 \\
\hline 13 & .00 & - & - & - & - & - & - & - & - & - & .00 & 1.52 \\
\hline 14 & .02 & - & - & - & - & - & - & - & - & - & .00 & .12 \\
\hline 15 & - & - & - & - & - & - & - & - & - & - & .00 & .04 \\
\hline 16 & - & - & - & - & - & - & - & - & - & - & .00 & .00 \\
\hline 17 & - & - & - & - & - & - & - & - & - & - & .00 & .00 \\
\hline 18 & - & - & - & - & - & - & - & - & - & - & .00 & .00 \\
\hline 19 & .00 & - & - & - & - & - & - & - & - & - & .00 & .00 \\
\hline 20 & .00 & - & - & - & - & - & - & - & - & - & .00 & .00 \\
\hline 21 & .02 & - & - & - & - & - & - & - & - & - & .00 & .00 \\
\hline 22 & .42 & 0.00 & - & - & - & - & - & - & - & - & .00 & .23 \\
\hline 23 & 1.03 & .00 & - & - & - & - & - & - & - & - & .00 & .12 \\
\hline 24 & 1.33 & .00 & - & - & - & - & - & - & - & - & .00 & .00 \\
\hline 25 & 3.93 & .00 & - & - & - & - & - & - & - & - & .00 & .04 \\
\hline 26 & .44 & .00 & - & - & - & - & - & - & - & - & .00 & .04 \\
\hline 27 & .08 & .00 & - & - & - & - & - & - & - & - & .00 & .00 \\
\hline 28 & .06 & .00 & - & - & - & - & - & - & - & - & .00 & .00 \\
\hline 29 & .27 & - & - & - & - & - & - & 一 & - & - & .00 & .00 \\
\hline 30 & .00 & - & - & - & - & 一 & - & - & - & - & .00 & .04 \\
\hline 31 & .00 & - & - & - & - & 一 & - & - & - & - & - & .00 \\
\hline Total & $14.62^{1}$ & $0.00^{1}$ & $0.58^{1}$ & - & - & - & - & - & - & - & $0.00^{1}$ & 7.81 \\
\hline \multicolumn{13}{|c|}{ Calendar year total $23.01^{1}$} \\
\hline
\end{tabular}


Table 53. Daily precipitation, Old Mammoth Meadow gage (OMM), 1996, Long Valley Caldera, California

[Values are in inches. - . no data]

\begin{tabular}{|c|c|c|c|c|c|c|c|c|c|c|c|c|}
\hline Day & Jan & Feb & Mar & Apr & May & Jun & Jul & Aug & Sep & Oct & Nov & Dec \\
\hline 1 & 0.00 & 0.04 & 0.00 & 0.82 & 0.00 & 0.00 & 0.00 & 0.00 & 0.00 & 0.00 & 0.04 & 0.08 \\
\hline 2 & .00 & .00 & .00 & .04 & .00 & .00 & .00 & .00 & .00 & .00 & .04 & .00 \\
\hline 3 & .00 & .00 & .00 & .00 & .00 & .00 & .00 & .00 & .00 & .00 & .00 & .00 \\
\hline 4 & .00 & 2.57 & .74 & .00 & .00 & .00 & .00 & .00 & .00 & .00 & .04 & .04 \\
\hline 5 & .00 & 1.25 & .86 & .00 & .00 & .00 & .00 & .00 & .00 & .00 & .00 & 1.87 \\
\hline 6 & .00 & .00 & .08 & .00 & .00 & .00 & .00 & .00 & .04 & .00 & .00 & .16 \\
\hline 7 & .00 & .00 & .00 & .00 & .00 & .00 & .00 & .00 & .00 & .00 & .00 & .00 \\
\hline 8 & .00 & .04 & .00 & .00 & .00 & .00 & .00 & .00 & .00 & .00 & .00 & .04 \\
\hline 9 & .00 & .00 & .00 & .00 & .00 & .00 & .00 & .00 & .00 & .00 & .00 & .74 \\
\hline 10 & .00 & .00 & .00 & .00 & .00 & .00 & .00 & .00 & .00 & .00 & .00 & 2.30 \\
\hline 11 & .00 & .00 & .43 & .00 & .00 & .00 & .04 & .00 & .00 & .00 & .00 & 1.21 \\
\hline 12 & .00 & .00 & .78 & .00 & .00 & .00 & .04 & .00 & .00 & .00 & .00 & .04 \\
\hline 13 & .00 & .00 & .19 & .04 & .00 & .00 & .04 & .00 & .00 & .00 & .00 & .04 \\
\hline 14 & .04 & .00 & .04 & .00 & .00 & .00 & .00 & .00 & .04 & .00 & .04 & .00 \\
\hline 15 & .00 & .00 & .00 & .00 & 1.48 & .00 & .00 & .00 & .00 & .00 & .00 & .00 \\
\hline 16 & 1.56 & .04 & .00 & 1.75 & 1.52 & .00 & .00 & .00 & .00 & .00 & .12 & .00 \\
\hline 17 & .08 & .04 & .00 & .82 & 1.09 & .00 & .00 & .00 & .04 & .00 & 3.82 & .00 \\
\hline 18 & .55 & .04 & .00 & .23 & .16 & .00 & .00 & .00 & .00 & .78 & .08 & .00 \\
\hline 19 & .43 & 2.61 & .00 & .04 & .00 & .00 & .00 & .00 & .00 & .23 & .55 & .00 \\
\hline 20 & .04 & 1.29 & .00 & .00 & .00 & .00 & .00 & .00 & .00 & .00 & .08 & .00 \\
\hline 21 & .58 & .47 & .00 & .00 & .00 & .00 & .00 & .00 & .00 & .00 & 1.91 & 1.68 \\
\hline 22 & .00 & .55 & .00 & .00 & .04 & .00 & .00 & .00 & .00 & .00 & 1.01 & 1.79 \\
\hline 23 & .04 & .08 & .04 & .00 & .12 & .00 & .00 & .00 & .00 & .00 & .00 & .16 \\
\hline 24 & .31 & .31 & .00 & .00 & .08 & .00 & .00 & .00 & .00 & .00 & .00 & .00 \\
\hline 25 & .35 & .47 & .00 & .00 & .00 & .00 & .00 & .00 & .00 & .08 & .04 & .00 \\
\hline 26 & .00 & .23 & .00 & .00 & .00 & .12 & .00 & .00 & .00 & .04 & .00 & .47 \\
\hline 27 & .70 & .00 & .00 & .00 & .00 & .04 & .23 & .00 & .00 & .00 & .00 & .55 \\
\hline 28 & .04 & .08 & .62 & .00 & .00 & .00 & .04 & .00 & .00 & .12 & .00 & .00 \\
\hline 29 & .04 & .00 & .12 & .00 & .00 & .00 & .04 & .00 & .00 & 1.17 & .04 & 1.21 \\
\hline 30 & .00 & - & .00 & .00 & .00 & .00 & .04 & .00 & .00 & .66 & .04 & .39 \\
\hline 31 & .55 & - & .00 & - & .00 & - & .00 & .00 & - & .04 & - & .04 \\
\hline Total & 5.31 & 10.11 & 3.90 & 3.74 & 4.49 & .16 & .47 & .00 & .12 & 3.12 & 7.85 & 12.81 \\
\hline \multicolumn{13}{|c|}{ Calendar year total 52.08} \\
\hline
\end{tabular}




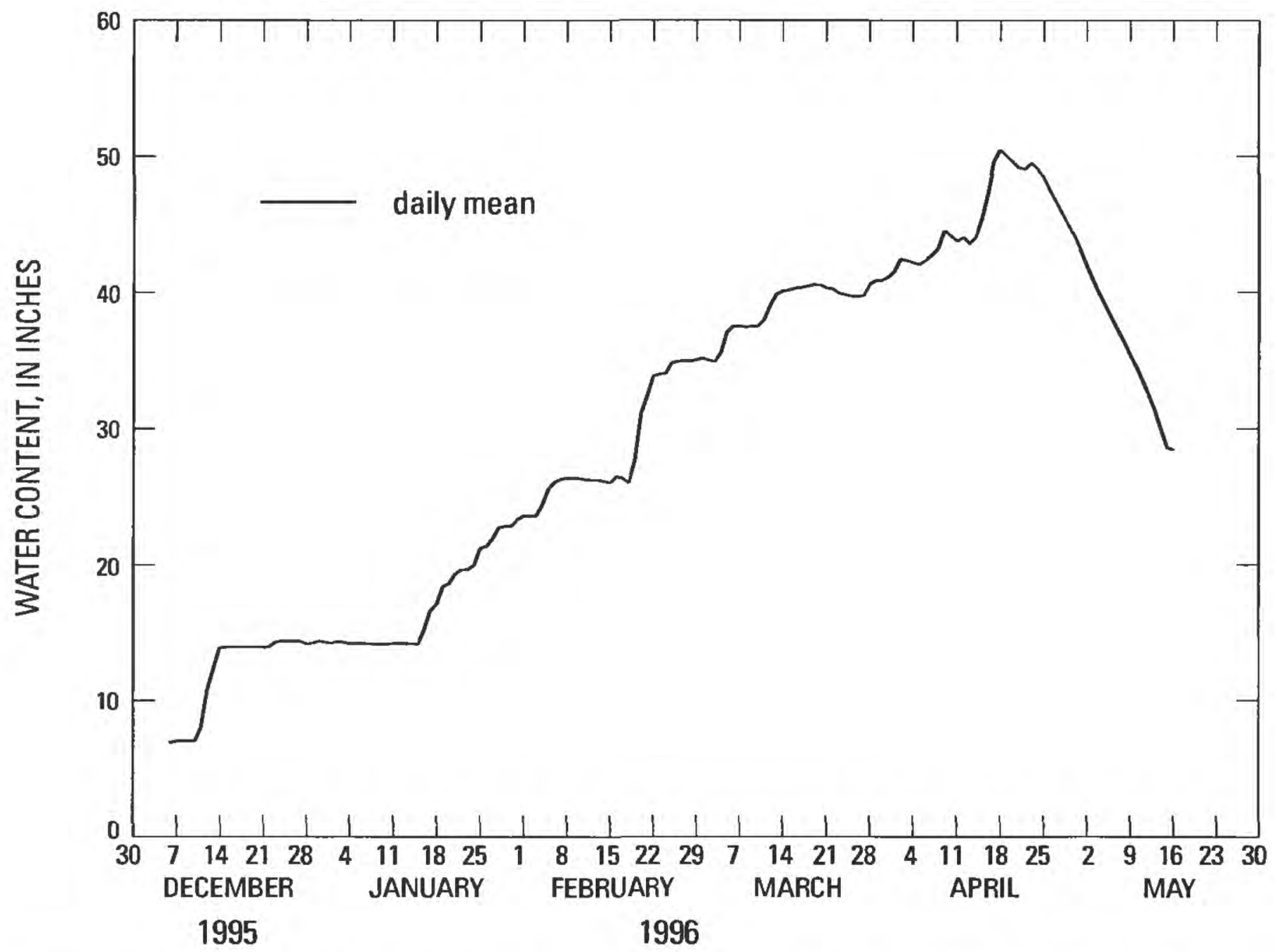

Figure 49. (A) Daily mean water equivalence of snowpack, Horseshoe Lake snowpillow site (HSL), 1995-96, near Mammoth Lakes, Mono County, California.

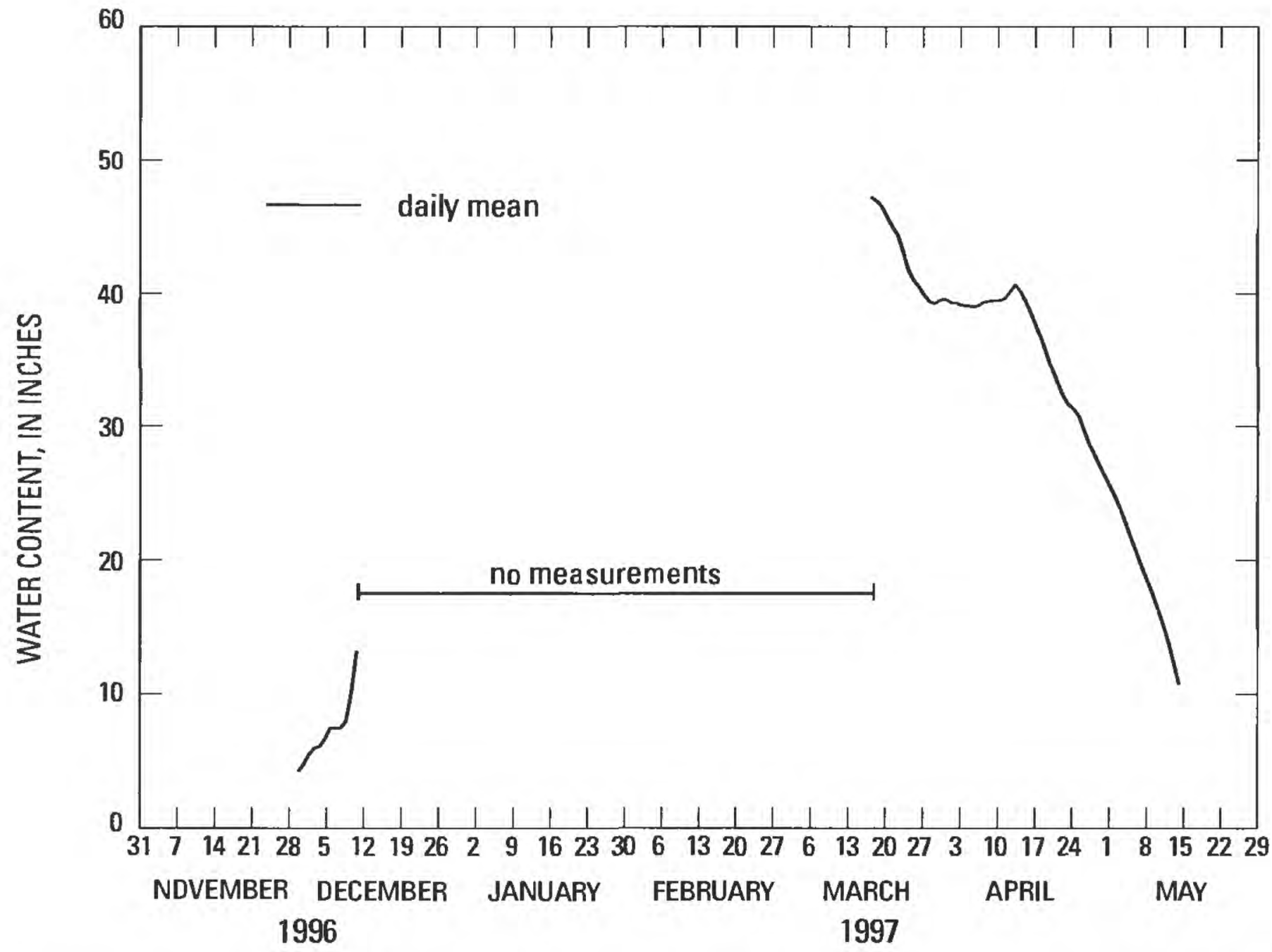

Figure 49. (B) Daily mean water equivalence of snowpack, Horseshoe Lake snowpillow site (HSL), 1996-97, near Mammoth Lakes, Mono County, California. 
Table 54. Daily mean water equivalence of snowpack, Horseshoe Lake snowpillow (HSL), December 1995 to May 1996, Long Valley Caldera, California

[Values are in inches of water. -, no data; Max, maximum; Min, minimum]

\begin{tabular}{|c|c|c|c|c|c|c|}
\hline Day & Dec & Jan & Feb & Mar & Apr & May \\
\hline 1 & - & 14.24 & 23.59 & 35.15 & 41.53 & 43.07 \\
\hline 2 & - & 14.35 & 23.59 & 34.99 & 42.45 & 41.94 \\
\hline 3 & - & 14.32 & 23.59 & 34.91 & 42.35 & 40.89 \\
\hline 4 & - & 14.21 & 24.36 & 35.55 & 42.19 & 39.92 \\
\hline 5 & - & 14.23 & 25.56 & 37.06 & 42.06 & 39.01 \\
\hline 6 & 6.91 & 14.22 & 26.08 & 37.50 & 42.37 & 38.11 \\
\hline 7 & 7.02 & 14.20 & 26.28 & 37.50 & 42.76 & 37.22 \\
\hline 8 & 7.02 & 14.18 & 26.39 & 37.42 & 43.24 & 36.36 \\
\hline 9 & 7.02 & 14.17 & 26.37 & 37.50 & 44.53 & 35.43 \\
\hline 10 & 7.02 & 14.17 & 26.35 & 37.50 & 44.17 & 34.53 \\
\hline 11 & 7.98 & 14.23 & 26.25 & 37.98 & 43.78 & 33.56 \\
\hline 12 & 10.76 & 14.23 & 26.24 & 39.06 & 44.04 & 32.49 \\
\hline 13 & 12.35 & 14.21 & 26.24 & 39.85 & 43.60 & 31.36 \\
\hline 14 & 13.88 & 14.21 & 26.13 & 40.10 & 44.08 & 29.94 \\
\hline 15 & 13.93 & 14.17 & 26.03 & 40.16 & 45.41 & 28.61 \\
\hline 16 & 13.93 & 15.21 & 26.51 & 40.32 & 47.31 & 28.42 \\
\hline 17 & 13.93 & 16.66 & 26.32 & 40.36 & 49.57 & - \\
\hline 18 & 13.93 & 17.12 & 26.00 & 40.46 & 50.45 & - \\
\hline 19 & 13.93 & 18.40 & 27.67 & 40.57 & 50.04 & - \\
\hline 20 & 13.93 & 18.65 & 31.12 & 40.56 & 49.58 & - \\
\hline 21 & 13.93 & 19.34 & 32.39 & 40.33 & 49.16 & - \\
\hline 22 & 13.97 & 19.65 & 33.84 & 40.27 & 49.05 & - \\
\hline 23 & 14.31 & 19.67 & 33.97 & 39.94 & 49.49 & - \\
\hline 24 & 14.41 & 19.98 & 34.03 & 39.83 & 49.09 & - \\
\hline 25 & 14.41 & 21.23 & 34.81 & 39.72 & 48.35 & - \\
\hline 26 & 14.41 & 21.37 & 34.91 & 39.69 & 47.40 & - \\
\hline 27 & 14.40 & 21.95 & 34.97 & 39.77 & 46.58 & - \\
\hline 28 & 14.17 & 22.76 & 34.94 & 40.62 & 45.72 & - \\
\hline 29 & 14.26 & 22.85 & 35.03 & 40.88 & 44.87 & - \\
\hline 30 & 14.41 & 22.88 & - & 40.89 & 44.08 & - \\
\hline 31 & 14.32 & 23.33 & - & 41.12 & - & - \\
\hline Mean & 12.33 & 17.24 & 28.61 & 38.95 & 45.64 & 35.68 \\
\hline Max & 14.41 & 23.33 & 35.03 & 41.12 & 50.45 & 43.07 \\
\hline Min & 6.91 & 14.17 & 23.59 & 34.91 & 41.53 & 28.42 \\
\hline
\end{tabular}

Table 55. Daily mean water equivalence of snowpack, Horseshoe Lake snowpillow (HSL), November 1996 to May 1997, Long Valley Caldera, California

[Values are in inches of water. -, no data; Max, maximum; Min, minimum]

\begin{tabular}{rrrrrrrr}
\hline Day & Nov & Dec & Jan & Feb & Mar & Apr & May \\
\hline 1 & - & 4.72 & - & - & - & 39.54 & 26.17 \\
2 & - & 5.46 & - & - & - & 39.28 & 25.40 \\
3 & - & 593 & - & - & - & 39.26 & 24.61 \\
4 & - & 6.06 & - & - & - & 39.09 & 23.64 \\
5 & - & 6.64 & - & - & - & 39.07 & 22.59 \\
& & & & & & & \\
6 & - & 7.45 & - & - & - & 38.98 & 21.50 \\
7 & - & 7.43 & - & - & - & 39.04 & 20.45 \\
8 & - & 7.44 & - & - & - & 39.32 & 19.41 \\
9 & - & 791 & - & - & - & 3939 & 18.42 \\
10 & - & 10.08 & - & - & - & 39.44 & 17.43 \\
& & & & & & & \\
11 & - & 13.19 & - & - & - & 39.45 & 16.30 \\
12 & - & - & - & - & - & 39.59 & 15.15 \\
13 & - & - & - & - & - & 40.11 & 13.86 \\
14 & - & - & - & - & - & 40.67 & 12.32 \\
15 & - & - & - & - & - & 40.12 & 10.71
\end{tabular}

$\begin{array}{llllllll}16 & - & - & - & - & - & 39.39 & - \\ 17 & - & - & - & - & - & 38.50 & - \\ 18 & - & - & - & - & 47.20 & 37.50 & - \\ 19 & - & - & - & - & 46.91 & 36.55 & - \\ 20 & - & - & - & - & 46.50 & 35.36 & -\end{array}$

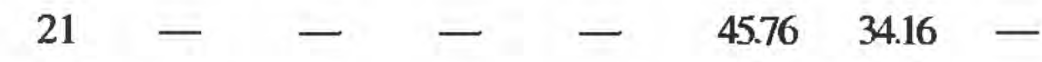

$22-\quad-\quad-\quad 45.0033 .13$ -

23 - $\quad$ - $\quad-\quad 4438 \quad 32.24 \quad-$

24 - $\quad$ - $\quad$ - $\quad$ - $43.17 \quad 31.62 \quad-$

25 - $\quad$ - $\quad$ - $\quad$ - 41.7731 .27 -

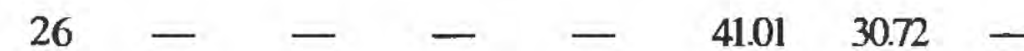

$\begin{array}{llllllll}27 & - & - & - & - & - & 41.01 & 30.72 \\ \end{array}$

28 - $\quad$ - $\quad$ - $\quad 39.8928 .60$ -

29 - $\quad$ - $\quad$ - $\quad$ - $39.36 \quad 27.81 \quad-$

$30 \quad 4.19 \quad-\quad-\quad 39.22 \quad 26.99 \quad-$

31 - $-\quad-3951$ -

\begin{tabular}{lrlllll} 
Mean & 17.48 & - & - & ${ }^{1} 42.87$ & 36.19 & ${ }^{1} 19.20$ \\
Max & 113.19 & - & - & ${ }^{1} 47.20$ & 40.67 & ${ }^{1} 26.17$ \\
Min & 14.72 & - & - & 139.22 & 26.99 & ${ }^{1} 10.71$ \\
\hline
\end{tabular}

${ }^{1}$ Incomplete record. 


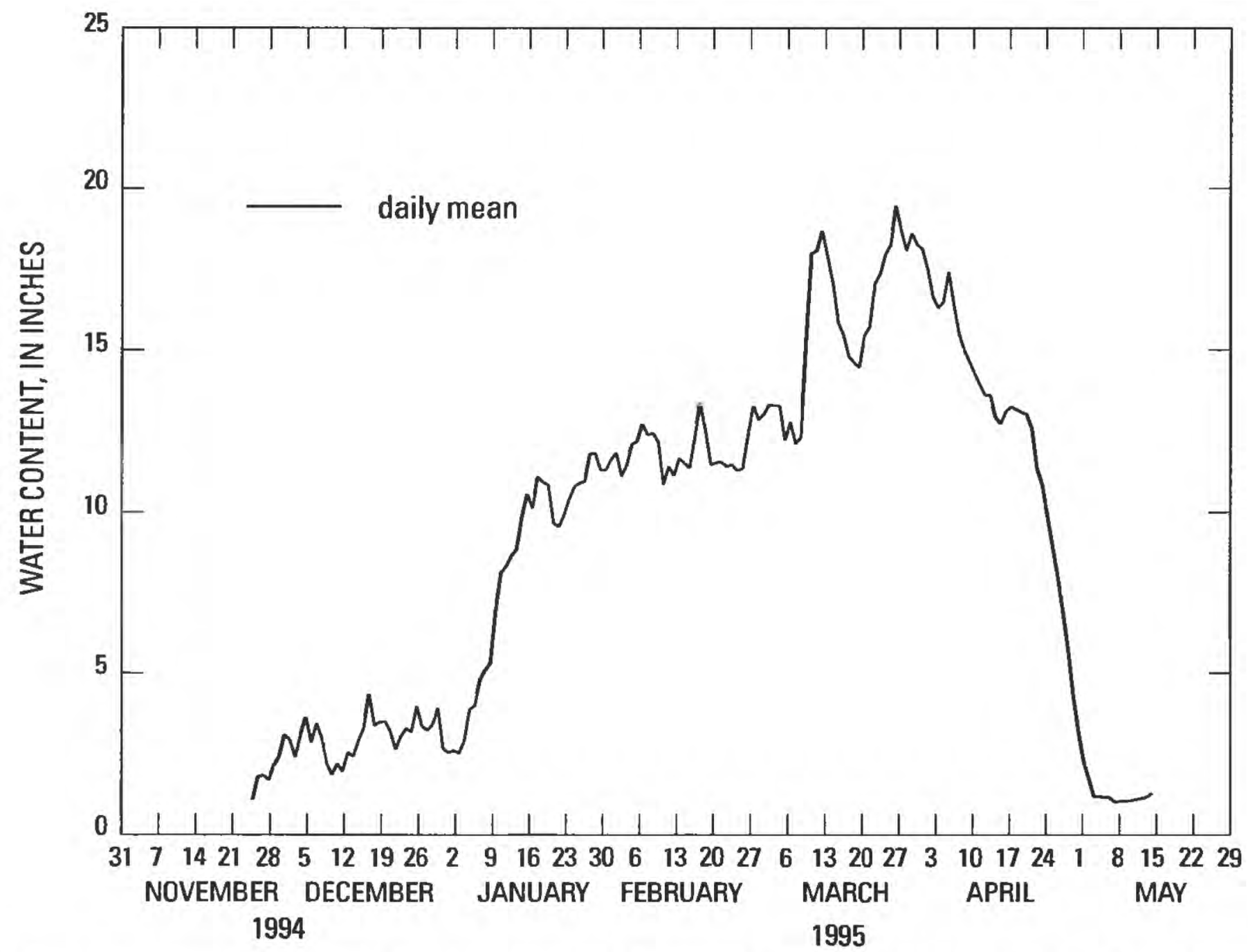

Figure 50. (A) Daily mean water equivalence of snowpack, Lookout Mountain (LKT-P), 1994-95, Long Valley Caldera, Mono County, California.

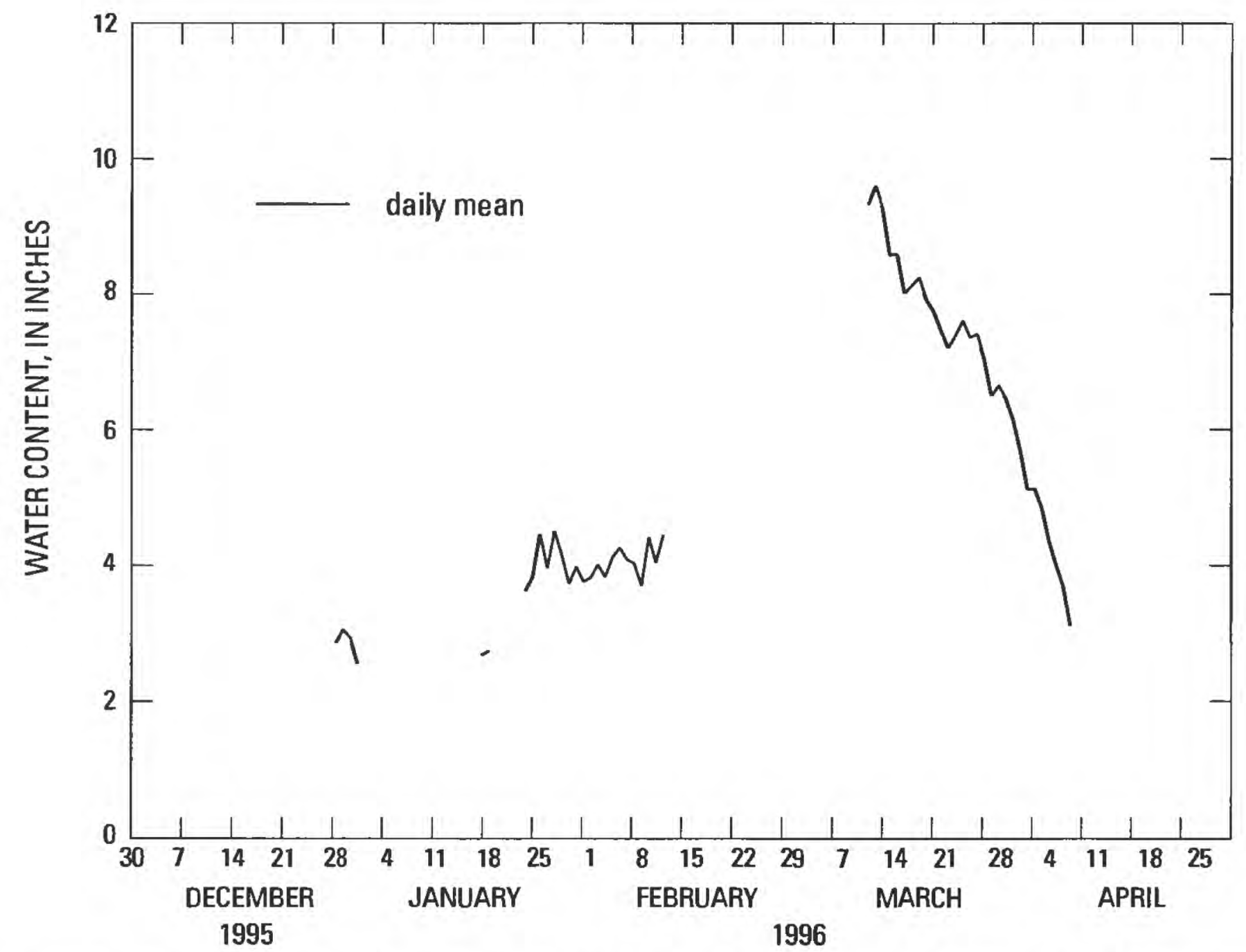

Figure 50. (B) Daily mean water equivalence of snowpack, Lookout Mountain (LKT-P), 1995-96, Long Valley Caldera, Mono County, California. Gaps in record December 1995 to April 1996 are due to equipment problems. 
Table 56. Daily mean water equivalence of snowpack, Lookout Mountain snowpillow (LKT-P), November 1994 to May 1995, Long Valley Caldera, California

[Values are in inches of water. -, no data; Max, maximum; Min, minimum]

\begin{tabular}{|c|c|c|c|c|c|c|c|}
\hline Day & Nov & Dec & Jan & Feb & Mar & Apr & May \\
\hline 1 & - & 3.06 & 2.52 & 11.62 & 12.85 & 18.11 & 3.23 \\
\hline 2 & - & 2.89 & 2.58 & 11.82 & 13.02 & 17.50 & 2.30 \\
\hline 3 & - & 2.38 & 2.50 & 11.10 & 13.30 & 16.64 & 1.74 \\
\hline 4 & - & 3.06 & 2.88 & 11.46 & 13.28 & 16.29 & 1.17 \\
\hline 5 & - & 3.61 & 3.84 & 12.09 & 13.28 & 16.48 & 1.17 \\
\hline 6 & - & 2.83 & 3.97 & 12.17 & 12.22 & 17.42 & 1.14 \\
\hline 7 & - & 3.41 & 4.77 & 12.72 & 12.78 & 16.38 & 1.14 \\
\hline 8 & - & 2.97 & 5.07 & 12.38 & 12.11 & 15.48 & 1.00 \\
\hline 9 & - & 2.18 & 5.29 & 12.44 & 12.31 & 14.97 & 1.02 \\
\hline 10 & - & 1.84 & 6.95 & 12.15 & 15.27 & 14.63 & 1.03 \\
\hline 11 & - & 2.18 & 8.11 & 10.85 & 17.97 & 14.26 & 1.04 \\
\hline 12 & - & 1.94 & 8.29 & 11.40 & 18.06 & 13.91 & 1.08 \\
\hline 13 & - & 2.52 & 8.62 & 11.12 & 18.68 & 13.60 & 1.11 \\
\hline 14 & - & 2.40 & 8.82 & 11.66 & 17.85 & 13.60 & 1.15 \\
\hline 15 & - & 2.88 & 9.82 & 11.50 & 17.04 & 12.95 & 1.27 \\
\hline 16 & - & 3.27 & 10.55 & 11.36 & 15.83 & 12.73 & - \\
\hline 17 & - & 4.33 & 10.11 & 12.33 & 15.45 & 13.11 & - \\
\hline 18 & - & 3.35 & 11.08 & 13.36 & 14.79 & 13.25 & - \\
\hline 19 & - & 3.45 & 10.92 & 12.50 & 14.61 & 13.17 & - \\
\hline 20 & - & 3.47 & 10.83 & 11.46 & 14.46 & 13.08 & - \\
\hline 21 & - & 3.17 & 9.65 & 11.52 & 15.44 & 13.02 & - \\
\hline 22 & - & 2.61 & 9.53 & 11.54 & 15.69 & 12.59 & - \\
\hline 23 & - & 3.02 & 9.89 & 11.40 & 17.04 & 11.34 & - \\
\hline 24 & - & 3.25 & 10.40 & 11.46 & 17.35 & 10.85 & - \\
\hline 25 & 1.06 & 3.15 & 10.78 & 11.27 & 17.96 & 9.91 & - \\
\hline 26 & 1.77 & 3.94 & 10.89 & 11.34 & 18.25 & 8.97 & - \\
\hline 27 & 1.82 & 3.33 & 10.94 & 12.35 & 19.45 & 8.02 & - \\
\hline 28 & 1.67 & 3.20 & 11.78 & 13.31 & 18.68 & 6.95 & - \\
\hline 29 & 2.12 & 3.37 & 11.81 & - & 18.07 & 5.77 & - \\
\hline 30 & 2.39 & 3.90 & 11.29 & - & 18.61 & 4.34 & - \\
\hline 31 & - & 2.64 & 11.29 & - & 18.25 & - & - \\
\hline Mean & 1.80 & 3.02 & 8.25 & 11.85 & 15.80 & 12.98 & 1.37 \\
\hline Max & 2.39 & 4.33 & 11.81 & 13.36 & 19.45 & 18.11 & 3.23 \\
\hline Min & 1.06 & 1.84 & 2.50 & 10.85 & 12.11 & 4.34 & 1.00 \\
\hline
\end{tabular}

Table 57. Daily mean water equivalence of snowpack, Lookout Mountain snowpillow (LKT-P), December 1995 to April 1996, Long Valley Caldera, California

[Values are in inches of water. -, no data; Max, maximum; Min, minimum]

\begin{tabular}{|c|c|c|c|c|c|}
\hline Day & Dec & Jan & Feb & Mar & Apr \\
\hline 1 & - & - & 3.82 & - & 5.13 \\
\hline 2 & - & - & 4.01 & - & 5.14 \\
\hline 3 & - & - & 3.83 & - & 4.85 \\
\hline 4 & - & - & 4.12 & - & 4.36 \\
\hline 5 & - & - & 4.26 & - & 4.00 \\
\hline 6 & - & - & 4.09 & - & 3.68 \\
\hline 7 & - & - & 4.03 & - & 3.11 \\
\hline 8 & - & - & 3.70 & - & - \\
\hline 9 & - & - & 4.42 & - & - \\
\hline 10 & - & - & 4.04 & 9.33 & - \\
\hline 11 & - & - & 4.45 & 9.60 & - \\
\hline 12 & - & - & - & 9.26 & - \\
\hline 13 & - & - & - & 8.58 & - \\
\hline 14 & - & - & - & 8.59 & - \\
\hline 15 & - & - & - & 8.02 & - \\
\hline 16 & - & - & - & 8.13 & - \\
\hline 17 & - & 2.68 & - & 8.25 & - \\
\hline 18 & - & 2.74 & - & 7.92 & - \\
\hline 19 & - & - & - & 7.75 & - \\
\hline 20 & - & - & - & 7.47 & - \\
\hline 21 & - & - & - & 7.21 & - \\
\hline 22 & - & - & - & 7.39 & - \\
\hline 23 & 3.15 & 3.62 & - & 7.61 & - \\
\hline 24 & - & 3.82 & - & 7.36 & - \\
\hline 25 & - & 4.46 & - & 7.41 & - \\
\hline 26 & - & 3.96 & - & 7.03 & - \\
\hline 27 & - & 4.51 & - & 6.50 & - \\
\hline 28 & 2.86 & 4.15 & - & 6.65 & - \\
\hline 29 & 3.05 & 3.73 & 一 & 6.45 & - \\
\hline 30 & 2.93 & 3.98 & - & 6.15 & - \\
\hline 31 & 2.55 & 3.76 & - & 5.70 & - \\
\hline Mean & ${ }^{1} 2.91$ & ${ }^{1} 3.76$ & ' 4.07 & ${ }^{1} 7.65$ & ${ }^{1} 4.32$ \\
\hline Max & 3.15 & ${ }^{1} 4.51$ & $' 4.45$ & ${ }^{1} 9.60$ & ${ }^{1} 5.14$ \\
\hline Min & '2.55 & ${ }^{1} 2.68$ & ${ }^{1} 3.70$ & ${ }^{1} 5.70$ & '3.11 \\
\hline
\end{tabular}




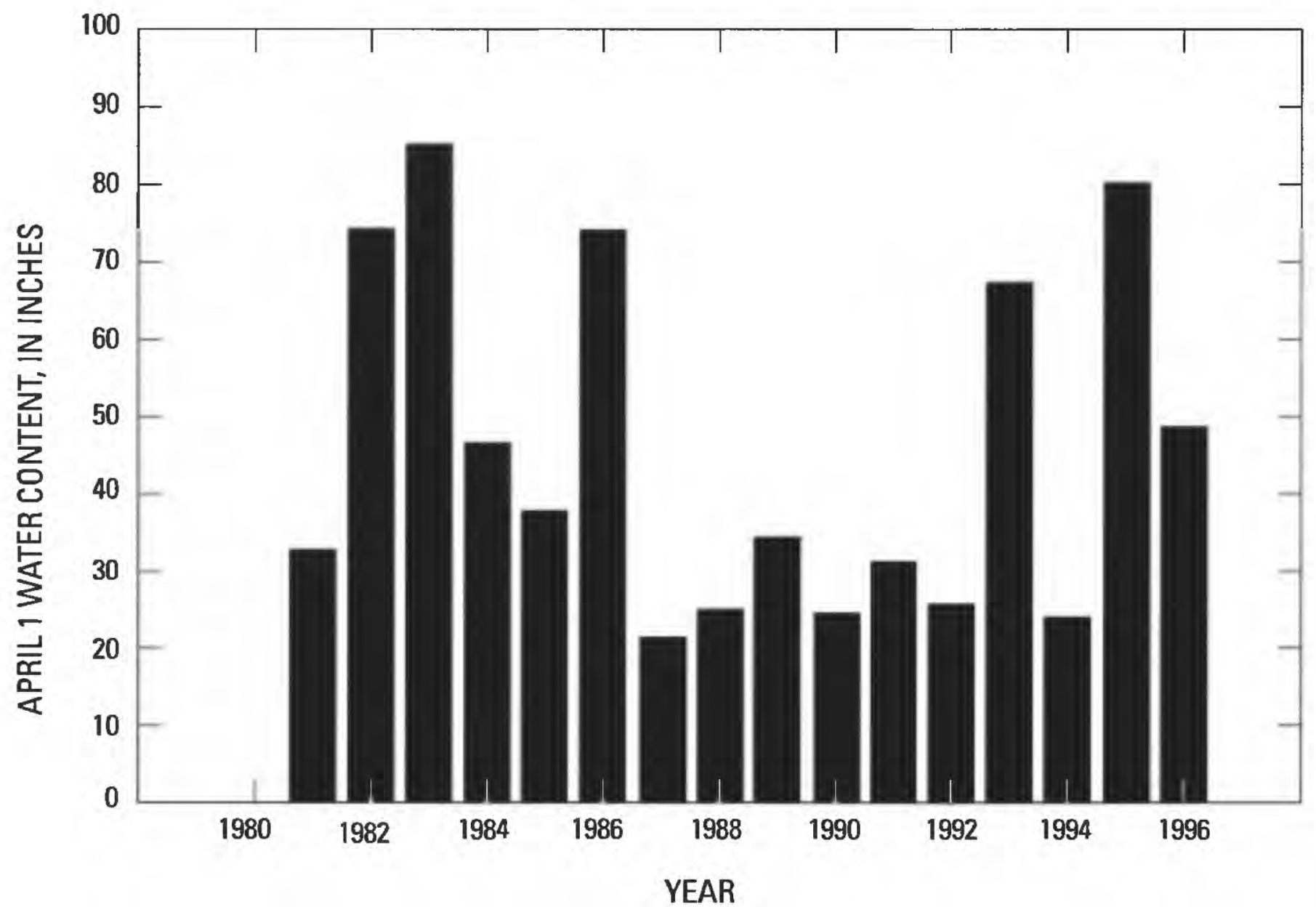

Figure 51. April 1 water equivalence of snowpack, Mammoth Pass Snow Course 205 (MP), 1981-96, near Mammoth Lakes, Mono County, California. Data from California Department of Water Resources. 


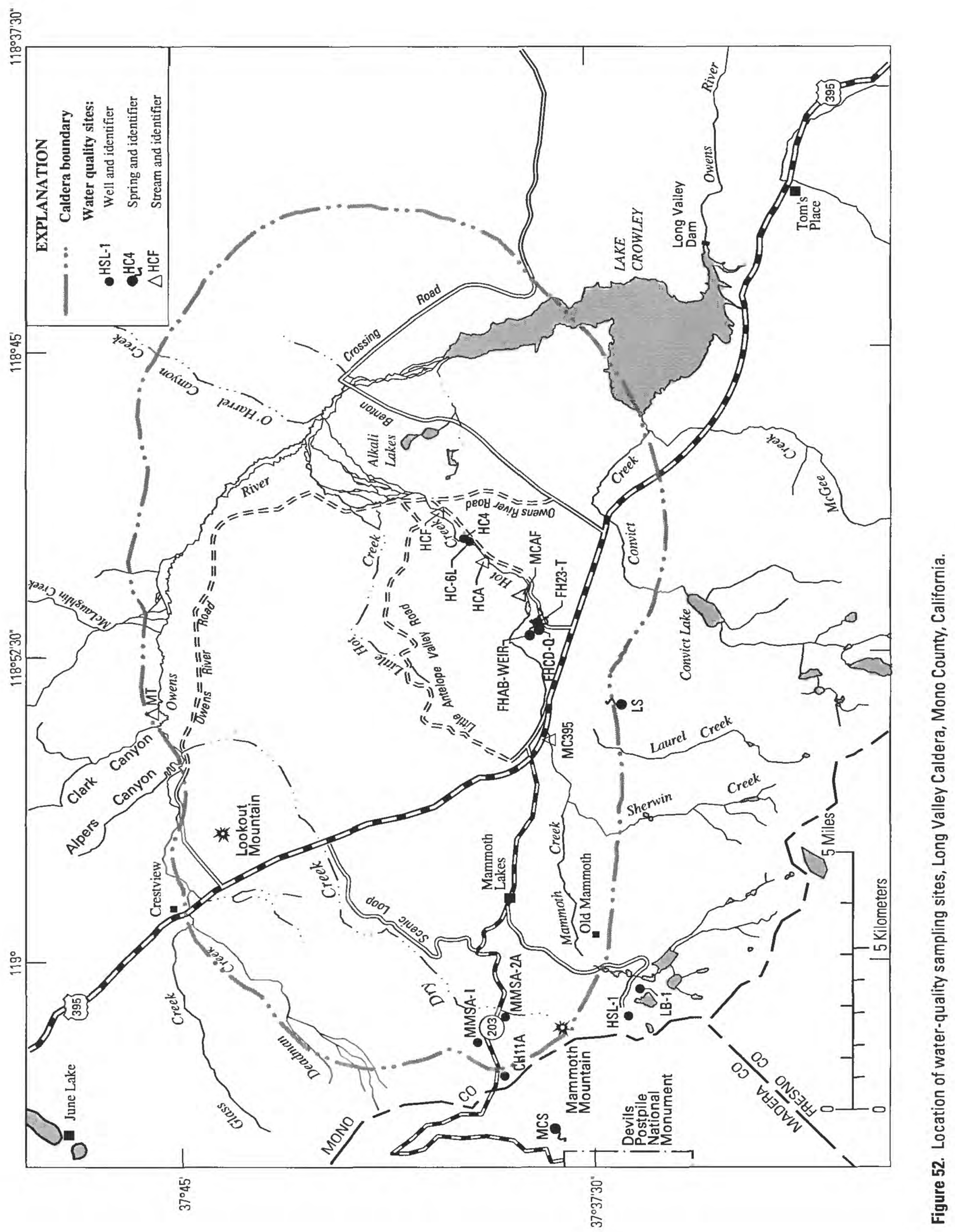


Table 58. Chemical and isotopic analyses of water samples collected at selected well, spring, and stream sites, 1994-96, Long Valley Caldera, California

[ ${ }^{\circ} \mathrm{C}$, degrees Celsius, $\mu \mathrm{S} / \mathrm{cm}$, microsiemens per centimeter at $25^{\circ} \mathrm{C}$; ANC is acid-neutralizing capacity, done in the field by incremental titration; mg/L, milligrams per liter; $\mu \mathrm{g} / \mathrm{L}$, micrograms per liter; ratio per mil, parts per thousand difference from Standard Mean Ocean Water; -, no data; <, less than. Temperature, specific conductance, $\mathrm{pH}$, and ANC measured in the field; other values are from laboratory analyses]

\begin{tabular}{|c|c|c|c|c|c|c|c|c|c|}
\hline $\begin{array}{l}\text { Station } \\
\text { identifier }\end{array}$ & Date & $\begin{array}{l}\text { Tempera- } \\
\text { ture, water } \\
\text { ( } \mathrm{C} \text { ' })\end{array}$ & $\begin{array}{c}\text { Specific } \\
\text { conduc- } \\
\text { tance } \\
(\mu \mathrm{S} / \mathrm{cm})\end{array}$ & $\begin{array}{c}\text { pH water } \\
\text { whole field } \\
\text { (standard } \\
\text { units) }\end{array}$ & $\begin{array}{c}\text { ANC water } \\
\text { unfiltered } \\
\text { (mg/L as } \\
\left.\mathrm{CaCO}_{3}\right)\end{array}$ & $\begin{array}{c}\text { Chloride, } \\
\text { dissolved } \\
\text { (mg/L) }\end{array}$ & $\begin{array}{l}\text { Fluoride, } \\
\text { dissolved } \\
\text { (mg/L) }\end{array}$ & $\begin{array}{c}\text { Nitrogen, } \\
\text { ammonia, } \\
\text { dissolved } \\
\text { (mg/L as } \\
\mathrm{NH}_{3} \text { ) }\end{array}$ & $\begin{array}{c}\text { Nitrogen, } \\
\text { nitrite, dis- } \\
\text { solved } \\
(\mathrm{mg} / \mathrm{L} \text { as } \\
\left.\mathrm{NO}_{2}\right)\end{array}$ \\
\hline \multicolumn{10}{|c|}{ Well sites } \\
\hline $\mathrm{CH}-11 \mathrm{~A}$ & $07-18-96$ & 4.5 & 163 & 5.1 & 80 & 0.2 & 0.9 & 0.020 & 0.010 \\
\hline HSL-1 & $07-18-96$ & 10.0 & 147 & 5.3 & 73 & $>.1$ & .1 & .020 & .010 \\
\hline \multirow[t]{2}{*}{ LB-1 } & $10-17-95$ & 6.0 & 158 & 7.0 & 82 & .5 & $<.1$ & - & - \\
\hline & $07-18-96$ & 5.0 & 189 & 7.0 & 98 & .6 & $<.1$ & .030 & $<.010$ \\
\hline MMSA-1 & $11-03-95$ & 4.5 & 276 & 5.7 & 91 & $<.1$ & 19.0 & $<.015$ & .010 \\
\hline MMSA-2A & $11-02-95$ & 5.5 & 256 & 5.6 & 112 & $<.1$ & 4.7 & $<.015$ & $<.010$ \\
\hline \multicolumn{10}{|c|}{ Spring sites } \\
\hline \multirow[t]{12}{*}{ FHAB-WEIR } & $01-11-94$ & 17.6 & 254 & 7.3 & 97 & 9.4 & .3 & .010 & $<.010$ \\
\hline & $04-12-94$ & 18.0 & 264 & 7.2 & 107 & 10 & .3 & .020 & $<.010$ \\
\hline & $07-12-94$ & 17.8 & 232 & 7.3 & 90 & 9.9 & .3 & $<.010$ & $<.010$ \\
\hline & $10-19-94$ & - & - & - & 一 & 12 & .3 & $<.015$ & $<.010$ \\
\hline & $10-19-94$ & 18.0 & 267 & 7.2 & 101 & 11 & .3 & $<.015$ & $<.010$ \\
\hline & 01-17-95 & 17.8 & 291 & 7.2 & 114 & 10 & .3 & $<.015$ & $<.010$ \\
\hline & 04-18-95 & 17.0 & 287 & 7.2 & 115 & 11 & .3 & $<.015$ & $<.010$ \\
\hline & $06-20-95$ & 16.3 & 217 & 7.3 & - & 8.2 & .3 & .020 & $<.010$ \\
\hline & $07-11-95$ & 16.2 & 212 & 7.2 & 85 & 7.1 & .4 & .020 & $<.010$ \\
\hline & $07-11-95$ & - & - & - & - & 7.1 & .3 & $<.015$ & $<.010$ \\
\hline & $01-24-96$ & 17.5 & 243 & 7.2 & 108 & 5.6 & .3 & $<.015$ & $<.010$ \\
\hline & $07-16-96$ & 16.7 & 224 & 7.2 & 94 & 4.7 & .3 & .030 & $<.010$ \\
\hline \multirow[t]{11}{*}{ FHCD-Q } & $01-11-94$ & 15.5 & 242 & 7.5 & 102 & 4.6 & .3 & .010 & $<.010$ \\
\hline & $04-13-94$ & 16.0 & 247 & 7.4 & 111 & 5.3 & .3 & .030 & $<.010$ \\
\hline & $07-12-94$ & 16.5 & 250 & 7.3 & 109 & 5.2 & .3 & $<.010$ & $<.010$ \\
\hline & $10-19-94$ & 15.5 & 251 & 7.2 & 109 & 5.1 & .3 & $<.015$ & $<.010$ \\
\hline & $01-17-95$ & - & - & - & - & 5.2 & .3 & $<.015$ & $<.010$ \\
\hline & $01-17-95$ & 15.0 & 258 & 7.3 & 109 & - & - & $<.015$ & $<.010$ \\
\hline & 04-18-95 & 15.0 & 249 & 7.3 & 108 & 5.2 & .3 & $<.015$ & $<.010$ \\
\hline & 04-18-95 & 15.0 & 250 & 7.3 & 109 & 5.2 & .3 & $<.015$ & $<.010$ \\
\hline & $07-11-95$ & 15.7 & 223 & 7.3 & 98 & 3.8 & .3 & .020 & $<.010$ \\
\hline & $01-24-96$ & 14.5 & 234 & 7.3 & 107 & 3.8 & .3 & $<.015$ & $<.010$ \\
\hline & $07-16-96$ & 15.5 & 232 & 7.3 & - & 2.9 & .3 & .040 & .010 \\
\hline
\end{tabular}


Table 58. Chemical and isotopic analyses of water samples collected at selected well, spring, and stream sites, 1994-96, Long Valley Caldera, California - Continued

\begin{tabular}{|c|c|c|c|c|c|c|c|c|c|c|}
\hline $\begin{array}{l}\text { Station } \\
\text { identifier }\end{array}$ & Date & $\begin{array}{c}\text { Nitrogen } \\
\mathrm{NO}_{2}+\mathrm{NQ} \\
\text { dissolved } \\
(\mathrm{mg} / \mathrm{L} \text { as } \\
\left.\mathrm{NO}_{3}\right)\end{array}$ & $\begin{array}{l}\text { Phospho- } \\
\text { rus } \\
\text { ortho, } \\
\text { dissolved } \\
\text { (mg/L as } \\
\mathrm{PO}_{4} \text { ) }\end{array}$ & $\begin{array}{l}\text { Sulfate. } \\
\text { dissolved } \\
\text { (mg/L) }\end{array}$ & $\begin{array}{l}\text { Calcium, } \\
\text { dissolved } \\
\text { (mg/L) }\end{array}$ & $\begin{array}{l}\text { Magne- } \\
\text { sium, } \\
\text { dissolved } \\
\text { (mg/L) }\end{array}$ & $\begin{array}{c}\text { Sodium, } \\
\text { dissolved } \\
\text { (mg/L) }\end{array}$ & $\begin{array}{l}\text { Potas- } \\
\text { sium, } \\
\text { dissolved } \\
\text { (mg/L) }\end{array}$ & $\begin{array}{c}\text { Silica, } \\
\text { dissolved } \\
\text { (mg/L) }\end{array}$ & $\begin{array}{c}\text { Boron, } \\
\text { dissolved } \\
\text { ( } \mathrm{gg} / \mathrm{L})\end{array}$ \\
\hline \multicolumn{11}{|c|}{ Well sites (continued) } \\
\hline CH11A & $07-18-96$ & 0.17 & 0.12 & 2.3 & 13 & 8.9 & 6.0 & 3.7 & 37 & 4.2 \\
\hline HSL-1 & $07-18-96$ & .11 & .04 & .30 & 11 & 6.4 & 6.2 & 6.7 & 55 & 12 \\
\hline \multirow[t]{2}{*}{ LB-1 } & $10-17-95$ & - & - & .8 & 17 & 4.9 & 7.3 & 4.4 & 32 & $<10$ \\
\hline & $07-18-96$ & .09 & .05 & .8 & 20 & 5.8 & 8.5 & 5.3 & 33 & 6.7 \\
\hline MMSA-1 & $11-03-95$ & 3.40 & .02 & 2.6 & 15 & 11 & 17 & 8.5 & 43 & 20 \\
\hline MMSA-2A & $11-02-95$ & 2.40 & .17 & 2.2 & 12 & 7.3 & 30 & 3.7 & 50 & 20 \\
\hline \multicolumn{11}{|c|}{ Spring sites (continued) } \\
\hline \multirow[t]{12}{*}{ FHAB-WEIR } & $01-11-94$ & .25 & .11 & 13 & 12 & 8.8 & 26 & 5.8 & 60 & 430 \\
\hline & $04-12-94$ & .28 & .13 & 13 & 12 & 8.6 & 25 & 5.3 & 56 & 470 \\
\hline & $07-12-94$ & .21 & .12 & 11 & 10 & 7.7 & 23 & 5.9 & 56 & 510 \\
\hline & $10-19-94$ & .25 & .14 & 12 & 13 & 9.1 & 28 & 6.0 & 59 & 540 \\
\hline & $10-19-94$ & .25 & .14 & 13 & 13 & 9.2 & 28 & 6.0 & 60 & 530 \\
\hline & $01-17-95$ & .26 & .15 & 12 & 14 & 10 & 28 & 6.0 & 61 & 490 \\
\hline & $04-18-95$ & .69 & .15 & 11 & 14 & 10 & 27 & 5.9 & 59 & 520 \\
\hline & $06-20-95$ & .52 & .12 & 9.4 & 10 & 7.5 & 22 & 4.9 & 59 & 410 \\
\hline & $07-11-95$ & .49 & .14 & 9.1 & 9.4 & 7.2 & 21 & 4.5 & 58 & 360 \\
\hline & $07-11-95$ & .49 & .14 & 9.1 & 9.4 & 7.3 & 21 & 4.8 & 59 & 360 \\
\hline & $01-24-96$ & .29 & .13 & 9.5 & 13 & 10 & 24 & 5.5 & 57 & 270 \\
\hline & $07-16-96$ & .33 & .16 & 10 & 11 & 8.3 & 22 & 5.1 & 58 & 277 \\
\hline \multirow[t]{10}{*}{ FHCD-Q } & $01-11-94$ & .29 & .15 & 11 & 12 & 9.3 & 24 & 5.2 & 56 & 240 \\
\hline & 04-13-94 & .28 & .16 & 11 & 12 & 9.4 & 23 & 5.2 & 56 & 260 \\
\hline & $07-12-94$ & .24 & .16 & 11 & 12 & 9.5 & 24 & 5.5 & 55 & 270 \\
\hline & $10-19-94$ & .27 & .17 & 10 & 13 & 9.6 & 25 & 5.3 & 57 & 260 \\
\hline & $01-17-95$ & .26 & .17 & 11 & 13 & 10 & 24 & 5.1 & 58 & 260 \\
\hline & $04-18-95$ & .43 & .17 & 9.5 & 13 & 9.4 & 23 & 5.1 & 56 & 250 \\
\hline & $04-18-95$ & .42 & .17 & 9.4 & 13 & 9.3 & 23 & 5.1 & 56 & 250 \\
\hline & $07-11-95$ & .39 & .15 & 10 & 11 & 8.5 & 21 & 4.7 & 55 & 200 \\
\hline & $01-24-96$ & .26 & .14 & 8.8 & 13 & 10 & 21 & 4.8 & 54 & 190 \\
\hline & $07-16-96$ & .25 & .17 & 10 & 12 & 9.1 & 21 & 4.8 & 55 & 176 \\
\hline
\end{tabular}


Table 58. Chemical and isotopic analyses of water samples collected at selected well, spring, and stream sites, 1994-96, Long Valley Caldera, California-Continued

\begin{tabular}{|c|c|c|c|c|c|c|c|c|c|}
\hline $\begin{array}{c}\text { Station } \\
\text { identifier }\end{array}$ & Date & $\begin{array}{c}\text { Arsenic, } \\
\text { dissolved } \\
(\mu \mathrm{g} / \mathrm{L})\end{array}$ & $\begin{array}{c}\text { Lithium, } \\
\text { dissolved } \\
(\mu \mathrm{g} / \mathrm{L})\end{array}$ & $\begin{array}{c}\text { Mercury, } \\
\text { dissolved } \\
(\mu \mathrm{g} / \mathrm{L})\end{array}$ & $\begin{array}{c}\text { Iron, } \\
\text { dissolved } \\
(\mu \mathrm{g} / \mathrm{L})\end{array}$ & $\begin{array}{c}\text { Manga- } \\
\text { nese, } \\
\text { dissolved } \\
(\mu \mathrm{g} / \mathrm{L})\end{array}$ & $\begin{array}{c}\text { Solids, } \\
\text { residue et } \\
180^{\circ} \mathrm{C} \text {, } \\
\text { dissolved } \\
\text { (mg/L) }\end{array}$ & $\begin{array}{c}\mathrm{H}-2 / \mathrm{H}-1 \\
\text { stable } \\
\text { isotope } \\
\text { ratio } \\
\text { per mil }\end{array}$ & $\begin{array}{c}\text { 0-18/0-16 } \\
\text { stable } \\
\text { isotope } \\
\text { ratio } \\
\text { per mil }\end{array}$ \\
\hline \multicolumn{10}{|c|}{ Well sites (continued) } \\
\hline CH11A & $07-18-96$ & $<1$ & - & - & 19 & 91 & 98 & - & -14.58 \\
\hline HSL-1 & $07-18-96$ & $<1$ & - & - & 35 & 46 & 100 & - & -14.04 \\
\hline \multirow[t]{2}{*}{ LB-1 } & $10-17-95$ & $<1$ & $<10$ & $<0.1$ & $<3$ & $<1$ & 98 & - & -14.9 \\
\hline & $07-18-96$ & $<1$ & - & - & $<3$ & $<1$ & 114 & - & -14.65 \\
\hline MMSA-1 & $11-03-95$ & $<1$ & 70 & - & 7 & 180 & 184 & - & -14.86 \\
\hline \multirow[t]{2}{*}{ MMSA-2A } & $11-02-95$ & $<1$ & 150 & - & 5 & 6 & 176 & - & -15.09 \\
\hline & \multicolumn{9}{|c|}{ Spring sites (continued) } \\
\hline \multirow[t]{12}{*}{ FHAB-WEIR } & $01-11-94$ & 44 & 100 & $<.1$ & 7 & $<1$ & 200 & - & - \\
\hline & 04-12-94 & 44 & 90 & $<.1$ & 3 & 1 & 200 & - & - \\
\hline & $07-12-94$ & 43 & 90 & $<.1$ & 4 & $<1$ & 164 & - & - \\
\hline & $10-19-94$ & 52 & 100 & $<.1$ & $<3$ & $<1$ & 192 & - & - \\
\hline & $10-19-94$ & 52 & 100 & $<.1$ & $<3$ & $<1$ & 192 & - & - \\
\hline & $01-17-95$ & 45 & 110 & $<.1$ & $<3$ & $<1$ & 208 & - & - \\
\hline & $04-18-95$ & 46 & 100 & $<.1$ & 12 & $<1$ & 214 & - & - \\
\hline & $06-20-95$ & 42 & 90 & - & 11 & $<1$ & 166 & - & - \\
\hline & $07-11-95$ & 43 & 80 & $<.1$ & 13 & $<1$ & 164 & - & - \\
\hline & $07-11-95$ & 48 & 80 & $<.1$ & 11 & $<1$ & 164 & - & - \\
\hline & $01-24-96$ & 39 & 100 & $<.1$ & $<3$ & $<1$ & 178 & - & - \\
\hline & $07-16-96$ & 36 & 76 & .1 & $<3$ & $<1$ & 150 & - & - \\
\hline \multirow[t]{10}{*}{ FHCD-Q } & 01-11-94 & 50 & 80 & $<.1$ & $<3$ & $<1$ & 184 & - & - \\
\hline & $04-13-94$ & 48 & 80 & $<.1$ & 5 & $<1$ & 186 & - & - \\
\hline & $07-12-94$ & 45 & 80 & $<.1$ & $<3$ & $<1$ & 168 & - & - \\
\hline & $10-19-94$ & 55 & 80 & $<.1$ & $<3$ & $<1$ & 180 & - & - \\
\hline & 01-17-95 & - & - & - & - & - & - & - & - \\
\hline & 04-18-95 & 48 & 80 & $<1$ & 7 & 1 & 186 & - & - \\
\hline & 04-18-95 & 49 & 80 & $<.1$ & 5 & 1 & 186 & - & - \\
\hline & $07-11-95$ & 45 & 70 & $<1$ & 6 & $<1$ & 168 & - & - \\
\hline & $01-24-96$ & 43 & 80 & $<1$ & 3 & 1 & 172 & - & - \\
\hline & $07-16-96$ & 37 & 68 & $<1$ & 5 & 1 & 150 & - & - \\
\hline
\end{tabular}


Table 58. Chemical and isotopic analyses of water samples collected at selected well, spring, and stream sites, 1994-96, Long Valley Caldera, California-Continued

\begin{tabular}{|c|c|c|c|c|c|c|c|c|c|}
\hline $\begin{array}{l}\text { Station } \\
\text { identifier }\end{array}$ & Date & $\begin{array}{l}\text { Tempera- } \\
\text { ture, water } \\
\text { ( } \mathrm{C} \text { ) }\end{array}$ & $\begin{array}{c}\text { Specific } \\
\text { conduc- } \\
\text { tance } \\
(\mu \mathrm{S} / \mathrm{cm})\end{array}$ & $\begin{array}{c}\text { pH water } \\
\text { whole field } \\
\text { (standard } \\
\text { units) }\end{array}$ & $\begin{array}{l}\text { ANC water } \\
\text { unfiltered } \\
\text { (mg/L as } \\
\mathrm{CaCO}_{3} \text { ) }\end{array}$ & $\begin{array}{l}\text { Chloride, } \\
\text { dissolved } \\
\text { (mg/L) }\end{array}$ & $\begin{array}{c}\text { Fluoride, } \\
\text { dissolved } \\
\text { (mg/L) }\end{array}$ & $\begin{array}{c}\text { Nitrogen, } \\
\text { ammonia, } \\
\text { dissolved } \\
\text { (mg/L as } \\
\mathrm{NH}_{3} \text { ) }\end{array}$ & $\begin{array}{l}\text { Nitrogen, } \\
\text { nitrite, dis- } \\
\text { solved } \\
\text { (mg/L as } \\
\mathrm{NO}_{2} \text { ) }\end{array}$ \\
\hline \multicolumn{10}{|c|}{ Spring sites (continued) } \\
\hline \multirow[t]{8}{*}{ FH23-T } & 01-11-94 & 11.5 & 219 & 7.3 & 83 & 0.2 & 7.5 & $<0.010$ & $<0.010$ \\
\hline & $04-12-94$ & 11.2 & 216 & 7.3 & 87 & .2 & 8.3 & .020 & $<.010$ \\
\hline & $07-12-94$ & 11.0 & 221 & 7.3 & 88 & .2 & 7.4 & $<.010$ & $<.010$ \\
\hline & $01-18-95$ & 10.1 & 224 & 7.3 & 85 & .2 & 8.3 & $<.015$ & $<.010$ \\
\hline & $04-18-95$ & 11.0 & 236 & 7.1 & 91 & .2 & 10.0 & $<.015$ & $<.010$ \\
\hline & $07-11-95$ & 11.0 & 237 & 7.1 & 94 & .2 & 8.7 & .020 & $<.010$ \\
\hline & $01-24-96$ & 11.5 & 220 & 7.2 & 91 & .2 & 6.8 & $<.015$ & $<.010$ \\
\hline & $07-16-96$ & 11.0 & 223 & 7.3 & 78 & .2 & 7.0 & .060 & .010 \\
\hline LS & $07-28-95$ & 11.5 & 115 & 8.7 & 39 & .3 & .1 & .020 & $<.010$ \\
\hline MCS & $07-14-94$ & 8.0 & 265 & 5.7 & 128 & .3 & .1 & $<.010$ & $<.010$ \\
\hline \multicolumn{10}{|c|}{ Stream sites } \\
\hline \multirow[t]{12}{*}{ MC 395} & $01-10-94$ & .1 & 126 & 8.0 & 60 & .4 & $<.1$ & .010 & $<.010$ \\
\hline & $04-12-94$ & 2.0 & 148 & 8.2 & 75 & .4 & .1 & .030 & $<.010$ \\
\hline & $07-11-94$ & 18.0 & 86 & 8.2 & 40 & .3 & $<.1$ & $<.010$ & $<.010$ \\
\hline & $10-19-94$ & 2.0 & 133 & 8.0 & 64 & .3 & .1 & $<.015$ & $<.010$ \\
\hline & $01-17-95$ & .1 & 130 & 7.8 & 60 & .5 & $<.1$ & $<.015$ & $<.010$ \\
\hline & $04-17-95$ & .5 & 148 & 8.1 & 71 & .7 & .1 & $<.015$ & $<.010$ \\
\hline & $07-10-95$ & 12.0 & 36 & 7.5 & 17 & - & - & $<.015$ & $<.010$ \\
\hline & $10-16-95$ & 7.5 & 93 & 7.9 & 43 & .2 & .1 & $<.015$ & $<.010$ \\
\hline & $01-24-96$ & .5 & 120 & 8.0 & 59 & 3 & .2 & $<.015$ & $<.010$ \\
\hline & $04-23-96$ & 3.0 & 128 & 7.9 & 61 & .4 & .1 & $<.015$ & $<.010$ \\
\hline & $07-16-96$ & 12.5 & 51 & 7.9 & 24 & .1 & $<.1$ & .050 & $<.010$ \\
\hline & $10-16-96$ & 6.0 & 129 & 8.2 & 61 & .3 & .1 & .020 & .010 \\
\hline \multirow[t]{12}{*}{ MCAF } & $01-10-94$ & 5.5 & 175 & 8.2 & 73 & 4.9 & .2 & .010 & $<.010$ \\
\hline & $04-12-94$ & 5.5 & 170 & 8.0 & 77 & 3.3 & .2 & .030 & $<.010$ \\
\hline & $07-11-94$ & 25.0 & 101 & 8.5 & 45 & 1.6 & .1 & $<.010$ & $<.010$ \\
\hline & $10-19-94$ & 5.5 & 168 & 8.1 & 73 & 4.2 & .2 & $<.015$ & $<.010$ \\
\hline & 01-17-95 & .1 & 166 & 7.7 & 66 & 5.0 & .1 & .020 & $<.010$ \\
\hline & 04-18-95 & .5 & 186 & 7.9 & 81 & 4.4 & .2 & $<.015$ & $<.010$ \\
\hline & $10-16-95$ & 14.5 & 127 & 8.2 & 55 & 2.2 & .1 & $<.015$ & $<.010$ \\
\hline & $10-16-95$ & - & - & - & - & 2.3 & $<.1$ & $<.015$ & $<.010$ \\
\hline & $01-24-96$ & .5 & 150 & 8.0 & 70 & 2.7 & .2 & $<.015$ & $<.010$ \\
\hline & $04-23-96$ & 13.0 & 141 & 8.0 & 65 & 1.4 & .1 & $<.015$ & $<.010$ \\
\hline & $07-16-96$ & 18.0 & 86 & 7.8 & 38 & 1.4 & .2 & .030 & $<.010$ \\
\hline & $10-16-96$ & 11.0 & 154 & 8.2 & 68 & 3.2 & .2 & .020 & .020 \\
\hline MT & $02-18-96$ & 11.9 & 414 & 7.8 & - & .4 & 6.7 & - & - \\
\hline
\end{tabular}


Table 58. Chemical and isotopic analyses of water samples collected at selected well, spring, and stream sites, 1994-96, Long Valley Caldera, California - Continued

\begin{tabular}{|c|c|c|c|c|c|c|c|c|c|c|}
\hline $\begin{array}{c}\text { Station } \\
\text { identifier }\end{array}$ & Date & $\begin{array}{c}\text { Nitrogen } \\
\mathrm{NO}_{2}+\mathrm{NQ} \\
\text { dissolved } \\
(\mathrm{mg} / \mathrm{L} \text { as } \\
\left.\mathrm{NO}_{3}\right)\end{array}$ & $\begin{array}{l}\text { Phospho- } \\
\text { rus } \\
\text { ortho, } \\
\text { dissolved } \\
(\mathrm{mg} / \mathrm{L} \text { as } \\
\left.\mathrm{PO}_{4}\right)\end{array}$ & $\begin{array}{l}\text { Sulfate, } \\
\text { dissolved } \\
\text { (mg/L) }\end{array}$ & $\begin{array}{l}\text { Calcium, } \\
\text { dissolved } \\
\text { (mg/L) }\end{array}$ & $\begin{array}{l}\text { Magne- } \\
\text { sium, } \\
\text { dissolved } \\
\text { (mg/L) }\end{array}$ & $\begin{array}{l}\text { Sodium, } \\
\text { dissolved } \\
\text { (mg/L) }\end{array}$ & $\begin{array}{l}\text { Potas- } \\
\text { sium, } \\
\text { dissolved } \\
\text { (mg/L) }\end{array}$ & $\begin{array}{l}\text { Silica, } \\
\text { dissolved } \\
\text { (mg/L) }\end{array}$ & $\begin{array}{c}\text { Boron, } \\
\text { dissolved } \\
(\mu \mathrm{g} / \mathrm{L})\end{array}$ \\
\hline \multicolumn{11}{|c|}{ Spring sites (continued) } \\
\hline \multirow[t]{9}{*}{ FH23-T } & $01-11-94$ & 0.30 & 0.09 & 12 & 18 & 6.2 & 16 & 4.1 & 38 & 110 \\
\hline & $04-12-94$ & .34 & .09 & 12 & 18 & 6.1 & 16 & 3.7 & 38 & 120 \\
\hline & $07-12-94$ & .25 & .09 & 11 & 18 & 6.1 & 16 & 4.1 & 38 & 130 \\
\hline & $01-18-95$ & .32 & .11 & 11 & 18 & 6.1 & 17 & 3.6 & 40 & 120 \\
\hline & 04-18-95 & .43 & .11 & 10 & 19 & 6.4 & 17 & 3.9 & 39 & 120 \\
\hline & $07-11-95$ & .60 & .10 & 11 & 20 & 6.7 & 17 & 3.9 & 40 & 120 \\
\hline & $01-24-96$ & .35 & .10 & 9.8 & 18 & 6.6 & 16 & 3.8 & 39 & 110 \\
\hline & $07-16-96$ & .46 & .12 & 12 & 18 & 6.4 & 18 & 3.9 & 42 & 113 \\
\hline & $07-16-96$ & 一 & 一 & 11 & 18 & 6.6 & 17 & 3.7 & 40 & 120 \\
\hline LS & $07-28-95$ & .13 & .01 & 15 & 16 & .57 & 5.7 & 1.2 & 21 & 10 \\
\hline MCS & 07-14-94 & $<.05$ & .13 & 9.2 & 14 & 13 & 15 & 8.6 & 65 & 20 \\
\hline \multicolumn{11}{|c|}{ Stream sites (continued) } \\
\hline \multirow[t]{12}{*}{ MC 395} & $01-10-94$ & .08 & .04 & 5.0 & 11 & 5.0 & 8.9 & 2.1 & 25 & $<10$ \\
\hline & 04-12-94 & $<.05$ & .04 & 6.0 & 12 & 6.1 & 10 & 2.3 & 27 & $<10$ \\
\hline & $07-11-94$ & $<.05$ & .02 & 3.5 & 8.1 & 2.7 & 5.1 & 1.4 & 9.6 & $<10$ \\
\hline & $10-19-94$ & $<.05$ & .03 & 4.1 & 11 & 4.8 & 9.0 & 2.0 & 17 & 10 \\
\hline & $01-17-95$ & .06 & .03 & 4.9 & 11 & 4.5 & 8.4 & 2.2 & 22 & 20 \\
\hline & 04-17-95 & .06 & .04 & 5.8 & 12 & 5.5 & 10 & 2.2 & 27 & 10 \\
\hline & $07-10-95$ & $<.05$ & .01 & - & - & - & - & - & - & - \\
\hline & $10-16-95$ & $<.05$ & .03 & 2.9 & 7.4 & 3.3 & 5.0 & 1.4 & 16 & $<10$ \\
\hline & $01-24-96$ & .06 & .03 & 3.8 & 10 & 4.9 & 7.8 & 1.9 & 24 & $<10$ \\
\hline & $04-23-96$ & $<.05$ & .03 & 5.5 & 11 & 4.7 & 7.8 & 1.9 & 25 & $<10$ \\
\hline & $07-16-96$ & .08 & .03 & 2.4 & 5.5 & 1.4 & 2.9 & .70 & 10 & 6.9 \\
\hline & $10-16-96$ & $<.05$ & .04 & 4.2 & 11 & 5.0 & 8.3 & 2.3 & 19 & 13 \\
\hline \multirow[t]{12}{*}{ MCAF } & $01-10-94$ & $<.05$ & .04 & 7.8 & 12 & 5.9 & 16 & 3.6 & 37 & 210 \\
\hline & $04-12-94$ & $<.05$ & .04 & 7.6 & 11 & 5.8 & 13 & 3.0 & 32 & 140 \\
\hline & $07-11-94$ & $<.05$ & .03 & 4.4 & 8.2 & 2.9 & 7.3 & 2.2 & 14 & 80 \\
\hline & $10-19-94$ & $<.05$ & .03 & 6.5 & 11 & 5.3 & 15 & 3.3 & 29 & 210 \\
\hline & $01-17-95$ & .06 & .04 & 8.1 & 12 & 5.0 & 14 & 3.3 & 28 & 170 \\
\hline & $04-18-95$ & $<.05$ & .05 & 8.3 & 13 & 6.3 & 15 & 3.2 & 32 & 170 \\
\hline & $10-16-95$ & $<.05$ & .03 & 4.0 & 8.2 & 4.2 & 8.8 & 2.3 & 23 & 100 \\
\hline & $10-16-95$ & $<.05$ & .03 & 3.9 & 8.4 & 4.4 & 9.0 & 2.3 & 24 & 100 \\
\hline & $01-24-96$ & $<.05$ & .03 & 4.7 & 11 & 5.7 & 12 & 2.7 & 29 & 110 \\
\hline & $04-23-96$ & $<.05$ & .04 & 6.0 & 11 & 4.9 & 9.6 & 2.4 & 27 & 60 \\
\hline & $07-16-96$ & .07 & .03 & 2.9 & 7.2 & 2.6 & 6.7 & 1.4 & 18 & 82 \\
\hline & $10-16-96$ & $<.05$ & .04 & 5.9 & 10 & 5.1 & 13 & 3.2 & 30 & 164 \\
\hline MT & $02-18-96$ & - & - & 6.3 & 41 & 17 & 22 & 3.1 & 54 & 290 \\
\hline
\end{tabular}


Table 58. Chemical and isotopic analyses of water samples collected at selected well, spring, and stream sites, 1994-96, Long Valley Caldera, California-Continued

\begin{tabular}{|c|c|c|c|c|c|c|c|c|c|}
\hline $\begin{array}{c}\text { Station } \\
\text { identifier }\end{array}$ & Date & $\begin{array}{c}\text { Arsenic, } \\
\text { dissolved } \\
(\mu \mathrm{g} / \mathrm{L} \mid\end{array}$ & $\begin{array}{l}\text { Lithium, } \\
\text { dissolved } \\
(\mu \mathrm{g} / \mathrm{L})\end{array}$ & $\begin{array}{c}\text { Mercury, } \\
\text { dissolved } \\
(\mu \mathrm{g} / \mathrm{L})\end{array}$ & $\begin{array}{c}\text { Iron, } \\
\text { dissolved } \\
(\mu \mathrm{g} / \mathrm{L})\end{array}$ & $\begin{array}{c}\text { Manga- } \\
\text { nese, } \\
\text { dissolved } \\
(\mu \mathrm{g} / \mathrm{L})\end{array}$ & $\begin{array}{l}\text { Solids, } \\
\text { residue at } \\
180^{\circ} \mathrm{C} \\
\text { dissolved } \\
\text { (mg/L) }\end{array}$ & $\begin{array}{l}\mathrm{H}-2 / \mathrm{H}-1 \\
\text { stable } \\
\text { isotope } \\
\text { ratio } \\
\text { per mil }\end{array}$ & $\begin{array}{c}\text { 0-18/0-16 } \\
\text { stable } \\
\text { isotope } \\
\text { ratio } \\
\text { per mil }\end{array}$ \\
\hline \multicolumn{10}{|c|}{ Spring sites (continued) } \\
\hline \multirow[t]{9}{*}{ FH23-T } & 01-11-94 & 15 & 50 & $<0.1$ & $<3$ & $<1$ & 152 & - & - \\
\hline & 04-12-94 & 21 & 40 & $<1$ & 9 & $<1$ & 148 & - & - \\
\hline & $07-12-94$ & 23 & 50 & $<.1$ & $<3$ & $<1$ & 132 & - & - \\
\hline & 01-18-95 & 25 & 40 & $<.1$ & $<3$ & $<1$ & 152 & - & - \\
\hline & 04-18-95 & 19 & 40 & $<.1$ & $<3$ & $<1$ & 168 & - & - \\
\hline & $07-11-95$ & 23 & 50 & $<.1$ & $<3$ & $<1$ & 158 & - & - \\
\hline & $01-24-96$ & 21 & 40 & $<.1$ & $<3$ & $<1$ & 154 & - & - \\
\hline & $07-16-96$ & 24 & 43 & $<.1$ & $<3$ & $<1$ & 140 & - & - \\
\hline & $07-16-96$ & 24 & - & - & $<3$ & $<1$ & 158 & - & 一 \\
\hline LS & $07-28-95$ & 4 & $<10$ & - & $<3$ & $<1$ & 82 & -127.0 & -16.79 \\
\hline \multirow[t]{2}{*}{ MCS } & $07-14-94$ & 1 & 50 & $<0.1$ & 5 & 3 & 182 & - & - \\
\hline & \multicolumn{9}{|c|}{ Stream sites (continued) } \\
\hline \multirow[t]{11}{*}{ MC 395} & $01-10-94$ & 3 & 40 & $<.1$ & 18 & $<1$ & 88 & - & - \\
\hline & $04-12-94$ & 3 & 30 & $<.1$ & 21 & 2 & 108 & - & - \\
\hline & $07-11-94$ & 3 & 20 & $<.1$ & 29 & 4 & 54 & - & - \\
\hline & $10-19-94$ & 3 & 20 & $<.1$ & 35 & 4 & 88 & - & - \\
\hline & 01-17-95 & 2 & 20 & $<.1$ & 27 & 1 & 88 & - & - \\
\hline & 04-17-95 & 3 & 30 & $<.1$ & 20 & 3 & 104 & - & - \\
\hline & $10-16-95$ & 3 & 10 & $<.1$ & 24 & 4 & 54 & - & - \\
\hline & $01-24-96$ & 4 & 20 & - & 30 & 1 & 82 & - & - \\
\hline & $04-23-96$ & 9 & 24 & $<.1$ & 41 & 4 & 80 & - & - \\
\hline & $07-16-96$ & 2 & 6 & $<.1$ & 45 & 5 & 20 & - & - \\
\hline & $10-16-96$ & 3 & 23 & $<.1$ & 32 & 11 & 86 & - & - \\
\hline \multirow[t]{12}{*}{ MCAF } & $01-10-94$ & 15 & 60 & $<.1$ & 100 & 110 & 142 & - & - \\
\hline & 04-12-94 & 17 & 50 & $<.1$ & 82 & 100 & 124 & - & - \\
\hline & $07-11-94$ & 16 & 30 & $<.1$ & 64 & 84 & 72 & - & - \\
\hline & $10-19-94$ & 23 & 50 & $<.1$ & 67 & 74 & 102 & - & - \\
\hline & 01-17-95 & 18 & 50 & $<.1$ & 100 & 97 & 110 & - & - \\
\hline & 04-18-95 & 18 & 60 & $<.1$ & 100 & 160 & 134 & - & - \\
\hline & $10-16-95$ & 16 & 40 & $<.1$ & 65 & 42 & 78 & - & - \\
\hline & $10-16-95$ & 17 & 40 & $<.1$ & 69 & 43 & 86 & - & - \\
\hline & $01-24-96$ & 17 & 50 & - & 71 & 110 & 102 & - & - \\
\hline & $04-23-96$ & $<1$ & 33 & $<.1$ & 54 & 54 & 90 & - & - \\
\hline & $07-16-96$ & 13 & 25 & $<.1$ & 80 & 47 & 50 & - & - \\
\hline & $10-16-96$ & 20 & 45 & $<.1$ & 61 & 82 & 114 & - & - \\
\hline MT & $02-18-96$ & 3 & 98 & - & 7 & 240 & 254 & -110 & -15.14 \\
\hline
\end{tabular}


Table 59. Partial chemical analyses of water samples collected at selected spring and stream sites, 1994-96, Long Valley Caldera, California $\left[{ }^{\circ} \mathrm{C}\right.$, degrees Celsius, $\mu \mathrm{S} / \mathrm{cm}$, microsiemens per centimeter at $25^{\circ} \mathrm{C} ; \mathrm{mg} / \mathrm{L}$, milligrams per liter; $\mu \mathrm{g} / \mathrm{L}$, micrograms per liter; -, no data. Where two analyses are given for same date, the second analysis was performed at a U.S. Geological Survey laboratory in Menlo Park, Calif., under direction of W. Evans, Chemist, and E. Colvard, Hydrologist. All other analyses shown were done by U.S. Geological Survey Central Laboratory in Arvada, Colo.]

\begin{tabular}{|c|c|c|c|c|c|}
\hline $\begin{array}{l}\text { Station } \\
\text { identifier }\end{array}$ & Date & $\begin{array}{l}\text { Tempereture weter } \\
\text { ( } \mathrm{C} \text { ) }\end{array}$ & $\begin{array}{c}\text { Specific } \\
\text { conductance } \\
(\mu \mathrm{S} / \mathrm{cm})\end{array}$ & $\begin{array}{c}\text { Chloride, } \\
\text { dissolved } \\
\text { (mg/L) }\end{array}$ & $\begin{array}{c}\text { Boron, } \\
\text { dissolved } \\
(\mu \mathrm{g} / \mathrm{L})\end{array}$ \\
\hline \multicolumn{6}{|c|}{ Spring sites } \\
\hline \multirow[t]{40}{*}{ FHAB-WEIR } & 01-11-94 & 17.6 & 254 & 9.4 & 430 \\
\hline & 01-11-94 & - & - & 9.2 & - \\
\hline & $02-16-94$ & 17.8 & 255 & 9.5 & 480 \\
\hline & $02-16-94$ & - & - & 9.9 & - \\
\hline & $03-09-94$ & 17.7 & 259 & 10 & 480 \\
\hline & 03-09-94 & - & - & 9.7 & - \\
\hline & 04-12-94 & 18.0 & 264 & 10 & 470 \\
\hline & 04-12-94 & - & - & 10 & - \\
\hline & $05-17-94$ & 17.6 & 271 & 12 & 570 \\
\hline & $05-17-94$ & - & - & 11 & - \\
\hline & $06-21-94$ & 17.7 & 215 & 9.6 & 470 \\
\hline & $06-21-94$ & - & - & 9.7 & - \\
\hline & $07-12-94$ & 17.8 & 232 & 9.9 & 510 \\
\hline & $07-12-94$ & - & - & 10 & - \\
\hline & 08-09-94 & 18.0 & 257 & 10 & 540 \\
\hline & 08-09-94 & - & - & 11 & - \\
\hline & 09-13-94 & 18.0 & 263 & 11 & 510 \\
\hline & 09-13-94 & - & - & 11 & - \\
\hline & $10-19-94$ & - & - & 12 & 540 \\
\hline & $10-19-94$ & 18.0 & 267 & 11 & 530 \\
\hline & $10-19-94$ & - & - & 12 & - \\
\hline & $11-10-94$ & 18.0 & 276 & 11 & 560 \\
\hline & $11-10-94$ & - & - & 12 & - \\
\hline & $12-15-94$ & 17.7 & 279 & 11 & 540 \\
\hline & $12-15-94$ & - & - & 11 & - \\
\hline & 01-17-95 & 17.8 & 291 & 10 & 490 \\
\hline & $01-17-95$ & - & - & 11 & 520 \\
\hline & $02-22-95$ & 17.5 & 299 & 11 & 520 \\
\hline & $02-22-95$ & - & - & 12 & 540 \\
\hline & $03-22-95$ & 17.2 & 282 & 9.8 & 460 \\
\hline & $03-22-95$ & - & 一 & 10 & 500 \\
\hline & 04-18-95 & 17.0 & 287 & 11 & 520 \\
\hline & 04-18-95 & - & - & 12 & 480 \\
\hline & $05-16-95$ & 17.2 & 283 & 9.9 & 440 \\
\hline & $05-16-95$ & - & - & 10 & 490 \\
\hline & 06-13-95 & 16.0 & 251 & 9.0 & 430 \\
\hline & $06-13-95$ & 一 & - & 9.0 & 470 \\
\hline & $06-20-95$ & 16.3 & 217 & 8.2 & 410 \\
\hline & $07-11-95$ & 16.2 & 212 & 7.1 & 360 \\
\hline & $07-11-95$ & - & - & 6.8 & 380 \\
\hline
\end{tabular}


Tabla 59. Partial chemical analyses of water samples collected at selected spring and stream sites, 1994-96, Long Valley Caldera, California-Continued

\begin{tabular}{|c|c|c|c|c|c|}
\hline $\begin{array}{l}\text { Station } \\
\text { identifier }\end{array}$ & Date & $\begin{array}{l}\text { Temperature weter } \\
\text { ( }{ }^{\circ} \mathrm{C} \text { ) }\end{array}$ & $\begin{array}{c}\text { Specific } \\
\text { conductance } \\
(\mu \mathrm{S} / \mathrm{cm})\end{array}$ & $\begin{array}{l}\text { Chloride. } \\
\text { dissolved } \\
\text { (mg/L) }\end{array}$ & $\begin{array}{l}\text { Boron. } \\
\text { dissolved } \\
(\mu \mathrm{g} / \mathrm{L})\end{array}$ \\
\hline \multirow[t]{31}{*}{ FHAB-WEIR (cont.) } & $07-11-95$ & - & - & 7.1 & 360 \\
\hline & 07-11-95 & - & - & 6.7 & 380 \\
\hline & $07-27-95$ & 16.5 & 213 & - & - \\
\hline & 08-10-95 & 16.4 & 220 & 5.3 & 270 \\
\hline & 08-10-95 & - & - & 5.2 & 290 \\
\hline & 09-11-95 & 16.4 & 223 & 4.3 & 280 \\
\hline & 09-11-95 & - & - & 4.8 & 250 \\
\hline & $10-17-95$ & 16.9 & 230 & 4.4 & 260 \\
\hline & $10-17-95$ & - & - & 5.2 & 260 \\
\hline & $11-08-95$ & 17.2 & 238 & 4.8 & 280 \\
\hline & $11-08-95$ & - & - & 5.3 & 280 \\
\hline & $12-12-95$ & 17.3 & 241 & 5.3 & 290 \\
\hline & $12-12-95$ & - & - & 5.6 & 260 \\
\hline & 01-24-96 & 17.5 & 243 & 5.6 & 270 \\
\hline & 01-24-96 & - & - & 5.9 & 310 \\
\hline & $02-13-96$ & 17.6 & 252 & 5.8 & 300 \\
\hline & $02-13-96$ & - & - & 6.3 & 320 \\
\hline & $03-12-96$ & 17.4 & 258 & 6.1 & 290 \\
\hline & $03-12-96$ & - & - & 5.6 & 320 \\
\hline & 04-23-96 & 17.5 & 256 & 6.2 & 330 \\
\hline & $04-23-96$ & - & - & 6.3 & 320 \\
\hline & $05-15-96$ & 17.0 & 231 & 6.9 & 350 \\
\hline & $05-15-96$ & - & - & 6.9 & 330 \\
\hline & $06-18-96$ & 17.0 & 219 & 5.9 & 318 \\
\hline & $06-18-96$ & - & - & 6.2 & 340 \\
\hline & $07-16-96$ & 16.7 & 224 & 4.7 & 277 \\
\hline & $08-20-96$ & 16.6 & 220 & 4.2 & 247 \\
\hline & $09-17-96$ & 16.8 & 234 & 4.0 & 226 \\
\hline & $10-16-96$ & 17.2 & 240 & 4.8 & 247 \\
\hline & $11-14-96$ & 17.1 & 268 & 5.4 & 292 \\
\hline & $12-19-96$ & 17.4 & 260 & 5.4 & 286 \\
\hline \multirow[t]{12}{*}{ FHCD-Q } & 01-11-94 & 15.5 & 242 & 4.6 & 240 \\
\hline & 01-11-94 & - & - & 4.2 & - \\
\hline & $02-16-94$ & 15.5 & 242 & 5.0 & 250 \\
\hline & 02-16-94 & - & - & 5.2 & - \\
\hline & 03-09-94 & 15.8 & 247 & 5.8 & 280 \\
\hline & 03-09-94 & - & - & 5.9 & - \\
\hline & 04-13-94 & 16.0 & 247 & 5.3 & 260 \\
\hline & 04-13-94 & - & - & 5.0 & - \\
\hline & $05-17-94$ & 15.5 & 255 & 5.3 & 290 \\
\hline & $05-17-94$ & - & - & 5.2 & - \\
\hline & $06-21-94$ & 16.0 & 253 & 6.6 & 330 \\
\hline & $06-21-94$ & - & - & 7.5 & - \\
\hline
\end{tabular}


Table 59. Partial chemical analyses of water samples collected at selected spring and stream sites, 1994-96, Long Valley Caldera, California-Continued

\begin{tabular}{|c|c|c|c|c|c|}
\hline $\begin{array}{c}\text { Station } \\
\text { identifier }\end{array}$ & Date & $\begin{array}{c}\text { Temperature water } \\
\left.\text { ( }{ }^{\circ} \mathrm{C}\right)\end{array}$ & $\begin{array}{c}\text { Specific } \\
\text { conductance } \\
(\mu \mathrm{S} / \mathrm{cm})\end{array}$ & $\begin{array}{l}\text { Chloride, } \\
\text { dissolved } \\
\text { (mg/L) }\end{array}$ & $\begin{array}{c}\text { Boron, } \\
\text { dissolved } \\
(\mu \mathrm{g} / \mathrm{L})\end{array}$ \\
\hline \multirow[t]{43}{*}{ FHCD-Q (cont.) } & $07-12-94$ & 16.5 & 250 & 5.2 & 270 \\
\hline & $07-12-94$ & - & - & 5.3 & - \\
\hline & $08-10-94$ & 15.8 & 251 & 4.7 & 250 \\
\hline & $08-10-94$ & - & - & 4.9 & - \\
\hline & 09-13-94 & 17.0 & 254 & 5.0 & 270 \\
\hline & $09-13-94$ & - & - & 5.3 & - \\
\hline & $10-19-94$ & 15.5 & 251 & 5.1 & 260 \\
\hline & $10-19-94$ & - & - & 5.4 & - \\
\hline & $11-10-94$ & 15.5 & 257 & 5.4 & 290 \\
\hline & $11-10-94$ & - & - & 5.7 & - \\
\hline & $12-15-94$ & 16.0 & 255 & 5.2 & 290 \\
\hline & $12-15-94$ & - & - & 5.7 & - \\
\hline & 01-17-95 & - & 一 & 5.2 & 260 \\
\hline & $01-17-95$ & 15.0 & 258 & 一 & - \\
\hline & $01-17-95$ & - & - & 5.7 & 270 \\
\hline & $02-22-95$ & 16.5 & 264 & 5.1 & 260 \\
\hline & $02-22-95$ & - & - & 5.9 & 270 \\
\hline & $03-22-95$ & 16.2 & 246 & 5.1 & 250 \\
\hline & $03-22-95$ & - & - & 5.4 & 260 \\
\hline & 04-18-95 & 15.0 & 249 & 5.2 & 250 \\
\hline & 04-18-95 & - & - & 5.5 & 230 \\
\hline & 04-18-95 & 15.0 & 250 & 5.2 & 250 \\
\hline & 04-18-95 & - & - & 5.5 & 240 \\
\hline & 05-16-95 & 15.8 & 249 & 5.1 & 210 \\
\hline & $05-16-95$ & - & - & 5.2 & 240 \\
\hline & 06-13-95 & 16.9 & 246 & 4.9 & 240 \\
\hline & 06-13-95 & - & - & 4.9 & 250 \\
\hline & $07-11-95$ & 15.7 & 223 & 3.8 & 200 \\
\hline & 07-11-95 & - & - & 3.5 & 220 \\
\hline & $07-27-95$ & 15.5 & 203 & - & - \\
\hline & $08-10-95$ & 16.0 & 206 & 2.5 & 140 \\
\hline & $08-10-95$ & - & - & 3.2 & 150 \\
\hline & $09-11-95$ & 14.4 & 216 & 2.5 & 140 \\
\hline & 09-11-95 & - & - & 3.0 & 250 \\
\hline & $10-17-95$ & 14.5 & 225 & 3.0 & 230 \\
\hline & $10-17-95$ & - & - & 2.9 & 170 \\
\hline & $11-08-95$ & 16.0 & 236 & 3.0 & 190 \\
\hline & $11-08-95$ & - & - & 3.0 & 180 \\
\hline & $12-12-95$ & 14.0 & 239 & 4.4 & 220 \\
\hline & $12-12-95$ & - & - & 4.8 & 190 \\
\hline & $01-24-96$ & 14.5 & 234 & 3.8 & 190 \\
\hline & $01-24-96$ & - & - & 3.9 & 200 \\
\hline & $02-13-96$ & 15.1 & 243 & 3.7 & 190 \\
\hline
\end{tabular}


Table 59. Partial chemical analyses of water samples collected at selected spring and stream sites,1994-96, Long Valley Caldera, California-Continued

\begin{tabular}{|c|c|c|c|c|c|}
\hline $\begin{array}{c}\text { Station } \\
\text { identifier }\end{array}$ & Date & $\begin{array}{l}\text { Temperature water } \\
\text { ( } \mathrm{C} \text { C) }\end{array}$ & $\begin{array}{c}\text { Specific } \\
\text { conductance } \\
(\mu \mathrm{S} / \mathrm{cm})\end{array}$ & $\begin{array}{l}\text { Chloride, } \\
\text { dissolved } \\
\text { (mg/L) }\end{array}$ & $\begin{array}{c}\text { Boron, } \\
\text { dissolved } \\
(\mu \mathrm{g} / \mathrm{L})\end{array}$ \\
\hline \multirow[t]{15}{*}{ FHCD-Q (cont.) } & $02-13-96$ & - & - & 3.7 & 210 \\
\hline & $03-12-96$ & 14.5 & 246 & 4.1 & 210 \\
\hline & $03-12-96$ & - & - & 4.4 & 220 \\
\hline & $04-23-96$ & 15.3 & 248 & 4.4 & 230 \\
\hline & $04-23-96$ & - & - & 4.7 & 220 \\
\hline & $05-14-96$ & 16.0 & 253 & 4.6 & 240 \\
\hline & $05-14-96$ & - & - & 5.0 & 220 \\
\hline & $06-18-96$ & 16.5 & 246 & 3.4 & 200 \\
\hline & $06-18-96$ & - & - & 3.7 & 200 \\
\hline & $07-16-96$ & 15.5 & 232 & 2.9 & 176 \\
\hline & $08-20-96$ & 15.0 & 226 & 2.5 & 153 \\
\hline & $09-17-96$ & 15.0 & 229 & 3.1 & 155 \\
\hline & $10-16-96$ & 15.0 & 228 & 3.3 & 167 \\
\hline & $11-14-96$ & 14.7 & 255 & 3.4 & 197 \\
\hline & $12-19-96$ & 14.5 & 243 & 3.3 & 190 \\
\hline \multirow[t]{16}{*}{ FH23-T } & $01-11-94$ & 11.5 & 219 & 7.5 & 110 \\
\hline & $01-11-94$ & - & - & 7.5 & - \\
\hline & $04-12-94$ & 11.2 & 216 & 8.3 & 120 \\
\hline & $04-12-94$ & - & - & 8.1 & - \\
\hline & $07-12-94$ & 11.0 & 221 & 7.4 & 130 \\
\hline & $07-12-94$ & - & - & 7.4 & - \\
\hline & $01-18-95$ & 10.1 & 224 & 8.3 & 120 \\
\hline & $01-18-95$ & - & - & 8.8 & 120 \\
\hline & $04-18-95$ & 11.0 & 236 & 10 & 120 \\
\hline & 04-18-95 & - & - & 11 & 110 \\
\hline & $07-11-95$ & 11.0 & 237 & 8.7 & 120 \\
\hline & $07-11-95$ & - & - & 8.6 & 130 \\
\hline & $07-27-95$ & 11.5 & 236 & - & - \\
\hline & $01-24-96$ & 11.5 & 220 & 6.8 & 110 \\
\hline & $01-24-96$ & - & - & 6.9 & 110 \\
\hline & $07-16-96$ & 11.0 & 223 & 7.0 & 113 \\
\hline \multirow[t]{4}{*}{ HC-4 } & $01-12-94$ & 93.4 & 1,563 & 180 & 8,000 \\
\hline & $01-12-94$ & - & - & 180 & - \\
\hline & $04-13-94$ & 94.0 & 1,558 & 190 & 8,800 \\
\hline & 04-13-94 & - & - & 192 & - \\
\hline \multirow[t]{8}{*}{ HC6L } & $03-10-94$ & - & 1,762 & 210 & 10,000 \\
\hline & $03-10-94$ & - & - & 216 & - \\
\hline & 04-13-94 & 94.3 & 1,711 & 210 & 9,900 \\
\hline & 04-13-94 & - & - & 215 & - \\
\hline & 07-13-94 & 94.4 & 1,790 & 210 & 10,000 \\
\hline & $07-13-94$ & - & - & 220 & - \\
\hline & $10-19-94$ & 90.6 & 1,780 & 210 & 10,000 \\
\hline & $10-19-94$ & - & - & 220 & - \\
\hline
\end{tabular}


Table 59. Partial chemical analyses of water samples collected at selected spring and stream sites, 1994-96, Long Valley Caldera, California-Continued

\begin{tabular}{|c|c|c|c|c|c|}
\hline $\begin{array}{l}\text { Station } \\
\text { identifier }\end{array}$ & Date & $\begin{array}{l}\text { Temperature water } \\
\text { ('C) }\end{array}$ & $\begin{array}{c}\text { Specific } \\
\text { conductance } \\
(\mu \mathrm{S} / \mathrm{cm})\end{array}$ & $\begin{array}{l}\text { Chloride, } \\
\text { dissolved } \\
\text { (mg/L) }\end{array}$ & $\begin{array}{c}\text { Boron, } \\
\text { dissolved } \\
(\mu \mathrm{g} / \mathrm{L})\end{array}$ \\
\hline \multirow[t]{13}{*}{ HC6L (cont.) } & 01-18-95 & 94.2 & 1,780 & 220 & 10,000 \\
\hline & $01-18-95$ & - & - & 210 & 10,000 \\
\hline & $04-19-95$ & 94.5 & 1,763 & 220 & - \\
\hline & $07-12-95$ & 94.2 & 1,810 & - & - \\
\hline & $07-12-95$ & - & - & 220 & - \\
\hline & $10-17-95$ & 95.2 & 1,720 & 210 & 10,000 \\
\hline & $10-17-95$ & - & - & 220 & 10,000 \\
\hline & $01-25-96$ & 94.5 & 1,680 & 210 & 10,000 \\
\hline & $01-25-96$ & - & - & 220 & 10,000 \\
\hline & $04-24-96$ & 94.5 & 1,775 & 230 & 10,000 \\
\hline & $04-24-96$ & - & - & 220 & 10,000 \\
\hline & $07-17-96$ & 93.4 & 1,793 & - & - \\
\hline & $10-17-96$ & 94.1 & 1,845 & 210 & 9,490 \\
\hline \multicolumn{6}{|c|}{ Stream sites } \\
\hline \multirow[t]{30}{*}{$\mathrm{HCA}$} & $01-12-94$ & 10.5 & 231 & 6.6 & 260 \\
\hline & $01-12-94$ & - & - & 6.5 & - \\
\hline & $02-15-94$ & 11.0 & 227 & 6.6 & 240 \\
\hline & $02-15-94$ & - & - & 6.8 & - \\
\hline & $03-10-94$ & 9.0 & 237 & 7.7 & 280 \\
\hline & $03-10-94$ & - & - & 7.5 & - \\
\hline & 04-13-94 & 11.5 & 233 & 7.8 & 250 \\
\hline & $04-13-94$ & - & - & 7.2 & - \\
\hline & $05-18-94$ & 10.0 & 149 & 4.0 & 150 \\
\hline & $05-18-94$ & - & - & 3.7 & - \\
\hline & $06-21-94$ & 19.5 & 190 & 5.8 & 240 \\
\hline & $06-21-94$ & - & - & 6.2 & - \\
\hline & $07-13-94$ & 19.0 & 208 & 5.7 & 250 \\
\hline & $07-13-94$ & - & - & 5.7 & - \\
\hline & 08-09-94 & 18.5 & 219 & 5.9 & 240 \\
\hline & 08-09-94 & - & - & 6.0 & - \\
\hline & 09-14-94 & 10.5 & 230 & 6.6 & 240 \\
\hline & 09-14-94 & - & - & 6.8 & - \\
\hline & $10-20-94$ & 8.0 & 233 & 6.9 & 270 \\
\hline & $10-20-94$ & - & - & 7.2 & - \\
\hline & $01-18-95$ & 9.5 & 232 & 8.0 & 270 \\
\hline & $01-18-95$ & - & - & 8.3 & 270 \\
\hline & 04-19-95 & 10.0 & 256 & 9.4 & 310 \\
\hline & 04-19-95 & - & - & 9.9 & 270 \\
\hline & $07-12-95$ & 10.0 & 93 & 1.7 & 80 \\
\hline & $07-12-95$ & - & - & 1.8 & 80 \\
\hline & $10-17-95$ & 15.0 & 202 & 4.7 & 160 \\
\hline & $10-17-95$ & - & - & 4.7 & 180 \\
\hline & $01-25-96$ & 5.0 & 245 & 6.2 & 240 \\
\hline & $01-25-96$ & - & - & 6.7 & 290 \\
\hline
\end{tabular}


Table 59. Partial chemical analyses of water samples collected at selected spring and stream sites, 1994-96, Long Valley Caldera, California-Continued

\begin{tabular}{|c|c|c|c|c|c|}
\hline $\begin{array}{l}\text { Station } \\
\text { identifier }\end{array}$ & Date & $\begin{array}{c}\text { Temperature water } \\
\text { ('C) }\end{array}$ & $\begin{array}{c}\text { Specific } \\
\text { conductance } \\
(\mu \mathrm{S} / \mathrm{cm})\end{array}$ & $\begin{array}{l}\text { Chloride, } \\
\text { dissolved } \\
\text { (mg/L) }\end{array}$ & $\begin{array}{c}\text { Boron, } \\
\text { dissolved } \\
(\mu \mathrm{g} / \mathrm{L})\end{array}$ \\
\hline \multirow[t]{4}{*}{ HCA (cont.) } & $04-24-96$ & 10.0 & 204 & 4.6 & 170 \\
\hline & $04-24-96$ & - & - & 4.8 & 170 \\
\hline & $07-17-96$ & 14.0 & 160 & 3.3 & 154 \\
\hline & $10-17-96$ & 15.0 & 219 & 5.4 & 195 \\
\hline \multirow[t]{34}{*}{$\mathrm{HCF}$} & $01-12-94$ & 26.0 & 577 & 50 & 2,400 \\
\hline & $01-12-94$ & - & - & 53 & - \\
\hline & $02-15-94$ & 30.0 & 559 & 51 & 2,400 \\
\hline & $02-15-94$ & - & - & 52 & - \\
\hline & $03-10-94$ & 31.0 & 572 & 53 & 2,500 \\
\hline & $03-10-94$ & - & - & 53 & - \\
\hline & 04-13-94 & 31.5 & 595 & 53 & 2,400 \\
\hline & 04-13-94 & - & - & 56 & - \\
\hline & $05-18-94$ & 22.5 & 362 & 29 & 1,400 \\
\hline & $05-18-94$ & - & - & 30 & - \\
\hline & $06-21-94$ & 34.0 & 469 & 40 & 1,900 \\
\hline & $06-21-94$ & - & - & 42 & - \\
\hline & $07-13-94$ & 31.0 & 538 & 47 & 2,300 \\
\hline & $07-13-94$ & - & - & 47 & - \\
\hline & $08-09-94$ & 34.0 & 583 & 54 & 2,600 \\
\hline & 08-09-94 & 一 & 一 & 53 & - \\
\hline & $09-14-94$ & 35.0 & 615 & 55 & 2,600 \\
\hline & $09-14-94$ & - & - & 59 & - \\
\hline & $10-20-94$ & 31.0 & 619 & 59 & 2,700 \\
\hline & $10-20-94$ & - & - & 60 & - \\
\hline & $01-18-95$ & 28.5 & 608 & 56 & 2,500 \\
\hline & $01-18-95$ & - & - & 57 & 2,500 \\
\hline & $04-19-95$ & 28.0 & 514 & 42 & 2,000 \\
\hline & $04-19-95$ & - & - & 45 & 1,800 \\
\hline & $07-12-95$ & 16.0 & 152 & 8.9 & 390 \\
\hline & $07-12-95$ & - & - & 8.5 & 420 \\
\hline & $10-17-95$ & 27.5 & 411 & 30 & 1,400 \\
\hline & $10-17-95$ & - & - & 31 & 1,400 \\
\hline & $01-25-96$ & 24.5 & 568 & 50 & 2,100 \\
\hline & $01-25-96$ & - & - & 50 & 2,400 \\
\hline & $04-24-96$ & 24.2 & 443 & 37 & 1,700 \\
\hline & $04-24-96$ & - & - & 35 & 1,600 \\
\hline & $07-17-96$ & 24.5 & 333 & 23 & 1,120 \\
\hline & $10-17-96$ & 30.0 & 508 & 40 & 1,840 \\
\hline
\end{tabular}

